\title{
Plant Health 2020 Abstracts of Presentations
}

\begin{abstract}
APS Plant Health 2020 Annual Meeting held virtually, August 10-14, 2020 (including abstracts submitted for presentation at the 2020 APS Pacific Division meeting). The recommended format for citing annual meeting abstracts, using the first abstract below as an example, is as follows:
\end{abstract}

Bahri, B. A., Sapkota, S., Ali, E., Vermeer, C. B., and Martinez-Espinoza, A. D. 2020. Identification of Clareeridia species causing dollar spot in turfgrasses in Georgia, USA. (Abstr.) Phytopathology 110:S2.1. https://doi.org/10.1094/PHYTO-110-12-S2.1

The abstracts are published as a supplement to Phytopathology for citation purposes. They were not reviewed by the Phytopathology Editorial Board and were not edited by the APS editorial staff.

https://doi.org/10.1094/PHYTO-110-12-S2.1

(C) 2020 The American Phytopathological Society

Identification of Clareeridia species causing dollar spot in turfgrasses in Georgia, USA

B. A. BAHRI (1), S. Sapkota (2), E. Ali (3), C. B. Vermeer (1), A. D. Martinez-Espinoza (2), (1) University of Georgia, Griffin, GA, USA; (2) Department of Plant Pathology, University of Georgia - Griffin Campus, Griffin, GA, USA; (3) University of Georgia, Tifton, GA, USA

Dollar spot is one of the most damaging fungal diseases of turfgrass in the US. The disease is characterized by "silver dollar size" spots at the early stages of infection. Recently, four species of the genus Clarireedia were identified worldwide as causal agents of the dollar spot disease, and were differentiated based on species-specific single nucleotide polymorphisms (SNPs) in the internal transcribed spacer (ITS) region of the ribosomal DNA. Clarireedia jacksonii and C. monteithiane, were the only species described in the US, on naturally infected cool-season (C3) and warm-season (C4) turfgrasses, respectively. However, no Clarireedia species identification has been carried-out in the state of Georgia and little is known about the cross-infection potential of both species. Four isolates from symptomatic blades each collected from bermudagrass (Cynodon dactylon), creeping bentgrass (Agrostis palustris), zoysiagrass (Zoysia japonica), and seashore paspalum (Paspalum vaginatum) in Georgia in 2019, were molecularly identified. Based on the ITS sequence alignments, the isolate from creeping bentgrass had all 28 SNPs specific to $C$. jacksonii, while the other isolates had the SNPs specific to C. monteithiana. This is the first report of $C$. jacksonii and C. monteithiana pathogens on turfgrasses in the state of Georgia. In addition, under artificial inoculations and controlled conditions, $C$. jacksonii and C. monteithiana were able to cross-infect $\mathrm{C} 4$ and $\mathrm{C} 3$ turfgrasses. Further pathogenicity tests are in progress to confirm these preliminary results and identify host ranges.

Advanced molecular approaches for Clarireedia spp detection causing dollar spot of turfgrasses T. L. STACKHOUSE (1), S. Waliullah (2), B. A. Bahri (3), A. D. Martinez-Espinoza (4), E. Ali (1), (1) University of Georgia, Tifton, GA, USA; (2) University of Georgia, GA, USA; (3) University of Georgia, Griffin, GA, USA; (4) Department of Plant Pathology, University of Georgia - Griffin Campus, Griffin, GA, USA

Dollar spot is one of the most economically important diseases of turfgrass. This disease is caused by the four recently differentiated species of the Clarireedia genera--C. homoeocarpa, C. bennettii, C. jasksonii, and C. monteithiana. While C. homoeocarpa and C. bennettii are currently only found in the United Kingdom, C. jasksonii and C. monteithiana can be found worldwide, including throughout the United States. The symptoms of this pathogen include white to straw foliar lesions which can result in entire plants blighted and forming small, sunken spots on the turf, killing the grass to the soil surface. More money is spent annually on dollar spot control than any other disease on golf turf. There is a critical need to be able to identify this pathogen quickly with molecular tools to allow for control measures. This research focuses on creating novel molecular assays for identification of dollar spot. Using the previously published gene sequences of several semi-conserved regions, including rDNA internal transcribed spacer (ITS) region and calmodulin $(\mathrm{CaM})$, we have created PCR primers for identifying C. jasksonii and C. monteithiana without having cross amplification to other close related genera. Future work includes further testing of these primers against $C$. homoeocarpa and $C$. bennettii and well as 
other turfgrass pathogens and the creation of loop-mediated isothermal amplification (LAMP) primers for rapid, sensitive onsite detection. These assays would allow for faster response and therefore reduced chemical control costs to turfgrass professionals.

Development of a Nicotiana benthamiana-tomato heterograft system for understanding graft transmitted resistance M. BRANDON (1), M. Kappagantu (2), Y. Tamukong (3), J. N. Culver (2,4), (1) University of Maryland, College Park, MD, USA; (2) Institute of Bioscience and Biotechnology Research, University of Maryland, College Park, MD, USA; (3) University of Maryland College Park, College Park, MD, USA; (4) Department of Plant Science and Landscape Architecture, University of Maryland, College Park, MD, USA

The application of grafting in crop production is an established horticultural method used to impart novel forms of pathogen resistance onto susceptible cultivars. While graft associated resistance often occurs against soilborne pathogens there is growing evidence that specific graft combinations can led to resistance within the scion against foliar infecting pathogens, including viruses. To better understand this phenomenon, we developed a heterograft system to examine the molecular components involved in the transmission of resistance against tobacco mosaic virus (TMV). This system uses scions from Nicotiana benthamiana, which are normally highly susceptible to TMV infection, grafted onto a range of tomato rootstock cultivars and allowed to grow for 3-4 weeks to insure graft stability. $N$. benthamiana scions are then inoculated with TMV and monitored for symptom development and virus accumulation via western immunoblot. Results show a range of disease responses with some tomato rootstocks conferring no resistance to the $N$. benthamiana scion while others confer a high level of resistance. Ongoing studies have isolated total mRNA from $N$. benthamiana scions heterografted onto either resistance conferring or non-resistance conferring tomato rootstocks and RNAseq analysis is being used to identify transcriptional changes that occur within newly resistant $N$. benthamiana scions. Combined these studies indicated that the transmission of resistance from rootstock to scion prior to infection represents a direct impact on host - pathogen recognition and responses.

\section{Horizontal transmission of the mycovirus SsHADV-1 from strain DT-8 to US isolates of Sclerotinia sclerotiorum} M. FU (1), Z. Qu (2), D. Jiang (2), P. Miklas (3), L. D. Porter (3), G. J. Vandemark (4), W. Chen (4), (1) Washington State University, PULLMAN, WA, USA; (2) Huazhong Agricultural University, Wuhan, CHINA; (3) USDA ARS, Prosser, WA, USA; (4) USDA ARS, Pullman, WA, USA

The mycovirus Sclerotinia sclerotiorum hypovirulence-associated DNA virus 1 (SsHADV-1) was discovered in the strain DT-8 of $S$. sclerotiorum in China. In order to explore the potential use of SsHADV-1 as a white mold biocontrol agent in the United States, attempts were made to transfer the mycovirus from strain DT-8 to US isolates. The efficacy of SsHADV-1 carrying isolates in managing white mold of various crops was tested. Strain DT-8 was used as the donor and co-cultured on a PDA plate for two weeks with the recipient strain WMA1 from US. A total of 11 WMA1-derived isolates were obtained. Slower growth rates on PDA and serrated colony edges suggested presence of the mycovirus, but the observed large-sized sclerotia suggested the isolates were derived from the recipient strain. Additionally, five nuclear SSR markers were developed and definitively confirmed the origin of the derived isolates was WMA1. Finally, ten of the 11 WMA1-derived isolates were confirmed containing the mycovirus SsHADV-1 using mycovirus-specific PCR primers. In pathogenicity tests, the derived isolates carrying SsHADV-1 became hypovirulent and functioned as a biocontrol agent against white mold on dry bean. Bean seeds were treated with the fermentation culture of hypovirulent $S$. sclerotiorum DT-8 and derived isolate A1V3-1, followed by inoculation of three-week-old plants from the treated and untreated bean seeds with virulent isolate WMA1. The results showed seed treatment with hypovirulent S. sclerotiorum DT-8 or A1V3-1 increased plant resistance to white mold under the experimental conditions.

\section{Meta-analysis of fungicide performance for managing frogeye leaf spot on soybean in the United States}

J. BARRO (1), E. M. Del Ponte (1), T. Allen (2), J. P. Bond (3), T. R. Faske (4), C. A. Hollier (5), Y. R. Kandel (6), H. M. Kelly (7), N. M. Kleczewski (8), D. S. Mueller (6), P. Price (9), E. J. Sikora (10), C. A. Bradley PhD (11), (1) Departamento de Fitopatologia, Universidade Federal de Viçosa, BRAZIL; (2) Delta Research and Extension Center, Mississippi State University, Stoneville, MS, USA; (3) Southern Illinois University, Carbondale, IL, USA; (4) University of Arkansas System Division of Agriculture Cooperative Extension, Lonoke, AR, USA; (5) Louisiana State Univ Agricultural Center, Baton Rouge, LA, USA; (6) Iowa State University, Ames, IA, USA; (7) West Tennessee AgResearch and Education Center, University of Tennessee, Jackson, TN, USA; (8) University of Illinois, Urbana, IL, USA; (9) LSU AgCenter, Macon Ridge Research Station, Winnsboro, LA, USA; (10) Department of Entomology and Plant Pathology, Auburn University, Auburn, AL, USA; (11) University of Kentucky, Princeton, KY, USA

Frogeye leaf spot (FLS), caused by Cercospora sojina, is a yield-reducing disease of soybean. Foliar fungicides have been applied for managing FLS and protecting yield. To help define the best options and the economic benefits from fungicide use, multi-state trials were established to evaluate fungicides for FLS management. Data from 41 trials conducted between 2015 and 2019 across eight states were gathered. The main goal of this study was to summarize and explore factors affecting efficacy and profitability of the following fungicides applied at the beginning pod development stage: azoxystrobin + difenoconazole (AZOX+DIFE), fluoxastrobin + flutriafol (FLUO+FLUT), pyraclostrobin (PYRA), pyraclostrobin + fluxapyroxad + propiconazole (PYRA+FLUX+PROP), tetraconazole (TTRA), thiophanate-methyl (TMET), thiophanate-methyl + tebuconazole (TMET+TEBU), and trifloxystrobin + prothioconazole (TFLX+PROT). A network meta-analytical model was fitted to the log transformed FLS severity mean and to the non-transformed yield mean for each treatment, including the control. The percent reduction in disease severity relative to the control ranged from $13.5 \%$ (PYRA) to 52.8\% (TMET+TEBU); the latter not differing from FLUO+FLUT (51.5\%), TFLX+PROT (50.4\%), AZOX+DIFE (50.3\%) and TMET (49.2\%). The mean yield response was greater for AZOX+DIFE (388.2 kg/ha), FLUO+FLUT (383.7 kg/ha), TFLX+PROT (379.4 kg/ha), TMET+TEBU (368.3 kg/ha), TMET (359.0 kg/ha), PYRA+FLUX+PROP (335.9 kg/ha), and TTRA (331.0 kg/ha) all performing better than PYRA (160.1 kg/ha). 
Influence of cultural practices and soybean maturity group on soil and root microbial community composition II: The bacterial perspective

A. Y. BANDARA, D. K. Weerasooriya, R. Trexler, T. Bell, P. Esker, The Pennsylvania State University, University Park, PA, USA

Cultural practices are integral to plant disease control. They can affect soil/plant health by modifying microbial structure. Soybean roots (R), rhizosphere soil (RS), and bulk soil (BS) were sampled at the V1 (one trifoliate) growth stage from 14 farms in Pennsylvania. Farms had diverse cropping histories related to cultural practices. DNA extracted from samples was subjected to PCR targeting 16S rRNA genes $(515 \mathrm{~F} / 806 \mathrm{R})$ and metabarcoded using the MiSeq system. Samples were tagged using total read count and cultural practices and analyzed using factor analysis of mixed data. Crop rotation and cover crops contributed the most to variation among BS samples while variation among RS and R samples were largely explained by soybean maturity group and crop rotation. Corn-soybean-corn (CSC) rotation increased the relative abundance of Kaistobacter and Flavobacterium while decreasing Streptomyces and Rhodoplanes in BS and RS samples compared to non-CSC rotations. Non-CSC rotations increased Streptomyces and Bradyrhizobium and decreased Flavobacterium in roots. Cover crops decreased the abundance of Bradyrhizobium, Flavobacterium, and Rhodoplanes in BS. RS from maturity group two (MG2) soybean had lower \% of Flavobacterium, Kaistobacter and greater \% of Bradyrhizobium compared to late maturity group three (MGL3). R from MG2 contained greater \% of Streptomyces, Flavobacterium, and lower \% of Bradyrhizobium compared to MGL3. Our study revealed the comparative impact of cultural practices on the relative abundance of important bacterial genera in soil and soybean roots.

Transcriptome analysis of grapevine powdery mildew to elucidate the molecular basis of resistance to metrafenone A. KUNOVA (1), A. Perniola (2), L. E. Cadle-Davidson (3), P. Cortesi (1), (1) DeFENS, Università degli Studi di Milano, Milano, ITALY; (2) DeFENS, Università degli studi di Milano, Milano, ITALY; (3) USDA Grape Genetics Research Unit, Geneva, NY, USA

Grapevine powdery mildew, caused by Erysiphe necator, is a serious disease widespread in all grape growing areas. The pathogen can infect all green parts of the plant, causing the major economic damage on bunches. Metrafenone is a valuable fungicide used in the powdery mildew disease management since 2006 especially in rotation with other chemistries on grapevine and cereals. Its exact mechanism of action is still not known, but based on cross-resistance studies it is different from other fungicides used in powdery mildew management. Early studies on barley and wheat powdery mildews suggest that it may interfere with hyphal morphogenesis, polarised hyphal growth and the establishment and maintenance of cell polarity. Obtaining RNA of a good quality and sufficient quantity from obligate plant pathogens like powdery mildews can be problematic especially due to their tight adherence to the host plant epidermis and low mycelium availability at early developmental stages. Here, we searched for the best methodology for tissue collection and the RNA extraction with the aim to perform RNA transcriptome analysis to compare the expression of $E$. necator strains sensitive and resistant to metrafenone, identified previously by our research group. The data of gene expression from these different treatments will be compared to identify possible biosynthetic pathways/enzymatic targets involved in the interaction with metrafenone.

Characterization of host resistance in strawberry to powdery mildew caused by Podosphaera aphanis

M. PALMER (1), G. J. Holmes (2), K. Blauer (3), (1) California Polytechnic State University, San Luis Obispo, CA, USA; (2) Strawberry Center, California Polytechnic State University, San Luis Obispo, CA, USA; (3) Cal Poly Strawberry Center, San Luis Obispo, CA, USA

Host resistance is an essential tool in plant disease management worldwide. Evaluations of strawberry cultivar resistance to powdery mildew (PM) have been done previously, but many new cultivars have been released and merit evaluation for today's growers. This study characterizes the level of host resistance to PM in 12 commonly grown cultivars from 4 breeding programs in California. PM-free plants were established in 6-inch pots under high plastic tunnels and moved after three weeks into a greenhouse where an active PM epidemic was present. These plants were at the 4- to 5-leaf stage and showed no visible symptoms or signs of PM. Evaluations began after 3 weeks when the first visible symptoms were observed. Plants were evaluated weekly in the greenhouse for disease incidence (\%) and severity (\%) over a 7-week period. Significant differences were found in disease incidence and severity among cultivars but none were fully resistant. The most resistant cultivar was Sweet Ann with only $11 \%$ of leaves showing visible symptoms of infection after 10 weeks of exposure. More susceptible cultivars include Royal Royce and BG 3.324 with 53\% and 54\% incidence at 10 weeks, respectively. This information is valuable to strawberry growers who select cultivars based in part on their susceptibility to economically important diseases.

Understanding bacterial wilt resistance in transgenic tomato plants expressing the $\boldsymbol{E F R}$ Pattern Recognition Receptor S. KUNWAR (1), M. L. Paret (2), S. Hutton (3), C. Allen (1), (1) University of Wisconsin-Madison, Madison, WI, USA; (2) North Florida Research \& Education Center, University of Florida, Quincy, FL, USA; (3) University of Florida, Wimauma, FL, USA

Transgenic expression of the Arabidopsis EFR pattern recognition receptor increases tomato resistance to bacterial wilt (BW) caused by the Ralstonia solanacearum species complex (RSSC). The mechanism(s) of EFR resistance are unknown. To determine if EFR plants show fewer symptoms because they suppress bacterial colonization, we measured pathogen population sizes and distributions in infected tomato stems. EFR plants contained $10^{4}$ fewer bacteria than near-isogenic controls five days after inoculation, and monitoring distribution of GFP-expressing bacteria revealed reduced radial colonization of $E F R$ stems. This indicates $E F R$ plants resist BW in part by restricting bacterial colonization and movement. We explored the tissue specificity of $E F R$ resistance using reciprocal-graft experiments with $E F R$ rootstocks grafted onto susceptible scions and vice-versa. Although root genotype was key to EFR resistance, bacterial growth and spread was also restricted in $E F R$ stems grafted onto susceptible rootstocks. The RSSC is a heterogenous group and BW resistance can be strain specific. We therefore used a naturalistic soil-soak disease assay to evaluate resistance of $E F R$ tomatoes to RSSC strains isolated from around the world. EFR plants resisted most tested strains but were completely susceptible to an African 
phylotype III strain. The discovery of a highly virulent natural variant that overcomes $E F R$ suggests that other RSSC strains may also infect $E F R$ plants. Thorough screening against endemic RSSC strains is thus necessary before deploying EFR across a wide geographical area.

Deposition pattern of charged and uncharged droplets on field grown Vitis vinifera differ based on tissue age M. MCCOY (1), G. Hoheisel (2), M. M. Moyer (1), (1) Washington State University, Prosser, WA, USA; (2) Washington State University Extension, Prosser, WA, USA

New sprayer technologies used in vineyards have limited information on in-field performance. This information is critical for appropriate use and optimal performance of these technologies. One such technology is electrostatic sprayers, which electrically charge the surface of spray droplets with the intent of improving deposition in otherwise drift-prone situations. Electrostatic performance in-lab can play out differently than in-vineyard. In Washington state, many vineyard sites are windy, and the climate is generally arid with high mid-season temperatures. This can result in evaporation of small spray droplets (30-50 microns), which are common in electrostatic spray technologies. In-field trials evaluated spray deposition patterns on leaves based on their age (i.e., the transition from a source organ to a sink organ) when sprayed with and without an electrostatic charge. There was increased deposition on source and sink organ tissue with charged spray as compared to without charge spray $(\mathrm{p}=0.0004$ at $\alpha=0.05)$, as to be expected with this technology. Deposition was highest on source organ tissue compared to sink organ tissue, regardless of spray with or without charge (Tukey HSD, $P$ $<0.05)$. Understanding how tissue age interplays with charge or leaf structure is important when utilizing a sprayer technology like electrostatic since it can assist in appropriate use, thus amplifying the usage of products being applied at a critical period of plant protection.

Characterizing the distribution of two onion virulence loci among a diverse Pantoea isolate collection S. STICE (1), B. H. Kvitko (1), B. Dutta (2), (1) University of Georgia, Athens, GA, USA; (2) University of Georgia, Tifton, GA, USA

Pantoea ananatis is a gram-negative bacterium and the primary causative agent of center-rot disease of onions in Georgia. Previous genomic studies identified two virulence loci, HiVir and alt, associated with center-rot disease. The HiVir locus induces necrosis on onion tissues with a predicted small molecule toxin. The alt locus aids in tolerance to thiosulfinates generated during onion tissue damage. WGS of other Pantoea isolates indicate that these loci are distributed beyond P. ananatis. To assess the distribution of these loci, and their accuracy in predicting associated phenotypes, two PCR primer sets were designed to detect the presence of HiVir and alt separately. Two hundred fifty nine isolates were phenotyped using the red-onion scale necrosis assay (ROA) and PCR tested for the presence of these genomic targets. A diverse panel of isolates from the Pantoea genus ( 24 species, 41 isolation sources, 23 countries, 1946-2018) were tested. ROA positive isolates (74) tested positive for HiVir (25.7\%), alt (16.2\%), both (52.7\%), or neither (5.4\%). ROA negative isolates (185) tested positive for HiVir (4.9\%), alt (31.9\%), both (0.5\%), or neither (62.7\%). HiVir was detected in: $P$. ananatis, $P$. stewartii subsp. indologens, and $P$. allii. Other Pantoea species tested positive for necrosis and negative for the HiVir primer set indicating a divergent HiVir cluster or a different pathogenicity mechanism. Future work will improve primary accuracy and develop a diagnostic assay workflow.

\section{A preliminary genome-wide association study of Fusarium head blight resistance in soft winter wheat in the southeast United States}

B. GHIMIRE (1), M. Mergoum (2,3), S. Sapkota (1,3), J. Youmans (1), S. Pradhan (4), M. A. Babar (4), A. D. Martinez-Espinoza (1), J. W. Buck (1), (1) Department of Plant Pathology, University of Georgia - Griffin Campus, Griffin, GA, USA; (2) Department of Crop and Soil Sciences, University of Georgia - Griffin Campus, Griffin, GA, USA; (3) Institute of Plant Breeding, Genetics, and Genomics, University of Georgia, Griffin, GA, USA; (4) Department of Agronomy, University of Florida, Gainsville, FL, USA

In recent years, wheat production in the southeast United States has been challenged by Fusarium head blight (FHB) that reduces both grain yield and quality due to harmful mycotoxin contamination. The objective of the current study was to identify and characterize the novel quantitative trait loci (QTL) associated with FHB host resistance types. A genome-wide association study (GWAS) was conducted in a 236 soft winter wheat association mapping panel using 27,466 single nucleotide polymorphisms (SNPs) obtained from genotyping-by-sequencing. The panel was phenotyped for seven traits including disease incidence, disease severity, FHB index, Fusarium-damaged kernel, deoxynivalenol, thousand kernel weight, and days to heading in two environments (greenhouse and field) in $2018 / 2019$. A total of 17 significant marker-trait associations (MTAs) were identified at the uniform cut off value of $-\log _{10} \mathrm{p}=4.00$ to all seven traits with 14 MTAs associated with active FHB resistance types alone across wheat chromosomes 2A, 2B, 3B, 3D, 4A, 4B, and 7A. The experiment is being currently repeated in both environments to collect additional data in order to identify novel QTL for FHB resistance. Subsequently, validation of these QTL is essential to use these valuable genetic sources to enhance FHB resistance in wheat breeding programs.

\section{What is needed to succeed in xylem? Comparative analysis of multiple in planta transcriptomic datasets of Ralstonia solanacearum}

C. HENDRICH (1), A. Truchon (2), C. Allen (1), (1) University of Wisconsin-Madison, Madison, WI, USA; (2) University of Wisconsin, Madison, WI, USA

Ralstonia solanacearum $(R s)$ causes bacterial wilt disease on a wide variety of plant hosts, including tomato, potato, and banana. $R s$ infects through roots and thrives in the low-oxygen, low-nutrient xylem fluid, where it grows to high densities. However, xylem tissue is 
difficult to access and experimentally manipulate, limiting insights into Rs biology inside its hosts. To obtain a bacterial perspective on life in xylem, the $R s$ transcriptome has been assessed during host infection under diverse conditions. To take advantage of this wealth of expression data, we aggregated multiple published transcriptomes, including recent data comparing $R s$ gene expression in tomato stems to that of $R s$ growing hypoxically in a nitrate-rich minimal medium designed to mimic conditions in planta. Comparing these datasets identified functions that are highly expressed in planta in multiple hosts and $R s$ strains. This increased our confidence that these traits, which include denitrifying respiration and stress tolerance, are vital during infection. We are mining this dataset to answer key questions such as: What nutrient sources are most important for $R s$ during infection, and what stressors challenge xylem-inhabiting bacteria? We found that $R s$ gene expression in culture is dramatically different from that of the pathogen growing in the dynamic and incompletely understood host xylem environment, even in culture conditions intended to mimic plant xylem. Comparing the pathogen's transcriptomic profiles across diverse hosts, tissues, and disease stages may be a better way to understand $R s$ biology.

Trichoderma cell-free culture filtrates effects on Fusarium virguliforme conidia germination M. FILGUEIRA PIMENTEL, A. Subedi, J. P. Bond, A. M. Fakhoury, Southern Illinois University, Carbondale, IL, USA

Fusarium virguliforme, the causal agent of sudden death syndrome (SDS), is a major threat to soybean in North America due to its aggressiveness. Our previous work demonstrated that some Trichoderma spp. isolates are able to suppress $F$. virguliforme growth and reduce SDS symptoms through a combination of mechanisms. Exploring the interaction between these Trichoderma isolates and $F$. virguliforme is important to optimize their efficacy and develop biocontrol tools that could be included in SDS management recommendations. The current study aimed to evaluate the effect of five Trichoderma spp. isolates cell-free culture filtrates on $F$. virguliforme conidia germination. Assays were conducted using 50-well silicone cover slips on a glass slide. Each well received $3 \mu 1$ of a suspension containing $F$. virguliforme conidia diluted in autoclaved and non-autoclaved Trichoderma filtrates. Conidia germination was observed 12,24 , and $48 \mathrm{~h}$ after adding the filtrate. Over ninety percent inhibition of $F$. virguliforme conidia germination was noted with most Trichoderma isolates after 24 and $48 \mathrm{~h}$ of incubation. Filtrates from a T. asperellum isolate also caused visual deformation of conidia $12 \mathrm{~h}$ after incubation. The antibiosis effects of Trichoderma filtrates on $F$. virguliforme conidia were evident through significantly reduced germination and reduced viability. Further efforts could be focused on the identification and characterization of the proteins and/or metabolites causing inhibition of $F$. virguliforme conidia germination.

Transcriptional responses to interactions among arbuscular mycorrhizal fungi, soybean cyst nematode, and soybean M. Willman (1), L. Taylor (1), Y. Isack (2), D. Levy (2), M. S. BENITEZ PONCE (1), (1) The Ohio State University, Wooster, OH, USA; (2) Groundwork BioAg, ISRAEL

The microbial inoculant industry is on the rise as it provides alternative or complementary plant health management solutions. To predict success of microbial inoculants, research is needed on mechanisms of action. The objective of this study was to characterize the three-way interaction among soybean, soybean cyst nematode (SCN), and arbuscular mycorrhizal fungi (AM). Seedlings were grown with a combination of SCN and one of two AM species in a greenhouse randomized complete block design. Plants were evaluated for biomass, SCN infestation, and AM root colonization. In addition, a subset of roots was sampled for RNA sequencing to evaluate transcriptional responses of the different members of this interaction. We observed a trend of greater seedling biomass upon AM inoculation, depending on AM mode of application. Also, AM effect on soybean biomass depends on AM species and soybean genotype; and the magnitude of AM impact depends on SCN presence. Further, RNA reads were mapped to representative genomes and transcriptional profiles consisting of 49,524, 14,299 and 12,335 genes were recovered for each soybean, SCN and AM, respectively. In addition, we identified differentially expressed genes (DEGs) between treatments. Specifically, for soybean, changes in RNA sequencing profiles were distinct between AM or SCN colonized roots; however, when colonized by AM, we observed a smaller number of DEGs between profiles of SCN infested and non-infested roots. These results allow to begin teasing out interactions that can contribute to crop responses to AM inocula upon SCN infection.

\section{Development and validation of specific primer-probes for the detection and quantification of two Meloidogyne species using} multiplex quantitative PCR

M. SOULE (1), A. Peetz (2), I. A. Zasada (3), (1) Oregon State University, Corvallis, OR, USA; (2) Revolution Crop Consultants, Albany, OR, USA; (3) USDA ARS, Corvallis, OR, USA

In the Pacific Northwest (OR, WA, and ID) the two most common root-knot nematodes are Meloidogyne hapla and Meloidogyne chitwoodi. Of these, $M$. chitwoodi poses a significant threat to the $\$ 1.8$ billion potato industry in the region. With the ability to rapidly proliferate, infestations of $M$. chitwoodi are devastating even when initial densities are low. The potato industry has zero tolerance for the pimple-like galling that the nematode causes and detection in just $6 \%$ of tubers can result in total crop rejection. In contrast, $M$. hapla is not as aggressive on potato, thus there is a higher tolerance for its presence in potato fields. To inform pest management decisions, growers rely on information from disease diagnostic clinics. Currently, clinics use labor-intensive microscopy to identify nematodes based on morphology. A faster, more reliable method is using quantitative PCR (qPCR) to identify nematodes based on genetic markers. To efficiently identify and quantify Meloidogyne species, existing primer-probes targeting the internal transcribed spacer (ITS) region were tested for viability and then adapted for use in multiplex PCR. Primers were validated using eggs and second-stage juveniles (J2) first using conventional PCR, then in qPCR at 1, 10, 50, 100, 250, 500, and 1,000 J2 per reaction for each species to generate standard curves, and finally in multiplex to confirm simultaneous detection and quantification. This effective, lowcost assay can improve IPM by allowing targeted management decisions that are proportional to infestation severity and Meloidogyne species. 
Discovery of negative sense RNA viruses in grapevine via high throughput sequencing

A. Diaz-Lara (1), K. Stevens (2), M. Hwang (3), D. A. Golino (3), M. AL RWAHNIH PHD (4), (1) University of California-Davis, Davis, CA, USA; (2) Department of Evolution and Ecology, University of California-Davis, Davis, CA, USA; (3) University of California, Davis, CA, USA; (4) University of California-Davis/FPS, Davis, CA, USA

Relatively few negative sense (ns)RNA viruses have been associated with infection in plants, including agricultural crops. However, in the last few years, the use of high throughput sequencing (HTS) has allowed the identification of new nsRNA viruses in plants. For example, the first nsRNA viruses identified and transmitted in grapevine, Grapevine Muscat rose virus (GMRV) and grapevine Garan dmak virus (GGDV), were only recently discovered because of HTS. The genomes of both viruses were comprised of three segments each containing a unique gene: RNA-dependent RNA polymerase (RdRp), nucleocapsid protein (NP) and movement protein (MP). Based on sequence identity and phylogenetic analysis, GMRV and GGDV represent new members of the family Phenuiviridae; most phenuiviruses are linked to diseases in vertebrates and are vectored by arthropods. Similarly, HTS was used to characterize nsRNA viruses in apple and citrus associated with different diseases. The discovery of these two nsRNA viruses in grapevine and the parallel report of additional viruses with this type of genetic material in other perennial crops suggests a more extensive distribution. Consequently, the characterization of nsRNA viruses in plants via HTS will remain an active area of research for some time.

Quantification of the total population of Candidatus Liberibacter asiaticus inside citrus trees F. NOGALES C. VASCONCELOS, N. Collins, J. Li, N. Wang, Citrus Research and Education Center, University of Florida, Lake Alfred, FL, USA

Huanglongbing is the most devastating citrus disease worldwide. The causal agent of HLB Candidatus Liberibacter asiaticus (Las) colonizes the phloem and is naturally transmitted by Asian citrus psyllid Diaphorina citri. Because Las has not been cultured in artificial media, the virulence mechanism of Las remains poorly understood. The symptom development caused by Las is relatively slow compared to most bacterial pathogens infecting the intercellular spaces. One hypothesis of the Las virulence mechanism is that it causes an overwhelming burden for the metabolism of the plant host. Here we used qPCR to quantify Las from entire trees and calculated the ratio of citrus cells to Las cells in different tissues. Our data showed a much lower total Las population inside a tree than non-phloem-colonizing bacterial pathogens. We also observed tissues with high bacterial titers. Our results shed light on the biology and virulence mechanism of Las.

Clade-specific RXLR effectorome of the cucurbit downy mildew pathogen Pseudoperonospora cubensis S. PURAYANNUR (1), L. M. Cano (2,3), M. Bowman (4,5), K. L. Childs (5), L. M. Quesada-Ocampo (3), (1) North Carolina State University, Raleigh, NC, USA; (2) University of Florida, UF/IFAS, IRREC, Plant Pathology, Fort Pierce, FL, USA; (3) Department of Entomology and Plant Pathology, North Carolina State University, Raleigh, NC, USA; (4) Ball Horticultural Company, West Chicago, IL, USA; (5) Department of Plant Biology, Michigan State University, East Lansing, MI, USA

The cucurbit downy mildew pathogen Pseudoperonospora cubensis causes significant crop losses every year ever since its reemergence in 2004. There are two distinct host-specific clades in P. cubensis, Clade 1 and Clade 2; the introduction of Clade 2 is thought to have enabled the reemergence of the pathogen. In an attempt to identify clade-specific effectors of $P$. cubensis, we predicted the secretome of one representative isolate each of Clade 1 and Clade 2 . There were $\sim 1,000$ secreted proteins in both clades. Using pattern search and sequence similarity analysis in the R package effectR and a Galaxy toolshed designed for oomycete effectors, 200 genes encoding RXLR motif-containing effectors were predicted. Orthology analysis revealed core effectors common to both clades, and effectors that were specific to each clade. RNA-seq analysis in the sporangia of selected Clade 1 and Clade 2 isolates showed varying transcript evidence for 2 effector-encoding genes. An RNA-seq time-course experiment with samples collected at various points of infection revealed 8 effectors that showed varying expressions in the two clades. Differential expression of these effectors was validated through RT-qPCR. A selected set of differentially expressed candidate effectors is being further functionally characterized for their virulence/avirulence activity using bioassays in the host. These candidate effectors could be used for effector-assisted breeding to identify new sources of resistance to downy mildew in cucurbits.

Searching for a solution to a major challenge to sesame production: Screening sesame varieties for resistance to Macrophomina phaseolina

K. A. COCHRAN, Texas A\&M University, Uvalde, TX, USA

Charcoal rot, caused by Macrophomina phaseolina, has presented a challenge to sesame production in the United States and globally, particularly when grown as a dry land crop. Symptoms include stunting, defoliation, reduction in yield, and possibly plant death. In the southwest Texas area, production losses have been reported to be over $30 \%$ in severely affected fields. This pathogen is particularly difficult to manage due to its soilborne nature and longevity in the soil. Additionally, the often utilized cultural control method of supplemental irrigation is not always appropriate for drought tolerant crops such as sesame. The objective of this work was to screen sesame varieties for genetic resistance to M. phaseolina. Fifty varieties were selected for analysis. Plants were established and inoculated with M. phaseolina grown on PDA at 35 dap using a cut stem method. Of the varieties analyzed, 9 had average stem symptom progression of less than $5 \%$ of the total stem length, while varieties thought to be relatively susceptible in other research efforts had symptom progression of over $60 \%$ of the stem length. With additional development, this research can help provide a valuable tool for managing charcoal rot in an IPM program for sesame producers to manage nationally and abroad. 
Transcriptional response of watermelon during its compatible and incompatible interaction with Fusarium oxysporum f. sp. niveum

A. PETKAR, B. Dutta, P. Ji, University of Georgia, Tifton, GA, USA

Fusarium wilt is one of the most economically important diseases of watermelon and is caused by a soil-borne fungal pathogen Fusarium oxysporum f. sp. niveum (FON). Limited options are available for its management. The most effective approach for managing the disease is the use of resistant cultivars; however, they are not available for all pathogenic FON races. Improving our understanding of the molecular mechanisms that confer resistance to FON would facilitate development of resistant watermelon cultivars. In this study, we used RNA-sequencing approach to profile the transcriptome of compatible (cv. Calhoun Gray) and incompatible (accession PI-296341-FR) interactions of watermelon seedlings with FON race 2 over a time course of 1, 3, 5 and 7 days post inoculation. A total of 4549 genes were identified in watermelon as differentially expressed genes (DEGs). Further analysis revealed that many of these DEGs are involved in biosynthesis of amino acids, plant-pathogen interactions, phenlypropanoid biosynthesis pathways, MAPK signaling pathway and plant hormone signal transduction, suggesting that innate immunity, phytochrome signaling and numerous phenlypropanoid compounds responsible for complex defense network in plants are involved in interactions of watermelon and FON. This study provides new insights in understanding the molecular mechanisms involved in response of watermelon plant to FON infection, which can potentially be a valuable tool for development and genetic improvement of watermelon cultivars.

\section{Metagenomic sequencing as a diagnostic tool for Xylella fastidiosa diseases such as olive quick decline syndrome and Pierce's disease of grapes \\ V. ROMAN-REYNA (1,2), E. Dupas (3), S. Cesbron (3), G. Marchi (4), M. A. Hansen (5), E. A. Bush (5), F. Rotondo (6), M. L. Lewis Ivey (6), S. A. Miller (6), F. Peduto Hand PhD (7), M. A. Jacques (3), J. M. Jacobs (1,2), (1) Infectious Diseases Institute, The Ohio State University, Columbus, OH, USA; (2) Department of Plant Pathology, The Ohio State University, Columbus, OH, USA; (3) IRHS, Agrocampus-Ouest, INRAE, Université d'Angers, Beaucouzé, FRANCE; (4) DAGRI, Universita degli Studi, Firenze, ITALY; (5) School of Plant and Environmental Sciences, Virginia Tech, Blacksburg, VA, USA; (6) Department of Plant Pathology, The Ohio State University, Wooster, OH, USA; (7) Ohio State University, Columbus, OH, USA}

The plant pathogenic bacterium Xylella fastidiosa causes Pierce's disease of grapevine and olive quick decline syndrome. Devastating $X$. fastidiosa $(X f)$ outbreaks in Europe on olive highlight this pathogen's capacity to expand its host range and geographical distribution. Primary control strategy for $X f$ diseases is whole crop eradication; therefore, faster and accurate detection tools are necessary to prevent pathogen dispersion. The current diagnostic approach requires amplification of one or two different $X f$ genome regions, but it does not consider new or emerging variants. To remove biases towards known sequences, we tested low-cost, user-friendly metagenomic nextgeneration sequencing as a molecular tool for $X f$ detection and diversity analysis. As a proof of concept, we identified $X f$ to strain level in artificially single- and mixed-infected plant samples at concentrations as low as one picogram per gram of tissue. We then tested naturally infected extracted DNA samples from France, Italy and the United States. We successfully identified $X f$ subspecies to subgroups in all samples. We classified these reads into core and unique $X f$ sequences based on average nucleotide identity variation. Interestingly, we detected $X f$ reads from samples that had tested negative with real-time PCR. We also used this approach to detect $X f$ in Italian mediterranean shrubs, which are considered potential pathogen reservoirs. Overall, we developed a diagnostics pipeline that could be easily extended to other pathogens and implemented for emerging plant threat surveillance.

Old virus new host: Defining the disease cycle of Alfalfa mosaic virus in peppers T. ALBRECHT (1), M. Bartolo (2), W. S. Cranshaw (1), P. Nachappa (1), (1) Colorado State University, Fort Collins, CO, USA; (2) Colorado State University, Rocky Ford, CO, USA

Alfalfa mosaic virus (AMV) (Alfamovirus, Bromoviridae) causes disease in over 50 plant families including several economically important crops such as alfalfa, potato and soybean. The virus can be transmitted mechanically, through seed and vectored by at least 15 species of aphids. In a test plot of peppers located at the Arkansas Valley Research Center in Rocky Ford, Colorado, USA, we observed virus symptoms including leaf deformation, mosaic and chlorosis on several pepper (Capsicum sp.) species and eggplant (Solanum melongena). In 2018, we tested both asymptomatic and symptomatic plant tissues for presence AMV from several pepper varieties, eggplant, alfalfa (Medicago sativa) and pea aphids (Acyrthosiphon pisum). Symptomatic plant tissue and multiple pea aphid samples were positive for AMV using both serological and molecular testing methods. Sequencing and mechanical inoculation were used to validate testing results. Peppers are a new host for AMV and studies on the impact and disease cycle in this crop are lacking. Assessment of varietal susceptibility and seed transmission is of primary importance to commercial pepper production. In 2019, a pepper variety trial was analyzed to identify varieties with tolerance to AMV by visual assessment of AMV symptoms, quantification of AMV in tissue and yield measurements. To assess the seed transmission rate of AMV in peppers, seed was collected from mechanically inoculated peppers and tested for AMV using different methods. These data will inform management strategies for AMV in this new host.

Seasonal variability in the titer and tissue distribution of Tobacco ringspot virus infecting plants from Malus, Prunus, and Vitis genera E. BEAVER-KANUYA, S. J. Harper, Washington State University, Prosser, WA, USA

Tobacco ringspot virus (TRSV), a family member of genus Nepovirus, subgroup A, infects both herbaceous and woody plants including Malus (apple), Prunus (cherries), and Vitis (grapes). TRSV is primarily transmitted by nematodes (Xiphinema species), but other invertebrates, seeds, and pollen are all potential vectors. TRSV detection is complicated by seasonal fluctuations in the titer and distribution of the virus. To study this phenomenon, we developed an RT-qPCR assay for the detection of TRSV. This RT-qPCR assay 
showed higher sensitivity and specificity than extant end-point assays and showed no host-reactivity. We used this assay to study titer and distribution within three different tissues (leaves, stems, and roots) from apple, grape, and cherry to evaluate the best time and tissue to sample for accurate detection. Preliminary results showed that the titer distribution not only fluctuated with seasonal patterns and within plant tissues, but also varied among hosts. At the end of summer/beginning of fall (August - November), the titer stabilized on leaves for all three hosts. At leaf drop in November, titer fluctuations in stems and roots were more pronounced, resulting in minimal or no detection at some points. After the dormancy period (January-February), virus titer was equal within all three tissues from grapes, while for apples the concentration remained higher on stems than roots and leaves. These data suggest that TRSV titer varies considerably throughout the year and that time and tissue selection need to be considered for accurate detection.

\section{Shading alters foliar symptom expression caused by Sudden Death Syndrome of soybean J. E. KURLE, G. M. Anderson, U of MN, Saint Paul, MN, USA}

Sudden Death Syndrome (SDS) caused by Fusarium virguliforme (Fv) is a damaging fungal soybean disease occurring in Minnesota. Varietal resistance is the most effective means of limiting losses to SDS; however. resistance phenotypes in field and controlled environments are frequently not correlated. In earlier greenhouse research, we had observed that results differed when experiments were conducted at different times of the year or in different greenhouses. In Minnesota summer sunlight intensity is ten times that occurring in winter. Supplemental lighting in greenhouses does not compensate for the difference. Light intensity has been reported to affect resistance phenotypes in a number of soybean diseases. Our objectives were to determine; 1) the effect of light intensity on the apparent resistance of soybean varieties, and 2) if the effect of light intensity interacted with the method used to determine disease resistance, root rot or foliar symptom severity. In this greenhouse experiment, five varieties, resistant, moderately resistant, and susceptible soybean varieties were evaluated for their response to light of differing intensities. Phenotypes evaluated included foliar symptom expression and root rot severity. Light intensity manipulated by shading soybean plants under shadecloth reduced light by 0,40 , and $70 \%$. Phenotypic differences, root and foliar, observed among varieties were substantial and ranking of a variety phenotypes, either root or foliar, relative to other varieties changed significantly as light intensity was altered.

Genetic population structure and trichothecene genotype composition of Fusarium graminearum populations causing Fusarium head blight in Pennsylvania

M. R. DUFFECK (1,2), A. Y. Bandara (3), D. K. Weerasooriya (3), E. M. Del Ponte (4), P. Esker (3), (1) Penn State University, State College, PA, USA; (2) Departamento de Fitopatologia, Universidade Federal de Viçosa, Viçosa, BRAZIL; (3) The Pennsylvania State University, University Park, PA, USA; (4) Departamento de Fitopatologia, Universidade Federal de Viçosa, BRAZIL

Fusarium head blight (FHB or scab) is the major head disease that impacts yield and grain quality of small grains in Pennsylvania (PA), mainly due to accumulation of the mycotoxin deoxynivalenol (DON). A native population of Fusarium graminearum sensu stricto (Fg) with the 15-ADON genotype has been historically responsible for FHB in North America. Although several studies have reported the temporal and spatial dynamics of the Fg population in the U.S., there is a lack of information regarding the trichothecene genotype composition, genetic diversity, and population structure of the Fg population causing FHB in PA. During Summer 2018 and Summer 2019, naturally FHB-infected spikes of different small grain hosts (wheat, barley, spelt, rye) were collected across PA using a standardized sampling procedure. A total of 349 Fusarium spp. isolates were recovered from these spikes and were morphologically identified as $F$. graminearum sensu lato. Trichothecene genotypes (3-ADON, 15-ADON, and NIV) were characterized for all isolates through multiplex PCR assays targeting portions of the Tri3 and Tri12 genes. From the 349 isolates, 94.5, 4.5, and 1\% belonged to 15ADON, 3-ADON, and NIV genotypes, respectively. Results revealed that the Fg population in PA is predominantly represented by the $15-A D O N$ genotype. Current work is focused on investigating the genetic diversity and population structure of a subset of Fg isolates using eight VNTR markers. Overall, findings will help to streamline FHB control strategies in PA.

\section{Screening of cultural media for sporulation of Tilletia barclayana}

L. WANG (1,2), E. Nysetvold (2), X. G. Zhou (3), (1) Meishan Vocational \& Technical College, BEAUMONT, CHINA; (2) Texas A\&M AgriLife Research Center, BEAUMONT, TX, USA; (3) Texas A\&M AgriLife Research Center, Beaumont, TX, USA

Rice Kernel smut, caused by Tilletia barclayana (also known as Neovossia horrida), is an important fungal disease in rice worldwide. It can cause considerable losses of yield and milling quality under conducive conditions. Having a cultural medium able to stimulate the sporulation of the fungus is desirable for in vitro, greenhouse and field studies. However, no detailed research has been conducted to develop optimal cultural media for the growth and sporulation of T. barclayana. In this research, in vitro studies were conducted using four isolates of the fungus to evaluate various agar media, carbon sources, and incubation times on the growth and sporulation of the fungus. Among the six cultural media evaluated, rice bran medium and potato D-sorbitol agar were the best two media producing secondary sporidia of up to $4 \times 10^{6}$ sporidia/mL while potato dextrose agar (PDA) produced the least number of secondary sporidia $(<2$ $\times 10^{5}$ sporidia/mL). PDA and PSA (potato sugar agar) were the best two media for stimulating mycelial growth compared to the others. Carbon source (sugar, dextrose and D-sorbitol) significantly affected the sporulation of the fungus but not the mycelium growth. Incubation at $28^{\circ} \mathrm{C}$ for 2 weeks produced greatest sporidia than the other incubation treatments of 1,3 and 4 weeks.

Multigene based TaqMan qPCR multiplex assay for sensitive and reliable detection of Dickeya solani S. DOBHAL (1), G. Santillana (2), M. J. Stulberg (2), G. Boluk (1), J. Rascoe (2), M. K. Nakhla (3), A. M. Alvarez (1), M. Arif (1), (1) University of Hawaii at Manoa, Honolulu, HI, USA; (2) USDA-APHIS-PPQ-S\&T-CPHST, Beltsville, MD, USA; (3) USDA-APHISPPQ-S\&T, Beltsville Laboratory, Beltsville, MD, USA 
A recent, increased loss in potato yield has been ascribed to a comparatively aggressive member of the Pectobacteriaceae family of soft rot pathogens, Dickeya solani. Sensitive and accurate detection of D. solani is important for monitoring, surveillance and seed certification programs. The whole genomes of $D$. solani and other species were retrieved, and a comparative genomics approach was used to select two specific target genes for primers and probes design. The in-silico analyses showed that all primers and probes had $100 \%$ identity with $D$. solani. The $D$. solani primers were multiplexed with the previously designed Dickeya genus primers and a universal internal control to enhance assay reliability. The specificity of the developed TaqMan qPCR was confirmed using 124 bacterial strains from different hosts and geographical locations around the world. No false positives or negatives were observed with any bacterial strain in exclusivity or inclusivity panels. The sensitivity of the developed multiplex TaqMan qPCR assay was 1 pg for detection of $D$. solani. The developed assay was also validated with plant samples naturally and artificially infected with Dickeya species and/or D. solani, and the assay detected the target in plant samples with $100 \%$ accuracy. The developed multiplex TaqMan qPCR has applications in routine diagnostics, disease management and agriculture biosecurity.

\section{Comparative study of the microbiome of bulk soil, rhizosphere, and endosphere of the native plant Ceanothus velutinus from different locations}

J. GANESH (1), Y. Sun (2), A. Thomas (2), A. Kaundal (2), (1) Utah State University, Logan, UT, USA; (2) Utah State University, Logan, UT, USA

Climatic change is one of the biggest threats to Earth's ecosystem and biodiversity by enhancing environmental stresses. Environmental stresses such as biotic and abiotic stresses affect plant health and reduce crop production. Rhizosphere microbiome of a plant plays a significant role in a plant's defense against various biotic and abiotic stresses and improve plant health. In this study, we are investigating the microbiome diversity of bulk soil, rhizosphere, and endosphere of Ceanothus velutinus, snowbrush. Ceanothus is an evergreen native plant that is usually found in dry areas and thrives well in harsh conditions. The snowbrush samples were collected from different locations and elevations from the Tony Grove area of the Intermountain West region of the United States. The DNA was isolated from all the samples. The sequencing of 16s rRNA (V3 and V4 region) for bacteria and ITS for fungi was performed. The obtained NGS data of $16 \mathrm{~s}$ rRNA has been analyzed by the QIIME tool to investigate microbial diversity in all the endosphere, rhizosphere, and bulk soil samples. The results revealed the dominance of Proteobacteria 57\%, 37\% and $41 \%$ in endosphere, bulk, and rhizospheric soil followed by Actinobacteria 27\%,33\%, and 28\% in endosphere, bulk and rhizospheric soil samples respectively. The other common major bacterial phyla are Acidobacteria Verrucomicrobia, Planctomycetes, Bacteroidetes, Gemmatimonadetes, Chloroflexi, and Firmicutes. The bioinformatic analysis for 16SrRNA and ITS is under process to investigate microbial diversity in detail.

\section{Novel inhibitors of Erwinia amylovora and Candidatus Liberibacter asiaticus C. VOSBURG, T. McNellis, Pennsylvania State University, University Park, PA, USA}

Fire blight and citrus greening disease (Huanglongbing, HLB) are two of the most economically important plant bacterial diseases. Our aim is to find novel inhibitors of fire blight and HLB pathogenesis that can be developed into new management options for growers. Versatility in management options helps reduce the risk of resistance development and targeting mechanisms of pathogenesis reduces harm to non-target organisms. Erwinia amylovora is the causal agent of fire blight, which affects apples and pears. Random mutagenesis and suppressor screening of E. amylovora has led to the discovery of a gene called yibD that inhibits production of the essential virulence factor amylovoran when overexpressed. Deletion analysis is being performed to determine which portions of yibD are sufficient for inhibition of amylovoran production. Preliminary results indicate that partial deletions of the predicted glycosyltransferase domain do not affect inhibitory activity. Candidatus Liberibacter asiaticus (CLas) is the presumptive causal agent of HLB. Transgenic grapefruit have been developed that express an antibody that binds to a predicted efflux pump in CLas called NodT. By blocking NodT, the antibody is expected to inhibit important functions needed for CLas growth and pathogenesis such as host defense evasion. The transgenic lines have been challenged with CLas using both vector-mediated and vegetative graft transmission to observe CLas growth and HLB symptom development. Potential utility of these approaches for fire blight and HLB management is discussed.

\section{A PCR-RFLP tool to differentiate between Parastagonospora nodorum and its sister species Phaeosphaeria avenaria tritici 1 M. Hafiz (1), R. ABOUKHADDOUR (2), (1) AAFC, CANADA; (2) AAFC, Lethbridge, AB, CANADA}

Septoria nodorum blotch (SNB) is an important foliar disease of wheat worldwide. It is caused by two fungal pathogens, Parastagonospora nodorum and Parastagonospora avenaria tritici. In P. avenaria tritici (Pat), three genetically distinct groups, Pat1, Pat2, and Pat 3 have been differentiated based on variation in the internal transcribed spacer (ITS) sequence. The two species are nearly identical in morphology and are highly similar at the molecular level. Here, we developed a PCR-RFLP tool to differentiate between them based on sequence polymorphism in the ITS region. ITS sequences from P. nodorum and Pat 1 were analysed separately with NEBcutter v2.0 to identify restriction enzymes that produce differential restriction patterns between $P$. nodorum and Pat1. Double digestion of ITS-PCR products with HpaII and XapI enzymes clearly discriminated between the two species. Digestion of the Pat 1 ITSPCR products ( $700 \mathrm{bp})$ with HpaII and XapI enzymes produced a characteristic pattern consisting of three fragments: $340+280+80$ $\mathrm{bp}$, while $P$. nodorum produced a different pattern consisting of four restriction fragments: $280+220+120+80 \mathrm{bp}$. The ITS-based PCR-RFLP tool was validated in vitro with large number of $P$. nodorum and Pat 1 isolates and the results confirms the capability of the tool to differentiate between the two species. The tool was also validated in silico using sequences retrieved from GenBank for $P$. nodorum, Pat 1 and other leaf spot pathogens and all tested sequences showed the expected restriction pattern for each species obtained during this experiment. 
First report of the hops powdery mildew pathogen, Podosphaeria macularis, on naturally infected marijuana (Cannabis sativa L.) plants in the field

Z. K. PUNJA, Simon Fraser Univ, Burnaby, BC, CANADA

Cannabis (Cannabis sativa L., marijuana) plants 'Chronic Rider' grown under field conditions in the Fraser Valley of British Columbia displayed symptoms of powdery mildew infection in September, 2019. The disease was observed on leaves, stems, shoot tips and bracts of inflorescences. Severely infected plants showed curling of the shoot tips, with mycelial growth observed on the adaxial and abaxial leaf surfaces. Necrotic areas developed underneath mildew colonies. These symptoms were atypical of those caused by the powdery mildew pathogen reported in Canada, Golovinomyces chicoracearum. DNA was extracted from healthy and diseased tissues and subjected to PCR using primers that amplified the ITS1-5.8S-ITS2 region of rDNA. A band size of approximately 650 bp was obtained from diseased plants that was absent in asymptomatic tissue samples. A significant homology (100\%) was obtained following BLASTn to Podosphaeria macularis, the powdery mildew pathogen reported to infect hop plants. Powdery mildew infected hop leaves originating from Abbotsford and Kelowna, BC had significant sequence homology (99-100\%) to the pathogen infecting cannabis plants but not to G. chicoracearum. Artificial inoculations onto leaves of cannabis strain 'Pink Kush' from diseased 'Chronic Rider' leaves under laboratory conditions gave rise to mildew infection after 18-24 days, which was confirmed as P. macularis by PCR. The development of $P$. macularis on cannabis plants is slower compared to G. chicoracearum, and the threat of spread to outdoor and indoor cultivation facilities needs to be assessed.

Peanut on the wild side -introgression of disease resistance from wild relatives

S. LEAL-BERTIOLI (1), D. Bertioli (2), C. Taborda-Ballen (3), D. Gao (4), (1) UGA, Plant Pathology Department, Athens, GA, USA; (2) UGA, Athens, GA, USA; (3) UGA - Institute of Plant Breeding, Genetics and Genomics, Athens, GA, USA; (4) Uga - Institute of Plant Breeding Genetics and Genomics, Athens, GA, USA

The peanut crop suffers from numerous pests and diseases. Development and adoption of resistant varieties is the most cost efficient and effective way to control the spread of the disease and reduce yield losses. Wild species form a secondary gene pool, and provide a source of strong resistance alleles, but they have undesirable agronomic traits, such as small seeds and spreading habit, that are a disincentive to their use in breeding. The identification of genomic regions that harbor disease resistances in wild species is the first step in the implementation of marker assisted selection that can speed the introgression of wild disease resistances and the elimination of linkage drag. We have identified genome regions that control different components of rust, Late leaf spot and nematode resistances in populations developed using various Arachis species. In breeding, in some cases, desirable traits were quickly recovered with a few cycles of backcrosses and selection. However, using new, higher resolution genotyping methods uncovered unexpected genomic instability. These findings highlight new mechanisms of introduction of diversity and increased disease resistance to the peanut crop.

Comparison of methods for detecting bacterial spot pathogens on tomato seed H. WANG (1), G. E. Vallad (2), J. B. Jones (3), W. W. Turechek (4), (1) Clemson University, Blackville, SC, USA; (2) Gulf Coast Research and Education Center, University of Florida, Wimauma, FL, USA; (3) University of Florida, Gainesville, FL, USA; (4) USDA, ARS, U.S. Horticultural Research Laboratory, Fort Pierce, FL, USA

Bacterial spot is a serious disease of tomato that is caused by four different species of Xanthomonas: X. euvesicatoria, X. gardneri, X. perforans, and $X$. vesicatoria. Contaminated and/or infected seed are a major source of inoculum, and big seed companies routinely test seed lots for these pathogens using standard culturing procedures on semi-selective media. However, this method has limited sensitivity and it cannot detect the viable but non-culturable cells. To address these problems, we developed a viability-qPCR assay for specific quantification of viable Xanthomonas pathogens on tomato seed. Here we compared the performance of this viability-qPCR assay with the industry-standard culturing method on quantifying viable pathogen cells in seed samples. Contaminated tomato seed were prepared by inoculating batches of seed with bacterial suspensions between $0-10^{8} \mathrm{cfu} / \mathrm{ml}$. The inoculated seed were mixed with clean seed in a range of ratios $(0.01-100 \%)$ and samples were then drawn from these batches for testing. Bacteria were extracted from these samples using the standard protocol of International Seed Federation, and the bacteria extracts were processed with both the PMA-qPCR assay and the standard culturing method on different semi-selective media. The Ct values from PMA-qPCR assay and the colony count results from the culturing method were compared using latent class analysis to determine their detection sensitivity and specificity. Results showed that the PMA-qPCR assay had a higher sensitivity for detecting the viable Xanthomonas pathogens on tomato seed.

Identification and management of Phytopythium vexans on Acer rubrum

M. PANTH (1), F. Avin (2), T. Simmons (2), F. Baysal-Gurel (2), (1) Tennessee State University, McMinnville, TN, USA; (2) Tennessee State University, McMinnville, TN, USA

Red maple (Acer rubrum L.) plants grown in containers and fields in Tennessee have shown root and crown rot symptoms with reddish/dark brown lesions in 2017 and 2018. The objective of this study was to identify the pathogen affecting maples in nursery production and develop management recommendations. Isolations were done from the infected roots. Several Phytophthora-like colonies with spherical zoospores, ovoid to globose oogoni, and whitish mycelium, were isolated on PARPH-V8. To confirm the identity of the pathogen, total DNA was extracted using the PowerSoil DNA Isolation Kit directly from 3-day-old cultures. The PCR and sequencing were done to confirm the pathogen identification followed by pathogenicity test to complete Koch's postulates. Following the identification of Phytopythium vexans, the efficacy of cover crops to suppress the disease severity caused by $P$. vexans was assessed in greenhouse trials. Soils from red maple plantations grown with and without cover crops (crimson clover or triticale) were sampled following the senescence of the cover crops. A greenhouse bioassay was conducted using red maple cuttings inoculated 
with $P$. vexans and non-inoculated field soils. Results showed that cover crop usage was beneficial for inducing soil disease suppressiveness. There was lower disease severity and pathogen recovery when the cover crops were used compared to bare soil treatment. Thus, cover crops can be integrated in field nursery production systems to be able to suppress newly reported Phytopythium root and crown rot disease.

Niche clearing following fumigation events leads to stochastic assembly of soil microbiome M. MILLICAN (1), J. P. Dundore-Arias (2), L. L. Kinkel (1), (1) University of Minnesota, MN, USA; (2) California State University Monterey Bay, Seaside, CA, USA

Understanding the response of microbial communities to disruption events, such as chemical pest management, is critical in developing enhanced management of agricultural microbiomes. Agricultural systems require diverse management practices to prevent disease and promote plant health. Fumigation is among the most ecologically disruptive management practices but is routinely used in potato production in the Upper Midwestern U.S. To determine how microbial communities re-establish following a fumigation event (vapam or chloropicrin), we monitored bacterial (v4 16S rRNA) and fungal (ITS-2) communities via amplicon sequencing in Fall (prior to fumigation), in spring (pre-planting), and at harvest. Then, we evaluated the relationship between the occupancy and relative abundance of ESVs. We also analyzed the pairwise distances of community compositions within treatments. The data suggest that community composition/structure in chloropicrin-fumigated communities follows a stochastic assembly process, consistent with fumigation imposing a 'niche clearing' event on the soil microbiome. In contrast, non-fumigated and vapam-fumigated soil microbiomes are consistent with a non-random assembly process, implying enrichment of specific taxa. Understanding soil microbiome assembly dynamics post-fumigation will provide important insights to reduce reliance on fumigation by 'rewiring' community reassembly. Establishing principles of microbial community assembly in agricultural systems will inform the development of disease suppressive and plant beneficial microbiomes.

Ensuring food safety and security by maintaining climate resilience of grain nutritional content in Fusarium head blight resistant wheat

W. HAY (1), S. Mccormick (1), J. A. Anderson (2), B. Michael (1), M. Hojilla-Evangelista (1), M. Berhow (1), R. Dunn (1), J. Teresi (1), M. Vaughan (1), (1) USDA-ARS, Peoria, IL, USA; (2) University of Minnesota, St Paul, MN, USA

Rising carbon dioxide $\left(\mathrm{CO}_{2}\right)$ can change the nutritional content of wheat and increase the severity of Fusarium head blight (FHB), a devastating fungal disease of wheat that reduces yield and contaminates grain with harmful mycotoxins. At elevated $\mathrm{CO}_{2}$, FHB susceptible and moderately resistant wheat had disproportionate losses in protein and mineral content, with moderately resistant cultivars more severely impacted. Furthermore, on the grain with the most severe loss of nutritional content some Fusarium graminearum strains increased production of the mycotoxin deoxynivalenol. Future climate conditions may provide a pathogenic advantage on hosts with lower nutritional content, threatening global food safety and security. Wheat growers may be less likely to choose moderately resistant cultivars with reduced end use quality. Therefore, FHB control strategies should consider the climate resilience of wheat nutritional content.

\section{Development of image-based potato blemish disease evaluation tools}

S. MACCHIAVELLI-GIRÓN, J. Spychalla, E. A. Monette, A. J. Gevens, University of Wisconsin-Madison, Madison, WI, USA

Blemish diseases of potato negatively impact the skin or periderm of the tuber, reducing quality in appearance and integrity. Silver scurf of potato, caused by the fungus Helminthosporium solani, has been a tuber blemish disease of great and increasing concern to the United States potato industry. Symptoms of this disease include gray, silver or golden lesions on the periderm. Current visual rating systems require highly trained observers and consistent overhead lighting, which limits consistency and sample size capacity. Our aim was to develop and evaluate image-based rating tools to characterize silver scurf severity and be modified for other potato blemish diseases. The tools that were evaluated and compared to the traditional visual rating method were Image J and R. A set of images generated from a Photosimile 200 Lightbox with a CanonEOS T5i camera was processed with ImageJ and compared with the traditional visual rating method. A true severity value was determined by tracing the lesions using Photoshop. Preliminary results show that the traditional visual rating was closer to the true value $\left(R^{2}=0.71\right)$ than the ImageJ rating was $\left(R^{2}=0.55\right)$. Image J overestimated surface area covered in blemish disease, as it couldn't distinguish skin defects or other blemishes. Ongoing work is aimed at improving this tool. Further, we investigated the use of R to process samples faster and with greater precision and accuracy. Resulting, we developed an accessible image-based tool to evaluate tubers for blemish diseases in support of the potato research community and industry.

\section{Determining limit of detection of high throughput sequencing diagnostics with $\mathrm{MiFi}^{\circledR}$}

H. WANG (1), J. Habiger (1), A. Espindola (1), K. F. Cardwell (1), T. Dang (2), G. Vidalakis (2), A. Roy (3), (1) Oklahoma State University, Stillwater, OK, USA; (2) University of California, Riverside, Riverside, CA, USA; (3) USDA-APHIS-PPQ-S\&T, Beltsville, MD, USA

High throughput sequencing (HTS) technology can be applied to plant disease diagnostics. Microbe Finder $\left(\mathrm{MiFi}^{\circledR}\right)$ is an online platform for detection of plant pathogens in HTS data, eliminating pathogen isolation, bioinformatics, amplification. Diagnostic sensitivity, specificity and Limit of Detection (LOD) are crucial metrics of any diagnostic tool. We present how to calculate LOD for HTS diagnostics with a statistical inference model using pathogen-specific e-probe matches in metagenomic data. The LOD calculates the lowest levels of the target pathogen that can be reliably detected. Here we used a quadratic discriminant analysis to calculate the LOD of three citrus pathogens in metagenomic HTS data. The LOD assumes that positive samples have a higher e-probe 'hit $\mathrm{x}$ percent 
identity score' and a different Normal distribution than the negative control scores. LOD, formally defined as the estimated Bayes decision boundary, is computed using the mean and variance of the positive and negative groups. The LOD of citrus leprosis virus C2, citrus tristeza virus, and citrus exocortis viroid were 4.7, 4.9, and 5.1 hits respectively, indicating when the chance of true positive is 50/50. The LOD results were consistent with the RT-qPCR results, however $\mathrm{MiFi}^{\circledR}$ was found to be more sensitive. In this scenario, the model is trained on a viroid and two RNA viruses, but is assumed to be true for all taxonomic groups. The development of the probability model for citrus graft transmissible bacteria and a citrus specific oomycete (Phytophthora spp) is on-going.

\author{
Modification of a late blight resistance gene to avoid effector-mediated suppression \\ D. A. HALTERMAN (1), H. S. Karki (2), S. Abdullah (3), Y. Chen (3), (1) USDA ARS, Madison, WI, USA; (2) USDA/ARS, \\ Madison, WI, USA; (3) University of Wisconsin-Madison, Madison, WI, USA
}

Microorganisms that cause plant diseases present a substantial burden to agriculture through yield losses due to plant stress, costs associated with disease control, and efforts to detect infections and limit disease epidemics. Plant breeders are interested in the identification and incorporation of simply inherited genes that confer robust resistance to diseases. These resistance $(R)$ genes typically encode proteins that recognize the presence of very specific pathogen molecules, termed effectors, resulting in the activation of defense responses. However, certain effectors can directly suppress R proteins to allow the pathogen to elude recognition and cause disease. Using the potato late blight pathosystem, we previously identified a Phytophthora infestans effector that can suppress RB activity through direct interaction with the resistance protein. By mining natural genetic variation of $\mathrm{RB}$ from wild potato relatives, we identified specific amino acids within the coiled-coil region of the $\mathrm{R}$ protein that allow it to avoid suppression and maintain its ability to activate defense responses in both transient and stable transgenic expression systems.

Using a high throughput bioassay to characterize Erysiphe necator tolerance to multiple fungicide classes A. T. WONG (1), W. Mahaffee (2), (1) Oregon State Univ, Corvallis, OR, USA; (2) USDA ARS, Corvallis, OR, USA

Leaf disk bioassay techniques to characterize grape powdery mildew (Erysiphe necator) fungicide tolerance to demethylation inhibitors (DMI) are not highly robust or repeatable and are time and resource expensive. A conidium has limited supply of essential components (e.g. ergosterol) and must begin to synthesize them prior to successful infection. DMI inhibition of this pathway would result in a reduction in hyphal biomass and could be used to characterize tolerance to DMI's and other fungicide classes. Conidia were dispersed onto a 96-well microtiter plate with $0.5 \%$ gellan gum amended with $20 \mu \mathrm{l}$ of various fungicides solutions (FRAC 3, 7, 11, 13, and 50). Each well was imaged 0 and 48 hours after deposition to determine relative biomass. Images were processed in ImageJ and analyzed using the "HyphaTracker" macro (Brunk et al., 2018). The difference in biomass from the $0 \mathrm{~h}$ and $48 \mathrm{~h}$ measurements was compared to the control to produce $50 \%$ inhibitory concentration estimates. Results show that this method can be a flexible, robust, inexpensive, and high-throughput method to characterize fungicide tolerance to many fungicide classes. Results have been comparable to leaf disk and germination bioassays, as well as qPCR genotyping results for DMI and quinone outside inhibitor resistance. This method can assess 5 isolates' responses to 24 fungicide treatments with only 4 hours of labor and a 48 -hour incubation period; as compared to leaf disk bioassays, which require 12 hours of labor, a 14-day incubation, and subjective estimations of infected area for 8 treatments.

Detection of 'Candidatus Liberibacter asiaticus' by recombinase polymerase amplification (RPA) assay J. W. Park (1), M. KUNTA (1), J. V. Da Graça (1), W. E. Braswell (2), (1) Texas A\&M University Kingsville Citrus Center, Weslaco, TX, USA; (2) USDA APHIS PPQ S \& T, Edinburg, TX, USA

Huanglongbing (a.k.a. citrus greening), one of the most destructive diseases affecting citrus trees, is associated with three phloemlimited 'Candidatus Liberibacter' species, $C a$. L. asiaticus (CLas), Ca. L. americanus (CLam) and Ca. L africanus (CLaf). Unlike CLam and CLaf which are geographically limited to South America and Africa, respectively, the incidence of CLas is widespread which poses a great threat to citriculture worldwide. Since no resistant commercial citrus cultivars are available yet, the current preventive measures include the control of insect vector population (Asian citrus psyllid; Diaphorina citri) and the removal of the diseased trees. Currently, the disease diagnosis is based on visual inspection of HLB symptoms in the tree canopy followed by real-time PCR to confirm the presence of CLas DNA in plant tissue extracts. As symptom development is a slow process taking several months to one or more years to appear, asymptomatic trees in the field go unnoticed and serve as a reservoir of CLas inoculum eventually leading to the disease outbreak. Isothermal amplification technology such as Recombinase Polymerase Amplification (RPA) offers an alternative to PCR for the development of rapid, sensitive, and field-deployable diagnostic detection assays. The current study developed an RPA assay targeting the $n r d B$ gene, of which there are five copies in the CLas genome and evaluated its applicability for CLas detection using DNA extracts prepared from citrus leaf and fibrous root tissue.

Fitness of field isolates of Phytophthora capsici sensitive or resistant to fluopicolide L. WANG, P. Ji, University of Georgia, Tifton, GA, USA

Phytophthora blight, caused by Phytophthora capsici, is one of the most serious diseases in the production of vegetable crops. Fluopicolide has been widely used to control the disease. Recently, we identified fluopicolide-resistant isolates of $P$. capsici from commercial vegetable fields in Georgia, USA. The objective of this study was to assess fitness of fluopicolide-resistant and sensitive isolates. The relative fitness of the isolates was compared by mycelial growth on V8 agar medium with 0,10,50 and $100 \mathrm{ppm}$ of fluopicolide. Sporangial production and zoospore germination were evaluated on agar media with 0 or 50 ppm of fluopicolide.

Aggressiveness of the isolates on bell pepper (cv. Aristotle and Paladin) was evaluated in a greenhouse. For resistant isolates, there was no significant difference in mycelial growth on medium with $0-100 \mathrm{ppm}$ of fluopicolide. Sporangial production and zoospore 
germination of sensitive isolates were reduced significantly on fluopicolide amended media compared to non-amended media; however, such reduction was not observed for resistant isolates. In the absence of fluopicolide, there were significant differences among individual isolates in mycelial growth, sporangial production, and zoospore germination, but the differences were not associated with resistance or sensitivity to fluopicolide. In greenhouse studies, there was no significant difference between resistant and sensitive isolates in disease intensity on pepper seedlings. The results suggest that fitness of the fluopicolide-resistant isolates was similar to the sensitive isolates.

Development of a new, organic, bactericide

B. FRANCE (1), W. Bell (1), J. Yuen (2), D. J. Norman (2), (1) TDA Research, Inc., Wheat Ridge, CO, USA; (2) University of Florida, Apopka, FL, USA

Bacterial plant pathogens present a formidable challenge to growers because there are few effective products. The most commonly used bactericides contain copper compounds or other metals. After decades of heavy use of copper products bacterial populations are becoming less sensitive to copper. The objective of this research is to develop a novel bactericide in response to growers' requests for products that can mitigate economically significant bacterial challenges. A further objective is a bactericide that is safe, organic, classified as a bio-pesticide, minimal to no phytotoxicity, and is as effective as commonly used copper-based products. The new bactericide was developed through a collaborative process to optimize the chemistry, with initial evaluation using in vitro laboratory work, followed by greenhouse phytotoxicity evaluations, and finally greenhouse efficacy testing. Test results have shown that our developed formulation is effective at mitigating bacterial leaf spot caused by Xanthomonas on both geranium and rose. In these cases, it has been shown to be as effective or more effective than copper hydroxide. Preliminary results on powdery mildew on gerbera daisy also indicate that the new bactericide may also be very effective on certain fungal pathogens. Growers require new, regulatory acceptable, bactericides to improve the health of their plants. Our formulation is not an antibiotic and all the components are on the FDA GRAS list and will be broadly accepted by all producers and consumers.

Importance of air-borne conidia in the epidemiology of Cercospora Leaf Blight on soybean N. N. GALAGEDARA (1), V. Doyle (2), P. Price (3), C. L. Robertson (2), S. Thomas-Sharma (4), (1) Department of Plant Pathology, North Dakota State University, FARGO, ND, USA; (2) Department of Plant Pathology \& Crop Physiology, Louisiana State University, Baton Rouge, LA, USA; (3) LSU AgCenter, Macon Ridge Research Station, Winnsboro, LA, USA; (4) Louisiana State University Agricultural Center, Baton Rouge, LA, USA

Cercospora leaf blight (CLB), primarily caused by Cercospora cf. flagellaris (and to a lesser extent by Cercospora cf. sigesbeckiae and Cercospora kikuchii) is the most prevalent foliar disease of soybean in Louisiana. CLB was historically known to be a seed-borne disease, and conidia observed on symptomatic leaves (close to harvest) were considered inconsequential in the disease cycle. However, with the discovery of $C$. flagellaris and $C$. sigesbeckiae, both generalist pathogens that can infect alternate hosts around soybean fields, air-borne conidia might play epidemiologically important roles. In order to understand the within season dynamics of air-borne conidia, spore samplers were set-up in a soybean field in Winnsboro, LA from May-November 2019. An active Burkard spore sampler and two types of passive spore samplers were used. The passive samplers included a glass slide coated with Vaseline in a $3 \mathrm{~cm}^{2}$ area and a windsock mesh-based sampler (Assured Bio Labs, TN). The samplers were setup in the center and edges of the field and a total of 336 samples were collected at weekly intervals. We are currently developing a TaqMan probe-based quantitative PCR assay to detect, differentiate, and quantify the Cercospora spp. associated with CLB. The role of weather variables in conidial release will also be studied. These studies will fill knowledge gaps about the disease cycle and species composition of the CLB pathogens in Louisiana. Further, spore peaks may serve as susceptibility windows to target fungicide applications and provide improved management solutions for growers.

Using fungicides and improved host resistance to manage Cercospora beticola in sugar beet M. F. R. KHAN, North Dakota State Univ \& Univ of MN, Fargo, ND, USA

Cercospora beticola causes Cercospora leaf spot (CLS), one of the most economically damaging foliar diseases of sugar beet. Growers use cultural practices, tolerant cultivars, and fungicides to manage CLS. Since 2016, the pathogen, which has the ability to produce large numbers of conidia and multiple generations during a growing season, has developed resistance to most of the fungicides used for its control. Inoculated field trials were conducted at Foxhome, MN in 2019 to evaluate efficacy of individual fungicides, mixtures; and mixtures in a rotation program at controlling CLS. Treatments that were effective in the fungicide programs were then used at different timings to control $C$. beticola on commercial cultivars (2) and improved resistant cultivars (2). Few fungicides provided effective control of $C$. beticola when used alone. Multiple applications of mixtures of multisite and site specific fungicides provided effective control, and when used in a fungicide rotation program resulted in significant yield and economic benefit. One of the improved resistant cultivars had significantly lower disease severity, even in the absence of fungicides, and its yield of recoverable sucrose was not significantly different from approved commercial cultivars that were protected with fungicides. Further research will be necessary to develop a program that will determine the need and best time to use fungicides for control of $C$. beticola in the new resistant varieties.

Aspergillus flavus transcriptional changes in response to co-culture with an atoxigenic $A$. flavus biocontrol isolate R. R. SWEANY (1,2), B. Mack (3), M. Gilbert (3), J. Cary (3), K. Rajasekaran (3), G. Moore (3), K. E. Damann (1), (1) Louisiana State University, Baton Rouge, LA, USA; (2) U.S. Department of Agriculture-Southern Regional Research Center-New Orleans, New Orleans, LA, USA; (3) U.S. Department of Agriculture-Southern Regional Research Center, New Orleans, LA, USA 
Aflatoxin (AF) is a carcinogenic mycotoxin produced by Aspergillus flavus in corn. Atoxigenic (Atox) isolates lacking aflatoxin production are deployed in corn fields as a biocontrol to reduce AF contamination. Direct contact or touch between toxigenic (Tox) and Atox isolates dramatically reduces aflatoxin production. To understand the mechanism of touch inhibition, an RNA-seq study was conducted to examine gene expression during their interaction. Total RNA and AF were extracted, mRNA was high-throughput sequenced and AF was quantified with HPLC for 3 biological replicates of Atox KD17 and Tox KD53 grown separately and in cocultures for 30 and $72 \mathrm{~h}, \mathrm{n}=18$. When grown separately, Tox represents $27 \%$ and $38 \%$ of the combined biomass at the 30 and $72 \mathrm{~h}$ time points. However, only $3 \%$ reads uniquely aligned to Tox during co-culture, indicating Tox growth and/or gene expression was inhibited in response to Atox. Few reads aligned to AF gene cluster during co-culture. Eighteen genes expressed during Atox mono-culture were further up-regulated during co-culture, indicating a response to contact. Of those genes, 7 belong to a putative secondary metabolite (SM) cluster, suggesting a potentially inhibitory compound is produced. Taken together, these results suggest that Atox strains inhibit growth and AF biosynthetic gene cluster expression in Tox strains. In addition, other SM genes are upregulated during biocontrol interaction. This study demonstrates a potential role of inhibitory SMs in the biocontrol mechanism and deserves further exploration to improve biocontrol formulations.

LsGRP1 induces systemic resistance to gray mold in a salicylic acid-dependent manner Y. T. Shih, T. W. Chen, C. H. Lin, C. Y. CHEN, National Taiwan University, Taipei, TAIWAN

Gray mold disease, caused by the necrotrophic fungus Botrytis species, is an economically important disease in numerous horticultural crops. In Oriental hybrid Lilium 'Star Gazer', the pretreatment of plant defense hormone salicylic acid (SA) is able to reduce Botrytis elliptica infection accompanied with an increased expression of $L s G R P 1$ which encodes a cell surface-localized class II glycine-rich protein. The involvement of LsGRP1 and SA in the induced systemic resistance to gray mold was highly suggested. In this study, silencing of $L S G R P 1$ caused reductions in gray mold resistance and defense responses including callose deposition and reactive oxygen species accumulation, indicating the contribution of LsGRP1 in lily immunity. The systemic leaves of LsGRP1 fusion protein-infiltrated lily plants showed mitigated symptom and enhanced defense responses. Besides, pre-drench with LsGRP1 fusion protein systemically protected different lily cultivars from B. elliptica and also strawberry from Botrytis cinerea. Afterwards, the B. cinerea resistance of Arabidopsis conferred by the constitutive expression of LsGRP1 was found to be abolished as the AtICS1 gene critical for pathogeninduced SA biosynthesis was silenced. In addition, silencing of AtICS1 homolog downgraded the levels of gray mold resistance and LsGRP1 expression in Lilium 'Star Gazer'. Thus, LsGRP1 elicits plant systemic resistance to combat Botrytis species, presumably related to $\mathrm{SA}$.

Deep convolutional neural networks and epidemiological criteria to support breeding tactics against the wheat blast disease M. FERNANDEZ CAMPOS (1), Y. Huang (2), T. Wang (3), S. Das (4), M. R. Kabir (5), J. Jin (3), B. S. Valent (6), M. R. Jahanshahi (7), C. D. Cruz (8), (1) Purdue University, West Lafayette, IN, USA; (2) Purdue University, School of Electrical and Computer Engineering, West Lafayette, IN, USA; (3) Purdue University, Agricultural and Biological Engineering, West Lafayette, IN, USA; (4) Uttar Banga Krishi Viswavidyalaya, West Bengal, INDIA; (5) Bangladesh Wheat and Maize Research Institute, Nishapur, BANGLADESH; (6) Kansas State University, Manhattan, KS, USA; (7) Purdue University, School of Electrical and Computer Engineering, West Lafayette, IN, USA; (8) Department of Botany and Plant Pathology, Purdue University, West Lafayette, IN, USA

Wheat blast is a threat to global wheat production. Current estimations of wheat spike blast $\left(\mathrm{W}_{\mathrm{S}} \mathrm{B}\right)$ intensity rely on human assessments. Reliable visual estimations paired with images of $\mathrm{W}_{\mathrm{s}} \mathrm{B}$ can be used to train deep convolutional neural networks (DCNNs) for disease intensity classification. Also, epidemiological parameters can support cultivar selection for resistance. Objectives were to develop accurate and reliable DCNNs models to classify $\mathrm{W}_{\mathrm{S}} \mathrm{B}$ intensity under controlled conditions (CC), and to evaluate ten cultivars for wheat blast resistance under field conditions (FC) using epidemiological parameters. WsB intensity was visually estimated and RGB images acquired from six cultivars with various levels of resistance under CC. Datasets of images were created, with maturing spikes $(\mathrm{n}=$ $2637)$ and non-maturing spikes $(n=2123)$, then classified by intensity categories to train the DCNNs models. The model trained with non-maturing spikes had higher precision (0.90-0.95), F-1 (0.87-0.95), and recall (0.84-0.96) than the model trained with maturing spikes $(0.75-0.95,0.79-0.95$, and $0.74-0.96$, respectively). WSB intensity classification models could be used as a phenotyping tool. Wheat blast leaf and spike severity were evaluated under FC. Total area under the disease progress curve (tAUDPC), final disease severity $\left(\mathrm{Y}_{\max }\right)$, and type of epidemic were estimated. Disease progress curves of ten cultivars were fitted by the logistic $\left(\mathrm{R}^{2}=0.70\right.$ $0.96)$ and Gompertz $\left(\mathrm{R}^{2}=0.64-0.94\right)$ models, alleging a polycyclic epidemic. tAUDPC, and $\mathrm{Y}_{\max }$ can support cultivar selection for wheat blast resistance.

\section{Host range of Fusarium sambucinum and management of Fusarium canker of hop}

T. BORLAND (1), W. Thomas (1), B. Claassen (1), S. Massie (2), C. M. Ocamb (1), D. H. Gent (3), (1) Oregon State University, Corvallis, OR, USA; (2) Washington Hop Commision, WA, USA; (3) US Department of Agriculture, Agricultural Research Service, Corvallis, OR, USA

Fusarium canker of hop, caused by Fusarium sambucinum, is a poorly understood disease with rising importance in hop yards in the Pacific Northwest region of the United States. The need to understand this pathosystem better increases as the number of documented cases continue to grow. Greenhouse and commercial-scale field studies are underway with a focus on cultural and chemical management tactics, as well as on the host range of the fungus. The effect of spring pruning methods and the fungicide mefenoxam on the severity of Fusarium canker were evaluated in a factorial design in a commercial hop yard over two years. Preliminary results suggest the rumored efficacy of mefenoxam for Fusarium canker has no merit, and that chemical pruning reduces the severity of Fusarium canker as compared to mechanical pruning methods. Separate experiments were conducted in a commercial hop yard to evaluate the impact of single versus double drip tube irrigation systems on disease development. It is hypothesized that using double 
drip rather than single drip irrigation systems may reduce canker incidence by reducing water pooling near the base of stems, given that moist conditions reportedly favor infection. In addition to management methods, experiments regarding the pathogenicity of $F$. sambucinum on hop, potato, corn, and wheat are underway, using isolates derived from each of the four plant species. Together, these studies will provide evidence-based management strategies that will aid hop growers in Fusarium canker management and risk mitigation.

\title{
Identification and evaluation of phytotoxic secondary metabolites produced by charcoal rot disease fungus, Macrophomina phaseolina
}

V. KHAMBHATI (1,2), H. K. Abbas (2), W. T. Shier (3), M. Tomaso-Peterson (1), J. Chen (2), N. Bellaloui (2), A. Mengistu (4), (1) Mississippi State University, Mississippi State, MS, USA; (2) United States Department of Agriculture, Agricultural Research Service, Stoneville, MS, USA; (3) University of Minnesota, College of Pharmacy, Minneapolis, MN, USA; (4) United States Department of Agriculture, Agricultural Research Service, Jackson, TN, USA

Charcoal rot disease fungus, Macrophomina phaseolina, is consistently one of the leading contributors to soybean crop yield loss in the Mid-South US, and globally, the pathogen infects over 500 species of commercially-important plants. The population of $M$. phaseolina exhibits considerable phenotypic and genotypic diversity and is reported to produce a range of secondary metabolites and toxins, including asperlin, phomalactone, phaseolinone, patulin, and (-)-botryodiplodin. Recent work on M. phaseolina suggests that the fungus infects hosts through a toxin-mediated process. To investigate this process, toxins produced by the fungus were first identified and then evaluated for phytotoxicity. Cultures were grown in Czapek-Dox liquid medium for 10 days, vacuum filtered, and tested for phytotoxicity utilizing a hydroponic soybean bioassay at 1:1 and 3:1 growth medium:filtrate ratios. After 72 hours, $50 \%$ of the isolates exhibited phytotoxic responses ranging from mild chlorosis to complete seedling death at both concentrations. An in-culture assay was used to identify M. phaseolina isolates that produced known mycotoxin, (-)-botryodiplodin. Phytotoxic filtrates were examined by bioassay-guided fractionation, LC-MS, and GC-MS analyses to identify phytotoxins. Chemical analyses of select isolates revealed the first report of mellein production by $M$. phaseolina. Serial dilutions of mellein were tested for phytotoxicity in the hydroponic bioassay. At higher concentrations, mellein inhibited root growth, but at mid to low concentrations, mellein induced increased uptake of water.

\author{
A unique region revealed through genome-wide analyses was used to develop an RPA assay for detection of the Select Agent \\ Ralstonia solanacearum R3bv2 \\ S. DOBHAL (1), S. Paudel (1), M. J. Stulberg (2), J. Rascoe (2), M. K. Nakhla (3), A. M. Alvarez (1), M. Arif (1), (1) University of \\ Hawaii at Manoa, Honolulu, HI, USA; (2) USDA-APHIS-PPQ-S\&T-CPHST, Beltsville, MD, USA; (3) USDA-APHIS-PPQ-S\&T, \\ Beltsville, MD, USA
}

Ralstonia solanacearum R3bv2 - quarantine pest and Select Agent under the Agricultural Bioterrorism Protection Act of 2002-causes brown rot of potato, which has been reported to affect 3.75 million acres in approximately 80 countries worldwide. An in-field detection tool for this high consequence pathogen is necessary for surveillance and rapid response. In this research we developed a recombinase polymerase amplification (RPA) for specific and rapid detection at point-of-need. The unique gene region was found through genomewide analyses of more than 80 genomes retrieved from NCBI GenBank genome database followed by design of robust primers and probe. Initially, the specificity assessed through in silico BLASTn analysis using NCBI GenBank showed $100 \%$ query coverage and identity only with $R$. solanacearum $\mathrm{R} 3 \mathrm{~b} 2$ strain sequences. The in vitro specificity was evaluated with representative strains of the $R$. solanacearum complex ( $R$. solanacearum, $R$. pseudosolanacearum, $R$. sygygii and $R$. solanacearum R3b2) and other genera and species including Xanthomonas, Pseudomonas and Clavibacter - no false positives or negatives were detected. The developed RPA assay was also assessed for sensitivity using 10-fold serially diluted genomic DNA; the detection limit for spiked (1 $\mu$ l of host potato sap was added in each reaction) and non-spiked samples was $1 \mathrm{pg}$. No effects of plant inhibitors were observed. The developed RPA assay has applications in quarantine, surveillance, surveys, biosecurity, and microbial forensics.

Precision management of soilborne pathogens of strawberry in California A. I. PUTMAN (1), F. N. Martin (2), S. A. Fennimore (3), O. Daugovish (4), A. Biscaro (4), M. Matson (2), M. Hang (5), T. R. Gordon (6), F. Melton (5), R. Goodhue (6), M. S. Stanghellini PhD (7), N. Dorn (8), (1) University of California, Riverside, Riverside, CA, USA; (2) USDA ARS, Salinas, CA, USA; (3) University of California, Davis, Salinas, CA, USA; (4) Univ. of California Coop. Ext. Ventura County, Ventura, CA, USA; (5) California State University-Monterey Bay/NASA ARC-SGE, Seaside, CA, USA; (6) University of California, Davis, Davis, CA, USA; (7) TriCal Inc, Hollister, CA, USA; (8) Food Origins, Salinas, CA, USA

The soilborne diseases Fusarium wilt, Macrophomina charcoal rot, and Verticillium wilt can cause significant economic loss in California strawberry production. The current standard of uniform pre-plant fumigation does not account for the typical clustered distribution of disease. The objective of this research is to evaluate a precision fumigation approach that first spatially assesses disease pressure and then fumigates at a rate proportional to pressure. Four single-season trials (A, B, C, and D) from 5.6 to 11.0 acres in size with three or four replicate plots were initiated in commercial fields in California. Plant mortality counts at the end of the previous crop were used to draw zones of pest pressure in precision plots that were assigned a high, medium, or low rate of chloropicrin of 350, 300, or $250 \mathrm{lbs} \mathrm{acre}^{-1}$, respectively, whereas the standard control treatment was $350 \mathrm{lbs}$ chloropicrin acre ${ }^{-1}$ applied uniformly. Data collected during the crop cycle included whole-plot and high-resolution yield, multi-spectral imagery, and mortality. At Field A, the amount of chloropicrin applied was $15.2 \%$ lower in precision compared to standard plots. Partial budget analysis of part-season whole-plot yield data revealed that average net returns were $6 \%$ higher in precision compared to standard plots. At Field C, whole-plot yield at this point in the season is $23.4 \%$ lower in precision compared to standard plots. Results to date suggest that a precision approach has the potential to reduce fumigant use while not sacrificing yield in select situations. 
Phenotype microarray profiling to characterize differences between Xylella fastidiosa subspecies C. M. WALLIS, USDA Agricultural Research Service, Parlier, CA, USA

Various Xylella fastidiosa $(X . f$.) strains or subspecies may cause Pierce's disease of grapevine $(X . f$. ssp. fastidiosa) or other bacterial leaf scorch diseases (X.f. ssp. multiplex) in California. Other $X . f$. subspecies may cause a variety of other serious diseases throughout the world including olive quick decline and citrus variegated chlorosis. Defining traits that vary between $X$. $f$. subspecies and strains is key to understanding inherit differences that result in host ranges and relative aggressiveness. Therefore, phenotype microarrays were conducted on different subspecies of $X . f$. (spp. fastidiosa or ssp. multiplex) to observe how bacterial growth is affected by 2,000 different growth conditions including exposure to different antibiotic or plant-derived compounds. Of all compounds tested, various phenolic compounds, such certain flavonoid glycosides, affected growth differently among $X$. $f$. subspecies, with $X$. $f$. ssp. fastidiosa growing well at levels greater or equal to $2 \mathrm{ppm}$ whereas $X$. $f$. ssp. multiplex did not grow at levels greater than $0.5 \mathrm{ppm}$. Grapevine xylem phenolic levels reach roughly 2 to $5 \mathrm{ppm}$, and therefore it would appear that $X$. $f$. ssp. fastidiosa would be adapted to infect grapevines whereas $X . f$. ssp. multiplex would not. Additional compounds and subspecies are undergoing testing. Completion of this research will improve understanding about requirements for $X$. $f$. infection and could result in the development of novel management techniques to control Xylella-caused diseases.

\section{There is still resistance: 20 years of Coffee leaf rust (Hemileia vastatrix Berk. \& Br.) monitoring of Timor Hybrid Coffee} collection in Colombia

C. A. ANGEL (1), M. Cristancho (1,2), J. D. Ocampo (1), A. L. Gaitan (1), (1) CENICAFE, FNC Colombia, Manizales, COLOMBIA; (2) Universidad de Los Andes, Bogotá D.C., COLOMBIA

Coffee leaf rust (CLR) is the most devastating disease of this crop worldwide, affecting the two main commercial species $C$. arabica and $C$. canephora. The Timor Hybrid $(\mathrm{TH})$ is a natural fertile hybrid $(2 \mathrm{n}=4 \mathrm{x}=44$ chromosomes) between the allotetraploid $C$. arabica and the diploid C. canephora. The Portuguese CIFC Institute collected TH germplasm, and since 1960s, many breeding programs have used it as major source for resistance to CLR and other diseases. The most used accessions are CIFC 832-1, 832-2, 2252 and 1343. FNC Cenicafé in Colombia has 53 accessions, 42 of them from 1343 hybrid, and utilized few of these to develop its multiline resistant varieties; Colombia, Castillo ${ }^{\circledR}$, Cenicafé-1 and Tabi. To study co-evolution of CLR races and resistance on TH, the Plant Pathology Discipline has annually monitored CLR incidence on TH accessions since year 2000 at Naranjal Station (Chinchiná, Colombia) at 1,400 m.a.s.1. Initially, only three accessions (5.7\%) showed CLR sporulation; by 2005 there were pustules on $34.6 \%$ of them (1 for 2252 , 3 for $832-1$ and 14 for 1343 hybrids). In 2010, 36\% did not have CLR, 11\% showed high incomplete resistance, and 15\% moderate to low resistance. By 2020, 26\% showed no CLR, 34\% had high incomplete resistance and 26\% low resistance, indicating segregation within TH collection. No all accessions were consistent for CLR reactions during these 20 years, but two of them (I567 and I573) from TH 1343 never showed CLR. To conclude, new CLR races are co-evolving on TH, and there is still resistance genes to incorporate into FNC Cenicafé's breeding program.

The effect of tractor speed, volume and a volute on spray deposition in tall pecan trees C. H. BOCK (1), M. W. Hotchkiss (2), (1) USDA ARS, Southeastern Fruit and Tree Nut Research Laboratory, Byron, GA, USA; (2) USDA ARS, Byron, GA, USA

Scab (caused by Venturia effusa) is the major disease of pecan in the southeastern US. In epidemic seasons yield loss is substantial in susceptible cultivars. Fungicide coverage from air-blast sprayers declines with height in the canopy. How application volume (L/ha), speed (kph) and use of a volute influence deposition (\% area) at different heights is unknown. Deposition was quantified using kromekote cards and vision pink dye at heights of 5, 7.2, 9.4, 11.6, 13.8, 15, 17.2 and $19.2 \mathrm{~m}$ using a patternator, and at 5, 7.5, 10, 12.5 and $15 \mathrm{~m}$ in tree canopies. A $3785 \mathrm{~L}$ air-blast sprayer was used at 468, 935 and $1871 \mathrm{~L} / \mathrm{ha}$ at 2.4 and $3.2 \mathrm{kph}$. A volute was also tested at 468 and $935 \mathrm{~L} / \mathrm{ha}$ at 2.4 and $3.2 \mathrm{kph}$ using the patternator. Nozzles were selected to provide proportionally similar volumes to the upper and lower canopy positions at set speeds. Speeds tested did not affect deposition. However, without a volute, higher volumes resulted in significantly greater deposition in the canopy, but most of that increase was at heights $<12.5 \mathrm{~m}$. Although significant differences existed at $\geq 12.5 \mathrm{~m}$, they were numerically small. In this study use of a volute resulted in more uniform deposition at all heights, but numerically or significantly more at heights $>15 \mathrm{~m}$, but less at heights $<15 \mathrm{~m}$ compared to other treatments. The study demonstrates that higher volumes result in more deposition, but the effect declines rapidly with height. Volutes may increase deposition at heights $>15 \mathrm{~m}$.

Chromosomally encoded copper resistance genes from Xanthomonas perforans reside on the genomic island S. BIBI (1), R. Bhandari (2), G. V. Minsavage Jr. (1), S. Timilsina (1), J. B. Jones (1), N. Potnis (3), (1) University of Florida, Gainesville, FL, USA; (2) Auburn University, Auburn, AL, USA; (3) Department of Entomology and Plant Pathology Auburn University, Auburn, AL, USA

Bacterial spot disease of tomato and pepper is a major problem in many tomato production areas. Growers have used copper-based bactericides to manage the disease, but this has resulted in the emergence of copper resistant $\left(\mathrm{Cu}^{\mathrm{R}}\right)$ strains. Most $\mathrm{Cu}^{\mathrm{R}}$ genes in plant pathogenic bacteria reside on plasmids; however, there are reports of $\mathrm{Cu}^{\mathrm{R}}$ genes associated with the chromosome. The goal of this study was to determine the dynamics of chromosome versus plasmid associated $\mathrm{Cu}^{\mathrm{R}}$ in an Xanthomonas perforans population. Using PacBio sequencing to assemble the complete genome of strain Xp2010 including plasmids, we found that the cop operon is harbored on the chromosome in a genomic island and that it lacks a large plasmid that has been reported to contain $\mathrm{Cu}^{\mathrm{R}}$ genes in $X$. euvesicatoria. The cop operon found in many X. perforans strains including Xp2010 and GEV1001 was also found to be associated with integrases, DDE transposase and phage related proteins which speculate the possibility of this genomic island to be phage integrated. The chromosomally encoded $\mathrm{Cu}^{\mathrm{R}}$ operon in $\mathrm{Xp2010}$ is identical in organization and sequence to plasmid-borne $\mathrm{Cu}^{\mathrm{R}}$ operon from $X$. 
euvesicatoria strain LH3. Analysis of the cop operons in many whole genome sequenced $X$. perforans strains, coupled with Southern hybridization of genomic and plasmid DNA of representative strains, revealed that the $\mathrm{Cu}^{\mathrm{R}}$ genes are present on the chromosome of many $X$. perforans strains including many strains from the early 1990's. This indicated its early presence in Florida following the original identification of $X$. perforans in 1991.

Modelling the phenology of Thripidae species associated with Soybean vein necrosis virus C. ZAMBRANA-ECHEVARRIA (1), S. Kaplan (1), B. Bradford (2), R. L. Groves (2), D. L. Smith (1), (1) Department of Plant Pathology, University of Wisconsin - Madison, Madison, WI, USA; (2) Department of Entomology, University of Wisconsin Madison, Madison, WI, USA

Changing weather patterns alter the distribution and abundance of plant virus vectors likely leading to emerging diseases. Soybean vein necrosis virus (SVNV; Tospoviridae) is an emerging virus first reported in 2008 as a pathogen of Glycine max (L.) Merr. SVNV is transmitted in a persistent propagative manner by Neohydatothrips variabilis, Frankliniella tritici, and Frankliniella fusca (Thripidae). An increase in thrips populations has been reported prior to the onset of symptoms. However, the relationship between SVNV vectors, concurrent virus detection, and environmental factors has not been determined in Wisconsin. Generalized additive mixed models using cumulative degree days were developed to describe the seasonal population dynamics of thrips and relate the timing of their flight activity with the detection of SVNV. Replicated sentinel plots were planted with Vigna unguiculata and G. max and repeated from 2017-2019 in Arlington, Wisconsin. Sticky panel traps were used to capture thrips and the number of adults were tabulated by random sampling. RT-PCR was performed to detect SVNV from asymptomatic and virus-like symptomatic plants, sampled weekly. Studying the phenology of SVNV vectors could influence planting dates to avoid peak flight periods, and insect management strategies to limit virus transmission. Modelling plant virus vectors is important for assessing the effect of the environment on vector abundance and its potential on future virus epidemics.

Metabolomic characterization of walnut bark and effects of methanolic extracts on walnut twig beetle feeding behavior J. SIMMONS (1), C. M. Wallis (2), S. Seybold (3), R. M. Bostock (1), (1) University of California, Davis, CA, USA; (2) USDA Agricultural Research Service, Parlier, CA, USA; (3) United States Department of Agriculture, Forest Service, Davis, CA, USA

Thousand cankers disease (TCD) affects members of the Juglandaceae, including economically important Juglans species in the U.S. and Italy. TCD is caused by the fungus Geosmithia morbida, an anamorphic species in the Bionectriaceae family, and its insect vector, Pityophthorus juglandis (walnut twig beetle, WTB). WTB adults inoculate host trees with G. morbida spores as the beetles penetrate the outer bark and form galleries within the inner bark. The fungus causes localized phloem lesions that coalesce and girdle branches. Certain walnut species (e.g., Juglans californica) appear to be more attractive to the WTB than other species (e.g., Juglans major). Volatile organic compounds (VOCs) from host trees likely influence WTB landing rates, and larger molecular weight compounds in the bark may act as feeding deterrents or stimulants. This study characterizes differences in bark metabolites between $J$. californica and $J$. major. VOC sampling of J. major and J. californica was done through solvent extraction of bark as well as sorbent collection followed by analysis with GC-MS. Analysis of larger molecular weight compounds was done by methanolic extraction of bark and quantitation using HPLC. A microfeeding assay measured differences in feeding behavior of WTB on J. californica and J. major extracts. Differences in metabolite content and WTB feeding activity between extracts were observed. This information will guide further studies into the functional role of specific compounds in the TCD pathosystem.

Fungicide sensitivity of Sclerotinia sclerotiorum from dry bean and soybean in the U.S.

E. H. NIETO-LOPEZ (1), T. J. J. Miorini (2), M. Chilvers (3), L. J. Giesler (4), T. A. Jackson-Ziems (1), M. Kabbage (5), D. S. Mueller (6), D. L. Smith (5), J. M. Tovar-Pedraza (7), J. F. Willbur (3), S. E. Everhart (1), (1) University of Nebraska, Lincoln, NE, USA; (2) Carrington Res Ext Center - North Dakota State University, Carrington, ND, USA; (3) Michigan State University, East Lansing, MI, USA; (4) University of Nebraska-Lincoln, Lincoln, NE, USA; (5) University of Wisconsin-Madison, Madison, WI, USA; (6) Iowa State University, Ames, IA, USA; (7) Centro de Investigación en Alimentación y Desarrollo, A. C., Culiacán, MEXICO

Increasing use of fungicides may lead to resistance, yet few studies have investigated this for S. sclerotiorum from dry bean (Phaseolus vulgaris) and soybean (Glycine $\max$ ) in the U.S. We sought to determine if there is a difference in fungicide sensitivity between: a) isolates from dry bean (DB) and soybean (SB), b) isolates exposed to intensive (fungicide trials; FT), moderate (producer fields; PF), and no fungicides (baseline), and c) isolates from PF that used single-site fungicides. Sensitivity to thiophanate methyl, tetraconazole, boscalid, and picoxystrobin was assessed using discriminatory concentrations. To compare groups by source (FT, PF, baseline) and host (DB vs. SB), sensitivity was assessed for a regional level of 165 isolates (1-3 per field), including 3 Mexican and 7 Brazilian isolates. SB isolates were less sensitive to boscalid than DB isolates ( 0.088 vs. $0.104 \mathrm{ppm} ; p<0.001)$ and PF isolates were less sensitive than baseline isolates $(0.088$ vs. $0.111 ; p<0.001)$. Moreover, FT isolates were least sensitive to picoxystrobin $(0.015 \mathrm{ppm} ; p<0.01)$. No difference was found for thiophanate methyl or tetraconazole $(p>0.05)$. Within-field sensitivity was assessed using an additional 188 isolates ( $\geq 9$ per field). Between fields, isolates from Mexico-Brazil group (0.017 ppm), Montcalm-1 (0.016 ppm), and Hancock (0.016 $\mathrm{ppm})$ were less sensitive to picoxystrobin than baseline isolates $(p<0.05)$. Cytochrome $b$ gene sequencing will determine whether mutations conferring resistance (G143A/S and F129L) are present among isolates least sensitive to picoxystrobin.

Effect of host and weather factors on the rate of lesion expansion of Septoria nodorum blotch on winter wheat U. ADHIKARI (1), C. Cowger (2,3), P. S. Ojiambo (4), (1) North Carolina state University, Raleigh, NC, USA; (2) USDA-ARS Plant Science Unit, Raleigh, NC, USA; (3) Department of Entomology and Plant Pathology, NC State University, Raleigh, NC, USA; (4) Department of Entomology and Plant Pathology, North Carolina State University, Raleigh, NC, USA 
Septoria nodorum blotch (SNB), caused by Parastagonospora nodorum, is a major disease of wheat occurring frequently in the southeastern US. Resistance to SNB in wheat is quantitative and is hypothesized to be partially determined by rate of lesion expansion, which can be affected by environmental factors. A study was conducted in North Carolina from 2018 to 2020 to quantify the effect of temperature and relative humidity on the rate of lesion expansion in moderately resistant and susceptible wheat cultivars. Disease was initiated by spreading $P$. nodorum infected wheat straw. Cohorts (i.e., groups of foliar lesions randomly selected and tagged as an observational unit) were sequentially selected and monitored throughout the season. Lesion area was measured at regular intervals, while weather data were collected from data loggers. Lesion area and rate of lesion expansion varied significantly $(P<0.05)$ among cultivars and cohorts. Final mean lesion areas ranged from 1.3 to $30.9 \mathrm{~mm}^{2}$ on susceptible and 0.3 to $8.6 \mathrm{~mm}^{2}$ on moderately resistant cultivars. Estimated rates of lesion expansion ranged from $0.19 \mathrm{~mm}^{2} /$ day for susceptible to $0.05 \mathrm{~mm}^{2} /$ day for moderately resistant cultivars. Covariance analysis showed that temperature and relative humidity significantly $(P<0.05)$ increased the rate of lesion expansion, the effect was greater on $\mathrm{S}$ than moderately resistant cultivars. This study shows that lesion expansion is an important component of SNB resistance in wheat and results are useful in breeding and tailoring spray programs to cultivars with varying levels of SNB resistance.

\section{Use of 'dry spores' of atoxigenic isolates of Aspergillus flavus simplifies commercial manufacturing of aflatoxin biocontrol} products in Africa

A. ORTEGA-BELTRAN (1), L. Kaptoge (2), S. Aikore (2), T. Falade (1), L. Senghor (3), R. Bandyopadhyay (4), (1) International Institute of Tropical Agriculture, Ibadan, NIGERIA; (2) International Institute of Tropical Agriculture, NIGERIA; (3) International Institute of Tropical Agriculture, Dakar, SENEGAL; (4) IITA, NIGERIA

Aflatoxin contamination of crops negatively impact human and animal health, and hamper trade and economic development. Aflatoxin contamination can be mitigated through use of atoxigenic isolates of Aspergillus flavus. In Africa, various biocontrol products under the tradename Aflasafe have been developed. Each product contains four atoxigenic isolates native to the target nation where the product is used. Private sector licensees manufacture Aflasafe in factories using spores of the atoxigenic isolates freshly produced in laboratories near the factory. Trillions of spores are produced daily on sterile sorghum, harvested and diluted to appropriate concentrations. Some manufacturing partners have had difficulties to obtain equipment and materials to produce their own spores. To overcome this challenge, a process was developed for producing high-quality dry inoculum. Spores suspensions produced as above were filtered through a special membrane and then dried. Viability and stability of the dry spores were monitored for 6 months. In 2019, Aflasafe SN01 was manufactured using dry inoculum produced by IITA in Ibadan, Nigeria and sent by courier to BAMTAARE, the manufacturer in Senegal. BAMTAARE produced the product with dry inoculum to treat circa 20,000 ha of groundnut in Senegal and The Gambia. Aflasafe SN01 manufactured with dry inoculum was as efficient to reduce aflatoxin contamination as the one manufactured with freshly produced spores. The dry spore innovation will make commercial manufacturing of aflatoxin biocontrol products more efficient in several African nations.

Estimating pathogen movement across complex agricultural landscapes from pathogen phylogenies F. GUO (1), D. Rasmussen (2), (1) NC State University, Raleigh, NC, USA; (2) North Carolina State University, Raleigh, NC, USA

Tracking the spatial spread of plant pathogens is key to understanding, preventing and controlling disease spread. Phylogeographic methods can help reveal the migration of sampled pathogen lineages between populations from genetic sequence data. However, uneven sampling across populations may lead to severe biases in our inference of movement patterns if additional demographic information is not incorporated. Here we develop a new method based on structured coalescent models for reconstructing movement through spatial networks. Unlike current methods, our new method allows us to consider a large number of sequences sampled across a potentially large number of sampling locations. Moreover, our methods allow for spatial covariates such as geographic distances, climatic variables and crop connectivity to inform estimates of migration rates. Using both simulated and empirical data sets, we show that our method can overcome biases caused by uneven sampling and accurately estimate migration rates between locations. We also demonstrate how our methods can be used to identify spatial covariates that accurately predict pathogen movement. Thus, our method provides a powerful tool for reconstructing the spread of plant pathogens through complex agricultural landscapes and to identify landscape features that drive spatial spread.

Sudden Fir Mortality in New Brunswick, Canada: History and dendrochronological analysis Y. ZHOU (1), K. K. L. Costanza (2), (1) University of New Brunswick, Fredericton; (2) US Forest Service, Missoula, MT, USA

Balsam fir (Abies balsamea) is an ecologically and economically significant species in New Brunswick. In 2018, sudden foliar drying and discoloration occurred in many balsam fir trees province-wide. Trees turned red and died in one growing season, causing approximately $\$ 6$ million loss. A similar phenomenon occurred in the 1980s, referred to as Sudden Fir Morality (SFM). Previous research suggests possible causes include winter drying, drought, and other climatic variables; insects; and fungal pathogens, principally Armillaria root rot (Armillaria spp.). However, a detailed history of this phenomenon has not been developed, nor has a tree-level response or predisposing factors been elucidated. To address these knowledge gaps, this study set out to 1) quantify annual growth in both asymptomatic and symptomatic trees; and 2) determine which geographical, climatic, and site-level conditions predispose trees. Across western New Brunswick, 18 plots were sampled. Tree cores $(n=79)$ were collected from both asymptomatic and symptomatic trees. Image analysis software was used to measure annual growth. Results indicate symptomatic trees are already in decline prior to the onset of SFM, compared to asymptomatic trees. Historical growing season droughts and rapid spring thaws have been associated with recent occurrences of SFM. Additionally, $89 \%$ of sampled balsam fir trees had evidence of Armillaria spp. The results of this study can improve understanding of tree- and site-level factors predisposing trees to SFM, which will be useful in managing SFM and reducing financial losses. 
Development and application of a qPCR assay for early detection and quantification of the root-lesion nematode Pratylenchus scribneri in potato roots

D. ARORA (1), G. Yan (2), (1) North Dakota State University, Fargo, ND, USA; (2) North Dakota State University, Department of Plant Pathology, Fargo, ND, USA

The endoparasitic root-lesion nematode, Pratylenchus scribneri impacts potato production globally. Effective management of this nematode is contingent on its accurate detection and quantification. Typically, the nematode population estimates are made from soil, but considering the endomigratory lifestyle of this nematode, it is indispensable to quantify the nematodes dwelling inside the host roots too. In this study, a novel SYBR green-based real-time quantitative PCR (qPCR) assay was developed for direct detection and quantification of $P$. scribneri in potato roots. The assay used a previously reported $P$. scribneri-specific primer pair (ITS-2F/ITS-2R) and proved to be specific and sensitive, detecting as low as $7.8 \times 10^{-3}$ of a $P$. scribneri individual per $0.2 \mathrm{~g}$ of root tissue. The robustness of the assay was evaluated using artificially inoculated potato roots, naturally infected field potato root samples, and infected potato roots from a greenhouse trial. A high co-relation was obtained between the real numbers of nematodes and the those detected by qPCR in both artificially inoculated $\left(\mathrm{R}^{2}=0.93\right)$ and naturally infected potato roots $\left(\mathrm{R}^{2}=0.73\right)$. A time-course experiment carried out in the greenhouse detected $P$. scribneri in potato roots as early as 7 days through qPCR and the data showed the completion of its one life cycle in about 5 weeks, with root staining further complementing the results. Overall, the assay is specific and sensitive to $P$. scribneri and sheds light on various important aspects, such as early detection and life cycle timings of this important nematode.

Evaluation of fungicides as part of an integrated approach for management of Fusarium head blight in durum A. K. KALIL (1), D. L. Fonseka (1), T. Gargouri Jbir (1), T. Tjelde (1), J. Ransom (2), C. Deplazes (2), D. Eisinger (2), E. Bauske (3), J. Halvorson (3), S. M. Meyer (3), C. Schuh (3), A. J. Friskop (3), (1) North Dakota State University Williston Research Extension Center, Williston, ND, USA; (2) North Dakota State University, Department of Plant Sciences, Fargo, ND, USA; (3) North Dakota State University, Department of Plant Pathology, Fargo, ND, USA

Northwest North Dakota and northeast Montana account for over $90 \%$ of the durum acreage in the United States. Fusarium head blight (FHB) is arguably the most devastating disease in this region. Varietal resistance is the most important FHB management tool, however there is very little resistance in durum. Five integrated management (IM) trials evaluating fungicides and varietal resistance were conducted in 2018 and 2019 on durum in North Dakota. A combination of fungicides (pydiflumetofen + propiconazole or prothioconazole + tebuconazole) applied at Feekes 10.3 - half-head emergence, Feekes 10.51 - early-anthesis, or 3 to 7 days after Feekes 10.51 were evaluated on susceptible and moderately susceptible varieties. Low to moderate levels of disease occurred in four trials while high levels of disease occurred in one trial. Susceptible varieties had higher disease levels, higher deoxynivalenol (DON) accumulation, and lower yield under both low and high disease environments. With few exceptions, fungicide applications resulted in statistically lower DON and FHB than the non-treated control. Application of pydiflumetofen + propiconazole at Feekes 10.51 resulted in less DON than when applied at Feekes 10.3. Fungicide application 3 to 7 days after Feekes 10.51 lowered DON and was not significantly different from fungicide applications made at Feekes 10.51 . These studies suggest that satisfactory disease control may not be obtained when pydiflumetofen + propiconazole is applied at Feekes 10.3, however more studies are needed to generate application timing recommendations.

Soilborne Fusarium population levels associated with sweet corn and snap bean field soils in western Oregon C. M. OCAMB (1), K. Buckland (2), W. Thomas (1), A. Rasmussen (2), T. Bates (1), T. Flodquist (1), M. Dietrich (1), (1) Oregon State University, Corvallis, OR, USA; (2) Oregon State University, Aurora, OR, USA

Fusarium spp. are well known pathogens of corn and beans, causing root rot as well as crown and stalk rot and other diseases. Fusarium populations in two sweet corn fields and one snap bean field were determined in representative soil samples collected every two weeks during the cropping cycle, and then monthly after harvest. Soil subsamples were evaluated for Fusarium species by plating serial dilutions onto a Fusarium-selective medium. Disease incidence and severity ratings were recorded for sweet corn and snap bean plants on each sampling date. We also monitored soil temperature and soil moisture in each field at 6" and 12" below the soil surface to describe overall field conditions throughout the sampling period. Fusarium crown and stalk node rot as well as root rot were present in both sweet corn fields monitored, and crown rot was severe by the first sampling date. Rot of the adventitious root system was not severe in the sweet corn plants while snap bean plants exhibited high levels of root rot. All snap bean plants sampled had lesions on the lower stem by four weeks prior to harvest. The number of Fusarium colony forming units per gram of oven-dried soil were high overall, especially in one sweet corn field where soilborne Fusarium population levels were consistently greater than $10^{6} \mathrm{~g}^{-1}$ soil, and populations remained high at two months after harvest. An evaluation of the potential impact of environmental conditions on Fusarium populations and subsequent disease in vegetable crops is planned as additional seasons of data are collected.

Transformation of Raffaelea lauricola with GFP to visualize its colonization of swamp bay, Persea palustris J. KONKOL, Q. Wang, J. A. Rollins, University of Florida, Gainesville, FL, USA

Laurel wilt disease, caused by the fungus Raffaelea lauricola, has devastated members of the Lauraceae family throughout the southeastern United States. It is an exceptionally aggressive vascular wilt disease. Mortality of large avocado trees (Persea americana) in the orchard can be achieved with artificial inoculations of 40 conidia. Colonization of swamp bay (Persea palustris) by the pathogen was investigated previously with a transformant of $R$. lauricola labeled with a green fluorescent protein (GFP) using the pCT74-sGFP vector under control of the ToxA gene promoter, but the strain did not fluorescence in planta and required enrichment on media before observation. A new transformant of $R$. lauricola produced using the pRIEF-sGFP vector under control of the endogenous translation 
elongation factor promoter produced strong fluorescence both on media and in planta. This strain allows for spatial-temporal investigations of pathogen morphology during host colonization and tissue-specific interactions within the host. Swamp bay trees were inoculated with 3,000 conidia of the GFP-labeled strain, and three trees per time point were harvested 3, 7, 10, 14, 21 and 28 days after inoculation. Trees were sampled every $10 \mathrm{~cm}$ above the inoculation point along the main stem and $10 \mathrm{~cm}$ below the inoculation point. Sections were taken at each $10 \mathrm{~cm}$ interval and observed by epifluorescence microscopy. Preliminary observations have demonstrated movement of spores through the xylem exceeding $20 \mathrm{~cm} /$ day, and revealed pathogen morphology associated colonization of host xylem tissue over time.

Antimicrobial activity of endophytic fungi from the silver frailejon (Espeletia argentea) against Phytophthora infestans D. DUPLAT, S. Restrepo, M. Cardenas, Universidad de Los Andes, Bogotá, COLOMBIA

The development of new antimicrobial compounds is a constant need, given the permanent emergence of microorganisms resistant to antibiotics and fungicides. Endophytic fungi are a potential source of these compounds. The interaction between plant and fungi implies a constant metabolic exchange that increases the production of secondary metabolites, some of them with antimicrobial activity. Longliving and endemic plants from ecosystems with extreme environmental conditions are ideal for the isolation of endophytes. These requirements are met by the silver frailejón (Espeletia argentea), that grows in the paramo ecosystem. The aim of this study was to evaluate the production capacity of secondary metabolites with antimicrobial activity from endophytic fungi isolated from $E$. argentea leaves against important plant pathogens present in Colombia, and to preliminarily assess the metabolite production of these fungi in solid media. Fifty-one different endophytic fungi were obtained and their antagonistic properties were tested against Fusarium sp. and a fungicide resistant $P$. infestans strain. Seven isolates were able to significantly inhibit the growth of $P$. infestans $(\mathrm{P}<0.05)$ and were identified. The three most promising isolates were further studied and their metabolite production in solid media was preliminarily assessed. This work explores the production of fungicides from natural origin as an alternative to the chemical pesticides commonly used in disease management.

Development of a qPCR assay for detection and quantification of Fusarium sambucinum in soil and plant tissue W. THOMAS (1), T. Borland (1), B. Claassen (1), T. Flodquist (1), T. Bates (1), C. M. Ocamb (1), D. H. Gent (2), (1) Oregon State University, Corvallis, OR, USA; (2) US Department of Agriculture, Agricultural Research Service, Corvallis, OR, USA

Fusarium sambucinum can infect a range of plant hosts and incites a number of diseases including Fusarium canker in hop, dry rot in potato tubers, root and crown rot in grass seed crops, and stalk, root, and crown rot in corn. In addition to the economic losses caused by damage to crops, $F$. sambucinum can produce several different types of mycotoxins that can pose a threat to human and animal health. Factors that can contribute to the development of $F$. sambucinum-mediated disease include wounding of plant tissues and buildup of $F$. sambucinum populations in soil. Here, we describe the development of a qPCR assay to allow for rapid and reliable detection and quantification of $F$. sambucinum in plant tissue, soil, and other environmental samples. Using a novel method of primer discovery adapted from RAPD PCR, we identified a species-specific sequence in $F$. sambucinum, which was used to design $F$. sambucinumspecific primers and a TaqMan probe. This qPCR assay has been used to detect $F$. sambucinum from as little as $1 \mathrm{pg}$ of $F$. sambucinum genomic DNA isolated from pure culture. Moreover, this method was specific to F. sambucinum when tested against six other Fusarium species. Finally, this method reliably detected $F$. sambucinum isolates from multiple plant hosts, including hop, potato, corn, fescue, and perennial rye grass, as well as soil. Additional work is being conducted to determine the utility of the assay in detecting and quantifying the pathogen in a background of genomic DNA isolated from plant tissue and from soil from commercial fields.

\section{Relationship between phytopathogenic nematode densities and soil microbial communities across different production zones in} Pennsylvania

A. Y. BANDARA, D. K. Weerasooriya, R. Trexler, T. Bell, P. Esker, The Pennsylvania State University, University Park, PA, USA

Phytopathogenic nematodes (PPN) can adversely affect crop yields. Spatial variation of soil microbial communities may be useful to understand the spatial variation of PPN. Bulk soils sampled in 2018 from 14 soybean farms across different production zones in Pennsylvania at the V1 (one trifoliate) growth stage were profiled for PPN (counts $/ 500 \mathrm{~cm}^{3}$ soil). Lesion, spiral, and lance were the most prevalent nematode types across samples. DNA extracted from a portion of each soil sample was subjected to PCR targeting fungal ITS regions (ITS1F/58A2R) and bacterial 16S rRNA genes (515F/806R). Amplicons were sequenced using MiSeq platform. Non-rarefied read counts were classified into genus level OTUs and the most abundant 50 fungal $(=\mathrm{F})$ and bacterial $(=\mathrm{B})$ genera were sorted. Nematode counts were regressed against sorted OTU counts using stepwise regression at $\alpha=0.025$. Lesion nematode counts were explained by + Acremonium $(\mathrm{F}),+$ Dendryphion $(\mathrm{F})$, -Ilyonectria $(\mathrm{F}),+$ Lasiosphaeriaceae $(\mathrm{F})\left(P<0.0001 ;\right.$ adjusted $\left.\mathrm{R}^{2}=0.68\right)$. Spiral nematode counts were explained by + Capnodiales $(\mathrm{F})$, -Phallus $(\mathrm{F})$, and + Verticillium $(\mathrm{F})\left(P<0.0001\right.$; adj. $\left.\mathrm{R}^{2}=0.72\right)$, while lance nematode counts were explained by + Leotiomycetes $(\mathrm{F}),+$ Ellin329 (B), +Haliangiaceae (B), -Pseudomonas (B), and -WD2101 (B) $\left(P<0.0001\right.$; adj. $\left.\mathrm{R}^{2}=0.79\right)$. These findings suggest potential synergistic and antagonistic biological interactions between specific PPN and fungal/bacterial taxa across broader geographical regions. Results also provide insights into potential avenues for biocontrolling lesion, spiral, and lance nematodes.

Characterisation of $\boldsymbol{F}$. musae strains obtained from plants and humans

V. TAVA (1), E. Calastri (2), A. Kunova (3), M. Saracchi (3), A. M. Tortorano (2), M. C. Esposto (2), P. Cortesi (3), M. Brock (4), G. Vande Velde (5), M. Pasquali (3), (1) Università degli studi di Milano, Milano, ITALY; (2) Università degli studi di Milano, Milano, ITALY; (3) DeFENS, Università degli Studi di Milano, Milano, ITALY; (4) University of Nottingham, Nottingham, ENGLAND; (5) KU Leuven, Leuven, BELGIUM 
Fusarium musae was described for the first time as a species in 2011. It appears to have its evolutionary origin within the African clade of the F. fujikuroi species complex. Recent reports have identified $F$. musae strains in human patients causing various diseases such as nail and skin lesions as well as systemic infections in immunocompromised patients. A worldwide collection of Fusarium strains attributed to $F$. musae obtained from banana and human patients was characterised phylogenetically using four informative genes $\beta$ tubulin, Translation Elongation Factor $1 \alpha$, DNA-directed RNA polymerase II subunit and calmodulin; appropriate classification of the different strains was then established. The draft genomes of two representative strains colonizing banana and humans were obtained (genome size: $45 \mathrm{Mb}$ ). Furthermore, the pathogenic behaviour of each strain on detached bananas was investigated, showing the ability of the strains of human origin to cause significant crown rot infection on banana fruit. To identify the virulence and the pathogenicity mechanisms differences of representative strains, Galleria mellonella model is currently being employed. Bioluminescent and fluorescent strains are currently being produced to study the interactions with the different hosts.

What can the ecology and evolutionary history of plant pathogens tell us about the contours of host range? C. E. MORRIS (1), G. Henry (2), C. Monteil (2), B. Moury (2), (1) INRAE, Montfavet, FRANCE; (2) INRAE, Pathologie Végétale, Montfavet, FRANCE

Plant pathogens are often classified into groups (pathovars, formae speciales, races) that purport to assemble strains of a same phylogenetic cluster that have distinct host ranges. However, there are many examples where host ranges cannot be clearly delimited, such as the overlapping continuum of host range in Pseudomonas syringae. The "co-evolutionary arms race" between plants and pathogens is supposed to structure plant and pathogen populations. Yet, the evolution of many plant pathogens at the scale of life on land (that begun ca. 450 million years ago) does not necessarily mirror that in their modern crop hosts. We are determining how the fitness of strains of $P$. syringae is delimited within and beyond angiosperms and if the fitness of bacteria varies relative to taxonomic groupings (order, family, genus) or the evolutionary proximity of plants. Plants inoculated for bacterial fitness measurements include $>50$ species of Polypodiales, Ginkgoales and five orders of angiosperms. Strains representing the most ancient and environmentally ubiquitous lineages of $P$. syringae were able to multiply in plants across all orders tested, with no apparent effect of the taxonomic groups of plants. Tests with more recent and less widespread lineages are in progress. The overall variability in fitness within plant species suggests a role for stochastic processes in microbial multiplication in plants.

Effect of subinhibitory doses of the fungicide iprodione on the plant pathogenic fungi Fusarium oxysporum and $\boldsymbol{F}$. proliferatum V. FREIRE-ZAPATA (1), S. Marek (1), H. A. Melouk (2), O. Arias Gaguancela (3), M. E. Gard (1), F. J. Flores (4), C. D. Garzon PhD (1), (1) Oklahoma State University, Stillwater, OK, USA; (2) USDA ARS, Stillwater, OK, USA; (3) Texas A\&M Agrilife Center in Dallas, Dallas, TX, USA; (4) Universidad de las Fuerzas Armadas ESPE, Sangolqui, ECUADOR

Subinhibitory doses of fungicides can stimulate growth rate, spore germination, toxin production and virulence in fungal plant pathogens. However, little is known about changes in gene expression occurring during hormetic stimulation. The objective of this study was to examine the effects of subinhibitory doses of iprodione on growth, virulence, and expression of selected genes of two Fusarium spp. Fusarium oxysporum and $F$. proliferatum were isolated from white onion and transformed to express tdTomato fluorescent protein. Wild-type and transformed isolates were grown in vitro on SNA medium amended with eleven iprodione concentrations, including subinhibitory and suppressive doses and a fungicide-free control. Mycelial area was measured at 24, 48 and 72 hours post inoculation (hpi). Additionally, transformed isolates were inoculated onto surface-disinfected onion bulb epidermis dipped in fungicide and their growth was measured using epifluorescence microscopy. Subinhibitory doses of iprodione stimulated the mycelial growth of both pathogens. $F$. oxysporum displayed an average growth stimulation of $20 \%, 10 \%$ and $8 \%$ after 24,48 and 72 hpi, respectively, while $F$. proliferatum displayed $26 \%$ stimulation at 24 and 48 hpi, and $19 \%$ at 72 hpi. On onion epidermis, $F$. oxysporum displayed growth stimulation of $32 \%$ and $19 \%$ at 48 and 72 hpi, while $F$. proliferatum had $20 \%$ stimulation at 24 hpi only. Further experiments will attempt to elucidate molecular changes during hormetic stimulation by assessing the differential gene expression of housekeeping and pathogenicity-associated genes.

\section{Pydiflumetofen as a wheat seed treatment for management of Fusarium culmorum and Pythium ultimum under wet and dry soil} conditions

T. BATES (1), D. H. Gent (2), T. Flodquist (1), M. Dietrich (1), C. M. Ocamb (1), (1) Oregon State University, Corvallis, OR, USA; (2) US Department of Agriculture, Agricultural Research Service, Corvallis, OR, USA

The soilborne organisms, Fusarium culmorum and Pythium ultimum, are well-known pathogens that affect wheat and cause symptoms leading to stand losses and reduced crop yields. Limited work has been done considering the synergism between these two organisms and the role of soil moisture. This study investigated F. culmorum and P. ultimum in both dry and wet soil conditions under glass house trials and their associated effects on shoot and root weights and plant emergence after 28 days. Additionally, the efficacy of a seed fungicide treatment, Miravis (pydiflumetofen), was investigated. Wheat seed treated with Maxim XL (mefenoxam + fludioxonil) + Vayantis (picarbutrazox) as well as Maxim XL + Miravis had the highest mean percentage of plant emergence (95\%) under wet soil conditions. Nontreated seed growing under both dry and wet soil conditions had the lowest percentage emergence $(64 \%$ and $48 \%$, respectively), which were both lower than fungicide-treated seed. Each fungicide seed treatment improved the percentage of plant emergence anywhere from $21 \%$ to $56 \%$ in the presence of $P$. ultimum and/or F. culmorum. Wet soil reduced percentage emergence by $13 \%$ in P. ultimum treatments, but increased plant emergence by $13 \%$ in F. culmorum. Fungicide seed treatments did not impact mean shoot weight or root weight but were highly effective for establishing a healthy plant stand. 
Host-selective toxins by Corynespora cassiicola are determinants of host-specificity to target spot disease of cotton and tomato M. A. SAMACO (1), R. Kemerait (2), A. E. Glenn (3), T. Mitchell (3), M. T. Brewer (1), (1) University of Georgia, Athens, GA, USA; (2) University of Georgia, Tifton, GA, USA; (3) USDA-ARS, Toxicology \& Mycotoxin Research Unit, Athens, GA, USA

Host-selective toxins (HSTs) are virulence factors that have been implicated in host-specificity. Target spot disease, caused by Corynespora cassiicola, is gaining importance in the southeastern United States (U.S.) due to considerable yield losses on cotton and tomato crops. Isolates of $C$. cassiicola collected from the southeastern U.S. possessed putative gene clusters of HSTs or secondary metabolites in their genomes. However, their roles on host-specificity and virulence is unknown. Our goal is to identify the HSTs produced by $C$. cassiicola and to define their function on host-specificity by establishing interactions with host plants. Culture filtrates from C. cassiicola isolates collected from cotton, tomato and soybean were extracted and infiltrated onto these hosts, subjected to Proteinase-K treatment and a bio-assay guided fractionation. Plants were evaluated for symptoms in a greenhouse assay. We found that lesion areas were statistically significant for fungal extracts infiltrated onto their host of origin, signifying host specificity. These fungal filtrates have non-proteinaceous components causing plant toxicity. We separated the active compounds involved in pathogenicity into distinct chemical fractions. Cotton and tomato C. cassiicola HSTs were found to have different chemical characteristics. In-depth identification is being conducted through HPLC-MS and NMR. The study of HSTs is a fundamental first step in understanding hostpathogen interactions in C. cassiicola pathosystems and in screening for resistant target spot varieties of these crops.

Deciphering the soil microbiome from native and exotic Ecuadorian Forests B. S. Quintero García, A. M. Rivera Casignia, A. C. Uvidia Calles, D. A. Román Robalino, J. H. Rodriguez Guerra, P. I. ALVAREZ ROMERO, Escuela Superior Politécnica de Chimborazo, Riobamba, ECUADOR

Ecuador has been considered a megadiverse country with different types of native forests. The microbiome of these plants may play an important, although largely unknown, role in forest health, providing a useful resource for studying beneficial microbial communities. Hence, this study aimed at analyzing the fungal microbiota composition and diversity of native and exotic Ecuadorian Forest soils in the Chimborazo province. Six samples from soils of native forests, three from soils of exotic forests and one from soil of an agriculturetransition area were collected. DNA was extracted from the soil samples and the fungal Internal Transcribed Spacer (ITS) region were amplified and sequenced using the Illumina Miseq platform. Members of the phyla Glomeromycota, Mortierellomycota and Rozellomycota were more abundant in samples of native forests. In contrast, members of the phylum Ascomycota were more frequently found in soil of exotic forests. Interestingly, the highest fungal diversity was found in the samples from native forests. The information on fungal composition and diversity in Ecuadorian forests is critical in the development of a more targeted sustainable forest management strategy.

Development of a sequential sampling plan using spatial attributes of Cercospora leaf spot epidemics in table beet in New York D. W. HECK (1), J. Kikkert (2), L. E. Hanson (3), S. J. Pethybridge (1), (1) Cornell University, Geneva, NY, USA; (2) Cornell Cooperative Extension, Canandaigua, NY, USA; (3) USDA ARS, East Lansing, MI, USA

Sampling strategies that effectively assess disease intensity in the field are important to underpin management decisions. To develop a sequential sampling plan for the incidence of Cercospora leaf spot (CLS), caused by Cercospora beticola, 31 table beet fields were assessed in 2017 and 2018 in New York. Assessments were performed in three to six transects per field. Transects were assessed in 51 sampling locations containing six leaves each on which the presence or absence of CLS symptoms were recorded. Incidence $(\hat{p})$ and spatial pattern analyses were performed for individual transects and results were used to develop sequential sampling estimation and classification models. Bootstrap simulations evaluated the level of precision of the models developed. CLS incidence ranged from 13\% to $92 \%$ with a median of $31 \%$ and the beta-binomial distribution, that is associated with aggregation, best described the spatial patterns observed. Sequential estimation models estimated disease incidence $(p)$ with a preselected coefficient of variation of the mean. At the preselected level of precision, as the Average Sampling Units (ASN) to be evaluated increased, and the difference between the true $\hat{p}$ and achieved $p$ decreased. Models based on prespecified thresholds $\left(p_{t}\right)$ classified the datasets as above or below $p_{t}$ across a range of CLS incidence. As $p_{t}$ increased, the ASN and the number of correct decisions decreased. Results indicated that the sequential sampling plans developed for CLS can be used to effectively assess disease incidence in table beet fields.

Exploring the potential of plant growth-promoting rhizobacteria (PGPR) as next biocontrol agents for controlling Phytophthora fruit rot on watermelon

M. K. MANDAL PHD (1,2), A. Boroujerdi (1), J. Taylor (1), S. Fleming (1), T. Pikes (1), R. Harris (1), C. S. Kousik (3), (1) Claflin University, Orangeburg, SC, USA; (2) U.S. Vegetable Laboratory, USDA ARS, Charleston, SC, USA; (3) USDA ARS, Charleston, SC, USA

Phytophthora fruit rot caused by the oomycete Phytophthora capsici, is one of the most devastating pathogen affecting watermelon production in most states in the Southeastern US and current management strategies are not very effective. The watermelon fruits can become infected at any stage of maturity, either from direct contact with the soil or from splashing rain or irrigation. Fungicides remain an important method to control losses due to P. capsici. However, due to high disease pressure in Southeastern states as a result of extreme weather conditions (e.g., flooding, hurricanes), and development of fungicide insensitivity/resistance in $P$. capsici fungicides applications do not always help manage the disease. Since fully restricting the movement of $P$. capsici in fields is often impossible, one of the best alternative sustainable approaches is to prevent fungicide insensitivity in $P$. capsici is the use of environmental friendly plant growth promoting rhizobacteria (PGPR) bacterial isolates as biocontrol agents with antifungal properties, which can suppress pathogen growth both in field as well as during storage and transport. We have identified several PGPR bacterial isolates from natural environments, which inhibit $P$. capsici growth on V8 media. Some PGPR bacterial isolates also suppressed Phytophthora fruit rot 
development on watermelon. We have further identified the secondary metabolites associated with specific PGPR extracellular extracts through NMR and LC-MS/MS based PCA and spectral buckets analysis showing potential antifungal properties against $P$. capsici.

The survivability of Trichoderma-based biocontrol agents within a Myakka fine sand in Florida L. YANG (1), J. D. Carrillo (2), E. Van Santen (3), A. Suazo Tejada (4), G. E. Vallad (2), (1) China Agricultural University, Beijing, CHINA; (2) Gulf Coast Research and Education Center, University of Florida, Wimauma, FL, USA; (3) University of Florida, Gainesville, FL, USA; (4) Department of Plant Sciences, Escuela Agrícola Panamericana El Zamorano, Tegucigalpa, HONDURAS

Trichoderma are ubiquitous in soils and are commonly used as biological control agents (BCAs). However, BCA efficacies are limited by different soil types and little is known about how they survive in Myakka fine sand in Florida. Therefore, a field trial was conducted on two fumigated beds, one bed with and one bed without fertilizer, to investigate the survival of different Trichoderma over time. Four Trichoderma BCAs and a non-treated control were arranged in RCBD and drip-applied at $6.8 \times 10^{7}$ colony forming units (CFU) per plot. The soil was sampled at a depth of $0-10$ and 10-20 cm, and at a distance of $15 \mathrm{~cm}, 30 \mathrm{~cm}$ and $45 \mathrm{~cm}$ away from the drip-tape at 1day then 2, 4, 6, 8, and 10 weeks post-application (WPA). The results of this study indicate Trichoderma did not significantly differ in recovery over 10 weeks. Trichoderma CFUs at 2 WPA reached the highest value with average $3.0 \times 10^{5}$ per g of soil, then decreased gradually over time. In addition, the sampling distance within each treatment plot significantly affected Trichoderma recovery $(P<$ 0.001 ), with the highest recovery at $15 \mathrm{~cm}$ away from the drip-tape and lower recovery at 30 and $45 \mathrm{~cm}$ away, respectively. Sampling depth also had a significant effect on Trichoderma recovery $(P<0.001)$, with recovery at $0-10 \mathrm{~cm}$ depths greater than at $10-20 \mathrm{~cm}$. The application of fertilizer had no significant effect on Trichoderma recovery. These results suggest that the survivability of Trichoderma BCAs is limited in Myakka fine sand over time, which may lead to reduction in their ability to target pathogens present in fields.

Sweetpotato resistant germplasm selection to Fusarium wilt pathogen using a modified screening method W. PARK (1), H. U. Lee (1), S. Goh (1), N. C. Paul (2), S. S. Nam (1), S. Lee (1), M. N. Chung (2), I. B. Lee (1), (1) Bioenergy Crop Research Institute, NICS, RDA, SOUTH KOREA; (2) Bioenergy Crop Research Institute, NICS, RDA, Muan, SOUTH KOREA

Fusarium wilt disease, in which the sweetpotato stems are cracked or wilted, is caused by Fusarium oxysporum f. sp. batatas (Fob), and it creates the problem in the sweetpotato cultivation. Therepore, this study was conducted to investigate the Fusarium wilt resistance of 519 sweetpotato germplasms collected from Korea and overseas by a rapid screening method via the hydroponic system. We selected the most virulent three pathogen isolates among ten obtained from wilting symptoms of sweetpotato field. To find optimal assay condition for determining the resistance to Fusarium wilt, we investigated and optimized the effects of pathogen inoculation media, media concentration, conidial concentration and culture period. Healthy sweetpotato stalks with no apparent disease symptoms were grown in suspensions containing $1 \times 10^{5} \mathrm{spores} / \mathrm{ml}$ conidial concentration of Fob at $28^{\circ} \mathrm{C}$, and assessed the disease development 14 days after inoculation. The disease severity index of the germplasms widely ranged from 0 (no symptom) to 5 (completely died caused by Fusarium wilt). Among all the germplasms, 63 including IT 232008 showed strong resistance (100\% survival from Fusarium wilt) and 47 including IT 232024, showed high level of susceptibility scoring 5 and other 110 showed high level of resistance or sensitivity to the disease, which could be possible to use for genetic and genomic studies and will accelerate breeding programs for sweetpotato with Fusarium wilt resistance.

Genome scale sequencing for rapid and efficient detection of pest nematodes in agricultural soils

J. WANG (1), S. Kumar (1), M. Song (1), S. F. Hanson (2), (1) New Mexico State University- Depart of Computer Science, Las Cruces, NM, USA; (2) New Mexico State University- EPPWS, Las Cruces, NM, USA

Plant parasitic nematodes are a leading cause of losses to agriculture worldwide. However, it is challenging to identify them from soil samples due to difficulty in using limited morphological characters to separate similar benign species from serious pest species, especially those with quarantine implications. Current identification pipelines rely on highly trained personnel to perform either detailed morphological analysis or pathogenicity testing. Both methods have serious time and capacity limitations. Here we report on the development of a genome scale sequencing approach that characterizes the entire nematode community in a soil sample. The method utilizes the long-read DNA sequencing platform which allows determination of contiguous rDNA sequences including the 18S and ITS regions without any in silico assembly. We also describe an optimized bioinformatic analysis pipeline that enables rapid and efficient detection of rare targets in complex populations of rDNA sequences. The results presented show that this pipeline is able to detect rare sequences of high interest from nematode populations in soil samples, even when related benign species are present in the same samples. Thus, this pipeline has a potential to replace current low-throughput systems for detection of pest nematodes, especially those of regulatory concern like D. africanus. This pipeline is broadly applicable for surveillance of most if not all nematodes of agricultural interest and regulatory importance.

Engaging with commodity boards: Understanding the proposal process to maximize your research J. MAYORQUIN, M. Klein, Citrus Research Board, Visalia, CA, USA

The Citrus Research Program, administered by the Citrus Research Board (CRB), is a grower-funded and grower-directed program established in 1968 under the California Marketing Act as a mechanism enabling California's citrus producers to ensure a sustainable California citrus industry for the benefit of growers by prioritizing, investing in and promoting sound science. The CRB's research proposal process differs from other funding programs (i.e., federal and academic) in several ways, most notably in its proposal review process which includes a lay audience. With a few exceptions where basic research is necessary to ultimately address the needs of the industry, the majority of proposed research should focus on specific, achievable outcomes or deliverables which can be implemented by 
the industry at the conclusion of the proposed research. To maximize a research team's proposed research, several considerations should be given to the delivery of the research's message. Proposed research should be communicated appropriately based on the audience (i.e., using non-technical language when engaging with a lay audience) and the research should clearly relate to the priorities of the program. Adhering to these considerations will improve the overall communication and success of proposed research between a research team and its funder.

Assessing the population genetic structure of corn tar spot pathogen Phyllachora maydis in Midwestern United States, 2019 T. J. ROSS (1), J. L. Chaky (2), N. M. Kleczewski (3), D. S. Mueller (4), A. E. Robertson (4), D. L. Smith (5), D. E. P. Telenko (6), (1) Purdue University, West Lafayette, IN, USA; (2) Corteva Agriscience, The Agriculture Division of DowDuPont, Johnston, IA, USA; (3) University of Illinois, Urbana, IL, USA; (4) Iowa State University, Ames, IA, USA; (5) University of Wisconsin-Madison, Madison, WI, USA; (6) Department of Botany and Plant Pathology, Purdue University, West Lafayette, IN, USA

Phyllachora maydis causes tar spot of corn, a disease that reduces corn grain yield, stover, husks and silage quality. P. maydis, an obligate fungal pathogen of corn, was restricted to Latin America, until 2015, when it was first documented in the United States in Indiana and Illinois. After its initial discovery no economic consequences had been documented, but in 2018, the first epidemic of $P$. maydis occurred causing corn grain yield loss of 4.5 million t. $P$. maydis is now documented in nine states namely; IL, IN, IA, MI, MN, $\mathrm{MO}, \mathrm{OH}, \mathrm{WI}$ and FL. However, information on the population genetics of $P$. maydis, and emergence in the U.S., remains largely unknown. To gain a better understanding of the population genetic structure, ten (10) microsatellite markers were developed from the sequenced genome of $P$. maydis to assess the genetic variation within and among U.S. populations. Seventy (70) $P$. maydis isolates from infected corn leaves from four U.S. geographical locations (IL, IN, IA and WI) were genotyped. Preliminary data from 70 isolates (populations) suggest a clonal population structure with low levels of genetic variation within and among populations. The genetic diversity, differentiation, and linkage equilibrium of each population will continue to be evaluated. Assessment of the genetic variation and population structure will help advance the understanding of $P$. maydis epidemics and emergence, which will aid in the development and deployment of effective management strategies to affected farmers in the United States.

\section{Identification and quantification of Pratylenchus vulnus from almond orchard soil using quantitative PCR} M. TO (1), A. Westphal (2), J. Wenger (1), M. L. Ellis (1), (1) California State University, Fresno, Fresno, CA, USA; (2) University of California Riverside, Dept. Nematology, Parlier, CA, USA

California leads the world in the production of almonds. In contrast to this production success, growers continually face challenges from several soil-borne pathogens in their orchards. Pratylenchus vulnus, or the walnut root lesion nematode, is currently a pest of great concern for California almond orchards. The objective of this research was to develop a qPCR assay for the detection and quantification of $P$. vulnus DNA, that can be adapted and utilized for the quantification of nematodes from naturally infested soil. DNA primers were designed using the internal transcribed spacer (ITS) region, and verified in silico to be species-specific for $P$. vulnus. A qPCR assay using SYBR Green was optimized at $95^{\circ} \mathrm{C}$ for denaturation and $55^{\circ} \mathrm{C}$ for annealing. A standard curve was developed using DNA of $P$. vulnus obtained from nematode carrot disk cultures. The developed primers only detected DNA of $P$. vulnus producing a $158 \mathrm{bp}$ amplicon. Four other species of Pratylenchus common in California did not produce an amplicon. In all qPCR assays, only samples with $P$. vulnus DNA provided and amplification signal, and produced a melting peak of $80.5^{\circ} \mathrm{C}$. The development of standard curves with soil spiked with different numbers of $P$. vulnus that are epidemiologically meaningful will be necessary to work towards determining economical threshold levels in almonds. Such soil tests could form the foundation for almond growers for making meaningful and economically sound management decisions in the future.

\section{Identification of a citrus Coguvirus in Texas associated with oak leaf patterns}

J. W. Park, M. KUNTA, J. V. Da Graça, M. Gonzalez, E. Louzada, Texas A\&M University Kingsville Citrus Center, Weslaco, TX, USA

Three graft-transmissible citrus diseases of unknown etiology, concave gum (CG), cristacortis and impietratura, all induce leaf flecking and oak leaf patterns in young leaves. Recently a novel virus detected in a CG-infected tree in Italy was determined to belong to the Phenuiviridae and was named CG-associated virus (Coguvirus). A second related virus was subsequently detected in a symptomless tree and named Citrus virus A (CiVA). None of these diseases has been found in Texas, but leaf flecks and oak leaf patterns appeared in 4 young shadehouse navel orange (NO) plants obtained by embryo recovery, which were similar to those observed on a Californian CG301 plant. The symptoms on the NO plants were graft-transmitted to sweet orange indicator seedlings. High-throughput sequencing data obtained with CG301 confirmed the presence of a bipartite coguvirus. Primers and a probe for 5' nuclease assay for coguvirus detection were designed based on the nucleotide sequence of coguviral contigs generated by de novo assembly. All embryo-rescued $\mathrm{NO}$, a graft-inoculated sweet orange and CG301 trees tested positive for coguvirus by the 5' nuclease assay. We determined the fulllength nucleotide sequence of the negative sense RNA 1 (6689 nt) and the ambisense RNA 2 (2739 nt) of both NO and CG301 isolates which showed $\sim 72 \%$ sequence identity to CG-associated virus and $\geq 95 \%$ sequence identity to CiVA. No other known citrus-infecting viruses or viroids were present in either the NO or the CG301 plants.

Using an intelligent sprayer and sulfur for management of grape powdery mildew

B. WARNEKE (1), L. Nackley (2), R. L. Rosetta (2), J. W. Pscheidt (1), (1) Oregon State University, Corvallis, OR, USA; (2) Oregon State University, Aurora, OR, USA

The focus of the Intelligent Sprayer Systems (ISS) team is to improve spray application technology in specialty crops. Current projects evaluate standard sprayers retrofitted with ISS components. The airblast sprayer used in this study was fitted with ISS sensors and was 
used in either intelligent (variable rate) or standard (full on/off) mode. In 2018 and 2019 trials on Pinot noir or Pinot gris in Corvallis, OR, when the sprayer was used in intelligent mode with default ISS settings and sulfur at 5lb/100gal water, mean grape powdery mildew cluster severity was $66 \%$ and significantly higher than the $20 \%$ found in the $5 \mathrm{lb} / 100 \mathrm{gal}$ standard mode treatments. In 2019 , when sulfur was used at $20 \mathrm{lb} / 100 \mathrm{gal}$ using default ISS settings, or the spray rate was increased in intelligent mode (with sulfur at 5lb/100gal), 3.3-4.4 and 1.3-1.4 times more sulfur was applied than the 5lb/100gal ISS default treatment, and powdery mildew cluster severity was 36 and $37 \%$, respectively. Use of synthetic fungicides in intelligent mode in 2019 resulted in $11 \%$ cluster severity, significantly lower than all sulfur treatments. The $5 \mathrm{lb}$ sulfur/100gal default intelligent mode treatment resulted in higher AUDPCs than sulfur applied at 20lb/100gal or 5lb/100gal using higher output settings. The ISS is an effective system to reduce pesticide quantity and water use in vineyard systems while achieving comparable control of powdery mildew to standard sprayers.

\section{Characterizing the sensitivity of Wisconsin Phytophthora infestans isolates to mefenoxam}

L. MIGLIANO, E. Larson, A. J. Gevens, University of Wisconsin-Madison, Madison, WI, USA

The dominant clonal lineage of Phytophthora infestans in WI, US-23, is classified as sensitive to mefenoxam. With the re-emergence and persistence of clonal lineage US-8, classified as resistant to mefenoxam, there is a need for monitoring and characterizing. The role of minor genes in tolerance to mefenoxam is poorly understood. Isolates of $P$. infestans collected from WI during the 2017 and 2018 growing seasons were tested for sensitivity to mefenoxam with discriminatory dose of $100 \mathrm{ppm}$. In 2017, both US-23 and US-8 were isolated. On average US-23 was significantly more sensitive than US-8 $(p=0.001)$. There were significant differences among the US-8 isolates $(\mathrm{p}=7 \mathrm{e}-07)$. A single isolate showed sensitivity (a reduction in hyphal growth $>50 \%$ at 100 ppm mefenoxam). There was significant difference among US-23 isolates $(\mathrm{p}=0.00017)$, with only two isolates showing tolerance (a reduction in hyphal growth $<50 \%$ ). In 2018 only US-23 was isolated. Isolates were tested at $0,0.1,1,10$, and $100 \mathrm{ppm}$. At $0.1 \mathrm{ppm}$ significant differences among isolates appeared $(\mathrm{p}=21 \mathrm{e}-09)$, a single isolate showed complete tolerance. Isolates from both clonal lineages and years that show a moderate level of tolerance (a reduction in hyphal growth by $40-60 \%$ ) do so with greater variability among replicates. The phenotype of this multi-genic trait comes through in the variability seen in isolates that are showing more tolerance. Continued screening of $P$. infestans for mefenoxam sensitivity will help track the development and mechanism of tolerance, as well as aid in development of best management approaches.

\section{Comparative analysis of bacterial communities in flue-cured tobacco affected and unaffected by Ralstonia solanacearum R. GARCIA, L. D. Thiessen, North Carolina State University, Raleigh, NC, USA}

In North Carolina, losses caused by Ralstonia solanacearum range from $\$ 10$ to $\$ 15$ million annually. The bacterium is not evenly spread throughout fields, but often is found affecting clusters of plants (hot-spots). Thus, the objectives of this study were: a) determine whether hot-spots are associated with the rhizospheric bacterial composition, edaphic factors or both; and b) compare the microbiome of healthy and diseased plants inside and outside hot-spots. Plants along with bulk soil samples were sampled from three fields. Bulk soil samples were sent for analysis and genomic DNA was extracted from the rhizosphere. Amplification and sequencing of the 16S rRNA hypervariable regions V4-V5 was performed using the illumina ${ }^{\mathrm{O}} 16 \mathrm{~S}$ Metagenomic Sequencing Library Preparation and MiSeq ${ }^{\mathrm{TM}}$ with paired-end reads, respectively. VSEARCH was used for quality filtering, dereplication, chimera removal, and OTUs clustering at $97 \%$ similarity threshold. The RDP classifier were used for taxonomic assignment of OTUs. Differential abundance analysis showed that healthy plants inside hot-spots had a significantly higher abundance of the Order Actinomycetales. Cation exchange capacity, calcium, potassium, phosphorous, and bulk soil $\mathrm{pH}$ significantly influenced on the rhizospheric microbial composition; however, it did not describe differences between healthy and diseased plants. Results obtained from this study could enhance our understanding on potential BCAs naturally occurring in commercial tobacco fields.

\section{Cotton variety screening against $F$ usarium oxysporum f. sp. vasinfectum and root-knot nematode resistance}

J. DOTRAY (1), J. Chagoya (2), T. Wheeler (3), C. Monclova-Santana (4), (1) Texas Tech University, Lubbock, TX, USA; (2) Texas A\&M AgriLife Extension Service, TX, USA; (3) Texas A\&M AgiLife Research, Lubbock, TX, USA; (4) Texas A\&M AgriLife Extension and Research Service, Lubbock, TX, USA

Texas produces approximately $30 \%$ of the total cotton production in the United States. With the aid of root-knot nematodes, secondary diseases such as Fusarium wilt caused by Fusarium oxysporum f. sp. vasinfectum (FOV) colonize the plant vascular tissue leading to severe losses. Although FOV races 1 and 2 require root knot nematodes for infection, resistance to root knot does not always correlate with resistance to Fusarium wilt. The objective of this study was to screen different cotton varieties to test the Fusarium wilt and root knot resistance complex. Forty-seven varieties were tested in the field in Dawson, Gaines, and Hall Counties in West Texas. Isolates collected from infected tissue confirmed FOV race 1 and 2 based on DNA sequencing. In Dawson and Gaines County, the top yielding variety was ST 4946 GLB2; yielding $877 \mathrm{lbs} / \mathrm{A}$ and $376 \mathrm{lbs} / \mathrm{A}$, respectively. In addition, the plant death averaged 14\% and 38\% lower than the susceptible check in both cases. The root-knot nematode density in Dawson County was $1,390 / 500 \mathrm{~cm}^{3}$ of soil, while root-knot nematode density in Gaines County was $1,145 / 500 \mathrm{~cm}^{3}$ of soil. ST 4946 GLB2 also had the lowest disease rating in a greenhouse trial. In Hall County, PHY 500 W3FE was the top variety with a yield of 2,163 lbs/A, plant death of 3.0\%, and a root-knot density of $80 / 500$ $\mathrm{cm}^{3}$ of soil. Both PHY $500 \mathrm{~W} 3 \mathrm{FE}$ and ST 4946 GLB2 carry root-knot nematode resistance. Variety selection is the most effective and sustainable way to produce cotton; however, under high FOV pressure, stand loss and stunting was observed in all varieties.

\section{Huanglongbing is present in residential citrus trees within Georgia's Coastal Plain}

E. ALI (1), T. L. Stackhouse (1), S. Waliullah (2), J. Price (3), J. E. Oliver (4), (1) University of Georgia, Tifton, GA, USA; (2) University of Georgia, GA, USA; (3) University of Georgia, Valdosta, GA, USA; (4) Department of Plant Pathology, University of Georgia, Tifton, GA, USA 
Huanglongbing (HLB), caused by the bacterium Candidatus Liberibacter asiaticus (CLas) and spread by the Asian citrus psyllid (ACP), is the world's most destructive disease of citrus. HLB leads to lower fruit quality and signficantly reduces the productive life-span of citrus trees. In recent years, plantings of commercial citrus trees have been established in at least 32 counties across southern Georgia, leading to concerns regarding the spread and distribution of HLB. Georgia has been quarantined for HLB since 2009 based upon confirmation of the disease in a residential citrus tree in Savannah, Georgia; however, surveys of citrus have not been conducted previously in Georgia and there is little known about the distribution of CLas. To better understand the distribution of CLas in Georgia citrus, a survey was conducted of commercial and residential citrus trees within Georgia's Coastal Plain. Molecular testing was used to screen 94 citrus leaf samples collected from 11 counties, and results revealed the presence of CLas in 9\% of the tested samples. CLas was detected in three counties not previously known to have infected trees, including two inland counties in southern Georgia. All trees positive for CLas were residential trees; however, the presence of HLB in Georgia's Coastal Plain represents a threat to commercial citrus production. Accordingly, commerial citrus growers must vigilantly monitor new plantings for HLB and ACP, and infected commercial and residential citrus trees should be removed promptly to prevent further spread of this disease within southern Georgia.

\author{
Effect of SNP calling and filtering methods on QTL analysis in a Brassica napus double haploid population inoculated with \\ Sclerotinia sclerotiorum \\ F. SHAHOVEISI (1), L. del Rio Mendoza (2), A. Oladzad (3), S. Ruud (3), S. Hosseinirad (1), B. Rissato (1), (1) North Dakota State \\ University, fargo, ND, USA; (2) North Dakota State University, ND, USA; (3) North Dakota State University, Fargo, ND, USA
}

A doubled haploid Brassica napus mapping population from a cross between Topas and DHNEP63 (susceptible and resistant to Sclerotinia sclerotiorum, respectively) was phenotyped using agar plug stem method. Lesion size and plant mortality were measured every other day between the third and eleventh day post inoculation. The area under mortality curve (AUMC) was calculated. Genotyping by sequencing was used to obtain single nucleotide polymorphism (SNP) markers. First, SNP markers were called without imputation of data (dataset1) and then imputation was done (dataset2) to increase the number of the markers for construction of linkage maps. Markers on random chromosomes were removed from both data sets. Linkage groups were constructed at LOD 10 and then LOD 4 with 460 markers for dataset1 and at LOD 20 and then LOD 4 with 782 markers for dataset2. Two unique QTL with LOD values greater than 2.5 were identified in each data. Two QTL on chromosome C01 and A02 from dataset1 and two QTL on chromosome C01 from dataset 2 were mapped. The $\mathrm{R}^{2}$ values had similar magnitudes in both data sets. From this outcome, it is fair to assume that imputing data may not be necessary. In fact, QTL from non-imputed data had twice as many candidate genes; and $\mathrm{R}^{2}$ values and LOD scores were slightly higher. Whether the benefit of imputation is population-dependent is not known.

Population structure of Phytophthora infestans in the Mediterranean region A. C. Saville (1), S. O. Cacciola PhD (2), J. RISTAINO (1), (1) North Carolina State University, Raleigh, NC, USA; (2) Univ of Catania, Catania, ITALY

The oomycete plant pathogen Phytophthora infestans causes late blight disease on potato and tomato and is prevalent throughout Europe. While populations in northern Europe have been characterized for many years, there has been little examination of populations around the Mediterranean, particularly southern Europe and northern Africa. Between 2018 and 2019 we collected 57 samples from late blight outbreaks in Italy, Tunisia, and Algeria on FTA cards and genotyped them using 12-plex microsatellites for $P$. infestans. These samples were compared with previously collected data from Euroblight and published sources. Four clonal lineages were identified among collected samples: 13_A2 (Blue 13),2_A1,23_A1, and 5_A1. The 13_A2 and 2_A1 lineages were found in southern Italy, including Sicily, and Algeria, while 5_A1 was only found in the Friuli Venezia Giulia region of northern Italy. The 23_A1 lineage was prevalent in all regions examined. Differences in genotypes suggest that there may be multiple sources of introduction across Mediterranean countries, possibly via infected tubers from northern Europe. Since the northern outbreaks show evidence of geographic substructure, this suggests spread from a single or a limited number of introductions. Understanding the sources of seed potatoes and tomato transplants may provide additional information into the sources of $P$. infestans in the Mediterranean.

Optimizing pollination of muskmelon in organic mesotunnel systems

K. MPHANDE (1), M. L. Gleason (2), (1) Iowa State University, Ames, IA, ZAMBIA; (2) Iowa State University, Ames, IA, USA

The cucurbit bacterial wilt pathogen, Erwinia tracheiphila, is vectored by cucumber beetles. Organic muskmelon growers lack reliable controls against cucumber beetles. In preliminary trials, a full-season barrier system called mesotunnels $-1 \mathrm{~m}$ tall and covered with nylon-mesh netting - increased yield in organic muskmelon compared to low tunnels $(0.5 \mathrm{~m}$ tall and covered with spunbond polypropylene). A key challenge for mesotunnels is optimizing pollination. In Iowa during 2019, muskmelon (cv. Athena) seedlings were transplanted into 46-m-long, 3-row subplots in a randomized complete block design. Treatments: 1) one bumble bee hive (Koppert, Inc.) per subplot; 2) two bumble bee hives per subplot; and 3) natural pollination (netting was removed when female flowers appeared and was replaced 2 weeks later). Bee activity was noted twice a day on 3 days/week during 2 weeks after female flowers appeared. The natural-pollination treatment resulted in marketable yield equivalent to that of mesotunnels that had remained covered all season. The natural-pollination treatment had the most flower visits by bees and other insects, followed by the two-bumble bee-hive treatment and the one-bumble bee-hive treatment. No bacterial wilt symptoms were observed during the trial. In the absence of cucumber beetle pressure and bacterial wilt, full-season mesotunnels supplemented with purchased bumble bees did not provide a yield advantage over removal of covers for 2 weeks during bloom. 
Identifying and evaluating integrated management tactics for Sudden Death Syndrome (SDS) in Northern Indiana 2019 M. BROWN (1), Y. R. Kandel (2), D. S. Mueller (2), D. E. P. Telenko (3), (1) Purdue University, West Lafayette, IN, USA; (2) Iowa State University, Ames, IA, USA; (3) Department of Botany and Plant Pathology, Purdue University, West Lafayette, IN, USA

Soybean [Glycine $\max (\mathrm{L}$.$) Merrill] is one of the world's most valuable cultivated crop; it serves as a source of protein and oil.$ However, soybean production is heavily plagued by numerous diseases which cause yield reduction and severe economic loss. Sudden death syndrome (SDS) is among the top five yield-limiting diseases of soybean. SDS is caused by a soilborne fungus, Fusarium virguliforme, which results in root necrosis followed by foliar symptoms of interveinal chlorosis and necrosis. Resistance for SDS is partial and complex. Several management options have been evaluated for their effectiveness; many of which are limited in ability to provide optimal SDS management. Therefore, identifying, evaluating, and integrating other strategies is of paramount importance for SDS management. The objective of this research was to examine potential outcomes of integrated management tactics for SDS in Indiana, to study their effects on $F$. virguliforme root infection, root rot, foliar severity and yield. Management tactics included seed treatments, variety selections and seeding populations. A standard rating scale was used for root rot and foliar ratings and Real-time Polymerase Chain Reaction (qPCR) was used to quantify $F$. virguliforme in the roots. In 2019, weather conditions were unfavorable for SDS development; nevertheless, resistant varieties had the least disease incidence compared to susceptible varieties. We plan to repeat this trial in the future in hopes to develop and provide growers with an integrated, effective and economic management program for SDS.

\section{Structure-function analysis reveals motifs necessary for TuMV VPg-eIF(iso)4E interaction are dispensable for CBSV VPg interaction with cassava $\mathrm{nCBP2}$}

Z. J. LIN (1), E. De Meyer (2), H. Tiley (2,3), R. Bart (2), J. C. Carrington (2), (1) Donald Danforth Plant Science Center, Saint Louis, MO, USA; (2) Donald Danforth Plant Science Center, St. Louis, MO, USA; (3) USDA/ARS/NCAUR, Peoria, MO, USA

Cassava is a crucial food security crop in sub-Saharan Africa. There, a key constraint on production is cassava brown streak disease (CBSD). CBSD is caused by independent or simultaneous infection with two species of positive sense RNA viruses belonging to the family Potyviridae, genus Ipomovirus: cassava brown streak virus (CBSV) and Ugandan cassava brown streak virus (UCBSV). Diseases caused by the family Potyviridae have been found to require the interaction of viral genome-linked protein (VPg) and host eukaryotic initiation translation factor $\underline{4 \mathrm{E}}$ (eIF4E) isoforms. Cassava encodes five eIF4E-family members: eIF4E, eIF(iso)4E-1, eIF(iso)4E-2, nCBP-1, and nCBP-2. We previously found that cassava $n c b p-1 / n c b p-2$ double mutants exhibited attenuated CBSD symptoms and reduced storage root viral titer. Interestingly, CBSV VPg was found to associate with all cassava eIF4E-family members by co-immunoprecipitation. Here we demonstrate through pull-down assays that CBSV VPg directly interacts with all cassava eIF4Efamily proteins. We also find that certain amino acids critical for Arabidopsis eIF(iso)4E interaction with TuMV VPg are dispensable for CBSV VPg interaction with cassava nCBP-2. Furthermore, threading of CBSV and TuMV VPg onto the NMR structure of PVY VPg reveals that the " $\alpha 1-\alpha 2$ loop" 4E-interaction domain of PVY and TuMV VPg are similar, but the length of this structure is shortened in CBSV VPg. Taken together, these data suggest that VPg of CBSV, compared to those of other potyvirids, has evolved different means of interacting with host susceptibility factors.

Insights into detoxification of tolaasins, the virulence factor of brown blotch mushroom disease, by Microbacterium foliorum NBRC 103072

S. TOMITA (1), A. Kajikawa (1), S. Igimi (1), H. Shinohara (2), K. Yokota (1), (1) Tokyo University of Agriculture, Tokyo, JAPAN; (2) Tokyo University of Agriculture, Kanagawa, JAPAN

Tolaasins are lipodepsipeptides secreted by Pseudomonas tolaasii, the causal agent of bacterial blotch on cultivated mushrooms. In our previous study, Microbacterium foliorum NBRC $103072^{\mathrm{T}}$ (103072) shows tolaasin-detoxification depending on hydrolysis at the 2specific sites in the peptide moiety of tolaasin I by its cells. The objectives of this work were to characterize the properties of tolaasindetoxification by 103072. The tolaasin I-hydrolyzing activity, which was confirmed by detection of degradation products from tolaasin I, was observed in the extracts of 103072 -bacterial cells by $0.5 \%$ Triton X-100 treatment. In the extracts, added tolaasin I was linearly degraded, and the degradation products were increased inversely, while, in the cell suspension, tolaasin I-elimination was rapidly occurred comparing with the increase of the degradation products. We also compared the tolaasin I-elimination in the cell suspension to the extract at different $\mathrm{pH}$ range. Tolaasin I-elimination was observed in the cell suspension below of $\mathrm{pH} 5$ whereas no tolaasin Idegradation in the extract. Our results suggested that the tolaasin-hydrolyzing enzyme(s) are localized in the cell wall of 103072 and the absorption of tolaasins to 103072-cells might enhance tolaasin-detoxification and carry out by unknown factor(s) which are differed from the tolaasin-hydrolyzing enzyme. This is the first report to demonstrate the inactivation of antimicrobial lipodepsipeptides depending on cell wall localizing hydrolyzing enzyme(s).

\section{Identification and characterization of SCN resistance genes encoded by novel QTLs from Glycine soja via CRISPR-Cas9 mutagenesis \\ J. Z. ZAYAS-RIVERA (1), M. Riga (1), K. J. Butler (1), C. Fliege (2), M. Hudson (2), B. Diers (2), A. F. Bent (1), (1) University of Wisconsin-Madison, Madison, WI, USA; (2) University of Illinois, Urbana, IL, USA}

Soybean cyst nematode (SCN) is the most damaging pathogen of soybean in the US, causing up to 20 billion dollars in losses per year. To this day, the locus Rhgl has been used as genetic resistance against this pathogen. This locus utilizes a novel Rhgl-alpha-SNAP ( $\alpha$ $\left.\mathrm{SNAP}_{R h g l}\right)$ that, instead of sustaining cellular vesicle trafficking through NSF interactions, accumulates in the nematode feeding sites causing toxicity to the plant. This $\alpha$-SNAP ${ }_{R h g l}$ has a co-adapted $R h g l$-associated NSF (NSFRAN07) that ameliorates $\alpha$-SNAP Rhgl $_{\text {toxicity, }}$ 
thus being vital for the viability of Rhgl-containing soybean lines. Although Rhgl-mediated resistance has been fundamental for SCN control in the field, resistance seems to be breaking. Pyramiding Rhgl with other QTLs for SCN resistance is a breeding priority. Through map-based cloning, here we report a Glycine soja-derived resistance source that also modifies SNAP expression. CRISPR/Cas9-mediated disruption of the resistant allele reduces SCN resistance. There is no amino acid polymorphism between the products of resistant and susceptible $c q S C N-006$ alleles, but we identified promoter and gene body DNA variants, variation in alternative splicing, and differing induction this gamma-SNAP ( $\gamma$-SNAP) protein between resistant and susceptible infection sites. This presents an independent example of disease resistance against cyst nematodes that once again involves alteration of cellular SNAP and/or NSF processes. We will also report our work to clone another resistance QTL, cqSCN-007.

Potential roles of secondary metabolites in the plant-microbe interaction of Calonectria pathogens and boxwood L. ROGERS (1), J. A. Crouch (2), M. A. Cubeta (1), A. Koehler (3), D. Shew (1), N. LeBlanc (1,2,4), (1) North Carolina State University, Raleigh, NC, USA; (2) USDA-ARS, Mycology and Nematology Genetic Diversity and Biology Laboratory, Beltsville, MD, USA; (3) University of Delaware, Georgetown, DE, USA; (4) Oak Ridge Institute for Science and Education, Oak Ridge, TN, USA

Boxwood blight is an emerging disease caused by the fungal pathogens Calonectria henricotiae and C. pseudonaviculata. Currently, little is known about the role of secondary metabolites as pathogenicity factors in the interaction between susceptible boxwood hosts and these Calonectria pathogens. To better understand secondary metabolism in these fungi, biosynthetic gene clusters (BGCs) associated with secondary metabolism were surveyed in genome sequences of six Calonectria species and eight additional species in the family Nectriaceae. Using the antiSMASH pipeline, total predicted BGCs ranged from 23 to 148 among the 14 genomes, with Calonectria species containing the largest numbers of clusters. C. henricotiae and C. pseudonaviculata had 74 and 66 predicted BGCs, respectively. Interestingly, certain BGCs in the $C$. henricotiae and $C$. pseudonaviculata genomes showed similarity to the wellcharacterized trichothecene pathway (TRI) of Fusarium graminearum. To infer potential homologies in pathway products, the TRI protein sequences from $F$. graminearum were compared to the proteomes of six Calonectria species using BlastP. Both $C$. henricotiae and C. pseudonaviculata had eight functionally similar proteins out of the 14 characterized TRI proteins, while the remaining Calonectria species had six or less functionally similar proteins. These results demonstrate wide variation in secondary metabolism potential among Calonectria species and provide a framework for investigating the role of these products in host infection by $C$. henricotiae and C. pseudonaviculata.

Modeling distribution of endemic and potentially invasive Armillaria species for the southeastern panhandle of Alaska J. W. HANNA (1), R. L. Mulvey (2), M. S. Kim (3), J. Stewart (4), B. C. Bright (1), M. V. Warwell (1), N. B. Klopfenstein (1), (1) USDA Forest Service, Rocky Mountain Research Station, Moscow, ID, USA; (2) USDA Forest Service, Forest Health Protection, State \& Private Forestry, Juneau, AK, USA; (3) USDA Forest Service, Pacific Northwest Research Station, Corvallis, OR, USA; (4) Colorado State University, Fort Collins, CO, USA

Armillaria root disease is a primary cause of lost productivity in northern forests of North America, and its impact is expected to increase under climate change due to tree maladaptation. Little is known about the distribution of accurately identified Armillaria spp. and their relationship with climate in southeastern Alaska. Root surveys of forest trees yielded 32 isolates of A. sinapina. Locations of these isolates served as the basis for Maximum Entropy bioclimatic models of species distribution using contemporary and projected future climate surfaces (years 2055 and 2085 for both RCP 4.5 and RCP 8.5 greenhouse gas concentration scenarios). Under contemporary climate, suitable climate space for $A$. sinapina was widespread in southeastern Alaska, which suggests a high likelihood for Armillaria root disease caused by this pathogen. Furthermore, the probability of suitable climate space for A. sinapina increases under RCP 4.5 in years 2055 and 2085, with analogous results for the RCP 8.5 scenario. Additional occurrence records of Armillaria species from the Pacific Northwest were used to model potential invasion of non-native species into this region. Under the RCP 8.5 scenario for the year 2085, it is especially noteworthy that southeastern Alaska is projected to have suitable climate space for both $A$. solidipes (primary pathogen in the Pacific Northwest) and A. cepistipes, which are not known to presently occur in this region. Based on these predictions, it appears that both species pose invasive threats to southeastern Alaska under conditions of predicted climate change.

The evolution of bacterial and fungal communities in take-all disease-suppressive and -conducive rhizospheres M. LETOURNEAU (1), D. M. Weller (2), (1) Wheat Health, Genetics and Quality Research Unit, Pullman, WA, USA; (2) USDA-ARS Wheat Health, Genetics and Quality Research Unit, Pullman, WA, USA

Take-all disease of wheat is a root rot caused by the soilborne fungus Gaeumannomyces graminis var. tritici. Take-all suppressive soils are known to develop in cereal monocultures, and soil microbial communities play a significant role in this process. Therefore, the objective of this study was to identify links between the diversity and composition of bacterial and fungal communities in wheat rhizospheres and take-all disease severity. Using next-generation sequencing of ribosomal amplicons, we tracked the evolution of bacterial and fungal communities in the rhizospheres of two wheat cultivars, 'Tara' and 'Buchanan' over five cycles of growth in suppressive and conducive soils. To test the impacts of soil microbial diversity and proliferation of the take-all pathogen, we also compared pasteurized and inoculated soils with untreated soils. All experimental factors significantly impacted the bacterial and fungal communities. The initial soil suppressiveness or conduciveness and pasteurization exerted the strongest effects while inoculation exerted the weakest effect, and the effect of cultivar was stronger for the fungi than for the bacteria. Disease ratings of the wheat root systems in the inoculated treatments were also significantly correlated with bacterial and fungal community compositions. Several differentially abundant ribosomal sequences were identified, and these could provide markers for disease suppressiveness and conduciveness in field soil to inform management strategies that seek to minimize soilborne root rots. 
Since 2017, undergraduate interns in the Plant Pathology program at the West Tennessee Research and Education Center have included 13 students from 3 U.S. universities and 5 international students from Brazilian universities. For most of them, this is the first opportunity for a research/Extension project and careful instruction is necessary in the form of a 6-step approach. Identifying the research question is primary, which is opportunistic based on multiple factors. Second, guidance on experimental design through coordination with the faculty mentor and research technicians and timeline for project completion is established. Third, execution of the experiment is done through trial set up (greenhouse, field, and/or laboratory), data collection and analysis, and collaboration as necessary (county agents and/or producers). Fourth, results are disseminated in the form of a report, article, poster, and/or presentation. Utilization of social media to promote research and/or extension material may also be a component of the project. Fifth, evaluation in the form of an exit interview is completed. Finally, future opportunities for the intern are discussed and may include enrollment in a graduate program, publication(s), or conference attendance to present their research. This will be accomplished with the definitive goal of teaching the intern how to approach and execute research in an agricultural setting and ultimately communicate their results to stakeholders through research and extension programs.

BS ToxA producing Bipolaris sorokiniana population on wheat in South Dakota Z. Shabbir (1), S. Sehgal (1), K. D. Glover (1), E. Byamukama (2), Z. Liu (3), S. ALI (1), (1) South Dakota State University, Brookings, SD, USA; (2) South Dakota State Univ, Brookings, SD, USA; (3) North Dakota State University, Fargo, ND, USA

Many necrotrophic leaf spot pathogens produce host selective effectors (toxins), which are associated with disease development. The fungi Pyrenophora tritici-repentis Parastagonospora nodorum and Bipolaris sorokiniana cause tan spot, S. nodorum blotch (SNB), and spot blotch, respectively in wheat and they impact crop production in the US. P. tritici-repentis and $P$. nodorum are known to produce a host-selective effector ToxA which is associated with tan spot and SNB development, however, recently, ToxA was reported from Bipolaris sorokiniana isolates from Texas and may play a role in spot blotch development. In this study, 60 B. sorokiniana isolates recovered from wheat in South Dakota were genotyped for the presence or absence of ToxA gene. Also, culture filtrates of two $B s$ isolates of each with and without BS ToxA gene were infiltrated for their reaction into leaves of two ToxA sensitive (Boost and Forefront) and two ToxA insensitive (Linkert and Prevail) spring wheat varieties. Nearly 42\% (25) of 60 isolates amplified the ToxA gene. Further, the culture filtrates of isolates with the ToxA gene produced necrosis symptoms in ToxA sensitive wheat cultivars. The culture filtrates of the isolates lacking in the ToxA gene did not produce any symptoms in all four wheat cultivars. Our results indicate that a diverse population of $B$. sorokiniana prevalent on wheat in South Dakota. Also, developing ToxA insensitive wheat cultivars may help in the management of all three-leaf spot diseases tan spot, SNB, and spot blotch management and hence reduce their impact on wheat productivity.

Evaluation of PMMoV resistance in a pseudoF2 generation for the introgression of $L 4$ resistance gene in sensitive pepper landraces of northern Spain

S. LARREGLA (1), M. Ojinaga (2), (1) NEIKER, Derio, SPAIN; (2) NEIKER. Basque Institute of Agricultural Research and Development, Derio, SPAIN

Pepper is one of the most important vegetable crops in the Basque Country (Northern-Spain). The main cultivated landraces are Gernika pepper and Ibarra yellow chilli peppers, both susceptible to Tobamovirus that cause important reductions of crop yield and profitability in this area. Thus, a marker assisted selection backcross plant breeding program was started in 2015 for the introgression of resistance gene L4 against Tobamovirus in both cultivars. Resistance to Tobamoviruses was evaluated by inoculating the $\mathrm{P}_{123}$ pathotype of PMMoV virus in plants of the $\mathrm{PseudoF}_{2}$ generation within the breeding program. Virus inoculation was done mechanically, rubbing the leaves with carborundum mixed with the virus suspension. Symptoms produced by the virus were observed in the inoculated plants; plants with mosaic symptoms in non-inoculated leaves were considered virus-sensitive; conversely, plants with necrotic spots in the inoculated leaves followed by leaf abscission were considered resistant. The average percentage of resistant plants was $72 \%$, very close to the expected value for this generation (75\%). At the end of the trial, plants that showed resistance symptoms or non-symptoms (59 plants) were genotyped and analyzed by DAS-ELISA test. The molecular marker associated with the L4 resistance gene was observed in 56 of 59 plants. In the DAS-ELISA test, only 7 of 59 plants were positive to PMMoV. The DAS-ELISA and molecular marker results were consistent with the inoculation trial results, concluding that inoculation assays can be a useful tool to select Tobamovirus resistant plants.

Temporal shifts in sensitivity of Pseudoperonospora cubensis populations in response to cucurbit downy mildew fungicides I. KIKWAY (1), A. P. Keinath (2), P. S. Ojiambo (3), (1) North Carolina State Univ, Raleigh, NC, USA; (2) Clemson University, Coastal Research and Education Center, Charleston, SC, USA; (3) Department of Entomology and Plant Pathology, North Carolina State University, Raleigh, NC, USA

Cucurbit downy mildew (CDM) caused by the oomycete Pseudoperonospora cubensis is an important disease affecting members of Cucurbitaceae family. Chemical control is the most effective method but $P$. cubensis has a high risk for developing resistance to fungicides. This study aimed to assess shifts in a growing season in sensitivity of $P$. cubensis to fungicides used for CDM control. Baseline sensitivity was determined for 25 isolates of $P$. cubensis. At the end of 2018 and 2019 growing seasons, a total of 24,20 , and 18 isolates from fluopicolide, propamocarb and cymoxanil treated plots, respectively, were collected from cucumber and squash and subjected to sensitivity screening. Isolates with a relative growth $>35 \%$ were considered resistant. Across seasons, $37.5 \%$ of isolates were sensitive, while $62.5 \%$ were resistant to fluopicolide. For propamocarb-exposed isolates, $75.0 \%$ were sensitive, while $25.0 \%$ were resistant. Among cymoxanil-exposed isolates, $11.1 \%$ were sensitive, while $88.9 \%$ were resistant. Based on the baseline populations, all 
isolates exposed to cymoxanil were resistant (baseline $=68.1 \%$; exposed $=63.1 \%$ ), while fluopicolide-exposed isolates shifted towards resistance $($ baseline $=22.8 \%$; exposed $=49.2 \%$ ). In contrast, propamocarb-exposed isolates shifted towards sensitive $($ baseline $=26.0 \%$; exposed $=13.5 \%$ ). These results highlight directional selection to CDM fungicides within a growing season and emphasize the need for regular monitoring of fungicide resistance in field populations of $P$. cubensis.

Transcriptome-wide identification of the mildew locus o (mlo) gene family from hop (Humulus lupulus) and multi-host phylogeny M. S. WISEMAN (1), N. Adair (2), A. Black (1), B. Kronmiller (1), R. Eriksen (2), J. Henning (2), D. Gent (2), (1) Oregon State University, Corvallis, OR, USA; (2) USDA-ARS, Forage Seed and Cereal Research Unit, Corvallis, OR, USA

Hop (Humulus lupulus), the bittering agent in beer, is a dioecious climbing plant with a large and complex genome. In the Pacific Northwest, annual losses from hop powdery mildew (Podosphaera macularis) are estimated to be 15\% on average, with sometimes catastrophic losses in yards due to complete crop rejections. Restrictions on pesticide use in certain export markets and consumer preferences require breeding efforts for durable disease resistance. A successful strategy employed in other hosts has been selecting for loss-of-function mutations in the recessively inherited mildew locus $\mathrm{O}$ ( $\mathrm{mlo}$ ) gene family. Resistance resulting from loss-of-function mlo susceptibility mutations is thought to involve prevention of fungal penetration, conferring durable broad-spectrum resistance in monocots and dicots. We used size-selected Isoseq sequencing to identify and describe 35 mlo transcripts representing five of the seven previously characterized clades, including clade $\mathrm{V}$ which has been correlated with mildew susceptibility in other hosts. From these 35 transcripts, broad and specific primers were designed for use in qPCR. We plan to use qPCR to look for $m l o$ genes that are overexpressed in susceptible hosts post-inoculation. Subsequently, we will examine if these overexpressed genes are correlated to powdery mildew susceptibility by screening US hop germplasm. Incorporating loss-of-function susceptibility gene mutations, quantitative trait loci, and resistance genes in breeding programs should enhance durable broad spectrum resistance against powdery mildew.

\section{Virome profiling and reconstruction of viral/viroid genomes using the available mRNAome and sRNAome datasets of Indian grapevines (Vitis vinifera $\mathrm{L}$.) \\ K. S. VENKIDUSAMY (1), V. K. Baranwal (2), A. M. Sevanthi (3), S. Jaiswal (4), (1) Indian Agriculture Research Institute, NEW DELHI, INDIA; (2) Indian Agricultural Research Institute, New Delhi, INDIA; (3) National Institute for Plant Biotechnology, NEW DELHI, INDIA; (4) Indian Agricultural Statistics Research Institute, NEW DELHI, INDIA}

Next generation sequencing technologies have become routine approaches for reliable detection of plant viruses and viroids. In this study, we identified viral/viroidal spectrum of Indian grapevine cultivars and reconstructed their whole genomes using publicly available mRNAome and sRNAome datasets. Twenty three viruses and viroids were identified from two tissues (fruit peels and young leaves) of three cultivars, among which 11 grapevine viruses and viroids were identified for the first time in India. Irrespective of the assemblers and tissues (one or more) used, mRNA based approach identified a greater number of acellular pathogens than sRNA based approach across cultivars. Further, the mRNAome was on par with the whole transcriptome in identifying viruses. Through de novo assembly of transcriptomes followed by mapping against reference genome, we reconstructed 19 complete/near complete genomes of identified viruses and viroids. The reconstructed viral genomes included four large genomes ( $>13 \mathrm{~kb})$, a DNA genome (RG GGVA), a divergent genome (RG GVB) and a genome for which reference is unavailable (RG GVL). The large number of SNPs detected in this study ascertained the quasispecies nature of viruses. Detection of recombination events and phylogenetic analyses using reconstructed genomes suggested possible introduction of viruses and viroids into India from several continents through the planting material. Whole genome sequences generated in this study can serve as a resource for reliable indexing of grapevine viruses in quarantine stations and certification programs.

Protist facilitated transport of plant growth promoting bacteria in the rhizosphere J. L. MICCIULLA, C. J. Hawxhurst, C. Bridges, L. Shor, D. J. Gage, University of Connecticut, Storrs, CT, USA

Plant roots secrete nutrient rich exudates that attract the native microbial community. Protists are a major member of this community, yet are vastly understudied. Soil protists are known to contribute to plant health in two ways: (1) by releasing otherwise stored nutrients into the soil to be taken up by plant roots and (2) they often feed on pathogenic bacteria, lessening the threat in the community. Here, we make use of the bacterivorous nature of soil protists and propose another mechanism by which protists enhance plant health. Ciliated protists, Colpoda sp., are known to move quickly through the rhizosphere, using water films on roots as a means of travel. Soil bacteria, however, often form biofilms and do not travel through the rhizosphere. Colpoda feed on such bacteria, but have been found to egest undigested bacterial meals which may go on to populate new regions of the root. Using 3D printed soil microcosms (termed urhizoslides), we imaged and quantified the transport of fluorescently labelled Sinorhizobium meliloti, a nitrogen fixing symbiotic soil bacterium, over time. Two weeks post inoculation, $S$. meliloti is seen $30 \mathrm{~mm}$ farther down the roots in the presence of Colpoda than in the bacteria alone treatments. S. meliloti deposited in novel regions has the opportunity to form nitrogen fixing nodules on the roots, providing the plant with a limited resource. Protist facilitated transport has the potential to be a sustainable alternative for distribution of beneficial bacteria in the rhizosphere, decreasing the overuse of fertilizers and agrochemicals in farming.

\section{Loss of a single gene enhances non-vascular Xanthomonas streak}

J. BUTCHACAS (1), V. Roman-Reyna (1,2), J. M. Jacobs (1,2), (1) Department of Plant Pathology, The Ohio State University, Columbus, OH, USA; (2) Infectious Diseases Institute, The Ohio State University, Columbus, OH, USA

Xanthomonas transluscens subgroups are important bacterial pathogens of barley. X. transluscens pv. translucens (Xtt) causes bacterial blight of barley by moving long distances in the water-transporting xylem; meanwhile, the bacterial leaf streak pathogen, $X$. translucens 
pv. undulosa (Xtu), remains restricted to the mesophyll, causing small, water-soaked lesions. The genetic factors determining $X$. transluscens host-tissue specificity still remain poorly understood. Here we described the role of a xylem pathogen-conserved cellobiohydrolase, CbsA, for tissue-specific Xtt barley infection. We mutated $c b s A$ in Xtt and infected barley plants to determine the role of this gene during infection. We observed that the Xtt $c b s A$ mutant displayed wild-type vascular blight development. However, this mutant significantly increased apoplastic water soaking symptoms downstream of vascular blight symptoms, similar to the mesophyll pathogen Xtu. This indicates that loss of CbsA enhances non-vascular leaf colonization. Further co-inoculation of the mutant and the wild-type suppressed water-soaking, suggesting that presence of this secreted enzyme in the plant dictates pathogen tissuespecificity. Overall, our results demonstrates that CbsA plays a major role in Xtt regulation of mesophyll colonization behavior.

Engineering resistance to the soybean cyst nematode via detection of SCN effector proteases A. C. MARGETS (1), R. W. Innes (1), T. J. Baum (2), R. Masonbrink (3), T. Maier (2,3), (1) Indiana University, Bloomington, IN, USA; (2) Iowa State University, Ames, IA, USA; (3) Iowa State University, IA, USA

The soybean cyst nematode ( $\mathrm{SCN}$ ) is one of the most economically significant pathogens of soybean in North America. As part of the infection process, SCN expresses effector proteins in its esophageal glands that are translocated into host cells. The goal of this project is to (i) identify effector proteases secreted by SCN that are required for infection and (ii) create decoy substrates for these proteases that will activate defense responses upon cleavage. Toward the latter goal, we have shown that proteolytic cleavage of the soybean PBS1 protein induces cell death. Furthermore, PBS1 modification enables cleavage by the NIa protease from soybean mosaic virus, and confers complete resistance to SMV, demonstrating that PBS1 decoys are functional in soybean. We have recently identified proteases expressed in the esophageal glands of SCN and are now assessing whether these proteases can suppress defense responses when delivered to, or expressed in, the host cell cytoplasm. Such suppression would indicate that these proteases function inside plant cells, rather than in the apoplast. Proteases that display immune suppression activity will be further characterized by identifying host substrates and cleavage site sequences. Cleavage sites will then be inserted into soybean PBS1 and assessed for activation of cell death when cleaved by an SCN protease. We hypothesize that this approach will inhibit the nematodes' ability to establish and/or maintain feeding sites. If successful, decoy engineering has great potential to address other agriculturally significant nematode pests.

\section{Tolerance of eggplant cultivars with different fruit types to Phomopsis blight}

A. P. KEINATH, Clemson University, Coastal Research and Education Center, Charleston, SC, USA

Phomopsis blight caused by Phomopsis vexans is the most serious disease on eggplant fruit in fall crops in the southeastern U.S. As few fungicides are registered to manage this disease, the objective was to determine if 7 eggplant cultivars, representing 7 horticultural fruit types, differed in susceptibility to P. vexans. Eggplant was transplanted in April 2018 and 2019, cut back in late July or early Aug. and harvested in Sep. and Oct. Phomopsis blight developed from naturally occurring inoculum. Black Beauty (globe type) had a greater AUDPC for leaf spot severity $(P<0.05)$ than other cultivars both years. Rosa Bianca (Italian heirloom) and Gretel (white) had lower leaf disease incidence $(P \leq 0.01)$ both years than other cultivars except Millionaire (Japanese) in 2018. Percentage defoliation at the end of the season was greater $(P<0.05)$ for Black Beauty than Patio Baby (Indian), Millionaire, Rosa Bianca and Gretel both years. Most $(\geq 97 \%)$ abscised leaves had pycnidia in one or more lesions. Mean length of leaf spots and stem cankers did not differ among cultivars. Percentage fruit rot both years was greater $(P \leq 0.01)$ for Black Beauty than Hansel (Chinese), Gretel, Patio Baby and Rosa Bianca. In 2018 Hansel had greater marketable fruit weight than other cultivars except Millionaire; in 2019 Millionaire, Hansel and Gretel yielded more $(P \leq 0.005)$ than Fairy Tale (graffiti or Sicilian type), Rosa Bianca and Black Beauty. In summary, several eggplant cultivars other than Black Beauty are less susceptible to Phomopsis leaf spot and fruit rot and may produce greater marketable weight.

cutC gene in the xylem-limited pathogen Xylella fastidiosa is involved in the response to acute Cu stress Q. GE, P. Cobine, L. De La Fuente, Auburn University, Auburn, AL, USA

Copper $(\mathrm{Cu})$ is a crucial element for many bacteria to survive, however it can also be toxic. Plant pathogenic bacteria have been exposed to $\mathrm{Cu}$ stress for years, due to wide application of $\mathrm{Cu}$-containing antimicrobials. This has led to the acquisition of $\mathrm{Cu}$ resistance mechanisms by the pathogens to allow for better survival. One protein related to $\mathrm{Cu}$ tolerance of $E$. coli and humans is $\mathrm{CutC}$, this is a "TIM-barrel protein" that has a single copper binding site and is thought to protect cells against accumulation of free Cu. The mechanisms of $\mathrm{Cu}$ tolerance/resistance in Xylella fastidiosa $(\mathrm{Xf})$, which is a Gram-negative, xylem-limited plant pathogenic bacterium that causes disease in many economically important crops worldwide, is barely studied. Here we focused on analyzing the $c u t C$ gene, a homolog of $c u t C$ from $E$. coli, in Xf by site-directed mutagenesis. The cutC mutant when compared wild type Xf strain, shows increased sensitivity to $\mathrm{Cu}$. The mutant showed decrease in colony forming units under short time exposure to high concentrations of $\mathrm{Cu}$, less accumulation of $\mathrm{Cu}$ in biofilm, delay of biofilm formation, and increase of biofilm attachment. Thus, we hypothesize that in Xf the $\mathrm{cutC}$ gene is involved with $\mathrm{Cu}$ resistance by binding $\mathrm{Cu}$ in cells, leading to $\mathrm{Cu}$ detoxification. Future studies will address the copper binding capacity of $\mathrm{CutC}$ and more detailed analysis of damaged caused by $\mathrm{Cu}$ in the mutant. This will help to develop disease management strategies against this plant pathogen.

Phenotypic variability in the grape powdery mildew pathogen, Erysiphe necator C. L. NEWBOLD (1), W. Mahaffee (2), (1) Oregon State University, Corvallis, OR, USA; (2) USDA ARS, Corvallis, OR, USA

One issue in the study of plant pathogens is the phenotypic variation seen among clonal isolates; this is especially the case in Erysiphe necator, an obligate biotroph with a worldwide distribution. Through the collection and maintenance of over 100 isolates from the Pacific Northwest we observed striking differences among and within isolates. To describe this variation we used germination and 
generation time data that exposed eight $E$. necator isolates to 8 to $32^{\circ} \mathrm{C}$ temperatures. Isolates were collected from Oregon vineyards during the 2017 growing season and included Quinone outside Inhibitor (QoI) fungicide resistant and wild type isolates for increased genetic variation. Percent germination was measured as the number of germinated spores out of fifty, and generation time in days from spore landing to the first fully formed spore. While no isolates showed a difference in generation time at any temperature, contrast analysis indicated that 3 of the 8 isolates had lower germination at temperatures above $8^{\circ} \mathrm{C}(\mathrm{P}$ value $<0.05)$ with high variability $( \pm 27 \%)$ around $14^{\circ} \mathrm{C}$. QoI resistant isolates had significantly greater germination than wild type isolates at $14^{\circ} \mathrm{C}(\mathrm{P}$-value $<0.05)$. Effect on colony and spore development are underway. These results indicate that there are large phenotypic difference among isolates that may influence conclusions drawn from experiments using a single isolate. It is not yet clear if fungicide resistance is related to the observed phenotypic variation.

\title{
Accurate diagnosis of sugar beet root diseases is critical for driving disease management decisions
} J. R. Brantner (1), A. K. CHANDA (2), (1) University of Minnesota, Crookston, MN, USA; (2) Department of Plant Pathology, University of Minnesota, St. Paul, MN, USA

Accurate diagnosis of soilborne sugar beet root diseases is critical for implementing effective disease management strategies and documenting field history. Disease management in the current growing season and the next time the field is planted to sugar beet all depend on knowing what pathogen(s) are present in the field. The objective of our field sample diagnosis is to identify root pathogens and provide information to growers. In the last ten years (2010-2019) the sugarbeet pathology lab at the University of Minnesota, Northwest Research and Outreach Center has diagnosed samples from 1094 fields from across the sugar beet growing areas of Minnesota and North Dakota. Diagnosis is performed by gathering and assessing pertinent information from sample submitters followed by isolation of pathogens from infected tissues where appropriate and microscopic observation. The most common diseases were damping-off and/or adult root rots caused by Rhizoctonia solani (559 samples $=51 \%$ ) and Aphanomyces cochlioides (332 samples $=30 \%)$. Diseases caused by Fusarium oxysporum or F. secorum have been found in 63 samples (6\%) during this period. Other pathogens appearing infrequently are Pythium spp., and Verticillium dahliae. In the last five years, both $R$. solani and $A$. cochlioides have been isolated from 45 of 444 samples $(10 \%)$. Accurate identification of pathogens in sugar beet fields through proper sampling and diagnosis will give growers information to make better disease management decisions including cultivar and seed treatment selection and in-season fungicide applications.

\section{Multiplex CRISPR tool kits for citrus improvement against diseases}

X. HUANG, Y. Wang, J. Xu, N. Wang, Citrus Research and Education Center, University of Florida, Lake Alfred, FL, USA

CRISPR technology has been utilized for citrus genome editing. However, the gene editing efficiency in citrus remains low and it is very difficult to obtain homozygous or biallelic knockout mutants in citrus. Here, we aim to develop multiplex CRISPR tool kits to further improve the efficacy of citrus genome editing. First, we constructed a GFP surrogate reporter system to test functionality of CRISPR/Cas9 system in citrus protoplasts. Next, we screened and selected a native citrus U6 promoter that is more efficient than Arabidopsis counterpart used in previous studies for citrus genome editing. Finally, we established tRNA processing system-mediated and Csy4-mediated multiplex genome editing systems in citrus. Importantly we showed that the multiplex CRISPR tool kits enable us to obtain homozygous or bi-allelic mutants in the first generation of citrus with high efficiency. In conclusion, multiplex CRISPR genome editing systems based on endogenous tRNA processing and Csy4 nuclease were established for citrus. The tool kits are being used to modify putative susceptibility genes against citrus canker or HLB to improve disease resistance of elite citrus varieties.

\author{
A better method for profiling the eukaryotic diversity of the phytobiome \\ L. R. TRIPLETT (1), S. Taerum (2), D. J. Gage (3), B. Steven (2), (1) Connecticut Agricultural Experiment Station, New Haven, CT, \\ USA; (2) The Connecticut Agricultural Experiment Station, New Haven, CT, USA; (3) University of Connecticut, Storrs, CT, USA
}

High-throughput sequence profiling of bacteria and fungi is the most common way to study the phytobiome, although this approach only captures a portion of the ecological diversity present. Eukaryotic microorganisms including heterotrophic protists, algae, arthropods, and nematodes are abundant in the rhizosphere, where they influence plant health by shaping the bacterial microbiome and recycling nutrients. However, these organisms are too diverse to profile with group-specific barcoding primers, and the abundance of plant DNA in the rhizosphere makes these organisms difficult to study using universal 18S rRNA gene primers. We developed a peptide nucleic acid (PNA) clamp to block the amplification of maize DNA from rhizosphere samples. In quantitative PCR tests, the clamp was highly efficient at blocking amplification of DNA from several grain crop species, but did not block amplification of soil protist DNA. 18S rRNA gene sequencing from field-grown maize rhizospheres showed that the clamp reduced the abundance of plant-derived reads from $67.5 \%$ to less than $1 \%$, and increased the number of sequence variants from 20,000 to 33,000. Clamp addition greatly expanded the taxonomic richness of protists, animals, and fungi that could be measured in the samples, and did not introduce any apparent bias in the overall composition of those groups. These results indicate that PNA clamping is a highly promising method for exploring the poorly understood eukaryotic members of the phytobiome.

Integration of system phenotypes in microbiome networks to identify candidate synthetic communities: A study of the grafted tomato rhizobiome

R. Poudel (1,2,3), A. Jumpponen (4), M. M. Kennelly (5), C. L. Rivard (6), L. Gomez-Montano (5), K. A. GARRETT (1,2,3), (1) Plant Pathology Department, University of Florida, Gainesville, FL, USA; (2) Institute for Sustainable Food Systems, University of Florida, Gainesville, FL, USA; (3) Emerging Pathogens Institute, University of Florida, Gainesville, FL, USA; (4) Biology Department, Kansas 
Understanding factors influencing microbial interactions, and designing methods to identify key taxa, are complex challenges for achieving microbiome-based agriculture. Here we use a grafted tomato system to study how grafting and the choice of rootstock influence root-associated fungal communities. We then construct a phenotype-OTU network analysis (PhONA) using random forest and network models of the observed fungal OTUs and associated tomato yield data. PhONA provides a framework to select a testable set of OTUs for follow-up studies. Three tomato rootstocks (BHN589, RST-04-106 and Maxifort) were grafted to a BHN589 scion and the associated endosphere and rhizosphere fungal communities were profiled by sequencing the fungal Internal Transcribed Spacer. There was evidence for a rootstock effect (explaining $\sim 2 \%$ of the total variation, $\mathrm{p}<0.01$ ) on the fungal community. The most productive rootstock, Maxifort, supported higher fungal species richness than the other rootstocks or controls. Differentially abundant OTUs specific to each rootstock were identified for both endosphere and rhizosphere compartments. PhONA integrates the information about individual OTU obtained via differential abundance tests and role analyses. It identified OTUs that were directly important for predicting tomato yield, and other OTUs that were indirectly linked to yield through their links to these OTUs. Future studies of taxa associated with effective rootstocks identified using PhONA could support the design of synthetic fungal communities for microbiomebased agriculture.

\section{Genomics of Ralstonia pseudosolanacearum infecting multiple hosts in Southeast Asia}

T. L. KLASS (1,2), M. Hayes (3), T. M. Tran (4), J. Long (2), C. An (5), S. Ong (6), K. H. Seng (7), A. Sanchez (8), S. A. Miller (1,9), C. Allen (3), J. M. Jacobs (1,2), (1) Infectious Diseases Institute, The Ohio State University, Columbus, OH, USA; (2) Department of Plant Pathology, The Ohio State University, Columbus, OH, USA; (3) University of Wisconsin-Madison, Madison, WI, USA; (4) Nanyang Technological University, SINGAPORE; (5) iDE Cambodia, Siem Reap, CAMBODIA; (6) Royal University of Agriculture, Phnom Penh, CAMBODIA; (7) iDE Cambodia, Phnom Penh, CAMBODIA; (8) University of San Carlos, Guatemala City, GUATEMALA; (9) Department of Plant Pathology, The Ohio State University, Wooster, OH, USA

Ralstonia solanacearum, the causal agent of bacterial wilt disease, is arguably the most important bacterial plant pathogen worldwide. $R$. solanacearum is part of a variable species complex that includes large differences in host range and genetics. As a result, management strategies and resistance breeding must be adapted for local $R$. solanacearum strains. $R$. solanacearum has long been present in Cambodia and Vietnam. However, information on the diversity and distribution of bacterial wilt in these countries is lacking. To fill this knowledge gap, we isolated Cambodian and Vietnamese $R$. solanacearum strains from various hosts, including tomato, long bean, bitter gourd, and hot pepper. Long bean and bitter gourd were not previously known hosts for $R$. solanacearum. Multiplex PCR and partial endoglucanase gene sequencing were used to determine phylotype and sequevar, respectively, of the isolates. All isolates were confirmed to be phylotype I, which is also known as Ralstonia pseudosolanacearum, and cluster into a unique sequevar most closely related to sequevar 48 . Our results overall characterize the diversity of $R$. pseudosolanacearum in a specific geographic region and a continually expanding host range. We are currently carrying out comparative genomics to understand the relationship of these Southeast Asian isolates to the $R$. solanacearum species complex. These results provide new insights on pathogen diversity, which lays the foundation for the development of resistant varieties effective against Southeast Asian bacterial wilt.

\section{Occurrence of distinct genetic variants of cotton leafroll dwarf virus in Texas suggests its multiple introduction or local evolution in the U.S. \\ O. J. ALABI (1), C. Villegas (1), T. S. Isakeit (2), (1) Texas A\&M University, Weslaco, TX, USA; (2) Dept of Plant Pathology and Microbiology, Texas A\&M University, College Station, TX, USA}

The aphid vectored cotton leafroll dwarf virus (CLRDV; Polerovirus) and pathogen of cotton blue disease (CBD) was first described in Africa and subsequently in Asia Pacific and South American countries. Recently, a unique CLRDV strain with a divergent P0 protein was detected in Alabama and Florida cotton fields affected by cotton leafroll dwarf disease (CLRDD), which resembles CBD. CLRDD/CLRDV have also been reported from other U.S. cotton producing states, including Texas and initial data suggested that CLRDV isolates in U.S. are genetically homogenous. Analysis of P0 sequences of 15 CLRDV isolates from Texas revealed their segregation into two distinct clades, typified by isolates TX12 and TX20. The near complete genome of CLRDV was RT-PCRamplified from each isolate with a pair of newly designed primers, cloned and Sanger sequenced. The obtained 5,579-nt sequence is putatively missing 27-nt 5'UTR and 79-nt 3'-UTR sequences. The near complete genomes of TX12 and TX20 were $96.7 \%$ identical to each other and $96.5 \%$ \& 98.1\% identical, respectively to isolate Macon_AL from Alabama. Notably, the P0 of TX12 and TX20 shared $93.6 \%$ nt \& $91.5 \%$ aa identities with each other and $94.1 \%$ nt $\& 91.9 \%$ aa and $99.4 \%$ nt $\& 99.6 \%$ aa identities, respectively with corresponding sequence of Macon_AL. Genome-wide and P0-based phylogenetic analysis also revealed the segregation of U.S. CLRDV isolates into two clades with TX12 forming a distinct clade from TX20, Macon_AL and other U.S. isolates. The results suggest that multiple introductions or local evolution of CLRDV in the U.S. may have occurred.

Attenuation of Tobacco mosaic virus (TMV) pathogenesis on the tomato plant growth by a nanoparticulated sanitizer L. M. VÁSQUEZ-SILLER (1), O. Cordero-Garcia (1,2), A. Lopez Benitez (3), A. Sanchez-Lopez (1), N. A. Ruiz-Torres (1), A. Mancera-Rico (1), G. A. Frias-Treviño (1), G. S. Leyva-Mir (4), E. Cerna-Chávez (1), (1) Universidad Autónoma Agraria Antonio Narro, Saltillo, Coahuila, MEXICO; (2) Corteva Agriscience, Los Mochis, MEXICO; (3) Universidad Autónoma Agraria Antonio Narro, Saltillo Coahuila, MEXICO; (4) Universidad Autónoma de Chapingo, Texcoco, Edo. de Mexico, MEXICO

TMV causes losses in tomato production around $15 \%$ and $65 \%$ in greenhouse. This work evaluated TMV-inoculated tomato plants response to spraying a nanoparticulated sanitizer, $360^{\circ}$ Agroker $^{\circledR}$, using varieties Sofimely and Villa Narro, during vegetative and 
anthesis stages; inoculum monitoring done with Enzyme-Linked-Immuno-Sorbent-Assay (ELISA PathoScreen-TMV-Agdia ${ }^{\circledR}$ ) readings (OD). Sprays applied on leaves with $360^{\circ}$ Agroker $(3.3 \mathrm{~mL} / \mathrm{L})$ at frequency of three and seven days; absolute blanks and TMV-withoutAgroker blanks, and four replications included. Four ELISA readings done after first spray with intervals of 15 days in each inoculated stage of growth. 11 variables were analyzed with ANOVA, Tukey 5\% (SAS-9.1), Conglomerated (CA) and Principal Components (PC) Analysis (Minitab-16). Highly significant differences $(P \leq 0.0001)$ in: Plant height; leaves number; $(O D)$; plant fresh and dry weight; seeds number per fruit; longitude, locules number; diameter, and weight per fruit, and yield. CA generated seven groups (Euclidian distance 0.76 ), in which, in some of them, $360^{\circ}$ Agroker attenuated TMV pathogenesis in some variables such as plant height reducing up to $17.2 \%$; yield $29.50 \%$; seed number $57.97 \%$ in Villa Narro, and in Sofimely, $21.4 \%$, 24.94\% and $47.11 \%$ respectively. PC generated three components explaining the whole bioassay variation and a negative correlation between OD and growing variables inoculated with TMV such as yield $\left(-0.965^{* *}\right) .360^{\circ}$ Agroker revealed potential to reduce TMV damages on tomato for what studies should be done involving other virus groups of this crop.

Venturia carpophila - Mating type idiomorphs and genetic diversity on peach in the Southeastern U.S.A. C. H. BOCK (1), C. A. Young (2), M. Zhang (3), C. Chen (1), P. M. Brannen (4), J. E. Adaskaveg (5), N. D. Charlton (2), (1) USDA ARS, Southeastern Fruit and Tree Nut Research Laboratory, Byron, GA, USA; (2) Noble Research Institute, LLC, Ardmore, OK, USA; (3) USDA-ARS-SEFTNRL, Byron, GA, USA; (4) University of Georgia, Athens, GA, USA; (5) Department of Microbiology and Plant Pathology, University of California, Riverside, CA, USA

Peach scab (caused by Venturia carpophila) is a major disease affecting peach production in the southeastern U.S.A. We identified the mating type idiomorphs and explored whether they were in equilibrium, and performed preliminary studies on the population genetic diversity using previously developed microsatellite markers. Mating type gene MAT1-1-1 was identified in an available genome sequence, and the MAT1-2-1 gene was subsequently PCR amplified. Mating type loci structures were consistent with those of other Venturia species ( $V$. effusa and $V$. inaequalis). Primers designed to each of the mating type genes and a reference gene TUB2 were used as a multiplex PCR reaction to screen 81 isolates from populations of $V$. carpophila in various locations in the eastern U.S.A. The mating type loci were in equilibrium. The 81 isolates were a genetically diverse group, comprising 69 multilocus genotypes. A discriminant analysis of principal components indicated limited differentiation with overlap among populations. Due to small population isolate numbers, the results are considered preliminary. The ability to identify mating types of $V$. carpophila provides a basis for understanding reproductive methods of the pathogen in different peach production areas and can be a basis for further studies of genetics of the peach scab pathogen.

Genome assembly and comparative genomics of plant associated Rhizoctonia spp.

J. GIL (1), M. Colle (2), T. D. Miles (2), A. Rojas (1), (1) University of Arkansas, Fayetteville, AR, USA; (2) Michigan State University, East Lansing, MI, USA

The Rhizoctonia species complex in the family Ceratobasidiaceae is commonly associated with its pathogenic lifestyle causing diseases in crops of economic importance. However, this family comprises saprobes and mycorrhizal symbionts associated with orchids. Within the family, the genera Ceratobasidium and Thanatephorus are teleomorphic stages of Rhizoctonia. Classification of this species is based on hyphal recognition and fusion distinguishing different anastomosis groups within 2 major groups: multinucleate (Thanatephorus spp.) and binucleate (Ceratobasidium spp.). While the genomes of multinucleate anastomosis groups have been assembled and their pathogenicity mechanisms studied, binucleate isolates have not been equally described. Isolates of different anastomosis groups differ in genome sizes, host specificity and number of secreted proteins, suggesting differences in pathogenesis. Given this diversity and the lack of information for binucleate isolates to date, studying the genome of representatives from all anastomosis groups remains necessary for a better understanding of the species. We assembled the genomes of 4 Rhizoctonia solani binucleate isolates from Arkansas using longread sequences and characterized their growing behavior, including isolates from anastomosis group AG-1, on different carbon sources in an in vitro assay. Based on the genomic sequences, CAZymes were predicted and the functional profile of pathogenic and symbiotic isolates was compared. These preliminary genomic resources provide a framework for future comparative analyses.

Correlating drought induced susceptibility of Austrian pine with pathogen aggressiveness S. K. GHOSH (1), E. Visser (2), M. Sovic (3), A. O. Conrad (4), J. Slot (5), S. Naidoo (2), P. Bonello (4), (1) The Ohio State University, Columbus, OH, USA; (2) Forestry \& Agricultural Biotechnology Institute, Pretoria, SOUTH AFRICA; (3) Center for Advanced Plant Sciences, The Ohio State University, Columbus, OH, USA; (4) The Ohio State University, Department of Plant Pathology, Columbus, OH, USA; (5) Department of Plant Pathology, The Ohio State University, Columbus, OH, USA

Increased drought and heat render trees more susceptible to the biotic stress imposed by pathogen invasions. This increased susceptibility may be due to poorly understood metabolic trade-offs between abiotic and biotic (pathogen) stress responses. The conifer model system Austrian pine-Diplodia spp. was used to investigate these tradeoffs. A split-plot design was applied to test the effects of simulated drought/elevated temperature and inoculations with aggressive $D$. sapinea and normally non-aggressive D. scrobiculata on 34 year old open-pollinated Austrian pine saplings. Phenotypic characterization of resistance (lesion length) was correlated with underlying metabolic and transcriptional profiles. As expected, under abiotic stress Austrian pine became much more susceptible to both pathogens and as susceptible to non-aggressive $D$. scrobiculata as it was to D. sapinea. Phenolic metabolites associated with defense, like cinnamic acids, flavonoids, and stilbenoids were reduced in concentration, reflecting an observed down-regulation of their associated pathways. This clearly demonstrates that pine defense is compromised against pathogenic attack in the presence of environmental stress. These results provide necessary inputs for delving deeper into tree-pathogen-environment interactions over time and space. The ultimate goal of these studies is to provide the basis for developing genotypes that are simultaneously tolerant towards the multiple stress factors that characterize real world scenarios, especially under conditions of global warming. 
Developing a phage cocktail to control bacterial blotch of mushroom in organic systems

A. A. AHMAD (1,2), K. Hockett (3), R. Herschlag (4), C. Bull (5), (1) Dept of Plant Pathology and Environmental Microbiology, Penn State Univ, University Park, PA, USA; (2) Dept of Plant Pathology, Faculty of Agriculture, Minia Univ, El-Minia, EGYPT; (3) The Pennsylvania State University, University Park, PA, USA; (4) Department of Plant Pathology and Environmental Microbiology Penn State, University Park, PA, USA; (5) Pennsylvania State University, University Park, PA, USA

Bacterial blotch of mushrooms is the most economically important disease complex of button mushrooms, Agaricus bisporus, in organic mushroom production. Blotch, characterized by brown lesions on the surface of mushroom caps, is caused by Pseudomonas tolaasii, as well as at least 10 other Pseudomonas species. Because of the limitations of conventional methods to control the disease, there has been an increasing interest in using bacteriophage as a biological control tool for managing bacterial blotch. In this study, we isolated phage that are able to infect blotch inciting Pseudomonas strains from mushroom casing samples. Pseudomonas species commonly isolated from diseased mushrooms were selected as hosts for phage isolation. Phages were isolated representing a variety of plaque morphologies on each host strain. Among these phages, at least seven demonstrated strong lytic activity against individual Pseudomonas strains, whereas the other phages were more limited in their ability to lyse various hosts. The host range of the phages with strong lytic activity were evaluated against a panel of Pseudomonas species. We are carrying out a morphological and molecular characterization of the isolated phages, as well as assessing the potential of using these phages as a biocontrol agent to control bacterial blotch of mushrooms.

The impact of climate and grapevine leafroll disease on vine productivity and fruit quality of 'Cabernet Franc' in Pennsylvania vineyards

A. VARAKSA (1), B. E. Hed (2), M. Centinari (3), C. Rosa (4), (1) Penn State University, State College, PA, USA; (2) Lake Erie Regional Grape Res \& Ext Ctr, North East, PA, USA; (3) Penn State University, University Park, PA, USA; (4) The Pennsylvania State University, University Park, PA, USA

Grapevine leafroll-associated viruses (GLRaVs) are economically important pathogens that cause grapevine leafroll disease (GLD) in Vitis spp. A survey to assess the extent of GLRaV-1 and GLRaV-3's presence in Pennsylvania vineyards was performed. From this survey, a subset of five 'Cabernet Franc' vineyards located in the three main climatic areas of the state were selected to determine the influence of disease and climate on vine productivity and fruit quality. To achieve this goal, 12 to 15 pairs of infected and healthy grapevines were selected per vineyard and data on grape yield, juice chemistry, anthocyanin production, bud cold hardiness, and pruning weight were collected. In 2018 berries from infected vines had lower juice brix across 2 vineyards, and higher titratable acidity in 1 vineyard $(p=0.031)$, indicating that the disease can affect fruit quality. Preliminary yield data in 2019 indicate that two vineyards each had an estimated $17 \%$ yield loss, of which one also had reduced cluster weights as well as a $21 \%$ decrease in pruning weights in infected vines. Another vineyard located in the northwest had minimal production differences between healthy and infected vines, suggesting that weather conditions may play a role on disease impact. Data from the other 2 vineyards is still being analyzed. Results from this study could be used to create a disease model for growers in Pennsylvania as well as regions with similar climates, with the goal to establish proper management strategies for growers.

Aspergillus mycotoxins and genetic characterization of Aspergillus flavus isolates from sesame

H. K. ABBAS (1), P. K. Chang (2), V. Khambhati (3), C. Accinelli (4), W. T. Shier (5), (1) United States Department of Agriculture, Agricultural Research Service, Stoneville, MS, USA; (2) USDA-ARS, SRRC, New Orleans, LA, USA; (3) Mississippi State University, Mississippi State, MS, USA; (4) University of Bologna, Bologna, ITALY; (5) University of Minnesota, College of Pharmacy, Minneapolis, MN, USA

Sesame is extensively cultivated in Asia, but more recently, the demand for sesame products and seeds has resulted in planting of more acreage in the US. Initial studies of sesame in the Mississippi Delta showed low levels of aflatoxin and cyclopiazonic acid (CPA), and a follow-up study on genetic characterization of Aspergillus isolates showed that most of isolates from the MS Delta lacked both the aflatoxin and CPA biosynthesis gene clusters. Close to 500 Aspergillus flavus isolates were collected from four sesame varieties planted in the MS Delta in 2014 and 2015 and in the Florida Panhandle in 2015 and were investigated for the cause of low aflatoxin contamination of sesame seeds. A rapid molecular fingerprinting method was developed to assess the influence of prior application to field soils of the atoxigenic strain Afla-Guard ${ }^{\circledR}$ (NRRL 21882) on A. flavus populations in harvested crops. Sesame seed samples harvested from Afla-Guard ${ }^{\mathbb{R}}$-treated soil in MS had $66.7 \%$ to $95.9 \%$ of $A$. flavus isolates belonging to the NRRL 21882-type (i.e., no biosynthesis gene clusters for aflatoxin or CPA), whereas only $5.0 \%$ to $32.5 \%$ of isolates from sesame seed samples from treated FL soil lacked both gene clusters. This high percentage of the NRRL 21882-type A. flavus in MS sesame samples may be attributed to prior applications of Afla-Guard ${ }^{\circledR}$ to soil in that region. The results suggest a tenacious survivability of this particular type of atoxigenic $A$. flavus biocontrol strain in field soil and supports sesame as a good candidate for crop rotation with other row crops.

Effect of soybean leaf-associated gemycircularvirus infected Sclerotinia sclerotiorum on soybean gene expression C. PEDERSEN, Z. Wang, S. Y. L. Marzano, South Dakota State University, Brookings, SD, USA

Soybean leaf associated gemycircularvirus 1 (SlaGemV-1) is a gemycircularvirus that infects and causes hypovirulence in Sclerotiniaceae fungi, a group of economically important plant pathogens. SlaGemV-1 infected Sclerotinia sclerotiorum have slightly slower growth and grows endophytically in plants without causing disease. A resistance to pathogenic S. sclerotiorum was observed in plants after initial seed colonization with nonpathogenic, SlaGemV-1-infected S. sclerotiorum (Ss-SlaGemV1). In this study, we aimed 
to determine the mechanisms of observed resistance. Four replications of soybean seeds were placed by the inoculum of Ss-SlaGemV1 or by the autoclaved media as a negative control in comparison. At V3, total RNA was extracted from the leaves and subjected to RNAseq to determine the mechanisms involved in the observed induced resistance. Afterward, the plants were challenged with pathogenic $S$. sclerotiorum and resistance was found induced in the Ss-SlaGemV1 colonized plants but not the control. RNA-seq analysis detecting fold-change of 2 for 113 differentially expressed genes including several involved in the systematically acquired resistance and salicylic biogenesis pathways. Furthermore, mutants of Arabidopsis thaliana which have deletions in the biosynthesis pathways of jasmonate, salicyclate, and ethylene, factors in SAR and ISR defense pathways, will be tested for their induction of resistance under Ss-SlaGemV1 treatment. Gemycircularviruses infected endophytes to induce resistance could be an effective strategy in the effort to control plant pathogenic fungi.

\section{The first whole genome sequence of 'Candidatus Liberibacter asiaticus' strain from Brazil}

P. Silva (1), J. Huang (2), N. A. Wulff (3), R. Krugner (4), J. CHEN (5), (1) Fundecitrus, Araraquara, BRAZIL; (2) South China Agricultural University, Guangzhou, CHINA; (3) FUNDECITRUS, Araraquara, BRAZIL; (4) United States Department of AgricultureAgricultural Research Service, Parlier, CA, USA; (5) USDA-ARS, Parlier, CA, USA

Huanglongbing (HLB) is a highly destructive citrus disease associated with infection of 'Candidatus Liberibacter asiaticus' (CLas), an unculturable $\alpha$-proteobacterium. CLas whole genome sequences have provided important information for HLB management. In this study, the whole genome of CLas strain 9PA from São Paulo, Brazil, was sequenced. CLas-infected leaf DNA was enlarged and subjected to Illumina NextSeq $500(1 \times 75$ cycle). A total of $95 \mathrm{Gbp}$ in $>400 \mathrm{M}$ reads (read size $=35$ to 85 bp) were generated. The 9PA genome sequence was assembled by referencing to the whole genome sequence of CLas strain psy62 (CP001677.5) using CLC Genomic Workbench 7.5 and Bowtie 2. A de novo assembling was also performed using the CLC software. Gap enclosure was performed using information from de novo contigs, read walking, and PCR-Sanger sequencing. The 9PA draft genome sequence has $1,150,691 \mathrm{bp}$ in 187 contigs with an average nucleotide coverage of $10.2 \mathrm{X}, \mathrm{N} 50$ of 11,457 bp, 1,321 ORFs and 52 RNA genes. By reference-mapping to prophage SC1 (Type 1), SC2 (Type 2), and P-JXGC-3 (Type 3), two prophages were identified: P-BSP1-1 with 39,126 bp (Type 1) and P-BSP1-3 with 31,794 bp (Type 3). Type 2 prophages were absent. Analysis of the terL locus showed that the 9PA strain was in the Asiatic and Californian CLas group, not the Floridian group. This is the first whole genome sequence of CLas from Brazil. The 9PA genome sequence will contribute to the advancement in HLB research and control in Brazil and around the world.

Comparative genome analysis of Spiroplasma citri isolated from different hosts, locations, and times in the U.S.A. R. RATTNER (1), F. Osman (2), D. Pagliaccia (3), S. Hajeri (4), J. Chen (5), G. Vidalakis (6), R. K. Yokomi (7), (1) USDA, ARS, San Joaquin Valley Sciences Center, Parlier, CA, USA; (2) University of California, Davis, Davis, CA, USA; (3) Dept of Botany and Plant Sciences, Riverside, CA, USA; (4) Central CA Tristeza Eradication Agency, Tulare, CA, USA; (5) USDA-ARS, Parlier, CA, USA; (6) University of California, Riverside, Riverside, CA, USA; (7) USDA ARS PWA, Parlier, CA, USA

Spiroplasma citri, the causal agent of citrus stubborn disease and brittle root disease of horseradish, is transmitted by the beet leafhopper (Neoaliturus tenellus) in the U.S.A. The first whole genome sequence of $S$. citri from California was isolated from Chinese cabbage and reported in 2020. Since then, five additional $S$. citri strains from the U.S.A. were isolated from citrus, horseradish, and beet leafhopper, sequenced and genomes assembled. Analysis of the sequences revealed a single chromosomal contig of similar size ( $\sim 1.6 \mathrm{Mbp})$ for each strain. Annotation of the genomes predicted between 1908 and 2569 coding sequences in the U.S.A. S. citri strains. The chromosome of each $S$. citri strain contained a repetitive region with numerous insertions of plectroviral sequences. Plant-derived $S$. citri strains contained between one and three extrachromosomal contigs, while beet leafhopper-derived strains possessed nine to ten extrachromosomal contigs. Phylogenetic position was analyzed utilizing nucleotide sequences of 16S ribosomal RNA (rRNA) and by concatenation of essential genes such as spiralin, p58, and phosphoglycerate kinase (PGK) genes. Phylogenetic analysis of these six strains, along with Moroccan strain R8-A2, revealed that these $S$. citri strains were nearly identical and were most closely related to $S$. phoeniceum and S. kunkelii. This influx of genomic data affords the ability to improve detection assays and further our understanding of the diversity that has arisen during the evolution of $S$. citri.

Extracellular enzyme activity of Colletotrichum species associated with apple bitter rot disease F. KHODADADI (1), J. B. González (2), S. Aćimović (1), (1) Cornell University, Hudson Valley Research Laboratory, Highland, NY, USA; (2) Cornell University, NY, USA

Multiple Colletotrichum species cause bitter rot of apple and lead to economically significant losses in New York ranging from 2 to $100 \%$. Pathogenicity and virulence capacity of Colletotrichum species could be associated with their ability to produce extracellular enzymes. We qualitatively evaluated 32 isolates of $C$. fioriniae, belonging to $C$. acutatum species complex, and $C$. $s p$. nov. and $C$. chrysophilum belonging to $C$. gloeosporioides species complex, using agar plate method for lipolytic, proteolytic, amylolytic and cellulolytic activities. All species showed lipolytic, amylolytic and cellulolytic activity after five days of incubation on PDA. However, no proteolytic degradation halo was observed for all species. Formation of a yellow halo around the colonies confirmed their cellulase activity, with $C$. chrysophilum possessing the significantly larger mean degradation halo $(8 \mathrm{~mm})$ compared to $C$. $s p$. nov. and $C$. fioriniae (6 and $6.5 \mathrm{~mm}$, respectively). Clear halo around the isolate colonies indicated their ability to produce lipase and amylase. $C$. chrysophilum isolates showed the lowest lipid degradation with mean halo of $17 \mathrm{~mm}$ compared to C. fioriniae and $C$. sp. nov. isolates, with the mean of 23 and $27 \mathrm{~mm}$, respectively. For amylase, C. chrysophilum and C. fioriniae showed the smallest halo size (3 mm), while $C$. sp. nov. isolates showed the largest $(8 \mathrm{~mm})$. Our findings demonstrate the differences between Colletotrichum species in production of extracellular enzymes and establishes a foundation for further studies on the enzyme activity-pathogenicity relation. 
Deletions in the coat protein of wheat streak mosaic virus facilitated differential interaction with wheat proteins compared to wild-type virus

J. ALEXANDER (1), S. Tatineni (2), (1) USDA ARS, Lincoln, NE, USA; (2) USDA ARS, Univ of Nebraska, Lincoln, NE, USA

Wheat streak mosaic virus (WSMV; genus Tritimovirus, family Potyviridae), an economically important virus of wheat with significant yield loss in the Great Plains. Previously, we reported that coat protein (CP) of WSMV tolerates extensive deletions for systemic infection of wheat. Deletion of CP amino acids (aa) 36-57 elicited symptoms similar to those of wild-type (WT) virus, while deletions comprising aa 58-84 elicited severe chlorotic streaks and acute chlorosis with accelerated virus movement and genomic RNA accumulation. In this study, we examined interaction of wheat proteins with WSMV CP deletion mutants by performing coimmunoprecipitation of proteins from WT- or CP deletion mutants-infected wheat with CP antibodies, followed by mass spectrometry. Analyses of mass spectrometry data revealed that severe-symptom inducing deletion mutants (SSID; $\Delta 36-84 \mathrm{aa}$ and $\Delta 58-84 \mathrm{aa}$ ) efficiently interacted with the CI protein of WSMV compared to WT virus or mutant with no severe symptom-inducing deletion mutant (NSSID; $\Delta 36-57 \mathrm{aa}$ ). Additionally, the SSID mutants, but not WT or NSSID mutant, interacted with NIa-Pro of WSMV. The SSID mutants caused 17 to 24 -fold enhanced interaction with dynamin-like proteins compared to WT or NSSID mutant. The SSID mutants facilitated interaction with nine wheat proteins but failed to interact at detectable levels with 16 wheat proteins compared to WT or NSSID mutant. Our data suggest that deletion of CP aa 58-84 caused differential interaction with wheat proteins, possibly contributing to enhanced symptom severity in wheat.

High-throughput sequencing of viruses and non-viral vascular-limited pathogens in blueberry (Vaccinium corymbosum) in Ohio D. MASSAWE (1), M. L. Lewis Ivey (2), P. A. Paul (2), S. A. Miller (2), (1) The Ohio State University, Department of Plant Pathology, Wooster, OH, Wooster, OH, USA; (2) Department of Plant Pathology, The Ohio State University, Wooster, OH, USA

Vaccinium corymbosum (blueberry) is among the food crops that contribute significantly to the US economy, with production valued at $\$ 720$ million. A previous survey conducted of blueberry farms in the Pacific Northwest, Northeast, Southeast and Midwest, excluding Ohio, uncovered important viruses infecting this vegetatively propagated crop. To understand the population of viruses and non-viral vascular-limited pathogens infecting blueberries in Ohio, a survey was conducted on blueberry farms in 2019. Symptomatic leaves were collected from 27 farms located in 19 counties. Total nucleic acid was extracted from the leaves and sequenced using NovaSeq highthroughput sequencing. Through bioinformatics analysis, nearly complete genomes of potential blueberry-infecting viruses were found, with $92-99 \%$ nucleotide identity to reference sequences in GenBank. The predominant viruses were Blueberry latent virus, Blueberry leaf mottle virus, Blueberry mosaic associated virus, Blueberry virus A, Tomato ringspot virus, Tobacco ringspot virus, and Blueberry leaf mottle virus. Partial genomes for Blueberry green mosaic associate virus and Tobacco Streak virus were also recovered. Citrus exocortis viroid was detected, with $99.7 \%$ nucleotide identity with isolate DQ318790.1. About 52586 sequence reads had hits to Blueberry stunt phytoplasma, Xylella fastidious and Spiroplasma spp. These results indicate a high diversity of viruses and non-viral vascular-limited pathogens from Ohio blueberry farms and highlight the need to study their biology and possible yield impact.

Management performance mapping and the value of information for regional prioritization of management interventions C. Buddenhagen (1,2,3,4), J. L. Andrade-Piedra (5), G. A. Forbes (5), P. Kromann (6), I. Navarrete (5), S. Thomas-Sharma (7), Y. Xing $(1,2,3)$, R. Choudhury $(1,2,3,8)$, K. A. GARRETT $(1,2,3)$, (1) Plant Pathology Department, University of Florida, Gainesville, FL, USA; (2) Institute for Sustainable Food Systems, University of Florida, Gainesville, FL, USA; (3) Emerging Pathogens Institute, University of Florida, Gainesville, FL, USA; (4) AgResearch, Ltd., Ruakura, Hamilton, NEW ZEALAND; (5) CGIAR Research Program on Roots, Tubers and Banana, International Potato Center, Lima, PERU; (6) CGIAR Research Program on Roots, Tubers and Banana, International Potato Center, Quito, ECUADOR; (7) Louisiana State University Agricultural Center, Baton Rouge, LA, USA; (8) University of Texas Rio Grande Valley, Edinburg, TX, USA

In agricultural extension and development, decisions must often be made by policymakers and donors about where to implement interventions to improve crop yield. Implementation in some regions may provide substantially higher payoffs from investment, and higher quality information may help to target the high-payoff locations. Management performance maps (MPMs) identify where interventions will be maximally effective. The value of information (VOI) in this context is based on comparing the results of decision making guided by MPMs and the results of acting without taking the information into account. In case studies of Andean and Kenyan potato seed systems, we evaluate seed health and yield information from farms, plots, and individual plant observations. We illustrate use of Bayesian network modeling and recursive partitioning to characterize the relationship between seed health and yield responses and the environmental and management predictors used in studies of seed degeneration. These analyses address the expected performance of an intervention based on geographic predictor variables. In our Andean example, the benefit of positive selection of seed from asymptomatic plants, compared to roguing or random seed selection, was greater at high altitudes in Ecuador. Targeting training to high performance areas would often provide more benefits than would random selection of target areas. We illustrate how assessing the VOI can help inform targeted development programs and support a culture of continuous improvement for interventions.

New North American isolates of Venturia inaequalis can breakdown apple scab resistance in Malus floribunda 821, a key source for resistance breeding A. KHAN PHD, D. Papp, J. Singh, D. M. Gadoury, Cornell University, Geneva, NY, USA

Apple scab, caused by Venturia inaequalis, is the most destructive fungal disease of apple cultivars. Therefore, development of scab resistant cultivars is one of the highest priorities for breeding programs. The key source of resistance for apple scab breeding has been the gene Rvi6 from the Japanese crabapple Malus floribunda sel. 821. Approximately $90 \%$ of modern scab resistant apple cultivars carry resistance derived from $M$. floribunda 821 . We recently discovered scab infection on M. floribunda 821 trees in a research orchard 
at Cornell AgriTech, Geneva, NY. Leaves and fruit of M. floribunda 821 had sporulating apple scab lesions, many of which had coalesced to cover entire leaves. We observed both chlorosis, typical to Rvi6, and pinpoint pitting symptoms typical to failed infections by $V$. inaequalis on hosts bearing the Rvi7 gene. We assessed genetic diversity and population genetic structure of $11 \mathrm{~V}$. inaequalis isolates of North American and European origin from M. floribunda 821, 'Nova Easygro', 'Golden Delicious', TSR33T239, 'Schone van Boskoop', and 'Prima'. Our results suggest that the new Rvi6 virulent isolates emerged within USA populations, rather than being transported from Europe. The complete resistance breakdown in $M$. floribunda 821 but not in descendant cultivars, suggests that durable resistance to apple scab will require a more comprehensive understanding of distribution and evolutionary processes of pathogen isolates and Rvi6 mediated resistance in diverse genetic backgrounds.

Soil and soybean roots from high and low yielding field sites are characterized by distinct co-association microbiome networks A. Y. BANDARA (1), D. K. Weerasooriya (1), M. R. Duffeck (1), R. Trexler (1), R. Poudel (2), T. Bell (1), P. Esker (1), (1) The Pennsylvania State University, University Park, PA, USA; (2) Plant Pathology Department, University of Florida, Gainesville, FL, USA

Prevalence of sites within a farm that are consistently producing high $(\mathrm{H})$ or low $(\mathrm{L})$ crop yields is a frequently observed phenomenon in soybean. We conducted a study to examine whether microbial communities are associated with this phenomenon. Bulk soil (BS), rhizosphere soil (RS), and soybean roots (R) were sampled from historically H and L yield sites at V1 (first trifoliate) and R8 (maturity) soybean growth stages (SGS) from eight selected Pennsylvania soybean farms. DNA extracted from samples were subjected to PCR targeting fungal ITS regions and bacterial 16S rRNA genes. Amplicons were sequenced using MiSeq platform. Read counts were classified into OTUs and subjected to network analysis based on pairwise spearman correlations using igraph package in R. OTUs $>30$ read counts were included in the analysis. Links with a correlation $>0.9(P<0.0001)$ were retained in the network. Based on both ITS and 16S OTU data, a greater BS network size was observed for H-sites compared to L-sites at both SGS. Conversely, for RS and R, smaller network sizes were evident for H-sites compared to L-sites at both SGS (except R-16S-V1). The number of linked-nodes and total number of links were greater in the network from L-sites compared to H-sites for three sample types at both SGSs (except BS-ITSV1, BS-16S-V1, RS-16S-R8). Differences of other network attributes were also evident between H and L-sites. Results suggested that niche sharing and the number of potentially true interactions among fungal/bacterial community members are lower in samples from $\mathrm{H}$ sites compared to L-sites.

Pipecolic acid-mediated signaling in systemic acquired resistance X. YUAN (1), D. Li (2), R. Liu (1), D. Singh (2), R. Raina (2), P. Kachroo (1), (1) University of Kentucky, Lexington, KY, USA; (2) Syracuse University, Syracuse, NY, USA

Systemic acquired resistance (SAR) is a form of broad-spectrum resistance that protects uninfected tissues from secondary infections. SAR signaling requires two parallel branches, one regulated by salicylic acid (SA), and the other by pipecolic acid (Pip), azelaic acid (AzA) and glycerol-3-phosphate (G3P). AzA and G3P function downstream of the free radicals nitric oxide (NO) and reactive oxygen species (ROS). Pip functions by increasing NO/ROS levels and acts upstream of G3P. The distal Pip levels are dependent on normal transport of SA and G3P to the distal tissues. The local Pip levels are dependent on Jumonji (JMJ)14 encoded H3K4 demethylase, which regulates pathogen-induced $\mathrm{H} 3 \mathrm{~K} 4 \mathrm{me} 3$ enrichments and expression of Pip biosynthetic gene $A L D 1$. Consequently, jmj 14 mutants accumulate reduced level of Pip and show compromised SAR. Notably, exogenous Pip is unable to completely restore defective SAR phenotype of jmj14 plants, suggesting that other downstream factors regulate SAR in jmj14 plants.

\section{A fitness study of different Alternaria alternata genotypes resistant to SDHI fungicides: A classical and biochemical approach P. D. S. F. LICHTEMBERG (1), J. Kleemann (2), A. Mehl (2), J. Derpmann (2), T. Carraro (3), R. Puckett (4), T. J. Michailides (4), (1) University of California - Davis, Parlier, CA, USA; (2) Bayer AG - Crop Science Division, Monheim, GERMANY; (3) Universidade Federal do Paraná, Curitiba, BRAZIL; (4) University of California, Davis, Parlier, CA, USA}

Alternaria late blight, caused mainly by Alternaria alternata, can significantly impact crop yield and fruit quality. To control the disease, seasonal sprays may include SDHI fungicides, which can select resistant genotypes, such as SdhC-H134R and SdhB-H227Y. Our study aimed to demonstrate fitness costs associated with field strains carrying H134R and H277Y mutations compared to wild-type (WT) isolates. The effect of successive colony transfers on epidemiological parameters were determined. Furthermore, the impact of mutations on the complex II of the respiratory chain (enzymatic activity and SDHI binding) was studied by performing biochemical assays using extracted mitochondria. Our results revealed a clear fitness disadvantage for the H134R genotype affecting conidiation ($62 \%)$, germination $(-8.6 \%)$, mycelial growth $(+12 \%)$ and lesion size $(+41 \%)$. There was a conidiation cost in $\mathrm{H} 277 \mathrm{Y}(-79 \%)$ while the WT was not affected. Both resistant genotypes showed a reduced biochemical activity of the complex II when compared to WT, with H134R being most affected. Enzyme inhibition studies at various inhibitor concentrations showed that both mutations affected the complex II inhibition by boscalid at an extent where dose-response was not anymore detectable. In contrast, Fluopyram could still inhibit mutant complex II carrying H277Y in a dose-dependent manner. Taken together, our results demonstrate different degrees of fitness penalties associated with H134R and H227Y mutations and support the concept of incomplete cross-resistance among SDHI molecules towards different mutations.

Small-scale population genetic structure of Claviceps purpurea in two cool-season grass seed cropping systems in Oregon Q. Cheng, J. K. S. DUNG PHD, Oregon State University, Madras, OR, USA

Ergot, caused by Claviceps purpurea, is a major disease of Kentucky bluegrass (KBG) and perennial ryegrass (PRG) seed crops in Oregon. The importance of airborne ascospores and splash- and insect-dispersed conidia in ergot epidemiology was investigated at a 
small spatial scale using a population genetics approach. Two $10 \mathrm{~m}$ by $10 \mathrm{~m}$ sampling plots were randomly placed in one field each of KBG and PRG in Madras and Hermiston, respectively, and one-meter grid sampling was conducted to obtain sclerotia at each sample point. Isolates $(n=400)$ were hyphal-tipped from sclerotia and characterized using 12 microsatellite markers. Three-hundred-seventyeight multilocus haplotypes were observed and the clonal fraction among the plots ranged from $0 \%$ to $13 \%$, suggesting that ascospores were the primary source of inoculum. There were moderate levels of genotypic diversity $(H=4.47$ to 4.61$)$ and gene diversity $(H$ exp $=$ 0.42 to 0.47 ) among the sites. Analysis of molecular variance attributed $76 \%$ of the genetic variation to differences between isolates in a sampling plot, while $24 \%$ was attributed to isolates from the different hosts/locations. Autocorrelation coefficients between genetic distance and spatial distance were weak $(r<0.1)$ for all four sampling plots. The standardized index of association ranged from 0.005 $(P=0.09)$ to $0.03(P=0.0001)$ for the four plots, indicating differences in the relative contribution of ascospores and conidia to ergot epidemics among the sites. These results provide novel insights into the epidemiology and genetic structure of $C$. purpurea at small spatial scales.

\section{Grafted tomatoes with late blight resistance for the organic market}

I. MEADOWS (1), A. L. Strayer-Scherer (2), S. Sharpe (1), R. G. Gardner (3), P. Shoemaker (4), D. Panthee (5), (1) North Carolina State University, Waynesville, NC, USA; (2) Auburn University, Auburn, AL, USA; (3) North Carolina State Univ, Mills River, NC, USA; (4) Holly Spring Farm, Horseshoe, NC, USA; (5) NC State University, Mills River, NC, USA

Late blight of tomato, caused by Phytophthora infestans, is difficult to control under reduced or organic pesticide regimes and, if present, limits tomato production to the early season. Several heirloom-type tomato hybrid cultivars with resistance to late blight have been or are soon to be released from the NC State University tomato breeding program. In recent studies, the use of grafting tomato plants onto vigorous rootstocks increased yield and fruit size of some scion varieties under field conditions. The objective of this study was to measure yield response to grafting late blight resistant scions onto a vigorous rootstock. In 2019, replicated trials were conducted at two research stations and one commercial farm in western North Carolina. 'Mountain Gem', 'Mountain Rouge' and three other late blight resistant experimental hybrids were evaluated for late blight resistance and yield. Each cultivar was either non-grafted or grafted onto the vigorous rootstock 'Maxifort'. A popular heirloom cultivar, 'Cherokee Purple', was planted as a late blight susceptible, nongrafted control. Resistant scions showed no symptoms of late blight where the disease occurred. In only one of three locations did grafting cause a response. There, marketable yield was increased for some grafted cultivars and mean fruit weight was increased for all grafted cultivars. The use of a vigorous rootstock may improve yield and fruit size, but the response is at least dependent on the scion cultivar and location.

Fungal pathogens associated with perennial cereals in New York

M. FULCHER (1), E. Law (2), S. Wayman (2), M. Ryan (2), G. C. Bergstrom (2), (1) University of Minnesota, Saint Paul, MN, USA; (2) Cornell University, Ithaca, NY, USA

Perennial cereals (Thinopyrum intermedium 'Kernza' and Secale cereale 'ACE-1') were recently introduced in New York, and their interaction with local fungal flora is unexplored. We surveyed three field experiments and four on-farm trials from 2017-18 to record the incidence of putative foliar and spike pathogens. Fungi were isolated from symptomatic tissue, overwintered crop debris, and harvested grain for morphological and DNA sequence-based identification. The mycotoxin deoxynivalenol (DON) was quantified in harvested grain, and germination rates were related to seedborne fungal communities. Fourteen putative host-pathogen combinations were recorded. Although disease incidence and severity were too low to draw comparisons between management practices, the presence of common small grain pathogens in symptomatic tissue and overwintered crop debris was confirmed. Differences were seen between crop species for pathogen overwintering rates, black point ratings, and DON content. Germination rates were correlated with fungal load, and seedborne community composition was significantly influenced by host and location. We identified several potentially concerning diseases requiring further study in New York perennial grain cropping systems (ergot, Claviceps purpurea; Fusarium head blight, Fusarium, spp.; black point, Alternaria spp., Bipolaris spp.; tar spot, Phyllachora graminis). Perennial cereals can provide several benefits (e.g. reduced soil erosion and labor requirements), though without crop rotation their adoption may bring new challenges to disease management.

\section{Alternative mechanisms of self-resistance to cercosporin in Cercospora leaf blight pathogens on soybean}

M. I. COSTA DE NOVAES (1), V. Doyle (1), C. L. Robertson (1), S. Thomas-Sharma (2), (1) Department of Plant Pathology \& Crop Physiology, Louisiana State University, Baton Rouge, LA, USA; (2) Louisiana State University Agricultural Center, Baton Rouge, LA, USA

Cercospora leaf blight, caused by Cercospora $\mathrm{cf}$. flagellaris, occurs annually and can limit profitability of soybean production in the southern US. The pathogen relies on the production of a perylenequinone toxin, cercosporin, to kill host cells. Cercosporin is lightactivated to produce reactive oxygen species (ROS) and is therefore universally toxic to cells, except for the cells of the producer. The current model of self-resistance to cercosporin is based on the ability of Cercospora spp. to maintain cercosporin in a chemicallyreduced state inside hyphae, preventing internal ROS production. In another perylenequinone producing fungus, the toxin was specifically sequestered inside lipid droplets (LD) to prevent ROS production. Our studies into self-resistance of $C$. cf. flagellaris have revealed differences from the current model of self-resistance in Cercospora spp. Using light microscopy, we initially identified hyphae of two thicknesses $(21$ and $12 \mu \mathrm{m}$ ), with red cercosporin-like pigments in thinner hyphae. The presence of different hyphae may suggest a switch from biotrophic to necrotrophic lifestyle as observed in other hemibiotrophs. Using confocal fluorescence microscopy, we confirmed that hyphae contained reduced cercosporin uniformly in the cytoplasm. However, contrary to previous studies, light-cultured hyphae also contained cercosporin, specifically in vesicles of thinner hyphae. Studies with LD-specific dyes and inhibitors are currently underway. If LD trap cercosporin, lipid-inhibiting fungicides may provide new chemical tools for CLB management. 
DNA methyltransferases from Cryphonectria parasitica are involved in fungal development and pathogenicity, and in the response to hypovirus infection

Y. H. KO, K. K. So, J. Chun, D. H. Kim, Jeonbuk National University, Jeonju, SOUTH KOREA

The representative fungal DNA methyltransferase (DNMTase) genes of Cryphonectria parasitica were identified as $C p D m t 1$ and CpDmt2, orthologous of repeat-induced point mutation (RIP) defective (RID) and DNA methylation (DIM-2) of Neuropora crassa, respectively. In the previous study, $C p D m t 1$ but not $C p D m t 2$ has been implicated in the sporadic occurrence of sectorization. Therefore, we have focused on the study of the biological function of both DNMTase genes. Conidia production was only affected by the mutation of $C p D m t 1$, not by the $C p D m t 2$ mutation alone. Reactions of both mutants to various stressors confirmed, that the CpDmt1-null mutant (TdDMT1) showed hypersensitivity to high temperatures. Both mutants showed significant changes in pathogenicity in the opposite direction, i.e., TdDMT1 showed increased pathogenicity whereas the CpDmt2-null mutant (TdDMT2) showed decreased pathogenicity. In addition, when both single- and double-null mutants were transfected with CHV1, CHV1-containing TdDMT1 and TdDMT2 showed severe growth retardation. The titer of the transfected CHV1 was higher than that of the wild type, suggesting that there was no defect in virus replication and the accumulation of the viral genome was even increased. As the culture progressed, spontaneous viral clearance was observed in colonies infected with CHV1, which had never been reported in fungal-viral interactions. The study showed that both DNMTases play an important role in fungal development and pathogenicity, as well as in virus clearance.

Efficacy of different phytosanitary products against Pucciniastrum americanum in raspberry

M. Delisle-Houde (1), F. Demers (2), R. J. TWEDDELL (1), (1) Département de phytologie, Université Laval, Québec, QC, CANADA; (2) Club les productions Écolo-Max inc., Lévis, CANADA

In the Québec province, late leaf rust [Pucciniastrum americanum (Farl.) Arthur] is problematic for fall-fruiting raspberry growers, especially when growing seasons are wet and rainy. Under these conditions, management of the disease relies more on chemical control. However, few phytosanitary products are currently registered in Canada. In this study, different phytosanitary products registered in Canada for the management of various diseases of horticultural crops were tested for the control of raspberry late leaf rust. Efficacy was first determined in vitro on raspberry leaf discs. Based on in vitro efficacy, tested products were ranked from the most to the least effective as follows: Nova (myclobutanil), Sirocco (potassium bicarbonate), Fullback 125 SC (flutriafol), Pristine WG (boscalid + pyraclostrobin), Phostrol (phosphites of Na, K, and $\mathrm{NH}_{4}^{+}$), Kumulus DF (sulphur), StorOx (hydrogen peroxide), Cabrio EG (pyraclostrobin), Serenade Opti (Bacillus subtilis strain QST 713), and Actinovate SP (Streptomyces lydicus strain WYEC 108) / Double Nickel 55 (Bacillus amyloliquefaciens strain D747). Field assay was then conducted with five products (Fullback 125 SC, Kumulus DF, Nova, Phostrol, Sirocco). Foliar applications of Nova and Fullback 125 SC significantly reduced late leaf rust severity as compared with the control. In this study, flutriafol and myclobutanil were shown effective to control late leaf rust of raspberry.

DNA sequencing of mating-type genes confirms the heterothallic nature of Podosphaera leucotricha L. GAÑÁN (1), T. L. Peever (2), A. Amiri (1), (1) Washington State University, Wenatchee, WA, USA; (2) Washington State University, Pullman, WA, USA

Apple powdery mildew, caused by Podopshaera leucotricha (Erysiphales: Erysiphaceae), is one of the most economically significant diseases of cultivated apple (Malus $\times$ domestica). Sexual fruiting bodies (chasmothecia) are commonly observed in Washington apple orchards, suggesting a role in the life cycle and that $P$. leucotricha populations may undergo genetic recombination. However, the role of these structures remains unclear given knowledge gaps in the pathogen reproductive system and life cycle. The objective of this study was to characterize the mating-type idiomorphs of $P$. leucotricha isolates from different apple-producing regions in the United States. Specific primers, identified from whole-genome shotgun sequences, were developed for PCR-amplification of the MAT1-2-1 gene. The MAT1-1-1 gene was amplified using degenerate primers that targeted conserved regions of $M A T 1-1$ in other powdery mildew species. Among 20 isolates tested, the MAT1-1-1 gene was carried by eight isolates while the MAT1-2-1 gene was carried by 12 isolates, and PCR was confirmed by Sanger sequencing. These results suggest that $P$. leucotricha is likely heterothallic with similarities to mating genes in other powdery mildew fungi. Specific primers will help investigate reproductive mode in P. leucotricha, which is essential to understand its population biology and epidemiology.

Integrating planting date, fungicide spray and variety choice for management of chickpea foliar diseases in Central Virginia S. Chawla, L. Rutto, Z. MERSHA, Virginia State University, Petersburg, VA, USA

Chickpea (Cicer arietinum L.) is nutrient-dense legume with a number of health and ecological benefits. Chickpea faces many biotic and abiotic challenges in Virginia where weather is highly conducive for many of its pathogens. In 2019, a split-split plot field trial with six biweekly planting dates (PD) as main plots, fungicide spray (F) (treated and non-treated) as subplots, and four chickpea varieties (V) (Myles, Frontier, Leader and Pedrosillano) as sub-subplots, was conducted at Randolph Farm of Virginia State University. Fungicide treated plots received 3-6 sprays of five fungicides that were applied alternatively. Severity (DS) of foliar diseases, such as blights and leaf spots caused by Ascochyta and others, was rated visually by estimating percent foliage covered with lesions on plants in the middle rows. Relative area under the disease progress curve (rAUDPC) was calculated to account for the variation in maturity dates. Data from a weather station was used to correlate weather favorability with DS. After harvest, pods were categorized into threshable (clean or symptomatic) and non-threshable (insect damaged, shattered or aborted). In terms of rAUDPC, all the three individual factors $(P<$ $0.0001)$ and interactions between PD*V $(P<0.0001)$ and PD*F $(P=0.0437)$ were significant. Accordingly, rAUDPC was highest for 
the Kabuli type Pedrosillano planted on 15-June planting and lowest on the Desi type Myles planted on 01-May. Studies on plant and row spacing will be added in 2020 .

Characterization of causing scab disease Streptomyces isolates in Colombia

L. HENAO (1), M. Guevara-Suarez (2), S. Restrepo (2), J. Husserl (2), (1) Universidad de los Andes, Bogotá, COLOMBIA; (2)

Universidad de Los Andes, Bogotá, COLOMBIA

Scab disease-causing Streptomyces sp. generates one of the main phytosanitary problems of potato crops in Colombia. To our knowledge, there are no studies evaluating the presence and diversity of scab-causing Streptomyces sp. in Colombia. This work aims to characterize pathogenic Streptomyces isolates obtained from potato tuber lesions associated with scab disease in Colombia. Pathogenicity was evaluated by inoculation of potato tuber disks with agar plugs of sporulated Streptomyces sp. cultures. Pathogenic isolates were characterized by phylogenetic analysis based on concatenated sequences of three genes (recA, rpoB, 16s rRNA), and assessment of the presence of $t x t A$ and necl genes by PCR. In addition, carbon source utilization, spore, and mycelia color determination were carried out for isolates of each clade. In total, 20 pathogenic Streptomyces sp. isolates were obtained. According to the phylogenetic analysis, the isolates were grouped in three different clades; two of them, had no pathogenic Streptomyces species identified before this study, and are not phylogenetically close to other Streptomyces species described to date. The PCR results suggest that none of the isolates carry the txtA or necl genes. Tests of carbon source utilization and spore and mycelia color determination show different behavior for all isolates belonging to different clades. Isolate characterization suggests that scab disease in Colombia may be caused by Streptomyces species not previously described in the literature.

\section{Inheritance suggests the role of genetic control in recruitment of mutualistic bacteria in tomato}

T. TRAN (1), E. French (2), A. Iyer-Pascuzzi (1), (1) Purdue University, West Lafayette, IN, USA; (2) Department of Entomology, Purdue University, West Lafayette, IN, USA

Utilizing the power of root microbiomes for improving crop productivity is an emerging area and a target for future agriculture. It is critical to understand how plant hosts shape their microbial community. We hypothesize that plant genetic variation functions in the recruitment of beneficial bacteria. We first examined the microbial profile in the roots of eight different genotypes of healthy tomato plants using $16 \mathrm{~S}$ rRNA amplicon sequencing. We discovered that genotypes account for $10 \%$ of the variation in the assemblage of root microbiota. Thirty-two members of two bacterial families were found highly abundant in the endosphere in six of the eight genotypes examined. The abundance of these thirty-two members varied quantitatively across the eight tomato genotypes. We then surveyed the root endosphere microbiome of twenty recombinant inbred lines (RILs), progenies of two of the eight genotypes which are significantly different from one another in abundances of the high-frequency colonizing taxa. The abundance of thirty-two high frequency colonizers showed transgressive segregation across the 20 RILs. We then inoculated 20 RILs and their two parents with a culturable isolate, which was $97.1 \%$ identical to one of the high-frequency colonizers. Inoculation of RILs with the isolates showed that the RILs quantitatively differed in their response rate, in terms of shoot and root growth. These data suggest that genetic factors in tomato may play a role in the enrichment of beneficial microbes. Our data hold promises for the optimization of using beneficial bacteria in agriculture.

Simultaneous detection of cucurbit infecting tobamoviruses using a two-step conventional multiplex RT-PCR C. Padmanabhan (1), T. Pitman (2), T. Tian (3), B. W. Falk (2), V. A. Mavrodieva (4), M. K. Nakhla (5), A. ROY (6), (1) North Carolina State University, Beltsville, MD, USA; (2) University of California, Davis, CA, USA; (3) California Dept of Food \& Agric, Sacramento, CA, USA; (4) USDA-APHIS-PPQ S\&T CPHST, Beltsville, MD, USA; (5) USDA-APHIS-PPQ-S\&T, Beltsville Laboratory, Beltsville, MD, USA; (6) USDA-APHIS-PPQ-S\&T-CPHST, Beltsville, MD, USA

There are six cucurbit infecting tobamoviruses (CITV) reported so far: Cucumber fruit mottle mosaic virus (CFMMV), Cucumber green mottle mosaic virus (CGMMV), Cucumber mottle virus (CMoV), Kyuri green mottle mosaic virus (KGMMV), Zucchini green mottle mosaic virus (ZGMMV), and the recently discovered Watermelon green mottle mosaic virus (WGMMV). All of these viruses produce a variety of symptoms on different cucurbits that include mosaic, mottle, necrosis, rolling leaf, stunted growth and deformed fruit. Based on the nucleotide sequence comparisons and phylogenetic analysis, CITV grouped into two separate sub-clades. To identify the known and unknown CITV in natural infections, a set of generic and specific RT-PCR primers were designed utilizing the aligned conserved and variable nucleotide CITV sequences. In the two-step multiplex (m) RT-PCR assay, a generic (176 nt) and six specific CITV amplicons (294-803 nt) were simultaneously amplified along with the Nad5 host internal control (133 nt). Assays were optimized using CGMMV, WGMMV and ZGMMV isolates and synthetic DNAs of CFMMV, CMoV and KGMMV. Both simplex and mRT-PCRs were tested using CITV isolates from UC Davis, CA and single and mixed infections of CITV were successfully detected. Generic and specific RT-PCR amplicons of all the CITV were identified on the basis of their sizes followed by bi-directional Sanger sequencing. The current developed mRT-PCR assay is simple, reliable, cost-effective and has great potential to be utilized in the state and federal diagnostic laboratories.

Fungal pathogens causing grapevine trunk diseases in patrimonial vineyards in Chile D. GRINBERGS, J. Chilian, J. Carrasco-Fernández, A. France, Instituto de Investigaciones Agropecuarias, INIA Quilamapu, Chillán, CHILE

Trunk diseases have been identified as a major problem, reducing the productivity, quality and longevity of the vineyards. In Chile, GTDs have been studied in commercial vineyards, however, in patrimonial cultivars like País, they haven't been identified so far. Thus, the objective was to identify them and study their pathogenicity. In 2019 a survey was conducted on different ancestral vineyards of 
Cauquenes and Itata valleys. Wood samples were collected from symptomatic plants of 14 patrimonial cultivars. Fungi were isolated plating disinfested wood discs on APDA and WA, and incubating them at $25^{\circ} \mathrm{C}$ until mycelial development. Fungal tips were transferred to PDA to obtain pure cultures. The isolates were identified by their cultural characteristics, morphometry and nucleotide sequences of the ITS, $\beta$-TUB, TEF and LSU regions. Pathogenicity tests were conducted for the most frequently isolated species, inoculating previously rooted healthy cuttings cv. Petit Syrah and incubating them both in humid chamber for 30-d and in aerated tap water for 50-d. 205 isolates were obtained, where Botryosphaeriaceae spp. were the most frequently isolated fungi (42\%). The second and third groups in frequency belonged to the Sporocadaceae and Hymenchaetaceae families, respectively, where previously unreported species were identified. The isolates pathogenicity was diverse, being Neofusicoccum parvum, Diplodia seriata and D. multila the most virulent ones. Fungi causing GTDs in patrimonial vineyards are diverse and pathogenic, however, ancient vineyards are apparently resilient to them.

\section{Strategies for isolation of myxobacteria and specificity of biological control of Pseudomonas syringae seedborne pathogens of chenopods and cucurbits \\ L. N. BOYD (1), J. Kikkert (2), C. Bull (3), (1) Penn State University, State College, PA, USA; (2) Cornell Cooperative Extension, Canandaigua, NY, USA; (3) Pennsylvania State University, University Park, PA, USA}

The seedborne bacterial pathogen Pseudomonas syringae causes foliar diseases of chenopods and cucurbits leading to substantial yield loss. Strategies are needed to reduce seed infestation and manage diseases in seed and food crop production. In this research, host specificity of predatory myxobacteria is being investigated to increase the potential use of myxobacteria as biological control agents for reducing Pseudomonas syringae pathogen populations. To study host specificity, a hierarchical enrichment experiment with Pseudomonas syringae prey strains was conducted to evaluate the variability in predatory performance and specialization of myxobacteria. Myxobacteria were isolated from agricultural soils with beet crops exhibiting severe bacterial leaf spot or with no cultivation history of chenopods and cucurbits. Isolation methods compared one or two rounds of enrichment to methods with no enrichment. Equal numbers of myxobacteria were isolated from soils which had undergone enrichment versus those that did not. To determine whether the prey strain on which myxobacteria were isolated influences the predatory performance, predation assays for all prey and myxobacteria combinations are being conducted. Myxobacteria with enhanced predation performance will be evaluated by in situ biological control experiments using pathogen inoculated seed. Effective isolation methods identified in these experiments could be replicated for other seedborne bacterial pathosystems, providing additional management options for difficult to manage bacterial pathogens.

Field-relevant pathogenicity and fungicide sensitivity of three under-studied fungi associated with grapevine bunch rots S. D. COSSEBOOM, A. Schoeneberg, M. Hu, University of Maryland, College Park, MD, USA

Late-season bunch rots (LSBR) are considered a primary threat in mid-Atlantic vineyards. Fungi including Alternaria alternata, Aspergillus uvarum, and Pestalotiopsis telopae were frequently found associated with LSBR samples, but little information is known regarding their infection behaviors and fungicide sensitivity. The pathogenicity of these three fungi was evaluated by inoculating flowers and fruit of Cabernet Franc, Chambourcin, Chardonnay, and Merlot at the development stages of bloom, veraison, and preharvest, and both wounding and non-wounding treatments were included at each stage. At harvest, the overall disease severity was highest for clusters inoculated with $A$. uvarum $(9.9 \%)$, followed by A. alternata (4.1\%), and P. telopae (3.3\%), respectively. Chardonnay was the most susceptible cultivar, and the wounding treatments resulted in the most disease for each pathogen. The highest and lowest average disease severity resulted from inoculation at bloom (6.4\%) and veraison (1.2\%) for $A$. alternata, at pre-harvest $(14.5 \%)$ and bloom (6.9\%) for $A$. uvarum, and pre-harvest (6.0\%) and veraison (1.0\%) for P. telopae, respectively. A. uvarum and $P$. telopae were also evaluated for their sensitivity $\left(\mathrm{EC}_{50}\right)$ to boscalid, difenoconazole, and pyraclostrobin. The sensitivity of $A$. uvarum averaged $1.25,0.42$, and $0.15 \mu \mathrm{g} / \mathrm{ml}$, while the sensitivity of $P$. telopae averaged $>100,1.74$, and $0.93 \mu \mathrm{g} / \mathrm{ml}$, respectively. These findings suggest that the pre-harvest stage may be a critical point for LSBR management, but control can be complicated due to the involvement of multiple pathogens.

\section{Draft genome of the citrus stem end rot fungal pathogen Diplodia natalensis (Lasiodiplodia theobromae)} Q. Zheng (1), E. Ozbudak (1), P. Hosmani (2), S. Saha (2), G. Liu (1,3), Y. Lin (1), J. Zhang (4), M. Flores (2), B. Liu (3), L. Mueller (2), M. A. Ritenour (4), L. M. CANO (1), (1) University of Florida, UF/IFAS, IRREC, Plant Pathology, Fort Pierce, FL, USA; (2) Boyce Thompson Institute, Ithaca, NY, USA; (3) Fujian Academy of Agricultural Sciences, Fuzhou, CHINA; (4) University of Florida, UF/IFAS, IRREC, Horticultural Sciences, Fort Pierce, FL, USA

Diplodia species are fungal plant pathogens that are widely distributed in tropical and subtropical regions and cause different diseases in citrus. The control of Diplodia fungal infection in citrus in the field may reduce HLB-associated pre-harvest fruit drop. The type species Diplodia natalensis is synonym with Lasiodiplodia theobromae, Botryodiplodia theobromaeand Botryosphaeria rhodina. Infection by Diplodia pathogens often results in soft brown rot of fruits shortly before or after harvest, and the symptoms are known as stem end rot (SER). In this study, we sequenced and assembled a draft genome equivalent to $43.3 \mathrm{Mb}$ for the strain Lteo_CITRA15 using SMRTPacBio. This genome contains 475 contigs with a N50 value of $178 \mathrm{~kb}$. We have identified using Signalp software a set of 1,203 secreted proteins out of 10,456 predicted proteins in the genome. The long term goal is to perform comparative genome analyses with other Diplodia species and determine candidate disease-related effector genes specific to Lasiodiplodia theobromae that could be used for functional analyses. 
Comparative analysis of extracellular proteomes reveals effectors of the boxwood blight pathogens, Calonectria henricotiae and C. pseudonaviculata

X. YANG (1), M. B. McMahon (2), S. R. Ramachandran (3), W. Garrett (4), N. LeBlanc (5), J. A. Crouch (5), N. Shishkoff (6), D. G. Luster (2), (1) Foreign Disease-Weed Science Research Unit, USDA, ARS - Fort Detrick, MD, Frederick, MD, USA; (2) USDA ARS

Foreign Disease Weed Science Research Unit, Fort Detrick, MD, USA; (3) Foreign Disease-Weed Science Research Unit, USDA, ARS - Fort Detrick, Fort Detrick, MD, USA; (4) USDA-ARS, Beltsville, MD, USA; (5) USDA-ARS, Mycology and Nematology Genetic Diversity and Biology Laboratory, Beltsville, MD, USA; (6) USDA ARS FDWSRU, Frederick, MD, USA

Calonectria henricotiae (Che) and C. pseudonaviculata (Cps) are destructive pathogens causing boxwood blight, a persistent threat to the nursery and landscape industry and historical gardens. In order to define extracellular proteome profiles and pathogenesis-related proteins, in vitro secreted proteins of $C h e$ and $C p s$ were analyzed using liquid chromatography-tandem mass spectrometry and bioinformatic prediction tools. A total of 630 extracellular proteins ( 305 of Che and 325 of Cps) and 251 cell membrane proteins (115 of $C$ he and 136 of $C p s$ ) were secreted through the classical secretion pathway. An additional 79 extracellular proteins (48 of Che and 31 of $C p s$ ) were identified in the non-classical secretion pathway. These proteins belonged to 364 OrthoMCL clusters with a majority of $225(62 \%)$ present in both species and $68(19 \%)$ and $71(20 \%)$ unique to Che and Cps, respectively. Among these extracellular and cell membrane proteins, 124 including 64 of Che and 60 of Cps were identified as effector candidates using EffectorP. Many of these putative effectors have previously identified roles in suppressing host defense and facilitating infection processes, such as SnodProt1like proteins in OrthoMCL cluster OG5_152723 and PhiA-like cell wall proteins in cluster OG5_155754. This study facilitates our understanding of the biological functions and pathogenic roles of proteomes and effectors of $C h \bar{e}$ and $C p s$.

\section{Efficacy of nematicides at managing nematodes in corn} M. W. TSEGAY, University of Florida, Gainesville, FL, USA

Synthetic pesticides are an effective way to control plant parasitic nematode populations. In order to determine which nematicide chemistries are most effective for specific plant parasitic nematodes, a field experiment was conducted in 2019 in Hastings, FL. The objective of this experiment was to compare the nematicides 1,3-dichloropropene (1,3-D) at rates of 3 gallons and 6 gallons per acre, terbufos at 6 ounces per 1000 row-foot and fluopyram at 8 and 14 ounces per acre, with untreated control for plant-parasitic nematode management. Terbufos and fluopyram were applied in-furrow at planting, whereas 1,3-D was applied two weeks before planting. Soil populations of plant-parasitic and beneficial (free-living) nematodes were assessed at mid-season and at harvest. Nematicides did not significantly $(P>0.05)$ affect soil nematode abundances of any nematodes at harvest. At midseason, terbufos and 1,3-D at either rate significantly $(P<0.05)$ reduced stunt nematode (Tylenchorhynchus spp.) soil abundances compared with control. Terbufos significantly reduced stubby nematodes (Paratrichodorus spp.) abundances compared to control at mid-season. All nematicide treatments significantly reduced the population of root lesion (Pratylenchus spp.) compared to control. Sting nematodes (Belonolaimus spp.) were not significantly affected by treatments. The population of free-living nematodes was highly reduced by any nematicide treatment at mid-season. This experiment showed that various nematicide chemistries can help manage plant-parasitic nematodes in corn production.

Survey of fungal species associated with stem and root diseases of soybean in Delaware and Maryland A. C. KESSLER, A. Koehler, University of Delaware, Georgetown, DE, USA

Fungal pathogens can be very damaging to soybean (Glycine max (L.) Merrill) production, reducing both yield and quality. Wet conditions across the Mid-Atlantic in 2018 favored widespread disease that initiated interest in a survey of fungal species affecting soybean production, particularly species within the genus Diaporthe. Diaporthe spp. cause numerous diseases of soybean including seed decay, seed rot, pod and stem blight, and stem canker, but there has been limited research to characterize species diversity in the Mid-Atlantic. In 2019, sixty soybean fields were surveyed for pathogenic fungi associated with stem and root tissue across Delaware and Maryland. Isolates recovered from stem and root tissues were identified to species based on morphology and amplification of the internal transcribed spacer, partial elongation factor 1-alpha, and beta-tubulin genes. Out of the eighty isolates characterized, three species of Diaporthe were recovered (31\% of total isolates). Within this grouping, species included D. aspalathi (southern stem canker, $8 \%)$, D. longicolla (88\%), and D. ueckerae (4\%). Other fungi isolated included Macrophomina phaseolina (25\%) and Fusarium spp. $(44 \%)$. Northern stem canker (D. caulivora) has been widely assumed as the dominant stem canker organism in DE and MD, these results confirm the presence of Southern Stem Canker and other Diaporthe spp. that warrant further survey work to continue to characterize the distribution and abundance of Diaporthe spp. in Mid-Atlantic soybean fields.

\section{A 5-year analysis of rust fungi intercepted at Arizona ports of entry D. C. SANDBERG, USDA-APHIS-PPQ, Nogales, AZ, USA}

Rust fungi are complex, highly specialized obligate parasites that can cause significant damage to agricultural and forestry crops, native plants, and natural ecosystems. Information regarding host range, aggressiveness to native hosts, and presence in the United States is scarce for many of these fungi, making them easily overlooked in ecosystem diversity management. Many of the rust fungi intercepted at Arizona ports of entry transit primarily from Mexico, and are considered quarantine significant pests that account for a substantial portion of pathogens requiring phytosanitary action. From January 1, 2015 to December 31, 2019, a total of 1,231 interceptions of rust fungi were found at 8 Arizona ports of entry. The 1,231 rust fungi belonged to 6 known rust families, 10 genera, and 35 unique species. The most prevalent quarantine significant rust fungus was Puccinia horiana Henn. (the causal agent of Chrysanthemum White Rust) with 824 interceptions, which accounted for over half of the rust fungi intercepted. A majority of the rusts that were intercepted are not known to occur in the United States, and those that are present often are limited in their distribution. Identifying and cataloging rusts 
intercepted at Arizona ports of entry is critical in assessing the risk of entry and establishment of exotic plant pathogens that may pose a threat to the health and biodiversity of ecosystems in the United States.

Host-pathogen interactions in the tan spot pathosystem

S. SUAREZ (1), R. M. Hunger (2), F. Flores (1), R. Sunkar (1), S. M. Marek PhD (1), (1) Oklahoma State University, Stillwater, OK, USA; (2) Dept. of Entomology \& Plant Pathology, Stillwater, OK, USA

Tan spot, which is a major foliar disease of wheat that causes severe yield loss in many wheat-growing areas worldwide, is caused by the fungus Pyrenophora tritici-repentis (PTR). The pathogenicity of PTR mainly depends upon the production of host selective toxins (HSTs). Ptr ToxA is the major necrosis-inducing HST and is found in almost all PTR populations in Oklahoma. Consequently, two PTR isolates each possessing $\left(^{+}\right)$and lacking $\left(^{-}\right)$the ToxA gene were transformed to express either the fluorescent protein GFP or tdTomato to examine the infection and colonization process on susceptible and resistant wheat differentials. Third leaf stage wheat seedlings were inoculated with conidial suspensions adjusted to $\sim 8$ conidia/ $\mu$ l. Symptoms were scored at 3,5 and 7 days post inoculation (DPI). Preliminary results with $\operatorname{ToxA}^{+}$isolates on susceptible cultivars show initial necrosis after 3 DPI corresponded with hyphal expansion into leaf tissues through epidermal and mesophyll cells. On resistant cultivars, Tox $A^{+}$isolates only penetrated epidermal leaf tissue at 3 DPI and at 5 and 7 DPI, reduced infection sites with intensifyingly autofluorescent borders and restricted hyphal expansion were observed. Additionally, the use of RNA interference using double stranded RNAs (dsRNAs) targeting ToxA expression was evaluated to reduce disease development. Co-incubation of the fungal spores with Cy3-labeled long dsRNA controls demonstrated that germinating spores and hyphae of PTR are capable of taking up exogenous dsRNA. Further studies will evaluate spray-induced gene silencing of ToxA in PTR.

Seed borne Botrytis species of pulse crops in Montana S. MOPARTHI (1), L. Parikh (1), B. Agindotan (2), C. Peluola (3), M. E. Burrows (1), (1) Montana State University, Bozeman, MT, USA; (2) CPHST Beltsville Laboratory, USDA APHIS PPQ, Beltsville, MD, USA; (3) Montana State University, Sidney, MT, USA

Montana is the principle producer of pulse crops (dry pea, lentil, and chickpea) in the USA. Botrytis is an important pathogen of pulse crops. It is seedborne and can lower the germination rate. In this study, 96 isolates of Botrytis spp. were recovered from pulse seed samples that were submitted to the Regional Pulse Crop Diagnostic Laboratory at Montana State University. Based on culture characteristics, amplifying portions of the G3PDH, HSP60 and RPB2 genes, and by phylogenetic analysis, seven Botrytis species were found to be associated with pulse seeds. $B$. cinerea $(\mathrm{n}=54)$ was the predominant species followed by B. euroamericana $(\mathrm{n}=22), B$. prunorum $(\mathrm{n}=11)$, Botrytis sp.1 $(\mathrm{n}=2)$, Botrytis sp.2 $(\mathrm{n}=3)$, Botrytis sp.3 $(\mathrm{n}=1)$ and Botrytis $\mathrm{sp} .4(\mathrm{n}=3)$. A detached leaf assay was conducted using all the isolates on leaves of pea cv. Lifter, chickpea cv. Sierra, and lentil cv. Richlea. Each crop evaluation was conducted as a separate experiment. The experiments were arranged in a completely randomized design with five replications per isolate and lesion diameter was measured three days after inoculation. The lesions produced by the various Botrytis isolates differed significantly from each other $(P<0.05)$ on all three hosts. $B$. cinerea isolate C36 produced the largest lesions on pea, chickpea and lentil leaves $(18.7 \pm 1.12 \mathrm{~mm}, 22.5 \pm 0.98 \mathrm{~mm}, 22.1 \pm 1.15 \mathrm{~mm})$. B. prunorum isolates produced small or no lesions on pea and chickpea. This study emphasizes the need for effective seed treatment practices and increased knowledge of the species responsible for disease in the field.

\section{Formulation optimization of boron-containing fungicides provides a novel management tool against Asian soybean rust (Phakopsora pachyrhizi) \\ L. STEERE, M. Aubrey, M. Samuels, T. Liu, Boragen, Inc., Research Triangle Park, NC, USA}

A novel boron-containing fungicide, BAG8, has exhibited broad-spectrum activity against agronomically relevant plant pathogenic fungi. In general, fungicide formulations have a direct influence on control efficacy of the active ingredient (a.i.). Formulation chemistry with boron-containing compounds presents its own set of challenges due to the lack of previous research in the field and the unique physiochemical properties of the boron moiety. Three formulations of BAG8 were tested in southern Georgia on soybeans against an artificially established population of Phakopsora pachyrhizi: a low-loading, high-solvent Early EC (2.2\% a.i.); a higherloading Rosie EC (10\% a.i.), and an early SC, Lydia SC (25\% a.i.). These formulations were evaluated relative to a multisite positive control fungicide (Mancozeb) and a two-way systemic positive control fungicide, Priaxor from BASF (fluxapyroxad+pyraclostrobin). The Early EC provided control at the same level of traditional multi-sites (50\% control), like mancozeb, while the Rosie EC and Lydia SC approached the disease controlling efficacy level of Priaxor ( $>85 \%$ control). Additionally, Rosie EC and Lydia SC provided a $\sim 250 \%$ increase in yield compared to the untreated control (similar yield to Priaxor). This level of efficacy with a novel multisite fungicide will provide another management tool to combat $P$. pachyrhizi for soybean growers globally. These results highlight the importance of formulation for the optimization of in vivo and field efficacy and for the future development of boron-containing crop protection chemistry.

Building an educational program for soybean cyst nematode in Pennsylvania A. MURILlO-WILliaMS (1), A. A. Collins (2), P. Esker (3), (1) Penn State Extension-Centre County, Bellefonte, PA, USA; (2) Southeast Agricultural Research \& Extension Center, Manheim, PA, USA; (3) The Pennsylvania State University, University Park, PA, USA

Soybean cyst nematode (SCN), caused by Heterodera glycines, is the number one pest/pathogen in the U.S. While the distribution is widespread around the U.S., SCN has only been documented once in a single county of Pennsylvania as part of surveys conducted by 
the Pennsylvania Department of Agriculture in 2002. With the development of the second Soybean Cyst Nematode Coalition, and with support of local to national soybean commodity boards, we have expanded our efforts to improve knowledge of the importance of SCN in Pennsylvania through a combination of approaches, including surveys to gauge grower and other stakeholders' knowledge of SCN and how to best manage this pest, a statewide survey program for nematodes, and through workshop efforts. Our preliminary survey work in 2019 indicated that more than $80 \%$ of stakeholders have never scouted or taken soil samples for SCN testing and that $80 \%$ were also not aware of the source of resistance to SCN in the varieties that they use. Our statewide survey has enabled us to collect samples in over 50\% of the counties in Pennsylvania, and we have documented suspected new finds of SCN in three additional counties. Furthermore, our survey program also emphasizes testing for other nematodes of importance in the state given the diverse, mixed cropping systems. Overall, our current efforts have enabled us to establish a baseline set of information that will help improve best management recommendations for SCN and other nematodes in Pennsylvania.

Investigating sources and fungicide resistance profiles for apple rot fungi in commercial apple production to aid postharvest decay management

J. CASTRO (1), W. M. Jurick II PhD (2), K. A. Peter (3), (1) Penn State University, State College, PA, USA; (2) USDA-ARS Food Quality Laboratory, Beltsville, MD, USA; (3) Pennsylvania State University, Fruit Research and Extension Center, Biglerville, PA, USA

Postharvest rots are prevalent in the United States, with up to $15 \%$ losses on apple fruits due to pathogens such as Penicillium spp. The inoculum sources for blue mold are known; however, less information is available showing the relationship between inoculum source and the composition of fungal species and their fungicide resistance profiles. This research aimed to determine the location of postharvest fungi in the production chain and their fungicide resistance profiles, in relation to the inoculum source. The following sources were sampled in Pennsylvania apple production systems: apple fruits, picking bags, harvest bins, and the air of the packinghouse and cold rooms. Petri plates containing Potato dextrose agar (PDA) or PDA amended with thiabendazole, fludioxonil, pyrimethanil or difenoconazole were used to isolate the fungi and determine their frequency in relation to the inoculum source. Penicillium spp. were prevalent in the bins and the air of the packinghouse facilities and cold rooms; however, they were not detected on the fruit surface and picking bags. With the exception of pyrimethanil, fungicide resistant isolates were found at frequencies between $10 \%$ and $80 \%$ in the air of the packinghouse and cold rooms. Five Penicillium spp. were identified by cultural and molecular methods. The prevalence of Penicillium spp. with fungicide resistant and non-resistant phenotypes in the packinghouse and cold rooms will guide new management strategies to reduce the frequency of this pathogen, helping producers maintain the efficacy of currently available chemical control.

Design and validation of a universal reverse transcription PCR (RT-PCR) assay for vitiviruses infecting grapevine A. Diaz-Lara (1), T. M. Ericson (2), M. AL RWAHNIH PHD (3), D. A. Golino (4), (1) University of California-Davis, Davis, CA, USA; (2) Foundation Plant Services, UC-Davis, CA, USA; (3) University of California-Davis/FPS, Davis, CA, USA; (4) University of California, Davis, CA, USA

The genus Vitivirus (famiy Betaflexiviridae) includes eleven viruses known to infect grapevine: grapevine vitiviruses A, B, D, E, F, G, H, I, J, L and M (GVA-GVM). Three of these viruses, GVA, GVB and GVD, are associated with the rugose wood disease in grapevine and cause economic losses. The others vitiviruses were more recently discovered and their effects on grapevine are undetermined. To certify grape material for propagation as virus tested, an updated RT-PCR assay to detect all known vitiviruses is desirable. To accomplish this, grapevine vitivirus sequences were aligned at the amino acid level to search for conserved motifs. Two highly conserved motifs were found at an ideal distance for RT-PCR detection in the RdRp region of the replicase protein. The motifs were back translated to create degenerate primers and used to amplify all eleven grapevine vitiviruses. The primers were used to test a panel of vitivirus-infected vines for inclusivity as well as a panel of vines infected with closely related viruses in the Betaflexiviridae family (grapevine pinot gris virus and grapevine rupestris stem pitting associated virus) for exclusivity. Broader use of these primers to detect vitiviruses in other plant hosts was investigated. In summary, an end-point RT-PCR assay that detects all the known grapevine vitiviruses and potentially other members of the genus Vitivirus has been developed. The universal assay represents an alternative to individual assays to reduce the work associated with the diagnosis of vitiviruses, including for regulatory purposes.

Applied Astrobiology: The effects of the nitrogen-fixing plant-microbe symbiosis with a Martian twist F. HARRIS (1), J. Dobbs (1), J. Ippolito (2), J. Stewart (3), (1) Colorado State University-Agricultural Biology, Fort Collins, CO, USA; (2) Colorado State University-Soil and Crop Sciences, Fort Collin, CO, USA; (3) Colorado State University, Fort Collins, CO, USA

Due to increasing population growth and declining arable land here on Earth, astroagriculture is vital to terraform Martian regolith for settlement. Nitrogen-fixing ( $\mathrm{N}$-fixing) plants and their symbionts have been shown to increase plant viability through their ability to increase soil fertility. Previous studies have successfully grown N-fixing plants in simulated Martian regolith without inoculation of their respective $\mathrm{N}$-fixing symbiotes. In this study, we tested plant-bacterial symbiosis for N-fixation in simulated Martian regolith to influence plant growth by inoculating clover (Melilotus officinalis) with its respective N-fixing symbiont (Sinorhizobium meliloti) and measuring plant biomass and root nodules. The objectives of this study were to examine 1) the plant-microbe symbiosis in Martian regolith, and 2) the effects this relationship has on plant growth and soil fertility. We hypothesized that when plants in Martian regolith were inoculated with their symbiont, an increase in plant growth and in regolith N content would occur. Results showed statistical significance between biomass of inoculated and uninoculated groups with little change in nitrogen composition in both potting mix and regolith simulant. Data showed that the plant-bacteria symbiotic relationship is more productive to plants in regolith, but $\mathrm{N}$ production was limited to plant uptake with no excess $\mathrm{N}$ present in regolith. 
Properties of an endornavirus infecting three Capsicum species

C. ESCALANTE GUARDADO (1), R. I. Alcala-Brise (2), R. Okada (3), R. A. Valverde (1), (1) Louisiana State University Agricultural Center, Baton Rouge, LA, USA; (2) University of Florida, Gainesville, FL, USA; (3) Tokyo University of Agriculture and Technology, Tokyo, JAPAN

Endornaviruses are RNA viruses that have been detected in several economically important crops. These viruses do not cause symptoms, lack cell-to-cell movement, and are transmitted only vertically. Bell pepper endornavirus (BPEV) has been reported in several Capsicum species. The objective of this research was to examine the diversity of endornaviruses in the genus Capsicum. Endornavirus replicative form dsRNAs were isolated from C. baccatum, C. chinense, and C. frutescens. DsRNAs were sequenced using Illumina MySeq (pair-end $2 \times 250$ ), and the viral genomes de novo assembled with Spades 3.7.1.2. Five full genome sequences were obtained, two from C. chinense, two from C. frutescens, and one of C. baccatum. All sequences showed the four putative conserved domains found in many plant endornaviruses (methyltransferase, viral helicase 1, glycosyltransferase and RNA-dependent RNA polymerase). One sequence from $C$. frutescens showed $97 \%$ amino acid (aa) identity to BPEV, while the other four showed low percent identity $(<70 \%)$. These four sequences showed an aa identity that ranged from $85-99 \%$ and therefore consist of four viral isolates of a putative novel Capsicum endornavirus which we provisionally named Capsicum chinense endornavirus 1 (CcEV1). Phylogenetic analysis revealed that CcEV1 clusters in the same clade with BPEV and hot pepper endornavirus. Endornaviruses have been reported to be host specific; however, in this study, we report a putative endornavirus infecting three different plant species.

\section{Detection of phytomelatonin in Huanglongbing-infected sweet orange (Citrus sinensis) plants and its correlation with stress- associated phytohormones \\ Y. NEHELA (1), N. Killiny (2), (1) Department of Plant Pathology, Citrus Research and Education Center, IFAS, UF, Lake Alfred, FL, USA; (2) Department of Plant Pathology, Citrus research and education center, IFAS, UF, Lake Alfred, FL, USA}

Huanglongbing (HLB), a destructive citrus disease, is associated with Candidatus Liberibacter asiaticus (CLas) and transmitted by Diaphorina citri. Citrus plants have developed a complex defensive system against HLB, which is mainly regulated by phytohormones. The role of melatonin in activation of phytohormones pathways is poorly understood, and it remains unclear how it regulates citrus response. Herein, we used integrated metabolic, transcriptomic, and bioinformatic approaches to investigate the role of melatonin in the induction of stress-associated phytohormones against HLB. Using a targeted GC-MS-SIM-based method, melatonin was detected for the first time in the leaves of sweet orange (C. sinensis) after derivatization with MSTFA. Additionally, the melatonin biosynthetic pathway was dissected using a comparative in silico analysis, which including two CsTDC, CsT5H, two CsSNAT, three CsASMT, and three CsCOMT genes. Both CLas-infection and D. citri-infestation increased the endogenous melatonin content and its biosynthetic pathway, with a greater effect of $C$ Las. Furthermore, exogenous melatonin application enhances the salicylates, auxins, trans-jasmonic acid, and abscisic acid contents of citrus leaves. We hypothesize that melatonin might play a protective role against HLB via upregulation the gene expressions and endogenous content of stress-associated phytohormones, as well as, it might act as a signaling molecule to regulate the citrus defense response.

Evaluating commercially available biological fungicides for managing Phytophthora root and crown rot on lavender L. SCHMITZ, G. Powell, S. N. Jeffers, Clemson University, Clemson, SC, USA

Phytophthora root and crown rot (PCRC) is an emerging disease of lavender (Lavandula spp.) that is impacting both nurseries and field production in the United States. The primary causal agent, Phytophthora nicotianae, is spread mainly by contaminated nursery plants. Chemical fungicides should effectively manage this disease, but most lavender growers in the U.S. would prefer non-chemical methods. As an alternative, there currently are numerous biological fungicides labeled for Phytophthora diseases on herbs. Therefore, our objective was to evaluate the efficacy of commercially available biofungicides for managing PRCR on lavender. Eleven treatments were compared - including eight biofungicides (five with bacteria and three with fungi as active ingredients), one chemical fungicide (Reliant), and two non-treated controls (inoculated and non-inoculated). Eight replicate L. angustifolia 'Hidcote Blue' plants were used for each treatment, and plants were completely randomized on a greenhouse bench. All products were applied to plants twice before inoculation with $P$. nicotianae. At 70 days after inoculation, Reliant provided the best disease management with only $4 \%$ of the foliage showing symptoms and no mortality. None of the biofungicides provided adequate disease management; foliage symptom severity was similar to that on inoculated control plants and plant mortality was between $38-75 \%$. Plants treated with Reliant also had the greatest fresh shoot weight, which was significantly greater than that of inoculated control plants and plants treated with six of the biofungicides.

Development of genus- and species-specific primers for identification and detection of canker-causing pathogens in almond H. F. AVENOT (1), R. Jaime (2), R. Travadon (3), L. A. Holland (3), D. P. Lawrence (3), F. P. Trouillas (4), (1) University of California, Kearney Agricultural Research \& Extension Center, Parlier, CA, USA; (2) University of California Davis, Kearney Agricultural Research \& Extension Center, CA, USA; (3) University of California, Davis, CA, USA; (4) University of California, Kearney, Parlier, CA, USA

Trunk and scaffold canker diseases (TSCD) of almond can cause significant yield and tree losses within orchards, while also reducing orchard life spans. TSCD often go unnoticed during the early stages of infection and symptoms appear more visible as trees age. Common symptoms of TSCD include discoloration of vascular tissues, wood necrosis and extensive gumming. Dieback of scaffold branches can occur and eventually the whole tree may die. In California, several pathogens are known to cause cankers in trunks and scaffold branches of almond. These include Botryosphaeria spp., Diaporthe spp., Eutypa lata, Ceratocystis destructans, Cytospora spp., 
Collophora spp., and Phytophthora spp. Morphological identification of canker pathogens is generally difficult and field diagnosis of TSCD remains challenging as symptoms delineation among these diseases is not clear. Thus, the aim of this study is to develop DNAbased identification techniques for rapid and reliable diagnosis of almond canker diseases. Several genus and/or species-specific primers were designed by exploiting differences in the translation elongation factor 1 (EF-1), $\beta$-tubulin (BT), or internal transcribed spacer (ITS) gene regions. Primer specificity was successfully tested on DNA templates extracted from pure cultures of target and non-target pathogens, as well as from DNA templates obtained from diseased wood samples. Further PCR validation tests from artificially and naturally infected almond wood samples are currently in progress.

Accumulation of zearalenone and alpha-zearalenol in swine liver and reproductive tissues E. PACK (1), J. Stewart (2), M. Rhoads (2), J. Knight (2), R. De Vita (3), S. Clark-Deener (4), D. G. Schmale III (5), (1) School of Plant and Environmental Sciences, Virginia Tech, Blacksburg; (2) Department of Animal Science, Virginia Tech, Blacksburg, VA, USA; (3) Department of Biomedical Engineering and Mechanics, Virginia Tech, Blacksburg, VA, USA; (4) Virginia-Maryland College of Veterinary Science, Blacksburg, VA, USA; (5) School of Plant and Environmental Sciences, Blacksburg, VA, USA

Mycotoxins produced by Fusarium species persist in animal feed products, compromising the health of economically important livestock. Zearalenone (ZON) is an estrogenic mycotoxin which has severe reproductive effects on swine, including decreased litter size, anestrus, and pelvic organ prolapse. Distillers' dried grains with solubles (DDGS) is a common ingredient in swine feed, and contains increased concentrations of ZON relative to maize. This study aims to determine how ZON and the related metabolite, alphazearalenol (ZOL), accumulates in the reproductive and liver tissue of swine fed ZON concentrations similar to those reported in DDGS. Thirty pubertal gilts were assigned to one of three treatment groups, with ten gilts in each group: (1) base feed only, (2) base feed spiked with $6 \mathrm{mg}$ ZON for 7 days, then only base feed for 14 days, (3) base feed spiked with $6 \mathrm{mg}$ ZON for 21 days. A method was developed for analyzing ZON and alpha-ZOL, in reproductive and liver tissue using gas chromatography-mass spectrometry (GC-MS). Using the developed GC-MS method, ZON was found in the anterior vagina, posterior vagina, cervix, ovary, and liver, with higher concentrations in cervical tissue. Alpha-ZOL was present in liver tissue. These results show how ZON accumulates in the reproductive and liver tissues of pubertal gilts. More work is needed to determine how increased ZON concentrations in feed ingredients may affect swine reproductive health. This work contributes to our understanding of the risks associated with Fusarium infections in grains and coproducts used in swine feed.

Drip irrigation application of nematicides for management of Columbia root knot nematode on potatoes B. B. WESTERDAHL (1), H. L. Carlson (2), (1) University of California, Davis, Davis, CA, USA; (2) Univ of California, Tulelake, CA, USA

The Columbia root knot nematode, Meloidogyne chitwoodi causes significant numbers of blemished and unmarketable potato tubers. The influence of irrigation on nematicide effectiveness was demonstrated in two field trials conducted in a silty clay loam soil with 12 percent organic matter, in a field with a history of nematode infestation. Planting dates, harvest dates, and crop cultural practices were consistent with normal potato production practices in the Tulelake Basin, CA. Experiments were conducted as randomized complete blocks with 4 replications per treatment. Trials 1 and 2, had 18 and 16 treatments, respectively. Drip irrigation applications of metham sodium, 1,3-dichloropropene, oxamyl, and sodium tetrathiocarbonate were applied in different volumes of water and compared to an untreated check. At maturity, the percentage of tubers exhibiting a surface blemish due to nematodes was determined, and nematode samples were taken and analyzed. In both trials, for all products, there was good agreement between reduction in tuber blemish, and in reduction of root-knot nematode present at harvest. In trial 1, all but 4 treatments reduced nematode blemish. All but 2 treatments reduced root-knot nematode numbers. Higher rates and longer application times provided the best results. In trial 2, all treatments reduced blemish and root-knot nematode levels at harvest. For the fumigants, better results were achieved when the same rate was applied over 6 hours rather than 3 hours. The opposite was true for the carbamate product.

CacaoBIO: Characterization of plant pathogens in native forest cocoa trees

N. BOLAÑOS (1), D. Burbano (2), A. Jurado (2), E. Rodríguez (2), C. González (2), Y. Jaimes (2), S. Restrepo (1), (1) Universidad de Los Andes, Bogotá, COLOMBIA; (2) Corporación Colombiana de Investigación Agropecuaria-AGROSAVIA, Mosquera, COLOMBIA

The CacaoBIO expedition seeks to characterize the biodiversity of the genus Theobroma, its wild relative, Herrania spp., and their possible plant pathogens in Colombian poorly explored areas related to the post-conflict. Genetic information of the plants and their pathogens was generated as well as cultivation strategies that meet economic and cultural needs. Therefore, this study determined the possible causal agents of the diseases present in cultivated and non-cultivated cocoa trees by isolating and characterizing phytopathogenic fungi and oomycetes present in Theobroma spp. and Herrania spp. found in native forests in six localities of the Colombian Amazonia $(n=5)$ and Pacific coast $(n=1)$. In each locality, a $13 \mathrm{~m} \times 13 \mathrm{~m}$ plot was conveniently sampled where the disease incidence range $34-82 \%$. We collected 118 samples among pods, flower cushions and diseased leaves. Samples were disinfected and grown on potato dextrose agar, malt extract agar, water agar, V8 agar and Plich media. A total of 26 isolates were obtained and were identified using the ITS region and ITS1/ITS4 primers. Most of them corresponded to Moniliophthora perniciosa a hemibiotroph basidiomycete fungus known as the causal agent of witches' broom disease. Interestingly, no Phytophthora isolates were obtained. This is the first attempt to evaluate the phytosanitary state of non-cultivated cacao trees in Colombian native forests. 
Transcriptomic and Metabolomic approaches to unravel the antagonistic mechanism of Chaetomium globosum against Bipolaris sorokiniana

D. K (1), R. Aggarwal (2), B. Maya (3,4), S. V (5), M. S. Gurjar PhD (5), A. U. Solanke (6), M. S. Saharan (7), (1) Indian Agricultural Research Institute, NEW DELHI, India, INDIA; (2) ICAR-Indian Agricultural Research Institute, New Delhi, INDIA; (3) ICAR-IARI, INDIA; (4) ICAR-IARI, Delhi, INDIA; (5) ICAR-IARI, New Delhi, INDIA; (6) ICAR-National Institute for Plant Biotechnology, New Delhi, INDIA; (7) ICAR-IARI, New Delhi, New Delhi, INDIA

Chaetomium globosum Kunze has been widely recognized as an emerging biocontrol fungus. It can mycoparasitize and produce antifungal metabolites to suppress the growth of many plant pathogens. In this study, we utilized transcriptomic and metabolomic analyses to explore gene expression pattern and associated antifungal metabolites, respectively, during interaction between C. globosum strain Cg2 and Bipolaris sorokiniana isolate BS112. The Illumina HiSeq platform yielded an average of 20-22 million reads with 50$58 \%$ GC. After de novo assembly, 45582 transcripts with 27957 unigenes were generated. Transcriptome analysis displayed distinct expression profile during the interaction, out of which 6109 unique genes were differentially expressed. The heat map and cluster categorization suggested an increase in the expression levels of genes encoding secondary metabolites and some CAZymes. We identified various genes of biotechnological value encoding proteins with functions such as polyketide synthase \& nonribosomal peptide synthetases (NRPSs) etc. To check the reliability of the RNA-Seq data, RT-qPCR was performed on 20 randomly chosen genes. Metabolomic profiling using GC-MS and LC-MS showed that C. globosum strain Cg2 produced variety of antifungal secondary metabolites such as trans-limonene oxide, dodecene, heptacosanol, octadecanoic acid, chaetomugilin, globoxanthene, chaetoviridin and chaetoglobosin etc., which may be involved in the antagonisms. To our knowledge, this is the first effort to unravel the biocontrol mechanism of C. globosum against B. sorokiniana.

Effect of rotation crops on Potato early dying complex and potato yield

D. CHEN (1), K. Nahar (2), C. Wagg (3), L. P. Comeau (3), C. Goyer PhD (2), J. Nyiraneza (4), M. Singh PhD (5), H. Tai (6), B. Zebarth (2), (1) AAFC-Fredericton Research and Development Centre, Fredericton, NB, CANADA; (2) Agriculture and Agri-Food Canada, Fredericton, NB, CANADA; (3) Fredericton Research and Development Centre, Fredericton, CANADA; (4) Charlottetown Research and Development Centre, Charlottetown, CANADA; (5) Agricultural Certification Services, Fredericton, NB, CANADA; (6) Agriculture and Agri-Food Canada - Fredericton Research and Development Centre, Fredericton, NB, CANADA

Potato early dying (PED) is a major yield-limiting factor in potato production in Canada. PED is primarily caused by Verticillium dahliae, and severity of PED is increased by the root-lesion nematode, Pratylenchus penetrans. A two-year potato-barley crop rotation is the most common practice in New Brunswick, Canada. This study evaluated the effect of various rotation crops in a two-year potato rotation on PED and potato yield in New Brunswick in a small plot trial with twelve treatments in 2018 (rotation phase) and 2019 (potato phase). One composite soil sample was collected from each plot pre-plant and post-harvest in each year for quantification of root-lesion nematodes and $V$. dahliae. In the potato phase, PED severity was assessed over time and potato yield was determined. In the rotation phase, brown mustard grown as a biofumigant had the greatest reduction in root-lesion nematodes, while forage pearl millet and marigold suppressed the root-lesion nematodes. In addition, all rotation crop treatments, except the multi-species and bare soil treatments, significantly reduced $V$. dahliae compared with the potato treatment. In the potato phase, the area under disease progress curve ranged from 667 to 1551, with the lowest value for the brown mustard treatment and the greatest value for the barley treatment. Marketable tuber yield was greatest for the mustard treatment, followed by forage pearl millet, marigold, field pea and sorghum sudan grass treatments. Preliminary results suggest that choice of crop rotation species is a tool to manage PED and improve potato productivity.

\section{Integrating climate, cropland connectivity, and trade data layers to understand phytosanitary risk for mango in Haiti and the Caribbean}

J. Fayette (1,2,3), Y. Xing (1,2,3), W. Dantes (1,2,3), K. F. Andersen (1,2,3), O. N. Carvil (4), T. V. da Silva Galdino (5), S. Kumar (6), C. J. Penca (7), A. M. Szyniszewska (8), K. A. GARRETT (1,2,3), (1) Plant Pathology Department, University of Florida, Gainesville, FL, USA; (2) Emerging Pathogens Institute, University of Florida, Gainesville, FL, USA; (3) Institute for Sustainable Food Systems, University of Florida, Gainesville, FL, USA; (4) Faculte D'Agronomie et de Medecine Veterinaire, Haiti, Port-au-Prince, HAITI; (5) Department of Plant Science, Universidade Federal de Viçosa, BRAZIL; (6) USDA-APHIS-PPQ, NC, USA; (7) USDA-APHIS, FL, USA; (8) Rothamsted Research, Harpenden, UNITED KINGDOM

Haiti is among the top twenty mango producers. The potential introduction of pests and diseases, including Ceratocystis fimbriata, causing mango sudden decline, and the Mediterranean fruit fly, Ceratitis capitata, is a significant threat to mango production in Haiti and the Caribbean region. The objective of this study is to guide management practices that could limit or delay the introduction of new pests in Haiti and the region, using an integrated risk analysis framework integrating three geographic risk layers: climate-based species distribution models, cropland connectivity, and trade networks. We evaluated climate-based risk using the Biomod2 package and maximum entropy species distribution modeling (Maxent). A cropland connectivity risk index was used to evaluate the connectivity between areas of mango production in the Caribbean. Trade networks were evaluated based on the UN Commodity Trade Statistics Database. There are patches of particularly suitable environment for C. capitata toward the northern and southern fringes of Haiti. Mango cropland connectivity in Haiti is high and likewise Haiti has a potential role as a bridge location connecting neighboring countries. Trade linkages between major ports in the region also contribute to the overall risk profile. Integrated risk maps can inform national strategies for invasive pest management, where focusing on higher risk areas will generally allow for more efficient use of limited resources for detection and mitigation. 
Evaluation of host specific virulence factors of Fusarium solani f. sp. pisi for garden pea (Pisum sativum L.) A. POKHREL, J. Coleman, Auburn University, Auburn, AL, USA

Fusarium solani f.sp. pisi $(F s p)$ is a soil borne filamentous fungus that is able to cause a root rot on garden pea. Genome sequencing of an Fsp isolate revealed that at least three (14,15 and 17) of the seventeen total chromosomes were supernumerary and encode host specific virulence factors. Previous studies using pulse field gel electrophoresis indicated a high degree of variability exists in the karyotype of putative supernumerary chromosomes of field isolates. Therefore, this research aims to evaluate the diversity of the accessory genome in a population of $F s p$ field isolates through whole genome sequencing. Genes involved in pea pathogenicity ( $P E P$ genes) are clustered on chromosome 14 (the $P D A 1$-chromosome) and the cluster is $\sim 20 \mathrm{~kb}$ in size and encodes five genes, $P E P 1, P E P 2$, $c D N A 3, P D A 1$, and PEP5. Most of the studies on the PEP cluster have been focused on PDA1 which encodes a cytochrome P450 conferring pisatin demethylase activity. It has been shown that in the absence of the entire $P D A 1$-chromosome, the virulence of $F s p$ is drastically reduced; however, targeted gene disruption of the $P D A 1$ gene only reduced the virulence of $F_{s p}$ by $\sim 20-40 \%$. The contribution of the entire $P E P$ cluster to virulence on pea has not been fully addressed. The goal of this research is to generate a deletion of the entire $P E P$ cluster on the $P D A 1$-chromosome using a CRISPR/Cas9 system.

\section{Determining the distribution of quinone outside inhibitor (QoI) fungicide-resistant Cercospora sojina in Indiana} N. PINEROS GUERRERO (1), D. Neves (2), C. A. Bradley PhD (2), D. E. P. Telenko (3), (1) Purdue University, Lafayette, IN, USA; (2) University of Kentucky, Princeton, KY, USA; (3) Department of Botany and Plant Pathology, Purdue University, West Lafayette, IN, USA

Frogeye leaf spot (FLS) is an important foliar disease in soybean (Glycine max L. (Merr.)) caused by Cercospora sojina K. Hara. FLS is commonly found in hot and humid regions in the southern United States, but recently has been found causing significant yield losses in Indiana. Foliar fungicide application of quinone outside inhibitors (QoIs) or strobilurins have been one of the major tools in the management of this disease. In 2010, the first report of QoI-resistant $C$. sojina isolates were identified in Tennessee. Subsequent reports revealed the presence of Qoi-resistant $C$. sojina populations in 14 states, including Indiana. QoI-resistant isolates have been documented in only four of the 92 counties in Indiana (Clark, Delaware, Fountain, and Pulaski Counties). We hypothesize that QoI-resistant populations are wide-spread across the state and further mapping of these resistance populations will help in directing current management strategies. In this study, 148 isolates of $C$. sojina were collected from soybean fields in 11 new counties across Indiana to evaluate their sensitivity to QoI fungicides. The collected isolates were characterized as resistant or sensitive by assessing their sensitivity to QoI fungicides amended poison plates. A PCR-RFLP method was also used to identify the presence of a single nucleotide mutation associated with QoI resistance, in the $c y t b$ gene of the collected $C$. sojina isolates. The nucleotide sequences of the $c y t b$ gene from potential resistant $C$. sojina isolates were also analyzed to corroborate the presence of the mutation.

\section{Foliar infiltration of virus-derived small hairpin RNAs (shRNAs) triggers the RNAi mechanism against the Cucumber mosaic} virus (CMV)

B. VILLEGAS, M. Sánchez, A. Valencia-Jiménez, Universidad de Caldas, Manizales, COLOMBIA

Post-transcriptional gene silencing (PTGS) is an evolutionarily conserved plant defense mechanism against pathogens, especially viruses. The aim of this paper was to evaluate a dsDNA construct (77 bp) as the template for in vitro production of virus-derived artificial small hairpin RNAs (shRNAs), without using methodological approaches associated with recombinant DNA technology, and test the resulting hairpins structures for their potential to trigger the RNAi mechanism in Nicotiana benthamiana plants against the Cucumber mosaic virus (CMV) after their foliar infiltration. This approach allowed the production of significant amounts of shRNAs (60-mers) in a fast and easy way as revealed by $1.5 \%$ agarose gel electrophoresis. No degradation of the synthesized shRNAs was observed when treated with DNase and RNase A, revealing its resistant hairpin-type secondary structure. The gene silencing triggered by shRNAs was confirmed using polymerase chain reaction (PCR), Immunological-based assays, and Quantitative Real-Time PCR (RT-qPCR). The highest levels of gene silencing were recorded for mRNAs coding for replication protein (ORF1a), the viral suppressor of RNA silencing (ORF2b), and the capsid protein (ORF3b) with $98 \%, 94 \%$ and $70 \%$ of total transcript silencing respectively. It was found that this protocol provides an interesting alternative to produce significant amounts of small RNA hairpins that can effectively trigger the plant RNAi mechanism against CMV.

\section{Evaluation of Miravis Top and two phosphite application methods to manage scab (Venturia effusa) on pecan (Carya} illinoinensis) in Georgia

T. B. BRENNEMAN (1), M. W. Hotchkiss (2), J. H. Brock (3), C. H. Bock (4), (1) University of Georgia, Tifton, GA, USA; (2) USDA ARS, Byron, GA, USA; (3) Department of Plant Pathology, University of Georgia, Tifton, GA, USA; (4) USDA ARS, Southeastern Fruit and Tree Nut Research Laboratory, Byron, GA, USA

The phosphite fungicides Kphite and Phostrol were compared at 2.4, 4.7, 7.0 and 9.4 L/ha to a nonsprayed control and to Miravis Top $(1.0 \mathrm{~L} / \mathrm{ha})$ applied at 14-day intervals to scab-susceptible pecan cultivars Wichita and Desirable in 2019. Single shoots were sprayed to runoff diluted in $935 \mathrm{~L} /$ ha water for a total of 10 applications. The two phosphite products provided similar scab control. Some leaf necrosis occurred at the $9.4 \mathrm{~L} /$ ha rate but phytotoxicity was minimal at lower rates. At $2.4-7.0 \mathrm{~L} / \mathrm{ha}$ the phosphite products gave similar leaf scab control to Miravis Top, but on Wichita were more effective at $9.4 \mathrm{~L} /$ ha than Miravis Top. Control of scab on fruit showed a rate response using Kphite or Phostrol, but even the $9.4 \mathrm{~L} /$ ha rate was less efficacious than the Miravis Top treatment. On Wichita fruit, the $9.4 \mathrm{~L} /$ ha rate of phosphite resulted in $72-77 \%$ fruit area diseased, whereas fruit receiving the Miravis Top treatment had only $8 \%$ area diseased (nonsprayed $=100 \%$ ). In a separate trial, Kphite $(9.4 \mathrm{~L} / \mathrm{A})$ was sprayed 7 times at 3-week intervals to the soil around microjet sprinklers under Wichita and Desirable trees prior to irrigation. Scab severity on both leaves and fruit were similar on the 
treated and nontreated trees. Although phosphites are systemic, soil applications had no effect on scab, even on young trees with small canopies. Foliar sprays of phosphites are highly effective on leaf scab, and applications with commercial sprayers have not resulted in phytotoxicity.

\section{Wild wild west: Emerging hemp viruses}

J. CHIGINSKY, K. Langemeier, S. White, W. S. Cranshaw, A. C. Fulladolsa, M. Stenglein, P. Nachappa, Colorado State University, Fort Collins, CO, USA

Hemp (Cannabis sativa L.), is a rapidly growing industry in the United States. Colorado is one of the biggest hemp growing states having over doubled its acreage from 35,950 in 2018 to over 75,000 in 2019. However, hemp has been understudied in the U.S. since its production declined in the late 1950s and information on the biological and agricultural factors affecting hemp under current conditions is limited. Disease identification and management is an increasing challenge for hemp growers across the country. For instance, beet curly top virus (BCTV), which is transmitted by the beet leafhopper, was recently shown to infect hemp plants in Colorado. Since its initial detection, we confirmed the virus in over 150 hemp samples from 9 counties in Colorado, with several fields reporting disease incidence levels above 50\%. We found BCTV-Worland and BCTV-CO strains and many mixed infections. Experiments are underway to confirm Koch's postulates and transmission efficiency. It is likely that there are several undiscovered viruses and viroids in hemp that can potentially impact the plant, but also serve as a reservoir for disease spread to other crops in the vicinity. Using next generation based metatranscriptomics, we are characterizing the virome of hemp. The results of this research will improve diagnostics through the development of and accessibility of species-specific primers. Accurate and efficient identification of viruses and viroids will help target control strategies through integrated pest management and interruption of transmission cycles, in turn reducing yield losses.

\section{Use of laser-guided intelligent sprayer technology to manage grape diseases} L. M. WODZICKI (1), H. Zhu, Ph.D. (2), E. Long (1,3), L. V. Madden (1), M. L. Lewis Ivey (1), (1) Department of Plant Pathology, The Ohio State University, Wooster, OH, USA; (2) USDA-ARS, Wooster, OH, USA; (3) Purdue University, IN, USA

Laser-guided intelligent sprayer technology (IST) uses lasers to visualize canopy architecture to determine the optimum spray volume and direct the pesticide onto the crop based on vine height, width, spacing and density. The goal of the study was to validate the use of IST as a more environmentally friendly and cost-effective pest management tool as compared to traditional, airblast sprayers for grape producers in Ohio. Fungicide distribution in the canopy was determined by calculating the percent area covered on water sensitive paper. Foliar disease severity was assessed throughout the season by quantifying percent disease on the foliage on a $0-100 \%$ scale. Total marketable yield was used to assess fruit quality and the profit margin for each application method was determined. Foliar disease was significantly higher in vines where fungicides were applied using IST versus airblast technology, however no difference in yield between the two application methods was observed. Total volume of fungicide applied during the season was five times less using the intelligent sprayer compared to the airblast sprayer, which corresponded to significantly less fungicide deposition and lower fungicide costs. The profit margin for the grapes harvested from vines treated using intelligent sprayer technology was twice that of vines treated using airblast technology. Even though foliar disease severity was higher using IST, less fungicide was sprayed onto the canopy, reducing costs while maintaining fruit quality, supporting the technology as a cost-effective option for vineyards.

High-Throughput Sequencing (HTS) of bees and pollen for biosurveillance of agricultural pathogens and invasive species G. J. BILODEAU (1), M. Rott (2), M. M. Guarna (3), S. F. Pernal (3), J. Griffiths (4), (1) Canadian Food Inspection Agency, Ottawa, ON, CANADA; (2) Canadian Food Inspection Agency, North Saanich, CANADA; (3) Agriculture and Agri-Food, Beaverlodge, CANADA; (4) Agriculture and Agri-Food, Vineland Station, CANADA

Bees including the European honey bee, Apis mellifera, are a vital link of agricultural systems, responsible for pollinating over 30 percent of the world's food crops including fruits, vegetables and oilseeds. While pollinating, bee species come into contact with plant pathogens and have the potential to become vectors of pathogens between individual plants and also carry viruses, bacteria and fungi back to their hives. Here, we exploit this interaction between pollinators and plants by using bees and bee-collected pollen to monitor pathogens. This approach has the potential to provide unprecedented information on the health of the plant-pollinator ecosystem, and provide critical information for the early detection of plant pathogens. Employing high-throughput sequencing technologies, we aim to determine the presence of pathogens related to bee pollination activities, including honey bee and plant pathogens. We have started to evaluate pathogen profiles for two bee-dependent agricultural crops, blueberries and apples from ON and BC, Canada. From the first year of sampling 44 samples from hive-mounted pollen traps, bee bread and foragers were sequenced for the ITS region for Fungi and $16 \mathrm{~S}$ for bacteria with more than 19M reads per run. Fungal plant pathogens identified from blueberry and apple fields included Monilinia species as well as bee pathogens such as Ascosphaera apis, the causative agent of chalkbrood disease. We aim to provide a framework for future screening methods that would allow for early detection of new and emerging pathogens with invasive species.

Identification of Colletotrichum spp. causing postharvest anthracnose of avocados in Chile M. BUSTAMANTE, Y. Fernandez, C. Osorio-Navarro, J. L. Henriquez, Departamento de Sanidad Vegetal, Universidad de Chile, La Pintana, Santiago, CHILE

Anthracnose caused by Colletotrichum spp. is an economically important postharvest disease affecting avocados (Persea americana Mill.) worldwide. In Chile, the disease has increased during the last decades along with the establishment of new orchards in humid areas. The objective of this study was to determine the etiology of the disease in the country, following the current taxonomy of the genus. A survey was carried out in four commercial orchards located in the regions of Valparaiso, Metropolitana and O'Higgins, where 
146 single-spore isolates were obtained and separated in four morphological groups. Fifty representative isolates were identified by multi-locus phylogenetic analyses using partial gene sequences of ITS, TUB2, GAPDH, GS and ApMat. ITS alone clustered the isolates in four species complexes while multi-locus analyses revealed 10 species causing anthracnose of avocados grown in Chile: $C$. kahawae ssp. cigarro, C. jiangxiense, C. gloeosporioides, C. fructicola and C. perseae (C. gloeosporioides species complex); C. beeveri, C. brassicicola and $C$. karstii (C. boninense species complex); C. pyricola (C. acutatum species complex) and C. anthrisci (C. dematium species complex). Five of these are new species associated with avocados worldwide. Pathogenicity was confirmed by completing Koch's postulates, inoculating healthy Hass avocados with $10^{6}$ spores $/ \mathrm{mL}$ from 2 isolates per species and successfully re-isolating them from anthracnose lesions that developed after 7 days at room temperature $\left(20^{\circ} \mathrm{C}\right)$.

Successful isolation of tumorigenic Rhizobium sp. from blueberry cane galls using tissue culture N. A. Khalib (1), G. Sanahuja (2), J. Umble (2), V. O. STOCKWELL (3), (1) Oregon State University, Corvallis, OR, USA; (2) Fall Creek Farm and Nursery, Lowell, OR, USA; (3) USDA ARS Horticultural Crops Research Unit, Corvallis, OR, USA

In 2016, an outbreak of aerial crown gall emerged in Oregon blueberry fields. Symptoms of aerial crown gall of blueberry are numerous galls along the length of older canes to the crown; root galls are rarely observed. Diagnostic PCR to detect pTi-borne virD2, associated with crown gall, yielded mostly negative results on stem galls or colonies isolated from galls on semi-selective media for agrobacteria. Our objective was to evaluate if the pathogen could be enriched or isolated from tissue culture plantlets initiated from canes of symptomatic plants. Canes were collected from commercial blueberry fields from symptomatic and non-symptomatic plants. Cane segments without visible galls were surface disinfested and 100 segments were placed in Woody Plant Medium (WPM). After a 6 week incubation period at $24^{\circ} \mathrm{C}$ with a 16-h photoperiod, tissues were transferred to new media. After 8 weeks, galls were observed on 12 of 37 in vitro microcuttings. Gall tissues were surface disinfested, ground in sterile phosphate buffer, and spread on $\mathrm{PDA}^{2}+0.5 \% \mathrm{CaCO}$. Uniform, small, white, glistening colonies were isolated from the galls. virD2 was amplified and partial virD2 sequences were similar to MK423929.1. Isolates were positive with flaA primers of Kuzmanovic et al. for Rhizobium tumorigenes. Tomato and sunflower stems inoculated with the blueberry isolates formed galls. Although our method to isolate gall bacteria from blueberry was time-intensive, many of the tissue culture starts from symptomatic canes developed galls, from which the pathogen was easily isolated.

\section{Major diseases in the commercial production of dragon fruit in Florida}

T. DE PAULA LELIS (1), C. F. Hong (2), S. Zhang (1), J. H. Crane (3), R. Gazis (4), (1) University of Florida, Homestead, FL, USA; (2) National Chung Hsing University, Taichung City, TAIWAN; (3) University of Florida - Tropical Research and Education Center, Homestead, FL, USA; (4) University of Florida, FL, USA

Dragon fruit is a vining cactus (Hylocereus spp.) native to the tropical forest regions of Mexico and Central and South America. Due to an expanding market, dragon fruit is now cultivated in tropical and subtropical regions worldwide. In the USA, in addition to Florida, dragon fruit is produced in California, Puerto Rico, Hawaii, and, more recently, Texas. As dragon fruit represents a relatively new introduction to Florida's tropical fruit industry, little is known about the impact of diseases on host fitness and fruit yield and quality. In this study, we assessed the prevailing diseases and physiological disorders affecting commercial dragon fruit production in South Florida. Four genera of fungal pathogens (Alternaria, Bipolaris, Colletotrichum and Neoscytalidium), one virus (Cactus virus X), and one bacterium (to be determined) were found to cause disease on stems and/or fruits of dragon fruit. The prevalence and severity of the different diseases varied throughout the year, peaking during the rainy season. Stem and fruit canker, caused by Neoscytalidium dimidiatum, was found to be the major disease affecting dragon fruit production in South Florida. Abiotic disorders, such as sunburn and root and basal stem root rot due to overwatering, were often found compromising plant growth and development. This study represents the first comprehensive assessment of current major diseases in commercial dragon fruit orchards in Florida and constitutes a baseline for understanding disease issues on dragon fruit and developing effective integrated management programs.

\section{Investigating the relationship between xylem anatomy and tolerance to Phaeomoniella chlamydospora and Phaeoacremonium} minimum on grapevine rootstocks

C. RAMSING (1), S. Mocholí (1), D. Gramaje PhD (2), F. Cabello (3), J. Agustí (4), J. Armengol (1), M. Berbegal-Martinez (1), (1) Instituto Agroforestal Mediterráneo, Universitat Politècnica de València, Valencia, SPAIN; (2) Instituto de Ciencias de la Vid y del Vino, Logroño, La Rioja, SPAIN; (3) Instituto Madrileño de Investigación y Desarrollo Rural, Agrario y Alimentario, Alcalá de Henares, Madrid, SPAIN; (4) Instituto de Biología Molecular y Celular de Plantas, Valencia, SPAIN

Fungal trunk diseases (GTDs) threaten grapevine production worldwide. Esca and Petri disease are especially devastating GTDs and are associated with several fungal pathogens. Among them, Phaeomoniella chlamydospora and Phaeoacremonium minimum are the most common and widespread species isolated from affected vines. Recent studies demonstrated xylem vessel diameter affects susceptibility of grapevine rootstocks to $\mathrm{Pa}$. chlamydospora. The objectives of this project were: (i) to screen over 25 grapevine rootstocks from Spanish germplasm collections for xylem traits and tolerance to Pa. chlamydospora and Pm. minimum and (ii) to determine the relationship between xylem anatomy and tolerance. Rootstock cuttings were inoculated using spore suspensions in a hydroponic growing system. Disease tolerance was assessed by fungal DNA concentration on wood fragments (quantified via qPCR), while histological analyses measured xylem diameter, density, and the proportion of stem surface covered by xylem. Rootstocks were grouped into classes based on xylem characteristics to observe any relationship between xylem traits and tolerance. Our results showed that there were significant differences among rootstocks in all xylem characteristics and DNA concentration of both pathogens. Moreover, the relationship between xylem diameter and tolerance to Pa. chlamydospora was corroborated. Rootstocks with the widest xylem diameter proved the most susceptible to Pm. minimum. This information will be useful for cultivar choice and future breeding programs to reduce the detrimental impact of GTDs worldwide. 
Annotomic comparisons to identify candidate pathogenicity/virulence factors within the Aspergillus genus K. PENNERMAN (1), G. Yin (2), J. W. Bennett (2), (1) USDA, ARS, Athens, GA, USA; (2) Rutgers University, New Brunswick, NJ, USA

Members of the genus Aspergillus display a variety of lifestyles, ranging from saprophytic to pathogenic on plants and animals. Increased genome sequencing of filamentous fungi permits efficient use of omic comparisons between closely related species and strains to identify candidate genes that may contribute to phenotypes of interest. We retrieved 223 strain genomes of 10 Aspergillus species and annotated them with InterPro, Gene Ontology and KEGG Ontology terms. After k-means clustering and principal component analyses, the three annotomes for each strain largely clustered by assigned species; dendrograms could be derived from the plot distances that was on par with one generated using in silico-identified ITS sequences. The species clusters separated by pathogenicity on or association with plants, animals or neither along the first dimensions, which accounted for about $20 \%$ of the variance for all three annotation sets. Annotations that highly correlated with this dimension predicted genes encoding pectinases, apopotosis-inducing factors and major facilitator superfamily membrane transporters at higher frequencies in the plant-associated species A. flavus and A. oryzae. Strains of the human pathogen A. fumigatus and the less virulent $A$. fischeri strains had higher counts of pheromone/mating factors. These genes may be subject to future manipulations to further refine understanding of pathogenicity factors. We currently anticipate repeating the computational method in other fungal genera.

Implementing high-throughput sequencing on imported fruit trees held under quarantine at the Plant Germplasm Quarantine Program of the USDA

O. P. HURTADO-GOnZALES (1), M. M. Malapi-Wight (1), L. Carvalho Costa (1), X. Hu (2), A. Wunsch (1), R. P. Jones (1), J. Mendoza (1), B. Atha III (1), L. Hendrickson (1), B. Adhikari (1), C. McFarland (3), J. A. Foster (1), (1) USDA-APHIS Plant Germplasm Quarantine Program, Beltsville, MD, USA; (2) USDA, Beltsville, MD, USA; (3) USDA-APHIS-PPQ-Field Operations, Raleigh, NC, USA

Improved varietal and product development in the apple and fruit tree industries relies on access to valuable imported germplasm which is mainly introduced into the U.S. through the USDA-APHIS Plant Germplasm Quarantine Program (PGQP) located in Beltsville, Maryland. The PGQP Pomes program screens the imported germplasm for a wide variety of well-characterized high-risk pathogens including viruses, viroids and phytoplasmas using PCR-based techniques, greenhouse bioassays, and indicators trees in the field. If pathogen(s) are detected, germplasm is treated to generate pathogen-free trees before their released into the U.S. Increased demand for imported germplasm might poses higher risks of pathogen escape during the screening process. In order to reduce these risks, the Pomes PGQP program is implementing high-throughput sequencing (HTS) technologies combined with modern bioinformatic pipelines for the rapid identification of quarantine pathogens or uncharacterized disease agents. Over 100 different apples and pear fruit trees kept in quarantine as well as rootstocks, field indicators, trees that had undergone therapy, were subjected to HTS using a metagenomics approach. Total RNA was extracted, ribosomal RNA was depleted, and HTS libraries were prepared using a TruSeq Illumina kit. Approximately 30-50 million reads of 75bp single-end reads were obtained per sample. Bioinformatic analysis identified a wide range of plant viruses and viroids as well as unreported agents. A summary of our findings and their implications in accurate diagnostics will be presented.

Fungal flora, Fusarium biomass, and mycotoxin profiles of malting barley grain in New York A. LUGO-TORRES, Cornell University, Ithaca, NY, USA

Production of malting barley for the craft brewing supply chain is a new priority in New York with acreage expanding each year since 2012. Fusarium head blight and grain contamination with trichothecene mycotoxins are already established as major constraints in the production of malting barley in this state. Little is known about the mycoflora of malting barley grain in New York and especially about the spectrum of Fusarium species associated with grain and mycotoxin contamination, so research was conducted to establish baseline data on grain mycoflora and mycotoxin profiles. A total of 41 malting barley grain samples (24 in 2018 and 17 in 2019) was collected from commercial producers and analyzed for fungal flora with a focus on Fusarium spp. Fungi were identified morphologically to genus, and Fusarium-like colonies were identified by morphological features and confirmed by TEF-1a gene sequencing and comparison to GenBank sequences. Fungal incidence, species diversity, and mycotoxin identities and amounts varied widely among grain lots. Fungal diversity was higher in 2018, a very dry production season, than in 2019, a production season with moderate moisture. Fusarium graminearum and $F$. poae predominated in both years. Biomass of these two Fusarium species in malting barley grain was estimated with Real-Time PCR using species-specific primers. The finding of significant nivalenol (NIV), especially in the drier year, suggests that NIV as well as deoxynivalenol (DON) should be quantified in grain lots bound for malting.

\section{Detection and occurrence of virus infecting chayote in Taiwan}

T. C. LIU (1), Y. C. Tsai (2), T. H. Hung (3), (1) Hualien District Agricultural Research and Extension Station, Taiwan, Hualien, Taiwan, TAIWAN; (2) Hualien District Agricultural Research and Extension Station, Taiwan, Hualien, TAIWAN; (3) National Taiwan University, Taipei, Taiwan, ROC., Taipei, TAIWAN

Chayote (Sechium edule (Jacq.) Swartz.) is the featured crops with picking the sprouts for vegetables knows as "dragon-whisker vegetable" in Ji-an, Hualien country, Taiwan. In recent years, symptoms of mottling、wrinkle and leaf curling on chayote were observed in field. The symptomatic leaves was tested positive for Squash leaf curl Philippines virus by PCR using specific primers SLCVup/SLCVdw. In order to confirm whether it is possible to spread the disease though mechanically transmission during harvesting, the diseased plant was tested with RT-PCR and negative for four RNA virus commonly found in cucurbits, including Cucumber green 
mottle mosaic virus, Zucchini yellow mosaic virus, Cucumber mosaic virus. The specific primers SLCV250F/SLCV250R of SLCPHV were designed from conserved sequences of DNA-A. It is more sensitive than the primer SLCVup/SLCVdw. The former can be detected after $10^{5}$ fold dilution with PCR. In field survey, we made a visual survey to investigate the incidence of viral disease in 10 chayote fields. The result indicate that the incidence of symptoms were highest $22.8 \%$ in August, and lowest $7.2 \%$ in December. Because symptoms of new leaves will gradually decrease to almost disappear between October to December. The farmers thought the plants became healthy and did nothing for control. Most young leaves with non-symptoms tested positive with PCR. We found the viral disease incidence in 2019 has tripled compared to 2013, thus it's crucial for farmers to take precautions (eg. control insect pest) in the future.

Preliminary investigation on integrated use of two systemic acquired resistance inducers against bacterial speck on tomatoes S. DA SILVA (1), S. Maxwell (1), F. B. Iriarte (2), J. H. Freeman (1), J. B. Jones (3), M. L. Paret (1), (1) North Florida Research \& Education Center, University of Florida, Quincy, FL, USA; (2) Univ of Florida - IFAS NFREC, Quincy, FL, USA; (3) University of Florida, Gainesville, FL, USA

Bacterial speck of tomatoes caused by Pseudomonas syringae pv. tomato affects tomatoes early in the spring season in Florida. In the spring of 2018 and 2019, field trials were conducted in north Florida to examine the impact of using a sequence of two systemic acquired resistance (SAR) inducers in reducing bacterial speck disease severity. The 2018 trial consisted of two treatments, an untreated water control and a treatment with 5 weeks of Acibenzolar S-Methyl (ASM, Actigard $50 \mathrm{WG}$, Syngenta) followed by 3 weeks of Methyl salicylate (MS, Leap, Valent Biosciences). The 2019 trial consisted of an untreated water control, ASM for 5 weeks, MS for 8 weeks, and ASM for 5 weeks followed by MS for 3 weeks. Disease evaluations were made weekly using the Horsfall-Barratt (H-B) scale and the area under the disease progress curve (AUDPC) was calculated. There was no statistical separation in disease severity between treatments in 2018 even though the ASM + MS treatment had numerically lower disease severity $(\mathrm{P}=0.056)$. In 2019, ASM + MS, ASM and MS alone significantly reduced disease severity compared to the untreated control $(\mathrm{P}=0.05$, LSD). Further field studies are needed to understand the benefit of using a combination of SAR inducers against bacterial speck and the potential impacts on plant growth and development.

\section{Spread of citrus tristeza virus in central California} R. K. YOKOMI (1), M. Sisterson (2), S. Hajeri (3), (1) USDA ARS PWA, Parlier, CA, USA; (2) USDA-ARS, Parlier, CA, USA; (3) Central CA Tristeza Eradication Agency, Tulare, CA, USA

Citrus tristeza virus (CTV) in California is regulated by a State Interior Quarantine. In abatement districts, roguing of CTV-infected trees is based on reactivity to MCA13, a strain-discriminating monoclonal antibody. To determine effectiveness of this program to reduce spread of MCA13-positive CTV, surveys were conducted in abatement plots with mandatory aphid control versus nonabatement plots under normal pest management practices. In abatement plots, overall CTV incidence between 2008 to 2018 increased by an average of $3.9 \%$ and only two MCA13-positive trees were detected and removed. In contrast, between 2015 and 2018 , CTV incidence in non-abatement plots (without rouging) increased by an average of $4.6 \%$ and trees infected with MCA13-negative isolates increased eleven times higher than that of MCA13-positive trees. Spatial and temporal analyses showed MCA13-negative trees were more randomly distributed suggesting primary spread; whereas, MCA13-positive trees were more aggregated suggesting some secondary spread. These results suggested spread of MCA13-positive isolates was limited by a combination of rouging and aphid vector suppression. MCA13-positive isolates were comprised of the VT genotype with some mixtures with T30 genotype. Citrus cultivars in the plots included Navel, Valencia and mandarin and were grown on Carrizo rootstock. All CTV-infected trees were asymptomatic.

Azoxystrobin sensitivity of Rhizoctonia solani AG2-2 populations affecting Michigan sugar beet J. F. WILLBUR (1), C. Bloomingdale (1), C. Pincumbe (1), C. Hendershot (1), D. H. Minier (1), L. E. Hanson (2), (1) Michigan State University, East Lansing, MI, USA; (2) USDA ARS, East Lansing, MI, USA

Rhizoctonia root and crown rot (RRCR) is caused by Rhizoctonia solani AG 2-2 and continues to be a major disease of sugar beets. In Michigan, azoxystrobin (Quadris) is widely applied one to two times per season to manage RRCR. Azoxystrobin, a quinone outside inhibitor, targets a single site to inhibit fungal respiration and so possesses a high risk of fungicide resistance development. Continued reliance on this product has justified recent investigations of azoxystrobin sensitivity in Michigan $R$. solani populations. From 20182019, isolates were collected from research and commercial fields in the Michigan sugar beet growing region. Two additional baseline isolates, collected prior to azoxystrobin use in sugar beet, were included for comparison. Isolates were screened in half-strength clarified V8 broth amended with salicylhydroxamic acid at $10 \mu \mathrm{g} \mathrm{ml}^{-1}$ and azoxystrobin at concentrations: $0,0.01,0.1,1,10$, and $100 \mu \mathrm{g} \mathrm{ml}{ }^{-1}$. Percent inhibition was calculated and effective concentrations for $50 \%$ inhibition of colony mass were determined using three-parameter logistic regression. In 2019, high frequencies of Fusarium spp. and Geotrichum sp. were also isolated from root samples; further molecular and morphological characterization will be conducted with these. Thus far, azoxystrobin insensitivity has not been observed in Michigan $R$. solani populations, however, additional testing is ongoing. In addition, evaluation of azoxystrobin efficacy, and potential alternatives, for RRCR management was conducted at the Saginaw Valley Research and Extension Center in Frankenmuth, MI.

Metagenomic analyses of ACP and citrus samples infected with "Candidatus Liberibacter asiaticus" in California J. Huang (1), D. Zehan (2), Z. Zheng (1), P. Silva (3), L. Kumagai (4), J. CHEN (5), X. Deng (1), (1) South China Agricultural University, Guangzhou, CHINA; (2) South China Agricultural University Resources and Enviroment College, Guangzhou, CHINA; (3) Fundecitrus, Araraquara, BRAZIL; (4) California Dept of Food \& Agriculture, Sacramento, CA, USA; (5) USDA-ARS, Parlier, CA, USA 
Huanglongbing (HLB) is a highly destructive disease affecting citrus production. The disease is associated with an unculturable proteobacterium "Candidatus Liberibacter asiaticus" (CLas), transmitted by Asian citrus psyllid (ACP, Diaphorina citri). HLB was first found in southern California in 2012. Since then, the disease has been found in multiple locations there. Understanding the microbiome associated with HLB has important implications for the disease management. In this study, citrus and ACP CLas samples recently collected from County of San Bernardino were analyzed. A metagenomics pipeline was developed that included DNA extraction, nextgeneration-sequencing (NGS) in Illumina NextSeq format, and bacterial sequence grouping using a metagenomic tool (Kaiju) along with appropriate validation. In addition to CLas, sequence groups of Bradyrhizobium, Burkholderia, Mesorhizobium, Paraburkholderia, and Pseudomonas were found in both the citrus and ACP samples. Some groups had relatively high abundancy. Draft genome sequences of both ACP and citrus CLas strains were obtained. Based on terL locus, the two CLas strains were in the Asiatic group with Type 1 prophages, i.e. PGT-1 group. Within the sequence groups of Bradyrhizobium and Mesorhizobium, sequences highly similar to the 16S rRNA gene of CLas were detected. These sequences could potentially impact the accuracy of CLas detection using 16S rRNA gene-based PCR. Characterization of Burkholderia, Paraburkholderia and Pseudomonas groups is currently underway.

A physiological and transcriptomic examination of little cherry disease
A. WRIGHT, S. J. Harper, Washington State University, Prosser, WA, USA

Little cherry disease, which causes cherries to be small, misshapen, and poor tasting, has reached epidemic levels in the Pacific Northwest. This epidemic is caused by Little cherry virus 2 (LChV2) and X-disease phytoplasma (XDP). To understand how these pathogens affect fruit development, a physiological and transcriptomic approach was taken. Fructose, glucose, and sorbitol content was determined for healthy and symptomatic cherries collected from orchards in eastern Washington. Although there were location and cultivar differences, the overwhelming trend was a decrease of 30 to $80 \%$ for fructose, 30 to $70 \%$ for glucose, and 40 to $80 \%$ for sorbitol in symptomatic cherries, which may in part account for the poor taste. There was no difference in malate content or total phenolics. Differences in total anthocyanins and citric acid depended upon cultivar. Transcriptomic analysis was performed on samples collected from healthy and XDP-infected trees at shuckfall and harvest and for LChV2-infected trees at harvest. Differentially expressed genes included CYP78A9, which has been shown to regulate fruit size in cherries. Other genes of interest included sugar transporters and enzymes involved in secondary metabolism. This work provides a foundation for further understanding of this disease. Understanding how symptoms arise following infection is important for developing tolerant or resistant cherry cultivars.

Phenotyping the sorghum nested association mapping (NAM) parents in two Kansas locations for charcoal rot resistance A. NOOR (1), G. P. Morris (2), C. R. Little (1), (1) Dept. of Plant Pathology, Kansas State University, Manhattan, KS, USA; (2) Dept. of Agronomy, Kansas State University, Manhattan, KS, USA

Charcoal rot (CR) is caused by the soilborne fungus Macrophomina phaseolina (MP), is one of the most destructive disease of sorghum root and stalks under favorable conditions. Kansas (KS) is the number one grain sorghum producer in the U.S. and CR is a major concern for growers. Understanding the genetic variation underlying host resistance is critical to confer CR resistance. The sorghum nested association mapping (NAM) population (10 global lines crossed with an elite line, RTx430) could be an effective background to dissect the genetic architecture of CR resistance. The NAM parental lines and CR resistant and susceptible checks were phenotyped in two KS locations in 2019. At 14 days after flowering, suspensions of MP inoculum were injected into stalks and sterilized, distilled water was used for mock-inoculations. Data was collected at 56 days after inoculation for morphological, disease severity, and yield traits. Results revealed significant differences among the genotypes. The genotype*treatment interaction was not significant for either location. Some of the genotypes showed higher charcoal rot progression at both locations. However, they weren't significantly different from the mock treatment, suggesting wounding sensitivity. Also, some lines showed consistently low charcoal rot progression, but were not significantly different than the control. To address these phenotypic variability a future research goal will be to implement a more precise and effective inoculation method to obtain consistent phenotyping results for CR resistance using the sorghum NAM lines.

Identification of superinfection exclusion elicitors of turnip mosaic virus

H. NUNNA (1), F. Qu (2), S. Tatineni (3), (1) Department of Plant Pathology, Lincoln, NE, USA; (2) The Ohio State University, Wooster, OH, USA; (3) USDA ARS, Univ of Nebraska, Lincoln, NE, USA

Superinfection exclusion (SIE) is defined as the phenomenon where prior infection of cells by a virus prevents subsequent infection by the same or closely related viruses. The SIE phenomenon has been illustrated in various viral systems infecting humans, plants, and animals. In this study, we identified SIE elicitors of turnip mosaic virus (TuMV), a member of the genus Potyvirus in the family Potyviridae. Individual proteins encoded by TuMV tagged with the hemagglutinin epitope at the N-terminus of each cistron were cloned in the binary vector pCASS4 under a double 35S promoter. These constructs were mobilized into Agrobacterium tumefaciens strain EHA105 through chemical transformation. A. tumefaciens harboring TuMV cistrons were co-infiltrated with wheat streak mosaic virus P1, an RNA silencing suppressor, into Nicotiana benthamiana leaves. Leaves of $N$. benthamiana agroinfiltrated with TuMV individual cistrons were challenge infiltrated with an Agrobacterium culture harboring pTuMV-GFP at 24 h post-agroinfiltration. At 3 days post-agroinfiltration, $N$. benthamiana leaves expressing TuMV P1, HC-Pro, 6K1, CI, 6K2, NIa-VPg, NIb, or CP cistrons showed efficient expression of GFP, suggesting that these cistrons enabled the superinfection by TuMV-GFP. However, N. benthamiana leaves expressing TuMV P3 or NIa-Pro efficiently prevented superinfection by TuMV-GFP, suggesting that P3 and NIa-Pro independently act as elicitors of SIE. Accumulation of TuMV-GFP genomic RNA copies in superinfiltrated $N$. benthamiana leaves was confirmed by realtime RT-PCR. 
Stone fruits are a globally important crop in the genus Prunus. Many viruses and virus-like pathogens infect stone fruit including sweet cherry, causing diseases that reduce crop yields and market value. In this study, two large contigs with high sequence similarities to two different viruses of the family Betaflexiviridae were identified from a symptomless sweet cherry accession undergoing quarantine testing by high-throughput sequencing technology. Two known pathogens, prune dwarf virus (Ilarvirus, Bromoviridae) and hop stunt viroid (Hostuviroid, Pospiviroidae), were also present in the accession. The complete genomic sequences of the two new viruses were determined. Analyses of the genetic information show that one new virus, cherry robigovirus 5 [CRV-5, 8,384 nucleotides (nt)], is member of the genus Robigovirus while the other virus, cherry latent virus 1 (CLV-1, 7,460 nt), belongs to the genus Trichovirus. Sequence identities are 52-57\% between CRV-5 and other robigoviruses and 60-73\% between CLV-1 and other trichoviruses at genome level. Phylogenetic analyses also support the grouping of these viruses. Screening of germplasm collection accessions indicates that CRV-5 infections are prevalent, but CLV-1 infections are infrequent. The study provides the information necessary to classify the two new betaflexiviruses, and to help develop detection methods for quarantine and certification programs.

PCR multiplex to differentiate Ralstonia solanacearum species complex, including $R$. solanacearum, $R$. pseudosolanacearum and Select Agent R3bv2 strains

S. PAUDEL (1), S. Dobhal (1), T. Lowe-Power (2), R. Schlub (3), C. Allen (4), A. M. Alvarez (1), M. Arif (1), (1) University of Hawaii at Manoa, Honolulu, HI, USA; (2) University of California Davis, Davis, CA, USA; (3) University of Guam, Mangilao, AP, USA; (4) University of Wisconsin-Madison, Madison, WI, USA

Bacterial wilt strains in the Ralstonia solanacearum species complex (RSSC) pose serious threats worldwide to economically important crops. In 2014, Safni and co-workers proposed that the four phylotypes of RSSC be reclassified into three genospecies: $R$.

pseudosolanacearum (Rps), R. solanacearum (Rs) and $R$. syzygii. The revision of RSSC into three genospecies necessitates the proper identification and differentiation of strains for characterization, diagnostics, and epidemiological studies. Therefore, this study aimed to develop an endpoint PCR multiplex for detection and differentiation of Rps, Rs, the Rs Select Agent R3b2 subgroup and RSSC strains with an undetermined phylotype. Genomes representing different phylotypes and hosts were retrieved from the NCBI GenBank database and utilized to search for unique gene regions using OrthoMCL. Designed primers for each group were validated in silico for specificity. AT-rich flap sequences were added at the 5' position of each primer to optimize the reaction thermodynamics. The in silico specificity of the assay was tested in vitro with representative strains of each group and other genera. Neither false positives nor false negatives were detected. The detection limit for each of the primers was $10 \mathrm{pg}$ (Rps), $10 \mathrm{pg}(\mathrm{Rs}), 100 \mathrm{pg}$ (R3b2), and 10 pg (RSSC) of genomic DNA. The use of GoTaq green in this multiplex PCR provides an easy and inexpensive option for routine diagnostics. The tool is highly specific, reliable, and economical for potential use in culture characterization, diagnostics, surveys, and quarantine decisions.

\section{Overview of fungicide resistance affecting the efficacy of single-site fungicides to control Botrytis fruit rot in Florida strawberry fields}

A. ZUNIGA (1), L. Cordova (1,2), M. Bolognesi de Morais (1), N. Peres (1), (1) University of Florida - Gulf Coast Research and Education Center, Wimauma, FL, USA; (2) Corteva Agriscience, Indianapolis, IN, USA

The efficacy of single-site fungicides registered to control Botrytis fruit rot (BFR) of strawberry is threatened by the rapid selection of Botrytis cinerea fungicide resistant populations. Thus, continuous monitoring is essential to evaluate the efficacy of these fungicides. Efficacy of different fungicides on BFR management and their resistance frequencies were monitored for 5 seasons. The single-site fungicides fludioxonil+cyprodinil (Switch), isofetamid (Kenja), and fluopyram (Luna Tranquility) were applied in rotation with the multi-site captan based on the Strawberry Advisory System. In the field trials, fruit were harvested twice a week to evaluate BFR incidence and yield. BFR incidence was reduced by 7 to $62 \%$ with Luna Tranquility, whereas greater reduction was observed with Switch (47 to 90\%) and Kenja (28 to 100\%). Although some differences were observed among years, the use of fungicides considerably increased yield. Resistance frequencies to the same fungicides were obtained from 127-329 isolates collected each season using conidial germination assays. Overall, resistance frequencies increased from 13 to $31 \%$ for fludioxonil and 0 to $11 \%$ for isofetamid, while a major increase was observed for fluopyram from $21 \%$ in 2015 to $68 \%$ resistant isolates in 2019 . The efficacy of registered single-site fungicides such as Luna Tranquility is decreasing over the years as the frequency of resistant $B$. cinerea is increasing in Florida strawberry fields. The use of alternative strategies to control BFR are urgently needed to preserve the existing efficacy of Switch and Kenja.

Understanding the morphological and genetic diversity of soilborne Fusarium species in Pennsylvania D. K. WEERASOORIYA, A. Y. Bandara, M. R. Duffeck, S. R. May, P. Esker, The Pennsylvania State University, University Park, PA, USA

The genus Fusarium includes important soilborne fungal pathogens causing seed and root rots, and wilt in soybeans. However, there is limited information on the diversity of soilborne Fusarium spp. within Pennsylvania (PA). The objectives of this study were to determine the morphological and genetic diversity among Fusarium spp. across PA. During Summer 2018, more than 100 putative Fusarium isolates were acquired using Nash and Snyder medium using soil samples collected from 22 soybean farmer fields across PA. For single sporing, each isolate was initially grown on PDA for 7 days to make a conidial suspension. Thirty microliters of the suspension were plated on water agar and incubated for $24 \mathrm{hrs}$ to facilitate conidia germination. A single germinating conidium was 
transferred to a PDA plate to obtain a single-spore colony. After 7 days, pure cultures were examined for texture, color, margins, and pigmentation. Conidial morphometrics were evaluated and recorded using compound microscopy. For species confirmation, DNA will be extracted from each isolate and subjected to PCR targeting TEF-1 $\alpha$ gene. PCR amplicons will be sequenced and the consensus contigs will then be searched at the FUSARIUM-ID v 1.0 and the NCBI nucleotide databases using BLASTn. Phylogenetic analyses will be performed using MEGA software. Strengthening the knowledge on Fusarium spp. identification and diversity in PA provides important insights to help determine the best management approaches to reduce the potential negative impact on soybean production.

\section{Evaluation of resistance of different varieties of alfalfa to Rhizoctonia solani Z. WANG, Lanzhou University}

Alfalfa is a perennial legume plant with good quality and high yield. However, the root rot of alfalfa caused by Rhizoctonia solani became an important factor of its decline in quality and yield. In this study, the resistance of four cultivars to Rhizoctonia solani was evaluated by soil inoculation method in green house. Evaluation indexes included root and root crown disease index, plant height reduction rate and biomass of alfalfa. The results showed that the difference was significant among the alfalfa varieties. The disease index of root and root crown of 4030 was the highest, while WL232 was the lowest. Both plant height reduction rate and biomass reduction rate showed that 4030 was the highest, followed by WL232 and 020, and 010 was the lowest. 020 had the greatest decreased in the root length, while WL232 had lowest root length reduction rate. Among the 4 alfalfa varieties, WL232 has strong resistance, while 4030 has weak resistance to disease. The results laid a foundation for the breeding of root rot resistant cultivars of alfalfa.

Cytological changes in sweetpotato storage root cambium in relationship with age-related resistance to Ceratocystis fimbriata C. H. PARADA ROJAS (1), K. Pecota (2), C. Yencho (3), L. M. Quesada (4), (1) NC State University, Raleigh, NC, USA; (2) North Carolina State Univ, Raleigh, NC, USA; (3) North Carolina State University, RALEIGH, NC, USA; (4) Department of Entomology and Plant Pathology, North Carolina State University, Raleigh, NC, USA

Ceratocystis fimbriata is an important soilborne fungal pathogen threatening sweetpotato production in the United States. Growers who export their crop are limited in their ability to employ fungicides to control $C$. fimbriata. Deployment of host resistance represents a desirable low-cost tool to decrease losses. Here, we aimed to characterize the effect of storage root development on susceptibility to $C$. fimbriata and determine whether cytological changes in the storage root cambium create an unfavorable environment for $C$. fimbriata infections. We discovered that younger, early developing storage roots were comparatively more susceptible than older, fully developed storage roots. Ceratocystis fimbriata cross-sectional lesion ratio was negatively correlated $(\rho=-0.63)$ with total cross-section root area, suggesting a strong case of age-related resistance. Cytological analysis of older and younger storage roots under wounded, nonwounded, and wounded inoculated conditions revealed structural differences in the primary cambium that potentially acts as a structural barrier against $C$. fimbriata. Our research highlights a potential morphological resistance mechanism against $C$. fimbriata and suggests an early fungicide application during storage root development would maximize fungicide efficacy for prevention of $C$. fimbriata infection.

The role of irrigation in disease development and management of Macrophomina charcoal rot on strawberries L. PEDRONCELLI (1), A. Biscaro (2), A. I. Putman (3), (1) University of California Riverside, Riverside, CA, USA; (2) Univ. of California Coop. Ext. Ventura County, Ventura, CA, USA; (3) University of California, Riverside, Riverside, CA, USA

Macrophomina charcoal rot (MCR; caused by the soilborne fungus Macrophomina phaseolina) is an economically significant disease threatening California strawberry production, with few management options. This disease is typically associated with drought, and studies in other crops have shown that irrigation practices can reduce disease severity. The objective of this research was to evaluate the role of irrigation in disease development and management of MCR on strawberries. A field study was conducted from October 2018 through June 2019 in Irvine, CA. Irrigation treatments were established at high, optimal (grower's standard), or low soil moisture content as determined by soil water potential thresholds monitored using tensiometers. Irrigation treatments were evaluated in a factorial split-plot design with two cultivars (Monterey, Petaluma) and two inoculation treatments (inoculated, control). MCR severity of each plant was assessed on an ordinal scale. Slicing the significant three-way interaction revealed evidence for an influence of irrigation on disease severity only for Petaluma inoculated plants $(P=0.0025)$. Within Petaluma inoculated plants and when compared to optimal soil moisture, MCR severity was higher in the low soil moisture treatment $(P=0.0006)$ but similar to the high soil moisture treatment $(P=0.1679)$. These preliminary results suggest high soil moisture may not reduce MCR and that the influence of irrigation on MCR may be cultivar-dependent. This experiment is currently being repeated.

Revisiting seed-transmissibility of Sweet potato leaf curl virus, a begomovirus infecting sweetpotatoes S. ANDREASON, O. Olaniyi, P. Wadl, L. Williams, D. M. Jackson, A. Simmons, K. S. Ling, USDA-ARS, Charleston, SC, USA

Sweet potato leaf curl virus (SPLCV), a begomovirus (Geminiviridae) transmitted via vegetative propagation of sweetpotato or by the whitefly species Bemisia tabaci, threatens global sweetpotato production. Complicating management of SPLCV is the disputed publication of seed transmission of this begomovirus, a group of viruses which before 2015 were not reported to be seed transmissible. That report prompted an investigation into the validity of seed transmission of SPLCV. In this study, experiments were conducted on over 26,000 individuals. Conventional and quantitative real-time PCR were performed to detect the presence of virus on the seed coat (seed-borne; contaminant) and subsequently in germinated seedlings. Virus was detected on seed coats in each year of the study. Year one, ca. 200 seedlings were tested, and no seed transmission was detected. Year two, ca. 5,000 seedlings were tested, and a small number of samples were positive for SPLCV; however, adult whiteflies were observed in the greenhouse, therefore transmission via the 
seed versus the vector could not be determined. Year three, ca. 20,000 seedlings derived from breeding seeds of SPLCV-infected plants were examined, and no seed transmission was detected. Year four, 900 seedlings were grown, again with no seed transmission detected. Vector transmission tests demonstrated successful transmission of SPLCV to seedlings by viruliferous $B$. tabaci, although at low efficiency $(0.02 \%)$. Repeated large-scale, multi-family seedling grow-out experiments over successive years did not support seed transmission of SPLCV.

\author{
Changes in feeding and reproduction of $R$. padi are associated with differential expression of biotic stress genes in three \\ accessions of wild barley \\ M. BANNISTER, Q. Smith, L. Van Eck, Augsburg University, Minneapolis, MN, USA
}

Aphids are phloem-feeding insect pests that affect global crop production. The bird cherry-oat aphid (Rhopalosiphum padi) is prevalent on cereals and of primary concern as a vector of the barley yellow dwarf virus (BYDV). Barley germplasm resistant to $R$. padi has proved elusive to date, and current pest management approaches rely on expensive chemical inputs that negatively impact the environment and select for resistant insect biotypes. This study investigated three accessions of Hordeum vulgare ssp. spontaneum-the wild progenitor of cultivated barley - for genetic resistance against $R$. padi. Estimated intrinsic rate of natural increase $(r \mathrm{~m})$ and aphid fertility measurements suggested impeded reproductive capacity among aphids feeding on wild accessions relative to barley cv. 'Morex'. Data from electrical penetration graph (EPG) analysis indicated that aphids on the wild barley host WBDC053 spent significantly less time ingesting phloem than those on WBDC117, WBDC336, or 'Morex' - indicative of a phloem-based resistance in WBDC053. Differential expression of plant biotic stress response genes was correlated with EPG and aphid fitness data. The genes $H v S O D 1, H v L O X 2.2, H v L O X 2.3, H v A O S 1, H v A O S 2$, and HvHSP70 were selected as representatives and their expression in both infested and non-infested accessions assessed via quantitative RT-PCR. This characterization of pest resistance in three wild barley accessions demonstrates the potential of crop wild relatives as sources of novel genetic variation for plant improvement.

\title{
Investigating prevalent pathogens causing grapevine trunk diseases in Central Coast of California
}

S. DING (1), S. Seybold (2), K. M. Gold (3), E. Alvarez (2), H. Burgess (2), (1) California Polytechnic State Univ, San Luis Obispo, CA, USA; (2) California Polytechnic State University, San Luis Obispo, CA, USA; (3) Cornell University, Geneva, NY, USA

Wine grapes are one of the most important crops in California, covering approximately 600,000 acres with a total value of about $\$ 3.5$ billion annually. Grapevine trunk diseases (GTDs) are a severe trunk problem worldwide that threaten the longevity and productivity of vineyards. Each year, at least \$260 million is lost caused by GTDs in California, posing a great threat to the wine industry. GTDs are considered as a disease complex, which is caused by one or more pathogens. The purpose of this study is to identify prevalent pathogens causing GTDs in wine grapes in the Central Coast of California. Grapevine trunk tissues were collected from 10 vineyards in San Luis Obispo County and Santa Barbara County. Pathogens were identified with morphological characteristics and genetic regions of ITS, $\beta$ tubulin, and TEF-1 $\alpha$. Pathogenic fungal species were isolated from $90 \%$ of the samples collected, and a majority of the infections were due to Botryosphaeria dieback. With the fungal isolates obtained, our diagnosis and speciation results showed a frequency of $54 \%$ of Botryosphaeria dieback, $1 \%$ of Eutypa dieback, and $45 \%$ of other trunk associated microbes. Of the Botryosphaeria dieback, $26 \%$ were caused by Neofusicoccum spp. and 25\% were caused by Diplodia spp. With such a high incidence of GTDs, prevention strategies such as removing infected tissue, protecting pruning wound, and double pruning may help the growers protect the vines against GTD infections.

\section{Identifying Pythium species associated with damping off of corn in Delaware} J. T. BICKEL, A. Koehler, University of Delaware, Georgetown, DE, USA

Wet Spring conditions and minimized tillage have resulted in seedling disease issues in corn (Zea mays L.) production in Delaware and the surrounding Mid-Atlantic region in recent years. Pre- and post emergence damping-off of corn is frequently associated with Pythium spp., but there has been little work to molecularly characterize species diversity or abundance in the region. Corn seedlings displaying symptoms of stunting and damping-off were collected from fourteen fields across Kent and Sussex counties in Delaware in 2019. A total of 79 samples were isolated and characterized to species through morphology and sequencing of the ITS and Cytochrome Oxidase II gene regions. Thirteen species were identified including: Pythium aristosporum, $P$. arrhenomanes, $P$. attrantheridium, $P$. balticum, $P$. coloratum, $P$. dissoticum, $P$. graminicola, $P$. inflatum, $P$. lutarium, $P$. periilum, $P$. sylvaticum, $P$. tardicresens, and $P$ torulosum. Pythium graminicola was the most frequently isolated species, followed by Pythium inflatum. With many species of Pythium potentially causing disease, molecular identification is the first step of characterization to improve management strategies and prevent stand loss from damping-off.

Antibacterial activity of sugar maple (Acer saccharum Marsh.) autumn-shed leaf extract: Geraniin M. Delisle-Houde (1), P. Dubé (2), R. J. TWEDDELL (1), (1) Département de phytologie, Université Laval, Québec, QC, CANADA; (2) Centre de recherche industrielle du Québec, Québec, CANADA

Ethanolic extract prepared from Acer saccharum autumn-shed leaves (ASASL) has recently shown potential to control lettuce (Lactuca sativa L.) bacterial leaf spot (Xanthomonas campestris pv. vitians) and varnish spot (Pseudomonas cichorii). In this study, ASASL ethanolic extract was fractionated using HPLC system and antibacterial activity of each fraction was tested with the disk diffusion method. Geraniin was revealed as the main antibacterial compound using an UPLC/Q-Tof-MS system. Geraniin was subsequently purified from ASASL ethanolic extract with a glass chromatographic C18-reversed phase silica gel column and a semi-preparative HPLC system equipped with $5 \mu \mathrm{m}$ XTerra Prep MS C18 column. Minimal lethal concentrations of purified geraniin against $X$. 
campestris pv. vitians and P. cichorii were $3.125 \mathrm{mg} / \mathrm{ml}$ and $6.25 \mathrm{mg} / \mathrm{ml}$, respectively. Geraniin is reported here for the first time as the main antibacterial compound in A. saccharum autumn-shed leaf.

EU reference laboratory for pests of plants on bacteria and a proficiency test for molecular detection of Xylella fastidiosa T. DREO, M. Pirc, Š. Alic, National Institute of Biology, Ljubljana, SLOVENIA

In 2019, the European Union designated EU reference laboratory for pests of plants on bacteria. Its first activity, a proficiency test (PT) on molecular detection of Xylella fastidiosa (Xyf), was organized in 2019 by the National Institute of Biology. The PT aimed to assess proficiency of the EU national reference (NRLs) and selected US laboratories. Each received 14 samples prepared by mixing DNA of Olea europaea, Coffea arabica, Polygala myrtifolia, Spartium junceum, Lavandula angustifolia, Prunus avium and Vitis vinifera with DNA Xyf subsp. pauca (3), multiplex (2) or fastidiosa (1). The concentrations of Xyf DNA corresponded to those reported for real-life samples and were about $10^{4} \mathrm{DNA}$ copies/mL, as determined with digital PCR. Altogether, 30 participants reported 420 individual results of which $97 \%$ were conforming. False negative results (3\%) were reported for subsp. multiplex and fastidiosa in lavender and grapevine, respectively. The subsp. pauca, causing olive quick decline in Italy, was detected with overall conformity of $100 \%$. Comparative analysis of results showed that, when properly optimized and applied, real-time PCR, PCR and LAMP all enabled correct identification of all test items. Improvement were identified for seven participants. Overall, results of the PT showed that cell densities of Xyf as anticipated in plant material are readily detected by the participants regardless of different methods, tests, instruments, reagents, dilutions, etc. This demonstrates that knowledge and competence overrule the need for strict standardization.

\section{Endophytic fungal communities associated with chestnut trees in a traditional breeding program} C. SALGADO-SALAZAR, USDA-ARS, Beltsville, MD, USA

Plants have a diverse endophytic mycobiome that is functionally important for their growth, development, and health. In this study, the diversity of culturable endophytic fungal communities was explored in chestnut trees in a traditional breeding program for resistance to chestnut blight (Cryphonectria parasitica). Fungi were isolated from surface-sterilized leaf fragments from 27 trees. In total, 881 endophytic isolates were recovered and identified by sequencing the ITS1-5.8S-ITS2 region of the nrDNA. Molecular identification of the fungi indicated the most abundant order was Diaporthales, with few representatives from orders Hypocreales and Xylariales. Within the Diaporthales, at least 25 genera were identified, the most frequent of which were Dendrostoma, Gnomoniopsis, Ophiognomonia and Tubakia. The most frequently recovered genus in the Xylariales was Anthostomella. Representatives of the genus Tubakia were isolated from all the trees examined and represented $33 \%$ of the total number of isolates obtained. However, boundaries among Tubakia isolates and those species published in the literature could not be unambiguously determined. The EF1 $\alpha$ marker indicated the majority of Tubakia isolates likely represent undescribed species or species not yet reported in the United States. Given the importance of fungal endophytes in improving plant performance and protection against biotic and abiotic stresses, further studies will help determine if the fungal endophytic communities present in these chestnut trees play a role in the success or failure of the breeding program.

\section{Effect of Radopholus similis and Meloidogyne spp. concomitant infestation at different population densities on plantain (Musa AAB) seedling growth \\ O. A. Guzmán-Piedrahita (1), C. Zamorano-Montañez (1), H. D. LOPEZ-NICORA (2), (1) Universidad de Caldas, Caldas, COLOMBIA; (2) Universidad San Carlos, Asunción, PARAGUAY}

There is scarce information regarding the effect of plant-parasitic nematodes on plantain (Musa AAB). In this study, concomitant effects of Radopholus similis and Meloidogyne spp. at different population density combinations were evaluated on plantain 'Dominico Hartón' seedling growth. The study was conducted in a nursery, under field conditions at the Montelindo Research Farm of the Universidad de Caldas, Colombia. Forty days after planting plantain seeds (corms) in sterilized soil, seedlings were infested with 750, 1,500, 2,250, and 3,000 R. similis alone. Similarly, using the same nematode densities, other seedlings were infested with Meloidogyne spp. alone. In addition, sixteen interaction treatments were applied by simultaneously combining the $R$. similis densities presented above with each of the Meloidogyne population densities. Uninoculated seedlings were included as control. Twelve weeks post infestation, plant total dry weight, plant height, and number of leaves were significantly reduced $(P<0.005)$ by $R$. similis alone, Meloidogyne alone and the interaction effect. Compared to the control, the highest population density of $R$. similis and Meloidogyne alone reduced dry weight of plants by over $40 \%$ and $50 \%$, respectively. Similarly, concomitant infestation significantly reduced total dry weight (with decreases ranging from $20 \%$ to $36 \%$ ) in any of the nematode density combinations. Results from this study will aid in the evaluation of nematode interaction effects and contribute to further research on plant-parasitic nematode management in plantain production.

\section{Deciphering the microbiome of Phytophthora foot rot infected citrus trees in Texas} C. YANG, V. Ancona, Texas A\&M University Kingsville, Citrus Center, Weslaco, TX, USA

Phytophthora spp. are important soil and water-borne phytopathogens that can cause foot rot and root rot of citrus. Foot rot-affected trees exhibit twig dieback, canopy thinning, and produce smaller fruit and reduced yield. Recent surveys in the Texas citrus producing region showed that foot rot is widely spread, affecting most orchards. Therefore, Phytophthora foot rot cause important production losses to the Texas citrus industry. Endophytes have been shown to positively influence plant growth and plant responses to pathogens. This study analyzed the microbiome of roots from Phytophthora foot rot-affected and healthy citrus trees by Illumina sequencing of the 16S rRNA and internal transcribed spacer (ITS) amplicons. Results indicate that the composition and structure of the endophytic communities in citrus roots were altered in response to foot rot. The population of the beneficial bacterial genera Pseudomonas and Streptomyces was decreased in infected trees. The relative abundance of Exophiala pisciphila, a symbiotic yeast associated with drought 
resistance, and Glomeraceae spp., arbuscular mycorrhizae, was significantly reduced in foot rot-affected trees. Therefore, Phytophthora foot rot leads to the reduction of the beneficial associations in citrus roots. This study provides a new insight to the understanding of foot rot of citrus and the endophytic microbial communities in response to Phytophthora spp. infection.

Utilizing comparative genomics to develop species-specific diagnostic markers for basil downy mildew J. R. STANDISH (1), M. Bowman (2,3), K. L. Childs (3), M. Tian (4), L. M. Quesada (5), (1) North Carolina State University, Raleigh, NC, USA; (2) Ball Horticultural Company, West Chicago, IL, USA; (3) Department of Plant Biology, Michigan State University, East Lansing, MI, USA; (4) University of Hawaii at Manoa, Honolulu, HI, USA; (5) Department of Entomology and Plant Pathology, North Carolina State University, Raleigh, NC, USA

Peronospora belbahrii is an obligate oomycete that causes basil downy mildew, one of the most destructive diseases affecting basil production worldwide. Managing this disease can be challenging due to airborne dispersal of sporangia and planting of contaminated seed. Resistant cultivars are available, but the majority of basil plantings are to downy mildew-susceptible cultivars; leaving pathogenfree seed and fungicides as key management tools. The ability to identify $P$. belbahrii in seed-lots before sale or planting, or in the field before symptoms develop would provide greater precision in deploying disease management tactics. To address this need, $P$. belbahriispecific markers were predicted based on an Illumina draft genome assembly. RNAseq data were used to determine presence/absence variation for genes present in five individual $P$. belbahrii isolates. Publicly available next generation sequencing reads from $P$. destructor, P. effusa, P. tabacina, and Pseudoperonospora humuli were used to remove genes that were present across species. Based on these data, 1,492 markers were present in $P$. belbahrii only. Candidate $P$. belbahrii diagnostic markers were identified in 383 genes, of which 114 were considered single-copy with strong transcript support. Once validated against a diverse panel of oomycetes, these 114 candidate markers will be valuable for diagnostics, surveying seed-lots, and detecting airborne sporangia, as well as to help predict incoming basil downy mildew epidemics.

Detection of potentially pathogenic effector genes in Fusarium oxysporum $\mathrm{f}$. sp. vasinfectum race 4 isolates from California M. URNER (1), M. Ulloa (2), R. B. Hutmacher (3), R. Nichols (4), M. L. Ellis (5), (1) California State University, Fresno, Merced, CA, USA; (2) USDA ARS, Lubbock, TX, USA; (3) University of California, Davis, Shafter Research and Extension Center, Five Points, CA, USA; (4) Cotton Incorporated, Cary, NC, USA; (5) California State University, Fresno, Fresno, CA, USA

Fusarium oxysporum f. sp. vasinfectum (FOV) race 4 is an important soilborne pathogen responsible for Fusarium wilt of cotton. So far, FOV race 4 is geographically limited, first officially confirmed in California in 2003, and later confirmed in Texas in 2017 and New Mexico in 2019. The objective of this research was to screen FOV race 4 isolates collected from cotton across the San Joaquin Valley of California for possible fungal effectors that might be associated with disease development in cotton. These effectors included the Six genes that are cysteine rich proteins secreted in the xylem (SIX) of infected plants. A total of 74 FOV race 4 isolates were screened for the following known fungal effector genes Six1, Six2, Six3, Six4, Six6, Pda1, Pep1, and Prt1 using PCR specific primers for those genes. The effector genes Six1, Six2, Six4, and Six6 were not present in the 74 FOV race 4 isolates tested, while Pep 1 was dispersed among isolates. The fungal effector genes Six3, Prt1, and Pdal were present in the majority of the 74 FOV race 4 isolates. Future work should examine the role of these three fungal effectors and their potential role in pathogenicity towards cotton. A better understanding of the fungal effectors involved in pathogenicity will also aid in the development of more durable and resistant cotton germplasm that can be used to manage this important wilt disease.

An outbreak of bud blight disease of spruce (Picea spp.) in Alaska: An epidemiological study of Gemmamyces piceae S. M. GABRIEL PERALTA (1), G. C. Adams Jr. (2), L. Winton (3), K. Cerny (4), S. E. Everhart (2), (1) University of NebraskaLincoln, Lincoln, NE, USA; (2) University of Nebraska, Lincoln, NE, USA; (3) Alaska Forest Health Protection, Anchorage, AK, USA; (4) Department of Biological Risks, Průhonice, CZECH REPUBLIC

Gemmamyces piceae causes bud blight disease on several spruce species (Picea spp.) leading to defoliation and death. Serious losses have been attributed to this pathogen since 2009 due the complete destruction of large regions of Picea abies in the Czech Republic. Since G. piceae has also been found in other regions of Europe, Asia and recently Alaska, it is critical to determine if the causal species present in Alaska is an invasive organism or an emerging pathogen that is native to Alaska. To understand the nature of this pathogen, genome sequencing of one isolate from the Czech Republic was used to identify candidate simple sequence repeats (SSR) loci. From the 18 loci evaluated on eight isolates from Alaska and the Czech Republic, only one locus amplified in an Alaskan isolate. Although these loci were polymorphic among isolates from the Czech Republic, they have failed to amplify in isolates from Alaska. Additionally, a region harboring the ITS1, 5.8 S, ITS2 and partial $28 \mathrm{~S}$ region was PCR amplified, sequenced, and used to build a neighbor-joining tree with 1000 bootstrap replicates. Alaskan and European $G$. piceae isolates were placed in different phylogenetic clades and involved nine nucleotide sequences changes. These results support the hypothesis that these isolates may represent different species.

Nevertheless, to determine if this pathogen is an invasive or an emerging pathogen native to Alaska, additional sequence analysis of the genes b-tub, rpb2 and EF-1a, studies on morphological features and cultural characteristics are required.

Evaluating Helminthosporium solani, causal agent of potato silver scurf blemish disease, for sensitivity to the fungicide azoxystrobin

E. A. MONETTE, S. Macchiavelli-Girón, A. J. Gevens, Y. Chen, University of Wisconsin-Madison, Madison, WI, USA

Silver scurf of potato, caused by the fungus Helminthosporium solani, is a tuber blemish disease causing negative impacts in potato production. Growers have utilized variably effective cultural controls and fungicides for disease management. Recently there has been 
an increase in disease severely affecting quality and storability. Genetic mutations associated with QoI fungicide resistance have been identified in other ascomycetes and we hypothesized that $H$. solani might have developed such resistance. We conducted azoxystrobin sensitivity assays to develop a standard screening protocol and to evaluate sensitivity in a test population of 8 isolates. Emphasis was placed on the impact of salicylhydroxamic acid (SHAM) amendment, to determine its necessity for the suppression of alternative respiration by $H$. solani, which would normally be inhibited by plant secondary metabolites. We evaluated traditional plate assays, two types of liquid culture assays, and a germination assay using a range of azoxystrobin concentrations from $0-100 \mu \mathrm{g} / \mathrm{mL}$. Liquid culture assays and germination assays were conducted to reduce assay trial length relative to the traditional plate assays, but both resulted in similar time investments. Results showed that SHAM is necessary for proper evaluation of azoxystrobin sensitivity. In addition, results showed significant differences in growth between isolates and between treatments, with one isolate showing some level of insensitivity. Our results could greatly influence continued use of azoxystrobin in potato production for the control of silver scurf.

Towards identifying sources of resistance to Fusarium root rot in soybean

G. MOREL GADEA, D. A. Mayfield, S. R. Cianzio, G. P. Munkvold, Iowa State University, Ames, IA, USA

Fusarium graminearum, Fusarium oxysporum, and Fusarium solani are the main species causing Fusarium root rot in soybeans. This disease causes widespread yield loss in North and South America, however, sources of resistance to this disease complex have not been discovered yet due to the diversity of the pathogens involved. In this study, we evaluated soybean germplasm with resistance to Sudden Death Syndrome (SDS), another major soybean disease caused by a member of the genus Fusarium (F. virguliforme). The aim of this work is to identify parental lines that may contain genetic resistance to these three species of Fusaria, as well as SDS. For this, parental lines with SDS resistance were evaluated phenotypically using three measurements (visual root rot severity, disease severity index (DSI), and root weight). The importance of identifying parental lines with resistance to Fusarium root rot relies on the potential use of populations derived from these parents to identify molecular markers associated with resistance to this disease complex.

Developing a rapid and equipment-free diagnostic assay for boxwood blight using recombinase polymerase amplification X. YANG (1), J. A. Crouch (2), D. G. Luster (3), (1) Foreign Disease-Weed Science Research Unit, USDA, ARS - Fort Detrick, MD, Frederick, MD, USA; (2) USDA-ARS, Mycology and Nematology Genetic Diversity and Biology Laboratory, Beltsville, MD, USA; (3) USDA ARS Foreign Disease Weed Science Research Unit, Fort Detrick, MD, USA

Boxwood blight is a globally important disease of Buxus species. Early and accurate detection of the causal pathogens, Calonectria henricotiae (Che) and C. pseudonaviculata $(C p s)$, is fundamental to the prevention and management of boxwood blight. Traditional diagnosis of boxwood blight replies on disease symptoms and pathogen identification by morphology, DNA sequencing, and quantitative PCR. However, infected plants without visible symptoms are not uncommon. Pathogen identification requires trained expertise and can be time-consuming. There is an urgent need for a rapid and accurate diagnostic assay for boxwood blight.

Recombinase polymerase amplification (RPA) is an isothermal amplification and detection technology that has been used to diagnose many human, animal, and plant diseases. RPA amplicons can be visualized using lateral flow dipsticks (LFD). In this study, an RPALFD assay for detecting the boxwood blight pathogens is under development. Five sets of RPA primers and probes were designed to target DNA fragments of the P.38 putative gene, which was found to be unique to the boxwood blight pathogens based on comparative genomic analyses. All five primer and probe sets amplified their respective DNA targets. Preliminary results of this study indicate that this RPA-LFD assay can be done within 30 minutes without the aid of microscope, thermal cycling, or sequencing. Additional evaluations are underway to determine the specificity and sensitivity of this assay, as well as its utility under field conditions.

Greenhouse evaluation of wild Arachis species for resistance to Athelia rolfsii

R. S. BENNETT (1), C. E. Simpson (2), S. Tallury (3), A. Pickering (4), N. Wang (5), (1) USDA-ARS, Stillwater, OK, USA; (2) Texas Agric Experiment Station, Stephenville, TX, USA; (3) USDA-ARS, Griffin, GA, USA; (4) Oklahoma State University, Stillwater, OK, USA; (5) Oklahoma State University, Department of Biosystems and Agricultural Engineering, Stillwater, OK, USA

Athelia rolfsii (Curzi) C.C. Tu \& Kimbr. is the one of the most damaging pathogens of cultivated peanut, causing the soilborne disease known regionally as white mold, stem blight, or southern blight. Because the genetic base for cultivated peanut is narrow, wild Arachis species may possess novel and useful sources of disease resistance. We evaluated 18 accessions representing 15 Arachis species (batizcoi, benensis, cardenasii, correntina, cruziana, diogoi, duranensis, herzogii, hoehnei, kempff-mercadoi, kuhlmannii, microsperma, monticola, simpsonii, williamsii) in the greenhouse for resistance to Athelia rolfsii. Inoculations were conducted using actively growing mycelial plugs on intact plants propagated from rooted cuttings. Plants were maintained in an enclosed chamber with high humidity, and lesion length was measured at 4, 6, 10, and 12 days after inoculation. Preliminary results indicate that Arachis batizcoi (PI 468326), Ar. monticola (PI 497260), and Ar. batizocoi (PI PI468327) produced the longest lesions with a mean lesion length $>40 \mathrm{~mm}$ at 12 days after inoculation. Arachis microsperma (PIs 666096 and 674407), Ar. diogoi (PI 468354), and Ar. simpsonii (PI 688957), along with the resistant controls Georgia-03L and U.S. mini-core accession CC650 (PI 478819), were the most resistant entries and had mean lesion lengths $<16 \mathrm{~mm}$ at 12 days after inoculation. These results should be useful to peanut breeders seeking additional sources of resistance to Athelia rolfsii.

First report of insensitivity to tebuconazole, tetraconazole, and propiconazole in Cercospora beticola isolated from beet from South Carolina

A. P. KEINATH (1), S. Zardus (1), V. B. Dubose (1), S. Toporek (1), D. W. Heck (2), S. J. Pethybridge (2), J. Ballew (3), (1) Clemson University, Coastal Research and Education Center, Charleston, SC, USA; (2) Cornell University, Geneva, NY, USA; (3) Clemson University Cooperative Extension Service, Lexington, SC, USA 
Cercospora leaf spot, caused by C. beticola and other species, reduces leaf yield and quality wherever beet (Beta vulgaris) is grown. Demethylation-inhibitor (DMI) fungicides are used to manage Cercospora leaf spot but insensitive isolates occur in northern U.S. states. Cercospora spp. were recovered from red beet leaves in three conventionally managed fields and one organic field to determine the sensitivity of South Carolina isolates to DMIs. Of 30 isolates identified, 20 were C. beticola and 10 were C. cf. flagellaris. C. beticola was found in all fields, and C. flagellaris was found in one conventional field. Isolates were grown on nonamended minimal media and media amended with four concentrations of tebuconazole, tetraconazole or propiconazole to estimate $\mathrm{EC}_{50}$ values by linear regression. Four of 39, 7 of 20, and 2 of 29 isolates tested against tebuconazole, tetraconazole and propiconazole had $\mathrm{EC}_{50} \mathrm{values}>10 \mathrm{mg} / \mathrm{liter}$ and were considered insensitive. All isolates of $C$. flagellaris were sensitive to all fungicides tested. Isolates of $C$. beticola insensitive to tebuconazole, tetraconazole or propiconazole were found in 2, 3 and 1 conventional field. $\mathrm{EC}_{50}$ values for tetraconazole were highly correlated $(P \leq 0.001)$ with values for propiconazole and for tebuconazole. However, the single isolate tested from the organic field was insensitive to tetraconazole and propiconazole but sensitive to tebuconazole. Because insensitive isolates of $C$. beticola are present in South Carolina, DMI fungicides applied to beet should be rotated with fungicides with different modes of action.

\section{Evaluation of selected fungi and bacteria recovered from grapevine tissues to protect pruning wounds from grapevine trunk diseases R. BLUNDELL, A. Eskalen, T. Gallagher, M. Arreguin, P. Byrne, University of California, Davis, CA, USA}

Grapevine trunk diseases, including Esca, Eutypa dieback and Botryosphaeria dieback, are currently considered one of the most important challenges for viticulture worldwide. Pruning wounds provide the main entry point for fungal pathogens responsible for these diseases following precipitation events. Chemical fungicides are available for protection but they have many limitations, prompting a need for sustainable control. Biocontrol is a promising alternative, which has been implemented in some vineyards to protect pruning wounds against Eutypa lata but there are few studies on the use of biocontrol to manage other grapevine trunk diseases. The objective of this study was to identify and characterize potential biocontrol agents isolated from different grapevine tissues, including xylem sap, pith and canes, and test their ability to protect pruning wounds against grapevine trunk disease pathogens. Recovered species exhibiting in vitro antagonism included Trichoderma, Bacillus, Aureobasidium and Fusarium spp. In vitro tests included dual culture inhibition, volatile and spore germination assays. Trichoderma spp. were the most effective, causing up to 85\% inhibition against Eutypa Lata and $52 \%$ inhibition against Neofusicoccum parvum in dual culture and volatile assays. Promising candidates were implemented in a greenhouse detached cane assay as well as field trials in two different environmental regions in California, to see how well potential biocontrol agents protected pruning wounds compared to potential and registered products.

Comparative genomics analyses of the bacterial blight pathogen of anthurium, Xanthomonas phaseoli pv. dieffenbachiae S. DOBHAL, D. Arizala, S. C. Chuang, K. Pal, T. Amore, A. M. Alvarez, M. Arif, University of Hawaii at Manoa, Honolulu, HI, USA

Xanthomonas phaseoli pv. dieffenbachiae (Xpd), the causal agent of anthurium blight, is classified as an A2 quarantine organism in the EPPO list due to its devastating effects on anthurium. In this study we sequenced strain D182, a strain representative of the Hawaiian anthurium blight outbreak (1981-1986) and a recently isolated anthurium strain PL36 using PacBio SMRT. High quality de novo assemblies of D182 (5.22 MB) and PL36 (5.18 MB) were generated with mean coverage of 616 and 351, respectively. Strains D182 and PL36 harbor one $(73.5 \mathrm{~kb}, \mathrm{GC}-60.8 \%)$ and two (1 $1^{\text {st: }} 39.7 \mathrm{~kb}, \mathrm{GC}-64.1 \%$; $\left.2^{\text {nd. }} 43.9 \mathrm{~kb}, \mathrm{GC}-60.9 \%\right)$ plasmids, respectively. Overall, GC content of both genomes was $65 \%$. Other genomes included in the comparative study were downloaded from NCBI GenBank genome database. ANI and dDDH values, which ranged from $99.68-99.86 \%$ and $96.8-98.9 \%$, respectively, showed close phylogenetic relationships with Xpd strain LMG695. The genomes were broadly analyzed for genomic features, codon and amino acid usage, and pan-core genome analysis; heterogeneity in different clusters was observed. Currently, the genomes are being evaluated for type secretion systems, TAL effectors, cell-wall degradation, EPS/lipopolysaccharide and other important clusters which play important role in virulence and pathogenicity mechanisms. This study will provide insight into the genomic biology, virulence mechanisms and evolutionary relationships of Xpd and other strains associated with anthurium blight outbreaks worldwide.

Fungicide resistance frequencies in Botrytis cinerea from poinsettia and calcium as a control measure N. LUKASKO, M. K. Hausbeck, Michigan State University, East Lansing, MI, USA

United States poinsettia (Euphorbia pulcherrima) production was valued at $\$ 149$ million in 2018. Gray mold, caused by Botrytis cinerea, is a common and destructive disease. The necrotrophic fungal pathogen is ubiquitous in greenhouse environments due to the warm and wet conditions. Managing gray mold includes sanitation, environmental manipulation, and fungicide application. Due to the high fecundity and variable genetic nature of $B$. cinerea, fungicide resistance is common. Our objective was to determine rates of resistance to site-specific fungicides for $B$. cinerea in Michigan greenhouses. Isolates were collected from poinsettia in 2018 (26) and 2019 (70), single-spored, and subjected to a germination-based fungicide sensitivity assay. We observed, high resistance frequencies to thiophanate-methyl (95\%), pyraclostrobin (68\%), and iprodione (71\%); intermediate frequencies to boscalid (51\%) and cyprodinil (41\%); and low frequencies to fenhexamid (23\%), fludioxonil (14\%), and fluopyram (5\%). Resistance to products representing five FRAC codes was the most common phenotype, suggesting other control measures are needed. Some growers use weekly calcium sprays to prevent gray mold and necrosis on the on poinsettia bracts, known as bract edge burn (BEB). Three poinsettia cultivars were evaluated for disease severity following foliar calcium sprays with and without fenhexamid, cyprodinil + fludioxonil, and fluopyram + trifloxystrobin. Results indicate that foliar calcium sprays applied at the rate commonly used does not provide significant protection from gray mold on poinsettia. 
Biotic stresses, including pests and pathogens, are a major challenge for crop production worldwide. Insecticide resistance, pathogen evolution, and consumer hesitation over genetically modified crops necessitate novel approaches to combat biotic stressors in agriculture. The plant-associated microbiome has been implicated in biotic stress protection, though inoculations of single microbial strains have shown mixed results. A promising new method for creating entire microbial communities that enhance stress tolerance involves guided selection, an iterative process of selecting plants with a desired trait and transferring associated rhizosphere microbiomes to a new generation of plants. However, levels of exposure to biotic stress and appropriate methods of microbial transfer needed for successful selection of beneficial microbiomes have yet to be determined. The goal of this study was to optimize guided selection for microbiome-mediated defense against insects. We chose the potato aphid (Macrosiphum euphorbiae) and wild tomato (Solanum pimpinellifolium) system to capture pre-domestication microbiome interactions with a specialist insect. Using 16S rRNA sequencing, we identified differences in rhizosphere bacterial communities of control and infested plants across soil type, time, and stress exposure level. In a follow-up experiment, we successfully selected for reduced aphid performance using the time point and stress exposure level that maximized community variation. This technique will enable development of microbial communities protective against many different stresses.

\section{System establishment for RT-qPCR of Fusarium oxysporum f. sp. medicadins} Q. TENG, Lanzhou University, Lanzhou, chengguan, CHINA

Fusarium oxysporum f. sp. medicadins (Fom) is the pathogenic fungus of worldwide soil-borne disease. Fom can cause root rot which is an important limiting factor to the alfalfa industry. Fom of infestation mainly causes alfalfa root rot and eventual wilt of the whole plant. In this study, primers were designed based on Pf (5'-CGCCAGAGGACCCCTAAAC-3') Pr (5'-ATCGATGCCAGAACCAAGAGA-3'), the conserved sequence of Fom, to establish RT-qPCR system for SYBR Green I. Conditions were optimized for primer specificity, primer concentration, annealing temperature, cycling parameters and stability. The optimized results of RT-qPCR reaction conditions showed that the melt curve in the RT-qPCR experiment was a single peak.it indicating that the primers have the characteristics of good amplification results, strong specificity and no specific amplification. Primer annealing temperature can be amplified at $50-60^{\circ} \mathrm{C}$, and $58^{\circ} \mathrm{C}$ is the best annealing temperature. The ideal number of reaction cycles is 40 cycles. The system has good stability and repeatability. The establishment of this system laid a foundation for the detection of this fungus in alfalfa and provided technical support for the healthy cultivation and comprehensive prevention and control of alfalfa.

\section{Genetic mapping of Spot form net blotch resistance using a bi-parental population}

A. F. ALHASHEL (1), R. Sharma Poudel (1), S. J. Clare (2), T. L. Friesen (3), S. Yang (3), R. S. Brueggeman (2), (1) Department of Plant Pathology, North Dakota State University, Fargo, ND, USA; (2) Washington State University, Pullman, WA, USA; (3) USDAARS Cereal Crops Research Unit, Northern Crop Science Laboratory, Fargo, ND, USA

Spot form net blotch (SFNB), caused by the necrotrophic fungal pathogen Pyrenophora teres f. maculata (Ptm) is a foliar pathogen of barley that causes significant yield losses in major barley growing regions worldwide. Understanding the host-parasite interactions between pathogen virulence/avirulence genes and the corresponding susceptibility/resistance genes in the host is important for the deployment of genetic resistance against SFNB. A recombinant inbred mapping population derived from CIho5791 (S) $\times$ Tifang $(\mathrm{R})$ was developed to characterize genetic resistance/susceptibility to the Ptm isolate 13IM8.3, which was collected from Idaho (ID). This isolate was selected based on its distinct virulence profile from others collected from three states: Idaho, Montana (MT), and North Dakota (ND). Quantitative trait locus (QTL) analysis of an F6 recombinant inbred line (RILs) population identified one resistance or susceptibility locus each on chromosome $4 \mathrm{H}, 6 \mathrm{H}$ and $7 \mathrm{H}$. However, phenotyping of $\mathrm{F}_{2}$ individuals showed a bi-modal distribution with $\sim 50 \%$ of the progenies having higher levels of susceptibility than the susceptible parent CIho5791. PCR-GBS analysis of the F $_{2}$ individuals revealed one heterozygous locus on the $5 \mathrm{H}$ chromosome correlated with high levels of susceptibility, indicating the susceptibility to Ptm isolate 13IM8.3 may be caused by an allelic interaction at this locus. Loci identified in this study will help facilitate the deployment of resistance in barley to SFNB.

\section{Genome resources and comparative genomics of four races of Fusarium oxysporum f. sp. vasinfectum that cause wilt diseases of cotton}

S. Srivastava (1,2), K. A. ZELLER (3), J. Sobieraj (1,3), M. K. Nakhla (4), (1) Department of Entomology and Plant Pathology, NC State University, Raleigh, NC, USA; (2) USDA-APHIS-PPQ S\&T Beltsville Laboratory, Beltsville, MD, USA; (3) USDA-APHIS-PPQ S\&T, Beltsville, MD, USA; (4) USDA-APHIS-PPQ-S\&T, Beltsville, MD, USA

Fusarium oxysporum f. sp. vasinfectum (FOV) is an important fungal wilt pathogen of cotton with multiple pathogenic races derived from four phylogenetic subgroups. To find useful diagnostic markers, we developed Whole Genome Sequencing assemblies for race 1 (FOV1), race 4 (FOV4), race 5 (FOV5), and race 8 (FOV8) using a combination of Nanopore (MinION) and Illumina sequencing technology (Mi-Seq). We used high quality Illumina reads to polish contigs that we initially assembled from MinION long-read data. This resulted in contigs with more than $100 \mathrm{X}$ coverage for each FOV of the races of interest and estimated genome sizes of FOV1=52 $\mathrm{Mb}, \mathrm{FOV} 4=68 \mathrm{Mb}$, FOV5=68 Mb and FOV8=55 Mb. We have estimated each FOV genome to have over 98\% genome completeness when compared to a core eukaryotic gene set. These genomes were deposited in NCBI under project PRJNA605737. We predicted 16,384 genes in FOV1, 20,259 in FOV4, 20,609 in FOV5 and 16,839 FOV8 using AUGUSTUS tools. Then we annotated these genes using pfam domain to characterize functional genes. We compared the predicted genes from each of the races to identify lists of genes unique to each of the pathogenic races that might allow us to unambiguously discriminate each of the races. From this list of genes, we 
then selected a set of approximately 500 genes that appear unique to FOV4. We have designed sets of PCR primers and probes for a subset of targets on this list and have now begun optimizing, testing and validating these targets as diagnostic markers for FOV4.

\section{Succinate dehydrogenase subunit B mutations conferring resistance to pydiflumetofen in Botrytis cinerea M. BOLOGNESI DE MORAIS, A. Zuniga, N. Peres, University of Florida - Gulf Coast Research and Education Center, Wimauma, FL, USA}

Botrytis fruit rot (BFR), caused by Botrytis cinerea, is a major disease in Florida strawberry fields. The intensive use of fungicides to control BFR has resulted in resistance to several fungicide groups, particularly the Succinate Dehydrogenase Inhibitors (SDHIs) or FRAC group 7. Resistance to this group has been associated with different mutations in the SDH subunit B (SDHB) such as H272R/Y, N230I, and P225F. These mutations are known to confer medium-to-high levels of resistance to SDHIs like boscalid, penthiopyrad, fluopyram, and isofetamid. Pydiflumetofen $\left(\right.$ Miravis $^{\circledR}$ ) is a new single-site fungicide within FRAC group 7, but its sensitivity to $B$. cinerea has not been determined. In this study, $\mathrm{EC}_{50}$ values of $10 \mathrm{~B}$. cinerea isolates with previously characterized genotypes were obtained using a spiral gradient dilution method. The wild type isolates showed $\mathrm{EC}_{50}$ values ranging from 0.08 to $0.25 \mu \mathrm{g} / \mathrm{ml}$. For the $\mathrm{H} 272 \mathrm{R} / \mathrm{Y}$ and $\mathrm{N} 230 \mathrm{I}$ mutations, $\mathrm{EC}_{50}$ values ranged from 0.32 to $0.55 \mu \mathrm{g} / \mathrm{ml}$, and from 0.51 to $0.88 \mu \mathrm{g} / \mathrm{ml}$, respectively. The low EC50 values $(<1 \mu \mathrm{g} / \mathrm{ml})$ indicate sensitivity of these isolates to pydiflumetofen. Differently, P225F mutations had higher EC 50 values (3.72 to $11.93 \mu \mathrm{g} / \mathrm{ml})$, similar to previous reports showing this mutation confers high resistance to other SDHI fungicides. The SDHB P225F mutation might be a significant threat to the efficacy of the FRAC group 7 against $B$. cinerea. Further studies with more isolates are needed to determine the frequency of these mutations and resistance to pydiflumetofen among isolates collected from different strawberry fields.

Detection and identification of Neopestalotiopsis species from strawberry using a high-resolution melting (HRM) assay C. SUGUINOSHITA REBELlO (1), N. Y. Wang (2), J. Baggio (2), N. Peres (2), (1) University of Florida, Wimauma, FL, USA; (2) University of Florida - Gulf Coast Research and Education Center, Wimauma, FL, USA

Neopestalotiopsis species have been reported to infect fruit and underground parts of strawberry such as roots and crowns. Severe crop losses were reported by Florida strawberry growers in the past three seasons due to outbreaks of Neopestalotiopsis spp. causing severe leaf and fruit symptoms. Molecular characterization and pathogenicity tests indicated a new emerging population of Neopestalotiopsis that is more aggressive to aboveground parts of the plants. Therefore, to aid in disease management decision making, a high-resolution melting (HRM) assay was developed to detect and differentiate Neopestalotiopsis spp. Primers designed based on beta-tubulin genes of $N$. rosae and the new Neopestalotiopsis sp. produced distinct melting peaks from individual populations and had a detection limit of 10 pg of genomic DNA per $10 \mu \mathrm{L}$ reaction for both populations. The specificity test showed no cross-reaction with other common strawberry pathogens. Moreover, the same primer pair generated a different melting peak in the presence of both species DNA, indicating the capability of detecting the coexistence of both Neopestalotiopsis populations in a single reaction. To validate the diagnostic use of this assay, a crude DNA of symptomatic tissues was extracted for HRM, and isolation from the corresponding samples was performed for verification. Our results revealed a 100\% correlation between HRM assays and fungal isolation. In conclusion, the HRM assay is an effective and reliable diagnostic tool for the rapid detection and differentiation of Neopestalotiopsis spp. on strawberry.

Why does heat make rice more susceptible to bacterial blight disease?

J. SHIPP (1), E. K. Luna (1), S. P. Cohen (2), S. Abrams (3), L. Lai (3), J. E. Leach (1), (1) Colorado State University, Fort Collins, CO, USA; (2) Department of Plant Pathology, The Ohio State University, Columbus, OH, USA; (3) University of Saskatchewan, Saskatoon, CANADA

Heat stress in plants increases susceptibility to major pathogens and threatens crop production worldwide. It is important to understand the mechanisms behind this susceptibility to develop strategies to protect food production. Plant responses to biotic and abiotic stresses rely on hormone signaling to initiate defense mechanisms. Previous studies identified up-regulation of genes in the abscisic acid (ABA) biosynthetic pathway as well as ABA-regulated genes in rice under simultaneous Xanthomonas oryzae (Xo) infection and high temperature stress. The $\mathrm{Xa} 7$ gene in rice increases resistance to Xo, and unlike other known resistance genes, this activity is enhanced at high temperature. Transcriptomics analysis shows that ABA biosynthesis and signaling are down-regulated in $\mathrm{Xa} 7 \mathrm{plants,} \mathrm{suggesting}$ that $\mathrm{Xa} 7$ preserves pathogen defense by minimizing responses to heat stress; interestingly, downstream response to salicylic acid (SA), a hormone involved in rice immunity to Xo, was also down-regulated. We found that endogenous treatment with ABA enhanced Xa7 activity, indicating its complex role during this interaction. We are continuing to investigate the roles of ABA and SA during simultaneous Xo infection and high temperature stress through treatment of rice with a novel ABA antagonist that has been shown to chemically inhibit ABA signaling and overcome ABA-induced inhibition of germination in rice seeds. Understanding rice hormone signaling during simultaneous stresses will improve plant breeding strategies to address environmental conditions currently restricting rice production.

The impact of potassium phosphite on Cephaleuros virescens and management of orange cane blotch disease of blackberry (Rubus fructicosis)

W. Hemphill (1), P. M. Brannen (2), J. E. OLIVER (3), (1) University of Georgia, Tifton, GA, USA; (2) University of Georgia, Athens, GA, USA; (3) Department of Plant Pathology, University of Georgia, Tifton, GA, USA 
Orange cane blotch (OCB) disease of blackberry, also called orange felt, is caused by the parasitic algal species Cephaleuros virescens $(\mathrm{Cv})$. OCB is prevalent within the Coastal Plain region of southeastern U.S. where it results in cane cracking, cane girdling, and yield loss on blackberry. Potassium phosphite applications can reduce OCB disease severity; however, the specifics of how disease severity is reduced and the optimal spray timings for the use of potassium phosphite have not been detailed previously. Accordingly, to better understand the impacts of potassium phosphite, three field trials and two in vitro experiments were conducted. Applications of potassium phosphite to primocanes significantly reduced algal blotch number and diameter (up to $95 \%$ and $42 \%$, respectively), while applications to floricanes in the following spring had no additional effect on disease severity. In addition, comparisons between different spray schedules indicated that potassium phosphite applications made during the algal sporulation period were most effective for management of OCB. Furthermore, the results from in vitro experiments indicated that potassium phosphite is directly toxic to $\mathrm{Cv}$, with algal colony growth being significantly reduced in potassium phosphite amended media. Taken together, these studies enhance our understanding of the specific impacts of potassium phosphite on $C$. virescens and will allow for the more effective use of this chemical for the management of OCB on blackberry.

\section{Genome-enabled characterization of the diversity of plant-pathogenic Streptomyces}

C. CLARKE (1), A. Weisberg (2), J. Chang (3), C. Kramer (1), (1) USDA, Agricultural Research Service, Beltsville, MD, USA; (2) Department of Botany and Plant Pathology, Oregon State University, Corvallis, OR, USA; (3) Oregon State University, Corvallis, OR, USA

Common scab is a major disease of potato caused by several species of Streptomyces. The primary virulence determinant of pathogenic Streptomyces is the phytotoxin thaxtomin A, which is encoded by a gene cluster on a mobile pathogenicity island (PAI). We recently sequenced the genomes of 140 Streptomyces isolates from North America and performed plant virulence tests on many of the isolates. In this collection, we identified multiple undescribed Streptomyces species. One of the novel species groups contains aggressive pathogenic strains, a strain that was only weakly pathogenic on potato despite producing thaxtomin, and a strain that did not produce thaxtomin despite encoding an intact thaxtomin PAI. Therefore, multiple genes beyond the thaxtomin PAI are necessary for common scab symptom development. As a first investigation into identifying additional virulence genes, we are functionally testing Streptomyces cellulases hypothesized to be involved in the transition from saprophyte to pathogen. Additionally, we are characterizing the role of thaxtomin in the development of Streptomyces-induced scab in the root crops carrot, beet, turnip, and radish. While thaxtomin is the primary driver of scab symptom development for these crops, there is remarkable diversity in the virulence of individual Streptomyces strains. Taken together, these results demonstrate that important strain-level diversity of pathogenic Streptomyces remains under characterized and that other Streptomyces-produced virulence weapons beyond thaxtomin remain to be discovered.

\section{Distinct phytohormone pathways activated in response to different caterpillar herbivores may reflect salivary effectors} Z. Zhang (1), A. Schedl (2), R. Sontowski (2), B. Driscoll (3), N. van Dam (2), J. BEDE (4), (1) McGill University; (2) German Centre for Integrative Biodiversity Research (iDiv), GERMANY; (3) McGill University, CANADA; (4) McGill University, Ste-Anne-deBellevue, QC, CANADA

Plant responses to caterpillar herbivory are shaped by effectors present in the insect oral secretions. When a caterpillar wounds the plant, a rapid burst of hydrogen peroxide $\left(\mathrm{H}_{2} \mathrm{O}_{2}\right)$ changes cellular redox balance that leads to downstream phytohormone-mediate defense responses. In this study, induced foliar Arabidopsis thaliana responses between two caterpillar species were compared; the beet armyworm, Spodoptera exigua, which secretes glucose oxidase, that generates $\mathrm{H}_{2} \mathrm{O}_{2}$, in its labial saliva, and the cabbage looper, Trichoplusia $\mathrm{ni}$, whose saliva contains catalase, an enzyme that catalyzes the decomposition of $\mathrm{H}_{2} \mathrm{O}_{2}$. In response to herbivory, both insect species induce an increase in foliar jasmonate phytohormones; however, different dynamic responses in host plant gene expression are observed that likely reflect salivary enzymes. Though feeding by both caterpillar species induced AtPDF1.2 transcription, there was a temporal difference in expression pattern where transcript levels remained high in response to $T$. $n i$ feeding but decreased over $36 \mathrm{hr} S$. exigua attacked plants. Crosstalk between the jasmonate/ethylene and salicylic acid/NPR1 pathways is known to negatively regulate $A t P D F 1.2$ expression. Expression of the salicylic acid/NPR1 marker AtPR1 was only observed in response to $S$. exigua herbivory. Studies are continuing to determine if salivary effectors are responsible for the activation of the different phytohormone pathways to shape the plant defense response.

\section{Diversity of Phyllosticta species associated with citrus in Florida}

E. L. MULANDESA (1), Q. Wang (2), J. A. Rollins (2), M. M. Dewdney (1), (1) University of Florida, Lake Alfred, FL, USA; (2) University of Florida, Gainesville, FL, USA

Phyllosticta, a genus in the Botryosphaeriaceae family includes plant pathogenic and nonpathogenic species. Phyllosticta spp., as endophytes or pathogens, have a wide range of plant hosts. The pathogenic Phyllosticta spp. are responsible for inciting leaf and fruit lesions. In Florida citrus, $P$. citricarpa is the only reported pathogenic species causing citrus black spot disease. P. capitalensis is an extremely common co-occurring endophyte of citrus. To date, other Phyllosticta spp. have not been identified from Florida citrus. The objective of this study was to determine the diversity of Phyllosticta spp. associated with citrus in Florida. Therefore, multilocus phylogenetic analyses using the internal transcribed spacer region (ITS), the 28S large subunit nrDNA (LSU), translation elongation factor 1- $\propto$ gene (tef1), and actin gene (actA) were conducted to characterize 59 Phyllosticta isolates from citrus fruit sampled in different groves in Florida. Preliminary results of the multi-locus phylogenetic analysis indicated that the tefl sequences of two isolates (Gc-6 and Gc-7) are highly polymorphic compared with $P$. capitalensis and $P$. citricarpa sequences. The greatest sequence identity was found with $P$. hymenocallidicola, a relatively obscure species. Characterization of the other loci is underway to conclusively determine the identity of these two isolates. Our preliminary results suggest that Phyllosticta species associated with citrus in Florida are more diverse than previously reported. 
The progress for genome resource of Ex-Types \& well-authenticated Phytophthora species using Ultra-long-read Sequencing for Comparative genomics

S. SRIVASTAVA (1,2), Z. G. Abad (1), L. M. Knight (1,2), M. K. Nakhla (3), (1) USDA-APHIS-PPQ S\&T Beltsville Laboratory, Beltsville, MD, USA; (2) Department of Entomology and Plant Pathology, NC State University, Raleigh, NC, USA; (3) USDA-APHISPPQ-S\&T, Beltsville Laboratory, Beltsville, MD, USA

The complete genome sequences for reference organisms permits more efficient identification of target sequences for diagnostics development. Using a long DNA sequence read technology, like MinION high-throughput sequencing (HTS), results in a more complete genome assembly because of its increased ability to read through regions of low genome complexity and to generate better link contigs. Phytophthora contains numerous important plant pathogens of regulatory concern. We have used MinION HTS to produce draft genomes from specimens of the Ex-types (ET), and well-authenticated specimens (SE) for eleven species. We used Phytophthora capsici as a model organism to predict genes for all Phytophthora species and to train our gene prediction model for AUGUSTUS software. This study resulted in assembly of genomes size of mega-bases $(\mathrm{Mb})$ and predicted genes of: P. boehmeriae SE (60 Mb, 16,162). P. citricola ET (291Mb, 16,662). P. constricta ET (42 Mb, 21,782). P. hibernalis SE (42Mb, 21,762). P. kernoviae ET (40Mb, 12,793). P. macrochlamydospora ET (85 Mb, 23,996). P. melonis ET (50Mb, 34,580). P. nicotianae SE (205Mb 29,271), P. quininea ET (191 Mb, 29,244). P. ramorum ET (42Mb, 16,838). P. syringae SE (76Mb, 23,331). We further analyzed these predicted genes for unique genes analyses using the BLAST algorithm and with a cut-off level for significance of $\mathrm{E} \leq 10-5$ to individual Phytophthora spp. and using this information for metagenomics, gene based comparative genomics, and for diagnostics development.

Genetic resistance to Pea enation mosaic virus in pea genetic resources L. D. PORTER, USDA ARS, Prosser, WA, USA

Pea enation mosaic virus (PEMV) is a major pathogen annually impacting dry pea (Pisum sativum) production areas in Idaho and Washington, USA. Leaves of symptomatic plants are usually distorted, are covered with small translucent spots and demonstrate a mosaic pattern. Pods are also often distorted with a warty appearance and seed quality and production are negatively impacted. The virus is transmitted mechanically and by aphids in a persistent manner. Host resistance is the most critical means to manage this pathogen in an economic and environmentally friendly approach. Viruliferous pea aphids, Acyrthosiphon pisum, were used to phenotype genetic resistance to PEMV of 314 pea accessions from the USDA National Plant Germplasm System, Pisum Core Collection, located in Pullman, WA and 35 commercial cultivars under controlled greenhouse conditions. Three pea lines, 'Hampton', 'Lifter' and PI 306591 were determined to have complete resistance to PEMV of the lines evaluated. Hampton has been released as the first dry pea cultivar with resistance to PEMV, and 'Lifter' is a formerly grown commercial green dry pea cultivar from which 'Hampton' was derived. PI 306591 was collected in Hungry and may be a new source of genetic resistance to PEMV. A mapping population has been developed with 'Hampton' and the PEMV-susceptible cultivar 'Granger' to identify molecular markers that can be used in marker-assisted selection to identify pea germplasm with genetic resistance to PEMV to be used in resistance breeding programs. A similar mapping population will also be developed for PI 306591.

Studies on Alternaria leaf blight of Little millet (Panicum sumatrense) B. PRAVEEN (1), A. Nagaraja (2), P. Kumar (2), K. B. Palanna PhD (2), (1) UAS, GKVK, BANGALORE, KS, INDIA; (2) UAS, GKVK, BANGALORE, INDIA

Little millet (Panicum sumatrense) is one of the hardiest minor cereal crops in the family Poaceae (Gramineae) and is indigenous to Indian sub-continent. The crop is cultivated by tribal and poor farmers on marginal soils for its high nutritional quality. Leaf blight is one of the emerging diseases of little millet threatening its cultivation. The causal agent of typical leaf blight was isolated and identified as Alternaria alternata based on ITS sequencing, original descriptions and koch's postulates. Studies with solid media revealed that highest radial growth was observed on host extract agar media while malt extract agar favored profuse sporulation. Host range studies were conducted under glasshouse conditions with artificial inoculation of A. alternata on different millets and cereals. Little millet, kodo millet, pearl millet, brown top millet, wheat and oats developed leaf blight symptoms, whereas finger millet, proso millet, barnyard millet, foxtail millet, maize, sorghum and rice did not develop any symptoms. One hundred forty two little millet germplasm accessions from different states of India were screened for resistance to leaf blight under natural field conditions for two seasons (Kharif -2018 and 2019). Among these accessions, 38 were resistant, 33 were moderately resistant, 53 were susceptible and 18 were highly susceptible to leaf blight. We evaluated nine botanicals and thirteen biocontrol agents for inhibition of A. alternata. Eucalyptus plant extract and P42 (Bacillus velezensis) recorded the highest inhibition of mycelial growth in vitro.

Are potato tubers gated communities? Cool virulence and tuber transmission of African potato brown rot strains A. TRUCHON (1), S. Ravelomanantsoa (2), P. C. Prior (3), C. Allen (4), (1) University of Wisconsin, Madison, WI, USA; (2) CIRAD, Saint Pierre, FRANCE; (3) INRA CIRAD, Saint Pierre Reunion Is, FRANCE; (4) University of Wisconsin-Madison, Madison, WI, USA

Potato Brown Rot (BR) caused by Ralstonia solanacearum is a devastating disease for subsistence and market growers in tropical highlands. Potatoes are native to the South American Andes, but they are now a staple carbohydrate crop in many countries. Coolvirulent Andean ('Race 3 Biovar 2') BRstrains of $R$. solanacearum have been globally disseminated in infected potato seed tubers. Around 2005, Andean BR strains were introduced into Madagascar. By 2011 they had replaced the endemic African BR strains, causing a major ongoing BR epidemic. This suggested that Andean $R$. solanacearum strains are more virulent or more effectively transmitted 
than native African BR strains. To understand the factors limiting $R$. solanacearum cool virulence and tuber transmission, we used bioinformatic and biological analyses of 11 African BR isolates from Madagascar and the well-studied Andean BR strain UW551. We measured the virulence of UW551 to that of two sequenced African BR strains on potato and tomato at cool temperatures, and their colonization of potato stems and tubers. While Andean BR strain UW551 was more cool virulent than African BR strain RUN2279 on tomato and potato, RUN2279 effectively colonized stems and caused latent infections. However, though African and Andean BR strains colonized stems similarly, the African BR strain very rarely colonized tubers, suggesting that Andean BR strains displaced African strains in Madagascar because they are more efficiently transmitted in seed tubers.

Interactive Virtual Conferencing: Shifting our meeting paradigm

M. BOLTON (1), M. Fulcher (2), M. Millican (1), M. Michalska-Smith (1), J. P. Dundore-Arias (3), J. Handelsman (4), J. Klassen (5), K. Milligan-Myhre (6), A. Shade (7), B. Wolfe (8), L. L. Kinkel (1), (1) University of Minnesota, MN, USA; (2) University of Minnesota, Saint Paul, MN, USA; (3) California State University Monterey Bay, Seaside, CA, USA; (4) Wisconsin Institute for Discovery, University of Wisconsin, Madison, WI, USA; (5) University of Connecticut, Storrs, CT, USA; (6) University of AlaskaAnchorage, Anchorage, AK, USA; (7) Michigan State University, East Lansing, MI, USA; (8) Tufts University, Medford, MA, USA

In-person scientific conferences have been a vital part of scientific visibility and career development, however as technologies advance, so does our need to better utilize technology for virtual, interactive conferencing. This is particularly important in light of COVID-19 and our need to restrict both travel and physical interactions. We argue that the time is now to shift our meeting paradigm. We report the success of a novel hybrid in-person/virtual meeting platform at a two-day NSF-sponsored workshop ("Deciphering the Microbiome," December 2019). By integrating Slack as a messaging app, Zoom as a webinar platform, Twitter for public communications, and Google Drive as a meeting repository, we engaged participation of 80 in-person attendees and 507 virtual attendees. All talks were livestreamed and available for anyone to watch on Zoom. Virtual participants with advance registration were organized into virtual community discussion groups (VC) composed of individuals representing diverse career stages and areas of expertise. Nearly 1,000 messages were generated through our meeting's Slack platform as a result of engaged interactions among all registered virtual participants and in-person attendees. Virtual attendance saved an estimated $300+$ Tons of $\mathrm{CO}_{2}$, and a post-workshop survey found that $>97 \%$ of participants would attend another virtual meeting. The time is up for scientific communities to solely rely on in-person meetings for scientific interaction and visibility. Interactive virtual meetings are the future of eco-friendly and health-conscious scientific engagement.

\section{Evaluation of ergot resistance in hard red spring wheat varieties in North Dakota}

L. R. LUX (1), A. J. Friskop (2), J. Halvorson (2), B. Hansen (1), (1) North Dakota State University, Fargo, ND, USA; (2) North Dakota State University, Department of Plant Pathology, Fargo, ND, USA

North Dakota (ND) is the leading producer of hard red spring wheat (HRSW) in the United States. Every year, growers are concerned with diseases that lead to losses in yield and quality. Ergot, caused by the fungus Claviceps purpurea, causes severe reductions in grain quality due to the production of toxic alkaloids. In 2018, higher levels of ergot were reported in HRSW fields in ND leading to several questions pertaining to varietal resistance. Therefore, the objective of this research was to evaluate HRSW varieties for ergot resistance. Greenhouse experiments were conducted using a complete randomized design with a factorial arrangement and five replications. Eight HRSW varieties (SY-Ingmar, SY-Valda, Bolles, Elgin-ND, Faller, Glenn, SY Rockford, and ND-VitPro) were selected based on acreage trends in ND. Twelve $C$. purpurea single colony isolates were derived from ergot samples collected from different hosts across the growing region. Two seeds were sown into soil filled cone-tainers and allowed to grow until half-spike emergence (Feekes 10.3). At this growth stage, total of 4-6 spikelets were inoculated with conidial suspensions from each of the C. purpurea isolates. Wheat spikes were evaluated for honeydew production, size of ergot sclerotia, and weight of ergot sclerotia. Results from the experiments will be used to update ergot management recommendations for HRSW growers in ND.

\section{Distribution and characterization of Pythium spp. in Pennsylvania soybean fields facing unique microclimatic and soil conditions \\ M. PAUKETT (1), D. K. Weerasooriya (2), A. Y. Bandara (2), A. A. Collins (2,3), P. Esker (2), (1) Penn State University, University Park, PA, USA; (2) The Pennsylvania State University, University Park, PA, USA; (3) Southeast Agricultural Research \& Extension Center, Manheim, PA, USA}

Pennsylvania (PA) soybean acreage increased over $40 \%$ in the past 10 years, during which time the PA Soybean On-Farm Network expanded research trials into all soybean-producing regions of PA to address how management techniques should vary to account for the different microclimates and soil types. Network participants indicated that Pythium disease remains an issue; therefore, the objective of this study was to identify the Pythium spp. distribution across the different microclimatic and soil conditions in PA. Bulk soil samples were collected in 2018 from seed treatment and yield limiting (YL) field sites located in over 15 PA counties at pre-planting and postharvest, with some YL R6 (full seed) and R8 (full maturity) samples. Over 500 tentative Pythium isolates were recovered using P5ARP selective medium and stored in glycerol at $-80^{\circ} \mathrm{C}$. A subset of these isolates underwent DNA extraction and PCR targeting ITS-1 and ITS-4 regions. Purified PCR amplicons will be Sanger sequenced to verify the Pythium species identity. Results will be mapped to the different production regions with annotations on soil and climatic variables. Future work will incorporate 2019 samples and characterize fungicide sensitivity and pathogenicity of identified species on soybean seeds and seedlings to relate back to farm conditions and identify best management practices in these regions. Overall, this project establishes a foundation for future Pythium research in PA related to fungicide resistance and best management practices, with climate change implications. 
Sclerotinia sclerotiorum is a necrotrophic fungal pathogen causing white mold disease on more than 600 plant species, including many economically important crops. The most prominent symptom of white mold is maceration of host tissue, suggesting the efficiency of the pathogen in degrading plant cell wall. Galacturonic acid is the major building block of pectin which is a main component of plant cell wall. Thus, during tissue maceration, galacturonic acid, is likely the nutrient source for $S$. sclerotiorum. The genome of $S$. sclerotiorum encodes the genes responsible for galacturonic acid catabolism. However, the roles of these galacturonic acid catabolic genes in the biology and virulence of $S$. sclerotiorum are unknown. In this research, we identified four S. sclerotiorum genes that encode the enzymes for the D-galacturonic acid catabolism, namely Ssgar1, Ssgar2, Sslgd1 and Sslgal. In an effort to characterize the functions of the galacturonic acid catabolic pathway genes, gene deletion mutants of these genes in S. sclerotiorum were generated using targeted mutagenesis. Mutants displayed a reduction in virulence in sunflower, common bean and pea leaves and lost the ability to utilize galacturonic acid as a carbon source. When cultured on pectin as the alternative carbon source, Sslgd1-and Sslgal-deletion mutants exhibited significantly reduced growth. Understanding the catabolic pathway will give more insight on the mechanism involved in the colonization and virulence of $S$. sclerotiorum on the countless crops globally.

Fusarium graminearum effectors suppress plant immunity and play a quantitative role during infection G. HAO (1), S. Mccormick (1), T. Usgaard (1), H. Tiley (2), (1) USDA/ARS/NCAUR, Peoria, IL, USA; (2) USDA/ARS/NCAUR, Peoria, MO, USA

Fusarium graminearum causes Fusarium head blight (FHB) on wheat and barley. It produces the mycotoxin deoxynivalenol (DON), which functions as a virulence factor and causes grain contamination. F. graminearum secretes hundreds of effectors that can interfere with plant immunity and promote FHB. However, the functions of many of these effectors remain unknown. We investigated the expression of three $F$. graminearum effector-encoding genes and their roles during FHB development. Our results revealed that effectors FGSG_01831, FGSG_03599 and FGSG_12160, were highly induced during wheat head infection. We generated single gene deletion mutants for these three effectors and performed FHB virulence assays with point and dip inoculations. The deletion mutants of all three effectors had reduced FHB spread in wheat heads but none of the reduction was significant by statistical analysis. In contrast, deletion mutants of FGSG_01831 caused significantly less FHB initial infection and produced significantly less DON in wheat and barley heads. The three effectors were transiently expressed in Nicotinana benthamiana leaves. $N$. benthamiana leaves expressing these effectors significantly reduced the production of reactive oxygen species induced by chitin, but not by flg22. Furthermore, FGSG_01831 and FGSG_03599 significantly suppressed Bax-induced cell death when co-expressed with Bax in N. benthamiana leaves. Our study provides new insight into the functions of the $F$. graminearum effectors and suggests that they may play a quantitative role during $F$. graminearum infection.

\section{Oxytetracycline resistance in Erwinia amylovora detected in commercial pear orchards in California}

H. C. FÖRSTER (1), R. J. Belisle (1), G. W. Sundin (2), J. E. Adaskaveg (1), (1) Department of Microbiology and Plant Pathology, University of California, Riverside, CA, USA; (2) Michigan State University, East Lansing, MI, USA

Oxytetracycline (OXY) and streptomycin (STR) have been widely used in the United States for the management of fire blight of pome fruit crops caused by Erwinia amylovora since the 1950s and 1970s, respectively. Resistance to STR was first reported in California in 1971 and has been an ongoing problem. Reduced sensitivity to OXY with minimum inhibitory concentrations (MICs) of up to 3.5 $\mu \mathrm{g} / \mathrm{ml}$ as compared to sensitive strains with $<1 \mu \mathrm{g} / \mathrm{ml}$ was found at several locations in 2007 to 2012 but did not persist in the following years. In orchard surveys in 2018 and 2019, high levels of resistance to OXY with MICs of $>40 \mu \mathrm{g} / \mathrm{ml}$ were detected for the first time. At one location, 6 of 7 strains were resistant in 2018, whereas in 2019, 4 of 12 strains were resistant. At another location, 1 of 8 or 12 strains was resistant in 2018 and 2019, respectively. Thus, these resistant strains persisted, and their fitness was confirmed in coinoculations of pear flowers with sensitive and resistant strains. All strains highly resistant to OXY were also highly resistant to STR. The $t e t B$ gene that was previously described from OXY-resistant non-target phyllosphere bacteria was confirmed to be present on a plasmid pending characterization. At the location with the highest incidence of OXY resistance, nine applications of the antibiotic were done between 2017 and 2018. High dependency on one antibiotic in a two-year period may be responsible for the selection of the resistance detected.

\section{Host status of the plant-parasitic nematodes Rotylenchulus reniformis and Meloidogyne javanica on six Hawaiian native plants species \\ L. Wong, B. S. SIPES, University of Hawaii at Manoa, Honolulu, HI, USA}

Diseases caused by nematodes affect the health of native plants and their failure to establish when reintroduced into native ecosystems. The host status of native plants to the plant-parasitic nematodes Meloidogyne javanica and Rotylenchulus reniformis was determined. In a series of greenhouse experiments, cuttings of Ipomea pes-caprae brasiliensis, Sida fillax, Myoporum sandwicense, and Vitex rotundifolia and seedlings of Scaevola taccada and Prichartidiata sp. were inoculated with $M$. javanica or $R$. reniformis. M. javanica was not tested on I. pes-caprae brasiliensis and Myoporum sandwicense. R. reniformis was not evaluated on $S$. taccada. Tomato seedlings were used as a comparative host for $M$. javanica and cowpea seedlings for $R$. reniformis. Each test had 4 plants and was repeated twice. Three months after inoculation, plants were collected, roots shaken in $\mathrm{NaOCl}$, and nematode reproduction calculated. Nematode reproduction on tomato and cowpea was $>1$ in all experiments. Prichartidiata, $S$. fillax, and $V$. rotundifolia had reproductive factors $<1$ for both nematodes. $M$. sandwicense had a reproductive factor $<1$ for $M$. javanica. $S$. taccada had a reproductive factor $<1$ 
for $R$. reniformis. For $R$. reniformis, I. pes-caprae brasiliensis had a reproductive factor $>1$. Prichartidiata sp., S. fillax, M. sandwicense, $S$. taccada, and $V$. rotundifolia are not good hosts to either nematode species. Further research on other native plants will give better understanding of the potential impact plant-parasitic nematodes have on reforestation.

\section{UAVs in agriculture: Detection of plant diseases and other problems in vegetables and berries C. NISCHWITZ, Utah State University, Logan, UT, USA}

Unmanned aerial vehicles (UAV) with light and/or near-infrared (Near-IR) cameras are increasingly used in agriculture, however little is known about their applicability for plant disease detection. This project sought to determine the abilities and limitations of UAVs to detect diseases and other problems in Utah vegetable and berry crops. A UAV with both light and Near-IR cameras was flown over fields for the last four years. Images were analyzed the same day. Plants showing potential disease symptoms by UAV imagery were collected from the field the next day, and the problem identified using culturing, molecular, antibody-based, or nutrition testing. The UAV use was very successful in detecting disease and nutrient problems early in vegetable and berry crops compared to scouting. One crop where the UAV was not suitable was onion, due to the growth habit of the plants. Suitability of camera type depends on the disease problem. For instance, chlorosis due to viruses or nutrient deficiency was more visible using a Near-IR camera as all green tissue will turn red but chlorotic tissue remains white or yellow. For diseases with distinct symptoms like WMoV in sweet corn, we were able to determine the cause by UAV image alone, saving time and diagnostic costs. Using a UAV for early plant disease detection reduces labor cost and scouting time. Symptomatic plants in the images can be collected and taken for testing to minimize yield loss. In cases with distinct symptoms, a grower will be able to identify the disease from the images, and remove affected plants or provide treatment.

\section{Quick Rot of Solanum tuberosum to test Streptomyces protection against fungal pathogens (Helminthosporium solani, Pythium} spp.)

I. BALLARD (1), S. Taylor (1), B. Geary (2), (1) Brigham Young University, Provo, UT, USA; (2) Brigham Young Univ, Provo, UT, USA

Pythium ultimum has been reported to rot potato crops and reduce their yield by as much as 5-9\%. We are researching non-pathogenic strains of Streptomyces that are suspected to combat oomycetes. Our experiment tested the viability of the strains as biological controls for P. ultimum. To test the effectiveness of the strains, we coated them onto potatoes and allowed the bacteria to colonize. The potatoes were bathed two days later in a liquid culture of P. ultimum. After one week, the potatoes were measured to determine the amount that the potato rotted. The Streptomyces strains were shown to combat the P. ultimum over the course of the week, resulting in less overall rot when compared to the control potatoes that were treated with only pathogen. The strains were catalogued by their performance in inhibiting the rot due to oomycete invasion. Their performance was compared to the amount they were able to inhibit P. ultimum growth on oat agar plates. Our team is now pursuing further research to isolate the secondary metabolites that may be responsible for the P. ultimum inhibition.

Multiple Botrytis and powdery mildew species associated with industrial hemp in Oregon A. GARFINKEL, Oregon CBD, Independence, OR, USA

Oregon is the second largest hemp (Cannabis sativa) producing state with acreage growing each year since its legalization in 2015. In 2019, Botrytis gray mold and powdery mildew were observed as causing significant economic damage to hemp plants in the Willamette Valley. Gray mold infections in the field and powdery mildew infections in the greenhouse reached up to $100 \%$ incidence, whereas powdery mildew in the field and gray mold in the greenhouse remained at low incidence even in the absence of disease management. Samplings were conducted to determine which species were responsible for disease. Pathogens were either isolated into pure culture or identified directly from infected host tissue. DNA extractions and sequencing of housekeeping genes for each genus were performed. Phylogenetic analyses indicated that at least three species of Botrytis are present on hemp: B. cinerea, B. pseudocinerea, and an unnamed Botrytis species. The majority of isolates consisted of $B$. cinerea in both the greenhouse and field environments, suggesting it is likely the most economically significant species. A Golovinomyces species was identified exclusively as the cause of powdery mildew in greenhouse plants, whereas a Podosphaera species was identified only from field-grown plants, possibly suggesting environmental specificity of these two genera. Pathogenicity trials confirmed the ability of all species to cause disease. Studies to determine differences in the biology and epidemiology of these pathogens will be vital to maintaining the health of the hemp industry as it continues to grow.

Diversity of Fusarium associated with fisheye lesions caused by tar spot disease in corn J. M. S. Luis (1), T. Lo (2), D. E. Plewa (3), H. L. Mehl (4), N. M. KLECZEWSKI (3), (1) Virginia Tech, VA, USA; (2) University of Illinois Department of Crop Sciences, Urbana, IL, USA; (3) University of Illinois, Urbana, IL, USA; (4) Virginia Tech Tidewater AREC, Suffolk, VA, USA

Tar spot, caused by Phyllachora maydis, is an emerging disease of corn in the USA. In some instances, stroma of $P$. maydis are surrounded by a necrotic lesion, known as a fisheye. Based on anecdotal accounts from Central America, fisheye lesions are hypothesized to be caused by Monographella (Microdochium) maydis. However, the association of M. maydis with fisheye lesions has not been well documented, especially for tar spot in the US. In 2018, 73 corn foliar samples with tar spot-associated fisheye lesions were obtained from production fields across Mexico, Illinois, and Wisconsin. A total of 305 isolates were obtained from fisheye lesions, with 98\% identified as Fusarium spp. based on morphology. Multi-gene (ITS, TEF1- $\alpha$, RPB1, and RPB2) phylogenies were constructed for a subset of 55 isolates using Tamura-Nei genetic distance model; Microdochium, Cryptostroma, and Fusarium sequences obtained 
from GenBank were included as references. All of the fisheye lesion isolates clustered within Fusarium lineages and were phylogenetically distinct from the Monographella/Microdochium clade. All Fusarium isolates from Mexico belonged to the $F$. incarnatum equiseti species complex, whereas $>85 \%$ of US isolates grouped within the $F$. sambucinum species complex. Results indicate that fisheye lesions are associated with a diversity of mycotoxigenic Fusarium spp. rather than M. maydis, and regional differences incidence of different species suggest environmental conditions favoring tar spot may be favorable for growth and development of local Fusarium spp. on fisheye lesions.

Identifying QTL in Cucumis melo associated with resistance to Pseudoperonospora cubensis

S. TOPOREK (1), A. P. Keinath (2), S. Branham (1), W. P. Wechter (3), (1) Clemson University, Charleston, SC, USA; (2) Clemson University, Coastal Research and Education Center, Charleston, SC, USA; (3) USDA, ARS, Charleston, SC, USA

Pseudoperonsopora cubensis, causal organism of cucurbit downy mildew (CDM), is the largest threat to cucurbit production in the eastern United States. Currently, no Cucumis melo (melon) cultivars have significant levels of resistance. Recombinant inbred lines (169) from a cross between the resistant melon cultivar MR-1 and susceptible cultivar Ananas Yok'neam were phenotyped for CDM resistance in a greenhouse and growth chamber. A genetic linkage map with 5663 binned SNPs created from the RIL population was utilized. Nine QTL, including two major QTL, were identified. $q$ Pcub-10.1 was stable across growth chamber and greenhouse tests, whereas $q P c u b-8.2$ was detected only in growth chamber tests. $q P c u b-10.1$ co-located with an MLO-like protein coding gene, which has been shown to confer resistance to powdery mildew and Phytophthora in other plants. This is the first report of genetic resistance to P. cubensis attributed to chromosome 10 in C. melo.

Development of a qPCR assay for hop powdery mildew field-specific differentiation and late-season dispersal patterns D. Gent (1), C. ROSS (2), M. Wiseman (3), (1) USDA-ARS, Forage Seed and Cereal Research Unit, Corvallis, OR, USA; (2) Oregon State University, Corvallis, OR, USA; (3) Botany and Plant Pathology, Corvallis, OR, USA

Podosphaera macularis is the causal agent of hop (Humulus lupulus) powdery mildew, a polycyclic disease, that causes severe economic damage annually across the Pacific Northwest through expensive fungicide applications, hop cone degradation resulting in yield loss and quality defects. The fungus emerges as focal infections on heavily diseased shoots after dormancy breaks and spreads throughout the season. Determination of the late-season dispersal patterns of powdery mildew between hop yards may improve management practices, potentially accelerating pathogen detection before disease onset and enabling more strategic use of fungicides. The development of a quantitative PCR (qPCR) assay for P. macularis needs to be highly sensitive and specific to be capable of detecting and differentiating $P$. macularis from other fungi. Various candidate gene regions, including $\beta$-tubulin and the $28 \mathrm{~S}$ nuclear ribosomal large subunit rRNA gene (LSU), were investigated for their suitability in a qPCR assay. Assays targeting single nucleotide polymorphisms specific to $P$. macularis are under development for both the $\beta$-tubulin, and LSU regions. Impaction spore traps were deployed near commercial hop yards in western Oregon from 2018 to 2020 to associate airborne inoculum density with disease levels on leaves. Development of the assay and its use as a component of a disease forecasting system for powdery mildew dispersal will be discussed.

The occurrence of mixed virus infections in a Texas Blanc du Bois vineyard D. N. APPEL (1), S. A. McBride (2), R. Davis (1), O. J. Alabi (3), (1) Texas A\&M University, College Station, TX, USA; (2) Texas A\&M AgriLife Extension Service, College Station, TX, USA; (3) Texas A\&M University, Weslaco, TX, USA

Grapevine viruses cause significant losses in terms of winegrape quality and vine health on a global scale. Infections of grapevines with multiple species is one of many poorly understood aspects of the influence of virus infections on winegrapes. A survey was conducted in a vineyard block of Blanc du Bois (Vitis spp.: 'Florida D 6-148' × 'Cardinal') on the Upper Gulf coast of Texas for grapevine viruses. There were 2,025 vines from which 51 were sampled systematically throughout the block and processed for the presence of viruses and viroids. Five viruses (GLRaV-3, GFkV, GVB, GVE, GVL, GVM) and three viroids (GYSVd-1, GYSVd-2, HSVd) were detected. Two of these, GLRaV-3 and HSVd, were detected in relatively high levels of vines ( $65 \%$ and $99 \%$, respectively). The others ranged in far lower incidences of $8 \%$ (GYSVd-1) to $20 \%$ (GVE). Nearest neighbor analyses (ArcGIS version 10.7.1) revealed GLRaV-3 and HSVd were significantly dispersed evenly throughout the block. Mixed infections were common. One vine had 5 viruses and a viroid, 39 had two to five viruses/viroids, eight were infected with only viroids, and two were negative for all viruses/viroids. There appeared to be a few weak, but significant correlations among certain viruses. With further analyses, these results will aid us in better understanding the impact of multiple infections on winegrape quality and vine health.

Detection and identification of apple mosaic virus in plants of Magnolia tripetala showing an obvious mosaic J. HAMMOND (1), M. Reinsel (2), R. L. Jordan (3), (1) USDA ARS Floral and Nursery Plant Research Unit, Beltsville, MD, USA; (2) USDA ARS, Floral and Nursery Plants Research Unit, Beltsville, MD, USA; (3) USDA-ARS, USNA, Floral \& Nursery Plants Research Unit, Beltsville, MD, USA

Obvious mosaic symptoms have been observed in Magnolia tripetala for several years. Whereas a novel carlavirus was detected in trees from North Carolina and Virginia, no carlavirus was detected by RT-PCR or transmission electron microscopy from several trees at Longwood Gardens (Pennsylvania) with similar symptoms. Cucumber mosaic virus is the only other virus reported from Magnolia spp. Total RNA from pooled symptomatic leaves of plants from Longwood Gardens was used to prepare a cDNA library for highthroughput sequencing (HTS). Illumina MiSeq paired-end protocols yielded 3,545,590 raw reads, which were trimmed to remove adapter linkers, and de novo assembled into contigs using Geneious Pro R9. Contigs were analyzed by BLASTX against viral sequences 
from GenBank. No carlavirus-related HTS sequence reads were detected. Ilarvirus-related contigs were further edited and assembled into three larger contigs representing RNA1 (3936 reads; $3434 \mathrm{nt}$ ), RNA2 (5468 reads; $2914 \mathrm{nt}$ ), and RNA3 (7306 reads, $2,002 \mathrm{nt}$ ) of apple mosaic virus (ApMV); the genomic RNAs lacked 19-35 nt at the 5' end, and 4-69 nt at the 3' end by comparison to other ApMV sequences. BLASTn revealed highest nt identities to other ApMV isolates - i.e. 92.3\% for RNA1 (to KY965061; 99\% coverage); $\sim 89.4 \%$ for RNA2 (to KY965062; 99\% coverage); and $\sim 94.3 \%$ for RNA3 (to AM490197; 96\% coverage). RT-PCR, utilizing primer sets designed from alignments of the sequences obtained by HTS with sequences from GenBank, detected ApMV in the HTS source, and from a symptomatic M. tripetala from a different location.

\section{Effect of relative cell number and timing of application of a biological control agent Rhizobium vitis ARK-1 on grapevine crown gall}

M. NITA (1), A. Nahiyan (1), A. Kawaguchi (2), (1) Virginia Polytechnic Institute and State University, Winchester, VA, USA; (2) National Agricultural and Food Research Organization, Fukuyama, JAPAN

Crown gall of grapevine, caused by the bacterium Rhizobium vitis, is an economically significant disease in many grape-growing regions worldwide. The objectives of this study are to determine the highest effective cell ratio between a mixture of four tumorigenic $R$. vitis strains (Ti-mix) and a biocontrol agent $R$. vitis ARK-1 and the best timing of ARK-1 application using in planta assays. Concentrations of ARK-1 and the Ti-mix were determined based on the optical density ( $\left.\mathrm{OD}_{600}=0.1, \sim 5 \times 10^{7} \mathrm{CFU} / \mathrm{ml}\right)$. In the ratio experiment, the treatments of 1:1,1:2,1:3,1:4, and 1:5 ratio of ARK-1 to the Ti-mix were examined. In the timing experiment, ARK-1 was applied 24, 3, 1, and 0 hours before or 5, 30, and 60 min after inoculation of the Ti-mix. Treatments were applied as $10 \mu l$ of inoculum injected in artificial wounds in grapevine woody cane tissue (Vitis vinifera, 'Chardonnay') or in tomato stem (Solanum lycopersicum, 'Beefsteak'). In both tomato and grapes, ARK-1 significantly $(P £ 0.05)$ reduced gall incidence and gall size up to a 1:4 ratio. When ARK-1 was applied 24 hours before the Ti-mix, it significantly reduced both gall incidence and size than other treatments. This study demonstrated that ARK-1 reduced the gall formation against four times higher cell numbers of tumorigenic strains when both were co-inoculated in planta, and ARK-1 was more effective when it was inoculated to the plant 24 hours before inoculation of the Ti-mix.

\section{Baiting techniques used for the direct isolation of the Fusarium wilt pathogen of cotton Fusarium oxysporum f. sp. vasinfectum} race 4 from field soil

J. GARCIA (1), C. Lara (1), T. Schramm (1), M. Ulloa (2), R. B. Hutmacher (3), R. Nichols (4), M. L. Ellis (1), (1) California State University, Fresno, Fresno, CA, USA; (2) USDA ARS, Lubbock, TX, USA; (3) University of California, Davis, Shafter Research and Extension Center, Five Points, CA, USA; (4) Cotton Incorporated, Cary, NC, USA

In California, Fursarium oxysporum f. sp. vasinfectum (FOV) race 4 is an important wilt and seedling pathogen of cotton. Since FOV race 4 was officially reported in the San Joaquin Valley of California in 2003, many confirmed infested fields have been transitioned into permanent tree crop production such as almond and pistachio. The goal of this research was to develop two different protocols for the direct isolation of FOV race 4 from soil collected from current and abandoned cotton production sites. The first method consisted of plating a dilution of field soil on a selective Komada medium. Fungal growth resembling Fusarium was transferred to potato dextrose agar for morphological identification. For the second method, a moderately susceptible Pima cultivar was used as bait and planted directly into the collected field soil. Symptomatic plant tissue was isolated onto Nash-Snyder medium for the isolation of Fusarium. For both methods, Fusarium isolates were tested using previously reported FOV race 4 specific primers. Current results from both methods were able to isolate FOV race 4 from collected field soil in current cotton production sites. Future work will include using these methods to determine if FOV race 4 can be baited from the soil collected in abandoned or tree production sites. If successful, the FOV race 4 from the abandoned and current production soils will be compared in future genetic studies to look for possible evolutionary changes in the fungus in the long absence or presence of a susceptible plant host.

Plant defensin antibacterial mode of action against Pseudomonas species A. E. SATHOFF (1), S. Lewenza (2), D. A. Samac (3), (1) Dakota State University, Madison, SD, USA; (2) University of Calgary, Calgary, CANADA; (3) USDA ARS, St Paul, MN, USA

Many plant defensins exhibit antibacterial activity, but little is known about their antibacterial mode of action (MOA). Antimicrobial peptides with characterized MOAs induce the expression of multiple outer membrane modifications, which are required for resistance to these membrane-targeting peptides. Mini-Tn5-lux mutant strains of Pseudomonas aeruginosa with Tn insertions disrupting outer membrane protective modifications were assessed for sensitivity against plant defensin peptides. Defensins displayed specific and potent antibacterial activity against strains of $P$. aeruginosa. These transcriptional lux reporter strains were also evaluated for lux gene expression in response to sublethal plant defensin exposure. A defensin from Medicago truncatula, MtDef4, induced dose-dependent gene expression of the aminoarabinose modification of LPS and surface polycation spermidine production operons. The ability for MtDef4 to damage bacterial outer membranes was visually verified with fluorescent microscopy. Another defensin from M. truncatula, MtDef5, appears to have a different antibacterial MOA. MtDef5 treatments failed to induce lux gene expression and limited outer membrane damage was detected with fluorescent microscopy. A plant pathogen, P. syringae pv. syringae was modified through transposon mutagenesis to create mutants that are resistant to MtDef4 treatments. The transposon insertion site on defensin resistant bacterial mutants was sequenced, and modifications of ribosomal genes were identified to contribute to enhanced resistance to defensin treatments. 
Diversity and pathogenicity of bacterial soft rot pathogens (Pectobacterium spp. and Dickeya spp.) isolated from potatoes grown in the Columbia Basin

H. RIVEDAL (1), J. Brazil (2), K. Frost (3), (1) Oregon State University, Hermison, OR, USA; (2) Oregon State University, Hermiston, OR, USA; (3) Oregon State University, Hermiston Agricultural Research and Extension Center, Hermiston, OR, USA

The Columbia Basin of Oregon and Washington is an important, high yielding potato production region of the U.S. and is impacted annually by the bacterial soft rot pectobacteriaceae (SRP). The diversity and pathogenicity of these pathogens has not recently been characterized in this region. A multiplex PCR assay was used to determine the identity of SRP causing disease in 51 plant samples submitted to the Oregon State University Plant Clinic in Hermiston, OR. Pectobacterium atrosepticum (Pba) was detected in $20.4 \%$, $P$. carotovorum subsp. carotovorum (Pcc) was detected in $74.1 \%$, and Dickeya spp. were detected in $5.6 \%$, with mixed infection caused by both $P b a$ and $P c c$ detected in $14 \%$ of samples. From the 51 plant samples, 28 SRP isolates were obtained in pure culture. The phylogenetic relationships of these isolates were examined by sequencing 16S, PELY, and DNAX genes. Two species unable to be differentiated from Pcc with the multiplex PCR, P. parmentieri (Pbp) and P. brasiliense, were detected. To evaluate the pathogenicity of the bacteria, the 28 isolates were stab inoculated into tubers $\left(10 \mathrm{ul}\right.$ at $\left.10^{8} \mathrm{CFU} / \mathrm{mL}\right)$ of 7 potato cultivars. Four isolates representing each bacterial species were stab inoculated as 6 mixtures into tubers and stems of 7 and 11 potato cultivars, respectively. Pathogenicity of isolates varied as a function of cultivar and tissue type. Disease incidence and severity increased in mixed inoculations, especially those comprised of $P b p$ or $P c c$ with Pba. In the Columbia Basin, Pectobacterium spp. appear to be the predominate SRP causing disease of potato.

Evaluation of cover crops and diploid potatoes to reduce $S$. subterranea soil inoculum M. ALARYAN, Y. Zeng, A. C. Fulladolsa, A. O. Charkowski, Colorado State University, Fort Collins, CO, USA

Potatoes are susceptible to a soil-born obligate plasmodiophorid, Spongospora subterranea. It causes tuber lesions and produces galls on the roots and stolons, filled with resting spores (sporosori). It also transmits potato mop-top virus, which causes necrotic arcs in potato tubers. There are no effective methods to manage these diseases and the host ranges of these pathogens include other crops in addition to potato. The aim of our work is to determine which cover crops and diploid potato lines decrease soil inoculum levels of $S$. subterranea. Eighteen cover crops and nine diploid potatoes were assessed for their ability to reduce soil inoculum. For each plant line, we grew five plants in pots with potting mix inoculated with 10 sporosori/g and five plants in pots with potting mix alone. qPCR was performed on potting mix after harvest and plant roots were stained and examined under the microscope. Root examination indicated that $S$. subterranea was able to invade the roots of all tested plants. Even though the pathogen could invade plant roots, post-harvest $S$. subterranea DNA levels in the potting mix indicated that the pathogen was unable to complete its life cycle on some of the cover crops. In contrast, all diploid potatoes increased the sporosori level in the potting mix, indicating completion of the pathogen life cycle. This experiment will be used to guide in-field cover crop experiments and to advise farmers on cover crops that might reduce $S$. subterranea levels in soil.

\section{Prevalent pathotypes of Plasmodiophora brassicae in North Dakota}

V. CHAPARA (1), S. E. Strelkov (2), (1) North Dakota State University, Langdon, ND, USA; (2) Univ of Alberta, Edmonton, AB, CANADA

Clubroot (Plasmodiophora brassicae) on canola (Brassica napus) is spreading faster than expected in North Dakota, causing significant economic losses. An integrated approach, including longer crop rotations, sanitation and cultivar resistance, are recommended management options to minimize the impact of this disease. Currently, cultivar resistance is the main management tool sought by growers for clubroot management, without longer rotations out of host crops. Short rotations with clubroot resistant (CR) canola in clubroot-infested regions may lead to resistance breakdown, eventually leading to a decline in canola hectares. The development of new CR cultivars, preferably carrying resistance to novel pathotypes of $P$. brassicae, is therefore important. To obtain cultivars resistant to the prevalent pathotypes, knowledge of pathotype distribution is necessary. Clubbed canola roots were collected from 32 infested fields in North Dakota, and representative samples were tested for pathotype designation on the hosts of the Canadian Clubroot Differential (CCD) Set. The P. brassicae pathotype composition in North Dakota was quite distinct from that reported previously from Alberta, Canada, where the clubroot outbreak is most severe. None of the pathotypes identified could overcome first generation resistance, and in North Dakota, clubroot may still be managed by planting CR canola in a minimum 3-year rotation.

Mapping of an anthracnose resistance locus in Andean common bean landrace Beija Flor L. XAVIER (1,2), G. Valentini (2), M. A. Pastor-Corrales (2), (1) Universidade Estadual de Maringa, Beltsville, MD, USA; (2) ARSUSDA, Beltsville, MD, USA

Anthracnose, a devastating disease of common bean, is caused by Colletotrichum lindemuthianum, a pathogen known to possess extensive virulence diversity comprised of Mesoamerican and Andean races. It has been proposed that resistance genes of Andean origin confer resistance to the highly virulent Mesoamerican strains of $C$. lindemuthianum. The purpose of this study was to map a resitance gene present in Andean common bean landrace Beija Flor that confers broad resistance to highly virulent Mesoamerican races of $C$. lindemuthianum. To that end, we inoculated an F2:3 population from the Beija Flor (resistant) $\times$ Cornell 49242 (susceptible) cross with race 1545 and an F2 population from Beija Flor $\times$ Crioulo 159 (susceptible) cross with races 321 and 1545 of $C$. lindemuthianum. We used the BARCBEAN12K bean chip with 11,292 single nucleotide polymorphism (SNPs) markers to perform bulk segregant analysis (BSA). Multiple SNPs associated with the resistance locus in Beija Flor revealed the physical location of this locus on the upper arm of chromosome Pv04 of common bean. Kompetitive Allele Specific PCR (KASP) markers poisoned the resistance locus between markers ss $120(164,385 \mathrm{bp})$ and ss198 (1’260,459 bp) ( $P v$ v2.1). We are using fine mapping to determine the precise physical 
location of the resistance locus in Beija Flor and to develop molecular markers tagging the resistance locus. This resistance would be most useful in countries such as the United States, Brazil, Mexico, and countries in Central America where Mesoamerican races of $C$. lindemuthianum predominate.

\section{Differential expression of genes encoding sugar transporters in the basil pathogen Peronospora belbahrii} E. JOHNSON (1), M. Tian (2), L. M. Quesada (3), (1) USDA ARS, Peoria, IL, USA; (2) University of Hawaii at Manoa, Honolulu, HI, USA; (3) Department of Entomology and Plant Pathology, North Carolina State University, Raleigh, NC, USA

Carbohydrate transport is an essential function for obligate pathogens such as Peronospora belbahrii, which infects sweet basil. Little is known about how $P$. belbahrii transports sugars from its host or within mycelium. To gain a better understanding of sugar transport in this pathogen we utilized bioinformatic methods to identify genes in the $P$. belbahrii genome from two families of transporters: 12 sugar transporter genes (ST proteins are part of the Major Facilitator Superfamily) and 6 SWEET genes (Sugars Will Eventually be Exported Transporter proteins are part of the MtN3/saliva protein clan). Expression of these genes was investigated with transcriptome samples of the pathogen infecting basil in Hawaii, Illinois, and North Carolina. Only 5 of the genes expressed full length transcripts in each transcriptome sample at each location, but 7 genes expressed full length transcripts in at least 9 of the 11 transcriptome samples. PbST8 was the most highly expressed sugar transporter gene, with transcript levels comparable to that of the enolase gene in the samples from Illinois and Hawaii, and to the tubulin B gene in the samples from North Carolina. These results indicate that many of the putative sugar transporter genes likely contribute to the infection and propagation of this pathogen. Some of the genes may be promising candidates for host induced gene silencing that could help reduce damage caused by basil downy mildew.

\section{Screening of fungicides for management of kernel smut of rice}

X. G. ZHOU (1), L. S. Uppala (2), Z. Guo (3), B. Liu (4), X. Lei (3), L. WANG (5), S. P. Gaire (1), (1) Texas A\&M AgriLife Research Center, Beaumont, TX, USA; (2) University of Massachusetts, East Wareham, MA, USA; (3) Lamar University, TX, USA; (4) Hunan Agricultural University, Changsha, CHINA; (5) Meishan Vocational \& Technical College, BEAUMONT, CHINA

Kernel smut, caused by Tilletia barclayana, has become an economically important disease of rice in the USA in recent years. Fungicides could be an effective tool for control of the disease. However, failures or reduced efficacy of fungicide control has been frequently reported across the rice-producing areas. Objectives of this research were to screen available fungicides and newly synthesized chemical compounds for control of the fungus in vitro and evaluate their efficacy in the field. Sensitivity of the fungus to 13 fungicides and 28 newly synthesized chemical compounds were evaluated at various concentrations on PDA plates. Fungicides containing propiconazole as the only active ingredient (Propimax ${ }^{\circledR}$ and Tilt ${ }^{\mathbb{B}}$ ) were highly effective and showed complete inhibition of all the fungal isolates evaluated at concentrations as low as $50 \mathrm{ppm}$. Other fungicides showed variable responses among the isolates and were not as effective as propiconazole. Among the newly synthesized chemical compounds screened, 11 had antifungal activities similar to or greater than Tilt. In the field trials of 2018 and 2019, azoxystrobin plus difenoconazole (Amistar Top ${ }^{\mathbb{R}}$ ) was most effective in reducing kernel smut incidence, followed by azoxystrobin plus propiconazole (Quilt Xcel $\left.{ }^{\circledR}\right)$, propiconazole $\left(\right.$ Tilt $\left.{ }^{\circledR}\right)$ and a newly synthesized chemical compound. Applications made at the mid-boot stage were effective in reducing the disease while applications made at the heading stage were ineffective.

\section{Prevalence and management of chickpea damping-off caused by metalaxyl-resistant Pythium ultimum in the US Pacific} Northwest

M. Wang (1), S. Van Vleet (2), R. J. McGee (3), T. C. Paulitz (4), L. D. Porter (5), K. L. Schroeder (6), G. J. Vandemark (3), W. CHEN (3), (1) Washington State Univerty, Pullman, WA, USA; (2) Washington State Univ, Colfax, WA, USA; (3) USDA ARS, Pullman, WA, USA; (4) USDA ARS, Wheat Health, Genetics and Quality Research Unit, Pullman, WA, USA; (5) USDA ARS, Prosser, WA, USA; (6) University of Idaho, Moscow, ID, USA

Metalaxyl (mefenoxam) has been the primary fungicide used in managing Pythium damping-off of chickpea and other pulse crops. Metalaxyl resistance among Pythium spp. has been often reported in production systems where metalaxyl is used multiple times in a growing season, but not from dryland agriculture where metalaxyl is used only in seed treatments. However, recent outbreaks of seed rot and damping-off of metalaxyl-treated chickpea seeds were found to be associated with emergence of metalaxyl-resistant Pythium populations in the dryland agriculture region in southeastern Washington and northern Idaho. Isolates of Pythium obtained from rotten seeds and associated soil showed high levels of resistance to metalaxyl. High densities of metalaxyl-resistant Pythium populations were found in areas where severe chickpea damping-off occurred and were observed in several commercial chickpea fields over the past five years in southeastern Washington and northern Idaho. All metalaxyl-resistant isolates were identified to be P. ultimum var. ultimum. The metalaxyl resistance trait measured by $\mathrm{ED}_{50}$ values is stable over 10 generations. Under controlled conditions, metalaxyl treatments failed to protect chickpea seeds from seed rot and damping-off following inoculation with metalaxyl-resistant Pythium isolates. Field and greenhouse tests showed that ethaboxam is effective in managing metalaxyl-resistant Pythium. Ethaboxam in combination with metalaxyl is now commonly applied in seed treatments in commercial chickpea production.

Tissue section and immunofluorescent staining in phloem tissue of red leaf infected susceptible and tolerant rootstocks N. BUZKAN (1), A. Walker (2), (1) Kahramanmaras Sutcu Imam University, Kahramanmaras, TURKEY; (2) University of California, Davis, CA, USA

Grapevine leafroll associated virus $1(\mathrm{GLRaV}-1)$ and Grapevine virus A (GVA) are the most important virus species within the viral disease complex; leafroll and rugose wood, subsequently. The intensity of symptoms depends on the scion/rootstock combination. It is 
very important to determine the effect of rootstocks in altering symptom expression. Greenhouse-forced Cabernet franc shoots as infected solely with GLRaV-1 and GVA as well as healthy rootstocks Freedom (susceptible) and St George (tolerant) were used for green-grafting ( 5 replicates/graft combination). They were kept in greenhouse until symptoms appeared. The isolates were LR131 (GLRaV-1) and LR132 (GVA). Movement and localization of the viruses in phloem tissue were investigated with immunofluorescent stained tissue sections with fluorescent microscopy. Virus specific-polyclonal antiserum and anti-sheep IgG-FITC antibody were used for immunocapturing and fluorescent staining, subsequently. Microscopy observation was done at 500-550 nm wavelength. GVA particles in St George were erraticly localized in phloem tissue up to 10-20 cm from graft point. Beyond that point, no virus particles were seen. It seems virus particles did not move upper level on tolerant rootstock. GLRaV-1 particles were visualized in phloem tissue of St George from grafting point up to $60 \mathrm{~cm}$. In distance between $20-40 \mathrm{~cm}$ above graft point, GLRaV-1 was not seen in phloem tissue. This might be due to irregular distribution and low virus titter in plant tissue. Viral presence in Freedom was remarkably high starting at graft point and more upward.

\section{Effectiveness of fungicide programs for control of potato early blight in Central Wisconsin, 2019} J. HAMMEL, University of Wisconsin-Madison, Madison, WI, USA

Early blight caused by the fungus Alternaria solani is a perennial foliar disease of potato (Solanum tuberosum) that affects potato production in Wisconsin. The disease can cause premature defoliation of susceptible cultivars, ultimately leading to losses in tuber quality and yield. Conventional potato growers in Wisconsin rely on routine fungicide applications for effective early blight control. There are many fungicides currently registered for managing early blight of potato in Wisconsin, with additional fungicides in various stages of development. Thus, we conducted a field trial with a randomized complete block design and 4 replications on 'Russet Burbank', an industry standard cultivar, at the UW-Hancock Agricultural Research Station to evaluate the effectiveness of 27 fungicide programs, including at-planting and weekly foliar treatments, for disease control. Early blight severity was assessed 6 times throughout the growing season using the Horsfall-Barratt (0-11) rating scale. The Area Under the Disease Progress Curve (AUDPC) was determined by trapezoidal integration and then converted into Relative AUDPC (RAUDPC). All data were analyzed using ANOVA ( $\alpha$ $=0.05$ ) and Fisher's LSD at $\alpha=0.05$. Compared to corresponding values of the non-treated control, 22 of the 27 treatments resulted in significantly less disease severity (measured by RAUDPC values) and 23 resulted in significantly greater marketable yields. These results are useful to WI potato growers in designing advanced disease control programs with consideration to fungicide resistance management.

\section{Detection of pathogenicity of Fusarium oxysporum causing root rot of alfalfa (Medicago sativa) in Gansu Province} W. CAI (1), X. Fang (2), (1) Lanzhou University; (2) Univ of Western Australia, AUSTRALIA

Root rot is one of the major constraints in alfalfa production, which influences the yield and quality of this pature, resulting in the decay and shortened-unitlization life of grassland. In this study, the effects of different Fusarium oxysporum strains on the growth of alfalfa seedlings were determined, using strains that were isolated and purified from alfalfa root rot plants in three different regions of Gansu province. Alfalfa seedlings were inoculated with soil inoculation, and then root diseases were scored with root length, shoot height, aboveground biomass and underground biomass being measured after 28 days. Results showed different strains exhibited significant effect $(P<0.05)$ on root disease severity and growth of alfalfa seedlings, but there were differences among the pathogenic intensities. After inoculation with different strains, the disease index of seedling roots ranged from $46 \%$ to $69 \%$, root length and shoot height decreased by $9 \%$ to $28 \%$ and $20 \%$ to $52 \%$ respectively; aboveground biomass and underground biomass decreased by $17 \%$ to $48 \%$ and $15 \%$ to $60 \%$ respectively. Among them, the strain QY3 had the strongest pathogenicity, followed by QY2, and strain LZ3 had the weakest pathogenicity. The strain QY2 caused the greatest decrease in root length and aboveground biomass, while the strain LT2 caused the greatest decrease in shoot height and underground biomass. This study would provide theoretical guidance for efficient control of alfalfa root rot disease.

\section{Characterization of diverse Xanthomonas spp. associated with bacterial leaf spot of watermelon in Florida}

P. ABRAHAMIAN (1), K. V. Xavier (2), J. Pereira-Martin (3), M. L. Paret (4), C. Lapaire Harmon (5), N. S. Dufault (3), J. B. Jones (3), G. E. Vallad (6), (1) USDA ARS Molecular Plant Pathology Laboratory, Beltsville, MD, USA; (2) University of Florida, Wimauma, FL, USA; (3) University of Florida, Gainesville, FL, USA; (4) North Florida Research \& Education Center, University of Florida, Quincy, FL, USA; (5) Univ of Florida PDC, Gainesville, FL, USA; (6) Gulf Coast Research and Education Center, University of Florida, Wimauma, FL, USA

Watermelon is widely grown in Florida with an annual value of 160 million U.S. dollars. During the spring of 2018, an unusual outbreak of bacterial leaf spot occurred with incidence of up to $50 \%$ reported in several Florida counties. Symptoms were observed in multiple cultivars and consisted of severe water-soaked leaf spots with chlorotic margins that quickly progressed into leaf blight. Isolated strains $(\mathrm{n}=17)$ were identified using biochemical and pathogenicity assays to the genus Xanthomonas. Multi-locus sequence analysis (MLSA) and virulence gene analysis resulted in the specific identification of the strains as X. arboricola $(\mathrm{n}=12)$, X. melonis $(\mathrm{n}$ $=4)$ and $X$. cucurbitae $(\mathrm{n}=1)$. $X$. arboricola strains had high intra-genetic diversity in the population and did not cluster with any specific $X$. arboricola pathovar. $X$. melonis strains were identical but differed from the type strain at fusA, gyrB, lacF and lepA loci, whereas $X$. cucurbitae was highly conserved based on MLSA loci. Pathogenicity assays in the greenhouse and field were conducted on different cucurbit hosts to study the host range and capacity to incite disease. In the greenhouse, $X$. cucurbitae consistently produced symptoms on different cucurbit hosts, while the ability of $X$. melonis and $X$. arboricola varied across cucurbits hosts and required extended periods of leaf wetness to cause symptoms. These results supply the first report of multiple Xanthomonas spp. associated with bacterial leaf spot of Florida watermelon, which may complicate diagnostics and management. 
Molecular characterization of Cucurbit yellow stunting disorder virus (CYSDV) infecting summer squash in Georgia, USA A. TABASSUM, S. Bag, University of Georgia, Tifton, GA, USA

Cucurbit yellow stunting disorder virus (CYSDV; genus Crinivirus, family Closteroviridae), was first detected in the Southwestern desert region (California and Arizona) of the US in Fall 2006. The virus was subsequently detected in Florida (Fall 2008) and recently in Georgia (Fall 2016). During August 2018, Yellow Squash (Cucurbita pepo) fruits showing large green sunken spots that coalesced to form green islands with raised warts were collected from Grady county, Georgia. Symptomatic fruit samples $(n=3)$ were tested by PCR for three different viruses. The samples tested positive for CYSDV and Potyviruses. CYSDV is transmitted by whiteflies and Potyviruses are transmitted by Aphids in a semi-persistent and non-persistent manner, respectively. Full-length genome of CYSDV was sequenced from this mixed infection and characterized. The genome of CYSDV sequenced in this study had RNA1 9127nt long and RNA2 7976nt long with opening reading frames distinctive of Closteroviridae. The genome sequenced in this study was 94-96\% identical with the Arizona isolate, indicating presence of same strains in the US. The occurrence of aphids and whitefly transmitted viruses that has broad host range in Cucurbits calls for further research to understand the etiology and ecology of mixed infections in commercial vegetable production.

\author{
Harnessing the wheat curl mite microbiome for pest and disease control \\ P. NACHAPPA, S. White, T. Albrecht, G. Borlee, B. Borlee, Colorado State University, Fort Collins, CO, USA
}

Insect-bacteria associations have been shown to influence vector competence for human pathogens; however, there is limited information on the role of bacterial symbionts in transmission of the plant viruses. The wheat curl mite (WCM) is a globally important pest that transmits several viruses affecting grain production in the Americas, Europe, and Asia. Current management strategies are not effective enough to reduce the risk of disease transmission. Hence, understanding the intimate relationships between bacteria and the arthropod vector may provide new insights into strategies for controlling the vector and reducing disease spread. Here, using nextgeneration sequencing (NGS)-based shotgun metagenomics and direct 16S rRNA sequencing, we characterized the diversity of microbiota in viruliferous and non-viruliferous mites. Our analysis revealed that viruliferous mites has greater proportion and diversity of bacteria compared to non-viruliferous mites. Serratia, Yersinia and Erwinia were the dominant species in viruliferous mites, whereas, Bacillus, Wolbachia and Burkholderia dominated in non-viruliferous mites. Moreover, bacteria cultured from the WCM displayed a variety of unique characteristics such as antipathogen, quorum sensing, quorum quenching, and DNase capabilities. Hence, continued research into this field represents a great deal of potential for novel discovery for mite and virus control. These data provide first insights into bacterial diversity in WCM and helps generate hypotheses for testing functional potential of the mite microbiome.

Recovery of diverse Fusarium head blight pathogens on 'ancient grain' Triticum spp. in New York includes a first report of $F$. armeniacum causing scab

M. FULCHER (1), J. B. Winans (2), D. Benscher (2), M. Sorrells (2), G. C. Bergstrom (2), (1) University of Minnesota, Saint Paul, MN, USA; (2) Cornell University, Ithaca, NY, USA

Fusarium head blight (FHB) of Triticum spp. is caused by diverse mycotoxigenic members of the genus Fusarium. In New York, F. graminearum is considered the primary FHB incitant on common wheat (T. aestivum), and as a result, disease management and mycotoxin screening practices for other Triticum spp. are designed around this pathogen. A limited survey of Fusarium spp. infecting 'ancient grain' crops (emmer, T. dicoccum; spelt, T. spelta; einkorn, T. monococcum) was conducted in NY from 2016-2017. The incidence and severity of FHB were estimated visually in small variety trials at two locations. Every symptomatic spike observed was collected, and Fusarium spp. $(\mathrm{n}=99)$ were isolated for identification by RPB2 sequence homology. A significant difference in mean severity and incidence was observed between crop varieties in one replicated trial. Seven Fusarium spp. were recovered, including the first instance of $F$. armeniacum from an FHB symptomatic small grain. Single-floret and spray inoculations of a susceptible spring wheat ('Norm') in a greenhouse confirmed the pathogenicity of $F$. armeniacum, a reported producer of mycotoxins T-2 and HT-2. The presence of diverse Fusarium spp. with the ability to produce an array of toxic metabolites raises the concern that mycotoxins other than deoxynivalenol, which alone is routinely tested for, may be found in high value 'ancient grains.'

The effects of planting population and nitrogen fertility on severity of tar spot of corn

J. C. CHECK (1), M. Chilvers (2), (1) Michigan State University Department of Plant, Soil and Microbial Sciences, East Lansing, MI, USA; (2) Michigan State University, East Lansing, MI, USA

Tar spot, caused by the fungus Phyllachora maydis, is a foliar pathogen of corn (Zea mays) recently introduced into the US. Tar spot was first identified in the US in 2015 and is affecting corn production in the Midwest and Florida as of the 2019 growing season. When plant pathogens become established in new regions, growers are vulnerable to yield losses due to a lack of information on best management practices backed by scientific studies. Planting population and nitrogen fertility have been proven to influence disease dynamics in several different pathosystems. This study was conducted to assess the relationship between planting population and nitrogen fertility with the severity of tar spot in Michigan to aid growers in understanding best management practices and to reduce losses due to tar spot. Field trials took place in multiple locations across Michigan in naturally infested fields. Disease severity ratings were collected at three different time points throughout the growing season and were measured using a visual scale to assess percentage of leaf area covered by tar spot stroma. 
Aggressiveness and fungicide sensitivity of ectotrophic root-infecting fungi causing take-all root rot on ultradwarf bermudagrass putting greens

C. STEPHENS, T. Gannon, J. P. Kerns, North Carolina State University, Raleigh, NC, USA

Five ectotrophic root-infecting fungi associated with take-all root rot on ultradwarf bermudagrass greens were evaluated for aggressiveness and in vitro fungicide sensitivity. Gaeumannomyces graminis $(\mathrm{Gg})$, Gaeumannomyces sp. (Gx), Gaeumannomyces graminicola (Ggram), Candidacolonium cynodontis $(\mathrm{Cc})$, and Magnaportheopsis cynodontis $(\mathrm{Mc})$ were grown on sterile oats and used to inoculate 'Champion' bermudagrass. Necrosis and turfgrass density were evaluated to determine pathogen aggressiveness. In vitro fungicide sensitivity assays were conducted with 15 fungicides across 3 chemical classes. Isolates were incubated in constant darkness at $25^{\circ} \mathrm{C}$ and colony diameter was measured in two directions on day 3 for $\mathrm{Gg}$ and Ggram, and day 6 for Cc and Mc. Fungicide sensitivity was expressed as relative mycelial growth (RMG). Necrosis and turfgrass density were negatively correlated with an $\mathrm{r}^{2}$ of 0.83. Bermudagrass inoculated with Ggram had the highest necrosis (33.3\%) and lowest plant density. Gg and Cc exhibited moderate aggressiveness and $\mathrm{Gx}$ and $\mathrm{Mc}$ were the least aggressive and were not statically different from the mock-inoculated control. All fungi evaluated against succinate dehydrogenase inhibiting fungicides consistently had the highest RMG values $(>82 \%)$. The lowest RMG values were observed when isolates were grown on azoxystrobin-amended media, regardless of pathogen. Differences in aggressiveness and fungicide sensitivity provide novel insight into biological factors of fungi associated with take-all root rot.

Shedding light on the evolutionary strategies of obligate biotrophic pathogens: The example of Phlox powdery mildew C. FARINAS (1), P. Jourdan (2), J. Slot (3), F. Peduto Hand PhD (3), (1) The Ohio State University, Columbus, OH, USA; (2) Ohio State University, Columbus, OH, USA; (3) Department of Plant Pathology, The Ohio State University, Columbus, OH, USA

Plant pathogens that lack tractable model systems have left scientists pondering over questions regarding their genetic and evolutionary bases. This is the case of many obligate biotrophic pathogens that cannot be grown in axenic conditions. In this study, we investigated the genotypic and phenotypic diversity of a population of powdery mildew (PM) pathogens of Phlox to better understand their capacity to adapt to new environments and new resistant hosts. To do this, we developed laboratory tools to grow and study the pathogens, used comparative genomics and multilocus sequence typing analysis to investigate pathogens' genetics, and studied phenotypic responses using Phlox germplasm screenings. Our results suggest that Golovinomyces magnicellulatus has larger genomes than most pathogenic fungi, high repetitive content, and a fewer number of protein-coding genes. We found a lack of population structure and genetic diversity, despite diverse phenotypic responses to Phlox germplasm screening. Interestingly, we identified 7 secreted proteins predicted to be involved in the infection process, that are differently distributed between the genomes analyzed. We hypothesize that variation in predicted secreted proteins underlies the contrast between genetic and phenotypic diversity. Additionally, our results suggest that pathogen aggressiveness changes over time, through selection of cultivar resistance in constructed landscapes. These observations shed light on the evolutionary mechanisms of PM pathogens adaptation to their host, a key tool to face plant health's future challenges.

Characterization of two new strawberry viruses in the national clonal germplasm repository collection A. WAIT (1), D. E. V. Villamor (1), R. Martin (2), I. E. Tzanetakis (1), (1) University of Arkansas, Fayetteville, AR, USA; (2) USDAARS Horticultural Crops Research Unit, Corvallis, OR, USA

The Rhabdoviridae is a diverse family of negative sense RNA viruses with genomes ranging from 11-15 kb, infecting plants, animals, and invertebrates. Members of the genera Cytorhabdovirus, Dichoravirus, and Nucleorhabdovirus have been reported in plants, with two cytorhabdoviruses infecting strawberry. In effort to better understand strawberry virus disease complexes, we discovered a cytorhabdovirus and a representative of a new genus in the family. Phylogenetic analysis using the replicase gene revealed that the closest relative of the 12956-nt cytorhabdovirus is Lettuce yellow mottle virus, the type member of the genus, whereas the 14432-nt nucleorhabdovirus is only distantly related to nucleorhabdoviruses. Both viruses were detected in the strawberry collection of the national clonal germplasm repository in Oregon and for this reason, we have expanded research to identify potential vectors and develop diagnostic tests. The strawberry (Chaetosiphon fragaefolii) and green peach aphids (Myzus persicae) are being evaluated as potential vectors whereas diagnostic tools will be developed and be available to clean plant programs and certification schemes; facilitating the release of high quality, virus-tested planting materials to the industry.

Multi-year comparison of foliar fungicide efficacy for soybean diseases in Indiana

A. CONRAD (1), J. Ravellette (1), D. E. P. Telenko (2), (1) Purdue University, West Lafayette, IN, USA; (2) Department of Botany and Plant Pathology, Purdue University, West Lafayette, IN, USA

Between 2010 and 2018, soybean in Indiana has seen an average production loss of 7.54\% (24.0 million bu/annually) to disease. Cultural practices such as tillage, variety selection, row spacing, and plant populations can all be used to manage disease. However, even with appropriate management strategies, under ideal environmental conditions disease can still occur. When disease does occur, chemicals can be used to control incidence and severity. The goal of this project was to evaluate the efficacy of five different foliar applied fungicides in field trials located in central Indiana over multiple years. In 2018 and 2019, the treatments were applied at the R3 (beginning pod) growth stage on 12 Jul and 5 Aug respectively. Disease ratings were collected at the R6 (full seed) growth stage on 1 Sept in 2018 and 20 Sept in 2019. In 2019 when disease pressure was low, none of the treatments had a significant effect on frogeye leaf spot severity. However, in 2018 when frogeye leaf spot was more prominent, all of the treatments were able to decrease disease severity over the non-treated control. These results indicate that in a year with severe frogeye leaf spot pressure it is beneficial to apply any of the five tested fungicides in order to reduce disease pressure. No statistically significant differences were seen between treatments for yield in both 2018 and 2019. 
Effect of temperature and leaf wetness duration on an emerging foliar disease of an invasive grass A. ADHIKARI, A. Kendig, S. L. Flory, P. Harmon, E. M. Goss, University of Florida, Gainesville, FL, USA

Microstegium vimineum is an invasive annual C4 grass in forests of the eastern USA. Stricker et al. (2016) reported a Bipolaris species, since identified as Drechslera gigantea, as a major fungal pathogen of Microstegium that causes characteristic eyespot lesions on leaves. However, the disease is not present throughout the invaded range of Microstegium. Our objective was to determine the temperature and moisture requirement for disease on Microstegium to better understand the epidemiology and distribution of this fungal pathogen. Experiments were conducted in controlled environments to measure the effect of temperature regimes and leaf wetness on $D$. gigantea infection and disease severity on Microstegium. Six weeks old seedlings were inoculated with suspensions of $2 \times 10^{4} \mathrm{conidia} / \mathrm{ml}$ and bagged to maintain leaf wetness. Inoculated plants were placed into one of three incubators set at different temperature regimes representing the mean average day and night temperature of June, August and September, respectively, at our southern Indiana field site. On $0,1,3,5$, and 7 days after inoculation, four plants from each incubator were randomly selected, unbagged, wet leaves were dried, and placed back into the incubator. For all three temperature regimes, one day of leaf wetness was enough to observe infection. Disease severity increased with increasing days of leaf wetness. Severity was higher for the June and August temperature regimes than September. These results highlight the importance of temperature and leaf wetness requirement for infection and disease progression.

\section{Comparison between high throughput sequencing (HTS) and standard protocol for virus detection in berry crops (Fragaria, Rubus and Vaccinium spp.) \\ D. E. V. VILLAMOR (1), K. Keller (2), R. Martin (2), I. E. Tzanetakis (1), (1) University of Arkansas, Fayetteville, AR, USA; (2) USDA-ARS Horticultural Crops Research Unit, Corvallis, OR, USA}

A comprehensive study was completed comparing virus detection between high throughput sequencing (HTS) and standard protocols in 30 berry selections (12 Fragaria, 10 Vaccinium and 8 Rubus) with known virus profiles. This study consisted of four sampling points encompassing two growing seasons, with samples collected in the spring and fall of each year. Detection of known viruses by HTS and standard protocols nearly mirrored each other except that (1) HTS missed detection of one or two viruses in either one or two sampling points in seven berry selections, and (2) five novel viruses were identified by HTS that were not detected by the standard protocols. More importantly, where the two protocols showed parallel virus detection, one to four viruses in six berry selections were not consistently detected in all sampling points. The underlying implications of these results on the utility of HTS for virus detection is presented.

\section{Evaluation of diversity, pathogenicity, mycotoxin production potential, and fungicide sensitivity of Fusarium spp. in North Carolina corn production \\ S. M. COCHRAN (1), L. D. Thiessen (2), M. Salazar (2), (1) Raleigh, NC, USA; (2) North Carolina State University, Raleigh, NC, USA}

Corn ear rot is caused by several fungi, including Fusarium spp., that can produce mycotoxins, reduce yield, and decrease profit for growers. North Carolina's warm, humid climate makes the environment favorable for corn ear rot outbreaks during the growing season. Corn ear rot samples were collected throughout North Carolina for two consecutive growing seasons. Six different Fusarium spp. have been identified from these samples using Sanger sequencing of the internal transcribed spacer, RNA polymerase II subunit, and beta tubulin gene regions. The isolated Fusarium spp. were evaluated for trichothecene and fumonisin mycotoxin production potential through PCR and gel electrophoresis. Of the Fusarium spp. collected, 23 isolates had fumonisin production potential and 3 isolates had trichothecene production potential. The pathogenicity of Fusarium spp. isolates were evaluated through a detached leaf assay on REV 25BHR26 and REV 23BHR55 corn varieties. Representative isolates were evaluated for sensitivity to DMI and strobilurin fungicides. The results of these experiments will be used to inform North Carolina corn growers of the diversity of Fusarium spp. across the state and potential management options that target these pathogens.

Evaluating sudden death syndrome in soybean using multispectral imagery L. MCKINZIE (1), A. M. Fakhoury (1), R. Li (2), J. P. Bond (1), (1) Southern Illinois University, Carbondale, IL, USA; (2) Southern Illinois University Carbondale, IL, USA

Multispectral imaging and remote sensing technologies have been shown to provide a non-traditional method of detecting diseases in many crops. In this study, sudden death syndrome (SDS), a soybean disease caused by a soil borne fungal pathogen, was used to test a workflow for disease detection and assessment. This disease results in foliar symptoms, including interveinal chlorosis and necrosis of the trifoliate leaves and ultimately defoliation. This disease is an excellent candidate to test remote sensing and multispectral technologies, given the symptoms it causes and the impact on yield. Multispectral imagery and SDS field ratings were collected, and GIS software was used to calculate mean vegetation indices based on zonal statistics. Based on the regression analysis, we found a strong relationship between normalized difference vegetation index (NDVI) and the SDS field ratings $\left(\mathrm{R}^{2}=0.75, \mathrm{P}<.0001\right)$.

In vitro growth dynamics of Liberibacter asiaticus, the causal agent of citrus huanglongbing Y. DUAN (1), C. Armstrong (2), D. Zheng (1), W. Yao (3), B. Wu (4), W. Luo (5), M. Zhang (6,7), W. Hunter (8), N. Killiny (9), F. Luo (10), (1) ARS, Fort Pierce, FL, USA; (2) ARS, Wyndmoor, PA, USA; (3) University of Florida, Fort Pierce, FL, USA; (4) School of Computer Science, Clemson University, SC, USA; (5) USDA ARS, Ft Pierce, FL, USA; (6) University of Florida, FL, USA; (7) 
Univ of Florida, Fort Pierce, FL, USA; (8) ARS, FL, USA; (9) Department of Plant Pathology, Citrus research and education center, IFAS, UF, Lake Alfred, FL, USA; (10) School of Computing, Clemson University, SC, USA

'Candidatus Liberibacter asiaticus' (Las) is one of the causal agents of huanglongbing, the most devastating disease of citrus worldwide. Due to the intracellular lifestyle and significant genome reduction of Las, it is extremely challenging to culture Las in vitro. In this study, we developed a semi-selective media based on Biolog assays and antibiotic screening and optimized the growth conditions for cultivation of Las in vitro. Under the optimized conditions, we were able to culture Las bacteria to a growth peak in 2-3 weeks in the liquid medium, which was estimated as $10^{6}-10^{7}$ cells per/ml with approximate 500 fold increase based on qPCR. The cultured Las bacteria remained in a dynamic growth for over 12 months and displayed limited growth in subcultures. The presence and growth of the Las bacterial cells were also confirmed by fluorescence in situ hybridization with Las-specific probes and gene expression profiling, which targeted ca. 500 Las metabolism-related genes. It is important to note that Las bacterial growth in the medium relied on the presence of a helper bacterium. Cultured Las was inoculated back to citrus seedlings via psyllid feeding and inoculation. Although only a low percentage (less than 5\%) of the fed psyllids and inoculated plants became positive, this is first demonstration of significant growth of Las bacteria in vitro and successful inoculation of cultured Las back to psyllids and citrus plants. Factors that affect Las growth in vitro and its ability to propagate in psyllid and citrus hosts are under further investigations.

\section{Quantifying the relationship between soybean sudden death syndrome, pathogen density and yield at different spatial scales} M. Raza, F. W. Nutter Jr., L. F. S. LEANDRO, Iowa State University, Ames, IA, USA

Sudden death syndrome (SDS), caused by Fusarium virguliforme $(F v)$, is one of the major yield-limiting diseases of soybean in grower fields. However, the relationship between disease levels, pathogen density, and yield is poorly understood, making it difficult to accurately predict disease risk and crop loss. The objective of this study was to quantify the relationship between SDS disease intensity, pathogen density and soybean yield at different spatial scales. SDS foliar intensity (severity and incidence), pathogen population (in roots and soil), yield, and yield components were assessed on individual soybean plants (2018) and quadrats (2016-2018), in commercial fields and in experimental plots located in Iowa, USA. In individual soybean plants, SDS foliar severity and root rot were positively associated with each other $\left(0.1<\mathrm{R}^{2}<0.53\right)$ and negatively associated with soybean yield components $\left(0.10<\mathrm{R}^{2}<0.50\right)$. In quadrats, SDS foliar symptoms had a negative relationship with soybean yield $\left(0.05<\mathrm{R}^{2}<0.46\right)$. At plant level, $F v$ population density in roots had an inconsistent relationship with foliar severity, root rot and yield components, and $F v$ population in soil had no significant relationship with any disease or yield measures. However, $F v$ populations in soil were positively related to foliar SDS intensity at quadrat level $\left(0.20<\mathrm{R}^{2}<0.42\right)$. This study expands current knowledge on the relationship between SDS intensity, pathogen density and soybean yield, which is an essential precursor for developing SDS risk and yield loss predictive models.

A forward genetic screen to dissect the regulation of pathogenicity and cercosporin production in Cercospora beticola S. SHARMA KHATIWADA (1), S. J. Pethybridge (2), (1) Cornell AgriTech at the New York State Agricultural Experiment Station, Geneva, NY, USA; (2) Cornell University, Geneva, NY, USA

Cercospora beticola Sacc. is a destructive foliar pathogen of Beta vulgaris subsp. vulgaris L. in the US. In table beet production in New York, C. beticola causes Cercospora leaf spot (CLS) resulting in defoliation and the inability to harvest using top-pullers. CLS has been traditionally controlled with fungicides. However, development of resistance to single site mode of action fungicides within the $C$. beticola population (e.g. strobilurins; FRAC 11), has threatened the durability of the approach and alternate management strategies are critical. Little is known about the biology and pathogenicity of $C$. beticola on table beet. To dissect the infection biology, and regulation of pathogenicity and cercosporin production, a forward genetic screen was undertaken. A population of 1,169 random insertional mutants were generated using Agrobacterium tumefaciens-mediated transformation of C. beticola Tb14-085 (wild-type isolate) and screened for pathogenicity on table beet cv. Ruby Queen and cercosporin in-vitro. We identified seven avirulent mutants, and 14 mutants with reduced virulence compared to Tb14-085. Four of the mutants also showed increased cercosporin production invitrocompared to the wild type isolate. Some mutants displayed atypical morphological phenotypes on V8 medium. A target-enrichment sequencing method is being developed to identify the site/s associated with mutation. Taken together our findings provide a framework for understanding the genetics of virulence and cercosporin production in C. beticola and contribute towards developing management strategies.

Exploring the natural genetic diversity of cacao's wild relatives for crop improvement A. M. BOSSA-CASTRO (1), M. L. Cepeda-Hernandez (1), W. Haerty (2), F. Di Palma (2), J. E. Richardson (3,4), M. Vives (1), (1) Universidad de los Andes, Bogota, COLOMBIA; (2) Earlham Institute, Norwich, UNITED KINGDOM; (3) Universidad del Rosario, Bogota, COLOMBIA; (4) Royal Botanic Garden Edinburgh, Edinburgh, UNITED KINGDOM

Cacao, Theobroma cacao L., is the main ingredient of chocolate. Only a small portion of the genetic diversity of cacao and its wild related species is currently used in the global production of this crop. In Colombia, cacao is a promising option for illicit crop substitution. However, producers face serious challenges from pathogens (Phytophthora palmivora, Moniliophthora roreri and M. perniciosa) and limits placed on the permitted level of cadmium in cacao beans. Our goal is to identify genetic regions associated with pathogen resistance and cadmium accumulation/translocation in cacao's wild relatives, and to understand the evolution of these genetic regions through comparative genomics and phylogenetic reconstruction. Theobroma belongs to the tribe Theobromeae, along with the genera Herrania, Guazuma and Glossostemon. We selected eight collections worldwide and collected 280 samples from of all 45 species of Theobromeae from 22 countries. Genomic DNA was isolated from each sample. Quality control and PCR amplification of eight genomic regions commonly used to reconstruct phylogenies were performed. Whole genome sequencing is in progress to generate draft genomes to be used for phylogenetic reconstruction of the Theobromeae, and to identify candidate genes associated with pathogen 
resistance and low cadmium accumulation/translocation. This knowledge will contribute to the development of pathogen resistant and low cadmium accumulating cacao varieties, thus improving the marketability and profit margins of Colombian cacao.

\section{Efficacy evaluation of demethylation inhibitors and mixtures against Colletotrichum spp. causing strawberry anthracnose A. SCHOENEBERG, M. Hu, University of Maryland, College Park, MD, USA}

Strawberry anthracnose, caused by Colletotrichum spp., is a major disease in strawberry production. Quinone outside inhibitors (QoIs) are the primary fungicides used for anthracnose control, but resistance is widespread. The aims of this study were to determine the sensitivity of three Colletotrichum species to six demethylation inhibitors (DMIs) labeled for use in strawberry in the U.S., and to test mixtures of DMIs for synergistic effects. $\mathrm{EC}_{50}$ values of $C$. fioriniae, $C$. siamense, and $C$. nymphaeae averaged $0.2,0.4,0.3 \mathrm{ppm}$, and $0.1,0.2,0.3 \mathrm{ppm}$ for propiconazole and difenoconazole, respectively. According to average $\mathrm{EC}_{50}$ values, the three species were found to be ten times less sensitive to myclobutanil, flutriafol, triflumizole, and tetraconazole compared to propiconazole and difenoconazole. All 15 combinations of the six fungicides were tested in vitro with two mixing ratios for $C$. nymphaeae. None of the combinations had a lower $\mathrm{EC}_{50}$ value than difenoconazole or propiconazole alone. Results from detached fruit assays with $C$. nymphaeae were largely consistent with the $\mathrm{EC}_{50}$ values. For example, propiconazole inhibited lesion development by $100 \%\left(\mathrm{EC}_{50}=0.3 \mathrm{ppm}\right)$, whereas the inhibition rate for triflumizole was $41.8 \%\left(\mathrm{EC}_{50}=4.6 \mathrm{ppm}\right)$, and a $1: 10$ mixture of both fungicides showed $85.1 \%$ inhibition $\left(\mathrm{EC}_{50}=1.7\right.$ ppm). This suggests that DMIs currently labeled for strawberry do not show synergistic effects in mixtures, but propiconazole and difenoconazole proved to be promising active ingredients for strawberry anthracnose management.

\section{Comparative Genomics analyses revealed distinct pathogenicity determinants and distinct features between Dickeya zeae strains from taro and pineapple}

G. BOLUK, D. Arizala, S. Dobhal, A. M. Alvarez, M. Arif, University of Hawaii at Manoa, Honolulu, HI, USA

Dickeya zeae, a bacterial plant pathogen in the family Pectobacteriaceae, is responsible for a wide spectrum of diseases on potato, maize, rice, banana, pineapple, taro and ornamentals and significantly reduces crop production; D. zeae causes soft rot on taro (Colocasia esculenta) and heart rot of pineapple (Ananas comosus). In this study, we used pacbio to sequence complete genomes two different strains of D. zeae: PL65 (size - $4.74997 \mathrm{MB}$; GC - 53.3\%) and A5410 (size - 4.7792 MB; GC - 53.6\%) isolated from two economically important Hawaiian crops, taro and pineapple, respectively. Other complete genomes of $D$. zeae representing various hosts - strain EC1 causing root rot of rice, Ech586 causing soft rot of philodendron and MS2 causing soft rot of banana-were retrieved from NCBI GenBank genome database. The genomic analyses indicated truncated type III and IV secretion systems (T3SS and T4SS) in the taro strain, which only harbors 4 and 2 genes of T3SS and T4SS, respectively, and also showed high heterogeneity in the type VI secretion system. Compared to the EC1 strain both PL65 and A5410 genomes harbor about half the number of the genes within the zeamines biosynthesis gene cluster, which plays a key role in bacterial virulence. The ANI and dDDH percentages between the two genomes were 94.47 and 57.00, respectively. Our study highlights genetic constituents of pathogenicity determinants and genomic heterogeneity that will assist in understanding the pathogenicity and aggressiveness of this plant pathogen.

Field deployable recombinase polymerase amplification assay for rapid and accurate detection of Ralstonia solanacearum species complex

S. PAUDEL (1), S. Dobhal (1), M. J. Stulberg (2), J. Rascoe (2), M. K. Nakhla (3), H. Seo (1), R. Schlub (4), A. M. Alvarez (1), M. Arif (1), (1) University of Hawaii at Manoa, Honolulu, HI, USA; (2) USDA-APHIS-PPQ-S\&T-CPHST, Beltsville, MD, USA; (3) USDA-APHIS-PPQ-S\&T, Beltsville Laboratory, Beltsville, MD, USA; (4) University of Guam, Mangilao, AP, USA

The Ralstonia solanacearum species complex (RSSC)—recently separated into three genospecies (R. solanacearum, $R$. pseudosolanacearum and $R$. sygygii) - is associated with bacterial wilt of numerous plant species and has high economic consequences worldwide. Prevention of pathogen dissemination in symptomless planting stocks necessitates rapid and sensitive point-of-need detection for all three species. Recombinase polymerase amplification (RPA), a relatively new isothermal technique, is becoming popular among diagnosticians due to its speed, sensitivity and ability to overcome reaction inhibitors. A rapid point-of-need Exo RPA assay was developed to detect multiple RSSC strains in field settings. A unique conserved genomic region was identified through a comparative genomics approach using OrthoMCL and Geneious to design robust primers and probe. The specificity of the assay was validated with representative strains from each of three genospecies and non-target genera. No false positives or false negatives were detected. The detection limit was assessed with 10-fold serially diluted genomic DNA and determined to be $10 \mathrm{pg}$. Sensitivity in spiked assays - $1 \mu \mathrm{l}$ sap from $100 \mathrm{mg}$ host tissue macerated in $500 \mu \mathrm{l}$ of TE buffer — was also $10 \mathrm{pg}$. The potato host tissue showed no adverse effects on the detection limit. The developed assay is useful in field settings with high accuracy and minimum instrument sophistication. The assay has wide-range applications in biosecurity, quarantine, routine diagnostics and the formulation of effective disease management strategies.

Integrated management of emerging seedborne Pseudomonas syringae pathogens of Cucurbitaceae and Chenopodiaceae M. NAMPIJJA (1), L. N. Boyd (2), S. Crane (1), J. P. Dundore-Arias (3), E. Gaulke (4), R. Herschlag (5), A. I. Huerta (6), E. Kulesza (5), Y. Kan (5), E. A. Newberry (7), N. Potnis (7), (1) Department of Plant Pathology Washington State University, Mount Vernon, WA, USA; (2) Penn State University, State College, PA, USA; (3) Department of Biology and Chemistry, California State University, Seaside, CA, USA; (4) Department of Plant Pathology University of Wisconsin, Madison, WI, USA; (5) Department of Plant Pathology and Environmental Microbiology Penn State, University Park, PA, USA; (6) Department of Entomology and Plant Pathology North Carolina State University, Raleigh, NC, USA; (7) Department of Entomology and Plant Pathology Auburn University, Auburn, AL, USA 
Bacterial leaf spot (BLS) is caused by multiple pathovars of Pseudomonas syringae and is responsible for significant economic losses in Chenopodiaceae and Cucurbitaceae crops. The disease has increased in incidence and severity in many regions of production, but identification of the pathogens and management of the disease have lagged behind. In 2017 alone, the incidence of BLS in New York table beet crops was 75\%, and in 2013-14, BLS affected approximately 3,000 ha of cucurbit production in Florida. Given the seedborne nature of these pathogens, their genetic similarity to non-pathogenic strains, their wide host range, and potential similarities in pathogen detection and disease management strategies across host species, a federally-funded research project was initiated in 2019. Over the next 4 years, 18 investigators from 9 institutions in 2 countries will work together to develop an economically-sound, integrated approach to manage BLS across seed and food production systems, to ensure high-quality seed and food crops. The project will elucidate the epidemiology of the pathogens in these crops and result in development of resistant plant lines as well as improved IPM strategies that incorporate research findings and new technologies. Biological and genomic data generated will enable development of accurate and sensitive pathogen detection and quantification methods, development of novel seed treatments to ensure clean seed, cost analysis for implementing practices developed, and translation of findings for growers, seed companies, scientists, students, and the public.

Field efficacy of fungicides for management of grape powdery mildew in the presence of fungicide-resistant populations of Erysiphe necator in Georgia

B. WARRES (1), T. Neill (2), W. Mahaffee (2), M. T. Brewer (1), P. M. Brannen (1), (1) University of Georgia, Athens, GA, USA; (2) USDA ARS, Corvallis, OR, USA

Grape powdery mildew (PM), caused by Erysiphe necator, is one of the most economically important diseases of Vitis vinifera wine grapes worldwide. Management is based on numerous fungicide applications each season, but $E$. necator has developed resistance to multiple fungicidal classes. We were interested in determining the efficacy of QoI and DMI fungicides and the extent of resistance to these fungicides by PM in commercial vineyards in Georgia. Seventy-five PM samples were collected from a study site in Blairsville, GA and genotyped for alleles associated with strobilurin (QoI) and myclobutanil (DMI) resistance. Both the G143A mutation in the mitochondrial cytochrome $b$ gene, associated with complete resistance to QoIs, and the Y136F mutation in the $14 \alpha$-demethylase (CYP51) gene, associated with tolerance to DMIs, were observed in this population. Eleven fungicides representing nine classes, including the QoIs and DMIs, were tested for efficacy against PM. Of these, azoxystrobin (QoI) and myclobutanil (DMI) had statistically similar disease compared to the untreated control. A survey of ten commercial vineyards from 2018 and 2019 also found substantive resistance to QoIs when they had been used extensively; of the ten vineyard locations sampled, eight showed QoI resistance. The information derived from this research provides researchers and wine grape producers with an understanding of the extent of fungicide resistance development in PM populations, while identifying the degree of control failure under Georgia environmental conditions when QoI or DMI resistance is present.

Fungal communities associated with wheat stem and grain samples collected from Alberta M. Hafiz (1), N. Schatz (2), M. Telfer (2), R. Gourlie (1), K. Turkington (3), R. ABOUKHADDOUR (4), (1) AAFC, CANADA; (2) University of Lethbridge, CANADA; (3) AAFC, Lacombe, CANADA; (4) AAFC, Lethbridge, AB, CANADA

Plant pathogens coexist in nature as part of complex communities. The accurate diagnosis of the causal pathogen is critical to manage these pathogens and reduce yield losses. In this study, stem and grain samples from wheat were collected in 2018 from three field plots in Alberta (Lethbridge, Lacombe and Beaverlodge) to investigate the impact of fungicide application on the fungal communities associated with wheat. Detection of fungal species was performed using both culture-based and molecular-based methods. The results showed that, in grain samples, $F$. avenaceum and $F$. poae were the most predominant species in the tested sites of Beaverlodge and Lacombe, respectively. Out of 775 fungal isolates, three genera represent 93\% of collected isolates: Alternaria (38.7\%), Fusarium (32.6\%) and Parastagonospora (21.7\%). A total of 253 Fusarium isolates belonging to nine Fusarium species were recovered. PCR assay based on the Tri5 gene was used to screen for trichothecene-producing species, and the results showed that, 114 Fusarium isolates (45\%) were found to amplify Tri5 gene (trichothecene producers). In conclusion, diversity and frequency of Fusarium species are much higher in stem than in grain samples, and fungicide application significantly reduced the number of Fusarium isolates recovered from grain samples. Our results showed that, in addition to F. graminearum, more attention should be given to the other Fusarium species that were frequently isolated from Alberta during the current study, and are also associated with Fusarium head blight on wheat.

Genetic structure of contemporary populations of the boxwood blight pathogen in the U.S.

V. CASTROAGUdin (1), J. E. Weiland (2), F. Baysal-Gurel (3), M. Cubeta (4), M. L. Daughtrey (5), N. A. Ward-Gauthier (6), J. A. LaMondia (7), D. G. Luster (8), F. Peduto Hand PhD (9), N. Shishkoff (10), J. L. Williams-Woodward (11), X. Yang (12,13), N. LeBlanc (4,13,14), J. A. Crouch (14), (1) NMDBG Lab, USDA-ARS, Beltsville, MD, Belstville, MD, USA; (2) USDA-ARS, Horticultural Crops Research Laboratory, Corvallis, OR, USA; (3) Tennessee State University, McMinnville, TN, USA; (4) North Carolina State University, Raleigh, NC, USA; (5) Cornell University, Long Island, NY, USA; (6) University of Kentucky, Lexington, KY, USA; (7) Connecticut Agric Exp Station, Windsor, CT, USA; (8) USDA ARS FDWSRU, Fort Detrick, MD, USA; (9) Ohio State University, Columbus, OH, USA; (10) USDA ARS FDWSRU, Frederick, MD, USA; (11) Department of Plant Pathology, University of Georgia, Athens, GA, USA; (12) Foreign Disease-Weed Science Research Unit, USDA, ARS - Fort Detrick, MD, Frederick, MD, USA; (13) ORISE, Oak Ridge, TN, USA; (14) USDA-ARS, Mycology and Nematology Genetic Diversity and Biology Laboratory, Beltsville, MD, USA

Calonectria pseudonaviculata (Cps) is currently the most devastating pathogen of boxwood (Buxus spp.) worldwide. In the U.S. the boxwood blight was first detected in North Carolina, Connecticut and Oregon in 2011, and is now present in 28 states. A previous study reported that Cps residing in the U.S. and Europe prior to 2014 had a clonal population structure, and only the MAT1-2 mating type 
(idiomorph) was present. However, the genetic diversity and structure of contemporary Cps populations in the U.S. remain unknown. We aimed to uncover the genetic structure of Cps populations in the U.S. after 2014 and to assess the relative contribution of sexual reproduction. A total of 218 isolates of $C p s$ sampled from 21 states were genotyped by sequencing 11 SSR loci (Illumina technology) and by PCR amplification of MAT1 idiomorphs. All isolates possessed $C p s$-specific alleles, indicating that $C$. henricotiae (that also causes boxwood blight in Europe) is not yet present in the U.S. The contemporary U.S. population of Cps is predominantly clonal, composed of 13 multilocus genotypes (MLG SSR) unevenly distributed with predominance of MLG SSR-G2 (88\%), and only the MAT1-2 idiomorph is present. DAPC and AMOVA indicated Cps is not geographically subdivided and is not differentiated from the U.S. population prior to 2014, yet it is significantly differentiated from the main European population. These new insights into the population genetics of Cps in the U.S. contribute to a better understanding of boxwood blight epidemics, and are critical for the breeding of durably resistant varieties.

Analysis of virulence factors and population dynamics of Xanthomonas cynarae pv. gardneri in New York J. L. GONZALEZ-GIRON (1), C. D. Smart (2), (1) Cornell Univ - Ithaca, NY, USA; (2) Plant Pathology and Plant-Microbe Biology Section, Cornell AgriTech, Geneva, NY, USA

Xanthomonas cynarae pv. gardneri (Xcg) is the predominant causal agent of bacterial spot of tomato and pepper in the Northeastern United States. Although this bacterial pathogen has recently caused epidemics in Michigan and other tomato-producing areas, there is currently no information regarding the pathogen's dynamics or genomic composition across New York State. To broaden our understanding of the population diversity of the pathogen, we made whole-genome comparisons of ten Nanopore-sequenced isolates collected in NY between 2004 and 2019. To understand the relevance of specific virulence factors in the pathogenesis, we correlated effector content and virulence in several accessions of tomato by measuring bacterial growth at three timepoints after infiltrating with a bacterial suspension. We also contrasted effector presence with genomes present in Genebank. Resistance to copper-based compounds was evaluated in-vitro for each $\mathrm{Xcg}$ isolate and correlated with the presence of copper-resistance genes in the sequenced genomes. Overall, this study provides an insight into the population diversity, virulence, and copper resistance of the pathogen in New York.

\section{Diseases affecting Theobroma cacao in Puerto Rico's emerging cacao industry}

A. PUIG (1), J. K. Brown (2), R. Ramos-Sobrinho (2), C. V. Keith (3), O. Gutierrez (4), R. Goenaga (5), (1) United States Department of Agriculture, Miami, FL, USA; (2) University of Arizona, Tucson, AZ, USA; (3) The University of Arizona, AZ, USA; (4) USDAARS, Miami, FL, USA; (5) USDA-ARS, Mayaguez, PR, USA

Cacao production is a rapidly expanding industry in Puerto Rico; new farmers have planted $\sim 20,000$ trees in the past few years. The first cultivation on the island in 1636 failed due to adverse weather and a disease of unknown etiology. The US Department of Agriculture (USDA) conducted cacao research throughout the 1900's but the large increase in commercial production occurred following an initiative in 2000 to develop a cacao industry. To determine the etiology and extent of diseases affecting Theobroma cacao in Puerto Rico, a survey was conducted in August 2019 at eight sites including commercial farms, field trials, and the USDA cacao germplasm collection. Pods exhibiting necrotic lesions were observed on cacao trees from all survey sites. Hyphal-tip cultures of isolated organisms were identified as Diaporthe spp. (60\%), Lasiodiplodia spp. (30\%), and Phytophthora palmivora (8\%) based on sequences of the internal transcribed spacer and large subunit regions. Only the latter two have been reported as cacao pathogens. Several pods from a commercial farm showed red blotchy discoloration symptoms, reminiscent of badnaviral infection. Amplification and sequencing of a $870 \mathrm{bp}$ fragment indicated the presence of a badnavirus $~ 95 \%$ identical to Cacao mild mosaic virus (CaMMV) (KX276640), previously identified from cacao trees in the Trinidad Gene Bank collection. In Trinidad, CaMMV was thought to have been eliminated from commercial cacao plantations during the 1950's. This is the first report of CaMMV in Puerto Rico or outside Trinidad, and of Diaporthe spp. as a pathogen of cacao.

Isolation of fungi from Phragmites australis

D. GALO, C. Escalante Guardado, J. Zhor, R. A. Valverde, Louisiana State University Agricultural Center, Baton Rouge, LA, USA

Roseau cane (Phragmites australis) occurs worldwide. In most places, it is considered an invasive plant, although in some areas, it plays an important role in protecting coastal infrastructures and marsh ecosystems. Recently, Roseau cane die-off has been reported in the lower Mississippi River area of Louisiana. It is suspected that insects and pathogens may be playing an active role in the die-off. The objective of this investigation was to identify fungal pathogens associated with Roseau cane in Louisiana. Leaves and stem tissues showing disease symptoms were collected from Roseau cane growing in the delta of the Mississippi River and Manchac, Louisiana. Fungi were isolated from surface sterilized plant tissue on potato dextrose agar. Pure cultures were generated, and then DNA was extracted and used to amplify the fungal DNA internal transcribed spacer region. To test their pathogenicity, spore suspensions of some isolated fungi were sprayed to healthy Roseau cane plants under laboratory conditions. Initial symptoms developed within 4 days of inoculation, and plants exhibited foliar lesions 10 days after inoculation. Re-isolations were conducted successfully from diseased tissues. Thirty-seven fungal isolates were identified, of which 25 were non-pathogenic. Twelve isolates were able to cause foliar symptoms under laboratory conditions. Some pathogenic fungi included Bipolaris sp., Fusarium incarnatum and Fusarium fujikuroi species complex. These results suggest that foliar pathogens can cause disease on Roseau cane and could contribute to Roseau cane dieoff.

An open tree-based (T-BAS) phylogeny of Phytophthora infestans and species of Phytophthora

A. COOMBER, M. Knight, A. C. Saville, J. Ristaino, North Carolina State University, Raleigh, NC, USA 
With more than 142 species, there is a need for an open bioinformatic tool that centralizes diverse streams of sequence data and metadata, and that allows integration of this information into a robust phylogenetic framework. We developed a T-BAS phylogeny of Phytophthora infestans using 12-plex microsatellite data and sequences of seven nuclear genes from Phytophthora species archived in GenBank from well-curated samples. For Phytophthora species, the phylogenetic tree was developed using RAxML. For P. infestans, a neighbor-joining tree and a structure analysis was used as the backbone of the tree. The T-BAS tool will provide the framework and allow users to visualize the phylogenetic placement of unknown sequences without the uncertainty of incorrect species id that often happen with a Blast search. Phytophthora infestans and other species sourced globally from over thirty years of collecting by J. Ristaino and a larger set of Phytophthora species obtained from Dr. Mannon Gallegly's collection. The tools developed will share metadata in the species phylogeny including: clade, host species, substrate, sexual characteristics, source paper, temperature range, and distribution. The tool will allow open data sharing by different research groups that are currently working on these species and the database will enable the global Phytophthora community to upload sequences and determine the phylogenetic placement of an isolate, identify a genotype (for $P$. infestans), create a tree, and download sequence datasets.

First complete genome sequence of Carnation latent virus, the genus Carlavirus type species R. L. JORDAN (1), E. Korolev (2), S. Grinstead (3), D. Mollov (3), (1) USDA-ARS, USNA, Floral \& Nursery Plants Research Unit, Beltsville, MD, USA; (2) Biotechnology Career Academy, Applications and Research Laboratory, Ellicott City, MD, USA; (3) USDAARS-NGRL, Beltsville, MD, USA

The genus Carlavirus [Family Betaflexiviridae, Order Tymovirales] includes 53 species recognized by ICTV. The NCBI GenBank database has 43 accessions of full-length (7,890 to 9,073 nt) carlavirus genomes. Surprisingly, the type species Carnation latent virus (CLV) only has accessions with 1313 or fewer nts. The goal of this study was to determine the full-length genome of CLV. Naturally infected greenhouse-grown 'Kiwi Lace' carnation plants that had been continuously vegetatively-propagated over the past 30 years and that tested positive for CLV by ELISA and RT-PCR were used as source plants for high-throughput sequencing (HTS). A cDNA library was generated from total RNAs and HTS performed on a NextSeq 500 Illumina platform. Single 75-bp sequence reads (23.7M) were processed and de novo assembled into 49,555 contigs. Seven contigs having homology to carlavirus sequences in BLASTX analysis against GenBank viral databases were trimmed and re-assembled into an 8,464 nt consensus sequence which was validated by 5' and 3' RACE and Sanger sequencing of CLV-specific RT-PCR generated amplicons. The complete CLV-KL sequence was determined to be $8,513 \mathrm{nt}$ [MN450069]. In pairwise analysis, the genome shares $40-46 \%$ identity with recognized carlaviruses and the six in silico translated proteins have $15-62 \%$ amino acid identities with their respective carlavirus orthologs. The CLV-KL coat protein shares 98.4\% identity with the CLV-UK NCBI Reference Sequence. In phylogenetic analysis, CLV clusters with Butterbur mosaic virus, Coleus vein necrosis virus, and Garlic common latent virus.

Stripe rust epidemics of wheat and barley and races of Puccinia striiformis identified in the United States in 2019 M. Wang PhD (1), X. CHEN (2,3), (1) Washington State University, Pullman, WA, USA; (2) USDA-ARS, Pullman, WA, USA; (3) Washington State University, Pullman, WA, USA

In 2019, stripe rust of barley, caused by Puccinia striiformis f. sp. hordei (Psh), occurred in Idaho, Oregon, and Washington, and stripe rust of wheat, caused by $P$. striiformis f. sp. tritici (Pst), occurred in 15 states. To monitor virulence changes of the pathogens, stripe rust samples were tested on 18 wheat and 12 barley differentials to identify Pst and Psh races, respectively. From 30 Psh isolates, 9 races were detected including one new race, PSH-116 (virulent on differentials Topper, Hiproly, Abed Binder 12, Trumpf, and Bigo). PSH-33 (virulent on Topper and Abed Binder 12) and PSH-116 were the predominant races with frequencies of $33.3 \%$ and $26.7 \%$, respectively. From 244 Pst isolates, 27 races were identified, including 3 new races (PSTv-323 virulent to $Y r 6, Y r 7, Y r 8, Y r 9, Y r 17, Y r 27, Y r 43, Y r 44$, YrSP, and YrExp2; PSTv-324 virulent to Yr1, Yr6, Yr7, Yr9, Yr27, Yr43, Yr44, YrTr1, YrExp2, and Yr76; and PSTv-325 virulent to Yr1, $\operatorname{Yr} 7, \operatorname{Yr} 8, \operatorname{Yr} 9, \operatorname{Yr} 27, \mathrm{Yr} 44, \mathrm{YrTr} 1, \mathrm{YrExp} 2$, and $Y r 76$ among $18 \mathrm{Yr}$ single-gene differentials). The top five predominant races were PSTv37 (43.9\%), PSTv-47 (9.4\%), PSTv-41 (8.2\%), PSTv-322 (6.1\%), and PSTv-4 (3.3\%). PSTv-37 was detected throughout the country, while PSTv-4, PSTv-41, PSTv-47, and PSTv-322 were mostly in the western U.S. Virulence frequencies were high $(70.5-90.2 \%)$ to Yr6, Yr7, Yr8, Yr9, Yr17, Yr27, Yr43, Yr44, YrTr1, and YrExp2; but low to Yr1, Yr10, Yr24, Yr32, YrSP, and Yr76 (7.4\% - 36.9\%). No virulence was detected to either $\operatorname{Yr} 5$ or $\operatorname{Yr} 15$. The results are useful for breeding resistant cultivars and control of stripe rust.

\section{Effects of micronutrient products from Earth Science Laboratories (ESL) on host transcriptional regulation and disease suppression in rice and soybean}

J. RICHARDS (1), B. Tubana (2), T. Ford (3), N. Filajdic (4), (1) Louisiana State University Agricultural Center, Baton Rouge, LA, USA; (2) Louisiana State University, LA, USA; (3) Earth Science Laboratories, AR, USA; (4) Earth Science Laboratories, Rogers, AR, USA

Micronutrient applications have been used as an effective disease management strategy. However, specific micronutrients may affect disease resistance or susceptibility differently, depending on the specific pathogen or host. Additionally, the functional mechanisms contributing to disease suppression following micronutrient treatments are not fully understood. The goal of this project was to examine the potential efficacy of four ESL products $\left(\mathrm{CuSO}_{4}, \mathrm{Zn}-\mathrm{Phire}, \mathrm{Zn}-\mathrm{Mn}\right.$, and $\left.\mathrm{Mn}\right)$ for the suppression of sheath blight (SB) of rice and frogeye leaf spot (FLS) of soybean. We also aimed to identify genes or pathways that were involved in the transport of the applied elements and/or potential mechanisms for the observed disease suppression. ESL Zn, Mn, and Zn-Mn products applied at both 16 oz/ac and $32 \mathrm{oz} / \mathrm{ac}$ significantly reduced SB in rice. Zn-Mn applied at both $16 \mathrm{oz} / \mathrm{ac}$ and $32 \mathrm{oz} / \mathrm{ac}$, as well as Mn applied at both rates, were effective for the suppression of FLS in soybean. Transcriptomics analyses identified a family of zinc transporters in soybean that likely facilitated the import/translocation of zinc into plant cells following application of ESL Zn and Zn-Mn. Components of the phenylpropanoid biosynthesis pathway and disease resistance-related genes were significantly upregulated following $\mathrm{CuSO}_{4}$ and $\mathrm{Zn}-\mathrm{Mn}$ 
treatment on soybean, indicating a potential priming of the immune response aiding disease suppression. These results have indicated efficacy of ESL products for the suppression of SB in rice and FLS in soybean and identified candidate mechanisms of disease suppression.

Characterization of the rhizosphere and endophytic microbiome across apple rootstock genotypes in replant orchard soils C. Van Horn, T. SOMERA, M. Mazzola, USDA Agricultural Research Service, Wenatchee, WA, USA

Apple replant disease (ARD), caused by a complex of soil-borne plant pathogens, limits tree productivity in new orchard plantings at sites previously planted to apple. Use of disease tolerant rootstocks may diminish the growth limiting effects of ARD, however the effect of rootstock genotype on modulating the rhizosphere and endophytic microbiome enabling ARD tolerance is not fully understood. Studies were conducted to characterize composition of the root rhizosphere and endophytic microbiome across a diversity of apple rootstock genotypes. A series of rootstocks were planted into orchard replant soil containing a known pathogen complex. Results from amplicon sequencing determined significant differences in microbial community composition between the rhizosphere and endophytic environments as well as across individual rootstock genotypes. In general, significant differences in fungal communities were observed between the Geneva and Malling rootstock genotypes. Among rootstock endophytic fungal communities, rootstock G.890 samples consistently harbored the highest percentage of arbuscular mycorrhizal fungal species ( $>5 \%$ of total). Ilyonectria spp., which may function as a pathogen of apple, were detected at high relative abundance in the endosphere of all genotypes, with the highest relative abundance in G.41. These results provide further insight into rhizosphere and endophytic microbial communities of apple rootstocks, which could be exploited or manipulated to improve tree fruit agricultural management practices with respect to plant nutrition and disease control.

The contribution of Sclerotinia sclerotiorum effectors to SSR development in Soybean D. SHAO (1), N. M. Westrick (2), M. Roth (3), M. McCaghey (4), D. L. Smith (1), M. Kabbage (1), (1) University of WisconsinMadison, Madison, WI, USA; (2) University of Wisconsin - Madison, Madison, WI, USA; (3) University of Wisconsin - Madison, Madison, WI, USA; (4) University of California, Davis, CA, USA

Sclerotinia stem rot (SSR) on soybean is a disease caused by the fungal pathogen Sclerotinia sclerotiorum. SSR has a major impact on yield in soybean production worldwide. Recent transcriptome analysis from $S$. sclerotiorum-infected soybeans suggests that effectors may be deployed during infection and provided a list of at least 57 such effector candidates. Six highly expressed candidates at various stages of infection were selected for further study. Deletion mutants were generated using a novel high-efficiency approach combining CRISPR/Cas9 and homologous recombination. Fungal mutants were examined for both developmental and virulence defects to identify effectors specifically required for disease establishment in soybean. Using a combination of confocal microscopy, reverse genetics, and protein-protein interaction approaches, we aim to place such effectors into their biochemical context in planta. This strategy contributes to a broader effort in our laboratory to identify $S$. sclerotiorum virulence factors that can be targeted using RNAi approaches to enhance resistance to this pathogen. We successfully employed RNAi approaches such as host-induced gene silencing (HIGS) to target oxalic acid production in S. sclerotiorum, thus confirming that such strategies are feasible against $S$. sclerotiorum in soybean. The goal of this project is to provide insights into the contribution of $S$. sclerotiorum effectors to SSR and further expand the repertoire of $S$. sclerotiorum virulence determinants that can be targeted via RNAi to enhance resistance to this broad host range pathogen.

\section{Distinct genetic lineages of Xylella fastidiosa carry conserved Type I Restriction-Modification systems with diverse specificity subunits M. O’LEARY, L. Burbank, D. C. Stenger, USDA-ARS, Parlier, CA, USA}

Xylella fastidiosa is a xylem-limited bacterial pathogen consisting of five subspecies (fastidiosa, morus, multiplex, pauca, and sandyi) which causes disease on numerous economically relevant plant hosts. $X$. fastidiosa is naturally competent, and recombination between strains is well-documented. Genomes of $X$. fastidiosa are enriched in restriction-modification (RM) systems, which can impede acquisition of foreign DNA. Transformation efficiency varies by strain and may be increased by inhibition or deletion of Type I RM systems. Activity of Type I RM systems relies on a specificity subunit (HsdS), containing two variable target recognition domains (TRDs) that each recognize a short (3-5 bp) nucleotide sequence. Recombination between $h s d S$ genes can generate alleles with novel target specificity if TRDs are replaced. In other pathogenic bacteria enriched in RM systems (e.g., Staphylococcus aureus), hsdS alleles in Type I RM systems are variable across distinct genetic clades. Genomes of $113 \mathrm{X}$. fastidiosa strains were evaluated for the presence of Type I RM systems, and $h s d S$ genes were categorized by TRD domains. Each $X$. fastidiosa strain contains two conserved and intact Type I RM systems; strains of subsp. multiplex and pauca contain a third conserved system. Comparison of hsdS gene TRDs reveals $12-$ 21 unique alleles per system; in total, at least $30 \mathrm{hsd} S$ allele profiles were identified. Allele profiles vary across strain types, and infrequently within strain type, but do not correlate with host of isolation or geographic origin.

The origin of the problem: Phylogeographic analysis of Septoria steviae, causal agent of Septoria leaf spot of stevia A. D. SANABRIA, D. Shew, North Carolina State University, Raleigh, NC, USA

Stevia, Stevia rebaudiana, is a semi-perennial plant of the family Asteraceae native to Paraguay. Commercial cultivation of this plant has rapidly expanded into many countries, including the USA. Multiple diseases have been reported on stevia, but the foliar pathogen, Septoria steviae, causes severe defoliation and can significantly reduce plant yields. Resolving the origin of $S$. steviae will enhance our understanding of the introduction and spread of the pathogen into new production areas. We performed discrete trait phylogeographic analysis on isolates from multiple countries to investigate the phylogeographic history and spread of $S$. steviae. We included sequences 
from S. steviae available in GeneBank from France, Japan, and the USA. We also obtained isolates from the center of origin of stevia in Paraguay and sequenced seven loci: actin, $\beta$-tubulin, calmodulin, ITS, LSU, RPB2, and TEF1 $\alpha$. A Bayesian coalescent constant population model was implemented in BEAST v2.6.2 and isolate locations were set as a discrete trait. Independent analyses with MCMC simulations of 10 million iterations were conducted for single locus and multi-locus alignments. Bayesian maximum clade credibility (MCC) trees with root state posterior probabilities supported an ancestral connection of isolates of $S$. steviae to Paraguay. The most immediate lineages to Paraguayan isolates were from Japan, followed by USA and French isolates. This suggests that $S$. steviae spread from Paraguay to other countries in which the stevia is cultivated, likely, together with plant material.

Time series transcriptome analysis reveals adaptable activation of defense related genes in tree tomato infected with late blight D. BAUTISTA, N. Guayazan, D. Botero, J. Duitama, A. J. Bernal, S. Restrepo, Universidad de Los Andes, Bogotá, COLOMBIA

Tree tomato (Solanum betaceum) is an emerging crop from the Andean region whose commercialization is of increasing interest. Despite this, sustainability and disease management have been a limiting factor for its production. Recently, a new species of the causal agent of late blight, Phytophthora betacei, was described in tree tomato. To characterize the response of the plant to the infection of $P$. betacei, we performed RNA-seq in a susceptible tree tomato variety in a time series after infection $(6,12,24,72,96$ hours post inoculation), aiming to perceive changes in the plant response to the pathogen. We provide the first transcriptome assembly for $S$. betaceum and a comprehensive expression profile across infection times. We performed a PCA of the transcriptional profiles for all times, which showed a marked separation of the early (biotrophic) and late stages (necrotrophic) of response of $S$. betaceum to the pathogen. We classified differentially expressed genes in 15 clusters according to similarities in their expression trajectories throughout the infection. We performed a Gene Ontology enrichment analysis to determine similar functions and discovered distinctive biological processes for each cluster, generally related to immune, stress response and plant-pathogen interactions. These results show the different infection strategies of $P$. betacei in a detailed time-based progression of the response of tree tomato.

Development and evaluation of sclerotia mediated pathogenicity to sugar beet cultivars

M. E. HAQUE (1), D. K. Lakshman (2), A. Qi (3), M. Z. R. Bhuiyan (4), Y. Liu (4), P. Hakk (5), M. F. R. Khan (6), (1) North Dakota State University, Dept of Plant Pathology, Fargo, ND, USA; (2) USDA ARS, Beltsville, MD, USA; (3) University of Hertfordshire, Hatfield, Hertfordshire, UNITED KINGDOM; (4) NDSU, Fargo, ND, USA; (5) North Dakota State University, Fargo, ND, USA; (6) North Dakota State Univ \& Univ of MN, Fargo, ND, USA

Rhizoctonia solani causes damping-off, and root and crown rot of sugar beet (Beta vulgaris L.) and may cause significant yield loss. Most research to understand the aggressiveness of $R$. solani is typically done using $R$. solani inoculum grown on sterilized grains such as barley. Since the pathogen is known to overwinter as sclerotia and mycelia, a study was conducted to determine how best to produce large quantities of sclerotia and mycelia, and compare the pathogenic potentials of sclerotia, mycelia, and colonized barley grains on several selected sugar beet cultivars. To find out a suitable culture media, an in vitro growth study was done using $R$. solani AG2-2 IIIB on six media including amended clarified V8 (ACV8), 50\% potato dextrose agar (PDA), 10\% PDA, methylene-benomyl-vancomycin (MBV), cornmeal agar (CMA), and water agar (WA) arranged as a completely randomized design (CRD) with four replicates. A greenhouse study was done to evaluate the pathogenic potential of $R$. solani as sclerotia, mycelium and on barley (one sclerotia/seed, one mycelial plug/seed, one barley-inoculum/seed, and autoclaved barley/seed as mock) on six sugar beet cultivars arranged as a CRD with four replicates. The highest mean number of sclerotia was observed in ACV8, followed by CMA. Sclerotia and mycelia mediated disease severity was significantly greater compared to barley inoculum. This study demonstrated that $R$. solani sclerotia can be produced in large quantities; it can be used as an alternative to barley inoculum for the evaluation of host resistance.

Crop host influences on outcomes of competition between Aspergillus section Flavi species co-infecting maize and groundnuts C. CHING'ANDA (1), J. Atehnkeng (2), R. Bandyopadhyay (3), K. A. Callicott (4), H. L. Mehl (5), M. Orbach (1), P. J. Cotty (6), (1) University of Arizona, Tucson, AZ, USA; (2) International Institute of Tropical Agriculture, Bukavu, CONGO, DEM. REP.; (3) IITA, NIGERIA; (4) USDA Agricultural Research Service, Tucson, AZ, USA; (5) Virginia Tech Tidewater AREC, Suffolk, VA, USA; (6) USDA ARS, Tucson, AZ, USA

Fungal species within Aspergillus section Flavi contaminate crops with aflatoxins. Prevalence of these species in soils varies across agroecologies, and multiple Aspergillus species frequently co-occur in agricultural fields and co-infect crops. Aspergillus flavus genotypes have been shown to vary in their ability to compete during crop infection and colonization, however competitive differences among section Flavi species have not been assessed. Maize and groundnuts are staple crops that are highly susceptible to aflatoxin contamination, and they differ in nutrient composition and physiology. The current study assessed the influence of host on outcomes of competition between four different aflatoxin-producing species during infection and colonization of these two crops. Maize and groundnuts were co-inoculated with all possible pairs of the four species and incubated at $31 \mathrm{C}$ for 7 days. Aflatoxins and conidia were quantified, and proportions of each species within a treatment were assessed with quantitative pyrosequencing. Regardless of the coinoculated species, aflatoxin production was greater on maize than on groundnuts, while groundnuts supported greater sporulation than maize. The two hosts also differentially influenced outcomes of competition between species with A. flavus generally more competitive on maize and the unnamed species referred to as the Lethal Aflatoxicosis Fungus more competitive on groundnuts. Results of this study demonstrate the potential for crop hosts to shape Aspergillus community structure and thus levels of aflatoxin contamination.

Lasiodiplodia spp. associated with stem end rot of mango (Mangifera indica)

L. Li (1), L. ZAKARIA (2), (1) School of Biological Sciences, Minden, MALAYSIA; (2) Universiti Sains Malaysia, Minden, Penang, MALAYSIA 
Stem-end rot is one of the main post-harvest diseases of mango fruits. The symptoms of the disease started with initial blemishes on the surface and as the fruit ripens, the rot symptom spread systemically to the other end of the mango fruits. The rot symptom reduced the quality of mango fruits and may directly lower the market value of the fruit crop. One of the causal pathogens of mango stem end rot is Lasiodiplodia spp. from the family Botryosphaeriaceae. Isolates of Lasiodiplodia were isolated from stem-end rot lesion of several mango cultivars, Chok Anan, Black Gold, Waterlily, Falan, Harumanis and Raja. The isolates were identified using three markers, ITS region, TEF1- $\alpha$ and $\beta$-tubulin. Based on BLAST search, all sequences of ITS, TEF1- $\alpha$ and $\beta$-tubulin produced $99-100 \%$ similarity with L. theobromae (CBS164.96), L. pseudotheobromae (CBS116459), L. iranensis (RB31) and L. mahajangana (CMW27801).

Pathogenicity test using wounded treatment showed that the four species of Lasiodiplodia were pathogenic on three mango cultivars tested, Chok Anan, Waterlily, and Falan with L. theobromae was the most virulent species. The rotting symptoms observed were similar of which circular brown lesion developed into watery soft rotting. This study indicated four species of Lasidioplodia were associated with stem end rot of mango in northern states of peninsula Malaysia. The knowledge of Lasidioplodia spp. causing mango stem-end is important to strategize more effective disease management as well as for quarantine purposes.

Temporal and spatial dynamics in the apple flower microbiome in the presence of the phytopathogen Erwinia amylovora Z. CUI, Q. Zeng, B. Steven, The Connecticut Agricultural Experiment Station, New Haven, CT, USA

Plant microbiomes have important roles in plant health and productivity. However, despite flowers being directly linked to reproductive outputs, little is known about the microbiomes of flowers and their potential interaction with pathogen infection. Here, we investigated the temporal dynamics and spatial traits of the apple stigma microbiome when challenged with a phytopathogen Erwinia amylovora, the causal agent of fire blight disease. We profiled the microbiome from the stigmas of a single flower, greatly increasing the resolution at which we can characterize shifts in the composition of the microbiome. Individual flowers harbored unique microbiomes at OTU level. However, taxonomic analysis of community succession showed a population gradually dominated by bacteria within the families Enterobacteriaceae and Pseudomonadaceae. The flowers inoculated E. amylovora established large populations of the phytopathogen, with pathogen specific gene counts of $>3.0 \times 10^{7}$ in $90 \%$ of the flowers. Yet, only $42 \%$ of inoculated flowers later developed fire blight symptoms. This reveals pathogen amount on the stigma is not sufficient to predict disease outcome. Our data demonstrate that apple flowers represent an excellent model in which to characterize how plant microbiomes establish, develop, and interact with biological processes such as disease progression in an experimentally tractable plant organ.

\author{
Abiotic soil factors associated with Verticillium dahliae inoculum density, root lesion nematode abundance, and potato yield in \\ the Columbia Basin \\ B. EVIN (1), E. Smith (2), V. Skillman (1), J. Stemke (2), A. Moore (2), K. Frost (3), (1) Oregon State University, Hermiston, OR, \\ USA; (2) Oregon State University, OR, USA; (3) Oregon State University, Hermiston Agricultural Research and Extension Center, \\ Hermiston, OR, USA
}

The Columbia Basin of Oregon and Washington is an important potato production region. Verticillium dahliae causes Potato early dying (PED), a soil borne disease exacerbated by Pratelenchus penetrans. Many growers conduct soil testing to determine crop management strategy and it would be useful to identify soil biological, chemical, and physical factors that correlate with PED and potato yield. The objective of this study was to assess on-farm soil factors that may serve as indicators of crop health. In 2019 , four potato fields were selected for soil sampling. A 7.3 ha area grid defining 20 sampling locations was established in each field. Soils were grid-sampled in April and June and assayed for abiotic characteristics, $V$. dahliae inoculum density and $P$. penetrans abundance. Potato yield was estimated at each location in September. Correlation analysis was used to examine relationships between soil biotic and abiotic properties and potato yields. In 3 fields, soil organic matter was correlated with $\mathrm{pH}\left(\right.$ Field $1 \mathrm{R}^{2}=0.73, \mathrm{p}<0.001$; Field $2 \mathrm{R}^{2}=0.60, \mathrm{p}<0.001$; Field $\left.4 \mathrm{R}^{2}=0.39, \mathrm{p}=0.003\right)$. Soil $\mathrm{pH}$ was correlated with $P$. penetrans $\left(\right.$ Field $\left.1 \mathrm{R}^{2}=0.27, \mathrm{p}=0.02\right)$ and $V$. dahliae (Field $3 \mathrm{R}^{2}=0.28, \mathrm{p}=0.02$; Field $\left.4 \mathrm{R}^{2}=0.43, \mathrm{p}=0.002\right)$. Field 1 yield correlated with organic matter $\left(\mathrm{R}^{2}=0.37\right.$, $\mathrm{p}=$ $0.004)$ and $\mathrm{pH}\left(\mathrm{R}^{2}=0.36, \mathrm{p}=0.005\right)$. Based on this preliminary analysis, soil $\mathrm{pH}$ may relate to $V$. dahliae and $P$. penetrans. In the future, we would like to examine how soil biotic and abiotic factors are related and how those relationships may vary spatially, within a field.

\title{
Ecological and molecular determinates of Candidatus (Ca.) phytoplasma asteris disease progress in susceptible carrot (Daucus carota L.)
}

S. B. PIPER (1), J. Clements (2), B. Bradford (3), M. Garcia (1), W. Huang (4), A. Zwolinska (5), S. Hogenhout PhD (4), R. L. Groves (3), (1) University of Wisconsin, Madison, WI, USA; (2) University of Idaho, Parma, ID, USA; (3) Department of Entomology, University of Wisconsin - Madison, Madison, WI, USA; (4) John Innes Centre, Norwich, UNITED KINGDOM; (5) National Research Institute, Poznan, POLAND

Aster Yellows phytoplasma (AYp; Candidatus ( $\mathrm{Ca}$.) Phytoplasma asteris) is an obligate bacterial pathogen that is the causative agent of multiple diseases in herbaceous plants, including commercially cultivated crop species. While this phytoplasma has been examined in depth for its disease characteristics, knowledge about the spatial and temporal dynamics of pathogen spread in crops is lacking. The phytoplasma is vectored by piercing-sucking insects within the group of leafhoppers (Cicadellidae: Hemiptera), including the aster leafhopper, Macrosteles quadrilineatus Forbes. Historical data suggests the aster leafhopper overwinters in the southern United States and migrates to north annually, transmitting and driving phytoplasma infection rates as they migrate. A more in-depth understanding of the spatial, temporal and genetic determinants of Aster Yellows disease progress will lead to better management strategies. Carrot, Daucus carota L., plots were established in two planting densities at the Hancock Agricultural Research Station in Wisconsin and monitored during the 2018 growing season for Aster Yellows disease progression. Symptomatic carrots were sampled and assayed for 
the presence of the Aster Yellows phytoplasma. Isolated tissue samples from infected carrots were examined for variations in aster yellows phytoplasma genotypes and for the presence of specific secreted AY-WB proteins (SAP effectors). Aster Yellows disease progression was determined to be significantly associated with calendar date, crop density, location within the field, and phytoplasma subgroup. Optimization of translating ribosome affinity purification for identification of phloem responses to Candidatus Liberibacter
asiaticus in citrus

T. COLLUM (1), M. Grando (2), A. L. Stone (1), D. Sherman (1), J. N. Culver (3,4), C. D. Dardick (5), E. Stover (2), E. E. Rogers (1), (1) USDA, Agricultural Research Service, Foreign Disease-Weed Science Research Unit, Frederick, MD, USA; (2) USDA, Agricultural Research Service, U.S. Horticultural Research Laboratory, Fort Pierce, FL, USA; (3) Department of Plant Science and Landscape Architecture, University of Maryland, College Park, MD, USA; (4) Institute of Bioscience and Biotechnology Research, University of Maryland, College Park, MD, USA; (5) USDA, Agricultural Research Service, Appalachian Fruit Research Station, Kearneysville, WV, USA

The bacterium Candidatus Liberibacter asiaticus (CLas) is associated with Huanglongbing (HLB), a serious disease of citrus. CLas is a phloem-limited pathogen that manipulates phloem gene regulation to facilitate its life cycle. However, accurately characterizing phloem responses to CLas has been challenging due to the technical difficulty of sampling phloem tissues. In this study, we have adapted translating ribosome affinity purification (TRAP) methodologies for use in citrus trees. TRAP utilizes tissue specific promoters to express FLAG-tagged ribosomal proteins for immuno-capture of cell-specific mRNAs. Unlike other phloem sampling methods, there is no disruption of the pressurized phloem system prior to mRNA harvesting. HLB-susceptible sweet orange, and HLB-resistant Poncirus and HLB-tolerant Carrizo (sweet orange $x$ Poncirus) were transformed to express the FLAG-tagged ribosome protein L18 (RPL18) under the control of three phloem-specific promoters (pSUC2, pSULTR2;2 and p396ss) as well as the constitutive CaMV 35S promoter. Anti-FLAG conjugated magnetic bead were used to immunopurify tagged ribosome complexes from citrus leaves. High quality translatome RNA was recovered from plants expressing FLAG-RPL18 but not from non-transformed control plants. The adaptation of TRAP methods for citrus will allow for the comparison of susceptible and tolerant phloem responses to CLas. Efforts are underway to identify phloem genes that are differentially regulated during infection. Identified genes will represent unique phloem specific targets for disrupting the CLas life cycle.

A science-mapping approach to investigate plant pathology's role in Integrated Pest Management J. RUWONA, H. Scherm, University of Georgia, Athens, GA, USA

Integrated Pest Management (IPM), an approach promoted by all pest control disciplines, seeks to incorporate multiple management strategies and tactics to minimize economic, environmental, and public health risks. Although the role of plant pathology in IPM has been discussed previously, a structured investigation of the evolution of phytopathological topics within the IPM continuum is lacking. Science mapping, a form of bibliometric analysis and visualization, can be used to investigate scientific landscapes, publication trends, and evolution of research topics. A Web of Science search for IPM yielded nearly 10,000 publication records for the period 1990-2019, of which over 6,000 were broadly related to plants and crops. A term map generated with VOSviewer software revealed four clusters, of which plant pathology topics fell largely within a broad cluster of farming and production factors. The other three clusters were dominated by entomology topics, the discipline in which IPM was pioneered. When the three decades of investigation were compared, key phytopathological themes for each were methyl-bromide alternatives, soil solarization, and biological control (1990-99); whitefly-transmitted virus diseases (2000-09); and climate change, citrus greening, and psyllid transmission (2010-19). The term maps also showed that pesticide use remains the dominant IPM strategy. The contribution of plant pathology journals to the IPM journal landscape will be discussed. This study demonstrates the utility of science mapping for generating insights for IPM researchers and policy-makers.

\section{Understanding the ecobiology of Sclerotium rolfsii, as a tuber rot of potatoes, to develop disease forecasting tools for chemical management}

M. MCCAGHEY (1), E. L. Helpio (1), N. McRoberts (1), J. Sidhu (2), B. J. Aegerter (3), C. L. Swett (1), (1) University of California, Davis, CA, USA; (2) University of California Cooperative Extension, Bakersfield, CA, USA; (3) University of California Cooperative Extension, Stockton, CA, USA

California is one of the largest producers of specialty potatoes in the United States. Southern blight (SB), caused by Sclerotium rolfsii, leads to tuber rot pre and post-harvest and results in major yield losses. Conventional wisdom postulates that SB does not develop until re-wetting immediately before harvest. However, ongoing studies indicate that disease can develop prior to canopy burn-down (up to 35 days pre-harvest), suggesting new opportunities for management. While chemical control can reduce SB losses in other crops, effective use in potatoes requires a better understanding of SB risk in California. Under controlled temperatures, we generated a growth response curve and identified that sclerotial germination is inhibited below $12^{\circ} \mathrm{C}$ and above $35^{\circ} \mathrm{C}$; these thresholds were validated with naturally infested field soil. Studies are underway to assess the robustness of the thresholds in $S$. rolfsii isolates from several locations in California. Based on these studies, we are developing a preliminary degree day model to predict germination in response to thermal time, which we are validating in field trials. In concert, efficacy of chemical applications in risk periods is being examined to determine optimal chemistries for SB management. Because temperatures above $35^{\circ} \mathrm{C}$ were found to reduce sclerotia viability up to $100 \%$, this work suggests opportunities for soil solarization as a management tool. We aim to use these studies to develop grower recommendations for risk-based fungicide tools and complementary methods to reduce SB losses in potatoes. 
Fruit rot: A bacterial disease affecting dragon fruit production in Florida

T. DE PAULA LELIS (1), C. F. Hong (2), S. Zhang (1), J. H. Crane (3), R. Gazis (4), (1) University of Florida, Homestead, FL, USA; (2) National Chung Hsing University, Taichung City, TAIWAN; (3) University of Florida - Tropical Research and Education Center, Homestead, FL, USA; (4) University of Florida, FL, USA

Dragon fruit (Hylocereus spp.), commonly known as pitaya or pitahaya, produces edible fruits that have become of great interest among U.S. consumers. Two main species commercially grown in South Florida are H. undatus (bright pink skin and white pulp) and $H$. costaricensis (bright pink skin and varying hues of pink pulp). Major challenges facing the industry and research and extension faculty include the accurate and rapid identification of diseases and their causal agents and the scarcity of control products. Recently, an outbreak of fruit rot was observed in several commercial orchards in South Florida. The disease occurred in summer 2019 and was estimated to affect 5 to $10 \%$ of developing fruits, leading to complete fruit loss. Isolations were made from the edges of water-soaked, rounded to irregular lesions. Fifteen bacterial strains were isolated from the diseased fruits. All isolates were gram-negative, rod-shaped bacteria. Based on the 16S rRNA gene sequencing and biochemical assays, four different genera were identified, i. e. Enterobacter sp., Kluyvera sp., Providencia sp., and Serratia sp. Pathogenicity tests on fruits and cladodes are currently in progress to determine the primary causal agent of fruit rot. Even though the four genera identified are known to be ubiquitous in nature and opportunistic pathogens, Enterobacter cloacae has been reported infecting cladodes and fruits of dragon fruit. Considering the economic losses this disease can cause, it is crucial to determine the causal agent of the disease for the development of effective management strategies.

Transcriptional response of the phytopathogenic oomycete Phytophthora infestans to the bacterial biocontrol agent Lysobacter capsici AZ78

S. TOMADA (1), G. Puopolo (2), P. Sonego (3), M. Moretto (3), K. A. Engelen (3), M. Perazzolli (2), I. Pertot (2), (1) Free University of Bozen/Bolzano, Bolzano, ITALY; (2) Università di Trento, S. Michele A/A, ITALY; (3) Fondazione E. Mach, S. Michele A/A, ITALY

In the soil ecosystem, the microbe-microbe interactions continuously stimulate the gene expression patterns of each microorganism. In the past, the fungus-bacterium model has been mainly studied within the ectomycorrhizal-mycorrhizal helper bacteria context, or to elucidate the mode of action implemented by the biocontrol agents, in the case of antagonistic interactions. By contrast, few studies reported the specific transcriptional responses of a phytopathogen to a biocontrol bacterium. The present study aimed to dissect the molecular mechanisms activated by the soilborne phytopathogenic oomycete Phytophthora infestans during the interaction with a biocontrol rhizobacterium Lysobacter capsici AZ78 via dual RNA-Seq approach. As a result of the study, the attempted defense reaction of $P$. infestans to $L$. capsici AZ78, such as the up-regulation of genes involved in detoxification and drug resistance processes, were overcome by the bacterial biocontrol activity. Indeed, $P$. infestans responded to the bacterium by the up-regulation of genes involved in putative apoptotic processes. The subsequent $P$. infestans cell death resulted in the suppression of the whole oomycete metabolism with the down-regulation of genes involved in primary metabolic pathways (carbohydrates, nucleic acids, and protein metabolisms). Overall, the response of $P$. infestans to $L$. capsici AZ78 was related to cell death processes nullifying the oomycete efforts to counteract the bacterium attack. The study provided new insights into the successful control of soilborne pathogens within sustainable agriculture.

Evaluation of antimicrobial compounds against Candidatus Liberibacter asiaticus using propidium monoazide and quantitative PCR

V. ANCONA (1), C. Yang (2), (1) Texas A\&M University, Weslaco, TX, USA; (2) Texas A\&M University Kingsville, Citrus Center, Weslaco, TX, USA

Huanglongbing (HLB) is an important citrus disease associated with the phloem-limited, unculturable bacterium Candidatus Liberibacter asiaticus (CLas). Many chemical compounds against CLas have been discovered from chemical library screens. However, field assessment of these compounds are time consuming, as they may take from several months to over a year to show results. The aim of this study is to develop a method for rapid evaluation of HLB therapies in field trials. To this aim, we optimized a DNA extraction method from citrus leaf tissues with propidum monoazide (PMA), a dye that binds covalently to dsDNA and blocks the DNA from dead bacteria in subsequent qPCR reactions. Thus, PMA allows distinguishing between viable and non-viable CLas cells. Results indicate that efficacy of PMA-qPCR is highly dependent on the primer set used. Primers targeting the 16S region, showed a clear distinction between qPCR from PMA-treated and non-treated samples, while RNR and J900 primers did not show significant differences between DNA extraction methods. PMA-qPCR viability analysis from different concentrations of penicillin and water showed a clear dose response effect four days after treatment, with pick differences at eight days post treatment. This method would allow for rapid assessment of new chemistries against CLas in citrus trees for implementation of effective management strategies against HLB.

A comparison of genome sequence data and pathogenicity among isolates of Sorghum mosaic virus from Louisiana sugarcane M. P. GRISHAM (1), A. Hale (1), J. W. Hoy (2), D. Mollov (3), K. Warnke (1), J. Maggio (1), (1) USDA, ARS, Sugarcane Research Unit, Houma, LA, USA; (2) Louisiana State University, Baton Rouge, LA, USA; (3) USDA, Beltsville, MD, USA

Mosaic of sugarcane is caused by Sorghum mosaic virus (SrMV) and Sugarcane mosaic virus (SCMV); however, in Louisiana is SCMV is rarely found infecting sugarcane. To determine the genetic diversity among the SrMV population in Louisiana, isolates were collected annually from 2012 to 2019 from released and near-release sugarcane cultivars exhibiting mosaic symptoms from natural infection. The infected plants were collected primarily from breeding trials at locations across the Louisiana sugarcane industry. A reverse transcriptase-polymerase chain reaction (RT-PCR) protocol was used to identify SCMV and SrMV isolates. All isolates collected during this time period were identified as SrMV. Genetic diversity was noted among the isolates. A phylogenetic analysis was 
performed using the RT-PCR sequences from 74 of the SrMV isolates. Isolates with were assigned to five groups based on sequence similarity. A mixture of four isolates were used to inoculate 41 sugarcane clones. Isolates recovered from clones expressing symptoms indicated that one of the isolated caused the majority of infections. Twenty SrMV isolates from among the five groups have been subjected to total nucleic acid sequencing and are being analyzed.

\section{Effects of selected soil amendments and mulch type on soil properties, yield, and disease development in organic vegetable} production

R. P. LARKIN, USDA-ARS, New England Plant, Soil, and Water Lab, Orono, ME, USA

The potential benefits of different soil amendments and mulch ground covers on soil chemical and biological properties, crop development and yield, and disease and pest issues in organic vegetable production, as represented by legume (green snap bean), cucurbit (green zucchini squash), and brassicaceous (turnip) vegetable crops, were evaluated in a two-year field trial in Maine. Soil amendments included a commercial organic fertilizer alone, composted dairy manure, compost plus fish meal, and compost plus Wollastonite, a natural source of silicon (Si). A paper mulch was also compared with a woven polypropylene fabric mulch for their performance and effects as weed barriers within these systems. The fabric mulch was associated with increases in soil moisture, organic matter, and several soil chemical and microbiological properties relative to the paper mulch, as well as earlier emergence and earlier harvests for bean and zucchini in at least one year. Compost amendments increased soil $\mathrm{pH}$, organic matter, and several nutrient concentrations, as well as crop emergence and yield of bean and zucchini relative to a fertilizer-only treatment. Additions of Si or fish meal also provided some effects, with fish meal increasing most nutrient element concentrations and microbial respiration, while silica increased emergence of beans, and reduced powdery mildew on squash and late season browning of beans. These results help define specific management practices to improve organic vegetable production and provide useful information and options for growers.

\section{A high-density transposon insertion screen in Dickeya dadantii 3937 to identify genes required for host colonization T. HELMANN, M. Filiatrault, P. Stodghill, USDA-ARS, Ithaca, NY, USA}

The soft rot pathogen Dickeya dadantii is capable of causing disease in diverse crop plants, primarily through pectinase-mediated plant cell wall degradation. Although several virulence genes have been identified in the model strain 3937, there is still very little known about the genes that contribute to fitness for soft rot pathogens in their diverse hosts. There is also a need for the development of improved molecular tools for functional genomics in these pathogens. To identify genes critical for host colonization and survival, we constructed a high-density barcoded transposon ( Tn) library in strain 3937. The association of each insertion with a 20-nucleotide barcode enables the use of a single Tn library under multiple conditions. We mapped over 200,000 insertional mutants, which enables the identification of non-essential genes, including small genes and sRNAs, that contribute to competitive fitness during the development of potato soft rot. By inoculating this Tn library into potato plants and tubers, we can identify functional categories of genes that are necessary for host colonization. This valuable new resource can be used in a number of ways, including screening in multiple host plants to better characterize genes required for its broad host-range lifestyle and comparing Tn libraries constructed in related phytopathogenic Dickeya strains, including D. dianthicola. These comparisons can be used to determine common virulence strategies and more generally the role of diverse genes required for necrotrophic colonization of host plants and post-harvest crops.

\section{Evaluation of Chlorothalonil alternative fungicide regimes for cranberry fruit rot and quality: Traditional methods vs high-} throughput image analysis

L. S. UPPALA (1), A. Marrano (2), B. Moyers (2), (1) University of Massachusetts-Amherst-Cranberry Station, East Wareham, MA, USA; (2) University of Massachusetts-Boston, MA, USA

Fruit rot has been the major challenge in Northeast cranberry cultivation. For the past two decades, $>90 \%$ NE cranberry acreage received broad-spectrum chlorothalonil fungicides for fruit rot management. However, in 2019, export markets targeted chlorothalonils for elimination and lowered maximum residue limits (MRLs) from 5 to 0.01 parts per million. This prompted the industry to shift towards the only three other effective classes (FRAC Group 3, 11 and M3) of registered fungicides for fruit rot management. Group 3 and 11 fungicides, although effective and less harmful to human and environmental health, they pose a much higher risk of selecting for resistance than multi-site fungicides. Group M3 mancozeb fungicides are believed to delay fruit color and reduce fruit size in some seasons. To preserve the effectiveness and durability of these fungicides, we evaluated 15 fungicide regimes, for their effects on fruit rot, fruit quality and yield. Fruit samples were analyzed both by traditional methods and high-throughput imaging using a handheld gimbal-mounted sensor system, the Sequoia Parrot+, which combines light sensors, GPS, an RGB camera, and four narrow-band sensors in the green, red, red edge, and NIR spectra. Using plot-level images extracted from an orthomosaic in each spectrum, we will explore image-analysis strategies for measuring fruit rot, fruit quality and yield, and compare to values obtained by traditional methods. We will use these comparisons to define and improve the accuracy of high-throughput phenotyping as a new tool for monitoring fruit rot in the field.

The effect of chemical control on the incidence and sensitivity of Phoma fungicola causing the pistachio fruit blight in Arizona P. D. S. F. LICHTEMBERG (1), J. Sherman (2), R. Puckett (3), M. Marzall-Pereira (1), J. Velasco (4), J. Brosin (5), T. J. Michailides (3), (1) University of California - Davis, Parlier, CA, USA; (2) University of Arizona, Willcox, AZ, USA; (3) University of California, Davis, Parlier, CA, USA; (4) EARTH University, Guacimo, COSTA RICA; (5) Universidade Federal do Paraná, Curitiba, BRAZIL

In the last seven years, the pistachio acreage has increased from 3,645 to 8,905 in Arizona. Over this period, Phoma blight, caused by Phoma fungicola, has been consistently isolated from buds, fruits and leaves causing growers concerns. Symptoms on fruit include dark 
brown to black coloration covered with small pycnidia. Our objective was to determine the effect of two spray programs (SDHI \& QoI $v s$. DMI \& AP) on disease incidence, to phenotype the pathogen sensitivity to 11 fungicides and to find the optimum temperature for growth of $P$. fungicola in-vitro. Four seasonal sprays applied, such as in April (Merivon vs. Quash), May (MBC-Topsin), July (Luna Sensation $v s$. Inspire Super) and August (Merivon vs. Quash) in two commercial orchards. Optimal mycelial growth of $P$. fungicola occurred at $22.2^{\circ} \mathrm{C}$. Relative growth (RG) range of 30 isolates tested at the discriminatory dose of $10 \mu \mathrm{g} / \mathrm{ml} \mathrm{revealed} \mathrm{higher} \mathrm{sensitivity}$ for sole fungicide formulations containing: pyraclostrobin $(0.1-1.4 \%)$, metconazole $(0.1-4.6 \%)$, trifloxystrobin $(0-7.8 \%)$, fluopyram (1-7.9\%), fluxapyroxad (1.5-8.3\%), penthiopyrad (6.6-10.4\%) and cyprodinil (2.1-11.8\%). Five more fungicides affected the RG range from 69 to $100 \%$. In the field, unsprayed trees showed 7- and 3-fold more disease incidence than trees sprayed with SDHI/QoI and DMI/AP, respectively. No spray program main effect was observed. However, significant interaction between spray time and program was observed for April with less incidence where SDHI/QoI was used. Higher disease incidence occurred in July and August when more precipitation occurs.

\section{Effects of cover crops on nematode community in pepper production}

C. LIU (1), Z. J. Grabau (2), G. Gabriel Maltais-Landry (2), J. Allar (3), (1) 459186, Gainesville, FL, USA; (2) University of Florida, Gainesville, FL, USA; (3) Unviversity of Florida, Gainesville, FL, USA

Cover cropping is a common practice in southeastern United States vegetable production. It can not only provide plant disease control, but also may affect soil ecology and the nematode community. This study tested the effect of sunn hemp, sorghum, buckwheat, sunflower, cowpea and their combinations as cover crops in pepper production in Florida. In 2018 and 2019, cover crops were planted in Gainesville, Florida during summer before Fall peppers, and rye was used as a uniform cover crop in winter after pepper. The soil nematode community was assessed at before cover crops, after cover crops, after pepper, and after rye. Treatments include sorghum sudangrass, sunn hemp, sorghum and sunn hemp ( 2 species), 2 species plus buckwheat ( 3 species), 3 species plus sunflower and cowpea ( 5 species), and weedy fallow. In 2018, treatments showed no significant differences on root-knot nematode (Meloidogyne spp.) and sting nematode (Belonolaimus spp.) at after cover crops. However, cover crops enriched bacterivore abundance as well as total freeliving nematodes in the soil compared to weedy fallow $(P<0.05)$. In 2019 , there were higher sting nematodes counts in the sorghum treatment compared to other treatments $(P<0.05)$ after cover crops, but no significant impacts of cover cropping on free-living nematodes or root-knot nematode was observed. In both years, sunn hemp significantly reduced total herbivore abundances in the soil $(P<0.05)$. In general, cover crops may influence nematode community in the soil, and sunn hemp may work as an effective cover crop against plant-parasitic nematodes.

Fits like a glove: Rapid, cost-effective sampling techniques to monitor Erysiphe necator S. LOWDER (1), A. Peetz (2), T. Neill (3), W. Mahaffee (3), (1) Oregon State University, Corvallis, OR, USA; (2) Revolution Crop Consultants, Albany, OR, USA; (3) USDA ARS, Corvallis, OR, USA

Detecting polycyclic Erysiphe necator, the causal agent of grape powdery mildew, in the field visually before it becomes a management problem can take trained scouts hours and yet there is still a low probability of detecting disease early in the epidemic. Impaction spore samplers have been used for early season detection and as a management decision aide but are expensive to operate. The use of cotton swabs to collect $E$. necator spores deposited on vineyard workers gloves (glove swab) during canopy manipulation was tested and compared with spore samplers during the 2019 growing season. Twelve vineyards in the Willamette Valley of Oregon were sampled 1 to 4 times between May and August. A glove swab sample consisted of a worker walking down the vineyard row, rustling the canopy, and rubbing the cotton swab over the hands of the worker and placing the swab in a tube. Samples were collected from the row with the spore trap and from two neighboring rows. Samples were subsequently analyzed using a TaqMan qPCR assay specific for $E$. necator (Thiessen et al. 2016). Comparison of each glove swab collected $(n=95)$ to its associated air sample from the same time period and location had $69.4 \%$ agreement and $91.3 \%$ sensitivity. When glove swabs within a block were aggregated $(\mathrm{n}=31)$, There was $81.3 \%$ agreement with $89.7 \%$ sensitivity. These results indicate that glove swabs may be a viable alternative to spore samplers for monitoring $E$. necator in vineyards, particularly given the significant reduction in costs, specialized materials, and time for swab collection and processing.

Isolation of fungal DNA from large soil samples using a $\mathrm{Fe}_{3} \mathrm{O}_{4}$ super paramagnetic nanoparticle extraction method E. BRANCH (1), S. J. Pethybridge (2), (1) Cornell University, Ithaca, NY, USA; (2) Cornell University, Geneva, NY, USA

Rhizoctonia solani AG 2-2 is an economically important soilborne pathogen in a broad range of agricultural systems resulting in crop loss through damping-off and reduced plant populations, reduced root volume, and lower quality roots. Disease caused by $R$. solani is often controlled by in-furrow fungicides without knowledge of inoculum densities and disease risk. Pre-plant DNA in the soil prior to planting may inform inoculum density and disease risk. Currently, most commercially available DNA extraction kits start with up to $250 \mathrm{mg}$ of soil. These small sample sizes limit their utility as they are unable to effectively capture soilborne plant pathogen populations due to high spatial heterogeneity. This project used enzymatic detergent lysis and super paramagnetic iron oxide nanoparticles (SPION) to isolate DNA from 25, 50, 75, and $100 \mathrm{~g}$ soil samples. After a polyvinylpolypyrrolidone (PVPP) purification step, the eluted DNA was compared to $R$. solani DNA extracted using commercial kits starting with $0.25 \mathrm{~g}$ and $10 \mathrm{~g}$ of soil. Conventional and SPION extraction methods produced similar quantities and qualities of DNA. Amplification using an AG group-specific primer confirmed the presence of R. solani AG 2-2. Bovine serum albumin (BSA) was used to bind PCR inhibitors. When coupled with DNA quantification techniques, this SPION extraction method may reduce variability and cost while supporting a platform for risk-based disease management.

Examining drivers of putting green rhizosphere bacterial communities

J. DOHERTY (1), J. A. Roberts (2), (1) University of Maryland, College Park, MD, USA; (2) Clemson University, SC, USA 
Putting greens have diverse rhizosphere microbiota and are susceptible to a number of root rot diseases. Little is known how these diseases or disease control applications alter the microbiota. To that end, a field study was implemented to examine the influence of root rot treatments on putting green rhizosphere microbiota. Field trials were established creeping bentgrass [Agrostis stolonifera L. (CBG)] at the Paint Branch Turfgrass Research Facility (i.e., sand-based root-zone and "T-1" CBG) and at Andrews Air Force Base (i.e., USGA sand-based root-zone and "Dominant" CBG). Treatments examined fungicides (cyazofamid and fosetyl-Al), a biofungicide (Bacillus subtilis QST-713), and combinations of a UMD experimental that were arranged in a RCBD with 4 replications and applied on a $14 \mathrm{~d}$ interval. On 19 July 2018 four $2.5 \mathrm{~cm}$ diameter soil cores were collected and homogenized to form a representative sample for each plot. DNA was extracted from each sample, PCR amplified using 16S primers, and sequenced on an Illumina MiSeq. Sequence data were analyzed using R. Non-metric multidimensional scaling showed strong clustering by location. Permutational multivariate analysis of variance showed significant differences between communities at each location. There were 50 differentially abundant taxa between locations. Within each location, bacterial communities were not different between treatments. These preliminary results indicate that location exhibits stronger influence on putting green rhizosphere microbiota than disease control treatments.

Spatio-temporal scales of soil bacterial communities in conventional and no-till wheat fields

D. C. SCHLATTER (1), J. Hansen (2), D. Huggins (3), B. Carlson (4), I. Leslie (5), T. C. Paulitz (6), (1) USDA-ARS, Wheat Health, Genetics and Quality Research Unit, Pullman, WA, USA; (2) USDA ARS, Northwest Sustainable Agroecosystems Research Unit, Pullman, WA, USA; (3) USDA-ARS, Northwest Sustainable Agroecosystems Research Unit, Pullman, WA, USA; (4) USDA-ARS, Northwest Sustainable Agroecosystems Research Unit, Pullman, WA, USA; (5) USDA-ARS Northwest Sustainable Agroecosystems Research Unit, Pullman, WA, USA; (6) USDA ARS, Pullman, WA, USA

Microbial community composition and diversity can be highly variable in both space and time. Tillage practices can impact the spatial distribution of microbial communities via redistribution of soil particles and burying plant residues. Thus, the spatial and temporal dynamics of soil microbial communities are likely to differ between conventionally tilled and no-till fields. We characterized the spatial and temporal variability of soil bacterial communities in paired fields managed with no-till or conventional tillage at the Cook Agronomy Farm Long-Term Agricultural Research site in Pullman, WA. Small-scale spatial variability in bacterial communities was evaluated in each field in March and then temporal trends were tracked over 11 time points from March to November at two depth increments $(0-10 \mathrm{~cm}$ and $10-20 \mathrm{~cm})$ using amplicon sequencing. We found that bacterial communities differed significantly among depths and between no-till and tilled fields at both depths but had similar dispersions of communities within fields. Some abundant bacterial taxa tended to follow similar temporal dynamics throughout the season in both no-till and conventional fields (eg. Planctomycetaceae, Sphingomonadaceae, Oxalobacteraceae, Burkholderiaceae), with higher abundance in the spring and fall, and lower abundance in the summer under dry soil conditions. Other groups demonstrated different temporal patterns under different tillage systems (eg. Chitinophagaceae, Caulobacteraceae, Gaiellaceae). These results suggest that temporal patterns of abundance may be affected by tillage.

\section{Effect of temperature and moisture on survival of Calonectria pseudonaviculata or $C$. henricotiae in boxwood tissue and as microsclerotia N. SHISHKOFF (1), M. Camp (2), (1) USDA ARS FDWSRU, Frederick, MD, USA; (2) USDA, MD, USA}

When leaves or twigs of boxwood infected with Calonectria pseudonaviculata (3 isolates) or C. henricotiae ( 1 isolate) were incubated in sand at two moisture levels (carrying capacity at 36\% water v:v and sand at 5\% water v:v) and at 5 temperatures $(-10,0,10,20$, and $30^{\circ} \mathrm{C}$ ) for $12 \mathrm{mo}$, sporulation from monthly tissue samples plated on GYET media declined significantly faster at -10 and $30 \mathrm{C}$ (reaching zero in 2-3 months) compared to 0,10 and $20 \mathrm{C} \mathrm{(3-12} \mathrm{months)} \mathrm{and} \mathrm{in} \mathrm{drier} \mathrm{sand} \mathrm{compared} \mathrm{to} \mathrm{moist} \mathrm{sand.} \mathrm{For} \mathrm{infected} \mathrm{twigs,}$ there was a small but significant difference in survival by individual isolate, but there was no significant difference by Calonectria species. Survival of the pathogen in twigs showed an interaction of temperature and moisture, with twigs incubated in dry sand at 0 , 10 , and $20 \mathrm{C}$ surviving more poorly than twigs in moist sand. Analysis of survival for discrete microsclerotia of Cps isolates grown on cellophane sheets showed significant differences in survival by temperature, moisture level, isolate, temp $\times$ moisture and temp $\times$ isolate, but not by species. Overall, this confirmed the persistent nature of Calonectria in soil.

Brief report of Gentian Kobu-sho associated virus in peony

C. SHAFFER (1), D. Michener (2), N. B. Vlasava (3), I. E. Tzanetakis (1), (1) University of Arkansas, Fayetteville, AR, USA; (2) University of Michigan, Ann Arbor, MI, USA; (3) Central Botanical Gardens NAS, Minsk, BELARUS

Peony (Paeonia lactiflora Pall.) is a highly coveted ornamental plant known for its large fragrant flowers. In the early 1900's, Lemoine's disease was described in peony (LDP) where the roots became gnarled by large galls that resulted in flowerless and stunted plants. The causal agent of LDP is unknown, yet anecdotally it is spread through pruning. During our investigations to determine whether LDP is caused by a virus, Gentian Kobu-sho associated virus (GKaV) was detected in two LDP symptomatic plants. GKaV is of particular significance because it is associated with tumor formation in ornamental gentian (Gentiana triflora Pall. and G. scabra Bunge) and is spread in the field in a pattern characteristic of soilborne pathogens. Using the peony isolate genome and others available on GenBank, primers were designed and used to screen 144 samples from the University of Michigan Nichols Arboretum Peony Garden. GKaV was found in 18/32 confirmed stunted plants and only found in 8 of the remaining plants. Further work, such as fulfilling Koch's postulates and virus epidemiology is needed to determine whether GKaV causes LDP in certain genotypes and to determine its potential impacts on other hosts. 
Screening of rootstock population for Grapevine leafroll associated virus-1

N. BUZKAN (1), A. Walker (2), (1) Kahramanmaras Sutcu Imam University, Kahramanmaras, TURKEY; (2) University of California, Davis, CA, USA

Grapevine leafroll disease is the most widespread and causes reductions in yield and quality in Vitis vinifera. Several serologically distinct members are associated with the disease. Grapevine leafroll-associated virus 1 (GLRaV-1) is one of the most important types worldwide. Its economic impact is still poorly understood, as is the implications of virus-resistant or -tolerant rootstock use. The breeding team at Department of Viticulture and Enology, University of California, Davis has produced seedling populations (08-180) from crosses of tolerant (St. George) and susceptible (Freedom) rootstocks that can lay the foundation for breeding to incorporate tolerance into new rootstocks. Budsticks of Cabernet franc vines with two different strains of GLRaV-1 (LR131, LR132) were benchgrafted onto 29 genotypes in 08-180 population and kept in greenhouse to evaluate the symptoms. ELISA test was performed to monitor virus accumulation starting from 3 month post grafting (MPG) up to one year. Leafroll symptoms were recorded in 6 and $7 \mathrm{mpg}$ for LR131 and LR132, subsequently. The symptoms were rated from 0 (no-symptom) to 4 (very severe). None of the genotypes were killed by both isolates. In general, the symptom rating on the majority of the plants with isolate LR131 was between 2 and 3 . The isolate LR132 was showing less severe symptoms (rating of 2) although the isolate was co-infected with GVA. The LR131 accumulation was high in $50 \%$ of the genotypes when first symptoms appeared, then remarkably dropped while LR132 accumulation always remained low compared to positive controls.

Impact of cropping history on seedling diseases causing fungal and phytopathogenic nematode counts in soil D. K. WEERASOORIYA (1), A. Y. Bandara (1), M. R. Duffeck (1), A. Murillo-Williams (2), A. A. Collins (3), P. Esker (1), (1) The Pennsylvania State University, University Park, PA, USA; (2) Penn State Extension-Centre County, Bellefonte, PA, USA; (3) Southeast Agricultural Research \& Extension Center, Manheim, PA, USA

Soilborne fungal pathogens and phytopathogenic nematodes cause significant yield losses in row crops. Bulk soil samples were collected from 21 soybean farms in Pennsylvania at V1 (one trifoliate) growth stage in 2018 to examine how cropping history affect fungal/nematode counts. Farms had different cropping histories in terms of cultural practices like crop rotation, cover crops, tillage, and manure application. Fusarium, Rhizoctonia, Pythium, and Phytophthora colonies (CFU/g soil) were enumerated using soil serial dilutions on standard selective media. Lesion, spiral, and lance were the predominant nematode types found across soil samples. At $\alpha=$ 0.1, Fusarium and lance nematode counts were not significantly affected by cultural practices. Tilled soil contained greater Rhizoctonia counts than non-tilled soil. In the presence of a cover crop, corn/soybean/corn (CSC) resulted in greater Rhizoctonia and Pythium counts than non-CSC rotations. In the absence of cover crops, soil receiving manure had greater Phytophthora counts than non-manured soil, while manure and CSC rotation resulted in lower lesion nematode counts. Soil with manure application contained greater Pythium counts than non-manured soil. Phytophthora count was greater under CSC than non-CSC rotations. Under non-CSC rotations, nonmanured and non-tilled soil contained lower spiral nematode count compared to manured and tilled soil. Our results suggest how cultural practices could be manipulated to reduce harmful fungal and nematode counts in soil.

The effects of Rhizoctonia solani on the growth of alfalfa

J. QU, Lanzhou University, Lanzhou, China, CHINA

Rhizoctonia solani (R. solani) is widespread in the soil and can cause great harm to plants such as alfalfa (Medicago sativa). In this study, R10 and R18 strains of R. solani and four different alfalfa varieties, which included Algonquin, GoldenQueen, Aribou and Salttolerant star, were selected to study the pathogenicity of $R$. solani on alfalfa. The four alfalfa seedlings were transplanted into the inoculated soil, and after three weeks of cultivation, statistical analysis of root neck disease level, plant height, root length and biomass was performed. The study results showed that: the root neck disease index of four varieties inoculated with R18 was significantly higher than that inoculated with R10 $(\mathrm{P}<0.05)$; the reduction in plant height and root length of all varieties after inoculation with $\mathrm{R} 18$ was greater than that with R10; after inoculation with R18, the underground biomass and above-ground biomass of all varieties were reduced by $100 \%$, except for GoldenQueen, they were significantly higher than the biomass reduction rate after inoculation with $\mathrm{R} 10$ (P $<0.05$ ). In conclusion, $R$. solani R18 is significantly more harmful to alfalfa seedlings than R10. The study results provide a basis for the prevention and treatment of diseases caused by $R$. solani.

Effect of planting into a green winter cereal rye cover crop on growth and development, seedling disease and yield of corn J. N. ACHARYA (1), T. Moorman (2), T. Kaspar (3), A. Lenssen (4), S. Gailans (5), A. E. Robertson (6), (1) Iowa State University, Department of Plant Pathology, Ames, IA, USA; (2) National Laboratory for Agriculture and the Environment (USDA-ARS-NLAE), Ames, IA, USA; (3) National Laboratory for Agriculture and the Environment, Ames, IA, USA; (4) Iowa State University, Department of Agronomy, Ames, IA, USA; (5) practical farmers of Iowa, Amea, IA, USA; (6) Iowa State University, Ames, IA, USA

We recommend farmers in Iowa terminate a winter cereal rye (Secale cereale L.) cover crop 10 or more days before planting corn to mitigate seedling disease and potential yield loss. In some years, however, cold temperatures and frequent precipitation prevents farmers from following our recommendation and are forced to plant corn immediately after or before terminating rye while the rye plants are still green, a practice commonly referred to as planting green (PG). A field trial was established to evaluate the effect of delaying termination of rye until shortly before or after corn planting on corn growth, development, seedling root disease, and yield of corn in 2019. A cover crop of rye was terminated 18 and 3 days before planting (DBP), and 6 and 12 days after planting (DAP) corn; corn planted following no cover crop was included as a control. Greater radicle disease incidence was observed when rye was terminated 3 DBP, 6 DAP, and 12 DAP corn compared to 18 DBP treatment and the no cover crop control. In general, the population of Pythium clade $\mathrm{B}$ detected in the radicle and seminal roots of corn seedlings sampled at V3 was greater when winter rye was terminated 6 or more 
DAP corn compared to the treatments where the rye was terminated $18 \mathrm{DBP}$ or $3 \mathrm{DBP}$ corn and the no cover crop control. All rye treatments reduced corn yield, with the lowest yield recorded in the treatment in which rye was terminated 12 DAP $(P<0.01)$. Our data suggest that PG increased corn seedling root disease caused by Pythium Clade B population and this may have contributed to reduced corn yield.

\section{A reevaluation of sulfur phytotoxicity in suppression of strawberry powdery mildew}

R. BORBA ONOFRE (1), G. Alonzo (1), D. M. Gadoury (2), N. Peres (1), (1) University of Florida - Gulf Coast Research and Education Center, Wimauma, FL, USA; (2) Cornell University, Geneva, NY, USA

Sulfur is a multi-site inhibitor of strawberry powdery mildew (Podosphaera aphanis) recommended for fungicide resistance management, yet growers may refrain from its use due to purported phytotoxicity under conditions often qualitatively defined as "hot", or "during periods of high temperature". Our objectives were to: (i) determine phytotoxicity of sulfur on three strawberry cultivars treated in controlled environments at temperatures of 30,35 and $40^{\circ} \mathrm{C}$ for periods of $1,2,4$, and $8 \mathrm{~h}$ after the sulfur application; and (ii) evaluate phytotoxicity and efficacy of sulfur on open-field and high-tunnel grown strawberries in Florida. Under controlled conditions, 'Florida 127' and 'Florida Beauty' exhibited minor sulfur-related phytotoxicity when exposed to at $30^{\circ} \mathrm{C}$ for 2 to $8 \mathrm{~h}$. 'Florida Radiance', however, was more sensitive, with greater phytotoxicity as exposure time increased. We found that weekly application of sulfur on cv. Florida 127 significantly reduced $P$. aphanis foliar and fruit disease as well or better than the standard fungicide treatment, and resulted in $80 \%$ to $300 \%$ higher yields (compared to non-treated controls) in open field and tunnel trials, respectively. Ambient temperature did not exceed $35^{\circ} \mathrm{C}$ in the field, where no phytotoxicity was observed. In the tunnel, where only mild leaf phytotoxicity was observed, temperature exceeded $40^{\circ} \mathrm{C}$ for a total of $20 \mathrm{~h}$ across 9 days. Our results suggest that sulfur has benefits for disease suppression and fungicide resistance management, and that phytotoxicity is minimal and does not affect yield at the above temperatures.

Evaluation of stink bug damage, Phomopsis infection, and grain quality of new soybean germplasm J. FOMBA (1), M. Tomaso-Peterson (2), T. Allen (3), D. Cook (4), J. R. Smith (5), S. Li (6), T. Wilkerson (7), (1) Mississippi State University, Stoneville, MS, USA; (2) Mississippi State University, Mississippi State, MS, USA; (3) Delta Research and Extension Center, Mississippi State University, Stoneville, MS, USA; (4) Delta Research Center \& Extension, Stroneville, MS, USA; (5) USDAARS, Stoneville, MS, USA; (6) USDA ARS CGRU, Stoneville, MS, USA; (7) MSU Department of Biochemistry, Molecular Biology, Entomology, \& Plant Pathology, Stoneville, MS, USA

Stink bugs, Hemiptera:Pentatomidae, are one of the most economically important insect pests of soybean (Glycine max) in the midsouthern U.S. Generally, green (Chinavia hilaris), southern green (Nezara viridula), and brown (Euschistus servus) stink bugs affect Mississippi soybean. Stink bugs attack soybean during late reproductive growth stages by feeding directly on developing seed through the pod wall, which extracts nutrients resulting in yield losses, shriveled seed, and reductions in grain quality. Wounds resulting from stink bug feeding can provide an entrance for important plant pathogens such as Phomopsis longicolla (PL), which causes Phomopsis seed decay (PSD). PSD can reduce soybean grain quality and produce symptoms including shriveled, elongated, cracked, and chalky kernels. The objective of this research was to determine the relationship between stink bug feeding and impact on seed infection by PL. In 2019, soybean plots were established consisting of three commercial PSD-susceptible checks and 18 new germplasm lines as single row plots, $3.6 \mathrm{~m}$ long replicated three times. Screened field cages surrounding individual plots were infested with adult and nymph stink bugs for 12 days following inoculation with a PL spore suspension at R5. Plots were harvested 2 weeks post-R8 with a one-row combine. Yield, visual damage, and feeding injury levels were evaluated. No differences were observed between yield ( $\mathrm{p}=0.0690)$, damage $(p=0.3438)$, or injury level $(p=0.1324)$; however, $65-414-132-1$ provided up to $45 \%$ less damage when compared to the susceptible checks.

\section{Genomic investigation of the Alternaria infectoria species group highlights potential pathogenicity of isolates collected during saprophytic growth \\ M. FULCHER (1), A. Taranto (2), P. Solomon (2), G. C. Bergstrom (3), (1) University of Minnesota, Saint Paul, MN, USA; (2) Australian National University, Canberra, AUSTRALIA; (3) Cornell University, Ithaca, NY, USA}

Members of the Alternaria infectoria species group are globally distributed and inhabit diverse ecological niches. Several species are known pathogens. Using closely related isolates, a comparative genomics approach is being taken to understand the basis for and evolution of pathogenicity in this group. A global collection of 15 type cultures, plus one isolate recently reported to cause a leaf spot of wheat in New York, were whole genome sequenced, resulting in $32.5-37.5 \mathrm{Mb}$ assemblies. Biosynthetic gene clusters coding for 15 known and at least 16 unknown secondary metabolites were predicted (antiSMASH), but these metabolites do not appear to explain virulence patterns within the group. Genomes were searched for known effectors (Phi-base). Thirteen isolates, representing both known pathogens and presumed saprophytes, contained a homolog of MoCDIP4 (Magnaporthe oryzae), known to cause plant cell death and to be present in other pathogenic fungi. Primary metabolic diversity was compared through genome modeling, and no pathways or profiles were linked to pathogenicity. Ongoing phylogenetic analysis with 36 related genomes examined species delimitation in this group, diversity at the mating type loci of isolates with known teleomorphs, and transposable elements common to Dothideomycetes. Our results so far have not uncovered a clear basis for differentiating lifestyles in this group but may suggest host and environmental factors strongly influence the incidence of diseases attributed to these species.

Impacts of artificial media on Phytophthora infestans virulence and effector expression E. LARSON (1), D. A. Halterman (2), A. J. Gevens (1), (1) University of Wisconsin-Madison, Madison, WI, USA; (2) USDA ARS, Madison, WI, USA 
Continuous sub-culturing of Phytophthora infestans on artificial media and revitalization of cultures through host plant tissues are standard laboratory practices. We investigated the virulence of $P$. infestans after continued sub-culturing on artificial media and analyzed changes in effector expression within the pathogen at select stages. We also investigated the effect of continued 'passage' through a host plant on pathogen virulence. $P$. infestans (US-8) was sub-cultured (6X) and maintained at $18^{\circ} \mathrm{C}$ on solid Rye-A medium. The $0,3^{\text {rd }}$, and $6^{\text {th }}$ sub-cultured isolates were used to inoculate late blight-susceptible potato plants and disease severity was evaluated. The area under the disease progress curve (AUDPC) significantly decreased from the 0 to $6^{\text {th }}$ sub-culture event $(p=0.0006)$, but not between the 0 and $3^{\text {rd }}$. RNA from infected tissue was used to assess effector expression. At 72 hours post inoculation, the relative expression of effector Avr3 was significantly reduced roughly 5-fold from the 0 sub-culture event to the $3^{\text {rd }}$. In testing the full range of sub-cultures (2-6) a significant reduction in AUDPC was observed between the $2^{\text {nd }}$ and $3^{\text {rd }}$ sub-culture $(\mathrm{p}<0.0001)$ with no further significant reductions. Continual passage through a potato host was found to decrease virulence, evidencing that the sub-culturing between each passage had a greater deleterious effect on virulence than any benefit conferred by re-inoculation onto a host. The impacts of sub-culturing on $P$. infestans virulence was rapid, highly significant, and poorly attenuated by re-infection on a host plant.

\section{Characterization of multiple dsRNA mycoviruses of Trichoderma harzianum NFCF305 and evidence for the related antifungal activity \\ B. NA (1), J. Chun (2), D. H. Kim (2), (1) Jeonbuk National University, Jeon-ju, SOUTH KOREA; (2) Jeonbuk National University, Jeonju, SOUTH KOREA}

We reported the 15 agarose gel band patterns of double-stranded RNA (dsRNA) from Trichoderma spp. Here, we describe that band pattern XV in Trichoderma harzianum NFCF305, which appeared to be co-infected with three mycoviruses of Macrophomina phaseolina hypovirus 1, Fusarium poae alternavirus 1, and Alphapartitivirus. Through repetitive single-spore isolation, we were able to cure mycoviruses, which resulted in a virus-free strain and strains with Macrophomina phaseolina hypovirus 1 or Fusarium poae alternavirus 1 and Alphapartitivirus. Regardless of mycovirus infections, strains did not show any significant difference in growth rate, colony morphology, and conidia production. However, the $\beta$-1,3-glucanase activity was increased in the strain with double infection of Fusarium poae alternavirus 1 and Alphapartitivirus. Moreover, growth inhibition of Rhizoctonia solani in media supplemented with culture-filtrate of Trichoderma harzianum NFCF305 was increased in the Fusarium poae alternavirus 1 and Alphapatitivirus-infected strain compared with other strains. Interestingly, virus-free strain and strains infected with all three or Macrophomina phaseolina hypovirus 1 did not show any difference in $\beta$-1,3-glucanase activity and growth inhibition. These results demonstrate that not all virus infection affected the fungal enzyme activity but specific combination of virus infection did. It will be of an interest to obtain strains infected with Fusarium poae alternavirus 1 or Alphapartitivirus.

Optimizing virus-based systemic expression of biologically active Clostridium perfringens phage endolysins in plants R. W. HAMMOND (1), P. Abrahamian (1), S. Swift (2), N. Kovalskaya (3,4), D. Donovan (2,5), (1) USDA ARS Molecular Plant Pathology Laboratory, Beltsville, MD, USA; (2) USDA ARS NEA BARC ABBL, Beltsville, MD, USA; (3) USDA ARS USNA FNPRU, Beltsville, MD, USA; (4) ORISE, Oak Ridge, TN, USA; (5) Morgan State University, Baltimore, MD, USA

Clostridium perfringens, a gram-positive, anaerobic, rod-shaped bacterium and the third leading cause of human foodborne bacterial disease and the cause of necrotic enteritis in poultry, is controlled using antibiotics, widespread use of which may lead to development of drug-resistant bacteria. Bacteriophage-encoded endolysins that degrade peptidoglycans in the bacterial cell wall are potential replacements for antibiotics. Phage endolysins have been identified that exhibit antibacterial activities against several Clostridium strains. Escherichia coli codon-optimized genes, including those encoding the glycosyl hydrolase endolysin $P l y C P 41$ and the chimeric, thermostable amidase endolysin PlyGVE2 $2_{c a t} C P 18 \mathrm{cwb}$, were engineered for expression in Nicotiana benthamiana plants using a agroinfiltration of a potato virus X-based transient expression vector. These endolysins accumulated to $\sim 1 \%$ total soluble protein without any obvious toxic effects on plant cells, and both the purified proteins and plant sap containing the proteins lysed $C$. perfringens strain Cp39 in a plate lysis assay. Optimal systemic expression of PlyCP41 and PlyGVE2 cat-CP18 cwb in plants was achieved at 2 weekspost-infection and the virus constructs were stable after passaging, contributing to our knowledge of plant viral genome expression. Our results demonstrate that functionally active bacteriophage endolysins can be produced in systemically infected plant tissue with potential for use in feed as an effective antimicrobial agent against $C$. perfringens.

\section{Fusarium cotton wilt pathogen Fusarium oxysporum f. sp. vasinfectum race 4 (FOV4) supports a diverse aerobic and anaerobic} bacterium on its hyphae

S. ANTONY-BABU (1), A. Dasgupta (2), E. Boak (3), E. A. Pierson (1), (1) Texas A\&M University, College Station, TX, USA; (2) Bidhan Chandra Krishi Viswavidyalaya, Mohanpur, INDIA; (3) Texas A\&M University, Kenosha, WI, USA

Fungal-bacterial interactions are central to a wide range of agriculturally relevant environmental processes. The key to understanding this interkingdom interaction is to decipher the microbiomes in the immediate vicinity of the fungal propagules. This dynamic interface, termed the mycosphere, is the fungal-bacterial interactions hotspot. In this study, we used an amplicon metabarcoding metagenomic guided approach to isolate a diverse bacterial community that is associated with mycosphere of Fusarium oxysporum f. sp. vasinfectum race 4 - Fov4. Our study shows that Fov4 supports the nutrient requirements of Actinobacteria, Bacillaceae, Enterobacteriaceae, Pseudomonadaceae and Rhizobiaceae on its hyphae. Interestingly, the fungal pathogen maintains anaerobic bacteria even when cultured under aerobic conditions. The functional implications of the pathogen's microbiome, a pathobiome, will be discussed. 
Incidence, distribution, and pathogenicity of fungi causing root rot on the top surface of long-term outdoor sugar beet storage piles in Idaho

C. A. STRAUSBAUGH, USDA ARS NWISRL, Kimberly, ID, USA

Sugar beet roots in Idaho are held in outdoor storage piles under ambient conditions which can lead to substantial sucrose losses and fungal root rot. The fungal rots associated with roots held inside piles has been recently established, but the incidence, distribution, and pathogenicity of fungi associated with root rot on the top surface has not been determined. Therefore, the fungal rot on the top surface of 14 Idaho sugar beet piles [tarped ventilated (TV) piles and piles with no tarps or ventilation (NTV) at 7 locations] was assessed in 9 one-square meter areas per pile using a stratified random sampling design in 2018-19 and 2019-20. Pathogenicity was evaluated through sugar beet root plug inoculations conducted in commercial storage buildings. Cladosporium spp. were the only fungi covering more than $1 \%$ of the root surface ( 2 to $48 \%$ ) on top of NTV piles both years. The surface of roots after tarp removal on TV piles was also dominated by Cladosporium spp. (13 to 60\%), but Penicillium spp. (0 to 35\%), an Athelia-like basidiomycete ( 0 to $2 \%$ ), and Botrytis cinerea ( 0 to $2 \%$ ) were also frequently present. NTV piles always had less fungal growth than TV piles regardless of variable. Roots placed on the pile first had more fungal growth than those in other areas based on Cladosporium spp., the Athelia-like fungus, and total fungal growth. In the plug assay, $B$. cinerea caused the most rot, while the Cladosporium spp. led to little or no rot. Thus Cladosporium spp. seemed to largely be opportunistic fungi despite being the most prolific on the pile top root surface.

Wheat streak mosaic virus virions contain four virus-encoded accessory proteins

S. PAL (1), S. Tatineni (2), (1) University of Nebraska-Lincoln, Lincoln, NE, USA; (2) USDA ARS, Univ of Nebraska, Lincoln, NE, USA

Wheat streak mosaic virus (WSMV; genus Tritimovirus; family Potyviridae) is an economically important virus on wheat in the Great Plains. In this study, we examined the content and composition of WSMV virions through mass spectrometry and Western blot using virions purified through $10-40 \%$ sucrose density gradient centrifugation. Mass spectrometric analysis of protein composition of purified WSMV virion preparation indicated that coat protein comprises $55-80 \%$ of total proteins in virion preparations. Surprisingly, virion preparations also contained four virus-encoded proteins as components of virions: HC-Pro (3-8\%), CI (0.8\%), P1 (0.8\%), and NIa-VPg (0.2-0.4\%). Western blot analysis confirmed the presence of CP, HC-Pro, and P1 as components of WSMV virions. Previously, we found that GFP was expressed as a fusion protein with HC-Pro in a GFP-tagged WSMV (WSMV-GFP)-infected wheat leaves.

Examination of purified virions from WSMV-GFP-infected wheat leaves revealed that virions also contained GFP as GFP-HC-Pro fusion protein. Additionally, purified virions from WSMV-GFP-infected leaves elicited fluorescence under a confocal microscope, suggesting that functional GFP was incorporated into WSMV virions as GFP-HC-Pro fusion protein. These data suggest that expression of specialty products on the surface of WSMV virions can be achieved by fusing the novel amino acid sequences to HC-Pro. Taken together, our data revealed that WSMV is unusual in incorporating four accessory proteins (HC-Pro, CI, P1, and NIa-VPg) along with $\mathrm{CP}$ (major protein) as components of virion.

\section{Discovery of two new viruses infecting blueberry}

D. E. V. VILLAMOR (1), A. Sierra (1), K. Keller (2), R. Martin (2), I. E. Tzanetakis (1), (1) University of Arkansas, Fayetteville, AR, USA; (2) USDA-ARS Horticultural Crops Research Unit, Corvallis, OR, USA

Recent assessment of the virus status of several blueberry genotypes revealed two new viruses belonging to the genera Luteovirus and Carlavirus. Genomic analyses revealed that the new luteovirus is related to nectarine stem pitting-associated virus whereas the new carlavirus closely resembles blueberry scorch virus. A survey to determine the distribution of these viruses focused on major production areas, blueberry germplasm repository, breeding programs, and nurseries. Results revealed presence of the novel luteovirus in all sites whereas testing is underway for the novel carlavirus. The population structure of these viruses is being investigated to develop sensitive protocols that allow detection of majority, if not all isolates of each virus that circulate in all agricultural systems. These protocols improve certification standards and consequential release of high quality virus-tested plants, thereby safeguarding the sustainability of one of the major berry crops of the country.

Prospects for areawide management of tomato yellow leaf curl virus

W. W. TURECHEK (1), S. T. Adkins (2), D. J. Anco (3), H. C. Mellinger (4), C. S. Kousik (5), P. D. Roberts (6), (1) USDA, ARS, U.S. Horticultural Research Laboratory, Fort Pierce, FL, USA; (2) USDA ARS USHRL, Fort Pierce, FL, USA; (3) Clemson University, Blackville, SC, USA; (4) Glades Crop Care Inc, Jupiter, FL, USA; (5) USDA ARS, Charleston, SC, USA; (6) University of Florida, Immokalee, FL, USA

Tomato yellow leaf curl virus (TYLCV) has become an economic problem for tomato production in the southeastern U.S. since its first detection in Florida in the late 1990s. Current management strategies for TYLCV - and its whitefly vector - rely on farm-centric strategies that have had limited success. Our recent spatiotemporal analysis indicated that management failures may be related to the areal extent that populations of whitefly and TYLCV occupy and biologically respond to over the agricultural landscape of southwest Florida, coupled with the highly favorable environmental conditions for epidemic development. Areawide pest management (AWPM) may be a viable management alternative because it directly addresses the spatial disparity between the expansive habitat occupied by the breeding populations of the insects and the relatively small management unit (e.g., a field). A single grower's field occupies an area that is considerably smaller than the area occupied by the breeding pest population; the targeted population usually spans multiple farms. Since farm managers do not often consult each other when making pest-management decisions, asynchronous execution of control strategies among farms - including the application of insecticides - is commonplace. This leads to only a portion of the pest 
population being treated at any given time, and rapid re-infestation of the crops by the insects from non-treated areas. In this talk, we provide a data-driven argument on how an AWPM might be implemented for management of TYLCV.

Genetic requirements for seedling colonization by Xanthomonas citri pv. malvacearum after cotton seed infection J. MIJATOVIC (1), R. Kemerait (2), R. R. Walcott PhD (3), B. H. Kvitko (4), (1) UGA Plant pathology, ATHENS, GA, USA; (2) University of Georgia, Tifton, GA, USA; (3) The University of Georgia, Athens, GA, USA; (4) University of Georgia, Athens, GA, USA

The re-emergence of bacterial blight of cotton, caused by Xanthomonas citri pv. malvacearum (Xcm) revealed many gaps in our understanding of this pathosystem. A shift in the proportion of US fields planted with susceptible cotton cultivars potentially explains recent epidemics. Infested cotton seeds can be an important source of Xcm inoculum. Our goal is to elucidate the process by which seedto-seedling transmission of $X \mathrm{~cm}$ occurs. We employed a Georgia field isolate of $\mathrm{Xcm}$ as a wildtype (WT) strain to generate a nonpathogenic, $h r c V$ mutant lacking a functional Type III secretion system (T3SS-). We tagged the WT and T3SS- strains with an autobioluminescent $\mathrm{Tn} 7$ reporter to compare colonization of susceptible and resistant cotton hosts using macroscopic imaging analysis and bacterial population estimates. WT and T3SS- Xcm strains colonized cotton cotyledons of resistant and susceptible cotton cultivars. However, Xcm populations were significantly higher in susceptible seedlings inoculated with the WT strain. Additionally, WT and T3SS- Xcm strains systemically colonized cotton true leaves, although at different rates. Finally, our data suggests that seed-to-seedling transmission of $\mathrm{Xcm}$ involves systemic dissemination through the vascular tissue of cotton plants. These findings yield novel insights into potential $\mathrm{Xcm}$ reservoirs.

\section{Black Rot of crucifers in New York State and the role of transcription activator-like effectors} Z. DUBROW (1), C. Audran (2), L. Noel (3), B. Szurek (4), H. W. Lange (5), C. D. Smart (6), G. Blanquet (7), I. Fuentes (8), A. J. Bogdanove (1), S. Carpenter (1), (1) Cornell University, Ithaca, NY, USA; (2) CNRS-INRA, UMR LIPM, Castanet-Tolosan, FRANCE; (3) LIPM Laboratoire des Interactions Plantes-Microorganismes UMR CNRS/INRA 2594/441, Castanet-Tolosan, FRANCE; (4) IRD, CIRAD, Université Montpellier, IPME, 34000 Montpellier, FRANCE; (5) Cornell University, Geneva, NY, USA; (6) Plant Pathology and Plant-Microbe Biology Section, Cornell AgriTech, Geneva, NY, USA; (7) CNRS-INRAE, Castanet-Tolosan, FRANCE; (8) CNRSINRAE, FRANCE

Xanthomonas campestris pv. campestris (Xcc) causes black rot of crucifers, the most important cabbage disease worldwide. We built a collection of Xcc strains isolated between 2004 and 2016 in New York to test varietal susceptibility and strain aggressiveness in field trials. There is no known major gene resistance to black rot in commercial cabbage varieties. Understanding of cabbage susceptibility is vital and can be gained via study of pathogen virulence mechanisms. Transcription activator-like effectors (TALEs) are a large family of type III secreted effectors present in some Xanthomonas species. They specifically target and directly upregulate plant susceptibility (S) genes. By PCR, and western and Southern blotting, we surveyed 124 New York Xcc isolates from our collection and found that 30\% express TALEs. Assays of TALE knockout strains in cauliflower revealed that across strains, different TALEs contribute to virulence. Long read sequencing of 20 Xcc isolate genomes identified TALE repeat sequences, allowing us to computationally predict TALE binding sites in cabbage and Arabidopsis promoteromes. In addition, we compared transcriptome profiles of cabbage inoculated with an Xcc strain containing cloned TALEs or an empty vector to determine TALE specific gene upregulation. Intersecting these computational and experimental data sets resulted in S gene predictions that we are testing via qRT-PCR and designer TALE effectors that target the candidate $\mathrm{S}$ genes. Identification of S genes could inform breeding of brassica crops for reduced susceptibility to black rot.

Identification of clade-specificity in the effector genes contributing to the virulence of two Erwinia tracheiphila clades O. I. OLAWOLE (1), M. L. Gleason (2), G. A. Beattie (2), (1) Iowa State University (ISU), Ames, IA, USA; (2) Iowa State University, Ames, IA, USA

Strains of Erwinia tracheiphila (Et), causal agent of bacterial wilt of cucurbits, fall into clades. Strains of the Et-melo clade rapidly wilt muskmelon but do not wilt squash, whereas strains of the $E t-C 1$ clade rapidly wilt squash and less rapidly wilt muskmelon. Both clades expressed the effector gene eopl in each of these plant species but exhibited expression differences of other effector genes in planta. A combinatorial mutant analysis demonstrated that the effectors Eop1, DspE, AvrB4, and OspG were the primary effectors contributing to rapid muskmelon wilt by the Et-melo clade strain SCR3. Loss of eop1 or ospG by themselves did not alter SCR3 virulence, whereas loss of $a v r B 4$ detectably reduced virulence, and loss of $\operatorname{ssp} G$ in combination with either eop 1 or $d s p E$ reduced virulence. Pyramided SCR3 mutants lacking $d s p E$, eopl, osp $G$ and/or $a v r B 4$ illustrated additive effects of these effectors on virulence. A combinatorial mutant analysis of the $E t-C 1$ strain BHKY also identified effectors critical to virulence. Surprisingly, loss of hopOl from BHKY did not alter virulence on either muskmelon or squash despite that it was expressed at an exceptionally high level in both hosts; however, its loss in combination with eopl reduced virulence. Similarly, although hopL1 expression was specific to muskmelon, only its loss in combination with eopl reduced virulence. Collectively, our results highlight roles for Eop1, DspE, HopO1 and HopL1 in BHKY virulence, and thus illustrate that strains representing two Et clades rely on overlapping but distinct sets of effectors for virulence.

Using MAGIC against Tan Spot disease: How Multiparent Advanced Generation Intercross wheat lines respond to Pyrenophora tritici-repentis infection

L. CARVALHO FERREIRA (1), F. M. Santana (2), M. Beckmann (3), L. Mur (4), (1) Aberystwyth University, Aberystwyth, Ceredigion, UNITED KINGDOM; (2) Embrapa, Passo Fundo, BRAZIL; (3) Institute of Biology, Environmental and Rural Sciences at Aberystwyth University, Aberystwyth, UNITED KINGDOM; (4) Institute of Biology, Environmental and Rural Sciences, Aberystwyth University, Aberystwyth, UNITED KINGDOM 
Pyrenophora tritici-repentis (Ptr) causes the Tan Spot disease of wheat, a major foliar disease. It is mainly managed through chemical control due to the lack of reliable resistant cultivars. Herein we explore Multiparent Advanced Generation Intercross (MAGIC) wheat populations for new sources of resistance and to develop our understanding of the wheat-P. tritici-repentis pathosystem. Seedlings were inoculated with toxin toxA positive Ptr strains and disease progression was assessed over 14 days after inoculation (dai). The eight parental lines exhibited differential responses where "Robigus" and "Hereford" were the most resistant and susceptible, respectively. Fluorescence microscopy indicated Ptr penetrating through the epidermis and stomata at 1 dai, followed by intra-/intercellular mycelial growth and localized cell death (1-3 cells) in both genotypes. However, at 3 dai extensive superficial and subcuticular growth was seen in Hereford but not in Robigus. Our initial omic characterization focused on metabolomics, which indicated the mobilization of glycerophospholipids, amino acids and sugars in Hereford after 3 dai. Resistance in Robigus was not associated with the production of phenylpropanoids or anti-microbial polyketides suggesting a novel defense mechanism may be being deployed. These results will be integrated into transcriptomics data in the near future. This program illustrates how genotypically varied populations such as MAGIC can be exploited to define new sources of resistance against important emerging diseases.

Examining the effects of Agrobacterium rhizogenes and biocontrol Pseudomonas on hydroponic tomato yield C. CHAGAS DE FREITAS, C. G. Taylor, The Ohio State University, Wooster, OH, USA

Hairy root disease (HRD; aka crazy-root disease) caused by Agrobacterium rhizogenes is an emerging problem for the hydroponic greenhouse industry in North America. HRD has been a problem in Europe since the 1990s but there have been few measures adopted for its control outside of expensive sanitation practices. The purpose of this study is to determine the impact of HRD as well as the impact of two biocontrol strains of Pseudomonas on tomato yield. In the first set of experiments, yield loss was determined for plants inoculated with $A$. rhizogenes or water. In the second set of experiments, plants were inoculated with A. rhizogenes; or one of two strains of Pseudomonas (strain 1B1 or strain 93G8). In the third set of experiments plants were inoculated with $A$. rhizogenes only or on plants previously treated with Pseudomonas (strain 1B1 or 93G8). Each set of experiments were repeated three times in a randomized block design. Tomato fruits were harvested once a week for four weeks when the first fruits were fully ripened. In the first set of experiments, we were able to confirm that HRD has a negative impact on yield and fruit weight. Fruit number was reduced by $14 \%$ and fruit weight was reduced by $20 \%$. In the second set of experiments we found that the applied Pseudomonas did not negatively impact yield. The third set of experiments are currently ongoing. Our results demonstrate how certain Pseudomonas strains can be used to control HRD which could help growers better manage an important disease of hydroponically grown vegetables.

\section{National Plant Diagnostic Network protocols and validation committee: Facilitating technology transfer from researchers to diagnosticians}

J. WOODHALL (1), L. M. Rodriguez-Salamanca (2), M. Moll (3), R. A. Bomberger (4), S. Bec (5), E. A. Bush (6), J. H. LaForest (7), M. Rahman (8), K. Ong (9), J. D. Olson (10), D. E. Plewa (11), L. Miles (12), E. Grimme (13), K. C. Obasa (14), S. Fieweger (15), S. R. May (16), K. L. Snover-Clift (17), D. Mollov (18), (1) University of Idaho, Parma, ID, USA; (2) Iowa State University, Ames, IA, USA; (3) University of Idaho, Idaho Falls, ID, USA; (4) Washington State University, Pullman, WA, USA; (5) University of Florida, Gainesville, FL, USA; (6) Virginia Tech, Blacksburg, VA, USA; (7) Southern IPM Center/ UGA, Tifton, GA, USA; (8) West Virginia University, Morgantown, WV, USA; (9) Texas A\&M AgriLife Extension Service, College Station, TX, USA; (10) Oklahoma State University, Stillwater, OK, USA; (11) University of Illinois, Urbana, IL, USA; (12) Michigan State University, East Lansing, MI, USA; (13) Montana State Univ, Bozeman, MT, USA; (14) Texas A\&M, Amarillo, TX, USA; (15) Wisconsin Department of Agriculture, Madison, WI, USA; (16) The Pennsylvania State University, University Park, PA, USA; (17) Cornell University, Ithaca, NY, USA; (18) USDA, Beltsville, MD, USA

Detection and diagnosis play a vital role in plant health. Early detection of exotic plant pathogens can enable the implementation of effective quarantines and eradication activities to control infectious disease outbreaks. Likewise, the success of disease management strategies greatly depends on the accurate detection of native or endemic plant pathogens in production systems. A wide range of testing methods are potentially available for diagnosticians to enable timely detection and diagnosis. However, this potential range of tests is not always fully utilized by the diagnostic community. A lack of training, validation data, suitable equipment or other resources required to implement the test may explain why assays often fail to cross the divide between research and diagnostic labs. The work of the NPDN Protocols and Validation Committee seeks to determine suitable diagnostic protocols for NPDN uptake, establish best practices for method development and implementation, and undertake outreach efforts to promote best practices for diagnostic development and delivery. A key output of the committee will be Plant Diagnostic Guidelines (PDG). PDGs will recommend specific diagnostic protocols which will be supported by validation data. The committee will also publish these validated protocols to aid in the implementation of diagnostic methods. The committee also enables closer working relationships between researchers and diagnosticians, which will increase the acceptance of fit-for-purpose diagnostic methods.

Small RNA and degradome sequencing identified microRNA-target gene pairs regulating apple root defense responses towards Pythium ultimum infection

Y. ZHU (1), M. Saltzgiver (1), R. Xia (2), (1) USDA ARS Tree Fruit Research Lab, Wenatchee, WA, USA; (2) South China Agricultural University, Guangzhou, CHINA

Small RNAs (sRNAs) play a key role in diverse plant biological processes including defense response to pathogens. The current study is part of a comprehensive transcriptomic analysis of molecular defense responses in apple root from infection by the soilborne necrotrophic oomycete pathogen Pythium ultimum. Thirty-six sRNA libraries, encompassing six apple rootstock genotypes with or without pathogen challenge, were constructed and sequenced. The deep sequencing and in-depth bioinformatic analysis of sRNA 
population uncovered novel characteristics of apple root defense response between resistant and susceptible genotypes. MicroRNA (miRNA)-target gene pair identification by degradome sequencing indicated that a broad range of cellular processes participate in defense responses including metabolite transportation and hormone synthesis. Notably, genotype-specific variations of miR393 family and miR393-triggered phasiRNAs (phased small interfering RNAs) through targeting auxin receptor TIR1, $A F B 2$, and $A F B 3$ appeared to be critical pathway regulating defense responses via hormone crosstalk. The miR482/2118 superfamily, which targets several $N B$ $L R R$ genes, seemed to enhance effector-triggered immunity (ETI)-based resistance. The current study presents a complete population profile of miRNAs and their triggered phasiRNAs in apple roots under the pathogenic pressure from $P$. ultimum. The dataset provided valuable insight, in addition to previously comparative transcriptome analysis, to elucidate the molecular mechanisms regulating apple root resistance to $P$. ultimum.

Plant microbiome engineering as a strategy to control early blight

P. I. ALVAREZ ROMERO (1), A. F. T. A. F. Ferreira (2), C. Carpio (1), E. S. G. Mizubuti (2), (1) Escuela Superior Politécnica de Chimborazo, Riobamba, ECUADOR; (2) Universidade Federal de Viçosa, Viçosa, BRAZIL

Reciprocal soil transplant was used to test the effect of putative suppressive Andean and Brazilian soils to potato and tomato early blight epidemics. Metabarcoding analyses were used to assess the temporal variation of the fungal and bacterial leaf-microbiota associated with potato and tomato plants grown in Brazilian and Andean soils and inoculated with different species of large-spored Alternaria. The leaf microbiota was affected by soil transfer. Thirteen families of fungi belonging to the Ascomycota phylum and 15 belonging to the Basidiomycota were found in potatoes associated with Brazilian soils. In contrast, eight families of fungi belonging to the Ascomycota and 17 belonging to the Basidiomycota were found in potatoes associated with Andean soils. Also, 15 families belonging to the Ascomycota and 10 belonging to the Basidiomycota were found in tomatoes grown in Brazilian soil while 14 and 16 families, respectively, were recorded in plants grown in Andean soil. The bacterial composition of potato microbiota in all treatments included members of three families: Moraxellaceae, Pseudomonadaceae, and Bacillaceae. For tomato in Andean soil members of Moraxellaceae, Pseudomonadaceae, Xanthomonadaceae, and Exiguobacteraceae were found whereas in Brazilian soil members of Moraxellaceae, Pseudomonadaceae, and Xanthomonadaceae were identified. The approach developed here can be viewed as a model for studying interactions in other pathosystems involving necrotrophic pathogens.

\author{
Characterization of Polerovirus genomic variation \\ K. LATOURRETTE, N. M. Holste, D. Negam, H. Garcia-Ruiz, University of Nebraska-Lincoln, Lincoln, NE, USA
}

The genus Polerovirus (family Luteoviridae) consists of 32 different species that collectively infect a large variety of agriculturally important crops, including potato, sugarcane, maize, and beets. Poleroviruses are transmitted by aphids and symptoms often include stunting, yellowing, and leaf malformations. The polerovirus genome consists of a single, positive-strand RNA that encodes for the proteins P0 through P7 in overlapping open reading frames. Here, using single nucleotide polymorphisms, we characterized genomic variation across all available Polerovirus genomes. We identified hypervariable and conserved regions for each species and across the genus. Hypervariable areas map to P0, the $\mathrm{N}$ - and $\mathrm{C}$-terminus of P1, and the coat protein read through (CP-RT). Across the genome, negative selection was 15 -fold higher than positive selection, suggesting that large portions of the genome are conserved. Specifically, the CP-RT and P1 are under the strongest negative selection pressure. However, the middle section of the genome (P2-P3) is under neutral selection. In contrast, the CP-RT and P0 are under strong positive selection. Consistent with the presence of both positive and negative selection sites, structural models show that CPRT is a highly disordered protein. Furthermore, the presence of both negative and positive selection sites suggests this protein is involved in host adaptation. Characterizing specific areas within the polerovirus genome can help improve diagnostics and provide target areas for host genetic resistance efforts.

From metabolomics to functional phenotypes: Revealing metabolic features of diploid potato species (Solanum chacoense) in response to blackleg disease

S. KARIM, J. R. Joshi, A. O. Charkowski, Colorado State University, Fort Collins, CO, USA

Potato (Solanum tuberosum L.) is the most important non-grain crop grown in worldwide. In 2015, there was an aggressive outbreak of blackleg disease of potato in the eastern half of the United States. This outbreak was caused by the bacterial pathogen Dickeya dianthicola, leading to large annual losses for potato growers. Commercial potato varieties lack resistance to blackleg disease. Conversely, some accessions of the diploid wild potato species Solanum chacoense are resistant to D. dianthicola. In this study, we analyzed resistant and susceptible accessions of $S$. chacoense with non-targeted liquid chromatography mass spectrometry (LC-MS). The metabolomic profile of $D$. dianthicola inoculated resistant and susceptible accessions were compared and characterized. Metabolites from infected and mock-inoculated stem tissue were taken 0- and 24-hours post inoculation (hpi) and detected using LCMS. There was a clear difference in the metabolites between the resistant and susceptible accessions. The analysis characterized significant metabolite differences between resistant and susceptible accessions of $S$. chacoense, which may potentially lead to tools for breeding new cultivars with resistance against blackleg disease.

Test performance study on the detection and identification of tomato spotted wilt tospovirus - re-emerging threat to vegetables and ornamentals

A. Vučurović (1,2), N. Jakoš (3), M. RAVNIKAR (2,4), N. Mehle (2), (1) University of Belgrade -Faculty of Agriculture, Belgrade, REPUBLIC OF SERBIA; (2) National Institute of Biology, Ljubljana, SLOVENIA; (3) Department of Biotechnology and Systems Biology, National Institute of Biology, Ljubljana, SLOVENIA; (4) University of Nova Gorica, Nova Gorica, SLOVENIA 
Tomato spotted wilt tospovirus (TSWV, Tospoviridae) is among "top ten" of the economically most important plant viruses infecting more than 1000 species. Yield losses caused by TSWV are estimated at over one billion dollars, annually. Because of its importance TSWV was recognized as one of 11 major pests that need comprehensive validation of diagnostic tests within VALITEST project (www.valitest.eu). At National Institute of Biology, in the frame of VALITEST project, 76 tests for TSWV diagnosis were evaluated. Following thorough literature review, in-silico analyses and in-house testing the most suitable tests for detection and identification of TSWV were determined. These are different enzyme linked immune-sorbent assays (ELISA), reverse transcription-polymerase chain reaction (RT-PCR), real-time reverse transcription-polymerase chain reaction (RT-qPCR) and tests applicable for on-site detection. Eight tests were selected for evaluation within test performance study where 21 proficient diagnostic laboratories from EU (18) and non-EU countries (3) participate. In March 2020, each participating laboratory received up to 5 batches with panel of 22 samples in each. Results obtained from the participants will be evaluated later in 2020 with the help of statistical analyses to gain better insights in obtained validation data. Results generated in this study will contribute in finding fast and reliable tests for detection and identification of TSWV. The on time and accurate detection is a crucial first step in overcoming the diseases caused by plant viruses.

\section{Passalora fulva is more than just a model fungus: Understanding the population diversity of the tomato leaf mold pathogen in high tunnels \\ M. SUDERMANN, C. D. Smart, Plant Pathology and Plant-Microbe Biology Section, Cornell AgriTech, Geneva, NY, USA}

High tunnels extend the growing season on high value crops such as tomatoes. Unfortunately, these structures are also conducive to the spread of the tomato leaf mold pathogen Passalora fulva (syn. Cladosporium fulvum). The disease leads to defoliation and ultimately to losses in yield. Despite research on P. fulva in molecular contexts, little is known about pathogen populations in growers' high tunnels. From 2016 to 2019, samples were collected in high tunnels in the Northeast and the fungus was isolated and characterized. Intriguingly, other Cladosporium species were also isolated and characterized simultaneously. We confirmed Koch's postulates to demonstrate that the Passalora fulva isolates we collected were the causal fungus. Given the qualitative nature of resistance, race assays were conducted over two summers by inoculating differential sets of tomatoes with each isolate to provide growers with information about tomato varieties that show resistance. The results show that most isolates we collected belong to just a small number of races. We also determined the presence or absence of five previously characterized effectors. Some isolates showed no amplification of the gene encoding the effector AVR2. This effector interacts with the tomato resistance protein CF-2. For greater insights on diversity, genotyping-by-sequencing results are being compared to the race assays and effector sequencing. Information about Passalora fulva population diversity will allow us to offer better management recommendations to growers as high tunnel production of tomatoes expands.

Characterization and population structure of Phytophthora palmivora in Theobroma cacao L. crops of Colombia J. RAMÍREZ-MARTÍNEZ (1), M. Guevara-Suarez (1), M. Cardenas (1), D. Duarte (2), E. Gutierrez (2), S. Restrepo (1), (1) Universidad de Los Andes, Bogotá, COLOMBIA; (2) Federación Nacional de Cacaoteros (FEDECACAO)-Fondo Nacional del cacao (FNC), COLOMBIA

Phytophthora palmivora is a relevant pathogen of Colombian cacao crops producing economic losses by causing black pod disease, stem canker and leaf necrosis. No study has been made regarding the morphological characteristics, pathogenicity or population structure of this pathogen in Colombia, so the aim of this work was to assess these topics. Samples were taken from pods, leaves, stems and rhizosphere of symptomatic plants in three main cacao regions. Eighty-eight isolates were obtained and their taxonomic identity was confirmed using ITS and coxI sequences. Morphological characteristics showed that the sporangia were smaller than expected from guidelines. Mating type assays showed that all isolates were A2 type, indicating a clonal reproduction. A pathogenicity test was made using leaves, inoculating a suspension of 104 esporangia/mL and measuring lesion area during 7 days. A few high virulent isolates were obtained from some localities, but no geographic pattern was found. For population analysis, DArTseq was used and reads were aligned to reference genome. High diversity was observed, DAPC showed there might be up to seven populations in the country, although they do not relate to geographic distribution, or to any factor studied. Overall, high genetic and phenotypic diversity was observed, but not related to geography. It is proposed that this might be related to the way plant material is handled and distributed in the country. Hence, future control programs of this pathogen need to consider this diversity.

Isolation and characterization of bacteria associated with sunflower resistance to Sclerotinia stalk rot M. B. GLASER (1), C. Pogoda (2), N. Kane (2), B. Hulke (3), C. A. Quandt (2), (1) University of Colorado, Boulder, Boulder, CO, USA; (2) University of Colorado Boulder, CO, USA; (3) USDA-ARS Sunflower and Plant Biology Research Unit, ND, USA

New research has linked the microbial community of sunflower (Helianthus annuus) with its resistance to Sclerotinia stalk rot caused by the fungus Sclerotinia sclerotiorum. The potential to identify, isolate, and use specific microbes associated with Sclerotinia resistance could have prophylactic implications. In our study, we have begun targeted isolation of sunflower field soil bacteria, which were previously correlated with resistance. We have isolated over 70 strains of bacteria thus far and performed competition assays with S. sclerotiorum for 50 of these. Major clades of bacteria isolated include Gammaproteobacteria, Firmicutes, Bacteroidetes, and Actinobacteria. We have identified several bacterial isolates that inhibit growth of the pathogen S. sclerotiorum when grown in coculture, and others that promote growth of the pathogen. Some of the samples that may inhibit Sclerotinia growth belong to genera (e.g. Serratia) which are known to have antifungal properties. This evaluation of inhibition in co-culture is particularly interesting because of the importance of these and other bacteria in explaining Sclerotinia resistance in field and greenhouse trials. Findings from this project will contribute to the development of alternative control efforts which take advantage of the crop-soil microbe interaction in Sunflower crops. 
Characterization of the roles of the monothiol glutaredoxin, grx4, in the cell biology and virulence of $U$ stilago maydis S. W. MCCOTTER (1), J. W. Kronstad (2), (1) The University of British Columbia, Vancouver, BC, CANADA; (2) University of British Columbia, Vancouver, BC, CANADA

The corn smut fungus, Ustilago maydis, is the premier basidiomycete model for studying biotrophic plant-pathogen interactions. Monothiol glutaredoxins are well-conserved, central regulators of functions such as iron homeostasis, cell wall integrity and cellular redox status. In this study we characterized the novel role of the monothiol glutaredoxin Grx4 in the pathogenesis of $U$. maydis on its host, Zea mays. Mutants expressing a conditional allele of grx4 under the control of the arabinose-induced/glucose-repressed promoter $P_{c r g}$ exhibited decreased virulence on maize. When grown in glucose, $P_{c r g}$ :grx 4 strains showed increased sensitivity to reactive oxygen stress and cell-wall damaging agents. These mutants also exhibited a proliferation of peroxisomes as measured by the peroxisomespecific stain, nitro-BODIPY, indicating increased reactive oxygen stress. Unlike the related basidiomycete Cryptococcus neoformans, grx4 mutants do not show increased sensitivity to iron-related stressors such as BPS, ferrozine and curcumin, suggesting a novel role for Grx4 in the cell biology of $U$. maydis. Together, these data suggest that glutaredoxins could play in important role in the virulence of plant pathogenic fungi, in addition to their established roles as key regulators of fundamental cellular processes.

\section{A network analysis framework for complex virome data}

R. I. ALCALA-BRISE (1), J. F. Kreuze (2), K. A. Garrett (3,4,5), (1) University of Florida, Gainesville, FL, USA; (2) CGIAR Research Program on Roots, Tubers and Banana, International Potato Center, Lima, PERU; (3) Institute for Sustainable Food Systems, University of Florida, Gainesville, FL, USA; (4) Plant Pathology Department, University of Florida, Gainesville, FL, USA; (5) Emerging Pathogens Institute, University of Florida, Gainesville, FL, USA

Complex data from microbial ecosystems are now commonly generated using sequencing technologies for eukaryotes, prokaryotes, and viruses. Biotic and abiotic factors determine the positive and negative interactions between species, impacting animal and plant health and productivity. These ideas inform a framework for the analysis of complex viromes, a Virome Network Analysis (ViNA) package, implementing the analysis of viromes in the agroecological landscape using data generated through viral metagenomics. We analyzed a survey of sweetpotato plants collected from eleven African countries in four consecutive years. Sweetpotato small RNAs were sequenced from individual sweetpotato accessions displaying a variety of symptoms, and bioinformatic analysis with VirusDetect revealed mixed infections. ViNA was used to assign data to two groups: georeferenced accessions (hosts) and sequence classification (viruses). The ViNA pipeline assigns viral sequences to a genus based on cluster identity (\%) based on species demarcation thresholds. The reads were normalized using RPKM quantification. We evaluated the network and geographic structure defined by the community of viruses over time. This revealed common virus-virus associations within the Pan-African sweetpotato virome, such as feathery mosaic, leaf curl, and symptomless mastrevirus, and new associations, along with sweetpotato virus community gradients across Africa. This bioinformatic pipeline can be applied to other complex virome datasets, and would help to identify possible threats and inform management strategies.

\section{A novel Partitivirus identified in Miscanthus sinensis}

X. HU (1), O. P. Hurtado-Gonzales (2), L. Carvalho Costa (3), R. Turner (2), J. A. Foster (2), A. V. Karasev (4), E. Sacks (5), T. Yamada (6), R. French Monar (2), M. O’Connell (2), M. M. Malapi-Wight (2), (1) USDA, Beltsville, MD, USA; (2) USDA-APHIS Plant Germplasm Quarantine Program, Beltsville, MD, USA; (3) USDA, Laurel, MD, USA; (4) University of Idaho, Moscow, ID, USA; (5) University of Illinois, IL, USA; (6) Hokkaido University, Sapporo, JAPAN

Chinese silvergrass (Miscanthus sinensis) is an ornamental grass native to eastern Asia. In 2015, a grass clone was imported by the USDA-APHIS PPQ Plant Germplasm Quarantine Program (PGQP, Beltsville, MD) and tested for pathogens. Total RNA was extracted from leaf tissue, and the associated ribosomal RNA depleted library was sequenced on an Illumina NextSeq 500 System. Sequence analysis of the assembled contigs revealed the presence of a putative Partitivirus in the plant. The three largest contigs were similar to three Partitivirus RNA segments. The lengths of the protein product of these three contigs were 478 amino acids (aa), 348-aa and 347aa for the RNA dependent RNA polymerase (RdRp), coat protein $1(\mathrm{CP} 1)$, and coat protein 2 (CP2) respectively, which is characteristic of the Deltapartitivirus group. The protein similarity between CP1 and CP 2 was found to be $37.0 \%$, suggesting that they are encoded by two different genes. The RdRp amino acid sequence shared 30.2\%, 18.2\%, 42.7\%, and 62.0\% identities with the Alphapartitivirus, Betapartitivirus, Gammapartitivirus, and Deltapartitivirus genera in Partitiviridae, respectively. Phylogenetic analyses based on RdRp sequences placed the novel virus in the genus Deltapartitivirus, closest to three other unclassified Partitiviruses identified in dicots. No evidence of another RdRp for a second virus was detected in this study. Therefore, this virus can be considered a new member of the Partitivirus, family Partitiviridae. The tentative name Miscanthus sinensis cryptic virus 1 is proposed for this new Partitivirus.

\section{Stripe rust resistance genes in U.S. wheat cultivars and breeding lines identified by molecular markers and genome-wide association studies}

L. Liu (1), J. Mu (1), Y. Liu (1), M. Wang PhD (2), D. See (1,3), X. CHEN (1,3), (1) Washington State University, Pullman, WA, USA; (2) Washington State University, Pullman, WA, USA; (3) USDA-ARS, Pullman, WA, USA

Stripe rust (caused by Puccinia striiformis f. sp. tritici) is one of the most destructive diseases of wheat in the United States. To identify genes for effective stripe rust resistance in U.S. wheat, 616 spring wheat and 857 winter wheat cultivars and breeding lines were studied using a genome-wide association approach and with markers for important previously reported genes. The accessions were phenotyped for stripe rust response at seedling stage in a greenhouse with multiple races of the pathogen and at adult-plant stage in multiple field environments under natural infections of the pathogen, and genotyped using a genotyping by multiplexed sequencing platform. In the 
spring panel, a total of 37 loci on 15 chromosomes (1A, 1B, 2A, 2B, 2D, 3B, 3D, 4A, 4B, 5A, 5B, 6B, 6D, 7A, and 7D) for resistance to stripe rust were detected including 10 new loci. In the winter panel, a total of 51 loci on 19 chromosomes $(1 \mathrm{~A}, 1 \mathrm{~B}, 1 \mathrm{D}, 2 \mathrm{~A}, 2 \mathrm{~B}, 2 \mathrm{D}, 3 \mathrm{~A}$, $3 \mathrm{~B}, 3 \mathrm{D}, 4 \mathrm{~A}, 4 \mathrm{~B}, 5 \mathrm{~A}, 5 \mathrm{~B}, 5 \mathrm{D}, 6 \mathrm{~A}, 6 \mathrm{~B}, 7 \mathrm{~A}, 7 \mathrm{~B}$, and 7D) were associated to stripe rust resistance, including at least 4 new loci. The frequencies of the resistant alleles of these loci in various regional nurseries indicated different use intensities in breeding programs in different regions. The information on wheat cultivars with identified resistance genes can be used for selecting and deploying resistant cultivars to manage stripe rust. The resistance loci and their markers should be useful in breeding programs for continually improving stripe rust resistance.

Establishing protocols to characterise Rhynchosporium commune infection in winter barley for the identification of susceptibility genes

T. O’CONNOR (1,2), E. Graciet (1), E. D. Mullins (2), (1) Maynooth University, Department of Biology, Kildare, IRELAND; (2) Teagasc Crops Research Centre, Carlow, IRELAND

Cool temperate climate bestows high yield potential for tillage crops in Ireland. However the climate that maintains yields also supports the prevalence of crop diseases. Rhynchosporium commune is a fungal pathogen that imposes a significant threat to barley yields. Resistant barley cultivars rely on their ability to recognise a range of the pathogen's molecules. Recognition of pathogen effectors by resistant proteins can trigger an immune response. However, high genetic diversity within $R$. commune populations can render resistance proteins susceptible after only a few growing seasons, therefore this mechanism is not durable. Recently, the importance of susceptibility genes (S-genes) encoded by the host's genome has emerged. The products of S-genes are exploited by pathogens to promote infection. Crops deficient for specific S-genes are more resistant to pathogens. Thus, inactivating S-genes could deliver a more durable resistant phenotype and provide a novel strategy for crop defence over existing systems that rely only on resistance genes. This project has established reproducible protocols to characterise $R$. commune disease and will generate datasets detailing the transcriptomic response of both resistant and susceptible barley varieties to different $R$. commune strains. RT-qPCR data generated show increased expression of defence related genes within the first $48 \mathrm{~h}$ post infection, before onset of symptoms in susceptible winter barley. Once identified, candidate barley S-genes targeted by $R$. commune will be determined using RNAi-based transient expression systems in barley.

An in vitro pipeline to screen and select citrus-associated microbiota with potential anti- Candidatus Liberibacter asiaticus properties

C. L. DROZD (1), A. Blacutt (1), N. Ginnan (1), T. Dang (1), S. Bodaghi (1), G. Vidalakis (1), P. Ruegger (1), B. B. Peacock (1), P. Viravathana (1), F. Campos Vieira (1), B. Jablonska (1), J. Borneman (1), G. McCollum (2), J. Cordoza (3), J. Meloch (3), V. Berry (3), L. L. Salazar (3), K. Maloney (3), P. E. Rolshausen (1), M. C. Roper (1), (1) University of California, Riverside, Riverside, CA, USA; (2) USDA, ARS, Fort Pierce, FL, USA; (3) Point Loma Nazarene University, Point Loma, CA, USA

Huanglongbing (HLB) is a serious disease of plants in the family Rutaceae and threatens citrus production around the world. In the United States of America, HLB is associated with the unculturable bacterium Candidatus Liberibacter asiaticus (CLas) and is a major threat to citriculture. In this study, we developed a culturing pipeline to isolate microbes from citrus tissues in Florida in areas under high disease pressure. These isolates were assayed for inhibitory activity against Liberibacter crescens, a culturable surrogate for CLas. Several bacteria and fungi produced secreted secondary metabolites that inhibited the growth of Liberibacter crescens. Bioassay-guided fractionation of culture supernatants of one fungal isolate, Cladosporium cladosporioides, revealed that the metabolites cladosporol A, $\mathrm{C}$ and $\mathrm{D}$ were inhibitory to $L$. crescens in a dose-dependent manner. We are continuing to screen our established citrus-associated microbial repository of $>1,300$ citrus-associated bacteria and fungi for inhibitory natural products. Our goal is to unravel the complex chemistries associated with the citrus microbiome, with a long-term goal of developing natural product based mitigation strategies for HLB and anti-CLas bioinoculants capable of persisting in citrus trees.

Identification of novel approach to control soybean aphid (Aphis glycines) by exploiting plant-insect-symbiont interaction S. R. ACHARYA, V. Nalam, Colorado State University, Fort Collins, CO, USA

Soybean aphid is one of the most important hemipteran pests that causes huge economic loss of soybean as they are phloem feeders and are vector of different plant viruses. These aphids have combated cultural, chemical and genetic control strategies overtime, suggesting that unique control management strategy is necessary. The current study targets nutrient pathway between soybean aphid and its bacterial endosymbiont, Buchnera aphidicola, it harbors in a specialized bacteriocyte cell. Soybean aphid compensates for essential amino acids, that are usually low in phloem sap, from their endosymbionts as these bacteria converts non-essential amino acids supplied by aphids to essential amino acids. The bi-direction flow of amino-acid at soybean aphid/Buchnera interface is controlled by yet unknown aminoacid transporters. However, two amino-acid transporters at this interface, ApGLNT1 and ApNEAAT1, has been functionally characterized in pea aphid. As soybean aphid shares multiple sequence orthologues with pea aphid, it is plausible that a similar membrane transporter exists in soybean aphid/Buchnera interface. Our RNA sequencing analysis of differentially expressed genes shows that three putative amino acid transporters: 72-RA, 86-RA and 91-RA are differentially expressed in the bacteriocytes. However, the functional characterization of these transporters remains to be tested in-vitro. Elucidation of transport function of these amino-acid transporters could aid in understanding aphid nutrient dependency on its endosymbiont and target them as a means of novel control strategy.

Real-time LAMP detection of Cucurbit leaf crumple virus and comparison with PCR-based techniques S. WALIULLAH (1), K. S. Ling (2), E. Cieniewicz (3), J. E. Oliver (4), P. Ji (5), E. Ali (5), (1) University of Georgia, GA, USA; (2) USDA-ARS, Charleston, SC, USA; (3) Clemson University, Clemson, SC, USA; (4) Department of Plant Pathology, University of Georgia, Tifton, GA, USA; (5) University of Georgia, Tifton, GA, USA 
A loop-mediated isothermal amplification (LAMP) assay was developed for rapid and efficient detection of an important begomovirus Cucurbit leaf crumple virus (CuLCrV) in infected leaf samples. A set of six primers targeting the DNA-A gene was able to detect $\mathrm{CuLCrV}$ infection using real-time LAMP amplification with the Genie ${ }^{\circledR}$ III system, which was further confirmed by gel electrophoresis and SYBR ${ }^{\mathrm{TM}}$ Green I DNA staining for visual observation. The optimum reaction temperature and time were determined to be $71^{\circ} \mathrm{C}$ for $60 \mathrm{~min}$. The LAMP assay could amplify CuLCrV in a mixture of viruses without cross-reactivity with other begomoviruses. The sensitivity of the LAMP assay was 10 times higher than conventional polymerase chain reaction (PCR) but slightly less sensitive than quantitative PCR (qPCR). Both LAMP and qPCR amplified asymptomatic CuLCrV-infected plant samples more efficiently than PCR. Although qPCR was the most sensitive assay, it is mainly applicable for laboratory-based detection rather than on-site detection. On the other hand, LAMP could be easily used in the fields with minimal equipment (a water bath or isothermal heat block). Thus, the LAMP assay shows excellent potential as a valuable diagnostic tool that could be utilized for disease surveillance in cucurbit production to detect $\mathrm{CuLCrV}$ infection.

Non-destructive mycorrhizal association detection with aerial hyperspectral imagery F. E. ROMERO GALVAN (1), J. Fisher (2), K. M. Gold (3), R. Pavlick (4), E. Brzostek (5), R. P. Phillips (6), (1) JPL, Sylmar, CA, USA; (2) NASA-JPL, Pasadena, CA, USA; (3) Cornell University, Geneva, NY, USA; (4) JPL, Pasaden, CA, USA; (5) West Virginia University, Morgantown, WV, USA; (6) Indiana University, Bloomington, IN, USA

Mycorrhizal symbionts provide a range of important services to their plant hosts, including breaking down inorganic matter, transporting water, and nutrient exchange. The need to detect and map mycorrhizal associations at scale is growing as their impacts on crop health, resilience to abiotic and biotic stress, global biogeochemical cycling, and $\mathrm{CO} 2$ fertilization continues to emerge. Historical efforts to measure wide-scale mycorrhizal distributions have been hampered by labor intensive and expensive sampling methods. Previously, we found that tree-mycorrhizal associations can be detected remotely using spaceborne multispectral measurements of canopy spectral and phenological signals. Aerial hyperspectral imagery has enormous potential to refine detection further through its capacity to connect mycorrhizal associations directly to resulting canopy nutrient levels. Here, we evaluate data from the Airborne Visible/Infrared Imaging Spectrometer (AVIRIS) NASA hyperspectral imaging platform (17 m pixels, 400-2400 nm) over multispecies mycorrhizal gradients in the United States to detect and map ratio of ectomycorrhizal to arbuscular mycorrhizal associations. Random forest regression explained $80 \%$ of the variability between ectomycorrhizal and arbuscular mycorrhizal associations at the Harvard Experimental Forest, Yosemite National Park, and Santa Cruz Forest Ecology Research Site. This work has great promise for the utility of spaceborne spectroscopic instruments, to non-destructively produce global coverage of fine-scale mycorrhizal associations.

\section{Navigating industry and university partnerships to advance plant pathology}

J. M. LANG, Colorado State University, Fort Collins, CO, USA

Strategic and successful partnerships between universities and industry can provide needed support for research and solutions to relevant, global challenges. Best practices for initiating and sustaining these relationships include creating a comprehensive partnership package that outlines goals and manages expectations. A holistic strategic partnership with industry requires key contacts from both institutions, committed faculty and clear timelines. A single point of contact can streamline solutions, efficiently manage deliverables and avoid confusion, particularly with large organizations. Student engagement with industry provides a mutually beneficial scenario whereby companies gain access to new talent and the ability to train on specific needs while students gain invaluable, real-world experience. Intellectual property is a critical issue to address in research-based relationships. Negotiation ahead of activity in a master agreement can allow for all subsequent research to be protected. The discipline of plant pathology is particularly well suited to integrate industry partnerships from pest management, diagnostic tools and advancing technologies to the development high-quality plant varieties. While federal support for research is becoming more competitive and challenging to capture, partnering with industry can be a viable compliment to any institutions' portfolio.

\section{Magnesium-Copper nanomaterials as alternative to conventional copper for tomato and pepper bacterial spot disease management \\ Y. Y. LIAO (1), Z. Huang (2), A. L. Strayer-Scherer (3), S. Santra (2), J. C. White (4), R. De La Torre-Roche (4), Q. Fan (5), S. Da Silva (5), G. E. Vallad (6), J. H. Freeman (5), J. B. Jones (1), M. L. Paret (5), (1) University of Florida, Gainesville, FL, USA; (2) University of Central Florida, Orlando, FL, USA; (3) Auburn University, Auburn, AL, USA; (4) Connecticut Agric Exp Station, New Haven, CT, USA; (5) North Florida Research \& Education Center, University of Florida, Quincy, FL, USA; (6) Gulf Coast Research and Education Center, University of Florida, Wimauma, FL, USA}

Bacterial leaf spot (BLS) of tomato, incited by Xanthomonas spp., is a devastating diseases worldwide. Copper (Cu) bactericides continue to be a standard choice for BLS management despite the emergence of $\mathrm{Cu}$-tolerant strains and potential environmental risks, as effective alternatives to $\mathrm{Cu}$ are limited. Prior research has led to the discovery of antibacterial properties of magnesium oxide nanoparticles (nano $\mathrm{MgO} ; 20 \mathrm{~nm}$ ) against Cu-tolerant X. perforans $(\mathrm{Xp})$. Nano $\mathrm{MgO}$ has been effective in management of bacterial spot disease of tomato under field conditions. In our current study, we evaluated formulated $\mathrm{Mg}$ and $\mathrm{Cu}$ nanomaterials (i.e., $\mathrm{Mg}$ - $\mathrm{Cu}$ and $\mathrm{Mg}$ $\mathrm{DB})$ with dual antimicrobial activities $\mathrm{Mg}$ - $\mathrm{Cu}$ and $\mathrm{Mg}$-DB significantly increased antibacterial efficacy against $\mathrm{Cu}$-tolerant $\mathrm{Xp}$ and $X$. euvesicatoria $(\mathrm{Xe})$, which cause bacterial spot disease of pepper, strains when compared to $\mathrm{Cu}$ bactericide in vitro $(\mathrm{p}=0.05)$. In a fall 2019 field study, $\mathrm{Mg}-\mathrm{Cu}$ and $\mathrm{Mg}-\mathrm{DB}$ applications at $500 \mu \mathrm{g} / \mathrm{mL}$ significantly reduced disease severity compared to untreated controls (UT), whereas conventional $\mathrm{Cu}$ bactericide was not significantly different from $\mathrm{UT}(\mathrm{p}=0.05)$. Additional field studies are required to determine consistent efficacy of these formulated $\mathrm{Mg}$ nanomaterials across multiple production seasons. 
Development of a multiplex high-throughput diagnostic assay for the detection of strawberry crown rot diseases using highresolution melting analysis

N. Y. WANG (1), A. B. Gama (2,3), M. Marin (1), N. Peres (1), (1) University of Florida - Gulf Coast Research and Education Center, Wimauma, FL, USA; (2) University of Florida, Wimauma, FL, USA; (3) University of Florida, Lake Alfred, FL, USA

Rapid and accurate disease diagnosis is a prerequisite for an effective disease management program in strawberry production. In Florida, Colletotrichum species, Phytophthora species, and Macrophomina phaseolina are the primary microorganisms causing strawberry crown rot. Even though the diseases can be caused by different pathogens, symptoms are indistinguishable and equally devastating. To timely inform strawberry growers of diagnostic results for effective deployment of control practices, we developed a multiplex high-resolution melting (HRM) assay to rapidly and accurately detect the above-mentioned pathogens. The multiplex HRM assays using three pre-designed primer pairs showed high specificity for individual species by generating specific melting peaks without cross-reaction between primers or with other common strawberry pathogens. The amplification limit of the assay was $1 \mathrm{pg}$ of Colletotrichum spp. and Phytophthora spp. and $100 \mathrm{pg}$ of $M$. phaseolina DNA per $10 \mu \mathrm{L}$ reaction. However, the presence of different melting peaks was observed in mixed DNA samples and was concentration- and target DNA-dependent. An improved crude DNA extraction protocol was developed to allow high-throughput screening by minimizing the inhibitory effects. Moreover, we applied the HRM assay to 522 plant samples and found high correlations between culture isolation and HRM results and between singleplex and multiplex assays. Altogether, this multiplex HRM assay is specific, cost-effective, and reliable for the timely detection of strawberry crown rot pathogens.

Incidence of Dahlia mosaic virus in Utah

T. COMPTON, C. Nischwitz, M. Stock, Utah State University, Logan, UT, USA

Dahlia (Dahlia variabilis) is an important crop in the expanding cut-flower industry in Utah. Growers have been sending increasing numbers of dahlia samples to the Utah diagnostic lab with viral symptoms including mosaic, stunting, and flower abnormalities. Some samples were positive for TSWV and TSV but negative for INSV. The results did not explain all the symptoms and symptoms did not match CMV. To determine if Dahlia mosaic virus (DMV) was the causal agent, a study was initiated to ascertain the presence and distribution of DMV in commercial cut flower production in Utah as well as in commercially available seed and tubers. DMV is a DNA virus in the genus caulimovirus. It is transmitted by aphids and seed, and is carried in tubers. There are three strains: DMV-D10, DMVHolland, and DMV-Portland. In total, 108 seed, leaf and tuber samples were tested for all three strains using PCR and strain specific primers. PCR results showed 54 percent and 44 percent of purchased seed and tubers, respectively, were positive for at least one of the three strains of DMV. Sixty-seven percent of leaf samples collected in the field tested positive for at least one of the three strains. Results were confirmed by sequencing of PCR products and BLAST search in the NCBI GenBank. DMV-D10 was most common, followed by DMV-Holland. The prevention of introduction of the virus into a cut flower operation is key to avoid secondary spread by aphids. The high incidence of DMV and the negative impact it has on commercial cut flower production suggest a screening program for DMV may be warranted.

Real-time loop-mediated isothermal amplification assay for rapid and accurate detection of fungal pathogens of wheat S. V. GOMEZ (1), S. B. Goodwin (2), (1) Purdue University, West Lafayette, IN, USA; (2) USDA ARS, West Lafayette, IN, USA

Wheat is an important cereal crop grown on more than 240 million ha. This crop is affected by the Septoria complex caused by Zymoseptoria tritici and Parastagonospora nodorum, as well as other pathogens like Pyrenophora tritici-repentis (tan spot), Cochliobolus sativus (spot blotch) and Magnaporthe oryzae (wheat blast). It is difficult to visually distinguish between these pathogens at the onset of infection. Accurate identification of the causal agent is decisive to make the right decisions about disease management. LAMP is a new isothermal nucleic acid amplification method that does not use a thermal cycler. It is emerging as a simple and highly sensitive diagnostic tool for early detection of plant pathogens. In this study we wanted to standarize a LAMP-based diagnostic assay for effective, quick and specific detection of pathogens in wheat. A set of six primers for each pathogen was designed by using Primer Explorer V4 software. Genomic DNA was extracted from Z. tritici and P. nodorum. The LAMP reaction was heated to $65^{\circ} \mathrm{C}$ for 60 min, $80 \mathrm{~min}$ and $120 \mathrm{~min}$ to standardize the reaction. The reaction at $65^{\circ} \mathrm{C}$ for $60 \mathrm{~min}$ was optimum and DNA amplification was obtained. Amplified bands of $200 \mathrm{bp}$ were detected by electrophoresis for $Z$. tritici and $P$. nodorum and no amplification was detected for the positive or negative controls. The correct identification of the species that are affecting a crop of interest is the first approach for phytopathology studies, making possible to study its biological features and conduct research to find an effective way to control the disease.

Virus and viroid cross-talk in solanaceous ornamentals

G. N. CHOFONG (1), M. Wassenegger (2), K. R. Richert-Poeggeler (1), (1) Julius Kühn-Institut, Braunschweig, GERMANY; (2) RLP AgroScience gGmbH AlPlanta - Institute for Plant Research, Neustadt, GERMANY

The devastating effects of viruses or viroids on plants are illustrated by a symptomatic phenotype. Invasion of new host, climatic change and changes in plant production can have a major impact on latent viruses and viroids. Viroids are small, unencapsidated singlestranded, circular RNA molecules (239-401nt) with no coding capacity. Pospiviroidae replicate in the nucleus using a rolling-circle mechanism. Seed and pollen-borne transmission of Potato spindle tuber viroid (PSTVd) the type member of Pospiviroidae has been reported in tomato. Viruses and viroids are intra-cellular parasites and thus may share the same niches for accession of host metabolic pathways to achieve their replication cycle. We used a microscopic-molecular biology approach to gain first insights in the dynamics and plasiticity of viroid-virus interactions in solanaceous hosts during latency. We analysed asymptomatic Solanum jasminoides mixed 
infected with a carlavirus and various pospiviroids. The amplification level of the carlavirus Potato virus $M$ seems to be modulated depending on which viroid is present. Molecular biological studies in the Netherlands as well as our own observation of naturally infected samples have shown that after eradication of PSTVd in S. jasminoides, plants showed infection by TASVd or CEVd. In grafting experiments to $P$. hybrida only TASVd had been transmitted to the stock. These suggest that, there may be a different basis for viroid-virus specificity in plant virus infection which might be well elucidated for exploration in practical agriculture.

Fusarium diversity in conifer tree nurseries of the contiguous U.S.A.: Who's at the party?

J. DOBBS (1), M. S. Kim (2), G. J. Reynolds (3), R. K. Dumroese (4), N. B. Klopfenstein (5), S. W. Fraedrich (6), M. M. Cram (6), J. J. Bronson (7), J. Stewart (8), (1) Colorado State University-Agricultural Biology, Fort Collins, CO, USA; (2) USDA Forest Service, Pacific Northwest Research Station, Corvallis, OR, USA; (3) US Forest Service, Albuquerque, NM, USA; (4) USDA Forest Service, Rocky Mountain Research Station, ID, USA; (5) USDA Forest Service, Rocky Mountain Research Station, Moscow, ID, USA; (6) USDA Forest Service, Athens, GA, USA; (7) USDA Forest Service, Asheville, NC, USA; (8) Colorado State University, Fort Collins, CO, USA

Conifer nurseries often serve as reservoirs for a diverse suite of native and exotic pathogens, e.g. Fusarium and Phytophthora spp. These pathogens can move undetected through nursery stock, causing mortality and increasing the risk of introduction into novel landscapes (e.g., restoration areas). Broad-spectrum chemical treatments are costly and affect non-target, beneficial microorganisms in the soil. Targeted management strategies can be developed for nurseries, if the pathogen composition and their pathogenicity are known. This project aims to address two complementary objectives: 1) evaluate the occurrence of Fusarium spp. in conifer tree nurseries across the U.S.A.; and 2) identify occurrence patterns among populations of pathogenic Fusarium, including host preferences, geographic distribution, and potential movement. Because diverse Fusarium spp. can cause damping-off and root rot diseases of conifer seedlings in forest nurseries, Fusarium isolates were collected from western, midwestern, and southern U.S.A. to determine diversity of Fusarium spp. Based on DNA sequences of mitochondrial small subunit gene and elongation factor 1-alpha, F. oxysporum, F. commune, F. redolens, $F$. fujikuroi, $F$. proliferatum, $F$. solani, $F$. equiseti, and $F$. tricinctum were among the 11 species found from conifer tree nurseries in these regions. This project will provide the framework for developing tools to quantify disease pressure by identifying pathogen populations and key mechanisms of disease development by determining roles of effector profiles shared among different pathogen genera.

High vs. low DON accumulating lines of barley evaluated by dip inoculation of Fusarium head blight

T. T. BALDWIN (1), S. M. Baldwin (2), E. Kress (3), E. Mndolwa (3), K. Esvelt Klos (3), J. M. Marshall (4), P. Bregitzer (5), (1) North Dakota State University, Fargo, ND, USA; (2) University of Idaho, Aberdeen, ID, USA; (3) USDA-ARS, ABERDEEN, ID, USA; (4) University of Idaho, Idaho Falls, ID, USA; (5) USDA ARS, Aberdeen, ID, USA

Fusarium head blight (FHB) is an important cereal disease. Field evaluation is complicated, which complicates identification of host resistance factors. Green house and growth chamber experiments could ameliorate these confounding factors given the right strategy for inoculation. Here we developed a simple dip inoculation method for high-throughput evaluation of FHB resistance in barley using conidia concentrations to separate the effects of resistance against initial infection from resistance to DON accumulation. Fifteen barley lines of genetically diverse backgrounds were evaluated for visual severity and accumulation of DON when inoculated with $F$. graminearum. Higher concentrations of conidia equated to better differentiation of lines based on accumulation of DON. From the fifteen barley lines tested, two high-DON and two low-DON-accumulating barley lines were identified that are parents of extant biparental mapping populations. Transcriptomic responses to wild type $F$. graminearum and $\Delta$ tri 5 , at 1 and 3 days post-inoculation, were assessed for the four lines. Low-DON lines showed differential expression of more genes than in response to pathogen infections. All lines showed differential expression of more genes when infected with the DON-deficient $\Delta$ tri5 mutant than when infected with the wild type, suggesting that DON suppresses pathogen response. Low-DON barley lines showed upregulation of genes related to pathogen response, including UDP-glycosyltransferases, pathogenesis-related proteins, and chitinases. These genes are potential breeding targets for future work.

Evaluation of field inoculation methods for Sclerotium rolfsii to facilitate epidemiological studies S. SANJEL (1), R. Barocco (2), N. S. Dufault (2), B. Tillman (3), D. Wright (4), I. M. Small (5), (1) University of Florida, Quincy, FL, USA; (2) University of Florida, Gainesville, FL, USA; (3) UF, Marianna, FL, USA; (4) Univ of Florida, Quincy, FL, USA; (5) University of Florida, Quincy, FL, USA

Stem rot disease, caused by Sclerotium rolfsii, is an important soil-borne disease of peanut. Efforts to investigate the epidemiology and management of stem rot are often constrained by variability of natural inoculum. The objective of this research was to evaluate the impact of inoculation methods and environmental variables on stem rot development. Inoculated field experiments were conducted at three locations in Florida during the 2019 season. At each location a split-plot design was implemented with variety as the whole plot factor and inoculum (isolate and method) as the sub-plot factor. Two varieties, Georgia-06G and TufRunner297, were inoculated with three isolates of S. rolfsii 3-4 weeks after planting. For each isolate, two inoculum types were tested: 1) laboratory-cultured sclerotia, or 2) oat and corn-based inoculum. Autoclaved oat and grain controls, as well as non-inoculated controls, were included in the experimental design. Stem rot progress was assessed. At all locations, stem rot symptoms were first observed approximately eight weeks after planting. Higher stem rot incidence was observed in Georgia 06G than TUFRunner297 at all locations. Both inoculation methods increased the incidence of stem rot, relative to the non-inoculated controls. Stem rot incidence significantly differed among isolates and inoculation methods, although these differences were not consistent across locations. These preliminary results suggest that early season sclerotial or grain-based inoculation are viable methods to ensure inoculum presence for field studies. 
Evolutionary relationships and phylogeny of Dickeya zeae strains based on phenotypic, biochemical and genomic characteristics G. BOLUK, S. Dobhal, A. M. Alvarez, M. Arif, University of Hawaii at Manoa, Honolulu, HI, USA

Dickeya zeae is the causal agent of bacterial heart rot and fruit collapse of pineapple, bacterial stalk rot of corn, bacterial foot rot of rice and bacterial soft rot of banana. In this research, we aimed to characterize and establish the evolutionary relationships among the $D$. zeae strains isolated from different sources including irrigation water. For multi-locus sequence analyses (MLSA), five housekeeping genes were analyzed: $d n a A$, gapA, gyrB, atpD, and purA. A total of 109 strains were included in the analyses (52 of $D$. zeae, 54 of other Dickaya sp., and 3 of Pectobacterium sp.). The phylogenetic tree revealed high genetic diversity among the $D$. zeae strains. The kale and taro strains showed close lineages with the strains isolated from corn and irrigation water, respectively. Comparative assays for different activities (cellulolytic, proteolytic, indigoidine and pectolytic enzyme production and motility), carbon utilization (BioLog) and pathogenicity tests on different hosts were also evaluated. All tested Dickeya and Pectobacterium strains showed higher activities of cell-wall degrading enzymes at $28^{\circ} \mathrm{C}$. All tested Dickeya strains except $D$. paradisiaca showed indigoidine production. The kale strain (PL47) showed different carbon utilization profiles among Dickeya species. All strains were motile and capable of swarming; the largest bacterial spreading zone was observed at $28^{\circ} \mathrm{C}$. Taro strain PL65 was the most aggressive on taro among all strains tested, indicating greater metabolic and/or genetic fitness during infection.

\section{Association of post-flowering non-senescence (Stay-green) and dhurrin levels in response to stalk rot pathogens in Sorghum bicolor \\ D. L. FUNNELL-HARRIS (1), S. Sattler (1), P. O’Neill (1), L. Bernhardson (1), M. Kilts (2), M. Khasin (1), (1) USDA-ARS, Lincoln, NE, USA; (2) USDA-ARS; Wheat, sorghum and forage research unit, Lincoln, NE, USA}

Sorghum (Sorghum bicolor (L.) Moench) is a diverse C4 crop used for grain- or juice-based bioethanol, with potential for lignocellulosic biofuels. Although drought-tolerant, sorghum is vulnerable to stalk rots caused by Fusarium thapsinum and Macrophomina phaseolina, especially during water-stress. Lines with post-flowering non-senescence (Stay-green), associated with drought and stalk rot resistance, were shown to have high levels of the cyanogenic glycoside dhurrin, unlikely to be a pathogen resistance factor. Lines with reported field stalk rot resistance, Stay-green with high dhurrin levels, or stalk rot-susceptibility and senescent, were screened in a greenhouse for response to the two pathogens. Of the four most resistant lines, Plant Introduction (PI) 534133 is known as Stay-green with high dhurrin levels and PI267379 as field resistant; there is little information on PI533882 and PI534053. Dhurrin assays using HPLC of greenhouse-grown leaves showed that PI267379 had significantly less dhurrin than all other lines while PI533882 and PI534053 had levels similar to PI534133 and a Stay-green check, PI656029. Dhurrin levels of field-grown leaves from the most resistant lines were similar to greenhouse-grown leaves; PI534053 and PI534133 had levels similar to a Stay-green check. Field-grown lines were scored at maturity for senescence with a 1 (non-senescent) to 5 (completely senescent) scale. PI534133 was non-senescent (1.2) while the other three resistant lines had low (2.0-2.4) senescence. The most resistant line, PI267379, had low field senescence and low dhurrin.

Diversity of polygalacturonase genes in Rhizoctonia solani AG 2-2 D. H. MINIER (1), L. E. Hanson (2), (1) Michigan State University, East Lansing, MI, USA; (2) USDA ARS, East Lansing, MI, USA

Rhizoctonia solani AG 2-2 affects a number of important crops, including sugar beets, in which it causes a root and crown rot (RRCR). While $R$. solani AG 2-2 functions primarily as a necrotroph, producing dark, macerated tissue, it also can survive for extended periods as a saprotroph. As part of this varied lifestyle and host range, $R$. solani AG 2-2 has a large repertoire of cell wall degrading enzymes. Our work is focused on those that degrade pectin, in particular, polygalacturonase (PG). The goal of this project was to compare pectin degrading genes from a diverse group of isolates and develop a list of candidate genes for further investigation as to their role in virulence. To accomplish this, we sequenced nine isolates from three phylogenetic subgroups within AG 2-2 on an Illumina HiSeq 4000. Assembled contigs were searched for putative genes using annotated assemblies found on GenBank (AG 2-2IIIB \& AG 3). We have identified 151 putative PG genes in nine isolates for an average of 16.8 genes per isolate. The average gene was predicted to produce a 374 amino acid protein contained in 7.9 exons with an average intron length of 59 bases. The number of PG genes per isolate reflected the average virulence of the AG 2-2 subgroups, with the most aggressive subgroup having the largest number of PG genes per isolate (22.3) and the least aggressive subgroup having the lowest number of PG genes per isolate (10.3). This work has identified putative PG genes that are unique to a phylogenetic subgroup and may help explain variation in virulence among AG 2-2 isolates.

Signaling function of antibiotics in the plant beneficial bacterium Pseudomonas protegens Pf-5 Q. YAN (1,2), C. Johnson (2), J. Chang (2), J. E. Loper (2), (1) Montana State University, Bozeman, MT, USA; (2) Oregon State University, Corvallis, OR, USA

Plant health is influenced by the plant-associated microbial community wherein diverse microbes constantly interact with each other. Antibiotics produced by plant-associated microbes play important roles in both competitive and cooperative microbial interactions. In this work, we focus on pyoluteorin, a potent antibiotic produced by many biocontrol strains of Pseudomonas protegens and Pseudomonas aeruginosa. Pyoluteorin has been shown to function as an intracellular signal regulating gene expression and antibiotic production. However, the protein(s) that perceive(s) the pyoluteorin signal has not been identified. Here, using P. protegens Pf-5 as a model, we showed that pyoluteorin directly binds to PltZ, a transcriptional regulator encoded in the pyoluteorin gene cluster. We further showed that PltZ protein directly binds to the promoter regions of pyoluteorin exporter genes and represses the exporter genes expression at transcriptional level. Binding of pyoluteorin to PltZ releases the repression of PltZ and induces the expression of pyoluteorin exporter genes. Overall, our results showed that pyoluteorin not only functions as a potent antimicrobial compound but also 
plays a role in intracellular signaling pathways in that pyoluteorin is directly perceived by a regulatory protein and regulates target gene expression in the producing bacteria.

\author{
Identification of early and extra-early maturing tropical maize inbred lines with multiple disease resistance for use in sub- \\ Saharan Africa \\ F. Bankole (1,2), B. Badu-Apraku (1), S. Abiodun Olusola (3), R. Akinwale (4), T. Falade (5), R. Bandyopadhyay (6), A. ORTEGA- \\ BELTRAN (5), (1) International Institute of Tropical Agriculture, NIGERIA; (2) IITA - Ibadan, Ibadan, NIGERIA; (3) Obafemi \\ Awolowo Univ, Ile-Ife, NIGERIA; (4) Obafemi Awolowo University, Ile-Ife, NIGERIA; (5) International Institute of Tropical \\ Agriculture, Ibadan, NIGERIA; (6) IITA, NIGERIA
}

Northern corn leaf blight (NCLB), southern corn leaf blight (SCLB), Curvularia leaf spot (CLS), and aflatoxin contamination by Exserohilum turcicum, Bipolaris maydis, Curvularia lunata, and Aspergillus flavus, respectively, are major biotic constraints to maize production in sub-Saharan Africa (SSA). Farmers in SSA would benefit tremendously if high yielding maize hybrids with multiple disease resistance (MDR) are developed and commercialized. Forty-nine early maturing (90-95 days to physiological maturity, EM) and 55 extra-early maturing (80-85 days to physiological maturity, EEM) inbreds developed by the Maize Improvement Program of the International Institute of Tropical Agriculture were found to be resistant to NCLB in field evaluations conducted in multiple agroecologies of Nigeria during 2017 and 2018. Thirty of the NCLB resistant inbreds from each maturity group were evaluated for resistance to SCLB and CLS using a detached leaf assay, and to kernel rot and aflatoxin contamination using a kernel screening assay. Seven inbreds (TZEI 1, TZEI 4, TZEI 5, TZEI 9, TZEI 13, TZEI 32, TZEI 83) were found to be resistant to the three foliar pathogens, while five inbreds (TZEI 9, TZEI 11, TZEI 86, TZEI 128, TZEI 269) supported significantly less aflatoxin accumulation. Inbreds with MDR should be tested extensively in hybrid combinations and commercialized. Large-scale use of maize hybrids with MDR would i) reduce losses caused by aflatoxin contamination, and ii) increase maize production and productivity to ensure food security and increased incomes of maize farmers in SSA.

Phenotypic evaluation of Miscanthus core collection for resistance to four sugarcane diseases S. SOOD (1), R. Bruyere (2), N. Labonte (2), V. Gordon (3), A. Lipka (2), J. C. Comstock (4), E. Sacks (2), (1) Sugarcane Field Station, USDA-ARS, Belle Glade, FL, USA; (2) University of Illinois, IL, USA; (3) National Agriculture Library, USDA, MD, USA; (4) USDA-ARS (Retired), Pensacola, FL, USA

Sugarcane is an economically important crop contributing to sugar, fodder, and energy; it has an approximately $\$ 3.2$ billion effect on Florida's economy and supports more than 12,000 jobs. Sugarcane is susceptible to many diseases that can cause significant yield losses. Growing resistant varieties to these diseases is the most sustainable and environmentally friendly method to limit losses. The Canal Point sugarcane breeding and development program is constantly developing resistant varieties and acquiring disease resistance from new sources. Miscanthus, a closely related genus to Saccharum, can provide novel resistance genes to sugarcane by breeding and selection. Therefore, we screened a collection of 74 genotypes, including a mini-core collection of 31 Miscanthus sacchariflorus, a mini-core collection of 35 M. sinensis, and 8 Miscanthus $\times$ cane hybrids (miscanes) for resistance to four important diseases of sugarcane: (1) ratoon stunt caused by a bacterium, Leifsonia xyli subsp. xyli; (2) sugarcane yellow leaf caused by Sugar Cane Yellow Leaf Virus (SCYLV); (3) orange rust caused by a fungus, Puccinia kuehnii and (4) smut caused by a fungus, Sporisorium scitamineum. Negative and positive control sugarcane cultivars for each disease were also included to rule out false negative and positive results. Most Miscanthus and miscanes genotypes were resistant to all four diseases. Only, 10\% of genotypes were susceptible to SCYLV. One miscane genotype was resistant to all four diseases. These resistant genotypes will be beneficial resources for the sugarcane-breeding program.

\title{
Development of a simple, rapid, and sensitive AmplifyRP isothermal assay for detection of Fusarium oxysporum f. sp. cubense Tropical Race 4
}

R. LI (1), B. Davenport (1), S. Zhang (2), K. Schuetz (1), T. Bai (3), G. Fu (4), S. J. Zheng (5,6), (1) Agdia, Inc., Elkhart, IN, USA; (2) Agdia Inc., Elkhart, IN, USA; (3) Yunnan Academy of Agricultural Science, Kunming, CHINA; (4) Guangxi Academy of Agricultural Sciences, Nanning, CHINA; (5) Yunnan, Yunnan Academy of Agricultural Science, Kunming, CHINA; (6) Bioversity International, Kunming, CHINA

Fusarium oxysporum f. sp. cubense Tropical Race 4 (Foc TR4) is threatening worldwide Cavendish banana production. Despite quarantine efforts, the pathogen continues to spread through transport of contaminated plants, human migration, and infested soil and water. Hence, a simple, rapid, and sensitive diagnostic assay is needed for implementing quarantine measures and mitigating spread of the pathogen. Agdia has developed an AmplifyRP assay for the detection of Foc TR4 based on recombinase polymerase amplification. The AmplifyRP assay targets a unique Foc TR4 virulent gene (Gene Bank ID: JX090598). Three DNA sequence regions from this gene were selected for designing AmplifyRP primers and probes to distinguish Foc TR4 from Foc R1, Foc R2, Foc R3, and Foc STR4. Two combinations of the primers and probes have been identified through extensive screening and shown to have specific reaction to $F O c$ TR4 but not to the other Foc races. The limit of detection is 4 copies of DNA amplicon fragment or 6-25 pg of Foc TR4 genomic DNA depending on the DNA purity. Detection results can be obtained within 20 minutes using a portable fluorometer such as AmpliFire. This assay was initially successful to distinguish five Foc TR4 DNA samples from ten other Foc DNA samples from Yunnan province of China. This assay can detect Foc TR4 directly from crude extract of banana tissue, infested soil, and contaminated water samples.

Analysis of leaf-associated bacterial and fungal microbiomes of corn inbred lines related with Tar Spot disease R. SINGH (1,2), S. B. Goodwin (3), (1) USDA-ARS, Whitestown, IN, USA; (2) Purdue University, West Lafayette, IN, USA; (3) USDA ARS, West Lafayette, IN, USA 
Tar Spot is a foliar disease of corn, caused by the obligate biotrophic pathogen Phyllachora maydis (P. maydis), that has recently emerged as an economic concern for corn production in the United States. We investigated phylloplane microbiomes in relation to corn cultivars with differential tar spot symptoms under P. maydis infestation in natural field conditions. Leaf samples from sixteen corn cultivars were assessed for tar spot development and both bacterial and fungal microbiomes were characterized by paired-end sequencing on the Illumina MiSeq platform. Comparative metagenomics analysis of the phylloplane microbiomes revealed clear differences in overall microbial communities between resistant and susceptible corn cultivars. Bacterial communities in both resistant and susceptible lines were dominated by a single operational taxonomic unit (OTU) identified as Methylobacterium spp. The leastabundant OTU in resistant lines was in the genus Pseudomonas, whereas species of Microbacterium and Deinococcus were the leastabundant OTUs in susceptible lines. For fungal communities, genera Fusarium, Genolevuria, Zymoseptoria and Colletotrichum were least abundant whereas Papiliotrema and Alternaria were the most abundant OTUs in the resistant lines. By contrast, fungal communities in susceptible lines were dominated by two OTUs in the genera Puccinia and Sphaerellopsis. Further analyses of differences in the microbiome composition between susceptible and resistant cultivars could lead to the development of novel and more effective biological control agents against Tar Spot.

\section{An alternative succinate dehydrogenase inhibitor to control the pistachio anthracnose caused by Colletotrichum fioriniae in California \\ P. D. S. F. LiCHTEMBERG (1), M. Marzall-Pereira (1), J. Brosin (2), T. Carraro (2), R. Puckett (3), T. J. Michailides (3), (1) University of California - Davis, Parlier, CA, USA; (2) Universidade Federal do Paraná, Curitiba, BRAZIL; (3) University of California, Davis, Parlier, CA, USA}

The pistachio anthracnose caused by Colletotrichum fioriniae was identified first in 2016 in northern California. If not controlled, the entire crop of a susceptible cultivar can be destroyed due to severe fruit blight and drop when peduncles are infected. Among four succinate dehydrogenase inhibitors (SDHI) registered for pistachio, only penthiopyrad (a.i. of Fontelis, Dupont) was able to inhibit $C$. fioriniae in in-vitro assays. The benzovindiflupyr (a.i. of Aprovia, Syngenta), which is not registered for nut crops, was reported to effectively inhibit Colletotrichum spp. The objectives of this study were to demonstrate the in-vitro and ex-vivo efficacy of benzovindiflupyr and to assess the role of single polymorphisms as a putative SDHI resistance mechanism. Mycelial sensitivity assays performed for 78 isolates revealed different mean $\mathrm{EC}_{50}$ values for penthiopyrad $(0.175 \mu \mathrm{g} / \mathrm{ml})$ and benzovindiflupyr $(0.025 \mu \mathrm{g} / \mathrm{ml})$. Preventative sprays on detached leaves of Aprovia at maximum label rate, and Aprovia at the concentration recommended for Fontelis resulted in a better anthracnose control than leaves treated with Fontelis or unsprayed control. Sequences of $s d h B, C$, and $D$ gene subunits revealed no nucleotide differences in regions already associated with SDHI resistance in other pistachio pathogens. After three successive UV irradiation of $C$. fioriniae conidia for different time regimes, the sequences of $\operatorname{Sdh} B, C$ and $D$ did not differ from that of wild-type isolates. UV irradiated colonies had no growth penalty or effect on benzovindiflupyr or penthiopyrad sensitivity.

\section{Virus-induced changes in the localization of nuclear proteins in Nicotiana benthamiana C. I. MATHIAS, R. Wang, M. M. Goodin, University of Kentucky, Lexington, KY, USA}

Viruses recruit host proteins that are required for processes such as replication and movement. This often results in a subversion from the normal site of protein localization to loci where virus-specific functions occur. To identify novel nuclear proteins involved in these processes, a Nicotiana benthamiana cDNA library was mobilized into the pSITE-2CA binary vector, for the expression of GFP fusions of random proteins. Over 1,080 of these tagged proteins were agroinfiltrated into transgenic $N$. benthamiana plants expressing RFP bound to histone H2B nuclear reference marker. Of these, 350 were found to localize in the nucleus. These proteins were then expressed in N. benthamiana plants challenged with Sonchus Yellow Net Virus (SYNV), a member of the genus Nucleorhabdovirus that forms its replication complex in the nucleus. These plants expressed RFP targeted to the endoplasmic reticulum in order to mark viral proliferations in the perinuclear space. It was determined that 15 of the 350 nuclear localized proteins had a modified localization pattern in response to SYNV infection, such as moving from the nucleolus to punctate regions throughout the nucleoplasm. For comparison, these proteins were also expressed in plants challenged with tobacco etch virus (TEV), a potyvirus which replicates in the cytoplasm. General and virus-specific changes in localization patterns were observed. This work serves as a demonstration of how visualization may be used to identify nuclear host proteins that are involved in viral infection, which will expedite downstream interaction studies.

Evaluation of sweetpotato rotational crops and weeds as hosts of Ceratocystis fimbriata, causal agent of sweetpotato black rot M. STAHR (1), L. M. Quesada (2), (1) North Carolina State University, Raleigh, NC, USA; (2) Department of Entomology and Plant Pathology, North Carolina State University, Raleigh, NC, USA

Ceratocystis fimbriata, causal agent of sweetpotato black rot, is responsible for severe yield losses in North Carolina, where over 50\% of United States sweetpotatoes are grown. Crop rotation is often used for disease control of this soilborne fungus, yet no studies have determined the host range of $C$. fimbriata amongst sweetpotato rotational crops. In this study, ten plants per 12 crop and weed type, were evaluated as hosts of $C$. fimbriata over two repetitions. Plants in 6-inch pots were drenched with $10 \mathrm{~mL}$ of sterile distilled water (SDW) or a $5 \times 10^{3} \mathrm{C}$. fimbriata spore/mL suspension one week after transplant. Disease incidence and mortality were recorded weekly for five weeks. Two additional repetitions were then performed with methods identical to the initial study, except $10 \mu \mathrm{L}$ of SDW or a $C$. fimbriata spore suspension were injected into a wound made with a sterile toothpick to assess wounding effects. Without wounding, $100 \%$ of sweetpotatoes and $10 \%$ of carrot and morning glory plants became infected after drench inoculation. However, when plants were wounded, $100 \%$ of sweetpotato plants died by the end of the trial, and $20-100 \%$ of inoculated carrot, cotton, lettuce, morning glory, soybean, and tobacco plants developed disease symptoms, and in severe cases resulted in plant mortality. Cabbage, peanut, 
squash, watermelon, and wheat failed to develop symptoms in all studies. These results indicate that many crops rotated with sweetpotato can serve as hosts for $C$. fimbriata, particularly when wounded, and that alternative methods are necessary to control this disease.

Development of a PCR-based detection assay for Erwinia aphidicola, a bacterial plant pathogen of concern in the United States A. NELSON (1), M. J. Stulberg (2), W. Cai (2), S. Albu (3), J. Rascoe (2), A. C. Fulladolsa (1), (1) Colorado State University, Fort Collins, CO, USA; (2) USDA-APHIS-PPQ-S\&T-CPHST, Beltsville, MD, USA; (3) California Department of Food and Agriculture, Sacramento, CA, USA

Erwinia aphidicola $(E a)$ was recently reported affecting legume, cucurbit, and solanaceous hosts in the U.S. It is a bacterial leaf spot pathogen first reported on common bean and pea in Spain in 2003-2007, causing up to 50\% crop loss. In May 2018, Ea was listed as reportable/actionable by USDA APHIS PPQ-PDEP. Its unknown establishment in the U.S. and pathogenicity on multiple hosts pose a threat to U.S. agriculture. Ea identification requires pathogen isolation, followed by a multi-locus sequence analysis (MLSA) to confirm the species. Although this procedure has been useful, a more efficient rapid screening method for $E a$ in suspect samples is needed. The CSU Plant Diagnostic Clinic is collaborating with the APHIS-PPQ Beltsville laboratory to develop and validate a rapid and costeffective PCR-based detection assay for $E a$. We isolated seven strains of $E a$ in symptomatic plants from Colorado, Ohio, and California. MLSA using rpoB, recA, and gapDH gene sequences showed all strains nested within a clade containing the Ea type strain. The genomes of six strains were sequenced and assembled de novo. We will use Uniqprimer, an automated primer design tool based on genome comparison analysis, to design real-time PCR primer sets specific to $E a$. The primer sets will be evaluated against a panel of related species. A real-time PCR assay will be optimized and validated in both laboratories. We will present the results of this research, a PCR-based assay that can be adopted by diagnostic clinics across the U.S. to improve our capacity to detect, monitor, and report Ea occurrence.

Epigenetic gene therapy strategies to manage citrus greening using the transient Citrus Tristeza Virus vectors C. A. A. EL-MOHTAR, T. Shilts, C. Robertson, W. O. Dawson, University of Florida, Lake Alfred, FL, USA

The citrus industry, especially in Florida, is facing the debilitating challenge of citrus greening, which is caused by a gram-negative bacteria Candidatus Liberibacter asiaticus (Clas). Clas is transmitted by the Asian citrus psyllid (ACP). Citrus tristeza virus (CTV) and Clas co-localize in phloem tissue of citrus where the ACP feeds. In Florida, CTV strains are relatively mild. Isolates of the T36 strain cause decline and death of trees on the sour orange rootstock but are symptomless in other scion/rootstock combinations. Isolates of the T30 strain are symptomless even in trees on the sour orange rootstock. Transient-expression vectors have been created based on CTV to express foreign genes in citrus or sequences to silence host genes. The exceptional feature of CTV-based vectors is their ability to express foreign genes or sequences at different locations within its genome for long periods of time (years). Thus, CTV-T36 based expression/RNAi vector/s have been used to screen therapeutic sequences in citrus seedlings to limit the pathogenic bacterium and the ability of the psyllid to transmit the disease. CTV-T36 expressing small peptides has been used to target the bacteria directly via antimicrobial peptides (AMPs) or induce plant resistance via expressing bacterial peptides. Furthermore, CTV RNAi expressing certain truncated ACP mRNAs prevented their reproduction on citrus seedlings. We now are using CTV RNAi vectors to screen potential plant CRISPR targets to engineer resistance. As a result, we have identified plant CRISPR targets to engineer resistance to HLB.

Unravelling the trans-kingdom function of dicer-independent tRNA-derived small RNAs

Y. CAO, C. Feng, A. Neupane, S. Y. L. Marzano, South Dakota State University, Brookings, SD, USA

In Sclerotinia sclerotiorum, white mold fungus, tRNA-derived small RNAs (tRFs) potentially belong to a new class of virulence effector because we previously reported two very abundant of larger size at 28-30 nt that had no fungal targets predicted based on transcriptome, degradome and small RNA-Seq of S. sclerotiorum. We also demonstrated that the tRFs in S. sclerotiorum are not generated by dicers because the double dicer knockout mutant of dcl-1 and dcl-2 genes does not have altered accumulation in these tRFs. Therefore, we hypothesize that these tRFs do not play a role in endogenous gene regulation in S. sclerotiorum, but instead are involved in pathogenesis by silencing specific plant gene expressions. The goal is to determine the roles of these major tRFs in white mold virulence towards plants. The invertase gene in Nicotiana benthamiana is one of the predicted target genes of tRF2. So far, our results showed that transient overexpression of the invertase gene in $N$. benthamiana improves plants' tolerance to $S$. sclerotiorum. Currently, the tRFs' function in a trans-kingdom manner are investigated by immunoprecipitation of argonaute protein for small RNASeq. Short tandem target mimics are also being expressed in planta or produced in vitro to block tRF functions. The study will help provide knowledge to devise ways disarming the novel class of effectors used by white mold to attack plants.

\author{
Hemp diseases in Arkansas \\ C. FENG, M. Villarroel-Zeballos, P. Ficheux, H. Zima, J. Correll, University of Arkansas, Fayetteville, AR, USA
}

Hemp (Cannabis sativa) had been grown in the U. S. as a fiber crop for 300 years before it was legally restricted and eventually banned as a schedule 1 drug in 1970. The strong interest in fiber, grain and potential medical use of hemp has driven the farm bill legislation in 2018, and hemp was reintroduced into AR as a crop, where approximate 700 ha were grown in 2019. To investigate what diseases may be present on hemp in AR, more than 120 symptomatic disease samples, from different age hemp plants, were collected from throughout the state for diagnosis. Isolations, morphological and molecular identification of various fungi, and pathogenicity tests were performed. Various fungal species were recovered from the diseased samples. Botrytis cinerea and Fusarium oxysporium were recovered from diseased hemp seedlings. A number of fungi were isolated from symptomatic leaf tissue, including Bipolaris sp., 
Curvularia sp., Nigrospora sphaerica, Epicoccum sp., and Phoma sp. Powdery mildew (Golovinomyces cichoracearum) was found at multiple farms and greenhouses. Pythium aphanidermatum, Fusarium oxysporum, and $F$. solani were recovered from hemp plants with crown rot symptoms. Fusarium chlamydosporum and Lasiodiplodia sp. were isolated from hemp tissue with stem canker. A dieback disease, caused by Neofusicoccum parvum, was confirmed. Although reported in Italy, this is the first report of this disease in the U. S. Pathogenicity of a number of fungal species are underway to determine if that are pathogens of hemp.

High-throughput sequencing analysis of the Basella alba virome in Hawaii X. WANG, University of Hawaii at Manoa, Honolulu, HI, USA

In 2018, Malabar spinach (Basella alba, Basellaceae) with leaves exhibiting symptoms of mosaic, rugose, and malformation on leaves were found in a community garden in Oahu, Hawaii. Preliminary studies using ELISA and RT-PCR identified basella rugose mosaic virus (BaRMV) in symptomatic plants. Sequence analyses of RT-PCR products with BLASTX/BLASTN from plants in different gardens suggested that additional potyviruses were present in some community gardens in Hawaii. High-throughput sequencing (HTS) analyses were conducted using a ribodepleted total RNA approach. Symptomatic samples from three community gardens were collected and the samples from each community garden combined as one HTS sample. About 40 million single-end reads were generated, and after quality control, trimming, and de novo assembly, 72,959 contigs were generated. Contig annotation with BLASTX/BLASTN revealed the presence of a complex virome and contigs resembling BaRMV, chilli veinal mottle virus (ChiVMV), alternanthera mosaic virus (AltMV), basella alba endornavirus (BaEV), broad bean wilt virus 2 (BBWV2), and iresine viroid 1 (IrVd-1). Pathogen-specific primer sets were then designed based on the retrieved contigs and used in RT-PCR, and Sanger sequencing further confirmed the presence of these viral and viral-like agents. Additional $B$. alba samples from other community gardens are being examined to determine the incidence, distribution, and diversity of these viruses and viroids, and their possible role in symptom expression in Hawaii.

Nutrient amendments and residue incorporation in long-term crop monocultures impact yield and pathogen-suppressive activity of soil Streptomyces

M. FULCHER (1), A. Dicko (2), S. Castle (3), L. L. Kinkel (3), (1) University of Minnesota, Saint Paul, MN, USA; (2) University of Segou, MALI; (3) University of Minnesota, MN, USA

Long-term crop monocultures are sometimes associated with increased pathogen-suppressive capacity among soil microbes. However, we have a limited understanding of variation in microbial population responses to management practices. A unique, 60-year, 5-crop (soy, corn, wheat, barley, oat) experiment with factorial fertilizer (NPK, +/-) and residue (+/-) treatments provides an opportunity to disentangle the effects of monoculture, nutrient, and residue management on pathogen-suppressive microbes and crop yields. The density of soil Streptomyces inhibitory to standard pathogen targets, Streptomyces inhibition strength, and crop yields were determined in all plots in two consecutive years. Yields increased with NPK, but not residue incorporation. Inhibitor density varied with year, crop, pathogen and residue, while inhibitory strength varied with year, crop, pathogen and fertilizer. However, the direction and magnitude of change in response to residue and NPK inputs differed among crops. Inhibitory strength was positively correlated with inhibitor density ( $\mathrm{r}=0.11, P \leq 0.001)$, suggesting a slight benefit to highly inhibitory Streptomyces in dense communities. Our data show pathogensuppressive activity in Streptomyces communities is altered by complex interactions between crops and management practices. The distinct effects of residue and NPK inputs highlight the potential for crop-specific management to promote pathogen-suppressive soil microbial communities.

Is there a relationship between root traits and susceptibility to fire blight in apple (Malus $\times$ domestica)? A. KHAN PHD (1), J. Singh (1), J. Fabrizio (1), E. Desnoues (1), K. J. Pereira Silva (1), W. Busch (2), (1) Cornell University, Geneva, NY, USA; (2) Salk Institute for Biological Studies, Plant Molecular and Cellular Biology, La Jolla, CA, USA

Fire blight, caused by Erwinia amylovora, is a systemic bacterial disease that leads to significant losses in apple production worldwide. Genetic resistance to fire blight exists in wild Malus species, however, due to long juvenility periods, high heterozygosity, and selfincompatibility in apples, it can take 20-25 years to achieve good fruit quality. Studies have indicated that root system architecture directly regulates upper plant growth, physiology and tolerance to fire blight, but the role of rootstock root traits in providing systemic defense against pathogens is unclear. We carried out several experiments to understand the relationship between root traits and fire blight susceptibility of grafted scions. We observed a significant $(p<0.05)$ negative correlation between fire blight susceptibility and root surface area. Furthermore, contrasting changes in gene expression patterns of diverse molecular pathways accompanied different levels of root-driven fire blight susceptibility. A singular co-expression gene network consisting of genes from defense, carbohydrate metabolism, protein kinase activity, oxidation-reduction, and stress response pathways seems to modulate root-dependent fire blight susceptibility in apple. We conclude that low root mass may incite resource-limiting conditions to activate carbohydrate metabolic pathways, which reciprocally interact with plant immune system genes to elicit differential levels of fire blight susceptibility.

Biology and management of blossom end rot disease of chestnut

A. C. MILlER (1), M. L. Lewis Ivey (2), (1) The Ohio State University, Wooster, OH, USA; (2) Department of Plant Pathology, The Ohio State University, Wooster, OH, USA

Blossom end rot (BER), caused by the Colletotrichum gloeosporioides species complex (CGSC), has become a significant economic detriment to chestnut production in Ohio since 2010, with 40\% crop loss in 2018, and no known methods of control. This study explores potential host resistance to BER and tests a fungicide known to be effective against the CGSC in other fruits. In commercial orchards in 
Ohio in 2019, disease incidence was compared in eight chestnut cultivars of high commercial value. Significant differences were found both among cultivars within a single site and within a single cultivar across different sites, suggesting that host susceptibility is influenced by environmental conditions. The bloom window of each cultivar was assessed, and daily precipitation and temperatures were recorded from a regional weather station. No significant correlations were observed between weather conditions at bloom time and disease incidence at harvest. The fungicide Sulforix ${ }^{\circledR}$ was applied to three separate orchards using late-dormancy application guidelines effective against the CGSC in other fruit crops, and disease incidence was compared to non-treated areas of those same orchards. No significant differences in BER incidence were found between trees in treated and non-treated areas. The search continues to discover parameters that predict chestnut BER incidence and effective management strategies for this disease.

Evaluating a Pseudomonas collection for antagonistic potential against Pythium spp. and generation of a consortia E. NAVARRO-MONSERRAT, R. Kimmelfield, C. G. Taylor, The Ohio State University, Wooster, OH, USA

Certain Pythium spp. are oomycetic plant pathogens which cause root rot in hydroponic vegetable production systems. Bacteria belonging to the Pseudomonas genus have been extensively studied for their capacity to suppress oomycetes via different modes of action and present themselves as a potential useful tool in the management of Pythium infections on hydroponically grown crops. Utilizing a collection of Pseudomonas strains we aim to; 1) utilize dual plating and volatile indirect exposure in vitro assays to identify strains with antagonistic activity against three Pythium isolates ( $P$. oopapillum isolates PO6-19 and PO7-20 and P. aphanidermatum isolate PA5-18; previously collected from spinach and lettuce hydroponic system), and 2) assess the compatibility of our Pseudomonas strains to determine compatibility using the in vitro top agar method. Shared-air and dual plating assays were carried out to assess the antagonistic indirect and direct activity against the Pythium isolates. In the dual plating assays, strains Darke and 1B1 were inhibitory $(>50 \%)$ to PA5-18 mycelial growth while strains 14B11 and 29G9 were inhibitory ( $>40 \%)$ against PO6-19. In the indirect exposure assay, six strains were inhibitory ( $>50 \%$ ) against PO6-19 and nine strains against PO7-20. Compatibility, or lack thereof, was observed between bacteria strains using the top agar method. Future experiments will revolve around determining the biocontrol potential of the best performing strains in single and multiple applications against Pythium spp. in hydroponic systems.

Endofungal Mycetohabitans spp. have TAL-like proteins that can alter the transcriptome and stress tolerance of their host M. CARTER (1), S. Carpenter (1), Z. Dubrow (1), M. Sabol (1), F. Rinaldi (1), O. Lastovetsky (1,2), S. Mondo (1,3), T. E. Pawlowska (1), A. J. Bogdanove (1), (1) Cornell University, Ithaca, NY, USA; (2) University College Dublin, IRELAND; (3) US Department of Energy Joint Genome Institute, CA, USA

From endophytes to mycorrhizae, diverse plant-associated fungi harbor bacteria. Despite increasing discoveries of endohyphal bacteria, the mechanisms by which bacteria manipulate fungal hosts are largely unknown. For example, Rhizopus microsporus causes rice seedling blight using a toxin synthesized by its symbiont, Mycetohabitans (formerly Burkholderia) rhizoxinica. M. rhizoxinica requires its Type III secretion system (T3SS) to infect $R$. microsporus, but no effector proteins have been characterized. Yet, the sequenced Mycetohabitans genomes each encode proteins resembling DNA-binding Transcription Activator-Like (TAL) effectors from phytopathogenic Xanthomonas and Ralstonia spp. In contrast to TAL effectors, Burkholderia TAL-like (Btl) proteins have truncated Nand C-termini, lacking predicted signals and domains critical for secretion, nuclear localization, and transcriptional activation; thus, their function in the symbiosis was unclear. Our evidence shows that a representative Btl protein, Btl19-13, has T3S and nuclear localization signals, can impact the host transcriptome, and increases the tolerance of $R$. microsporus to cell membrane stress. We found that a Btl protein from another bacterial strain is not functionally interchangeable with Btl19-13. Through Southern blotting and whole genome sequencing, we are investigating $b t l$ gene content across geographically diverse Mycetohabitans isolates. Btl proteins offer a way to probe the underpinnings of a bacterial-fungal symbiosis related to plant health and may inform our understanding of a unique effector family.

Characterizing Huanglongbing-pH interaction to improve management of root function S. SHARMA, T. Vashisth, E. G. Johnson, University of Florida, Lake Alfred, FL, USA

Huanglongbing (HLB), a problematic citrus disease worldwide caused by the phloem-limited 'Candidatus Liberibacter asiaticus' (CLas) shows early symptoms of reduced root health. Prior studies suggest that $C$ Las infection alters the pH response of root function in citrus affecting overall tree health. Additionally, Las infection has systemic effects on root dieback. Two experiments were set up using split root rhizotrons with different $\mathrm{pH}$ fertigation on each side. The first experiment was set to determine the effects of differential $\mathrm{pH}$ from managed and unmanaged zones on HLB-affected tree health. The second experiment was set to determine if sensitivity to $\mathrm{pH}$ is a systemic or local effect. Bud inoculation was done with healthy and HLB-affected bud on 'US-942' (pH tolerant) and 'Swingle' (pH sensitive) rootstocks arranged in a randomized complete block design of 8 blocks, each with HLB and healthy rootstocks in 2:1 ratio. Plants were irrigated every 3-4 days with high bicarbonate well water at $\mathrm{pH}$ adjusted to 5.5, 6.5, 7.5 with $\mathrm{H}_{2} \mathrm{SO}_{4}$. Unexpectedly, successful infection occurred in only $30.3 \%$ of US 942 seedlings compared to $69.7 \%$ for Swingle. Average log CLas genomes per mg of root was 2.59 for US942 and 4.16 for Swingle. The results suggest a partial resistance of US942 to CLas. Biweekly root tracing and preliminary data on physiological parameters also indicated a possible interaction of $\mathrm{pH}$ and $C$ Las infection. Further research could direct to optimize acidification treatments for HLB management while also providing rootstock specific targets for soil $\mathrm{pH}$.

Improving control of sclerotinia stem rot in soybean by integrated management R. WEBSTER (1), M. Roth (2), B. Mueller (1), J. Gaska (3), D. S. Mueller (4), M. Chilvers (5), S. Conley (1), D. L. Smith (1), (1) University of Wisconsin-Madison, Madison, WI, USA; (2) University of Wisconsin - Madison, Madison, WI, USA; (3) Arlington Research Station, Arlington, WI, USA; (4) Iowa State University, Ames, IA, USA; (5) Michigan State University, East Lansing, MI, USA 
Sclerotinia stem rot (SSR) of soybean is caused by the fungal pathogen Sclerotinia sclerotiorum and can result in significant yield losses. There is interest in optimizing management solutions for SSR, which includes using a fully integrated approach. This study examined the effects of integrating row spacing, planting population, and foliar fungicide applications on SSR disease severity index (DIX) and soybean yield potential using multi-state, multi-year field trials from 2017-2019. The interaction of row spacing and planting population had a significant effect on both DIX $(P=0.04)$ and yield $(P<0.01)$. DIX was lowest with a planting population of less than 345,000 seeds/ha in a $76-\mathrm{cm}$ row spacing. Conversely, DIX trended higher in the $38-\mathrm{cm}$ row spacing and was highest when a planting population of 420,000 seeds/ha was used with a $38-\mathrm{cm}$ row spacing. However, yields were highest in 38 -cm rows and decreased as planting populations were reduced at both row spacings. Fungicide application had a significant effect on DIX $(P<0.01)$ and yield $(P<$ 0.01). The greatest reduction of DIX and the highest yields were observed when fungicide was applied at both R1 and R3 growth stages. While our analysis suggests wide row spacing and lower planting populations can reduce disease, it can also decrease yield potential. Therefore, additional factors such as field history and environmental factors need to be considered for field specific SSR management, but the combination of wide row spacing and low populations is recommended for high-pressure SSR fields.

Predicting present and future distributions of invasive forest pathogens under changing climates

M. S. KIM (1), J. W. Hanna (2), J. Stewart (3), N. B. Klopfenstein (2), (1) USDA Forest Service, Pacific Northwest Research Station, Corvallis, OR, USA; (2) USDA Forest Service, Rocky Mountain Research Station, Moscow, ID, USA; (3) Colorado State University, Fort Collins, CO, USA

Invasive forest pathogens are expected to have a greater impact on forest health under future climate-change scenarios as rapidly changing climates will likely alter the distribution of emerging pathogens and predispose trees to other disturbances (e.g., fire, insects, etc.). Predictions of current and future distributions of invasive forest pathogens will guide forest managers and regulatory agencies for appropriate management practices to minimize impacts on forest ecosystems. Two invasive forest pathogens, Phellinus noxius (brown root rot) and Austropuccinia psidii (myrtle rust) are used to demonstrate bioclimatic prediction models (maps) of both current and future distribution. Both invasive pathogens cause destructive disease on diverse tree species that are integral parts of forest ecosystems, economies, and native culture in tropical/subtropical regions. Contemporary prediction models were produced using Maximum Entropy algorithms that couple climatic data with 179 and 403 georeferenced and DNA sequence-confirmed occurrence locations for $P$. noxius and $A$. psidii, respectively. From the contemporary models, data were extrapolated through future climate scenarios to forecast areas at risk for invasion by these pathogens. In addition, combining population and phylogenetic studies with bioclimatic modeling show that distinct genetic groups within both species pose distinct threats, which should be recognized by regulatory agencies to limit damage from these invasive forest pathogens.

Baseline sensitivity to adepidyn of isolates of Passalora arachidicola causing early leaf spot on peanuts G. OLAYA (1), K. L. Stevenson (2), (1) Syngenta Crop Protection, Vero Beach, FL, USA; (2) University of Georgia, Tifton, GA, USA

Early leaf spot, caused by Passalora arachidicola, is one of the major foliar diseases of peanuts. Multiple fungicide applications are needed to manage the disease. Adepidyn (pydiflumetofen) is the active ingredient of the fungicide Miravis ${ }^{\circledR}$ that was recently introduced to the peanut fungicide market. Adepidyn is a member of the succinate dehydrogenase inhibitor class of fungicides (FRAC Code 7), has a broad spectrum of control, and contains a high intrinsic activity due to its binding properties to the complex II enzymes. The sensitivity of 45 isolates of $P$. arachidicola to adepidyn was determined using a conidial germination inhibition assay. Isolates were obtained from symptomatic lesions from peanut fields in Georgia. Sensitivity tests were conducted in vitro using water agar medium amended with adepidyn at the following concentrations: $0,0.0001,0.0005,0.001,0.01$ and $0.1 \mathrm{mg}$ a.i./L. The distribution of EC 50 values of these adepidyn baseline isolates of $P$. arachidicola ranged from 0.00004760 to $0.00116692 \mathrm{mg}$ a.i./L with a geometric mean of $0.00020654 \mathrm{mg}$ a.i./L and a range of $24.5 \mathrm{X}$. This adepidyn baseline sensitivity distribution can be used as a point of reference in future resistance monitoring studies of $P$. arachidicola.

\section{Estimation of the residue content in streptomycin-treated citrus plants infected by Candidatus Liberibacter asiaticus using HPLC}

J. LI (1), Z. Pang (1), D. Lee (2), N. Wang (1), (1) Citrus Research and Education Center, University of Florida, Lake Alfred, FL, USA; (2) University of Florida, Lake Alfred, FL, USA

Streptomycin (STR) is currently being used to control citrus Huanglongbing (HLB) in Florida by foliar spray. However, the effective residue content of STR against the HLB pathogen Candidatus Liberibacter asiaticus (Las) in planta after treatment remains unknown. Here we developed and validated an analytical method for the determination of STR in citrus using reversed-phase high performance liquid chromatography (RP-HPLC) with ultraviolet (UV) detection. With this method, we determined the residue dynamics of STR in leaf tissues (midribs) of treated citrus plants following application by trunk injection in a field trial. We also determined Las titers in leaf midribs using qPCR assays at various times after treatment. The results showed that STR injection at $2.0 \mathrm{~g} /$ tree resulted in a decline of Las populations by $2.36 \mathrm{log}$ unit at 28 days post treatment, with an observed level of STR residue at 1.45 ppm. Nonlinear regression analyses and statistical modeling indicated that the levels of suppression of Las population were positively associated with STR residue levels in leaves, and, predicated the threshold for STR to suppress Las populations in planta to below the detection limit is $\geq 1.90$ ppm in leaf tissues. These findings provide useful information for citrus growers in decision making regarding controlling HLB with STR.

Characterization of Alternaria spp. as postharvest rot pathogens in pears

J. DESHIELDS, Q. Luong, A. N. KC, Southern Oregon Research and Extension Center, Oregon State University, Central Point, OR, USA 
The genus Alternaria is categorized into 26 sections and represented by more than 200 species. Many Alternaria species are saprophytes and pathogens of cultivated plants, such as A. alternata, A. tenuissima, and A. arborescens, which have been reported to cause core rot in apples, and fruit rot in blueberries and persimmons. While several fungal species cause postharvest rot on European pears, Alternaria spp. are frequently isolated and considered weak postharvest rot pathogens on pear. Here we characterize pear-isolated Alternaria spp. using DNA-based and morphological approaches and compare the pathogenicity of particular Alternaria sections on four pear varieties, Anjou, Bartlett, Comice, and Bosc. Thirteen isolates of Alternaria spp. were categorized into Alternaria sect. Alternata, sect. Infectoriae and sect. Ulocladioides, which were determined using the DNA sequences of the Alternaria major allergen (Alt a 1), plasma membrane ATPase, glyceraldehyde-3-phosphate dehydrogenase (gpd), Actin and Calmodulin loci, in addition to morphological features. Alternaria rot varied in severity on wound-inoculated fruits contingent on the Alternaria section of the isolate used but was uncommon amongst spray-inoculated fruits. Isolates from Alternaria sect. Alternata were pathogenic across all cultivars. Isolates from Alternaria sect. Ulocladioides only caused rots on Anjou and Bosc pears, while isolates from Alternaria sect. Infectoriae developed rots on only Bosc pears. In the presence of wounds, some Alternaria spp. appear to cause a significant amount of postharvest rot on pears.

\section{Microscopy and associations of Litylenchus crenatae (Anguinata) in beech Fagus grandifolia (Fagaceae) infested by beech leaf disease in North America \\ L. K. CARTA (1), Z. Handoo (2), G. Bauchan (3), D. Burke (4), (1) USDA ARS NEA MNGDBL, Beltsville, MD, USA; (2) USDA- ARS NEA MNGDBL, Beltsville, MD, USA; (3) USDA-ARS NEA Electron and Confocal Microscopy Unit, Beltsville, MD, USA; (4) The Holden Arboretum, Cleveland Botanical Garden, Kirtland, OH, USA}

A new disease of American beech trees, a major component of many deciduous forest areas in eastern North America, was discovered near Cleveland, Ohio, in 2012. Beech leaf disease (BLD) caused dark green and yellow stripes in leaves and could kill mature trees within seven years of detection. Its cause was recently demonstrated by researchers from the USDA-ARS, Beltsville, MD; Holden Arboretum; the state of Ohio and other Northeastern US states; and Canada. They isolated parasitic roundworms known as nematodes from diseased leaves and transmitted symptoms to previously healthy seedlings, primarily through buds. In this work, freeze-fracture low-temperature scanning electron microscopy images of the nematodes from symptomatic leaf and bud tissue illustrated the extensive damage to leaves and buds. Nematodes were also imaged entwined around the bodies of mites. Birds were also implicated through a microbiome marker for bird mites as a possible means of nematode distribution. DNA markers established that the nematode is closely related to a nematode population on beech trees in Japan where the disease associated with the nematode is not lethal. No previously known foliar tree diseases in North America have been caused by nematodes, so these BLD nematodes are likely to have been transported from Asia. These results are important because the disease is spreading primarily eastward and identification of transmission agents are needed to stop BLD progression. The information is useful to foresters, ecologists, geneticists and plant pathologists for integrated control of BLD.

\section{Molecular and functional analyses of the barley NLR immune receptor PBR1}

M. HELM (1), A. Myers (2), T. Frailie (3), R. W. Innes (3), S. R. Scofield (1), (1) USDA-ARS, West Lafayette, IN, USA; (2) Purdue University, West Lafayette, IN, USA; (3) Indiana University, Bloomington, IN, USA

Plant disease resistance is often mediated by intracellular innate immune receptors known as nucleotide-binding leucine-rich repeat receptors (NLR) whose central function is to detect the activity of pathogen-secreted effectors. Upon recognition, the NLR protein initiates an immune response known as the hypersensitive reaction (HR), which is characterized by rapid, localized cell death at the infection site. NLR proteins typically consist of an amino-terminal coiled-coil (CC) domain, a central nucleotide binding (NB-ARC) domain, and a carboxy-terminal leucine-rich repeat (LRR) domain. We previously identified an NLR gene from barley known as AvrPphB Response 1 ( $P b r 1)$, whose protein product confers recognition of the Pseudomonas syringae cysteine protease, AvrPphB. However, little is known about the molecular mechanisms that lead to PBR1-dependent cell death in response to AvrPphB. Here, we investigated PBR1 signaling after Agrobacterium-mediated transient expression in Nicotiana benthamiana. We show that PBR1 encodes a protein of 939 amino acids with an N-terminal CC domain, a central NB-ARC domain, and an LRR domain containing 12 repeats at the C-terminus. Co-expression of PBR1 with AvrPphB resulted in robust cell death, confirming that PBR1 is indeed the cognate NLR that recognizes AvrPphB protease activity. Further, none of the individual domains or fragments of PBR1 induced an HRlike cell death response as strong as full-length PBR1 when co-expressed with AvrPphB, indicating that the individual domains and fragments of PBR1 are insufficient to trigger the HR.

\section{Getting a jump on new banana diseases: Risk assessment for Haiti and the Caribbean}

W. Dantes (1,2,3), Y. Xing (1,2,3), R. Choudhury (1,2,3,4), J. Fayette (1,2,3), K. F. Andersen (1,2,3), G. Blomme (5), M. A. Dita Rodriguez (6), W. Ocimati (7), O. N. Carvil (8), J. F. Hernandez Nopsa (9), K. A. GARRETT (1,2,3), (1) Institute for Sustainable Food Systems, University of Florida, Gainesville, FL, USA; (2) Emerging Pathogens Institute, University of Florida, Gainesville, FL, USA; (3) Plant Pathology Department, University of Florida, Gainesville, FL, USA; (4) University of Texas Rio Grande Valley, Edinburg, TX, USA; (5) Bioversity International, c/o ILRI, Addis Ababa, ETHIOPIA; (6) International Center for Tropical Agriculture (CIAT), Cali, COLOMBIA; (7) Bioversity International, Kampala, UGANDA; (8) Faculty of Agronomy and Veterinary Medicine, Port-auPrince, HAITI; (9) Corporación Colombiana de Investigación Agropecuaria, AGROSAVIA, Mosquera-Bogota, COLOMBIA

Banana (including plantains), an important staple in the Caribbean region, is threatened by several emerging pathogens, including Xanthomonas vasicola pv. musacearum, banana bunchy top virus, Ralstonia solanacearum, and Fusarium oxysporum $\mathrm{f}$. sp. cubense tropical race 4. To assess the risk of these emerging pathogens for banana production in the Caribbean region, we evaluated several key risk factors for their spread and interactions. First, the risk of introduction of the last two pathogens is very high, because they have been 
formally reported to cause losses in banana production in countries that trade with Haiti and the Dominican Republic. Second, cropland connectivity analysis represents disease risk due to spread among banana farms and regions. Plantations that are clustered or act as bridges between banana plantations likely have important roles in the risk of spread. The Caribbean, especially Haiti, has particularly dense banana plantations. Third, many locations in the region have suitable climate conditions, contributing to a high risk of pathogen establishment. We evaluated how these system components interact to create higher and lower disease risk regions. Most countries in the Caribbean region are at risk for these diseases. Based on expert elicitation in Haiti, we found that places along the HaitianDominican border are at higher risk for pathogen introduction and should be under surveillance. This risk analysis can be used by National and Regional Plant Protection Organizations to reduce risks of pathogen introduction and establishment in the Caribbean.

Characterization and diversity of endophytic fungi in different parts of cowpea using Illumina Sequencing Approach T. ROSEMARY KINGE (1), G. T. Pambuka (2), M. Gryzenhout (2), (1) The University of Bamenda, Bambili, North West Region, CAMEROON; (2) The University of the Free State, Bloemfontein, SOUTH AFRICA

Cowpea is an economically important indigenous African legume crop. Different parts of the vegetative organs can carry endophytic fungal communities that can be beneficial or deleterious to the health and productivity of the plant. Next Generation Sequencing technologies have enabled intensive characterization of the mycobiome from plants. Endophytic fungi were characterized, and their diversity was determined in symptomless tissues of the leaf, main stem, crown stem and roots of cowpea. DNA was extracted and the ITS2 region was sequenced using the Illumina MiSeq. Data were analyzed using qiime, principal coordinates analysis and phylogenetic analyses. The highest fungal diversity was recorded for the Ascomycetes followed by Basidiomycetes. The highest fungal species richness was recorded from the roots and the least from the main stem. Some endophytes showed tissue specificity. Thirty fungal genera were identified in one or more of the four cowpea plant parts with Fusarium, Cladosporium and Phoma being the dominant genera. The result is a concern since some endophytic species might become pathogenic with time. Environmental sequencing is thus a useful tool for fungal community diversity comparisons. Taxa can be compared and monitored over time, aiding strategies for disease prevention and increased plant health.

Virulence assessment of Xylella fastidiosa subsp. fastidiosa strains from grapes using the model host Nicotiana tabacum L. M. GÓMEZ (1), Q. Ge (1), R. P. Naegele (2), R. P. P. Almeida PhD (3), L. De La Fuente (1), (1) Auburn University, Auburn, AL, USA; (2) USDA ARS, Parlier, CA, USA; (3) University of California Berkeley, Berkeley, CA, USA

Xylella fastidiosa $(X f)$ is a gram-negative plant pathogenic bacterium that lives in xylem vessels of infected plants and foregut of insect vectors like sharpshooters. $X f$ causes diseases in many important crops worldwide, and Pierce's Disease has a great economic impact on the California grape industry. More than $100 \mathrm{Xf}$ subsp. fastidiosa isolates (named "JeXX") were recently obtained from symptomatic grapevines of five geographic locations across California. To characterize the virulence variability among this collection, Nicotiana tabacum (tobacco) SR1 was used as a model plant since different subspecies of $X f$ are capable of colonizing and causing leaf scorch symptoms in this host. Inoculation of 20 strains of $X f$ in tobacco plants in greenhouse conditions showed that strains Je1, Je60, Je98, and control strains Temecula1 and WM1-1 reached 100\% incidence after five weeks of evaluation, while Je13, Je22, Je28, Je63, Je78 and Je100 had only $80 \%$ incidence, the rest of the strains shows less incidence. Percentage of leaf scorch showed that Je1, Je35, Je60, Je63, Je98, Je100, Je103 and Temecula1 were virulent as WM1-1 (previously characterized as highly virulent strain). When considering two independent experiments, AUDPC values showed that strains Je1, Je35, Je43, Je60, Je63 and Je78 were the most virulent. More isolates are currently being tested, and virulence is being assessed in the context of genomic variability and geographic location.

Bacteriocin-mediated antagonism provides a fitness benefit in planta for Pseudomonas syringae H. EHAU-TAUMAUNU (1), K. Hockett (2), (1) The Pennsylvania State University, University Park, PA, USA; (2) The Pennsylvania State University, University Park, PA, USA

For Pseudomonas syringae, the exposed nature of agriculture increases the possibility of two or more distantly related strains converging on a host. To antagonize competitors $P$. syringae can produce and release bacteriocins and gain a fitness benefit. To compare antagonistic interactions in planta we performed co-infiltrations of $P$. syringae pv. syringae (Psy) and $P$. syringae pv. phaseolicola $(P p h)$; both cause disease on common bean. Importantly, Psy encodes a bacteriocin that inhibits $P p h$. Populations of each strain were measured for an 8-day period. In 1:1 co-infiltration, Psy reduced Pph populations by 100-fold at 4 dpi, whereas $P s y$ bacteriocin-deficient mutant (Psy Dbac) reduced Pph by 10-fold. Surprisingly, there was no detectable bacteriocin-mediated benefit for $P s y$. In 1:9 co-infiltration, Psy at a low cell density (Psy 1: Pph 9) achieved a 2-fold population increase at 6 dpi compared to $P s y$ Dbac, showing a bacteriocin-mediated benefit. $P s y$ also reduced $P p h$ to a larger extent for all sample days. To assess how the host plant affects bacteriocin interactions, avirulent $P$ sy Type III Secretion System (T3SS) mutants were co-infiltrated 1:1 with Pph resulting in no reduction of $P p h$ at all dpi. Both Psy T3SS mutant strains also significantly increased in population size when co-colonizing with a virulent strain. Our data demonstrates a bacteriocin-mediated fitness benefit for Psy when antagonizing at a low cell density compared to Psy Dbac. Additionally, Pph inhibition due to bacteriocin production was observed at early day post-infiltration.

Identification of novel drought tolerance genes, $R D 29 A$ and $R D 29 B$, which concurrently improve crop growth and yields S. W. PARK (1), W. Liu (1), A. Sanz-Saez (2), E. J. Sikora (1), (1) Department of Entomology and Plant Pathology, Auburn University, Auburn, AL, USA; (2) Department of Crop, Soil and Environmental Sciences, Auburn University, Auburn, AL, USA

In recent decades, the scarcity of water (drought) has become a global problem of food security and agricultural productions. A variety of strategies have been developed to enhance drought tolerance, but these were largely unsuccessful because most drought tolerance 
genes stimulate the stomata closure, and in turn suppress plant growth and yield productions. Thus, our initial goal was to find out if and how plants can enhance drought tolerance without compromising their growth and development. We first were able to isolate a strain of plant growth promoting rhizobacteria (PGPR, Panebacillus polymyxa), capable of $\boldsymbol{i}$ ) priming (enhancing) drought tolerance and $\boldsymbol{i}$ ) concurrently stimulating the growth and productivity in plants (e.g., soybean and Arabidopsis). The results indicate a presence of plant genes that can mediate an action of $P$. polymyxa in synergistically promoting plant drought tolerance and growth processes. In the end, we employed transcription analyses, and uncovered that the drought tolearance occurs in parallel to the induced expression of two genes (i.e. RD29A and RD29B) by P. polymyxa. In agreement, we recently have isolated, and demonstrated that T-DNA insertion KO mutant Arabidopsis plants of $R D 29 A$ or $R D 29 B$ displayed enhanced susceptibilities towards drought stress, without any change in stomata behaviors and growth rates, comparing to WT plants. These results together elucidate a discovery of unique generic repertoires that potentially aid in upgrading plants' own survival capacity against drought conditions without trading off their yields and productions.

Impacts of silicon and sheath blight inoculation on the microbiome of rice rhizosphere R. CALDERON, J. Leonard, J. Hyun Ham, Dept of Plant Pathology and Crop Physiology, LSU Agricultural Ctr, Baton Rouge, LA, USA

Silicon ( $\mathrm{Si}$ ) has been widely known to promote rice disease resistance against major pathogens such as Rhizoctonia solani causing sheath blight; however, the effect of silicon on the microbial communities within the rice rhizosphere is still poorly understood. Using high-throughput sequencing, we extracted metagenomes in different treatment combinations: non-Si-treated without (S-/R-) and with inoculation $(\mathrm{S}-/ \mathrm{R}+)$, and Si-treated without $(\mathrm{S}+/ \mathrm{R}-)$ and with inoculation $(\mathrm{S}+/ \mathrm{R}+)$. Results revealed varied responses of microbial communities to Si-treatment. Bacterial amplicon sequence variants (ASVs) were highly diverse in Si-treated samples, while fungal ASVs were more abundant in non-Si-treated samples. The microbial structures clustered distinctly for both bacterial and fungal microbial communities among treatments, especially between inoculated and non-inoculated samples. Moreover, we identified differentially abundant ASVs among treatments between bacterial and fungal communities, suggesting significant microbial responders to Si-treatment and pathogen inoculation in the rice microbiome. Furthermore, co-occurrence analysis identified microbial hubs that regulate the network structure of the microbiome as influenced by the different treatments. Our study provides an understanding on the effect of silicon in the microbial interaction with and without pathogen in the rice rhizosphere, and how silicon may regulate favorable microbiome for plant health.

Comparative genome-wide analyses to develop a robust and sensitive method for race differentiation of Fusarium oxysporum f. sp. niveum

O. HUDSON (1), P. Ji (1), S. Waliullah (2), E. Ali (1), (1) University of Georgia, Tifton, GA, USA; (2) University of Georgia, GA, USA

Fusarium oxysporum f. sp. niveum (FON) is the causal agent of Fusarium wilt of watermelon, one of the most significant diseases and limiting factors of watermelon production worldwide. Detection methods are limited due to high genetic similarity with other formae speciales within the Fusarium oxysporum species complex. This is complicated by the multiple races of FON $(0,1,2,3)$ which have varying levels of virulence on a specific set of watermelon cultivars. The traditional method of race differentiation is a bio-assay where different cultivars of watermelon seedlings are inoculated with the pathogen and disease intensity is scored. Compared to molecular assays, this method is time-consuming, labor-intensive and often inaccurate due to differences in the greenhouse and experimental conditions. Recently, a PCR primer set was developed to differentiate race 2 of FON from the other races based on the absence of the avirulence gene $S I X 6$, but the information is not available for differentiation of the other races. In this study, Illumina wholegenome sequencing of FON races 1, 2, and 3 conducted and analyzed. By targeting unique, yet conserved, regions in the race 3 genome, we developed an assay for the differentiation of race 3 from the other races. To our knowledge, this is the first molecular assay with the ability to differentiate FON race 3 from the other races, which, when used in concert with SIX6, can differentiate races 1 , 2 and 3.

Lettuce dieback associated virus, a putative member of the Phenuiviridae and likely causative agent of lettuce dieback disease W. M. WINTERMANTEL (1), L. Hladky (1), V. Camelo (1), S. T. Koike (2), D. Mollov (3), (1) USDA-ARS, Salinas, CA, USA; (2) TriCal Diagnostics, Gilroy, CA, USA; (3) USDA, Beltsville, MD, USA

Lettuce dieback disease (LDD) causes foliar necrosis, stunting, and death of lettuce plants throughout all western US lettuce production regions. The disease is more prevalent in poorly drained soils and was traditionally believed to be caused by either of two viruses from the genus Tombusvirus: Tomato bushy stunt virus (TBSV) and Moroccan pepper virus (MPV). However, in recent years, neither tombusvirus has been found in symptomatic plants collected from the field, nor have degenerate primers detected any divergent tombusviruses from diseased lettuce. To identify additional viruses that may be associated with LDD, lettuce samples were collected from symptomatic fields and leaves were used to mechanically passage infectious agents to an array of test plants, resulting in symptoms in N. benthamianadistinct from those caused by TBSV or MPV. High throughput sequencing of RNA extracts from symptomatic lettuce field samples and Nicotiana benthamianatest plants consistently identified a previously unknown ssRNA virus with a tripartite RNA genome distantly related to others in the family Phenuiviridae, order Bunyavirales. RT-PCR using primers designed to a portion of the polymerase gene identified the new virus in nearly all symptomatic field samples and in archived diseased lettuce samples dating to 1999. The new virus, lettuce dieback associated virus (LDaV) has been reintroduced from infected $N$. benthamianato lettuce and reproduced LDD symptoms, but additional experiments are needed for validation that LDaV causes LDD, and to clarify its relationship with MPV and TBSV. 
Water contamination by Phytophthora capsici - advanced onsite diagnosis drives more profitable vegetable production O. Hudson (1), S. Waliullah (2), J. Hand (3), R. Gazis (4), F. Baysal-Gurel (5), E. ALI (1), (1) University of Georgia, Tifton, GA, USA; (2) University of Georgia, GA, USA; (3) University of Georgia Cooperative Extension, Tifton, GA, USA; (4) University of Florida, FL, USA; (5) Tennessee State University, McMinnville, TN, USA

Irrigation water contamination by Phytophthora capsici is a serious threat to vegetable production. The pathogen can survive in irrigation water that serves as an inoculum source and causes disease outbreaks, resulting in severe yield loss ofvegetable crops. This pathogen produces spores on diseased plant tissues that can be easily washed out by rain and splashing water andcontaminate nearby irrigation sources like ponds and lakes. Due to lack of efficient diagnosis systems, the production of vegetables is severely impacted by contaminated irrigation water. Detection of $P$. capsici in irrigation water is difficult using traditional culture-based methods because other microorganisms present in the environment, such as Pythium spp., which usually overgrow on culture media making $P$. capsici undetectable. To detect the presence of $P$. capsici spores in water sources (irrigation ponds, runoff, etc.), we developed a hand pumpbased filter paper $(8-10 \mu \mathrm{m})$ method that captured zoospores and was used to amplify DNA of the pathogen through a novel loopmediated isothermal amplification (LAMP) assay designed for specific amplification of $P$. capsici. This method amplified and detected DNA from a concentration as low as $1.2 \mathrm{x}$ zoospores $/ \mathrm{ml}$, which was 40 times more sensitive than conventional PCR. No crossamplification was obtained when closely related species were tested. This protocol could be adapted to other pathogens that reside, accumulate, or are dispersed incontaminated irrigation systems.

Steam-based thermotherapy - a promising management tool for common plant-parasitic nematodes on strawberry transplants M. GU (1), C. Khanal (2), H. Regmi (1), J. Desaeger (1), (1) University of Florida, Wimauma, FL, USA; (2) Clemson University, Clemson, SC, USA

Aphelenchoides besseyi, Meloidogyne hapla and Pratylenchus penetrans are commonly found on strawberry transplants shipped to Florida from out-of-state nurseries. Our previous in-vitro studies determined $100 \%$ mortality of these three nematodes when immersed in water at $44^{\circ} \mathrm{C}$ for $2 \mathrm{~h}$. Greenhouse experiments were conducted to evaluate the efficacy of steam-based heat treatment on controlling the three nematode species inhabiting strawberry transplants. Transplants were first separately infested with each of the three nematode species, and then heat-treated in a steam chamber (aerated steam of $44^{\circ} \mathrm{C}$ was applied for $0,1,2$ and $\left.4 \mathrm{~h}\right)$. Significant reductions $(P \leq$ $0.05)$ in nematode reproductions were consistently observed on all three nematode species when transplants were exposed to steam for 2 $\mathrm{h}$ and longer. Aphelenchoides besseyi and M. hapla on transplants were eradicated after $4 \mathrm{~h}$ of steam exposure. Pratylenchus penetrans was more tolerant to the heat treatment and some nematodes were still able to survive inside roots after $4 \mathrm{~h}$ of steam treatment. Results were encouraging and indicated that thermotherapy can be a promising management tool for common plant-parasitic nematodes on strawberry transplants.

\section{Getting in through the out door: Lytic phage PhiAP1 needs a functional Type II secretion system to infect the wilt pathogen} Ralstonia solanacearum

A. da Silva Xavier (1), A. G. De Melo (2), C. Hendrich (3), D. Tremblay (2), G. M. Rousseau (2), P. L. Plante (2), J. Almeida (4), P. Alfenas-Zerbini (5), S. Moineau (2), C. ALLEN (3), (1) Universidade Federal do Espírito Santo, BRAZIL; (2) Université LavalQuebec, CANADA; (3) University of Wisconsin-Madison, Madison, WI, USA; (4) Faculdade de Pará de Minas, BRAZIL; (5) Universidade Federal de Vicosa, Vicosa, BRAZIL

Soilborne pathogens such as the bacterial wilt agent Ralstonia solanacearum $(R s)$ are notoriously difficult to manage. Lytic phages like phiAP1, isolated from an $R s$-infested tomato field in Brazil, could provide sustainable bacterial wilt disease control. However, evolution of bacterial resistance can undermine effectiveness of phage therapy. To understand the possible consequences of $R s$ resistance to phiAP1, we screened a library of spontaneous bacteriophage insensitive mutants (BIMs). BIM phage resistance was not dependent on either CRISPR or abortive immunity. Rather, genetic and phenotypic evidence indicated that most spontaneous phiAP1-resistant Rs strains had lost the Type II secretion system (T2SS). Consistent with this idea, a T2SS-deficient $\Delta g s p L R s$ mutant was also phage resistant. To determine if phiAP1 infection requires only an assembled T2SS or if the secretion system must also be functional, we changed a key amino acid in the ATP binding site of the GspE ATPase. This mutation eliminated T2 secretion but should still permit T2SS assembly. This GspE K274A mutant was phage resistant, suggesting that phiAP1 needs a functional T2SS to infect Rs. However, loss of the T2SS, which secretes several major virulence factors, severely reduces the ability of $R s$ to cause bacterial wilt disease. As a result, all T2SS-deficient BIMs were nearly avirulent on tomato plants. If $R s$ strains must give up virulence to gain resistance to this phage, phiAP1 may offer sustainable bacterial wilt control, especially if combined in a cocktail with other phages.

Geastrumia-like species associated with sooty blotch and flyspeck of apple from three continents A. Mushtaq (1), J. C. Batzer (2), M. L. GLEASON (2), (1) UAF, Okara, PAKISTAN; (2) Iowa State University, Ames, IA, USA

Sooty blotch and flyspeck (SBFS) is a disease caused by a complex of approximately 100 fungal species in 30 genera, mostly in the order Capnodiales. SBFS causes blemishes on the surface of waxy fruit that reduce fresh market value up to $90 \%$. Geastrumia polystigmatis was first reported as a SBFS pathogen in North Carolina and is a dominant species of SBFS in the eastern U.S. Surveys of apple orchards in Germany, Spain, Brazil, and the U.S. (Georgia, Kentucky, Michigan, Massachusetts, New York, North Carolina, Ohio, Pennsylvania, and Virginia) found 148 SBFS isolates identical to or closely related to Geastrumia polystigmatis. Maximum likelihood analysis of the large subunit region of rDNA placed these fungi in the order Pleosporales class Dothideomycetes. Parsimony analysis of the internal transcribed spacer region of rDNA showed several base pair indels and substitution across the sequences that suggested multiple putative species. Eight representative isolates from the U.S., Spain, and Brazil were selected for further study. These 
isolates are being examined for morphological differences including colony growth, color and conidia morphology. To our knowledge, this is the first study to characterize micro- and macro-morphology of Geastrumia species that colonize apple fruit.

Use of high-resolution melting analysis to identify species in the Colletotrichum gloeosporioides species complex from strawberry M. OLIVEIRA (1), N. Y. Wang (1), N. Peres (2), (1) Gulf Coast Research and Education Center, University of Florida, Wimauma, FL, USA; (2) University of Florida - Gulf Coast Research and Education Center, Wimauma, FL, USA

Recent multi-gene studies defined Colletotrichum gloeosporioides as a species complex and identified three species within the complex that cause Colletotrichum crown rot (CCR) of strawberry in Florida. Our objective was to develop a high-resolution melting (HRM) assay to aid in the diagnosis and identification of the species of C. gloeosporioides species complex causing CCR. Symptomatic strawberry plants were received by the Plant Diagnostic Clinic at the Gulf Coast Research and Education Center, and $C$. gloeosporioides was isolated. In 2016, 2017, and 2018, C. gloeosporioides was diagnosed in 39, 35, and 32\% of symptomatic strawberry samples, respectively. For the development of an HRM assay, primers for the calmodulin (CAL) and glyceraldehyde 3phosphate dehydrogenase (GAPDH) genes were designed. CAL primers (Cg-CAL-F2/Cg-CAL-R1) proved to be the most efficient for C. gloeosporioides species complex identification. To avoid DNA extraction and accelerate the diagnostic process, we tested spore suspensions from $10^{2}$ to $10^{7}$ conidia/mL for the HRM assay. The optimum concentration of 1000 conidia/10 $\mu \mathrm{L}$ reaction was used to screen 85 isolates collected from 2016 to 2018 . We found that $84.7 \%$ of the isolates were C. siamense, $14.1 \%$ C. theobromicola, and $1.2 \%$ C. fructicola. Our study developed an HRM method to rapidly identify species within the C. gloeosporioides species complex. This technique will be helpful to aid in diagnosis and disease management recommendations for Florida strawberry growers, considering the species have different fungicide resistance profiles.

Virome screening of tomatoes and wild plants in Slovenia and Serbia using high-throughput sequencing M. P. S. RIVAREZ (1), D. Kutnjak (1), A. Vučurović (1,2), O. Maksimović Carvalho Ferreira (1), K. Bacnik (1), I. Stanković (2), G. Seljak (1), N. Mehle (1), I. Gutierrez Aguirre (1), M. Ravnikar (1,3), (1) National Institute of Biology, Ljubljana, SLOVENIA; (2) University of Belgrade -Faculty of Agriculture, Belgrade, REPUBLIC OF SERBIA; (3) University of Nova Gorica, Nova Gorica, SLOVENIA

Viruses are one of the most important causal agents of emerging diseases in major crops, including tomato. The presence and diversity of viruses in agroecosystems can be elucidated using viromics approaches, i.e., analysis of all viral genomes in samples collected from the ecosystem. The aim of this study was to examine the presence of viruses in tomato fields and in wild flora in the major tomato producing areas in Slovenia and Serbia, using high-throughput sequencing-based virome screening. A total of 186 symptomatic and asymptomatic tomato and weed samples were collected. Total RNA was extracted from the samples, pooled according to the location and symptomatology and sequenced using Illumina approach. The data were examined for presence of viral sequences using a pipeline in CLC Genomics Workbench (Qiagen). Several known and possibly new virus species were detected in tomato and weed samples. Analysis showed that at least tobamoviruses, amalgaviruses, tombusviruses, ophioviruses, nucleorhabdoviruses and orthotospoviruses were present in the samples. Lastly, we detected several possibly novel virus strains and species in weeds growing near tomato greenhouses. This study presents a proactive surveillance approach in studying virus presence and emergence in extended areas and will give epidemiological insights vital for the monitoring and prevention of disease outbreaks.

Evaluation of corn hybrids for resistance to the root-lesion nematode Pratylenchus neglectus G. YAN (1), N. Akhter (1), J. Ransom (2), (1) North Dakota State University, Department of Plant Pathology, Fargo, ND, USA; (2) North Dakota State University, Department of Plant Sciences, Fargo, ND, USA

Root-lesion nematodes (RLN), Pratylenchus spp., are among the major plant-parasitic nematodes that cause significant yield losses in corn. Nematode surveys conducted in North Dakota (ND) revealed that Pratylenchus neglectus together with two other Pratylenchus spp. are present in corn fields. Host resistance is considered to be environmentally friendly and agronomically sustainable. However, the resistance of corn hybrids in ND to these RLN species has not been reported. The objective of this study was to evaluate corn hybrids used in ND for resistance to $P$. neglectus. Five replications of ten commercial corn hybrids adapted to ND and one non-planted control were grown in a greenhouse experiment, which was repeated once. Soil naturally infested with $P$. neglectus was used to infect corn plants and the experiments were harvested after 15 weeks. Reproductive factor (RF: final/initial populations) was calculated for each corn hybrid. Data from both trials were combined and the average RF for each hybrid was compared to the hybrid with the highest average RF to classify corn hybrids for resistance. The hybrids identified as moderately resistant to P. neglectus were 1392 VT2P, PFS74K89, and 4913 VT2RIB, those moderately susceptible were L-2916 VT2PRO, GX89 VT2P, and X5B-8801, and those susceptible were DK 43-48-RIB, DKC 44-13, DKC 43-46, and LR-9487 VT2PRIB. The knowledge from this study will be useful to accelerate the selection of suitable hybrids for resistance to $P$. neglectus and develop effective and specific nematode management strategies.

First step in the development of novel clay protectants to minimize whiteflies that transmit Tomato yellow leaf curl virus R. CARVALHO (1), S. Da Silva (2), N. Johnston (2), X. Martini (2), S. Maxwell (2), J. H. Freeman (2), J. B. Jones (1), M. L. Paret (2), (1) University of Florida, Gainesville, FL, USA; (2) North Florida Research \& Education Center, University of Florida, Quincy, FL, USA

The silverleaf whitefly, Bemisia tabaci is the vector for Tomato yellow leaf curl virus (TYLCV) that have a high negative impact on Florida tomato production. This virus is responsible for severely damaging tomatoes causing stunting of plants, unmarketable fruits, and 
thereby reduced yield. The high whitefly population in Florida, and minimum number of commercially available resistant varieties are challenges for disease management. Decreasing the whitefly numbers attracted to and probing on the plants could be a first step in developing an approach for reduced TYLCV transmission. In this study, we investigated the potential of novel clay particles to create a barrier on the plant with the goal of disturbing visual and olfactory cues for whiteflies to find the plant precluding a viral transmission. In fall 2019, a field trial was conducted in which tomato plants were treated with five clay protectants including Kaolin. Untreated plots served as the control. The whitefly counts of adults and nymphs, and disease incidence was assessed weekly. There were significant differences in the cumulative whitefly and nymph numbers for Kaolin, but not for the other treatments in this study compared to the untreated control at the end of the trial $(\mathrm{P}=0.05$, LSD). Although the viral incidence did not show statistical difference, more field studies are needed using modified versions of the clay formulations.

Genetic mapping of bacterial spot resistance gene against Xanthomonas gardneri in Hungarian pepper

A. SHARMA (1), G. V. Minsavage Jr. (1), U. Gill (2), S. Hutton (3), J. B. Jones (1), (1) University of Florida, Gainesville, FL, USA; (2) North Dakota State University, Fargo, ND, INDIA; (3) University of Florida, Wimauma, FL, USA

Cultivation of cultivars containing recessive resistance is an effective method to control bacterial spot of pepper, but currently deployed resistance gene $b s 5$ is ineffective against Xanthomonas gardneri. Resistance against $X$. gardneri was identified in the Hungarian pepper accession PI 163192, and the objective of this study was to map the resistance gene(s) in this accession. An F2 population of 72 individuals was developed by crossing PI 163192 with the $X$. gardneri susceptible line, ECW50R. DNA was extracted from individual resistant (R) and susceptible (S) F2 plants, and R and S DNA bulks were created and sequenced. Bulk segregant analysis (BSA) was carried out using a custom BWA-GATK pipeline. The resistance present in PI 163192 was recessive in nature. The F2 segregated in approximately 1:6:9 ratio for resistant, intermediate and susceptible phenotypes respectively, suggesting that the resistance is conferred by two genes with additive effects. The BSA analysis found a major QTL in an approximately $6 \mathrm{Mb}$ interval in the sub-telomeric region of chromosome 11. Genotyping of more advanced backcross F2 populations with CAPS markers spanning the region further delimited the resistance locus within a $2.75 \mathrm{Mb}$ interval. Further fine-mapping will help to narrow down the position of the chromosome $11 \mathrm{QTL}$, and identification of the gene(s) underlying resistance in PI 163192 will contribute towards development of commercial pepper varieties with improved bacterial spot resistance.

Boxwood blight infection and symptom development under coastal Northern California conditions W. SCHWEIGKOFLER (1), K. G. Suslow (1), C. Hong (2), (1) Dominican University of California, San Rafael, CA, USA; (2) VPI \& State University, Virginia Beach, VA, USA

Boxwood blight (BB), caused by Calonectria pseudonaviculata (Cps), is a destructive disease of Buxus spp. Symptoms include circular, $\tan$ leaf spots and necrotic stem lesions, followed by sudden defoliation. This disease was first reported in the United Kingdom and New Zealand in the 1990s, and has since spread to numerous countries, including the USA. Severe outbreaks occurred in the Eastern US with temperate temperatures and abundant rainfall. In California, BB was found in residential areas but not in nurseries. Here we report the symptom expression on English boxwood (B. sempervirens cv. 'Suffructicosa') at the NORS-DUC quarantine facility in Northern California. Eighteen plants were first inoculated on December 17, 2018 by spraying each with $50 \mathrm{ml}$ of $C p s$ suspension at 2200 conidia/ml, then immediately enclosed by a plastic tarp for $48 \mathrm{~h}$ to facilitate infection. There was 5.9 mm rainfall with temperature ranging from 4.4 to $16.1^{\circ} \mathrm{C}$ during this 48 -h period. As no symptom developed after 93 days, the plants were inoculated again with the same Cps concentration on April 10 (rainfall: $0.3 \mathrm{~mm}$; T: $7.2-22.2^{\circ} \mathrm{C}$ ) and June 18, 2019 (rainfall: $0 \mathrm{~mm}$; T: $13.3-21.7^{\circ} \mathrm{C}$ ). Typical boxwood blight leaf spot symptoms were first observed on three of the eighteen plants on June 4. By October 7, diseased plants increased to nine with more severe foliar symptoms including necrotic lesions on twigs. Heavy defoliation started in mid-January 2020. These observations help understand the field epidemiology of this emerging disease. Their practical implications are also discussed.

Silverleaf disease: Foliar symptoms reversion in apple and their relation with transcriptional reprogramming J. CHILIAN (1), D. Grinbergs (2), A. France (2), (1) INIA Quilamapu, Chillan, CHILE; (2) Instituto de Investigaciones Agropecuarias, INIA Quilamapu, Chillán, CHILE

Silverleaf disease, caused by the fungus Chondrostereum purpureum, is considered a major economic disease for several fruit crops in Chile. Apple is one of the most susceptible species and silverleaf occurs throughout all the production area, having a negative impact on fruit yield and quality, and longevity of apple orchards and nurseries. As there are no curative methods for its control, the reversion of foliar symptoms, observed in apple plants, becomes an interesting phenomenon. Reversion is associated to the plant endophytic microbiota and involves the transcriptional reprogramming of genes related to different responses to pathogens. Thus, the objective of this work was to compare the transcriptional profile of silverleaf diseased and reverted plants. RNA was isolated from sawdust collected from the trunk of diseased and reversed apple plants cv. Brookfield and candidate genes which participate in different defense mechanisms were analyzed by quantitative RT-PCR (qPCR). Results showed that the photosynthesis-related-genes and genes related to the primary and secondary metabolism, were repressed in diseased plants, compared to those in reversed plants. Meanwhile, pathogenesis-related (PR), genes were upregulated in reversed plants. The identification of the genes that are differentially regulated in reversed plants and their relationship with the endophytic microbiota will help to understand the reversion phenomenon and will also allow the development of future strategies for silverleaf disease management using biological control agents from reverted plants.

Identification of organisms associated with cane dieback of cultivated blackberry (Rubus fructicosis) in Georgia W. Hemphill (1), P. M. Brannen (2), J. E. OLIVER (3), (1) University of Georgia, Tifton, GA, USA; (2) University of Georgia, Athens, GA, USA; (3) Department of Plant Pathology, University of Georgia, Tifton, GA, USA 
Many disease issues impact blackberry production in the southeastern U.S., and the decline and dieback of blackberry canes has been an emerging concern. Cane dieback is frequently attributed to Leptosphaeria coniothyrium, the causal agent of cane blight disease of blackberry; however, the causes of blackberry cane dieback in the southeastern U.S. have not been extensively studied. To better understand the causal agents of cane dieback of blackberry, blighted cane samples were collected from commercial blackberry production sites across eight counties in Georgia. Fungal isolates cultured from these samples were identified via morphology and sequencing, and their ability to cause dieback was assessed through inoculation of potted blackberry plants. In total, 126 fungal isolates belonging to 34 species were collected. Most isolates belonged to the Pestalotiopsidaceae ( 34 isolates), Botryosphaeriaceae (28 isolates), or Nectriaceae (21 isolates) families, and only a single isolate of L. coniothyrium was identified. Isolates of Fusarium oxysporum, Pestalotiopsis microspora, Neofusicoccum kwambonambiense, N. parvum, Lasiodiplodia pseudotheobromae, L. theobromae, and Colletotrichum siamense were determined to cause significant dieback on cut blackberry canes and Koch's postulates were fulfilled for these isolates. Collectively, this information suggests that other fungal species besides L. coniothyrium are capable of causing cane dieback of blackberry in the southeastern U.S., and additional work is needed to determine appropriate management strategies for these organisms.

Phenotypic evaluation and genotyping of snapbean lines for resistance against two distinct whitefly-transmitted begomoviruses G. AGARWAL (1), S. Kavalappara (1), S. Gautam (2), R. Srinivasan (2), B. Dutta (1), (1) University of Georgia, Tifton, GA, USA; (2) University of Georgia, Griffin, GA, USA

Snap bean (Phaseolus vulgaris) production and quality have been negatively impacted by two whitefly-transmitted begomoviruses; "cucurbit leaf crumple virus (CuLCrV)" and "sida golden mosaic Florida virus (SiGNFV)", which often appear as a mixed infection in Georgia. Currently, information is not available with respect to resistance to these two viruses in commercial cultivars. In two field seasons (2018 and 2019), we screened 84 snap bean lines including cultivars, advanced breeding lines and entries from germplasm collections (USDA, GRIN) to leaf crumple disease. Twenty four lines with moderate levels of resistance (disease severity ranging from $5-40 \%)$ to leaf crumple disease were identified. The whole genome of these snap bean lines were sequenced. Over 900 giga-base (Gb) of filtered data were generated and $>88 \%$ of this data were mapped on the reference genome to obtain structural variants (SNPs and Indels). A total of 645,729 SNPs and 68,713 Indels including 30,169 insertions and 38,543 deletions were identified, which were distributed in 11 chromosomes. Further, genome-wide association studies will be done to identify the genes/variants associated with resistance.

Investigating the role of Candidatus Liberibacter solanacearum effectors activity in solanaceous hosts P. REYES CALDAS (1), C. Casteel (2), G. L. Coaker (1), (1) University of California, Davis, CA, USA; (2) Cornell University, Ithaca, NY, USA

Candidatus Liberibacter solanacearum (Lso), the causal agent of Zebra Chip of potato and Psyllid Yellows in tomato, is an obligate, phloem-limited, Gram-negative bacterium. Lso is transmitted by psyllids, including Bactericera cockerelli, and proliferates in both plant and insect. In recent years, Lso has significantly impacted potato-tomato production in North America. To gain insight into how Lso causes disease, we have focused on characterizing its SEC effector repertoire. We have predicted Lso core effectors from eight published genomes. Expression of 20 effectors that are conserved in Lso haplotypes infecting solanaceous plants was tested in both tomato and B. cockerelli. Specific effectors were preferentially expressed in host and vector and effector expression dynamically changed over time in tomato. Effectors that were highly expressed in planta were evaluated for their subcellular localization and ability to suppress early and late defense hallmarks in Nicotiana benthamiana. Lso effectors exhibited diverse subcellular localizations, including the cytoplasm, nucleus, and chloroplast. Bacterial effector's ability to localize in eukaryotic subcellular compartments hints at interesting effector activity. Unlike characterized Type 3 effectors from plant pathogenic bacteria, most Lso SEC effectors were unable to suppress MAMP induced immune responses or programmed cell death A greater understanding of novel roles of Lso effectors will contribute to elucidate how this pathogen manipulates its hosts and can ultimately be used to develop targeted disease control strategies.

Study on carrier water volume for fungicide application to manage sheath blight in rice Y. WAMISHE (1), J. Hardke (2), T. Gebremariam (1), S. B. Belmar (1), C. Kelsey (1), T. B. Mulaw (1), (1) University of Arkansas, Stuttgart, AR, USA; (2) University of Arkansas, Little Rock, AR, USA

Sheath blight caused by Rhizoctonia solani AG1-1A is the most prevalent disease of rice in Arkansas where nearly $50 \%$ of rice in the U.S. is produced. Fungicides are used in most fields to manage sheath blight. However, the disease is poorly suppressed in some fields where low water volume is used as a carrier. In 2019 a field experiment was conducted on rice variety, CL163, where approximately 28, 94, and 187 liter/ha (LPHa) rates of water were used to deliver Amstar Top and Quilt Xcel at 1.1 and 1.5 LPHa, respectively. The fungicides were applied using a Mud Master sprayer a week after plots were inoculated with $R$. solani AG1-1A. Vertical and horizontal disease progress were assessed three times across the season using the standard 0 to 9 scale and percentage, respectively. Analysis of disease index and grain yield data showed no significant differences between Amistar top and Quilt Xcel in the degree of sheath blight suppression. Overall, there were differences between the unsprayed and the sprayed plots in disease and grain yield. However, plots that received the fungicides with $28 \mathrm{LPHa}$ water were not significantly different from the unsprayed plots. On the other hand, plots sprayed with 94 or 187 LPHa water were significantly different both in disease level and grain yield from the unsprayed plots and those that received 28 LPHa. This study confirmed the importance of using enough water to adequately cover the crop canopy to benefit from fungicide application in rice. 
Are small non-coding RNAs of Dickeya dadantii involved in detection and response to plant hosts?

J. GONZALEZ-TOBON (1), Y. Liu (1), M. Filiatrault (1,2), (1) Cornell University, Ithaca, NY, USA; (2) USDA-ARS, Ithaca, NY, USA

Dickeya sp. causes potato "blackleg", an economically important disease with billions of dollars lost per year in the U.S. Similar to other plant pathogenic bacteria, it uses methyl-accepting chemoreceptors (MCPs) to find an opening, enter the plant and sense plantdefense compounds, using chemotaxis. Interestingly, Dickeya possesses an unusually high number of MCPs (47) compared to other Enterobacterales (29), which appear to be crucial for its virulence. Long untranslated regions exist in the 5' extreme of most MCPs in Dickeya. RNASeq data from our lab shows most of these regions are transcriptionally active in planta. We hypothesize that these 5'UTRs encode for regulatory sequences such as small non-coding RNAs (ncRNAs) that control expression of downstream genes. The objective of this study was to identify if ncRNAs or other regulatory elements reside in 5'UTRs of Dickeya dadantii's MCPs. Bioinformatic analyses were used to identify 5'UTRs, scan for known regulatory sequences and predict secondary structures. Based on our previous in planta RNASeq data, 16 of these 5'UTRs were notoriously expressed. None contain known sRNAs, but all have the potential to fold into secondary structures. These expression levels were also tested experimentally, in vitro and in planta (in susceptible and tolerant potato lines). Future experiments will further investigate if Dickeya's ncRNAs are involved in its chemotaxis during infection. This provides important insights into regulatory mechanisms which could potentially be used to control how, or even if, Dickeya enters the plant.

The foliar endophytic microbiome points to potential causal agents of beech leaf disease C. EWING (1), J. Slot (2), M. S. Benitez Ponce (3), C. Rosa (4), A. Malacrino (1), A. Bennett (1), L. R. Stewart (5), P. Bonello (6), (1) The Ohio State University, Columbus, OH, USA; (2) Department of Plant Pathology, The Ohio State University, Columbus, OH, USA; (3) The Ohio State University, Wooster, OH, USA; (4) The Pennsylvania State University, University Park, PA, USA; (5) USDA-ARS Corn, Soybean and Wheat Quality Research Unit, Wooster, OH, USA; (6) The Ohio State University, Department of Plant Pathology, Columbus, $\mathrm{OH}$, USA

Beech leaf disease (BLD) is an emerging disease affecting American beech (Fagus grandifolia), a foundational tree species in the northeastern United States. Recently published work suggests that the presumably alien nematode, Litylenchus crenatae ssp. mccannii is the causal agent. Our group concurrently initiated a separate study to identify the causal agent of the disease using a comparative approach based on characterization of the foliar microbial endophytic communities. To do so, we are analyzing the communities of all plant pathogen types on symptomatic and asymptomatic American beech foliage. Our study uses a metabarcoding approach with high throughput sequencing to analyze total beech leaf RNA and DNA. Information on viruses is not yet available, and phytoplasmas were not found in any samples. However, differential abundance analyses focused on the remaining plant pathogens showed that the operational taxonomic unit (OTU) associated with Litylenchus crenatae ssp. mccannii is present in both asymptomatic and symptomatic samples, while bacterial and fungal OTUs associated with the genera Erwinia, Pseudomonas, Xanthomonas and Wolbachia (bacteria), and Heterocephalacria, Paraphaeosphaeria and two other unidentifiable Ascomycota (fungi) were only detected in symptomatic material. The presence of $L$. crenatae ssp. mccannii in all symptom types, coupled with the recently published evidence, suggests that the nematode may be a necessary but non-sufficient condition for BLD development, which instead may require the presence of both the nematode and microbial pathogens.

Deletion of nitric oxide reductase, NOR1, eliminates $\mathrm{N}_{2} \mathrm{O}$ production in Fusarium verticillioides, a mycotoxin-producing causal agent of maize ear rot

B. A. OAKLEY (1), L. Pierce (2), T. Mitchell (2), G. Hibbs (1), K. Pennerman (2), S. E. Gold (2), A. E. Glenn (2), (1) University of Georgia, Department of Plant Pathology, Athens, GA, USA; (2) USDA-ARS, Toxicology \& Mycotoxin Research Unit, Athens, GA, USA

Fungal denitrification is of major concern due to the dissimilatory reduction of plant-available nitrogen that results in fertilizer waste and the production of nitrous oxide $\left(\mathrm{N}_{2} \mathrm{O}\right)$, a potent greenhouse gas and an ozone degrader. Fungi may use the denitrification pathway under hypoxic conditions to tolerate otherwise uninhabitable macro- and micro-environments in the soil and the plant, respectively. Fusarium verticillioides $(F v)$ is a globally distributed soil-borne mycotoxigenic fungal pathogen of maize. The denitrification pathway in $F v$ has been shown to function dually as an alternative respiration pathway and a nitric oxide (NO) detoxification pathway. NOR1, a gene encoding a protein that reduces $\mathrm{NO}$ to $\mathrm{N}_{2} \mathrm{O}$ is a core component of the canonical denitrification pathway in bacteria and fungi. Our objective is to characterize the biological function of NOR1 in $F v$ and determine its role in $\mathrm{N}_{2} \mathrm{O}$ production. We hypothesized that NOR1 is solely responsible for nitrous oxide production in $F v$. To test this hypothesis, a single-gene deletion mutant $(\Delta n o r 1)$ was generated in $F v$ via homologous recombination. The $\Delta$ norl mutant was evaluated against a panel of other strains to assess $\mathrm{N}_{2} \mathrm{O}$ production via GC-ECD. Deletion of NOR1 significantly reduced $\mathrm{N}_{2} \mathrm{O}$ production below a detectable level when compared to the complement and the wild-type strain FRC M-3125. This information will be helpful for a) biochemical studies that determine the active binding sites of NOR1 in $F v$ and $b$ ) future development of chemical inhibitors of NOR1 that aim to reduce $\mathrm{N}_{2} \mathrm{O}$ production and impair NO metabolism of $F v$.

Evaluation of spinach and Beta vulgaris cultivars for differential susceptibility to two pathogenicity groups of Fusarium oxysporum f. sp. spinaciae

A. BATSON, S. Gyawali, L. J. du Toit, Washington State University, Mount Vernon, WA, USA 
Fusarium wilt of spinach (Spinacia oleracea), caused by Fusarium oxysporum f. sp. spinaciae (Fos), results in greatest losses during warm conditions in areas with acid soils. Most spinach inbreds and cultivars are moderately to highly susceptible to Fos. Recently, two distinct pathogenicity groups of Fos were demonstrated based on quantitative differential reactions of isolates to two spinach inbred lines. The objective of this study was to assess whether commercial spinach and Beta vulgaris subsp. vulgaris cultivars display differential reactions to isolates of each Fos pathogenicity group. Seed of spinach $(n=68)$, sugar beet (1), Swiss chard (1), and table beet (2) cultivars were sown in potting medium inoculated with isolate Fus254 (Fos group 1), Fus167 (Fos group 2), or water (control treatment). By 35 days, Fus254 had caused more severe wilt than Fus 167 on 20 (29\%) of the spinach cultivars, Fus 167 had caused more severe wilt than Fus254 on 13 spinach cultivars (19\%), and the two isolates caused similar wilt severity on 35 spinach cultivars (52\%). The correlation of mean severity of Fusarium wilt caused by the two isolates was significant across cultivars $(r=0.40, P<0.001)$. The sugar beet cv. MA709 was susceptible to Fus254 but not to Fus167, whereas the Swiss chard and table beet cultivars were not susceptible to either isolate. Understanding the susceptibility of spinach, sugar beet, table beet, and Swiss chard cultivars to the two pathogenicity groups of Fos is valuable for growers and will enhance efforts to breed for robust resistance to Fusarium wilt.

\section{Quantification of Erwinia amylovora survival in apple cankers and the impact of dormant copper spray applications with bark penetrants \\ R. Delgado Santander, F. Khodadadi, C. Meredith, S. AĆIMOVIĆ, Cornell University, Hudson Valley Research Laboratory, Highland, NY, USA}

We used viability digital PCR (v-dPCR) to quantify Erwinia amylovora (Ea) survival in fire blight cankers over time and after application of dormant copper $(\mathrm{Cu})$. V-dPCR combines the viability stain propidium monoazide and the dPCR technology for selective detection and quantification of only live-cell DNA. From August 2016 to April 2017 we monitored viable Ea populations in cankers originating from natural infections occurring in spring 2016 on apple cvs 'Cortland' and 'Honeycrisp', from an orchard in northern NY. About 70-100\% of the cankers were positive for Ea detection in August, but detection percentages decreased to 50-67\% in April. Viable $E a$ populations in August were about $10^{6}$ and $10^{7}$ live cells/g of canker tisue in 'Honeycrisp' and 'Cortland', respectively. In October, $E a$ populations decreased to ca. $10^{4}$ ('Honeycrisp') and $10^{5}$ ('Cortland') live cells/g and increased again up to $10^{6}$ live cells/g in April on both cultivars. In addition, we quantified the effect of $\mathrm{Cu}$ (Bordeaux mix, BM, $12.7 \mathrm{lb} \mathrm{Cu} / \mathrm{A}$; Champ WG, CWG, 8 lb Cu/A) and $\mathrm{Cu}$ plus bark penetrants (Bark Oil Blue, BOB; Bark Oil Clear, BOC; Basal Oil, BO; Loveland Bark Oil, LBO; CideKick II; Pentrabark) on the $E a$ survival in cankers on cv 'Cortland'. Bark penetrants were prepared as per manufacturer's instructions. Fifteen days after treatment application, $\mathrm{BM}+\mathrm{BOC}, \mathrm{BM}+\mathrm{BO}, \mathrm{BM}+\mathrm{LBO}$ and $\mathrm{CWG}+\mathrm{BOB}$ led to significant percentage reduction of live $E a$ cells by $67,85,94$ and $97 \%$, respectively. Our results show the potential of v-dPCR to elucidate unknown aspects of fire blight biology and help develop new control options.

Genome-wide association study of the soybean cyst nematode resistance in the USDA common bean core accessions A. Shi (1), T. Michaels (2), S. CHEN (3), (1) University of Arkansas, Fayetteville, AR, USA; (2) University of Minnesota, St. Paul, MN, USA; (3) University of Minnesota, Waseca, MN, USA

Common bean (Phaseolus vulgaris) is the most important edible legume crop worldwide. The upper Midwest USA is a major common bean (dry bean) production region. The soybean cyst nematode ( $\mathrm{SCN}$, Heterodera glycines) has been widely spread in the region and become the major yield-limiting biological factor in soybean production. Common bean is also a good host of SCN, and its production in the region is challenged by this emerging pest. Use of the host genetic resistance has been the most effective and environmental friendly method to manage SCN in soybean. The objectives of this study were to evaluate the SCN-resistance in USDA common bean core collection, and to conduct genome-wide association study (GWAS) to identify SNP markers associated with SCN resistance. A total of 322 accessions of USDA common bean core collection were phenotyped for resistance to SCN Type 0 (race 6). The common bean core set was genotyped with the BARCBean6K_3 Infinium BeadChips consisted of 4,790 SNPs. GWAS was conducted using four mixed linear models (MLM) TASSEL 5, GAPIT, FarmCPU, and BLINK. Seventeen accessions were resistant to SCN with Female Index (FI) at 4.8 to 10, and 64 accessions were moderately resistant $(10<\mathrm{FI}<30)$. Eleven SNP markers located on chromosomes 4, 7, 9 , and 11 were strongly associated with resistance to SCN HG Type 0 . This study provided basic information for developing SCNresistant common bean cultivars using the USDA core germplasm accessions.

What is behind Pythium disease variability in tobacco hydroponic greenhouses? Diversity of the Pythium community X. ZHANG (1), C. S. Johnson (2), T. D. Reed (2), (1) Virginia Polytechnic Institute and State University, Blackstone, VA, USA; (2) Virginia Tech Southern Piedmont AREC, Blackstone, VA, USA

Pythium disease is common in hydroponic greenhouse production of tobacco seedlings, lettuce, tomato, etc. Although its typical symptom is root rot, symptoms on upper plant parts often vary. The causes of this symptom variation remain unclear. To solve this mystery and study the ecology of the Pythium community in tobacco greenhouses, 424 samples were collected from seven environments within 41 greenhouses in four states. Pythium isolates were collected using PARP medium and initially identified using morphological traits. Internal Transcribed Spacer 1 and 2 regions were sequenced for further species identification and neighbor-joining phylogenetic analyses. Representative isolates of each species were used to inoculate tobacco TN 90LC seeds and seedlings. The survey yielded 360 Pythium isolates, $67 \%$ of which were $P$. dissotocum. The remainder belonged to $P$. myriotylum, $P$. catenulatum, $P$. coloratum, $P$. porphyrae, $P$. irregulare, $P$. adhaerens, $P$. inflatum, $P$. torulosum, $P$. aristosporum, $P$. attrantheridium and $P$. pectinolyticum. Pythium was isolated from $67 \%$ of bay water samples, with multiple species co-existing in $13 \%$ of the samples. Pythium was also recovered from tobacco seedlings, weeds and a center walkway. Virulence varied among Pythium species, with $P$. myriotylum the most aggressive. Intraspecific virulence variation and genetic diversity were observed within P. dissotocum. Aggressive Pythium 
species were recovered from bay water, tobacco seedlings and weeds. These results revealed the diversity of Pythium species and their niches in tobacco hydroponic greenhouses.

\section{The relationship of foliar symptom severity and root rot severity to yield lost to Sudden Death Syndrome of soybean J. E. KURLE, G. M. Anderson, U of MN, Saint Paul, MN, USA}

Sudden Death Syndrome (SDS) caused by Fusarium virguliforme (Fv) is a damaging fungal disease of soybean occurring in Minnesota. SDS develops as a root infection followed by expression of foliar symptoms of phytotoxins translocated from infected roots. Resistance can be expressed both to root infection and to foliar symptoms; however, breeders emphasize resistance to foliar symptom expression. Resistance to root infection is uncorrelated with resistance to foliar symptoms. The contribution of either resistance to root rot or resistance to foliar symptoms to grain yield is poorly understood. Our objective was to determine the relative contribution of both resistance to root rot and resistance to foliar symptom expression to grain yield. In a greenhouse experiment, fifteen soybean varieties, resistant or susceptible to either root rot (RRr or RRs) or foliar symptoms (Fr or Fs) of SDS, were planted in a randomized complete block design with two treatments, inoculated with Fv or uninoculated. The plants were grown to physiological maturity, evaluated for foliar symptom severity, and harvested for seed and dry matter yield. The percentage loss in seed yield resulting from inoculation was calculated. Foliar symptom severity was poorly correlated with yield loss. When inoculated with Fv, seed yield of RRr varieties was reduced $37 \%$ while seed yield of RRs varieties was reduced $49 \%$; seed yield of Fr varieties was reduced $36 \%$ while seed yield of Fs varieties was reduced $52 \%$. Resistance to root infection by Fv, provided a yield benefit equal to resistance to foliar symptoms.

\section{Effect of Phomopsis longicolla seed infection on soybean seed composition}

J. C. RUPE, A. Rojas, L. Mozzoni, R. Holland, E. Gbur, University of Arkansas, Fayetteville, AR, USA

Wet weather in fall of 2018 led to high soybean seed infection levels by Phomopsis longicolla. A test containing 45 maturity groups 4.1 to 5.7 soybean cultivars was planted on 1 June 2018 in a randomized complete block design with four replications at the Lon Mann Cotton Research Station, Marianna, AR. Seeds were harvested on 31 October. Levels of visibly damaged seed ranged from 2-44\% and P. longicolla infected seed from 9-76\%. Seed composition from each plot was determined with a Perten DA 7250 using near-infrared spectroscopy fitted with a small-sample dish. A total of 42 traits were measured, including protein, oil, moisture, and individual fatty acids, sugars, and amino-acids. Using PROC REG (SAS, Inc.) an all-possible subset screening regression method using r-square, adjusted R-square, Mallows $\mathrm{Cp}, \mathrm{A} / \mathrm{C}$, and mean square error identified multiple regression equations four candidate models with 7, 8, 13, and 19 independent variables, respectively. These variables included total protein and oil as well as various sugars, lipids and amino acids. Beginning with the 19 variable model, each candidate model was analyzed separately using standard regression diagnostics to remove non-significant terms, outliers and any adjustments to the analysis needed to satisfy the model's statistical assumptions (e.g., potential non-constant variance of the response). Only results from the final model are presented. Establishing the effect of seedborne pathogens on seed traits (i.e. oil, protein, and sugar) could help to understand differences in seed vigor and germination.

The impact of chicken-grazed soils on the tomato plant microbiome

A. M. VAZQUEZ-CATONI, M. S. Benitez Ponce, The Ohio State University, Wooster, OH, USA

By using culture-dependent and culture-independent methods to characterize the endophytic bacteria of tomato plants grown in chickengrazed pasture plots (CGPP), we will provide insight into the impact of crop-livestock rotation in plant and soil microbiome. We hypothesize that bacterial endophyte diversity is higher in plants grown in CGPP, as opposed to those grown in pasture only plots (POP). A field trial was conducted in the Summer of 2019 where tomato plants were grown in soils a year after chicken grazing. During the field trial, tomato plant tissues (stem, leaves, roots, fruit) and rhizosphere soil were collected at two time points: before flowering and at harvest. Plant tissues were measured for biomass and surface-sterilized for endophyte isolation. The stem, root, rhizosphere soil, seeds and leaves from individual tomato plants were stored for further 16SrRNA amplicon sequencing. Tomato plants from CGPP, POP and both time points had no significant biomass changes. Using Sanger sequencing of 16SrRNA gene, 124 bacterial endophytes from the stem and fruit were classified at genus level. 50 isolates were considered unique sequences, with 37 found only in stem and seven only in fruit. While Bacillus sp. and Pseudomonas sp. composed 50\% of the isolates across treatments, the remaining isolates, belonging to 17 genera, differed in each treatment. Therefore, management strategy, plant's life cycle stage and tissue can influence endophyte diversity. We will acquire a greater understanding of the impact of CGPP and POP on the microbiome of this highly demanded commodity.

\section{Development of a barcoded transposon library in Xanthomonas vasicola}

J. LONG (1), J. M. Lang (2), D. Mackey (3), J. E. Leach (2), J. M. Jacobs (1), (1) Department of Plant Pathology, The Ohio State University, Columbus, OH, USA; (2) Colorado State University, Fort Collins, CO, USA; (3) Ohio State University, Columbus, OH, USA

Xanthomonas species are a diverse group of plant pathogenic bacteria infecting hundreds of different plants. Interestingly, even pathovars of the same species may occupy different host and tissue niches, despite genetic similarity across the bacteria. We intend to identify the genetic determinants of host range and tissue specificity in Xanthomonas vasicola pathovars: vasculorum and holcicola, which are pathogens of corn and sorghum, respectively. Libraries of $X$. vasicola mutagenized by randomly barcoded mariner transposon insertions (Tn-libraries), will be examined by RB-Tnseq to uncover potential determinants of tissue specificity in these bacterial pathogens. In our preliminary work, we established optimal conditions to conjugate the mariner transposon library into $X$. vasicola. Once the Tn-library reaches our target size of over 100,000 individual mutants, deep sequencing will be used to confirm sufficient 
genome coverage and enable mapping of each barcode to its genomic locus. We also have quantified bacterial population sizes after different inoculation methods in the two native hosts to define conditions that will avoid exposing the Tn-library to bottlenecks during inoculation, growth, and harvest in planta. This work will enable RB-Tnseq experiments to identify bacterial genes required for in planta growth, including those that confer host-specificity.

Integrated management strategies to improve Huanglongbing-affected trees in Florida

J. D. VELOSO DOS SANTOS PULICI (1), D. Kadyampakeni (2), E. G. Johnson (2), (1) University of Florida, Lake ALfred, FL, USA; (2) University of Florida, Lake Alfred, FL, USA

Huanglongbing (HLB), caused by 'Candidatus Liberibacter asiaticus' (CLas) and other related species is the major threat to global citrus production. CLas moves downward in the phloem and infects the roots soon after transmission causing over $70 \%$ root loss after symptoms develop in the canopy. Any strategy that can help to improve the health of the root system and the tree over all is essential to the citrus industry in HLB-era. The objective of this work was to determine if fertilizer nutrient sources coupled with management of other root pathogens and pests reduces decline and extends the productive life of HLB-affected trees. An ongoing trial has been carried out using Valencia oranges on Swingle rootstock trees with 8 treatments composed of 2 nutrient and micronutrient mixes; made of different NPK sources; and 4 crop protection: psyllid control (PC); $\mathrm{PC}+$ aliette soil; $\mathrm{PC}+$ velum prime soil; $\mathrm{PC}+$ aliette and velum, divided in factorial combination with 4 randomized blocks. In two years of treatment, root densities have improved in all combination, with treatments utilizing PC+Aliette soil, in different nutrient mixes, returning to pre-HLB root densities $\left(>1.7 \mathrm{~g}\right.$ dry weight $\mathrm{L}^{-1}$ of soil). However, the disease severity increased across all treatments. No significant treatment differences in leaf or root nutrient concentrations were observed. These results demonstrate that some symptoms of HLB can be reduced in two years, but if an economic yield benefit is possible it would take many years to be realized.

\section{Effects of fungicide spray programs for powdery mildew on fungicide resistance in non-target Botrytis species from grape vineyards in California \\ H. C. YANG (1), J. DeLong (1), S. Saito (1), F. Wang (1), T. D. Miles (2), C. L. Xiao (1), R. P. Naegele (1), (1) USDA ARS, Parlier, CA, USA; (2) Michigan State University, East Lansing, MI, USA}

Grapevine powdery mildew (PM), caused by Erysiphe necator, is a major disease that causes economic loss worldwide. The Central Valley of California contributes $98 \%$ of the table grapes in the U.S. and thus disease management is crucial in California. Currently, PM control heavily relies on fungicide applications. Several fungicides commonly used for powdery mildew management may also affect Botrytis spp., primarily B. cinerea, which causes postharvest gray mold in table grapes. Therefore, this study aims to evaluate the effects of PM fungicide spray programs on the non-target Botrytis spp. in terms of their resistance to three fungicides: pyraclostrobin (QoI; FRAC 11), boscalid and fluopyram (SDHI; FRAC 7). Six fungicide spray programs (treatments) for PM were implemented in a grower's field in Dinuba, CA in 2019. Two additional fields in Fresno, CA with one spray program each were also included. Leaf samples were collected from each treatment between the bloom and the fruit set growth stages. A total of 328 single-spore cultured Botrytis isolates were obtained from the leaf samples. The fungicide resistance of each isolate to the three fungicides was tested using a conidial tube growth assay on the fungicide-amended media. The results showed that Botrytis isolates obtained from different PM fungicide spray programs in the same field displayed different fungicide resistance profiles to pyraclostrobin, boscalid, and fluopyram, suggesting non-target effects of PM spray programs on fungicide resistance in Botrytis species.

Cercosporin production and pathogenicity in Psuedocercosporella capsellae

H. YOKOTA (1), K. Kato (2), T. T. Tsurushima (3), Y. Imura (2), Y. Fujita (2), N. Kita (2), (1) Nihon univercity, Hiratsuka, JAPAN; (2) Nihon University, Fujisawa, JAPAN; (3) Hannan Univ, Osaka, JAPAN

Pseudocercosporella capsellae, a causal agent of white leaf spot of cruciferous plants, was reported to produce a phytotoxin, cercosporin, though the role in the pathogenesis has not been fully elucidated. We isolated $P$. capsellae from white leaf spots of shepherd's purse (Capcella brusa-pastoris), N417, and mustard green (Brassica juncea), KR2, in Japan, both of which showed distinct pathogenicity each other: N417 could infect only shepherd's purse while KR2 could not and, instead, was pathogenic not only to $B$. juncea but also B. rapa. When N417 and KR2 were cultured using malt extract broth, reddish purple pigmentation was observed in the media. Then, the culture filtrates and mycelia were subjected to extraction of cercosporin (Gunasinghe et al., 2016) followed by silica gel TLC. By comparing the chromatographic behavior in HPLC analyses and ESI mass spectra with those of authentic compound, purified compounds from both N417 and KR2 were identified as cercosporin. To determine the role of cercosporin in the pathogenesis, nine $P$. capsellae isolates were obtained from different Brassica species and the virulence was evaluated using cotyledons of Komatsuna green (B. rapa) in combination with the cercosporin productivity. As a result, strong correlation was detected between the virulence level and cercosporin productivity among 9 isolates, suggesting that $P$. capsellae produces differential amount of cercosporin that corresponds to the virulence. Cercosporin, thus, is considered to be a virulence factor but not a host determinant.

Soybean yield response to foliar fungicides evaluated from 2005 to 2018 in the United States and Canada Y. R. KANDEL (1), C. Hunt (2), K. A. Ames (3), N. Arneson (4), C. A. Bradley PhD (5), E. Byamukama (6), A. Bryne (7), M. Chilvers (7), L. J. Giesler (4), J. Halvorson (8), D. Hooker (9), N. M. Kleczewski (3), D. K. Malvick (10), S. G. Markell (11), B. Potter (12), W. Pedersen (3), D. L. Smith (13), A. U. Tenuta (14), D. E. P. Telenko (15), K. A. Wise (5), D. S. Mueller (1), (1) Iowa State University, Ames, IA, USA; (2) The Ohio State University, Columbus, OH, USA; (3) University of Illinois, Urbana, IL, USA; (4) University of Nebraska-Lincoln, Lincoln, NE, USA; (5) University of Kentucky, Princeton, KY, USA; (6) South Dakota State Univ, Brookings, SD, USA; (7) Michigan State University, East Lansing, MI, USA; (8) North Dakota State University, Department of Plant Pathology, Fargo, 
ND, USA; (9) Univ of Guelph Ridgetown, Ridgetown, ON, CANADA; (10) University of Minnesota, Department of Plant Pathology, St. Paul, MN, USA; (11) North Dakota State University, Fargo, ND, USA; (12) University of Minnesota, Lamberton, MN, USA; (13) University of Wisconsin-Madison, Madison, WI, USA; (14) Ontario Ministry of Agric \& Food, Ridgetown, ON, CANADA; (15) Department of Botany and Plant Pathology, Purdue University, West Lafayette, IN, USA

Meta-analyses were performed on data from 239 field trials conducted over 13 years between 2005 and 2018 across nine U.S. states and Ontario, Canada, to quantify the yield response of soybean following application of foliar fungicides at the R3 soybean growth stage. Meta-analysis showed that the overall mean yield response to fungicide was $105 \mathrm{~kg} / \mathrm{ha}$ (a $2.6 \%$ over non-treated). Nine moderator variables including fungicide class, growing season, trial location, planting date, weather variables (temperature and precipitation) and base yield were used to further explain increased yield gains. All moderator variables significantly influenced the yield response, except trial location. Fungicide products containing multiple modes of action including QoI increased the yield response by $3.5 \%$ (152 $\mathrm{kg} / \mathrm{ha}$ ). The greatest yield response to foliar fungicides occurred when soybeans were planted earlier than was typical for a location (3\% increase over not treating). Warmer temperature and higher precipitation than historic averages during the season also provided a greater yield response when foliar fungicide was used. Baseline yield (mean yield in a non-treated plot) significantly influenced the yield response with a greater yield observed in the average-baseline yield category compared to the highest- and lowest-baseline yield categories. The probability of covering fungicide and application costs was low in most scenarios, which suggest that the use of foliar fungicides is less likely to be profitable when foliar diseases are absent or at low levels.

Using whole genome sequencing to develop improved molecular diagnostics for detection of Phytophthora quercina K. JENNINGS (1), S. Nunziata (1), Y. Rosado-Rivera (1), J. C. Bienapfl (2), (1) USDA-APHIS-PPQ-S\&T, Beltsville, MD, USA; (2) USDA APHIS PPQ, Riverdale, MD, USA

Phytophthora quercina has been considered a priority pest in the commodity-based survey listing for the USDA Cooperative Agriculture Pest Survey. It is also ranked as the \#1 exotic Phytophthora species of concern to the USA by PPQ for the potential damage that it could cause if introduced into the country. P. quercina has been reported as a prevalent root rot pathogen of multiple oak species in several European countries and was officially confirmed in the USA for the first time by PPQ during 2016. Screening and confirmatory diagnostic methods for detection of $P$. quercina have been validated at the Beltsville Lab, but they involve lengthy nested PCR assays and require sequencing due to a lack of assay specificity with closely related species. As part of our efforts to enhance detection of Phytophthora species of concern for the USA we have used Illumina and Nanopore whole genome sequencing data to assemble and compare the partial genome of $P$. quercina with five other closely related species. The $P$. quercina genome size is estimated at $71.7 \mathrm{Mb}$ distributed in 100 contigs from a hybrid Illumina-Nanopore assembly using Canu and Pilon and have estimated 97.9\% genome completeness with BUSCO. The genome sizes among the other four species range between 69.7 to $116.9 \mathrm{Mb}$. We are using comparative genomics for marker identification to develop new and improved diagnostic methods for the detection of $P$. quercina. Results on method selection, optimization, and validation will be presented.

\section{Low or no risk of infection by Aspergillus flavus and aflatoxin contamination of almond during the time of drying on the orchard ground}

R. JAIME (1), R. Puckett (1), A. Papagelis (2), M. T. García López (3), J. Moral (3), T. J. Michailides (1), (1) University of California, Davis, Parlier, CA, USA; (2) University of California, Davis, CHALKI / LARISA, GREECE; (3) Universidad de Cordoba, Cordoba, CA, SPAIN

Aflatoxins are carcinogenic metabolites produced by fungi in Aspergillus section Flavi, which are strictly regulated in food and feed worldwide. California almonds are very occasionally contaminated with aflatoxins, and the almond industry has taken drastic measures to reduce contamination even more. The aflatoxigenic A. flavus and A. parasiticus normally infect crops during fruit maturing in the field. However, when harvested crops are subjected to conditions conducive to infection it results in a rapid increase in aflatoxin content. Almond growers are concerned by the possibility of nuts being contaminated with aflatoxin while drying on the ground. The study objective was to evaluate the risk of infection by Aspergillus and increase of aflatoxins while almonds are drying on the ground. An experimental plot with almonds drying on the ground was subjected to irrigation and inoculation treatments during the 2016 and 2017 seasons. Half of the plot was inoculated with a $5 \times 10^{5}$ spores $/ \mathrm{ml}$ suspension of toxigenic A. flavus after irrigation, simulating a 15 $\mathrm{mm}$ rain. Irrigation was applied to half of each inoculated and not inoculated areas. Incidence of navel orange worm (NOW) and aflatoxin content was determined. Results from both seasons indicate that there was no significant effect of moisture content in the almonds on the ground for either aflatoxin contamination or incidence of NOW damage, and that there was no aflatoxin increase in nuts inoculated with $A$. flavus. Therefore, drying nuts on the ground may not pose any additional risk for aflatoxin contamination of almonds.

\section{Fusarium oxysporum causing post-harvest meltdown on Zinnia flowers}

R. BIKA (1), F. Baysal-Gurel (2), T. Simmons (2), L. Alexander (3), (1) Tennessee State University, Mc Minnville, TN, USA; (2) Tennessee State University, McMinnville, TN, USA; (3) USDA-ARS U.S. National Arboretum, McMinnville, TN, USA

Ornamental growers of Eastern and Southern states are threatened with post-harvest zinnia meltdown leading to reduced yields, income, and opportunities for production expansion. Disease symptoms like wilting and bent neck are associated with post-harvest meltdown on zinnia cut flowers. The objective of this study was to diagnose problems related with zinnia meltdown and determine the possible sources. A survey was conducted, and samples of zinnia were collected from Tennessee, Ohio, and Massachusetts. Several Fusariumlike colonies with micro and macro conidia were isolated from flowers and stem on PDA and Fusarium selective media. PCR and sequencing were used to identify pathogens. Sequence identity for ITS and EF-1 $\alpha$ was $99.4 \%$ identical to Fusarium oxysporum. To accomplish Koch's postulates, pathogenicity test was performed on three zinnia cultivars (Giant Golden Yellow, Giant Lime, and Giant 
Pink) at vegetative (one month old) or flower-bud stage by soil drench, stem injection and foliar spray of spore suspension $\left(10^{5}\right.$ conidia/ml). Control plants received sterilized water. Flowers were harvested at normal maturity dates and maintained at room temperature. Symptoms of zinnia meltdown i.e. floral axis bending just below flower head were observed on inoculated zinnia cultivars two days after harvesting. F. oxysporum was re-isolated from flower stems of all three cultivars. This study confirms that $F$. oxysporum is the causal agent of zinnia flower meltdown. In the future, possible sources of pathogen will be screened and disease management recommendations will be developed.

Why isn't rice susceptible to Sclerotinia sclerotiorum?

J. SHARKEY (1), J. E. Leach (2), F. Martin (3), (1) Colorado State University, Colorado Springs, CO, USA; (2) Colorado State University, Fort Collins, CO, USA; (3) Colorado State University, Fort Collins, CO, USA

Why cereal crops, such as rice, are resistant to broad-host range, necrotic fungal pathogens such as Sclerotinia sclerotiorium, is a major question in plant pathology. One hypothesis is that monocots such as rice are resistant to $S$. sclerotiorum because they produce oxalate oxidases, enzymes that degrade the major S. sclerotiorum virulence factor oxalic acid (OA). The rice genome contains four tandemly duplicated oxalate oxidase encoding genes that map to a disease resistance QTL. Silencing of the OsOxo genes rendered plants more susceptible to multiple pathogens indicating that they function in QTL-based resistance. Interestingly, activation of only one of the four genes, $\mathrm{OsOXO4}$, is associated with higher levels of resistance to the rice blast pathogen, and this resistance is correlated with faster and higher expression of the $\mathrm{OsOxo}_{4}$ gene and the presence of a $26 \mathrm{bp}$ insertion in the gene's promoter. To determine if the $\mathrm{OsOxO}_{4}$ gene and its expression are factors in rice resistance to $S$. sclerotiorum, we developed rice plants with varying deletion alleles within the 26 bp promoter insertion by using genome editing. The edited lines are being tested using a detached leaf assay to determine the relationship of resistance and the composition of the $\mathrm{OsOxo4}$ promoter insertion. Results from this analysis will help define why monocots are resistant to OA-producing pathogens and will enhance our understanding of the positive and negative elements that mediate transcriptional activation during plant-pathogen interactions.

\section{Resistance to late blight of potato accessions from the N. I. Vavilov Institute of Plant Industry (VIR) in the Toluca Valley, México \\ G. ENCISO, H. Lozoya-Saldana, Chapingo Autonomous University, Texcoco, MEXICO}

Wild potato species are a vital resource in breeding, for many of them have pest and disease resistance genes, including, among other, to late blight (Phytophthora infestans). The Toluca Valley, in México, provides the ideal climatic conditions for the optimal manifestation of the pathogen each year, and the two compatibility groups of the pathogen are present. The objective of this work was to identify sources of resistance to $P$. infestans in wild potato species in the Toluca Valley. Under field conditions and natural infection, we evaluated the development of disease in 104 accessions of wild potatoes of 13 species (Solanum acaule, S. albicans, S. berthaultii, S. brevicaule, $S$. chacoense, $S$. demissum, $S$. guerreroense, $S$. juzepczukii, $S$. pinnatisectum, $S$. stoloniferum, $S$. vernei and $S$. verrucosum) of the Vavilov Institute of Plant Research. Variation in the resistance response between species and between accessions within the same species was observed. S. demissum and S. guerreroense were resistant to infection ( $0 \%$ severity), while $S$. pinnatisectum, $S$. michoacanum and $S$. microdontum were intermediate, reaching final severity values between 60 and $70 \%$ with an area under the disease progress curve (AUDPC) between 1200 and 2300. The other accessions reached severity values above 87\% and AUDPC values above 2500. Most accessions showed variation in resistance within the population. S. demissum, S. guerreroense were immune to the disease.

\section{Characterizing the wheat virome: Discovery of novel plant and fungal-associated viruses}

C. D. REDILA, S. Nouri, Kansas State University, Manhattan, KS, USA

Important wheat viruses including Wheat streak mosaic virus (WSMV), Triticum mosaic virus (TriMV), Wheat mosaic virus (WMoV), and Barley yellow dwarf virus (BYDV) cost substantial losses in crop yields. Although there have been extensive studies done on known viruses, novel viruses associated with humans, animals, and plants are being discovered every day. Currently, there is limited information about the components of the wheat virome. The objective of this study was to investigate the composition of the wheat virome and characterize novel viruses. Twenty symptomatic and asymptomatic field-collected samples were utilized for total RNA deep sequencing. Sequences mapped to the available wheat reference genome were removed. The unmapped reads were de novo assembled and a BLASTx search against these assembled contigs were conducted. The BLASTx results indicated the presence of viral sequences sharing $<45 \%$ similarity at the amino acid level with Barley mild mosaic virus (BaMMV). Additionally, the presence of Virga- and Narna-like viral sequences were also detected through BLASTx. Fungal-associated viral sequences, such as Puccinia striiformis mitovirus, were also found in plants that were infected with the wheat rust. The presence of all viral sequences was confirmed in original RNAs using RT-PCR. The discovery and characterization of novel viruses is important to determine if these novel viruses may pose a threat to the wheat industry or have the potential to be used as new biological control agents.

Integrated disease management options for tar spot of corn in northern Indiana, 2019

T. J. ROSS (1), J. Ravellette (1), D. E. P. Telenko (2), (1) Purdue University, West Lafayette, IN, USA; (2) Department of Botany and Plant Pathology, Purdue University, West Lafayette, IN, USA

Tar spot of corn is a foliar disease that has emerged as an economic threat in Indiana. Tar spot is caused by Phyllachora maydis. Little is known about tar spot management in the U.S. and is currently based on strategies implemented in Latin America. Although fungicides are available to manage tar spot of corn, integration with susceptible verses moderate resistant hybrids is unknown. In this study, a VT/R1 (tassel/silk) growth stage fungicide application (Trivapro at $13.7 \mathrm{fl} \mathrm{oz/A}$ on $9 \mathrm{Aug}$ ) was compared to non-treated (NTC) across 
three hybrids with various levels of susceptibility to tar spot. Hybrids compared were W2585SSRIB, RPM-4329AM and P0589AMT. Disease was assessed during the season and a final rating taken at the R6 (maturity) growth stage. Tar spot was rated by visually assessing the leaves for percentage of stroma and symptomatic tissue (chlorosis and necrosis). Percent stay green, lodging, and yield were also calculated. In 2019, tar spot severity was moderate to high. Both RPM-4329AM and P0589AMXT had significantly less tar spot stroma, symptoms, and increase percentage of the canopy staying green compared to the susceptible W2585SSRIB. Trivapro significantly reduced tar spot stroma and symptoms on W2585SSRIB over the NTC, but no differences were detected on RPM4329AM and P0589AMXT. Highest yield was achieved with the RPM-4329 AM hybrid with Trivapro but was not significant from RPM-4329 NTC or W2585SSRIB with Trivapro. The results indicate that if a hybrid is susceptible to tar spot, a fungicide application may improve tar spot management and protect yield.

\section{Determining the cause of fisheye lesions in the tar spot complex using fungal community associations}

E. ROGGENKAMP (1), A. Mccoy (1), Z. Noel (1), C. J. Harbach (2), N. M. Kleczewski (3), P. A. Paul (4), A. E. Robertson (5), D. L. Smith (6), D. E. P. Telenko (7), G. Bonito (1), M. Chilvers (1), (1) Michigan State University, East Lansing, MI, USA; (2) University of Illinois, IL, USA; (3) University of Illinois, Urbana, IL, USA; (4) Department of Plant Pathology, The Ohio State University, Wooster, OH, USA; (5) Iowa State University, Ames, IA, USA; (6) University of Wisconsin-Madison, Madison, WI, USA; (7) Department of Botany and Plant Pathology, Purdue University, West Lafayette, IN, USA

Phyllachora maydis is a fungal pathogen that causes the disease tar spot of corn in North and South America. The disease causes black stroma on the foliage of corn, also known as tar spots. Another symptom that can occur is a necrotic ring surrounding the tar spot, also known as a "fisheye" lesion. Fisheye lesions are reported to be caused by the fungal pathogen Monographella maydis in Latin America, however this pathogen has never been isolated in the United States. A previous study conducted in Michigan looked at the fungal communities associated with fisheye lesions. They reported that M. maydis was not necessary to cause fisheye lesions and identified several other candidates that may cause fisheye lesions: Neottiosporina paspali, Paraphaeosphaeria neglecta, Fusarium sp., or even Phyllachora maydis. To confirm these results and further understand the cause of fisheye, samples containing tar spots with and without fisheye lesions and asymptomatic samples were collected in Iowa, Illinois, Indiana, Michigan, Ohio, and Wisconsin in 2019. Through the use of high-throughput sequencing of the internal transcribed spacer, fungal communities associated with fisheye lesions will be determined and compared between states. We hope to identify the causal agent(s) of fisheye lesions in the United States. Validation of these species will be conducted by culturing and co-inoculating with Phyllachora maydis.

\section{Epidemiology of tar spot of corn under different management strategies in Honduras}

C. E. PUERTO HERNANDEZ (1), L. Henriquez-Dole (2), M. C. Avellaneda Barbosa (3), C. C. Gongora-Canul (4), C. D. Cruz (5), (1) Zamorano Escuela Agricola Panamericana Biblioteca, San Antonio de Oriente, Francisco Moraz, HONDURAS; (2) Escuela Agrícola Panemericana El Zamorano, San Antonio de Oriente, HONDURAS; (3) Louisiana State University, Baton Rouge, LA, USA; (4) Department of Botany and Plant Pathology, Purdue University, West Lafayette, IN, USA; (5) Purdue University, West Lafayette, IN, USA

Tar spot of corn, caused by Phyllachora maydis, currently a major disease in Latin America and the Caribbean. There is limited epidemiological information about this disease, which makes it difficult to manage. The objective of this study was to characterize the temporal dynamics of tar spot at three canopy levels, and yield impact under field conditions in Honduras. The experiment consisted of a randomized complete block design RCBD, with two fungicide treatments (with and without application) and two commercial corn hybrids (DICTA-96 and DK390-RR). Results showed significant $(\mathrm{p}<0.0001)$ differences among the area under the disease progress curves $($ AUDPC) at three canopy levels, low $=979.2, \mathrm{mid}=753.1$, and upper $=432.0$. Hybrid DICTA-96 had lower AUDPC $(189.8)$ than DK390-RR (1248.3) ( $<<0.0001)$. Application of fungicide reduced $(\mathrm{p}<0.0001)$ the AUDPC (195.3) compared to the untreated control (1242. 9). The Gompertz model described the temporal disease progress curves (DPC's) at low $(p=0.049)$ and upper canopy $(\mathrm{p}=0.036)$ of hybrid DK390-RR without fungicide. The rates of tar spot progress were faster in the lower canopy $\left(\mathrm{r}^{*}=0.06 \mathrm{day}^{-1}\right)$ than in the mid $\left(\mathrm{r}_{\mathrm{G}^{*}}=0.049\right.$ day $\left.^{-1}\right)$ and upper $\left(\mathrm{r}_{\mathrm{G}^{*}}=0.03\right.$ day $\left.^{-1}\right)$ canopies. There was a $(\mathrm{p}<0.0001)$ negative correlation between yield $\left(\mathrm{Kg} \mathrm{plot}^{-1}\right)$ and AUDPC and yield and Ymax at each canopy level $(\mathrm{r}=-0.60,-0.60$ and -0.61$),(\mathrm{r}=-0.55,-0.57$ and -0.60$)$ respectively. Results can be the basis for adequate development of tar spot management strategies in Honduras.

\section{Diversity and mycoparasite activity of Trichoderma spp. on Fusarium oxysporum f. sp. lycopersici recovered from tomato growing regions in Florida}

J. D. CARRILLO (1), L. Yang (2), G. E. Vallad (1), (1) Gulf Coast Research and Education Center, University of Florida, Wimauma, FL, USA; (2) China Agricultural University, Beijing, CHINA

Fusarium wilt of tomato, caused by Fusarium oxysporum f. sp. lycopersici (FOL), is a major soilborne disease affecting tomato production in Florida. Commercial strains of Trichoderma spp., mainly in the Harzianum and Viride clades, are reported to have antagonistic activity against $F O L$. Although they are applied in a variety of agricultural settings, commercial strains are more likely to be derived from a different soil type, host, and environmental condition. Therefore, we conducted a survey to recover local Trichoderma antagonists from commercial tomato fields in major growing regions in the west (6 fields) and south (4 fields) of Florida. In total, 81 Isolates recovered from tomato roots, mulched fumigated beds, as well as non-fumigated soil within tomato fields represented 7 different species clades based on multigene phylogenetic analysis using translation elongation factor 1- $\alpha$ (TEF1- $\alpha$ ) and RNA polymerase II (RPB2) loci. Antagonism was assessed by introducing Trichoderma isolates to fully colonized $F O L$ (race 3) plates on 1/4 PDA and then rated over 40 days. Significantly more fruiting bodies were observed from some Trichoderma isolates recovered locally than commercial products, with $T$. asperellum isolates containing the most among isolates screened $(P<0.001)$. In addition, image analysis of the plates showed a significantly higher proportion of local Trichoderma colonizing FOL than commercial products tested 
$(P=0.02)$. This study suggests local Trichoderma isolates may be ideal candidates to explore control of $F O L$ in commercial tomato fields in Florida.

New races and novel strains of the spinach downy mildew pathogen

C. FENG, M. Villarroel-Zeballos, P. Ficheux, H. Zima, J. Correll, University of Arkansas, Fayetteville, AR, USA

Downy mildew, caused by the obligate pathogen Peronospora effusa (Pfs), is the most economically important disease of spinach. Growing resistant cultivars is the most effective way to manage this disease, especially for organic spinach production, which accounts for approximately $50 \%$ of total production. New races and novel strains of the pathogen frequently appear that overcome the deployed resistance which threatens spinach production. Monitoring the race dynamics of the Pfs population is critical for the spinach industry. Since 2018, more than 50 isolates collected from various production areas have been tested on a standardized set of spinach differentials for their virulence phenotype. Four isolates each collected in 2018 and 2019, and two isolates collected in 2020, were found to be unique and different from the 17 reported races and previously known novel strains based on the disease reactions. Four novel isolates could infect the cotyledons, but not the true leaves of the differential cultivar Hydrus, which is resistant to all races and known novel strains. However, 14 isolates with a unique pathotype (UA202001E) were recently collected from AZ, CA and ME, from Dec. 2019 to March 2020. This pathotype was able to infect the cotyledons and true leaves of Hydrus, and many other newly released Pfs1-17 resistant commercial cultivars, resulting in a significant concern to the industry. A few resistance loci were found to be effective to this novel strain which has tentatively been named Pfs race18 upon sanctioning by the International Working Group on Peronospora (IWGP).

\section{Csp1, a cold-shock protein homolog in Xylella fastidiosa is involved in biofilm formation}

L. Burbank, W. WEI, USDA-ARS, Parlier, CA, USA

Bacterial cold shock proteins (CSPs) are conserved nucleic acid binding proteins that help facilitate translation at low temperatures. Bacterial CSPs are important for adaptation to stress, including conditions unrelated to temperature. Csp1, a temperature-independent cold shock protein homolog, is a virulence factor in grapevine pathogen Xylella fastidiosa. Csp1 binds single-stranded nucleic acids and contributes to $X$. fastidiosa survival in high salt conditions and at low temperatures, in vitro. However, little is known about the specific function(s) of $X$. fastidiosa Csp1. To investigate the role(s) of Csp1 on gene expression, we compared the transcriptomes of wild-type $X$. fastidiosa strain Stag's Leap to a cspl deletion mutant $(\Delta c s p 1)$ under standard $\left(28^{\circ} \mathrm{C}\right)$ and cold $\left(15^{\circ} \mathrm{C}\right)$ conditions. We observed changes in expression of several genes important for motility and biofilm formation in $\Delta \operatorname{csp} 1$ compared to wild-type. One gene of interest, pilA1, encodes a type IV pili subunit protein and was up-regulated in $\Delta c s p 1$ compared to wild-type. $X$. fastidiosa type IV pili is required for twitching motility, and contributes to virulence by facilitating bacterial movement inside the host. Other studies showed deleting pilA1 in $X$. fastidiosa lead to increased biofilm formation. The $\Delta \operatorname{csp} 1$ mutant showed significant decrease in biofilm formation compared to wild-type, which may be the result of increased pilAl expression. Xylem vessel occlusion caused by biofilms is one method of $X$. fastidiosa pathogenicity, and Csp1 may act as a virulence factor by influencing biofilm formation and motility.

"Earth Fever": A college classroom exercise to teach climate change, plant diseases, and scientific collaboration A. M. SHIGENAGA, G. Kroh, C. Argueso, Colorado State University, Fort Collins, CO, USA

Challenges of food security and climate change are current agricultural socio-scientific issues that are important for creating a sustainable future. Currently, there is a need to teach students how scientific research can inform policy to improve food security in the face of climate change. "Earth Fever" is an active learning activity designed to give college students experience in synthesizing information and developing a solution, in the context of plant pathology. "Earth Fever" uses the issue of rice susceptibility to a bacterial pathogen under increased temperature conditions to improve student understanding of the interactions between abiotic and biotic factors impacting rice yield and the importance of communication between different fields to produce solutions to major issues affecting global food security. For the activity, students are invited to a "Food Security Summit" at the International Rice Research Institute in the Philippines, which will act as a conference to bring together student experts from different fields (Plant Pathology, Climatology, Agricultural Economy, and Agronomy). At the Summit students are asked to synthesize data, construct a concept map, and develop a policy to combat the threat climate change poses to rice production. By using an international agricultural pathosystem, we aim to inform students how environmental pressures can impact economically important plant systems, the role scientists and experts play in policy making to preserve food security, and the importance of agriculture on a global scale.

Evaluation of ultraviolet light for suppression of Botrytis fruit rot (Botrytis cinerea) in vitro, in open-field production, and postharvest

P. MELLO (1), R. Borba Onofre (1), U. Lopes (2), A. Zuniga (1), D. M. Gadoury (3), N. Peres (1), (1) University of Florida - Gulf Coast Research and Education Center, Wimauma, FL, USA; (2) Universidade Federal Rural de Pernambuco, Garanhuns, BRAZIL; (3) Cornell University, Geneva, NY, USA

Resistance across several fungicide modes of action has increased worldwide demand for alternative management strategies for Botrytis Fruit Rot (BFR) of strawberry. Our objective was to evaluate the efficacy of ultraviolet C (UVC) light against Botrytis cinerea in vitro and on fruit in open-field and post-harvest experiments. In open-field trials, weekly and twice-weekly UVC night applications at $200 \mathrm{~J}$ did not reduce incidence of BFR ( $\mathrm{p}>0.05$ ). Doses ranging from 950 to $9500 \mathrm{~J}$ were required to reduce in vitro conidial production of $B$. cinerea by 96 to $99 \%$. At the same doses, conidial viability and mycelial growth were reduced by 21 to $81 \%$, and 28 to $41 \%$, respectively. When berries were detached, inoculated, and then treated once with UVC at $9500 \mathrm{~J}$, incidence of BFR was reduced by up 
to $30 \%$. However, post-harvest applications of UVC at 500 and $3000 \mathrm{~J}$ did not reduce BFR $(\mathrm{p}>0.05)$ in non-inoculated, field-collected berries. Neither was BFR reduced $(\mathrm{p}>0.05)$ when strawberry fruit harvested from fields treated weekly or twice weekly with UVC at $200 \mathrm{~J}$ (effective in suppressing powdery mildew) received additional post-harvest UVC treatments of 500 and $3000 \mathrm{~J}$. Phytotoxicity was not observed on plants or treated fruit, even at the highest UVC doses. Our results suggest that suppression of BFR on strawberry grown in open-field production systems commonly used in Florida might require UVC doses exceeding $9500 \mathrm{~J}$. Latent infections and inaccessibility of berry undersurfaces on plastic-covered beds in field plantings were unresolved challenges to the use of UVC against BFR in our studies.

Implementation of a problem based learning approach in an introductory plant pathology course M. E. MCKELLAR (1), K. D. Cox (2), (1) Cornell University, Ithaca, NY, USA; (2) Cornell University, Geneva, NY, USA

Student feedback on course evaluations for an upper level introductory plant pathology course has been a valuable tool to assess the effectiveness of currently used teaching methods and content. Student feedback over the last five years have included requests for authentic fieldwork and understanding how to apply disease management strategies using an integrated approach. We redesigned our course to incorporate a problem based learning (PBL) approach to foster a student centered learning environment and address student feedback. PBL's are a student-centered learning strategy in which student groups work on a real-world task or problem to construct a solution. PBL activities implemented in the course over the last two years included working with local stakeholders to develop an integrative plant disease management plan based on current disease issues for a specific crop and location, role-playing authentic plant disease scenarios, and alternative summative assessments including two-stage exams. Learning outcomes included content driven outcomes as well as professional skill outcomes including identification of target audiences for effective communication and proficient self-regulated learning for project development and management. End of semester student surveys were administered to assess student perception of the impact of PBL activities on their learning. Student responses indicated that PBL activities were most beneficial to their learning the content and the course helped improve their communication and teamwork skills.

Disease resistance of alfalfa varieties to Rhizoctonia solani

H. TIAN, Lanzhou University, Lanzhou, China, CHINA

Alfalfa (Medicago sativa) is a legume pasture with largest planting area in China \& the World. Root rot is one of the major constraints in alfalfa production. Rhizoctonia solani (R. solani), as one of the main pathogens of the disease, can survive for a long time in soil and diseased plants, making it difficult to control alfalfa root rot. The moderate virulence of $R$. solani R10 was inoculated into 77 alfalfa varieties by soil inoculation method in greenhouse. The resistance of different alfalfa varieties to $R$. solani was evaluated by measuring the disease index, plant height and root length, aboveground and underground biomass, and further screen out disease-resistant alfalfa varieties. The results show that the cluster analysis of alfalfa varieties based on 5 indicators, 77 varieties can be divided into 3 categories, corresponding to the three resistance performance of susceptibility (S), medium resistance (MR) and resistance (R). There are 43 susceptible varieties, accounting for $56 \%$ of the total tested varieties, such as Aurora, Gannong No.6, Baoding alfalfa; 27 medium resistant varieties, accounting for $35 \%$ of the total tested varieties, such as salt-tolerant star, Zhonglu No.1 and Victoria; 7 disease resistant varieties, accounting for $9 \%$ of the total tested varieties, were Gannong No.7, Hunter Field, Arc, UC-1465, WL316, 4020 and 4030. The decrease in plant height, root length, aboveground and underground biomass of resistant varieties was significantly lower than that of susceptible varieties.

\section{Horizontal chromosome transfer and convergent evolution drive diversification in the global population of Fusarium oxysporum} f. sp. fragariae

P. HENRY (1), D. Pincot (2), B. Jenner (3), S. Knapp (2), T. R. Gordon (4), (1) United States Department of Agriculture, Salinas, CA, USA; (2) University of California, Davis, Dept. of Plant Sciences, CA, USA; (3) University of California, Davis, Dept. Plant Pathology, CA, USA; (4) University of California, Davis, Davis, CA, USA

The observation that host specific pathogenicity could be acquired through horizontal chromosome transfer (HCT) fundamentally changed how evolution within the Fusarium oxysporum species complex (FOSC) is understood. A decade after this evolutionary mechanism was first described, we still have little knowledge on the frequency with which it occurs in nature or its potential to explain why so many host specific forms (i.e. formae speciales) in the FOSC are polyphyletic. We explored the role of HCT in the diversification of a globally representative population of Fusarium oxysporum f. sp. fragariae, the cause of Fusarium wilt of strawberry. We identified a predicted pathogenicity chromosome that has been transferred up to seven times in this forma specialis. Sequence diversity suggests this chromosome existed in multiple phylogenetic groups prior to the first observation of this pathogen in Japan, which indicates that selection pressure for this chromosome occurred outside of the strawberry wilt pathosystem. Fusarium oxysporum f. sp. fragariae strains from Australia and Spain do not carry this chromosome and caused distinct symptoms on a different cultivar host range. We discovered FWl-mediated resistance breaking isolates prior to their arrival in California, where this resistance locus is critical for disease management. Surprisingly, a relatively large number of heirloom cultivars and wild ecotypes were resistant to both race 1 and race 2 genotypes, and there is evidence that many genes for resistance to Fusarium wilt are available for breeding.

Optimal timing for preventative Pythium root rot management on golf course putting greens H. D. HAMPY (1), J. Kerns (2), (1) North Carolina State Univ, Raleigh, NC, USA; (2) North Carolina State University Plant Pathology, Raleigh, NC, USA 
Pythium root rot (PRR) is a detrimental disease of creeping bentgrass golf course putting greens in almost all regions of the world. The disease is typically managed by spraying fungicides throughout the summer months. However, the timing of preventative applications is poorly understood. Therefore, the objective of this study is to evaluate the most efficacious time period to initiate a fungicide program in order to manage Pythium root rot symptoms on creeping bentgrass putting greens. Five treatments were arranged in a randomized complete block design on a creeping bentgrass putting green previously inoculated with Pythium spp. Cyazofamid (Segway SC) was sprayed every 28 days and immediately followed with $0.64 \mathrm{~cm}$ of post-application irrigation. Each treatment was initiated at different dates and all treatments received applications through the end of August. Cyazofamid applications starting in March, April, and May or before soil temperatures reach $25^{\circ} \mathrm{C}$ resulted in improved turfgrass quality when compared to the applications sprayed in June and July, or after soil temperatures increased above $25^{\circ} \mathrm{C}$. Treatments initiated after soil temperatures reached $26^{\circ} \mathrm{C}$ did not reduce disease severity when compared to the control plots and therefore suggests that cyazofamid applications should be initiated before soil temperatures reach $26^{\circ} \mathrm{C}$.

Field trials demonstrate biological control of Pierce's disease in Florida, Georgia, and California and citrus greening in Florida D. L. HOPKINS (1), Y. Benyamini (2), (1) University of Florida, Apopka, FL, USA; (2) Luxembourg Industries Pumol Ltd, Tel Aviv, ISRAEL

Pierce's Disease (PD) caused by the xylem-limited, bacterium Xylella fastidiosa subsp. fastidiosa and citrus greening caused by the phloem-limited Candidatus Liberibacter spp. are severe threats to the grape and citrus industries in the United States. Injection of a benign strain of Xylella fastidiosa (EB92-1) into the xylem vessels controlled PD in Vitis vinifera 'Cabernet Sauvignon' for 14 years in the University of Florida vineyard. In 8-year field trials in Central Florida, strain EB92-1 provided significant control of PD in $V$. vinifera 'Cabernet Franc', 'Chardonnay', 'Chenin Blanc', and 'Ruby Cabernet', as well as V. aestivalis 'Cynthiana' and a FrenchAmerican interspecific hybrid 'Chambourcin.' EB92-1 provided biological control of PD in a new planting of 'Malbec' for 7 years and in a mature planting of 'Mourvedra' for 5 years in Dahlonega Georgia. In the UC Riverside vineyard, EB92-1 provided significant control of PD for 6 years in 'Cabernet Sauvignon' and 'Pinot Noir'. Trials were established in Florida in 2014/2015 to evaluate EB92-1 for the biocontrol of citrus greening. In sweet orange treated as 2-year-old trees, only $8 \%$ of the treated trees had severe symptoms after 6 years compared with $40 \%$ of the untreated trees. After 6 years, yield was reduced by $40 \%$ in the untreated compared with the EB92-1 treated. While a single treatment with EB92-1 at planting can provide PD control for several years, citrus greening may require treatment every 18-24 months to sustaining disease suppression.

Investigating biological mechanisms of resistance against root-knot nematodes H. V. BAKER (1), C. Gleason (1), C. Clarke (2), (1) Washington State University, Pullman, WA, USA; (2) USDA ARS, Beltsville, MD, USA

Plant-parasitic nematodes (PPN) are economically important pests because of their ability to parasitize a wide range of susceptible hosts. The most commonly used methods for control of nematodes are pre-plant fumigation or non-fumigant nematicides which are harmful to human and environmental health. Root-knot nematodes (RKN) are the most damaging of the plant parasitic nematode because most crop plants lack RKN resistance. There is a need for new tools of resistance against RKN to reduce our reliance on nematicides. This research is investigating two forms of resistance, including the use of the trap crop Litchi tomato, Solanum sisymbriifolium, and the use of non-pathogenic strains of Streptomyces that produce chitinases. Litchi is a well-known trap crop that has been shown to significantly reduce populations of potato cyst nematode, Globodera pallida (PCN), by up to 98\%. We hypothesize that Litchi roots are also resistant to RKN and that the nematodes elicit a hypersensitive response (HR) similar to the HR caused by PCN infection. Our data demonstrates the ability of RKN to enter the Litchi roots but being unable to establish feeding sites. Another biological mechanism of control is using non-pathogenic strains of Streptomyces that produce chitinases. Current data has shown treating eggs with chitinases extracted from various Streptomyces strains can be effective at controlling RKN infections of tomato. We are investigating if these Streptomyces may be useful for biological nematode control in the future.

The role of hydrophobins in Pencillium expansum biology and virulence D. LUCIANO-ROSARIO, N. P. Keller, University of Wisconsin, Madison, WI, USA

Penicillium expansum is the causative agent of blue mold, a post harvest disease of apples and pears. This filamentous fungal pathogen poses both economic and food safety threats as this ascomycete produces patulin, a mycotoxin that contaminates processed food products. Research efforts have largely focused on elucidating the regulation of patulin and in developing methods for its decontamination but with limited knowledge on the molecular mechanisms of disease for this pathosystem. In a recent study, CreA, a carbon catabolite repressor was identified and described as a $P$. expanusm virulence factor. We used an RNA-seq dataset from that study to identify other potential genes that may contribute to virulence in this pathosystem. We identified a hydrophobin gene to be significantly differentially downregulated in the DcreA mutant when compared to WT strain. Preliminary data suggests this gene may contribute to virulence although to a minimal extent. In order to account for functional redundancy, we are currently generating single and poly deletion mutants for the 5 putative hydrophobin encoding genes in the $P$. expansum genome. These mutants will be compared to the WT strain and will be assessed in different aspects of fungal physiology and disease including in vivo virulence assays, attachment and dispersal.

Role of type III effectors from Ralstonia solanacearum in bacterial wilt disease R. R. HILES, D. Caldwell, A. Iyer-Pascuzzi, Purdue University, West Lafayette, IN, USA 
Host perception of phytopathogenic bacteria, like Ralstonia solanacearum, triggers PAMPs (pathogen-associated molecular pattern)triggered immunity (PTI), which is a cascade of immune responses. One example of a PTI response is a transient burst of reactive oxygen species (ROS). Ralstonia solanacearum can suppress PTI through the use of type III effectors (T3Es), or virulence proteins, that are injected into the host cell. Once inside the cell, T3Es can achieve immune suppression through various strategies such as organelle disruption or vesicle trafficking interference. Therefore, there is an arms race between T3Es and the host proteins. To understand this battle, the localization and PTI suppression ability of $R$. solanacearum K60 T3Es was first established. A small list of unknown T3Es from $R$. solanacearum strain K60, endemic to the United States, was selected to be studied. The first step in this experiment was to determine the subcellular localization of these T3Es, which will help in determining protein function. Fluorescently tagged T3Es were transiently expressed in two systems: the tomato hairy root system and the Nicotiana tabacum leaf system. We found that RipBD localizes at the plasma membrane, RipAM at the nucleus, RipU localizes at actin filaments, and two, RipR and RipC1, localize to tonoplasts. All T3Es, except RipC1 and RipBD, can suppress ROS bursts. Future work will use secretion assays to confirm the secretion of the T3Es, as well as yeast two-hybrid and mutant analyses to determine the interacting partners and function of the individual T3Es.

Loop-mediated amplification as promising on-site detection approach for Pecan Root-Knot Nematode Meloidogyne partityla J. BELL (1), S. Waliullah (2), T. L. Stackhouse (1), G. B. Jagdale (3), A. Hajihassani (1), T. B. Brenneman (1), E. Ali (1), (1) University of Georgia, Tifton, GA, USA; (2) University of Georgia, GA, USA; (3) Department of Plant Pathology, University of Georgia, Athens, GA, USA

Meloidogyne partityla is the dominant root-knot nematode (RKN) species parasitizing and causing losses in Georgia pecan production. Rapid and accurate diagnosis of this RKN is required to control this nematode disease. In this study, a loop-mediated isothermal amplification (LAMP) method was developed for simple, rapid and on-site detection of $M$. partityla in infested plant roots. This novel tool can detect the nematode in both laboratory and field conditions. M. partityla and other Meloidogyne spp. are distinguished with specific primers based on sequence distinction of internal transcribed spacer (ITS)-18S/5.8S ribosomal RNA gene. The LAMP detection technique can detect the presence of $M$. partityla genomic DNA at a concentration as low as 1 pg, with no cross reactivity of DNA from other major RKN species such as M. javanica, M. incognita and M. arenaria, and M. hapla. The LAMP assay provides more rapid results, amplifying the target nematode species in less than $60 \mathrm{~min}$ at $65^{\circ} \mathrm{C}$. These results are 100 times more sensitive than conventional PCR ( 2-3 hrs). These features have greatly simplified the operating procedure and make the assay a powerful tool for rapid, on-site detection of M. partityla. The LAMP assay will facilitate accurate pecan nematode diagnosis in the field and contribute to the management of the pathogen.

\section{Molecular mechanisms associated with fungicide sensitivity in the Irish populations of the oilseed rape pathogen Pyrenopeziza brassicae}

D. BUCUR (1,2), Y. Huang (3), B. Fitt (4), F. Ritchie (5), S. Kildea (2), (1) University of Herfordshire, UNITED KINGDOM, Hatfield, Hertfordshire; (2) Teagasc, Carlow, IRELAND; (3) University of Hertfordshire, Hatfield, UNITED KINGDOM; (4) University of Hertfordshire, Hatfield, Hertfordshire, UNITED KINGDOM; (5) ADAS Boxworth, Boxworth, UNITED KINGDOM

Light leaf spot (LLS) disease of brassicas, caused by the hemibiotrophic fungal pathogen Pyrenopeziza brassicae (anamorph Cylindrosporium concentricum), is amongst the most damaging diseases of winter oilseed rape (Brassica napus spp.) in Northern Europe. In Ireland, although believed to be a significant threat to oilseed rape crops due to the climatic conditions, which are ideal for the development and spread of fungal pathogens, the epidemiology of $P$. brassicae is poorly understood, and fungicides are relied upon to provide control. As limited data are available on the fungicide sensitivity of the Irish $P$. brassicae population, we aimed to investigate if the cropping strategies currently used are the best for the disease control. To do this, we screened different populations of isolates sampled from the main producing regions of Ireland for alterations in the genes targeted by azoles and Methyl benzamidazole carbamates (MBC), two classes of fungicides used in controlling the development of LLS. As the results do confirm the presence of alterations previously associated with a decrease in fungicide sensitivity, following the detection of the different inserts and mutations targeted, the next steps are to determine the sensitivity of the Irish population to these classes of fungicides, to confirm the degree of sensitivity established in the P. brassicae populations.

Evaluating the efficacy of two organic fungicides, copper and EF400, in controlling tomato late blight T. WU, E. Silva, A. J. Gevens, University of Wisconsin-Madison, Madison, WI, USA

Phytophthora infestans, causal agent of late blight, continues to be an economically important phytopathogen of tomato and potato. In organic systems, copper-based fungicides are considered the most effective compound for chemical control. However, organic farmers avoid regular copper applications due to concerns about negative environmental effects and potential human health risks. Organic certified biopesticides have not been studied extensively as alternatives to copper but are of increasing interest to growers. Previous studies of EF400, a commercial plant extract-based biopesticide, found this product to be more effective in controlling late blight than copper (Seidl et. al., 2015). To expand our understanding of the current formulation of EF400, we evaluated the efficacy of copper (Champ WG) and EF400 in controlling late blight and compared preventative and curative application timings. Whole tomato plants were grown in a complete randomized block design in a growth chamber maintained at $>90 \%$ relative humidity with a 12 -hour photoperiod at $21^{\circ} \mathrm{C}$ day/night. Treatments of EF400, Champ WG, and water control were applied 24 hours before and after inoculation with a sporangial suspension of $P$. infestans US-23 isolates. Preventative applications of copper were significantly more effective at controlling late blight than all other treatments, although the efficacy of EF400 varied between experiments. These data suggest that the efficacy of EF400 for late blight management is variable and less consistent than copper. 
Effect of environmental factors on infection of soybean seedlings by Fusarium solani and $\boldsymbol{F}$. tricinctum H. YAN, B. D. Nelson Jr., Plant Pathology NDSU, Fargo, ND, USA

Fusarium solani and F. tricinctum are important root rot pathogens of soybean in North Dakota. The role of environment in disease development is poorly documented. The effects of soil type, temperature and moisture on infection of soybean seedlings were examined in a growth chamber. Soil types were Fargo clay, Glyndon sandy loam and La prairie silt loam, which represent soils of the soybean production region in the Red River Valley. Disease incidence and lesion length were evaluated at 3 weeks after planting in artificially infested soil. Soil types affected disease development, with higher severity in Glyndon sandy loam and La Prairie silt compared to Fargo clay. Soil types also interacted with Fusarium species, in which the maximum severity was observed in Glyndon sandy loam for $F$. solani, and in La Prairie silt loam for $F$. tricinctum. Among the three soil types, $F$. solani infected plants at temperatures of $10-20^{\circ} \mathrm{C}$ while $F$. tricinctum at $15-20^{\circ} \mathrm{C}$. The most severe disease with $F$. solani was at $20^{\circ} \mathrm{C}$ while it was $15^{\circ} \mathrm{C}$ for $F$. tricinctum. At lower compared to higher temperatures there was a broader range of soil moistures resulting in infection. At $18^{\circ} \mathrm{C}$, infection occurred at 20 $80 \%$ soil moisture while at $28^{\circ} \mathrm{C}$ it was $40-80 \%$. Fusarium solani caused greater disease over a broader range of soil moistures (40$80 \%$ ) compared to $F$. tricinctum. Maximum infection by $F$. solani at $80 \%$ soil moisture occurred at $28^{\circ} \mathrm{C}$ in Glyndon sandy loam, but at $18^{\circ} \mathrm{C}$ in Fargo clay.

Expression pattern of SWN genes and analysis of its mycotoxins, slaframine and swainsonine level at different time points in Slafractonia leguminicola

S. DAS, R. Creamer, New Mexico State University, Las Cruces, NM, USA

The fungal pathogen Slafractonia leguminicola, the causal agent for black patch disease in red clover plants produces two important mycotoxins: slaframine and swainsonine. The indolizidine alkaloid swainsonine is a deadly mycotoxin to livestock causing locoism while slaframine causes slobbers syndrome. Other than $S$. leguminicola, swainsonine is also produced by fungi such as the locoweed symbiont Alternaria oxytropis, fungi from Swainsona canescens, and the insect pathogen Metarhizium robertsii. Genome sequence analyses revealed all the swainsonine-producing fungi, including S. leguminicola share orthologous gene clusters, "SWN," which include 7 genes: a multifunctional swnK gene (NRPS-PKS) with domains for the initial steps of swainsonine biosynthesis, $s w n \mathrm{~N}$ and $s w n \mathrm{R}$ (reductase genes), and $s w n \mathrm{H} 1$ and $s w n \mathrm{H} 2$ (nonheme iron dioxygenase gene). This study aimed to investigate the mRNA levels of all the genes of SWN clusters and level of toxin production in S. leguminicola at different time points. cDNAs from total mRNA were isolated from the mycelia at 5 time points $(24 \mathrm{~h}, 48 \mathrm{~h}, 72 \mathrm{~h}, 5$ days and 15 days) post-inoculation and expression pattern were analyzed using RT-qPCR. The total level of swainsonine and slaframine production from this fungus at these 5 time points were also examined. using liquid chromatography mass spectrometry. Knowledge on how the age of the mycelia affects toxin production by this fungus is an important step toward developing swainsonine management.

Analyzing populations of cotton seedling disease and evaluating seed treatment efficacy for 2018 and 2019 S. PATE (1), H. M. Kelly (2), R. Guyer (2), K. S. Lawrence (3), A. Rojas (4), R. Kemerait (5), P. D. Colyer (6), P. Price (7), T. Wilkerson (8), T. Allen (9), L. D. Thiessen (10), T. S. Isakeit (11), H. L. Mehl (12), (1) University of Tennessee, Jackson, TN, USA; (2) West Tennessee AgResearch and Education Center, University of Tennessee, Jackson, TN, USA; (3) Entomology and Plant Pathology, Auburn University, Auburn, AL, USA; (4) University of Arkansas, Fayetteville, AR, USA; (5) University of Georgia, Tifton, GA, USA; (6) Louisiana State Univ Agric Center, Bossier City, LA, USA; (7) LSU AgCenter, Macon Ridge Research Station, Winnsboro, LA, USA; (8) MSU Department of Biochemistry, Molecular Biology, Entomology, \& Plant Pathology, Stoneville, MS, USA; (9) Delta Research and Extension Center, Mississippi State University, Stoneville, MS, USA; (10) North Carolina State University, Raleigh, NC, USA; (11) Dept of Plant Pathology and Microbiology, Texas A\&M University, College Station, TX, USA; (12) Virginia Tech Tidewater AREC, Suffolk, VA, USA

For over 25 years, the goal of the National Cottonseed Treatment, NCST, program has been to analyze seed treatment efficacy and stand persistence, in relation to pathogens present in the soil and on seedlings of cotton plots across the United States Cotton Belt. Through executing the existing protocol and analyzing the data collected from the program for the 2018 growing season, it was apparent that changes were needed to enhance the sampling process and improve data accuracy. Our improved protocol now includes plating seedlings from each field trial location on different selective media for the morphological identification of Thielaviopsis basicola, Pythium spp., and Fusarium spp., as well as soil baiting for Rhizoctonia solani, and enzyme-linked immunosorbent assays for Pythium spp. and $R$. solani detection. To further enhance Pythium data, isolates' pathogenicity were evaluated using a seed germination assay. With more accurate data, we are able to better interpret seed treatment efficacy in relation to pathogens managed at each field site location and will use this to build risk models. In the presentation, data yielded from detection techniques across both years will be compared outlining the most accurate, time efficient, and cost effective techniques and further utility of the data for modeling. In addition to this, similarities of significant locations will be discussed across 2018 and 2019.

Evaluation of disease resistance to early and late leaf spot in wild-derived peanut allotetraploids M. GONZALES (1), R. Kemerait (2), A. K. Culbreath (2), M. T. Brewer (1), D. J. Bertioli (1), S. Leal-Bertioli (3), (1) University of Georgia, Athens, GA, USA; (2) University of Georgia, Tifton, GA, USA; (3) UGA, Plant Pathology Department, Athens, GA, USA

Early (ELS) and Late (LLS) leaf spots caused by Passalora arachidicola and Nothopassalora personata, respectively, are the most common and important diseases in peanut. These diseases can cause substantial yield loss of up to $50 \%$ or greater. Effective chemical control programs are available but disease-resistant cultivars remain the best way to mitigate these diseases. To date, there is no cultivated peanut with strong resistance to these pathogens. Several peanut wild relatives show strong resistance, but they are diploid, therefore, incompatible with the cultigen. By crossing diploid species and duplicating their chromosome number, synthetic 
allotetraploids are induced and can be utilized as genetic resources for disease resistance. This study aims to determine disease resistance levels of wild-derived allotetraploids to ELS and LLS. A detached leaf bioassay was conducted to evaluate 13 synthetic allotetraploids for possible sources of resistance to ELS and LLS and to determine their disease resistance levels. Eight peanut cultivars with various degrees of resistance to the pathogens and one wild peanut relative (Arachis ipaensis) were used for comparison. Different components of resistance were measured: incubation period, lesion number, number of sporulating lesions, percent diseased leaf area and defoliation. Results showed several synthetic allotetraploids have resistance to ELS and LLS, which can be used as donors in breeding programs. Development of advanced lines with various disease resistance segments will provide long-term protection to these fungal diseases.

Agent-based modeling to evaluate the impact of citrus landscapes on ACP/HLB management performance W. LUO (1), D. Posny (1), N. McRoberts (2), T. R. Gottwald (1), (1) USDA ARS, Ft Pierce, FL, USA; (2) University of California, Davis, CA, USA

Since the initial HLB + find, subsequent ACP/HLB findings have increased, indicating epidemic development and amplifying the risk of HLB introduction into commercial citrus. This poses a major threat to the viability of the citrus industry, emphasizing the urgent need for proactive multiscale intervention. Understanding ACP and HLB spread across California's residential, commercial, and mixed citrus landscapes is crucial in successfully designing/prioritizing mitigation efforts/resources while minimizing economic losses. Therefore, we developed a spatially explicit agent-based model to investigate how ACP and HLB spread in actual CA landscapes, and conduct scenario-based simulations analyzing the effectiveness of current and proposed control strategies in these landscapes. Given ACP/HLB incidence or actual findings for a particular region and a CA-recommended/proposed management program (grove-level, residential/dooryard, multiscale, area-wide), we can estimate the likelihood of ACP/HLB establishment and predicted spread to evaluate the effectiveness and expected benefit of control. Control effectiveness can vary greatly across different citrus landscapes; offering fixed control programs for the entire region may not be appropriate, albeit easier to implement. Moreover, we assessed resource allocation and minimum coordination level needed for different citrus regions in Southern CA. Informative analyses/outputs can assist in guiding spatiotemporal ACP/HLB targeted management strategies across CA, and provide unique insight into the effectiveness of HLB mitigation.

Genomic trends of bacteriocin repertoires in Pseudomonas syringae pv. tomato M. SMITH, Pennsylvania State University, PA, USA

Pseudomonas syringae pv. tomato (pto), is the causal agent of bacterial speck of tomato. Competition between members of the same species(intraspecific) and between members of different species(interspecific) play a role in pto's ability to colonize a plant. One common way pto competes with members of the same species is by producing narrow-target antimicrobial compounds called bacteriocins. Bacteriocins presumably evolved because of the advantages they provide within a community context. Many bacterial genomes are predicted to encode multiple bacteriocins, thus, potentially allowing for targeting of multiple distinct competitors by the same strain. Therefore, the combination of bacteriocins a strain encodes should impact the microbial community dynamics differently than a dual-strain interaction. This study examined 17 publicly available and predicted pto to have three combinations of bacteriocins within its genome that correlate with the whole genome phylogeny of this pathovar. Each clade encodes a single combination of 3 or 4 of the following bacteriocins; two HNH endonuclease bacteriocins, syringacin M, carocin-D like bacteriocin, and a R-type tailocin. The results support the hypothesis of maintaining specific combinations of bacteriocins to compete in the microbiome and could elucidate dynamic interactions that are important in order for pto to cause disease. Future genomic analysis and microbiome transplant experiments will be conducted with 56 strains of pto to further disentangle these dynamic interactions.

\section{An outreach program to engage and promote careers in Plant Health in rural Florida high schools} S. BARRY, P. Digennaro, University of Florida, Gainesville, FL, USA

The first image of a scientist that comes to the minds of most high school students is someone in a lab coat conducting biomedical research. All too often, plant related careers are not at the forefront of these young minds due to a lack of exposure in high school classrooms and popular culture. Here we present an outreach program designed to demystify plant science career options in high school classrooms by bringing young scientist 'Ambassadors', postdocs and graduate students in the plant sciences, into high school classrooms to discuss their career paths and ambitions, as well as pre-made modules on DNA and its importance to plant science. The program, UF DNA Day, participates as a National DNA Day program and addresses the apparent gap in high school students' perceptions of what a scientist can do and what a scientist can be. Utilizing anonymous surveys before and after the single period presentations, we're able to assess the impact of the program and potential changes in high school students' perspectives on careers in science and scientists. This outreach program also serves to encourage and train outreach skills in our Ambassadors to further advance and communicate the diversity of plant science careers.

The role of polyacetylenic falcarins in the defense of carrot (Daucus carota) against necrotrophic and biotrophic pathogens S. LEE (1), G. Frihse (2), S. Kennedy (3), K. Malyszka (2), A. Melchor (2), D. Kosma (2), P. Santos (4), (1) University of Nevada, Reno, Reno, NV, USA; (2) Department of Biochemistry and Molecular Biology, University of Nevada, Reno, NV, USA; (3) University of Nevada, Reno, NV, USA; (4) University of Nevada Reno, Reno, NV, USA

Plant pathogens are responsible for huge losses of agriculturally important crops in the field and during post-harvest storage. It is estimated that by 2050 crop production needs to double in order to accommodate the exponential growth of the population, therefore it 
is imperative to have resilient crops to increase yields and provide a sustainable food source. "Chemical warfare" is a viable answer to improve crops against plant pathogens. Carrot (Daucus carota) and other Apiaceous species, produce polyacetylenic lipids (falcarins) in the form of falcarinol and falcarindiol, $\mathrm{C}_{17}$ aliphatic compounds derived from linoleic acid $\left(\mathrm{C}_{18: 2}\right)$. Falcarins have been described as playing a role in plant defense against pathogens, but their mode of action is still poorly understood. Since carrot produces and accumulates high levels of falcarins and is an agronomically important vegetable crop, it was selected to be the model system for this work. Different plant pathogens that employ different mechanisms of pathogenicity (i.e., effector proteins, toxins, quorum sensing, cell wall degrading enzymes, oxalic acid, ROS, etc.) are being used to study the defense responses of the host concerning the involvement of polyacetylenic falcarins. Bioassays, transcriptomic and metabolomic analyses are currently in progress. The knowledge generated from this project will serve as a platform for breeding programs and biotechnology strategies that will help mitigate crop losses.

Elucidating the role of MORC1 during plant immunity against Phytophthora infestans N. JACKSON (1), A. Buehlman (1), J. C. Nam (2), H. G. Kang (3), P. M. Manosalva PhD (1), (1) University of California, Riverside, CA, USA; (2) Texas State University, San Marcus, TX, USA; (3) Texas State University, San Marcos, TX, USA

Phytophthora infestans (Pi), the causal agent of potato and tomato late blight, is a global threat to agriculture and food security, causing multibillion-dollar losses due to reduction in crop production and expenditures in disease control. Microrchidia (MORC) proteins belong to the GHKL (Gyrase, Hsp90, Histidine Kinase, MutL) ATPases superfamily and have been shown to play critical roles during plant immunity against virus, bacterial, fungal, and oomycete pathogens. This study focuses on understanding how MORC1 regulates plant immunity to $P i$ by the identification of key MORC1-interactors (MIPs) using biochemical, genomics, and proteomics approaches. Through a yeast-two hybrid screen, a basic leucine zipper (bZIP) transcription factor (MIP10) was found to physically interact with MORC1. Moreover, the interaction between these two proteins occurs at their C-terminal regions. To determine whether MIP10 contributes to the plant's defense response against $P i$, MIP10 was transiently silenced and overexpressed in Nicotiana benthamiana (Nb). Silencing MIP10 enhanced $P i$ susceptibility and compromised cell death triggered by Infestin 1 (INF1), a $P i$ elicitor while its overexpression enhanced $P i$ resistance relative to the control plants. Together, these results suggest that MIP10 is a positive regulator of plant immunity against $P i$. Future studies will utilize the MORC1-MIP10 interaction to effectively develop durable $P i$ resistant traits in crop plants.

Uncovering known unknowns: Localization and quantification of viroids in hops S. J. Harper, N. KHUU, A. Wright, Washington State University, Prosser, WA, USA

Symptoms of viroid infection of hops can manifest as dwarfing, reduction of branching, decreased cone yields, and lower bittering acids compared to non-infected plants. The most cost-effective measure to maximize productivity throughout the lifespan of a hop yard is by planting with virus-tested propagation material. Viroids remain recalcitrant against existing therapies and may preferentially proliferate at elevated temperatures of traditional thermotherapy. The aim of this study was to understand how cold and dark therapy affects the accumulation and localization of two viroids, HSVd and HLVd, in the hop cultivars 'Comet,' 'Chinook,' 'Canadian Red,' and 'Wuerttemberger' for application with meristem-tip excision. Experiments in vivo compared spatial distribution of the viroids in the apical meristem, petiole, and internode after cold treatment by in situ hybridization and RT-qPCR. In vitro experiments investigated the titer changes of the viroids after cold or dark treatment by RT-qPCR. Cold treatment reduced viroid accumulation in vivo and in vitro while dark treatment increased viroid titer. HLVd titer dropped immediately after cold therapy while HSVd titer took longer to show statistically significant reductions. In situ hybridization indicated that the viroids were diffentially localized, but neither were present in the tunica cells. Hops free of HSVd and HLVd infection were produced by excision of $0.1 \mathrm{~mm}$ apices containing the tunica layer without additional therapies. This study helps to bring clarity to currently opaque virus elimination therapy.

\section{Investigating the role of quorum sensing in Xanthomonas translucens pv. undulosa infection in barley and wheat: A class research experience}

M. M. LEWANDOWSKI (1), J. Long (1), J. Butchacas (1), T. L. Klass (1), N. Heiden (1), C. Olmos (2), G. E. Valero-David (1), C. V. Garcia (3), S. P. Cohen (1), V. Roman-Reyna (1), J. M. Jacobs (1,4), (1) Department of Plant Pathology, The Ohio State University, Columbus, OH, USA; (2) The Ohio State University, Columbus, OH, USA; (3) Department of Horticulture and Crop Science, The Ohio State University, Columbus, OH, USA; (4) Infectious Diseases Institute, The Ohio State University, Columbus, OH, USA

We developed a laboratory class to teach the scientific method around the study of plant-microbe interactions. Novel aspects include involving students in the development of a hypothesis and research plan, and executing the research. In this course, students chose to investigate cell-to-cell signaling, also known as quorum sensing, in Xanthomonas translucens pv. undulosa (Xtu), the causal agent of bacterial leaf streak and black chaff in wheat and barley. Xanthomonas spp. use small molecules called diffusible signal factors to regulate quorum sensing. However, the role of quorum sensing in Xtu-host infection is unknown. Here we aimed as a class to understand the basics of bacterial genetics and host-microbe interactions by defining the role of quorum sensing in Xtu. Our team created insertional and clean deletion mutants in canonical quorum sensing regulatory genes (e.g. the rpf signaling system) or target pathways (e.g. motility, biofilm). We determined that quorum sensing and regulated behaviors are critical for Xtu foliar pathogenesis. In addition, we are developing methods to assess student understanding of scientific method, and mastery of laboratory techniques through planning, repetition and problem solving. This will allow us to deploy a curriculum plan that instructors can use to develop their own hypothesis-driven research courses, based on their own expertise and available resources. 
Inoculum sources of Phytophthora spp. and thermotherapy for management of Phytophthora crown rot in strawberry M. MARIN, J. Baggio, N. Peres, University of Florida - Gulf Coast Research and Education Center, Wimauma, FL, USA

Phytophthora crown rot (PhCR) is an important disease of strawberry worldwide. In Florida, it is caused mainly by Phytophthora cactorum, but $P$. nicotianae has recently been reported. Mefenoxam is the most effective fungicide for PhCR management. However, the recent report of resistant isolates puts chemical management at risk. Surveys conducted in commercial fields with history of PhCR in the previous season found that Phytophthora spp. were rarely recovered from the soil prior to planting. In trials performed in a research farm where soil was sampled every month during two strawberry seasons, neither species was recovered one month after the crop had been destroyed. Since Phytophthora spp. were not able to survive in the soil over summer in Florida, strawberry transplants acquired form different nursery sources were examined for the presence of the pathogens. $P$. cactorum was recovered from plants of 77 and $100 \%$ of the nurseries surveyed during two seasons, respectively. In vitro, thermotherapy at $40^{\circ} \mathrm{C}$ for 60 min inhibited germination and colony formation of $P$. cactorum zoospores but failed to inhibit oospore germination. However, in field trials, the thermotherapy treatment at $44^{\circ} \mathrm{C}$ for 4 hours reduced mortality on plants inoculated with either reproduction structure. Therefore, strawberry transplants are the main source of Phytophthora spp. inoculum in Florida production fields and the use of thermotherapy could be an effective non-chemical approach for managing PhCR in production and nursery fields, while limiting building up of mefenoxamresistant populations.

\section{Host and strain factors contribute to variation in colonization potential of enteric bacteria during Pseudomonas syringae plant disease} A. LOVELACE, S. Lee, S. Ayoub, D. Downs, B. H. Kvitko, University of Georgia, Athens, GA, USA

With an increase in food-borne illness outbreaks associated with fresh produce consumption, understanding the risk factors that contribute to produce contamination is important for mitigating future outbreaks. Although plant innate immunity can restrict the proliferation of food-borne illness causing enteric pathogens, compatible plant pathogens can suppress this immune response, thus providing a favorable environment for enteric colonization. We demonstrate that enteric strains can benefit from co-inoculation with the plant pathogen, Pseudomonas syringae, in some but not all combinations of enteric strains and plant hosts. Opportunistic growth of enteric pathogens is therefore dependent on both host and strain factors. Differential biphasic growth in Nicotiana benthamiana apoplastic fluid was observed between two Salmonella enterica strains, DM10000 (DM10K) and 14028S. This growth phenotype was suppressed in the presence of exogenous glucose which is indicative of catabolite repression. We hypothesize that differential metabolism of sugar sources in the apoplast by DM10K and $14028 \mathrm{~S}$ contribute to differential colonization in the apoplast during plant disease. Mutations in the sigma factor, RpoS, in DM10K could explain observed differences in sugar metabolism and ultimately plant colonization. We demonstrate that $P$. syringae is a weaker competitor during co-inoculation with $S$. enterica strains in apoplastic fluid and therefore inter-bacterial competition for shared nutrients likely does not explain the observed lack of opportunistic growth of DM10K in N. benthamiana.

Pathogenicity testing of fungal isolates associated with olive trunk diseases in South Africa M. Van Dyk (1), C. Spies (1,2), L. MOSTERT (1), M. van der Rijst (2), I. Du Plessis (1), P. Moyo (1), W. Van Jaarsveld (1), F. Halleen $(1,2),(1)$ Stellenbosch University, Matieland, SOUTH AFRICA; (2) ARC Infruitec-Nietvoorbij, Stellenbosch, SOUTH AFRICA

A diverse fungal complex was associated with olive trunk diseases in the Western Cape Province, South Africa (SA). Pseudophaeomoniella globosa was the most prevalent fungus, while other fungal species of the Phaeomoniellaceae, Botryosphaeriaceae, Phaeosphaeriaceae, Diaporthaceae, Togniniaceae, Valsaceae, Coniochaetaceae, Calosphaeriaceae, Diatrypaceae and Basidiomycota occurred at much lower frequencies. Many of the species recovered had not been reported from olive trees and their pathogenicity needed to be determined. Pathogenicity tests were conducted on detached shoots and in field trials. From the detached trial, 58 isolates comprising 38 species were selected for field trials. Two-year-old olive branches of 15-year-old trees were inoculated by inserting colonised agar plugs into artificially wounded tissue. Measurements were made of the internal lesions after 8 months. Species that formed lesions significantly longer than the control were considered pathogens. These included known olive trunk pathogens as well as Biscogniauxia rosacearum, Celerioriella umnqumae, Coniochaeta velutina, Coniothyrium ferrarisianum, isolates of the Cytospora pruinosa complex, Didymocyrtis banksiae, Neofusicoccum stellenboschiana, Neophaeomoniella niveniae, Phaeoacremonium spp. (P. africanum, P. oleae, P. prunicola and P. spadicum), P. globose, Punctularia atropurpurascens, Vredendaliella oleae and nine undescribed species. Pseudophaeomoniella globosa can be regarded as an important olive trunk pathogen in SA, due to its high incidence from olive trunk disease symptoms and high virulence.

\section{Different groups of Pseudomonas syringae are responsible for vein clearing disease of zucchini}

C. LACAULT (1), M. Briand (1), M. A. Jacques (2), A. Darrasse (1), (1) IRHS, Agrocampus-Ouest, INRAE, Université d'Angers, SFR 4207 QuaSaV, Beaucouzé, FRANCE; (2) IRHS, Agrocampus-Ouest, INRAE, Université d'Angers, Beaucouzé, FRANCE

Pseudomonas syringae strains are affecting zucchini (Cucurbita pepo subsp. pepo) during plantlet development by inducing vein clearing, stunting and necroses. The aim of this study was to characterize phylogenetically and phenotypically the strains responsible for vein clearing of zucchini (VCZ strains). A multilocus sequence analysis (MLSA) based on four housekeeping genes was conducted on a worldwide collection of $58 \mathrm{VCZ}$ strains and whole genomes were sequenced for a subset of 23 strains. The pathogenicity of this strain subset was evaluated on zucchini and some representative strains were assessed on 11 cucurbit species. Most VCZ strains were included into clades $2 \mathrm{a}$ and $2 \mathrm{~b}-\mathrm{a}$ within phylogroup 2 of $P$. syringae species complex together with diverse strains previously isolated from cucurbits. Whole genome analysis allowed to reveal three clusters in each clade comprising only cucurbit strains; the VCZ strains belonged to four of them. All VCZ strains are responsible for a common disease on zucchini but present variations in their host ranges 
on other cucurbits. Indeed, one cluster of VCZ strains presented a narrow host range including only squash while other strains have a wide host range including squashes, cucumbers, melons and sometimes watermelons. Specific rerpertoires of type III effectors were associated to the pathogenic features of the strains. Genome sequences were mined to identify three ubiquitous genes that enlarge MLSA scheme in order to improve strain identification. This MLSA scheme will favor the $P$. syringae epidemics monitoring in cucurbit producing areas.

\author{
Diversity of grapevine endophytic bacteria and their potential as biocontrol agents against fungal pathogens causing cankers \\ and dieback in grapevine \\ M. BUSTAMANTE, S. Wells, K. Elfar, A. Eskalen, Department of Plant Pathology, University of California, Davis, Davis, CA, USA
}

Grapevine trunk diseases (GTD) are one of the major challenges in viticulture worldwide. Currently there is a lack of effective control strategies against GTDs. Endophytic bacteria have been extensively studied due to multiple beneficial effects to their plant hosts, including antifungal activity. The aim of this study was to isolate and analyze endophytic bacteria within grapevines affected by GTD that could be beneficial to control trunk diseases. In 2019, twenty vineyards in common grape growing regions of California were sampled for their microbial communities. Over 1,200 culturable bacteria were recovered from trunk and rhizospheric soil from GTDsymptomatic and healthy-looking vines and further tested against GTD-causing pathogens including Neofusicoccum parvum, Diplodia seriata, Eutypa lata and Diaporthe ampelina byantagonism assays in vitro. Preliminary results indicate that 72 bacterial isolates from different geographical areas exert strong mycelial inhibition (over 40\%) against $N$. parvum, D. seriata, E. lata and D. ampelina. Partial 16S rRNA gene sequences revealed that the majority (95.8\%) of isolates belong to the Bacillus subtilis species complex whereas three isolates were identified as Pseudomonas chlororaphis and Stenotrophomonas maltophilia. These grapevine endophytic bacteria, which may serve as potential biocontrol agents, are currently being tested on dormant propagation material to determine their protection ability against the causal agents of cankers and dieback of grapevine.

The outer membrane proteins of Candidatus Liberibacter asiaticus and their roles in interactions with citrus plants Y. HUANG, N. Wang, Citrus Research and Education Center, University of Florida, Lake Alfred, FL, USA

Huanglongbing (HLB, also called greening) is one of the most devastating diseases of citrus in the world. It is caused by Candidatus Liberibacter asiaticus (CLas) and transmitted by Asian citrus psyllid (ACP) Diaphorina citri in the United States. Typical HLB symptoms include blotchy mottle leaves, branch dieback, and lopsided fruits. CLas has not been cultured in artificial media that hinders our understanding of its interaction with both plant and insect hosts. Interestingly, CLas lives inside the sieve element cells, a specialized living cell. We hypothesize that the outer membrane proteins (OMP) of CLas might play important roles in its interactions with both the plant and psyllid hosts. In this study, we predicted all the OMPs based on the genome sequences of CLas and conducted yeast two hybrid (Y2H) assays to identify their targets in citrus. How those OMPs are involved in CLas interactions with citrus plants will be discussed.

Foliar fungicide efficacy for tar spot management in northern Indiana, 2019

C. R. DA SILVA (1), T. J. Ross (1), J. Ravellette (1), D. E. P. Telenko (2), (1) Purdue University, West Lafayette, IN, USA; (2) Department of Botany and Plant Pathology, Purdue University, West Lafayette, IN, USA

Tar spot caused by Phyllachora maydis is an emerging foliar disease of corn in the U.S. Signs of P. maydis infection include small (0.21 in.), raised, brownish-black, stromata that resembles 'spots of tar' embedded in the surfaces of leaves. As the number of stroma increases in number on a leaf or infected tissue, the tissue will turn chlorotic, then necrotic, and if severe enough leads to a leaf blight. Management information for tar spot includes the use of foliar fungicides. However, information on fungicide efficacy is limited in U.S. In this study, we assessed the efficacy of nine foliar fungicides against a non-treated control in Indiana. All fungicides were applied at VT/R1 (tassel/silk) growth stage and disease ratings were assessed during the season and a final rating was taken at the R6 (maturity) growth stage. Tar spot was rated by visually assessing leaves of five plants for percentage of stroma, and the percentage of symptomatic tissue (chlorosis and necrosis). Percent stay green, lodging, and yield were also calculated. In 2019, weather conditions were favorable for disease. Tar spot reached moderate to high severity and all fungicide treatments significantly reduced the percentage of tar spot stroma and chlorotic and necrotic symptoms on the ear leaf as compared to non-treated control. All fungicides treatments significantly increased percent stay green, and reduced percent lodging over non-treated control. Seven of the nine fungicides tested significantly increased yield of corn over the non-treated control and yields were increased from 18.3 to $24.9 \mathrm{bu} / \mathrm{A}$.

\title{
Novel and significant virulences detected from a sexual population of Puccinia graminis in Spain
}

P. D. OLIVERA FIRPO (1), D. Villegas (2), C. Cantero-Martinez (3), D. G. Luster (4), Y. Jin (5), (1) University of Minnesota, St. Paul, MN, USA; (2) Sustainable Field Crops, IRTA, SPAIN; (3) Universitat de Lleida, SPAIN; (4) USDA ARS Foreign Disease Weed Science Research Unit, Fort Detrick, MD, USA; (5) USDA-ARS Cereal Disease Laboratory, Saint Paul, MN, USA

Wheat stem rust is a re-emerging disease, posing a threat to wheat production worldwide. In Spain, stem rust has not been common since 1970s, but infection was observed in wheat fields in 2018. A sample collected from a field in close proximity to barberry -the alternate host of the stem rust pathogen - yielded a race with a unique and significant virulence combination. This observation motivated a more extensive survey for rust infection on barberry and on cereal crops in the provinces of Huesca and Teruel, where indigenous barberries, Berberis vulgaris var. seroi, and intermediate forms of var. seroi and var. vulgaris are distributed. In 2019, multiple aecial samples from barberry, and uredinial samples from infected durum, bread wheat, barley, and triticale were collected at 13 sites. Over 260 single pustule isolates derived from both aecial and uredinial samples were analyzed for reactions on a number of stem rust 
resistance genes. The isolates were highly diverse in virulence, including novel virulence combinations that have not been detected anywhere else. The virulence diversity was not a result from selection pressure as most of these resistance genes have not been deployed in Spanish wheat cultivars. These results indicated that the indigenous barberries are functional as the alternate host of $P$. graminis, and sexual cycles on the alternate host play an important role in generating novel virulence.

\section{Development of a SCAR marker and real-time PCR assay for detection and quantification of Fusarium oxysporum f. sp. carthami in host tissue and soil}

N. SINGH, R. Kapoor, University of Delhi, Delhi, INDIA

Safflower wilt, caused by Fusarium oxysporum f. sp. carthami $(F o c)$ is a devastating disease of safflower (Carthamus tinctorius) worldwide. In India, the disease has been recorded from all safflower growing areas with up to $80 \%$ incidence. Early and specific detection and quantification of Foc can be of high importance for addressing disease epidemics and management. Seventy-five isolates of Foc, 25 isolates of non-carthami formae speciales of $F$. oxysporum, 17 isolates of Fusarium spp., and seven isolates of other pathogens of safflower were used for identification of band specific to Foc using inter-simple sequence repeat (ISSR) markers. Post screening with 70 ISSR primers, the one that specifically produced a $490 \mathrm{bp}$ amplicon from all the Foc isolates was chosen. Based on the sequence of the $490 \mathrm{bp}$ band, sequence characterized amplified region (SCAR) primers, FocScF/FocScR were designed. The primer set unambiguously amplified a DNA band of $c .213 \mathrm{bp}$ in all the Foc isolates, but not from any other non-Foc tested isolates. The detection limit of the assay was $10 \mathrm{pg} F o c$ genomic DNA using conventional PCR, while the SCAR primers when combined with realtime PCR were able to detect as low as $1 \mathrm{pg}$ of genomic DNA and 1000 conidia/g of soil. The assay expedited Foc detection at $72 \mathrm{~h}$ post inoculation in latent infected seedlings, and enabled diagnosis of Foc in soil samples collected from infested fields. This approach can be used for fast and reliable detection of $F o c$ in soil and plant samples, which will facilitate early forecast of outbreaks and timely control of the disease.

\section{An optimized PCR-based protocol to detect the Laurel Wilt pathogen from environmental samples}

P. P. PARRA GIRALDO (1), W. Dantes (2), A. Sandford (1), C. De la Torre (1), J. Pérez-Martínez (1), D. Hadziabdic (3), B. Schaffer (4), R. Gazis (5), (1) Plant Pathology, University of Florida, Homestead, FL, USA; (2) Plant Pathology Department, University of Florida, Gainesville, FL, USA; (3) Entomology \& Plant Pathology, University of Tennessee, Knoxville, TN, USA; (4) Horticultural Sciences, University of Florida, Homestead, FL, USA; (5) University of Florida, FL, USA

Laurel Wilt (LW), caused by Raffaelea lauricola (RL), is a deadly vascular fungal disease affecting species in the Lauraceae. This disease has spread rapidly across the U.S. and is now present in 11 states. Since its introduction, the disease has caused significant tree loses in natural forests and Florida's commercial avocado orchards. RL spreads across from tree to tree through ambrosia beetle vectors and root-grafts. Early detection and eradication are recommended to contain outbreaks. LW diagnosis can take up to 10 days and involves pathogen isolation and the amplification of two microsatellite regions. We optimized this PCR-based detection technique to reduce the diagnosis time. We further screened the primers IFW and CHK against RL strains of different host and geographic origin, 12 lineages of Raffaelea spp., a strain collection of avocado-associated fungi, and multiple host genotypes. Sensitivity was tested using pure cultures and RL-infected avocado sapwood. There was no cross-amplification in non-RL samples, confirming the specificity of the primers. The amplification rate for IFW and CHK, using pure cultures, was $100 \%$ and $99 \%$, respectively. IFW primers were more suitable than CHK primers for RL detection with a threshold of $0.01 \mathrm{ng} / \mathrm{ul}$. Using IFW primers, we were able to detect RL in $89 \%$ of RL-infected sapwood samples (76/85). Our optimized PCR-based technique allowed RL detection directly from infected tissue and reduced the diagnosis time to 24 hours. This protocol represents a powerful tool for sentinel networks and high throughput pathogen screening.

Effect of four initial densities of the potato cyst nematode, on the physiology and yield of the diploid yellow tuber flesh potato genotype Colombia

L. T. Mayorga (1), C. GARCÍA (2), (1) Universidad Nacional, Bogota, BOGOTA, COLOMBIA; (2) Universidad Nacional de Colombia, Bogotá, COLOMBIA

Colombia is the main producer and exporter of the diploid Andean potato Solanum tuberosum group phureja landrace "criolla". The presence of the cyst nematode, Globodera pallida, is likely to be a major phytosanitary problem, but there are no quantitative data on the role of this nematode on the crop. This study aimed at evaluating the effect of four initial densities of G. pallida on the physiology and yield of genotype Colombia. Plants were inoculated with 0, 1, 2 and $4 \mathrm{eggs} / \mathrm{cm}^{3}$. Plant height and relative chlorophyll content were assessed weekly. At the end of the experiment, fresh and dry shoot weight and fresh tuber weight were measured, and eggs counted. The experiment was conducted twice, with five replicates per treatment. Final results were different between the two experiments: reduction in fresh tuber weight ranged from 55.9 to $19.5 \%$ with $1 \mathrm{egg} / \mathrm{cm}^{3}, 23.4$ to $0.04 \%$ with $2 \mathrm{eggs} / \mathrm{cm}^{3}$ and 41.4 to $30.7 \%$ with 4 eggs $/ \mathrm{cm}^{3}$ for each experiment. The final egg counts expressed as number of egg $/ \mathrm{cm}^{3}$ were 409.4 and 201.4 with $1 \mathrm{egg} / \mathrm{cm}^{3}, 358.5$ and $343.2 \mathrm{with} 2$ eggs $/ \mathrm{cm}^{3}$ and 657.4 and 284 with 3 eggs $/ \mathrm{cm}^{3}$. Data indicate a relationship between egg count and fresh tuber weight. There was no effect on plant height, fresh and dry shoot weight and relative chlorophyll content. Greater attention should be paid to this nematode, because evidence shows that even at low densities, it could affect yield without aerial symptoms. This information is critical for farmers because the presence of $G$. pallida could pass unnoticed making impossible to carry out timely phytosanitary management.

Comprehensive analysis of the apple rhizobiome as influenced by different Brassica seed meals in the same soil/plant system T. SOMERA (1), S. Freilich (2), M. Mazzola (1), (1) USDA Agricultural Research Service, Wenatchee, WA, USA; (2) ARO, Rishon LeZion, ISRAEL 
Tree fruit replant disease is encountered when attempting to establish the same or related species on old orchard sites. Apple replant disease results from the concerted action of multiple soil-borne pathogens which build up in soil over time. Different Brassicaceae seed meal (SM) soil amendments provide varying levels of replant disease control depending on apple rootstock genotype used and pathogen complex present. Thus, understanding relationships between Brassica SM-based disease control, rootstock genotype, and the rootassociated microbiome, particularly in regard to long term disease control, is a difficult task. Numerous trials examined the effect of individual seed meals on microbiome composition; however, this study is the first to simultaneously examine bacterial, fungal, and oomycete communities as influenced by different Brassica seed meals $(B$. juncea $+S$. alba $(\mathrm{BjSa}), B$. juncea, and $B$. napus) in the same soil/plant system. In accordance with previous studies, amendment type was a major factor structuring the root-associated microbiome. Pythium and Phytopthora were predominant in apple rhizospheres regardless of system type; however, multiple pathogenic oomycete and fungal groups were clearly supported by apple roots in a treatment $\times$ rootstock genotype dependent manner. This study also provided further support for the hypothesis that bacteria and fungi with biocontrol and bioremediation potential are amplified in BjSa SM-structured microbiomes, a finding with important implications for improving orchard health in many ways.

The current status of plant-related outreach across the United States and what APS is doing to help J. KLEIN (1), T. Wu (2), J. A. Delgado (3), F. Peduto Hand PhD (4), F. Baysal-Gurel (5), R. L. Hirsch (6), T. L. Friesen (7), N. M. Donofrio (8), (1) University of Florida, Gainesville, FL, USA; (2) University of Wisconsin-Madison, Madison, WI, USA; (3) Dow AgroSciences, Indianapolis, IN, USA; (4) Department of Plant Pathology, The Ohio State University, Columbus, OH, USA; (5) Tennessee State University, McMinnville, TN, USA; (6) University of Kentucky, Lexington, KY, USA; (7) USDA-ARS Cereal Crops Research Unit, Northern Crop Science Laboratory, Fargo, ND, USA; (8) Plant and Soil Sciences Department, University of Delaware, Newark, DE, USA

To engage the public regarding what plant pathologists do and the importance of plant health, it is critical that scientists at all career levels engage in outreach-related activities. So, what are plant pathologists already doing, and where are the gaps for more effective engagement? The APS Office of Public Relations and Outreach (OPRO) Board set out to answer these questions. We developed a 13question survey that was sent out to 54 plant pathology-related academic departments across the United States and one representative was asked to complete the survey on behalf of their department or we compiled responses into one representative response. From 40 total responses, a minimum of 2,385 plant-related outreach events occurred in 2018 across the United States, with over 90,000 total people in attendance. We identified that the major limitations to outreach are financial support, staffing, and time. Over half of the respondents requested financial support or readily sent materials, as well as outreach ideas from APS. To address this need, the OPRO Board will now offer several annual awards, to be given out in $\$ 500$ awards, to fund outreach events organized by any APS member. The OPRO will also begin a monthly Phytopathology News column to advertise new resources, highlight resources already on the APS website, and feature outreach efforts conducted by APS members.

\section{Potential of cannabis aphid to transmit potato virus $\mathbf{Y}$}

W. PITT, E. Villa, P. Nachappa, Colorado State University, Fort Collins, CO, USA

Potato virus Y (PVY) is one of the most significant threats to the cultivation of potatoes (Solanum tuberosum) in the U.S. and worldwide. There are over 50 aphid species that transmit PVY and most have low transmission efficiencies. However, these aphids can be important contributors to PVY prevalence. With the increasing acreage of hemp in Colorado and the western United States, we were interested to know if the cannabis aphid (Phorodon cannabis Passerini) is a potential vector of PVY. There is evidence that hemp can serve as a reservoir plant for PVY. Our results show that the cannabis aphid can acquire PVY from an infected host and can transmit the virus to hemp. Experiments are underway to determine the aphid's ability to vector PVY to potato. In addition, feeding behaviors of aphids are being analyzed using the Electrical Penetration Graph (EPG) technique. This is the first report of the cannabis aphid as a vector for PVY. The results of this study will provide insights on both the efficiency of the cannabis aphid as a vector of PVY and possible virus induced effects on aphid feeding behaviors, which will be useful in the development of management strategies for aphids and PVY.

Population genomics and potential temperature adaptation in the charcoal rot fungus Macrophomina phaseolina from the US, Puerto Rico, and Colombia

V. ORTIZ LONDONO (1), H. Sang (2), H. X. Chang (3), K. A. Wise (4), M. Chilvers (1), (1) Michigan State University, East Lansing, MI, USA; (2) Chonnam National University, Gwangju, SOUTH KOREA; (3) National Taiwan University, TAIWAN; (4) University of Kentucky, Princeton, KY, USA

Macrophomina phaseolina is an important pathogen of crops worldwide. Charcoal rot caused by M. phaseolina can significantly reduce yield and seed quality in soybean and dry bean mainly in tropical and subtropical regions, but more recently in temperate zones as well. Little information is available on the population structure of M. phaseolina. Genomes of $96 \mathrm{M}$. phaseolina isolates from 13 states across the US, Puerto Rico, and Colombia, isolated from soybean and dry bean were sequenced to determine whether populations are structured by host or geographic origin. Sequencing was performed on an Illumina HiSeq 4000 with 150 bp paired-end reads to an average depth of $23 \mathrm{X}$. Linkage disequilibrium analyses indicated that $M$. phaseolina isolates are predominantly clonal which is supported by its biology since $M$. phaseolina reproduces asexually. Inference on population structure revealed clustering of Puerto Rican and Colombian isolates from dry bean, while isolates from the US regardless of dry bean or soybean origin were assigned to a separate cluster and exhibited lower diversity. Limited genome-wide differentiation among populations by host and geographic origin was observed. To investigate temperature adaptation, mycelial growth at $15^{\circ} \mathrm{C}$ and $35^{\circ} \mathrm{C}$ was measured and some isolates from the 
states of Michigan, Wisconsin and Minnesota grew faster at $15^{\circ} \mathrm{C}$ than isolates from southern states. The candidate genes associated to cold adaptation in the model fungus Neurospora crassa were assembled to investigate signatures of selection in M. phaseolina.

Ecological implications of an emerging pathogen on the invasive grass Microstegium vimineum B. R. LANE (1), A. Kendig (1), C. Wojan (2), K. Stricker (1), A. Adhikari (1), M. Ascunce (3), M. Smith (1), K. Clay (4), S. L. Flory (1), P. Harmon (1), E. M. Goss (1), (1) University of Florida, Gainesville, FL, USA; (2) Indiana University, Bloomington, IN, USA; (3) Department of Plant Pathology, University of Florida, Gainesville, FL, USA; (4) Tulane University, New Orleans, LA, USA

In initial stages of the invasion process, invasive species may escape pathogens present in their native range that are not present in the invaded region. This phenomenon, called 'enemy release,' may diminish over time as local pathogens colonize the invasive species. Recently, a foliar disease emerged on the invasive C4 annual grass, Microstegium vimineum. Initial reports characterized the causal pathogen as an undescribed Bipolaris species, but we have now identified it as Drechslera gigantea. Drechslera gigantea was first isolated in 1909 from Cynodon dactylon in Texas, prior to the introduction of $M$. vimineum, but the pathogen has also been documented throughout the eastern USA. Multi-locus phylogenetic analysis placed our isolates within the genus Bipolaris and distant from other Drechslera species. We conducted a field experiment in invaded sites to evaluate the effect of this pathogen on invasive $M$. vimineum and a co-occurring native perennial grass, Elymus virginicus. Symptoms associated with Bipolaris gigantea were observed on nearly all $M$. vimineum but only half of $E$. virginicus. Fungicide application reduced disease on both grass species. Disease also reduced the biomass of invasive $M$. vimineum. While previous experiments have found reductions in $M$. vimineum seed production in the presence of disease, we did not observe a significant effect of fungicide application on M. vimineum or E. virginicus seed production. However, our results suggest that disease caused by $D$. gigantea may alter the competitive interactions between $M$. vimineum and $E$. virginicus.

Influence of spatial planting arrangement of a winter cereal rye cover crop on corn productivity S. KURTZ, T. Kaspar, A. Lenssen, A. E. Robertson, Iowa State University, Ames, IA, USA

Farmers are hesitant to plant cover crops because of reported yield declines in corn. A potential reason for this yield decline is seedling disease. A winter cereal rye cover crop serves as a 'green bridge' for corn seedling pathogens. We hypothesized that close proximity of corn seedling roots to decaying rye cover crop roots contributes to corn seedling disease. A field experiment was done to evaluate growth, development, and disease severity of corn seedlings planted at various distances from decaying rye cover crop plants. The experimental design was a randomized complete block of four winter rye cover crop treatments and five replications. The treatments were 76-cm rye rows, $19-\mathrm{cm}$ rye rows, broadcasted, and no cover crop control. Corn was planted on 76-cm rows 8 days after terminating the rye cover crop resulting in corn being planted $38-\mathrm{cm}, 9.5-\mathrm{cm}$ away from, or within decomposing rye. Root rot severity was assessed at growth stage V3; corn height was assessed at V12; and yield was collected at harvest. Quantitative PCR was used to estimate the abundance of Pythium Clade B and Clade F members present in corn roots, and soil samples taken in the corn row and the inter-row. There was no difference in root rot severity among treatments $(P>0.05)$, however corn height and yield were greater in the control and $76-\mathrm{cm}$ rye compared to $19-\mathrm{cm}$ rye and broadcast rye $(P=0.0002)$ This study improves our understanding of the effect of a cereal rye cover crop on growth, development, seedling disease, and yield in corn, and enables us to develop improved management practices for farmers.

Use of cow manure for multiplication of Trichoderma for control of soil-borne diseases in sunflower J. RAJA, ANNAMALAI UNIVERSITY, ANNAMALAINAGAR, INDIA

Charcoal rot (Macrophomina phaseolina (Tassi) Goid.) and basal stalk and root rot (Sclerotium rolfsii Sacc.) are the major soil-borne diseases affecting sunflower (Helianthus annuus L.). This study was conducted to determine the rate of multiplication of Trichoderma on cow manure and shelf-life of the formulation; the rate and time of application of Trichoderma fortified cow manure for the control of soil-borne diseases in sunflower. Trichoderma asperellum S97 multiplied on sorghum grains was used as source of inoculum.

Trichoderma grown on sorghum grains mixed with cow manure at 1:25, 1:50, 1:75 and 1:100 proportions. Trichoderma population was increased with increased manure proportions and extended shelf-life up to 9 months. The effects of cow manure incorporated 1, 2 and 4

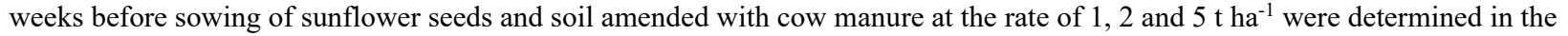
glasshouse and field studies. At 45 and 60 days after sowing, soil-borne disease incidence reduced as well as plant stand and plant growth was increased as rates of Trichoderma fortified cow manure increased. Trichoderma population fluctuated during experiments, but generally had positive linear relationships with manure proportions. A strong correlation was recorded between disease suppression and Trichoderma population activity in the sunflower rhizosphere. Now, it is possible to multiply Trichoderma on cow manure so that farmers themselves can prepare necessary quantity of inoculum required for their cultivation.

\footnotetext{
Activation of the rice disease response gene OXO4 is modulated by polymorphisms in its promoter sequence during pathogen infection

F. MARTIN (1), J. E. Leach (2), A. J. Bogdanove (3), J. Belanto (4), D. Voytas (4), (1) COLORADO STATE UNIVERSITY, Fort Collins, CO, USA; (2) Colorado State University, Fort Collins, CO, USA; (3) Cornell University, Ithaca, NY, USA; (4) University of Minnesota, St. Paul, MN, USA

Single gene resistance against pathogens in economically important crops has proven to be insufficient for long term protection because pathogens progressively adapt and overcome these defenses. Identifying and understanding QTL for disease resistance have the potential to improve plant defense responses against diverse pathogen pressure, providing a more durable alternative. While rice $($ Oryza sativa) is a highly diverse species, transfer of this diversity to agronomically relevant cultivars has proven difficult by conventional
} 
breeding techniques because of the complexity of these traits. The candidate gene (CG) approach for breeding is an accurate and rapid method to booster broad defense responses. Cultivars containing favorable alleles of key CGs, including oxalate oxidase and germinlike protein genes, show stable reductions of disease against multiple rice pathogens. These genes are found in clusters with multiple members that show differential activation upon pathogen infection. For example, out of the four copies of OXO genes, OXO4 is the only gene expressed during pathogen infection. This is the result of polymorphisms found in the promoter region, which controls transcriptional activation of the gene. Using RNAi silencing and genome editing approaches, we demonstrate OXO4s influence as a broad-spectrum defense response gene and highlight the importance of specific cis-regulatory motifs for fast and strong transcriptional activation during pathogen attacks.

Understanding genetic variability in Aphanomyces cochlioides isolates from the United States J. R. BOTKIN (1), C. Hirsch (2), F. N. Martin (3), A. K. Chanda (2), (1) University of MN, CFANS, Dept. of Plant Pathology, Corcoran, MN, USA; (2) Department of Plant Pathology, University of Minnesota, St. Paul, MN, USA; (3) USDA ARS, Salinas, CA, USA

Aphanomyces cochlioides, the causal agent of seedling damping-off and root rot of sugar beet, causes yield losses in major sugar beet growing regions. Information about genetic variation of this plant pathogen is lacking, but the current state of sequencing allows the production of massive genomic data sets at low costs. While analysis of large data sets presents challenges, bioinformatic pipelines can be used to extract biologically meaningful information. As no reference genome for $A$. cochlioides is currently available, we used the closely related $A$. euteiches as a reference genome in our study. We obtained paired-end DNA sequence data for $A$. cochlioides isolates from Minnesota (2), North Dakota (1), and Texas (2), with approximately 150x coverage of the genome. We identified variation among A. cochlioides isolates, such as single nucleotide polymorphism (SNP) counts by type, location, allele frequencies, and small in/dels. The SNP variation in this set of isolates was utilized to understand the genetic structure of $A$. cochlioides isolates in the U.S. In addition, we looked at the influence this variation could have on important genes and pathways known to be involved in pathogenicity. We are also interested in developing diagnostic molecular markers based on this variation which could be used to identify A. cochlioides isolates from a specific region. These molecular markers could be useful for breeders to select a representative and diverse set of pathogen isolates when screening germplasm for resistance to $A$. cochlioides.

Understanding the role of environmental nitrate on Xanthomonas translucens evolution and host interactions N. HEIDEN (1), V. Roman-Reyna (1,2), J. Slot (1), J. M. Jacobs (1,2), (1) Department of Plant Pathology, The Ohio State University, Columbus, OH, USA; (2) Infectious Diseases Institute, The Ohio State University, Columbus, OH, USA

Environmental nitrogen is a driver of microbial evolution and symbiosis. As nitrate is a common nitrogen source found at elevated levels in cropping systems, we hypothesize that this nitrogen source influences the evolution and biology of plant pathogens. Many bacteria in the gamma-proteobacterial genus Xanthomonas cause diseases on a broad diversity of agriculturally important crops. We investigated the evolution of three genes in a Xanthomonas nitrate assimilation cluster: nitrate reductase (nasA), nitrite reductase (nirB), and nitrate transporter $(\operatorname{nar} K)$. We constructed maximum likelihood phylogenetic trees each gene and found that the placement of Xanthomonas homologs conflicts with the species phylogeny. Our analysis provided support for Xanthomonas nitrate assimilation genes to be within a clade of beta-proteobacteria. This suggests that the cluster was horizontally transferred from the relatively phylogenetically distant beta-proteobacteria, possibly due to a shared evolutionary pressure. To search for this evolutionary pressure, we are currently using traditional mutagenesis approaches to investigate the role of nitrate assimilation for the bacterial blight and leaf streak cereal pathogen Xanthomonas translucens. We are looking at its role both in vitro and in interactions with its plant hosts. We hypothesize that the pathway enables $X$. translucens to obtain nitrogen when nitrate is prominent in the pathosystem. This research will generate deeper understanding about how the metabolism of Xanthomonas pathogens is influenced by their environment.

\section{Complete sequence of three novel Xylella fastidiosa subsp. pauca genomes reveals striking similarities to $X$. fastidiosa subsp. pauca type strain 9a5c \\ A. Sechler (1), E. E. ROGERS (2), (1) Foreign Disease-Weed Science Research, USDA-ARS, Ft Detrick, MD, USA; (2) USDA, Agricultural Research Service, Foreign Disease-Weed Science Research Unit, Frederick, MD, USA}

Xylella fastidiosa is a gram negative plant pathogen capable of infecting over 100 species of plants around the world. Transmitted by plant-sucking insects like sharpshooters and spittlebugs, this xylem-limited bacterium kills the host plant by restricting movement of water and nutrients though the xylem. X. fastidiosa has five recognized subspecies: fastidiosa, multiplex, pauca, morus, and sandyii. X. fastidiosa subsp. pauca strains cause citrus variegated chlorosis (CVC), coffee leaf scorch (CLS), and olive quick decline syndrome (OQDS). We have sequenced the complete genomes of three archival strains of $X$. fastidiosa subsp. pauca: FK 67, FK 81 (both isolated from Citrus sinensis), and FK 89 (isolated from Coffea arabica). Genomes of all three strains are $2.7 \mathrm{MB}$ in length, with a GC content of 52.6-52.7\%. De novo assemblies of all three strains show high levels of similarity and synteny with $X$. fastidiosa subsp. pauca $9 \mathrm{a} 5 \mathrm{c}$, including a plasmid similar to pXF51 from 9a5c. Additional details and comparisons among the newly sequenced strains, 9a5c and the OQDS CoDiRO strain will be presented.

Evaluation of the virulence of Phytophthora betacei sporangia produced on artificial media and Solanum betaceum leaf lesions D. ORTIZ (1), J. Gonzalez-Tobon (2), G. Danies (2), W. E. Fry (3), S. Restrepo (2), (1) Universidad Nacional de Colombia, Bogotá, COLOMBIA; (2) Universidad de Los Andes, Bogotá, COLOMBIA; (3) Cornell University, Ithaca, NY, USA 
The genus Phytophthora is responsible of causing serious plant diseases. A new species was recently described, Phytophthora betacei infecting tree tomato crops (Solanum betaceum) in Colombia. In recent years, it has been a controversy on whether P. infestans' sporangia behavior differs according to their production source, either produced in vivo or in vitro. The aim of this study was to determine if there are differences between $P$. betacei sporangia coming from culture media with those produced on its natural host, $S$. betaceum. To this end, we evaluated the morphology of sporangia, the number of hours until the first symptoms appeared in the host, the lesion area and sporulation rate. This was done by exposing $P$. betacei to two temperature conditions, favoring the sporangia's direct $\left(21^{\circ} \mathrm{C}\right)$ or indirect $\left(4^{\circ} \mathrm{C}\right)$ germination. We observed that sporangia obtained either from media or plant lesions were not able to release zoospores. In addition, there were no differences in sporulation rates or lesion area. On the other hand, the time until the appearance of symptoms was shorter for sporangia coming from leaflets. Furthermore, the sporangia that had been previously incubated at $4^{\circ} \mathrm{C}$ generated symptoms faster for the appearance of symptoms. We also observed morphological differences in the sporangia, being wider those produced on leaflets than those obtained in culture media. Such results are in agreement with what had been recently reported for $P$. infestans suggesting that $P$. betacei sporangia from plant and culture media might be functionally similar.

\section{Into the new era of decision support in crop protection: Multifaceted disease management advisors based on machine learning} and open science

M. CUCAK (1,2), F. Dalla Lana (3), P. S. Ojiambo (4), E. D. De Wolf (5), D. A. Shah (5), P. A. Paul (6), P. Esker (7), (1) Teagasc, Carlow, IRELAND; (2) Geography Department, Maynooth University, Maynooth, IRELAND; (3) The Ohio State University, Wooster, OH, USA; (4) Department of Entomology and Plant Pathology, North Carolina State University, Raleigh, NC, USA; (5) Kansas State University, Manhattan, KS, USA; (6) Department of Plant Pathology, The Ohio State University, Wooster, OH, USA; (7) The Pennsylvania State University, University Park, PA, USA

Growers and other stakeholders continue to have questions about the best management approaches for crop diseases to improve both inseason management, as well as between-season decision-making. Multiple sources of knowledge and information would help answer these questions exist, but are often scattered, not easily accessible to growers, and hard to interpret. Hence, the goal of this project is to integrate these sources into a new-generation-disease management advisory platform (= decision support tool, DST), based on big data and advanced statistical learning techniques. This advisory tool will provide answers to disease management-related questions to growers while leaving the decision-making in their hands. The model crop disease management systems will be for wheat and corn. The DST will integrate advice on best-management practices while considering economic factors, where farmers will be able to ask specific questions based on their local observations and concerns for disease management. A feedback mechanism will be incorporated. This will help growers learn what are their best strategies for long-term management of the major diseases that impact their production situation, improving production and profitability. Furthermore, tools developed will integrate open-source software and data collection to maximize and extend the type of research questions that can be asked as more data are inputted into the system. Such an approach will enable training of the next generation of both crop protection experts as well as growers to "think epidemiologically".

Brome mosaic virus causes yield reduction through growth-stage dependent mechanisms B. A. HODGE (1), P. A. Paul (2), L. R. Stewart (3), (1) The Ohio State University, Wooster, OH, USA; (2) Department of Plant Pathology, The Ohio State University, Wooster, OH, USA; (3) USDA-ARS Corn, Soybean and Wheat Quality Research Unit, Wooster, $\mathrm{OH}, \mathrm{USA}$

Brome mosaic virus (BMV) causes yield loss in soft red winter wheat (SRWW) from either fall or spring infection. Fall infections of BMV induce mosaic symptoms, stunting, and reduced tiller count leading to yield loss. When infection occurs in early spring (at the Feekes 5 growth stage), BMV instead induces chlorosis symptoms on the flag leaf, a primary source of photosynthates for grain fill. To assess the impact of flag leaf chlorosis on grain yield, field trials were conducted during the 2018 and 2019 growing seasons in which SRWW cultivars Hopewell and Truman were inoculated at Feekes 5 with a dilution series of BMV concentrations. BMV infection induced up to $25 \%$ incidence of chlorotic flag leaves, $20.5 \%$ reduction in mean chlorophyll content, and $22.5 \%$ yield reduction relative to mock-inoculated checks. The main effects of cultivar, BMV concentration, and their interaction were statistically significant ( $<<$ 0.05 ) for incidence of chlorotic leaves and chlorophyll content, but only BMV concentration was significant for yield in both years. Results from linear mixed model regression analyses indicated that the incidence of chlorosis and mean chlorophyll content were significantly associated with the variation in observed grain yield. These findings indicate that BMV infection of wheat at Feekes 5 reduces the photosynthetic green area of the flag leaf, thus causing yield loss through a different mechanism than results from fall infection. More research is needed to develop management strategies to minimize BMV-induced yield reduction from infection at all growth stages.

\section{Revision of Potato late blight forecasting in the Republic of Ireland}

M. CUCAK (1,2), S. Kildea (1), R. de Andrade Moral (3), P. Esker (4), R. Fealy (2), (1) Teagasc, Carlow, IRELAND; (2) Geography Department, Maynooth University, Maynooth, IRELAND; (3) Department of Mathematics and Statistics, Maynooth University, Maynooth, IRELAND; (4) The Pennsylvania State University, University Park, PA, USA

Potato late blight (PLB), caused by oomycete Phytophthora infestans (Mont) De Bary, is arguably the most important disease of potato in terms of economic losses and environmental and economic impact of the disease management. The Irish Rules model was proposed in the 1950s and is operationally used by the national meteorological agency, Met Éireann, to aid identifying periods of blight conducive weather. Changes in the pathogen population and potato production systems prompted the need to evaluate the model criteria and decision thresholds. The Modified Irish Rules (MIR) in combination with varietal resistance was further evaluated under field conditions to showcase and empower the use of integrated pest management tools to reliably inform the standard PLB management practice under growing conditions and contemporary $P$. infestans population in the Republic of Ireland. An extensive evaluation has 
shown that the MIR model represents an optimum modelling compromise due to the uncertainty of the model input variables, which currently demand simpler modelling approaches, such as the MIR. However, constant technological advancements will lead to improvements such as, in numerical weather prediction, and will provide possibilities for more complex risk prediction modelling. Hence, a new model which provides a more realistic interpretation of the $P$. infestans lifecycle is proposed. To facilitate a shorter timegap between PLB model evaluations, the entire analysis is implemented in a single open source programming environment, reproducible and hosted on an open access server.

\section{Pathogenicity of five Phytophthora species causing rhododendron root rot in Oregon}

G. SACHER (1), C. Scagel (2), A. Davis (2), B. Beck (2), J. E. Weiland (3), (1) Oregon State University, Botany \& Plant Pathology, Corvallis, OR, USA; (2) USDA ARS, Corvallis, OR, USA; (3) USDA-ARS, Horticultural Crops Research Laboratory, Corvallis, OR, USA

Phytophthora root rot is a destructive disease of rhododendron, causing substantial losses in infested fields and container production areas of this important ornamental nursery crop. Historically, Phytophthora cinnamomi was considered the main causal agent of the disease. However, a recent survey of soilborne Phytophthora species from symptomatic rhododendrons in Oregon revealed that $P$. plurivora is now more abundant than $P$. cinnamomi and that several other less common Phytophthora species are also involved. We investigated the ability of the four most abundant species identified from the survey to cause root rot: $P$. plurivora, $P$. cinnamomi, $P$. cryptogea, and $P$. pini. We infested the soil of containerized Rhododendron catawbiense 'Boursault' in a greenhouse experiment using three to four isolates of each species obtained from six Oregon nurseries. Each species caused root rot, with P. plurivora, $P$. cinnamomi, and $P$. pini causing frequent, severe symptoms ( $\geq 90 \%$ incidence of severe root rot) and $P$. cryptogea causing fewer, less severe symptoms ( $46 \%$ incidence of severe root rot). Little difference was observed between isolates of the same species. Results show that these four Phytophthora species, which were representative of $86 \%$ of the isolates from the survey, are capable of causing significant damage but that not all species are equally pathogenic. Further research on Phytophthora root rot of rhododendron should consider whether the efficacy of disease control strategies, such as host resistance and chemical control, differ among the causal agents of this disease.

Evaluation of damage by high densities of four common plant-parasitic nematodes on the growth of hop, Humulus lupulus (cv. Centennial)

E. DARLING (1), E. Cole (2), M. Quintanilla (2), (1) Michigan State Univ, East Lansing, MI, USA; (2) Michigan State University, East Lansing, MI, USA

Yield loss on hop from plant-parasitic nematodes within the United States remains widely unstudied and undetermined. The lack of literature on plant-parasitic nematodes of hop emphasizes the importance for studies involving this specialty crop. Behind the Pacific Northwest (Washington, Oregon, Idaho), Michigan ranks $4^{\text {th }}$ nationally in hop production, with an estimated 900 commercial acres. Michigan hop growers already face a myriad of known pathogen and pest challenges. Large gaps in knowledge exist on the impact by plant-parasitic nematodes on hop and how to control these pests. In Michigan soil samples, we have determined that the dagger nematode (Xiphinema americanum), root lesion nematode (Pratylenchusspp.), northern root knot nematode (Meloidogyne hapla) and hop cyst nematode (Heterodera humuli) have the potential to impact American hop production. Plant-parasitic nematode infestation can inhibit nutrient uptake and cause serious stress on the plant. In a greenhouse experiment, we measured the impacts of high densities of these four plant-parasitic nematodes on young hop plants (cv. Centennial). Hop bine measurements, root weight, and hop production were recorded to measure how nematodes impacted plant growth.

\section{Epidemiological characterization of a new phase of lettuce drop (Sclerotinia spp.) identifies soft stem as a host susceptibility factor in lettuce}

B. E. MAMO (1), R. Eriksen (2,3), R. J. Hayes (2,3), I. Simko (2), K. V. Subbarao (1), (1) University of California At Davis, Salinas, CA, USA; (2) USDA-ARS, Crop Improvement and Protection Research Unit, Salinas, CA, USA; (3) USDA-ARS, Forage Seed and Cereal Research Unit, Corvallis, OR, USA

Two species of Sclerotinia cause one of the most damaging diseases of lettuce (Lactuca sativa L.) worldwide. Infections caused by $S$. sclerotiorum are initiated through airborne ascospores. In contrast, S. minor, the predominant species in coastal California, Canada, and New Zealand, causes infections through mycelial germination of soilborne sclerotia. S. minor was not known to form sclerotia in the aboveground parts of infected Lactuca spp. In March 2017, production of sclerotia in the stems of cultivated lettuce genotypes infected with S. minor was observed in a greenhouse in the U.S. We investigated lettuce-environment-Sclerotinia interactions to identify possible risk factors for lettuce drop and targets for disease control. Significant variations were observed among lettuce genotypes in height to which sclerotia formed in stems, days to various visible signs/symptoms on stems, leaves, and shoots and other variables measured. We also detected host plant architecture characteristics significantly correlated with height of sclerotia formation and associated symptoms. Infections also had significant effect on carbon assimilation and stomatal conductance. Monitoring of microenvironment under host plant canopy did not provide conclusive insight into the influence of relative humidity and temperature on lettuce drop. The results elucidate interactions of $S$. minor with lettuce and broaden our understanding of disease processes and resistance in lettuce. Soft stem is a host susceptibility factor for lettuce drop and could be targeted in resistance breeding.

Development of a triplex AmplifyRP molecular assay for a reliable detection of Tomato brown rugose fruit virus R. LI (1), B. Davenport (1), S. Zhang (2), K. Schuetz (1), K. S. Ling (3), (1) Agdia, Inc., Elkhart, IN, USA; (2) Agdia Inc., Elkhart, IN, USA; (3) USDA-ARS, Charleston, SC, USA 
Tomato brown rugose fruit virus (ToBRFV) is an emerging Tobamovirus initially identified infecting greenhouse tomatoes in Israel and Jordan in 2014-2015. ToBRFV has since been reported in many countries in Asia, North America, and Europe indicating it has become widely distributed throughout the world. The serious damage to tomato and pepper crop productions, the Tm- $2^{2}$ resistance-breaking ability, and the seedborne nature make ToBRFV a serious concern to vegetable growers, seed production industries, and governmental organizations responsible for plant biosecurity. With cross serological reactivity, serological detection using TMV or ToMV antibodies can make a general detection of a Tobamovirus infection. However, in order to facilitate reliable detection of ToBRFV, Agdia has developed a ToBRFV-specific isothermal AmplifyRP assay. The AmplifyRP assay targets two virus-specific genes of ToBRFV and a host-specific gene as an internal control. Screening primer and probe combinations led to the discovery that one set of primer/probe from the RNA dependent RNA polymerase (RdRp) gene and another set from the coat protein $(\mathrm{CP})$ gene exhibited specific and sensitive detection for ToBRFV, plus one set of primer/probe specific for the plant ribosomal RNA as internal control. The preliminary experiments showed that both the RdRp-specific and the CP-specific assays can detect ToBRFV from infected leaves using a simple diluted crude tissue extract. No cross reactions were observed to other Tobamoviruses. Data from the optimization of this assay will be reported in this meeting.

\author{
Effects of a plant defense-inducing bacterial fermentation media product, ACS-811, on conidial germination and appressorium \\ formation \\ E. A. FEALKO, E. E. Pfeufer, P. Kachroo, University of Kentucky, Lexington, KY, USA
}

Biofungicide products are a rapidly expanding sector of the plant protection market, but thorough evaluation of their direct and indirect effects on plant pathogens is often unclear. In ongoing work, it has been established that the fermentation product, ACS-811, contains naturally occurring plant defense metabolites that prime for the induction of defense genes in tomato and Arabidopsis plants. However, this fermentation extract may also possess additional bioactivity. ACS-811 was examined for its ability to inhibit Colletotrichum higginsianum conidial germination by drop assays, which included the positive control $10 \%$ hydrogen peroxide, $10 \%$ glycerol, salicylic acid at the following concentrations; $500 \mu \mathrm{M}, 250 \mu \mathrm{M}, 100 \mu \mathrm{M}, 10 \mu \mathrm{M}$, and $1 \mu \mathrm{M}$; and ACS-811 at the following concentrations: $12 \%$, $8 \%, 4 \%, 2 \%, 1 \%$, and $0.5 \%$. Conidia were evaluated using $200 \mathrm{x}$ microscopy for germination and appressorium formation after 14 hours and the experiment was replicated 3 times. Treatments as low as 4\% ACS-811 completely inhibited conidial germination of $C$. higginsianum. At concentrations lower than 4\% ACS-811, C. higginsianum was characterized by abnormal morphology compared to the negative control. Alternaria tomatephila and Magnaporthe oryzae conidia were also evaluated. Together with the known plant defense gene induction properties of ACS-811, this suggests a product with at least two modes of action.

Evaluation of soybean breeding lines for resistance to Phomopsis seed decay and for high seed germinability S. LI (1), J. R. Smith (2), (1) USDA ARS CGRU, Stoneville, MS, USA; (2) USDA-ARS, Stoneville, MS, USA

Phomopsis seed decay (PSD) of soybean is a major cause of poor seed quality in most soybean production areas, especially in the midsouthern region of the United States. PSD is caused by the seed-borne fungal pathogen Phomopsis longicolla (syn. Diaporthe longicolla). Breeding for PSD-resistance is the most cost-effective long-term strategy to control this disease. The goal of this research is to develop soybean breeding lines with PSD resistance, high germinability, and reduced mature seed damage under Phomopsisinoculated field conditions. In 2016, a total of 221 heterogeneous breeding lines derived from crosses between lines with PSD resistance and high germination were planted at Stoneville, MS. These breeding lines were derived from six pedigrees and utilized five sources of PSD-resistance. Seed quality assays, including seed plating for percentage of Phomopsis seed infection and standard germination tests, were conducted following the harvest of plants selected for improved agronomic traits. Twenty-seven homogeneous breeding lines with resistance to PSD and high seed quality were identified. Seed of seven improved soybean lines, 11043-225-72, 11043-224-91, 11030541-28, 10061-236-11, 10076-121-11, DS65-1, and DS31-243, have been transferred under Material Transfer Agreements to public and private soybean breeders for developing high yielding varieties with less mature seed damage. The improved soybean lines will reduce price-discounting at the grain elevator due to damaged seed and be capable of producing high quality seed beans for planting.

\title{
AWaRe: An online learning tool for the assessment of Wheat streak mosaic risk in Montana U. MCKELVY, T. Seipel, R. K. D. Peterson, M. E. Burrows, Montana State University, Bozeman, MT, USA
}

Wheat streak mosaic (WSM) is a persistent viral disease of wheat which causes sporadic epidemics in Montana. The causal agent, Wheat streak mosaic virus, is vectored by the eriophyid wheat curl mite Aceria tosichella. Through extensive research over the past century, much information on WSM and factors contributing to disease risk has been uncovered. However, this multitude of complex information can be overwhelming for wheat growers to analyze and translate into management practices for mitigating WSM risk. Therefore, the objective of this study was to develop an application that assists users in identifying and evaluating crucial components contributing to WSM disease risk. AWaRe - Assessment of Wheat streak mosaic Risk - is an online learning and risk assessment tool developed for wheat growers, extension agents, and industry professionals in Montana. It was built using the Rshiny package in the R statistical environment. The tool emphasizes the most important factors contributing to WSM risk in Montana through specific and applied questions. These factors relate to the history of WSM disease, landscape composition, and constitution of the green bridge in a specific location. Through interaction with AWaRe, users can explore the individual and combined impact of different factors on the overall WSM risk. WSM risk is expressed as numerical score which is associated with a categorical classification: low, medium, or high risk. To our knowledge, AWaRe presents the first and only risk assessment tool that integrates complex information on the dynamics underlying WSM disease. 
Alternative management practices for the northern root-knot nematode (Meloidogyne hapla) in daylily (Hemerocallis spp.) production

A. HOWLAND, E. Cole, K. Poley, M. Quintanilla, Michigan State University, East Lansing, MI, USA

In the United States, the floriculture industry was valued at $\$ 4.77$ billion in 2018 . Daylily (Hemerocallis spp.) production is a major component of the floriculture industry, but one of its main pathogens is plant-parasitic nematodes. In Michigan, the northern root-knot nematode, Meloidogyne hapla, can cause over 20\% yield loss in daylily production. Previous, ongoing field trials testing different management strategies showed four treatments with promising control of $M$. hapla that also increased plant growth. Therefore, a threeyear field trial was established in 2019 in Zeeland, MI with the objective to test these top treatments to determine which would work best for managing M. hapla in daylilies. The treatments included in the trial are Indemnify Root Dip (Dip) + TerraClean 5.0, Dip + Indemnify, Dip + AzaGuard, Dip + Compost, and an untreated control. In the field, the five treatments were arranged in a randomized block design with five replications. Daylilies were dipped in Indemnify and immediately planted; the additional treatments were then applied to each respective plot. To monitor $M$. hapla population levels, soil samples were taken at treatment, 21-day post treatment, mid-season, and end-season. Mid-season plant height data and root samples were also taken. Preliminary results show the Dip + TerraClean 5.0 having the best M. hapla control and the Dip + Indemnify treatment having the best plant growth. The field trial will continue in 2020 and 2021 to provide daylily producers the best management strategy to reduce root-knot nematodes populations in their field.

\author{
Biocontrol agent, biofumigation, and anaerobic soil disinfestation suppress soil-borne disease and improve yield of strawberry \\ in perennial system \\ M. RAHMAN, West Virginia University, Morgantown, WV, USA
}

Black root rot complex and crown rot of strawberry caused by soil-borne fungi limit sustainable strawberry production in the Northeast especially in perennial matted row systems. Many organic and small growers who can't use chemical fumigants due to new regulations and potential health hazards need alternative management options. This study was conducted by growing strawberry plug plants with beneficial microbes inoculated or regular planting mix followed by transplanting in fruiting field plots that either were bio-fumigated with mustard cover crop, anaerobically disinfested or left untreated. Different combinations of plug plants and field plot treatments were used to determine efficacy of individual treatment or synergistic effect of combination treatments. Plug plants were transplanted in pretreated plastic mulched raised beds and grown following a typical organically-recommended production method. Plug plants grown on inoculated planting mix showed enhanced plant vigor in fruiting field compared with non-treated plants. Bio-stimulant/beneficial microbe treated plants had significantly $(P \leq 0.045)$ higher fruit yield. Plant survival in treated plots were also significantly higher in the second year compared with non-treated control. Results indicated that alternative fumigation methods could provide soil-borne disease suppression and improve yield.

Predicting the worldwide climate suitability for mycoparasites of Sclerotinia sclerotiorum S. D. COHEN, Center for Regulatory Research LLC, Roseville, MN, USA

White mold disease is caused by a fungal plant pathogen, Sclerotinia sclerotiorum. This disease is particularly difficult to control as it produces sclerotia that survive in soil debris for many years. Sclerotia are known to be susceptible to biological control from mycoparasites, Paraphaeosphaeria minitans, Trichoderma harzianum and Trichoderma viride. This study compares the geographical and climate distribution range of the mycoparasites with $S$. sclerotiorum, and predicts areas suitable for biological control. Data for each fungus included geographic occurrences and coordinates retrieved from the Mycology Collections Portal (mycoportal.org) and scientific literature. Fungal data were imported into Quantum GIS (QGIS ver. 3.4.12) software to check accuracy of coordinates. Potential fungal distribution was modeled using Maximum Entropy Analysis (MAXENT ver. 3.4.1) with 8 bioclimatic variables (WorldClim.org). Bioclimatic variables with the highest influence on geographic predictions included annual mean temperature and precipitation of driest month for S. sclerotiorum and Paraphaeosphaeira minitans; annual mean temperature and precipitation of coldest quarter for $T$. harzianum; and annual mean temperature, annual precipitation, and precipitation of coldest quarter for T. viride. Overall, mycoparasite prediction maps were similar for areas of climate suitability, but some differences were noted based on kappa statistics analysis.

The impact of saline rich soil on the fungal and bacterial microbiome of plant roots and soil D. JAKUBOWSKI, A. Neupane, Z. Wang, L. Gu, S. Y. L. Marzano, South Dakota State University, Brookings, SD, USA

The microbiome found within soil and the roots of plants has large impact on the growth of the plant. When soil has too high of a salt content, it is diminishing to plant growth. Samples were gathered from plants grown in salty soil and good soil. Also, bulk soil samples from good and salty soil were gathered for a second year in a row. With the use of 16S and ITS amplicon sequencing techniques, the microbiomes of bacteria and fungi present in the samples gathered were analyzed. The diversity of the microbiome in roots as well as the diversity of bulk soil over time were compared based on the salt content of the soil. This data gives us an idea of how different these microbial communities really are. Using the same root samples, metabolites were identified with a GCMS. Even with a small sample size there were significant differences in the regulation of certain metabolites, such as (R)-Pantothenate. This metabolite is found in a pathway responsible for oxidative stress. Furthermore, endophytes isolated in the same root samples are screened for their effects on salt tolerance. The difference within these microbial communities helps us harness the utility of microbial communities when faced with an abiotic stressor like high salt tolerance because some species within the microbiome are beneficial for the plant's growth. Using this information, we are able to identify the taxonomy of the microbiome and functionally determine specific species that benefit the salt tolerance of a plant. 
Characterization of a novel resistance gene, $G m P A P 2.1$, encoding the purple acid phosphatase against soybean mosaic virus K. WIDYASARI, J. Lee, K. H. Kim, Seoul National University, Seoul, SOUTH KOREA

Soybean mosaic virus (SMV) is one of the most prevalent viral pathogen that infects soybean plants and occurs all around the world. Management of SMV is depends on a good agricultural practices and uses of resistant cultivars. However, the emergence of resistancebreaking strains of SMV urges the characterization of alternative resistance genes on soybean pants. GmPAP2.1 is member of purple acid phosphatases (PAPs) in soybean cultivar L29 which carrying an Rsv3 resistance gene. Overexpression of GmPAP2.1 on cultivars that susceptible to the infection of SMV strain G5H resulted in significantly reduced viral RNA accumulation in inoculated leaves and prevented virus spread into upper systemic leaves. GmPAP2.1-overexpressed plants also showed less severe mosaic symptom in inoculated leaves. In addition, coexpression of GmPAP2.1 delayed infection of more virulent SMV strain G7H. Construction of deletion and amino acids substitution mutants identified C-terminal region as important region for maintaining resistance to SMV strains. Transient expressions of GmPAP2.1 in experimental host Nicotiana benthamiana revealed that this protein is localized exclusively in the chloroplast. Furthermore, expression analyses of endogenous salicylic acid (SA)-related genes in GmPAP2.1-overexpressed soybean cultivar Lee74 exhibited higher expression levels of SA-related genes compared to those in the mock plants. Together, our finding provides new evidence and insight of the host protein which is GmPAP2.1 as a resistance factor in the defense response against SMV strains.

Inhibition of Erwinia amylovora strains by novel antagonistic pseudomonads

A. M. JIMENEZ MADRID (1), C. Chagas de Freitas (1), M. L. Lewis Ivey (2), (1) The Ohio State University, Wooster, OH, USA; (2) Department of Plant Pathology, The Ohio State University, Wooster, OH, USA

Fire blight, caused by the bacterium Erwinia amylovora (Ea), is a devastating disease of apples worldwide. Current management strategies include cultural practices and the use of antibiotics, such as streptomycin, oxytetracycline, and kasugamycin. However, streptomycin resistant (SmR) Ea have been recovered in the United States, including $\mathrm{OH}$. Resistance in $\mathrm{OH}$ strains is conferred by the linked StrA-StrB genes on the non-conjugative plasmid pEA29. The emergence of SmR Ea generates a need to identify alternative strategies to control fire blight disease. Biological plant disease control can be integrated into fire blight management programs; reducing the reliance on antibiotics. A collection of 50 novel Pseudomonads were screened in vitro to look for inhibitory activity against resistant and sensitive $E a$ strains from $\mathrm{OH}$. Moderate to high inhibitory activity was observed for 38\% of the Pseudomonads tested. Two strains of $P$. protegens (1B1 and Darke) and one $P$. brassicacearum strain (93G8) had significantly larger zones of inhibition against both resistant and sensitive Ea strains compared to the other Pseudomonas spp. screened. These strains have been previously reported to produce antimicrobial compounds, which make them strong candidates for biocontrol. The efficacy of these strains will be tested alone and in combination with plant immune boosters in planta with the long-term goal of providing sustainable alternatives to antibiotics.

How to develop and select the right disease risk model for leaf blotch disease management in wheat? A. FICKE, A. G. Hjelkrem, I. S. Hofgaard, G. Brodal, NIBIO, Aas, NORWAY

In Norway and Denmark, precipitation-based risk models have been used to guide fungicide application timing to control leaf blotch diseases in wheat. Recent field trials showed that both models overestimated disease risk under Norwegian conditions. Our objective was to develop a model with higher accuracy, that would be widely used by Norwegian advisors and farmers. Several tree-based models and one K-nearest neighbor (KNN) model were fitted to 283 field data sets including disease severity, weather variables, and host phenology. Disease severity was assessed visually by different individuals at growth stage (GS) 70-80 and climate factors were recorded from weather stations at varying distances from the trials. Selection of the new model was generally based on its accuracy, biological relevance of the variables included, availability of the data needed, simplicity, and farmers preference (few false negatives). The KNN model had the highest accuracy (83-85\%) and a low percentage of false negatives (5-6\%), however, this algorithm is based on variable similarity and not pre-classification of relevant variables. A simpler tree-based model, based on temperature and precipitation during tillering (GS 30-40) and humid hours during booting (GS 50-60), with slightly less accuracy (76-80\%) and an even lower percentage of false negatives (1-2\%) was selected. In the final analysis, the most accurate model might be of less value to endusers if it is overly complex, increases the risk of missing essential fungicide sprays and if the input data itself is of variable quality.

\section{Investigating the genetic variation of wheat streak mosaic-associated viruses in Kansas fields through high-throughput} sequencing C. D. REDILA, S. Nouri, Kansas State University, Manhattan, KS, USA

Wheat streak mosaic (WSM), a viral disease affecting cereals and grasses, costs substantial losses in crop yields. Wheat streak mosaic virus (WSMV) is the main causal agent of the complex, but is sometimes found in mixed infections with Triticum mosaic virus (TriMV) and Wheat mosaic virus (WMoV). Although resistant varieties help control the disease, WSMV resistant-breaking isolates have been reported, suggesting that viral populations are genetically diverse. The objective of this study is to examine the genetic variation and evolutionary mechanisms of WSM-associated viruses in Kansas fields. Twenty historic and field samples were used for total RNA sequencing. Sequences were mapped to available NCBI genomes of the WSM-associated viruses. Preliminary results showed WSMV as the predominant virus followed by mixed infections of WSMV+TriMV in Kansas fields. Phylogenetic analysis of WSMV isolates from this study reveals four different clades. Isolates from the USA, Turkey, and Argentina were placed in a defined clade and divided into distinct sub-clades. Unlike other US isolates, Kansas is widely distributed in these sub-clades. However, the historic and fieldcollected samples were clustered in different sub-clades, inferring that there is a degree of genetic diversity among these isolates. The 
phylogenetic relationship among TriMV isolates is currently under study. The findings of this study will provide insights into the genetic structure of WSM-associated viruses and in turn, help in improving strategies for disease management.

Coordinated modulation of gene expression mediate the immune response to stalk rot in a resistant maize inbred line A. F. SALCEDO (1), J. Al-Haddad (2), C. R. Buell (2), F. Trail (2), E. Góngora-Castillo (3), L. M. Quesada-Ocampo (4), (1) North Carolina State University, Raleigh, NC, USA; (2) Michigan State University, East Lansing, MI, USA; (3) Centro de Investigación Científica de Yucatán, Chuburná de Hidalgo, MEXICO; (4) Department of Entomology and Plant Pathology, North Carolina State University, Raleigh, NC, USA

Under favorable conditions, maize stalk rot caused by Fusarium graminearum can cause severe losses and accumulation of mycotoxins in livestock feed. Since few management strategies are available, the development of resistant varieties is considered the most costeffective way to control the disease. However, the stalk-tissue specific mechanism underlying the resistance to $F$. graminearum is not entirely understood and strongly influenced by the environment. In this study, we performed a temporal transcriptome analysis of two maize inbred lines with contrasting resistance to stalk rot using RNA-seq technology. We also evaluated the resistant phenotype under two environmental conditions that differ in temperature and relative humidity. We observed a noticeable downregulation of gene expression in the resistant inbred line during the first two weeks of infection. Time-course gene ontology enrichment analysis suggests that resistance may be caused by a modulation of gene expression associated with oxide-reduction, hormone biosynthesis, cytoskeleton activity, and cell wall remodeling. We validated the transcriptome analysis by evaluating the expression patterns of ten representative differentially expressed genes by RT-qPCR confirming the environmental influence on gene expression. This research provides relevant information about the molecular events behind resistance to stalk rot in maize and the effect of environmental conditions. Our findings can be exploited for the development of resistant maize varieties.

Searching for biological control agents of Ailanthus altissima

E. CALASTRI (1), V. Tava (2), A. Kunova (3), C. Pizzatti (3), M. Pasquali (3), P. Cortesi (3), M. Saracchi (3), (1) University of Milan, Milan, ITALY; (2) Università degli studi di Milano, Milano, ITALY; (3) DeFENS, Università degli Studi di Milano, Milano, ITALY

Ailanthus altissima Mill. Swingle, is a deciduous tree indigenous to China and, since its introduction to North America and Europe, has become an invasive species spreading worldwide both in urban and natural ecosystems. Neither mechanical nor chemical techniques have demonstrated to be fully effective in its management, therefore, biological control is considered to be a promising approach. With the aim of scouting potential Biological Control Agents from the group of wilt-inducing fungi, the endemic mycoflora strictly associated with $A$. altissima from wilting or decaying ailanthus trees was collected across Northern Italy. Discolored and brownish xylem and cortical tissue samples were excised from trunk section and cultured. Developing fungal colonies were observed, colony characteristics were recorded and presence of reproductive structures was described by optical microscopy observations. Forty-seven strains, related to 10 morphotypes, were finally isolated and purified. Molecular identification by sequencing of DNA ITS regions confirmed morphological identifications. Indeed, based on a database search the isolates were traced back to putatively 9 fungal genera: Alternaria, Bipolaris, Cladosporium, Coniothyrium, Curvularia, Diaporthe, Fusarium, Neofusicoccum, and Verticillium. Strains from the most frequently isolated genera were tested on A. altissima plants both in laboratory and field trials. Preliminary pathogenicity tests with Fusarium and Verticillium strains showed promising results.

Sensitivity of Corynespora cassiicola isolates in Alabama to DMI and QoI fungicides and the G143A mutation M. N. RONDON (1), K. S. Lawrence (2), (1) Auburn University, Auburn, AL, USA; (2) Entomology and Plant Pathology, Auburn University, Auburn, AL, USA

Corynespora cassiicola, the causal agent of target spot in cotton and soybean, is a pathogen responsible for significant yield losses. Fungicides are a crucial tool in disease management; although, there are reported cases of $C$. cassiicola resistance to systemic fungicides. QoI-resistance has been detected in the cytochrome b ( cytb) gene which a nucleotide substitution confer resistance to QoI fungicides (e.g. G143A, F129L, and G137R). Our objective was (1) to determine the in vitro sensitivity of C. cassiicola isolates from Alabama to prothioconazole (DMI) and pyraclostrobin (QoI), and (2) to assess the occurrence of point mutations in the $c y t b$ gene. The $\mathrm{EC}_{50}$ of $C$. cassiicola isolates was tested by in vitro mycelial growth assay in amended media with prothioconazole and pyraclostrobin $(0,0.01,0.1,0.5,1,10$, and $100 \mathrm{mg} / \mathrm{L})$. EC 50 were estimated by nonlinear regression in SAS 9.4. EC 50 of prothioconazole ranged between $0.32-1.43 \mathrm{mg} / \mathrm{L}$ for cotton isolates and ranged between $0.29-2.35 \mathrm{mg} / \mathrm{L}$ for soybean isolates. EC 50 of pyraclostrobin ranged between 3.39-13.77 mg/L for cotton isolates and ranged between 9.59-72.12 mg/L for soybean isolates. Multiple alignment analysis of the $c y t b$ gene sequences revealed a mutation at position $143(\mathrm{G} 143 \mathrm{~A})$ in four isolates from soybean plants that exhibited $\mathrm{EC}_{50}$ of pyraclostrobin above $46 \mathrm{mg} / \mathrm{L}$. The G143A mutation was not found on isolates from cotton plants. F129L and G137R mutations were not found in our isolates. Our results will be useful to monitor resistance to fungicides of $C$. cassiicola and help with fungicide resistance management.

Originally mefenoxam-sensitive isolates of Phytophthora infestans are able to quickly acquire and lose resistance M. REGNIER, J. Gonzalez-Tobon, M. Cardenas, S. Restrepo, G. Danies, Universidad de Los Andes, Bogotá, COLOMBIA

Phytophthora infestans is an oomycete that causes potato and tomato late blight disease, a worldwide concern for farmers given its explosive epidemic potential. In order to control the disease, an integral management approach, which includes the use of fungicides, has been implemented. Unfortunately, populations of $P$. infestans resistant to fungicides have been documented. Furthermore, it has been shown that originally sensitive isolates of $P$. infestans are able to acquire resistance to mefenoxam in vitro, after a single exposure 
through sublethal concentrations of this widely used fungicide. The effect on sporangial production and mycelial radial growth of two consecutive exposures on mefenoxam-amended media was assessed. For originally sensitive isolates there was increased mycelial radial growth on the second exposure but no significant differences in sporangial production were observed. On the contrary, for originally resistant isolates there was no effect on mycelial radial growth, but significant differences in sporangial production were detected. Isolates were then repeatedly exposed to mefenoxam-amended media (four additional times). However, no significant changes in sporangial production or mycelial radial growth were observed. Isolates that had acquired resistance to mefenoxam became sensitive after being transferred from one to five times through unamended media. Interestingly, resistance could be re-induced on isolates that had reverted or partially reverted their acquired resistance to mefenoxam.

\section{Screening for resistant plant genotypes and identification of cold temperature survivability for the management of Meloidogyne enterolobii \\ T. SCHWARZ, A. M. Gorny, Department of Entomology and Plant Pathology, North Carolina State University, NC, USA}

Meloidogyne enterolobii, the guava root-knot nematode, is a highly pathogenic root-knot nematode species that is capable of causing significant damage to a broad range of host plants. Current knowledge of this species is limited, especially regarding the biology, host range, and available effective management tools. The availability and use of nematicides has been decreasing, therefore, the identification of non-hosts and resistant plant genotypes is essential for long-term management. Greenhouse screening tests were conducted to identify susceptibility or resistance status of important field crops, such as sweetpotato and soybean, grown in North Carolina that can be utilized to assist in management of this root-knot nematode species. In addition, the egg hatching rate (proportion of eggs successfully hatching to second-stage juveniles) and cold-temperature tolerance (lowest temperature at which half of the population survives following exposure) of $M$. enterolobii was investigated. Our results indicate differential hatching rate and temperature tolerance compared to other major root-knot nematode species found in North Carolina. These results have implications on over-wintering capabilities and formulation of integrated pest management plans to help mitigate the impact of this species on important North Carolina field crops.

\section{Impact of cover crop usage on soilborne disease suppressiveness in field nursery production} M. Panth (1), T. Simmons (2), K. Addesso (2), F. BAYSAL-GUREL (2), (1) Tennessee State University, Mc Minnville, TN, USA; (2) Tennessee State University, McMinnville, TN, USA

Soilborne diseases are one of the major limitations to field grown nursery production. The use of cover crops is a newer area of research in woody ornamental nursery production as it has been previously explored most extensively in row crop, vegetable, fruit and flower production. The objective of this study was to determine the impact of cover crops on soilborne disease suppressiveness in woody ornamental nursery production systems. Soils from red maple plantation grown with and without cover crops (crimson clover or triticale) were sampled following the senescence of the cover crops. Greenhouse bioassays were conducted using red maple cuttings on inoculated (with Rhizoctonia solani or Phytophthora nicotianae) and non-inoculated field soils. The results showed that cover crop usage was beneficial for inducing soil disease suppressiveness against those pathogens. There was lower disease severity and pathogen recovery when the cover crops were used compared to the bare soil treatment for both pathogens. However, there were no significant differences in plant fresh weight and root weight within the treatments. Implementation of cover crop usage may improve field nursery production efficiency by reducing pressure from soilborne diseases. Therefore, the stakeholders can consider cover crop usage as an alternative sustainable management tool for soilborne diseases in field nursery production systems.

\section{Co-managing soil health and plant disease: Effects of soil health practices on persistence of plant pathogens with differing modes of survival \\ K. R. PAUGH, N. Tautges, K. Scow, C. L. Swett, University of California, Davis, CA, USA}

Soil health practices, such as cover cropping and compost amendments, have grown in popularity due to their ability to enhance soil physicochemical and biological characteristics. However, impacts on survival of soilborne plant pathogens remain unclear. Synthetic fertilizer (conventional) vs. compost + cover crop-based (organic) nutrient management methods were evaluated for effects on survival of two tomato pathogens with differing modes of survival: Fusarium oxysporum f. sp. lycopersici (Fol), which persists within plant tissue (cellulose-based), and Sclerotium rolfsii, which survives as sclerotia (chitin-based). In a greenhouse pot study using soils managed with either organic or conventional methods for 25 years, Fol-infested tissue decomposed more rapidly in organically managed soil; this effect was lost when microbes were killed in soil via steam treatment before tissue incorporation. In contrast, survival of $S$. rolfsii sclerotia was enhanced in organically managed soil. In a field study, compost amendment influenced survival of $S$. rolfsii but not of Fol. Viability of $S$. rolfsii sclerotia was greater in soil amended with poultry manure compost vs. green waste compost or synthetic fertilizer. The combination of chitin with poultry manure counteracted this effect. These studies indicate that compost enhances survival of chitin-based structures but decreases persistence of fungi residing in cellulose (plant tissue). Expanded studies are needed to test this hypothesis for the purpose of identifying and mitigating disease risks associated with soil health practices.

Gene expression in the pathogenic interaction between brown rust (Puccinia melanocephala) and sugarcane J. CORTES (1), J. W. Hoy (2), N. Baisakh (3), (1) Plant Pathology and Crop Physiology Department, Baton Rouge, LA, USA; (2) Louisiana State University, Baton Rouge, LA, USA; (3) School of Plant, Environmental, and Soil Sciences, Baton Rouge, LA, USA

Brown rust (Puccinia melanocephala) can reduce sugarcane yield by $22 \%$ in Louisiana. Host plant resistance is the preferred disease management strategy. To obtain a better understanding of this host-pathogen interaction, a comprehensive gene expression analysis 
using RNA-seq was performed using three resistant (L 99-233, L 01-299, and L 11-172) and three susceptible (Ho 95-988, HoCP 96540, and LCP 85-384) cultivars inoculated with a mixed population of urediniospores. For all resistant and susceptible cultivars pooled over time (12 h, $24 \mathrm{~h}, 48 \mathrm{~h}$, and $1 \mathrm{wk}$ after inoculation), 36 common genes were up-regulated, and three common genes were downregulated. Thirty genes were up-regulated among all three resistant cultivars through all times, and genes involved in catalytic activity and oxidation-reduction process were highly enriched. For the susceptible cultivars, 16 common genes were up-regulated with high representation in oxidation-reduction process and transcriptional regulation. Genes (19) including known defense-related genes, such as NPR4, LRR protein, PR10, WIR1A, chalcone synthase, beta-glucosidase, peroxidase, and lipase were induced only in the resistant varieties. Transcripts encoding WRKY70, bHLH148, E3 ubiquitin ligase, serine/threonine kinase, chtinase, PR1, MAPKKK, defensin etc. were specifically upregulated in L 99-233, which may be important candidates for its quantitative brown rust resistance. Ongoing analyses will provide clues to the metabolic pathways and associated networks underlying compatible/incompatible interactions in sugarcane-brown rust system.

Effect of geraniin on Xanthomonas campestris pv. vitians viability and ultrastructure M. Delisle-Houde (1), M. Blais (2), R. J. TWEDDELL (1), D. Rioux (2), (1) Département de phytologie, Université Laval, Québec, QC, CANADA; (2) Natural Resources Canada, Canadian Forest Service, Laurentian Forestry Centre, Québec, QC, CANADA

Geraniin extracted from Acer saccharum Marsh. leaves was investigated for its impact on the causal agent of bacterial leaf spot of lettuce (Lactuca sativa L.), Xanthomonas campestris pv. vitians (Brown) Dye. Firstly, the impact of geraniin on the viability of $X$. campestris pv. vitians was highlighted using SYTOX Green nucleic acid stain and plate counts. Secondly, bacteria treated with geraniin were examined in transmission electron microscopy (TEM) to determine the effects of the compound on bacterial ultrastructure. Incorporation of SYTOX Green and plate counts showed an increase of bacterial mortality of $37 \%$ and $62 \%$ respectively, when treated with geraniin $\left(3.125 \mathrm{mg} \mathrm{mL}^{-1}\right)$. Observations of treated $X$. campestris pv. vitians in TEM showed some alterations of its morphology while untreated bacteria kept their typical rod-shaped form. It was possible to note cell deformation, breaking of cell walls, cytoplasm leakage, and cytoplasmic vacuolization after geraniin treatment. Moreover, formation of multi-membranous and/or -wall systems, and some extracellular electron-dense material were observed. This study highlights for the first time, the effect of geraniin on viability and ultrastructure of $X$. campestris pv. vitians.

Penicillium species associated with blue mold rot of apple in South Tyrol (northern Italy)

S. BARIC, T. Drexler, J. Tappeiner, G. Amaral Carneiro, Free University of Bozen-Bolzano, Bozen-Bolzano, ITALY

Apple is the most important agricultural commodity of the Autonomous Province of Bozen-Bolzano - South Tyrol (northern Italy) and the major part of the harvest is stored under controlled atmosphere conditions for several months. During storage, apples can be affected by different postharvest pathogens, mainly of fungal origin. As the knowledge on infectious postharvest diseases of apple in South Tyrol is rather limited, an investigation was performed in spring 2018. More than 800 decayed apples were sampled in five packinghouses, representing four major production areas in South Tyrol and comprising both apples from integrated and organic production. From each apple, the pathogens were isolated on potato dextrose agar (PDA) nutrient medium and around 700 isolates were obtained. Based on colony morphology, Penicillium spp. was identified in $28 \%$ of the isolates and thus represented the most frequent pathogen of stored apples in this area. In order to determine the isolates to the species level, a DNA-based analysis was performed, which included DNA Sanger sequencing of the internal transcribed spacer (ITS) region and fragments of the $\beta$-tubulin and calmodulin genes. The analysis of 83 isolates revealed that $P$. expansum was the most common species isolated from apples with symptoms of blue mold rot in South Tyrol, while three other species of this genus, identified as P. paneum, P. carneum and P. bialowiezense, were found sporadically. Accurate identification of pathogen species involved in a disease is a prerequisite for the implementation of appropriate control measures.

Occurrence and management of apple bitter rot in Illinois

F. Acheampong (1), A. Miller (2), M. BABADOOST (1), (1) University of Illinois - Crop Sciences Dept, Urbana, IL, USA; (2) University of Illinois - Illinois Natural History Survey, Urbana, IL, USA

During 2016-2019, bitter rot of apple occurred in more than $60 \%$ of commercial orchards in Illinois. Incidence of fruits with bitter rot ranged from 0.3 to $63 \%$ in affected commercial orchards. Percent infected fruits in 'Honey Crisp' and 'Empires' was higher (1-63\%) than other cultivars. The incidence of fruits with bitter rot ranged from 0.3 to $36 \%$ in 'Gala', 'Golden Delicious' 'Jonathan', and 'McIntosh' apples. The fungus Colletotrichum acutatum has been identified in most of the orchards. Studies to identify additional causal species of Colletotrichum are underway. In 2019, a trial was conducted in a 'Honey Crisp' orchard that had more than 50\% of fruits with bitter rot in 2018. Prior to the bloom, all mummified apples left from 2018 and prunes and dead wood in the orchard were collected and burned. The trees were sprayed with mancozeb plus Inspire Super alternated with mancozeb plus Fontelis at 7-day intervals, from the green-tip growth stage to one week before the petal fall stage, to control spring diseases (powdery mildew, rusts, scab). Several fungicides, including Aprovia, captan, Merivon, potassium phosphides, and Topsin-M, were tested for their effectiveness for managing summer diseases of apples (sooty blotch/flyspeck and fruit rots). Spray-applications of the fungicides began at petal fall stage and ended 21 days prior to harvest, at 7- and 10-, and 14-day intervals. Only bitter rot developed on fruits. Aprovia, captan, and Merivon were effective fungicides in preventing development of bitter rot on fruits.

Cercospora sojina sensitivity to DeMethylation Inhibitors and Methyl Benzimidazole Carbamates fungicides in Tennessee E. ZUCHELLI, E. Schütz, R. Guyer, H. M. Kelly, West Tennessee AgResearch and Education Center, University of Tennessee, Jackson, TN, USA 
Quinone outside inhibitor (QoI) resistance in Cercospora sojina, agent of frogeye leaf spot disease in soybean, was first reported in 2012 in Tennessee. Besides QoI fungicides, DeMethylation Inhibitors (DMI) and Methyl Benzimidazole Carbamates (MCB) also have been used to manage $C$. sojina. Development of resistance may be expected after several years of using the same compound and multiple resistances may develop. This project aimed to investigate fungicide sensitivity to DMI and MCB fungicides using two methods: analysis of field trials and in vitro mycelial growth assays. Reduction of C. sojina from application of pyraclostrobin (QoI), flutriafol (DMI), tetraconazole (DMI), and thiophanate-methyl (MCB) at beginning pod development compared to control was evaluated from TN field trials conducted across 7 years (2013 to 2019). Disease severity was assessed at R6 growth stages. In addition, the EC50 of the same two DMI, single MBC, and a QoI fungicide with mycelial growth assay was conducted using TN isolates collected from 2017 to 2019. Preliminary analysis indicates DMI and MCB fungicides were effective at managing C. sojina in field trials, although control varied by year and from 2015 to 2019. QoIs were not effective.

\section{Determining inter- and intraspecific competitiveness of Fusarium virguliforme for nutrient utilization}

R. HALL (1), K. Bushley (2), D. K. Malvick (3), (1) Department of Plant Pathology, University of Minnesota, St Paul, MN, USA; (2) Department of Plant and Microbial Biology, University of Minnesota, St Paul, MN, USA; (3) University of Minnesota, Department of Plant Pathology, St. Paul, MN, USA

Fusarium virguliforme $(F v)$ is an invasive soilborne fungal pathogen that causes sudden death syndrome (SDS) of soybean and root rot of other legumes. SDS symptoms include root rot and interveinal necrosis and chlorosis, often resulting in significant economic losses. To help understand the competitive abilities of this pathogen, nutrient utilization profiles were analyzed for $F v$ and other common soilborne organisms found in association with soybean roots. Carbon, nitrogen, phosphorus, and sulfur utilization of $F v$ isolates were compared with isolates of Fusarium solani, F. oxysporum, F. prolieratum, F. acuminatum, Clonostachys rosea, and Pythium ultimum using Biolog Phenotype MicroArray ${ }^{\mathrm{TM}}$ plates. After incubation, fungal growth in the presence of each nutrient was measured based on the optical density (OD) within each well of each plate. Results suggest that $F v$ isolates are highly similar in nutrient utilization, whereas nutrient utilization differed more between $F v$ and the other organisms studied. Large niche widths for each of the nutrient type suggests that $F v$, as well as most of the other fungi tested, are generalists in nature. Results from this study suggest the ability for this invasive pathogen to outcompete other organisms for selected nutrients and its' potential to survive in soil with various nutrient resources.

Discovery to development: The journey of dsRNA-based foliar fungicides

M. L. MILNER (1), A. Arango-Velez (2), J. Eldridge (2), C. Lawrence (2), K. McCorkle (2), A. Smartt (2), (1) Greenlight Biosciences, Research Triangle Park, NC, USA; (2) GreenLight Biosciences, Research Triangle Park, NC, USA

RNAi is a biological solution for pest control that is target specific, biodegradable, and has the capability for rapid discovery of candidate sequences for gene knockdown. GreenLight Biosciences has developed a proprietary, scalable process for producing costeffective RNA products for agricultural management of insect pests and plant pathogenic fungi. GreenLight's first exogenously applied dsRNA product for Colorado potato beetle (CPB) control has shown comparable defoliation and yield protection to leading synthetic chemistry market products. Success with insect control has prompted research of dsRNA application for fungal pathogen management by targeting essential and pathogenicity genes. A high-throughput method of producing hundreds of dsRNAs and screening them in an in vitro assay for control of a necrotrophic foliar fungal pathogen was developed and implemented. Actives from the in vitro screen were tested for activity on host leaves and fruit with the most efficacious leads selected for field testing in 2020. The use of exogenously applied dsRNA can be expanded to additional target fungal pathogens and other agricultural pests. The future use of dsRNAs could give growers an additional disease management tool that is safe, sustainable, and effective.

Development of an RGB image-based system for detection and quantification of tar spot disease intensity on corn leaves D. Y. LEE (1), D. Y. Na (2), C. C. Gongora-Canul (1), C. E. Puerto Hernandez (3), C. Schloemer (1), A. Cruz (1), D. E. P. Telenko (1), C. D. Cruz (1), (1) Department of Botany and Plant Pathology, Purdue University, West Lafayette, IN, USA; (2) School of Electrical and Computer Engineering, Purdue University, West Lafayette, IN, USA; (3) Zamorano University, San Antonio de Oriente, HONDURAS

Tar spot of maize, caused by Phyllachora maydis, is a prevalent disease in Latin America and the Caribbean. It was first reported in the U.S. in 2015, has since spread to nine U.S. states. In 2018, a major TS epidemic occurred in the Midwest, causing significant yield losses to U.S. corn production. In order to suppress the progression and escalation of TS epidemics, it is of utmost importance to conduct rapid surveillance and early detection of disease signs such as $P$. maydis fruiting bodies (stromata). Our study aims to develop an automated system to detect and quantify $P$. maydis stromata in both the early and mature stages of infection using Red-Green-Blue (RGB) images of TS-infected corn leaves as input. A model utilizing K-means clustering in the L*a*b* color space and contour analysis was constructed, enabling the quantification of disease intensity (stromata count and proportion of stromata relative to leaf area). In order to measure agreement between visual and digital estimates of TS intensity, both Lin's concordance correlation coefficient and Cohen's Kappa statistic were measured. The outcomes of this research will help build a tool that can minimize human visual subjectivity and increase time and labor efficiency, while also providing high-throughput and reliable data for constructing tar spot prediction and surveillance models. 
Determining yield response to foliar fungicide application in sunflower (Helianthus annuus L.)

N. Braun (1), S. G. Markell (2), F. M. MATHEW (1), C. Carlson (3), B. Hansen (2), J. Halvorson (4), T. Patrick (3), A. Rickey (3), (1) South Dakota State University, Brookings, SD, USA; (2) North Dakota State University, Fargo, ND, USA; (3) University of Nebraska, NE, USA; (4) North Dakota State University, Department of Plant Pathology, Fargo, ND, USA

Diseases are a yield-limiting factor in sunflower (Helianthus annuus L.) production. Foliar application of Quinone outside Inhibitor (QoI) fungicides at R1 growth stage of sunflower development (when the miniature floral head is formed on the plant) may provide efficacy against diseases. The objective of this study is to determine the yield response to QoI fungicide application at R1 growth stage in sunflower in the presence of diseases. For this study, 12 field trials were planted to susceptible and/or moderately-susceptible hybrids belonging to oilseed or confection types of sunflower using a uniform protocol in Nebraska, North Dakota and South Dakota from 2016 to 2019. The plot size for the two treatments (fungicide and non-fungicide treated) varied across locations, however, each plot had four rows planted at $0.76-\mathrm{m}$ row spacing and was at least $6-\mathrm{m}$ in length and 3-m wide. Yields were harvested from the center two rows of each plot and subjected to a mixed linear model analysis of variance. Our results suggest that year, location and treatments had a significant effect $(P=0.05)$ on yield between the fungicide treated and non-fungicide treatments. Also, QoI fungicide application resulted in a range of yield responses in sunflower when compared to not treating with fungicides, with $72.2 \%$ and $50.0 \%$ of the yield responses being positive for confection and oilseed hybrids respectively. Further data analysis will be performed to determine if yield increases are profitable for sunflower farmers when QoI fungicides are used to manage diseases.

\section{Sensitivity of Helminthosporium solani to three classes of fungicides}

S. DESOTELL (1), G. Olaya (2), N. Rosenzweig (1), (1) Michigan State University, East Lansing, MI, USA; (2) Syngenta Crop Protection, Vero Beach, FL, USA

Helminthosporium solani is the cause of silver scurf (SS) on potatoes. Silver scurf causes cosmetic lesions and shrinkage in stored potatoes which negatively impact the crop's marketability in storage. Thus, effective fungicides may control SS and extend the storability of potatoes. The objectives of this study included: 1) Determine in vitro sensitivity of $H$. solani to three classes of fungicides: DMI fungicides [difenoconazole (DFZ)], SDHI fungicides [Adepidyn (APN), solatenol (STL) and sedaxane (SDX)], and phenylpyrrole fungicides [fludioxonil (FDL)]; 2) compare the accuracy and precision of the spiral gradient dilution method to a standard ten-fold agar plate dilution method to estimate the effective fungicide concentration at which $50 \%$ of the fungal growth is inhibited (EC 50$)$ and 3 ) compare the two methods to a standard ten-fold agar plate dilution method to estimate $\mathrm{EC}_{50}$ sensitivity values. The relative growth and germination of spores for all mono-conidial H. solani isolates are being recorded to determine fungicide sensitivity. Preliminary results from screening of a limited number of isolates $(n=19)$ collected from CA, ID, MI and WI have been completed. Mean EC50 values (mg.ai/L) based on the spiral gradient dilution method were $0.58,0.62,1.08,0.61$ and 91.4 for APN, SDX, DFZ and FDL respectively. Mean EC50 values (mg.ai/L) based on relative growth were $0.20,0.83,4.20,0.92$ and 1.80 for APN, STL, SDX and DFZ respectively. Mean EC50 values (mg.ai/L) based on relative germination were $0.20,0.32$ and 0.58 for APN, STL, and SDX respectively. This study is ongoing.

Supraoptimal temperature influences on powdery mildew susceptibility in the hop cultivar 'Cascade' D. H. GENT (1), B. Claassen (2), M. S. Wiseman (2), S. Wolfenbarger (2), (1) US Department of Agriculture, Agricultural Research Service, Corvallis, OR, USA; (2) Oregon State University, Corvallis, OR, USA

Powdery mildew resistance (Podosphaera macularis) in the hop cultivar 'Cascade' was overcome by a virulent race of the fungus that emerged in the western U.S. in 2012. Powdery mildew now occurs annually at damaging levels in many hop yards in the region and can be difficult to manage because of lack of historical experience with the disease on this cultivar. We conducted growth chamber experiments to understand how constant and transient supraoptimal temperature influences the susceptibility of Cascade to powdery mildew and development of the disease. A complex relationship between cultivar, temperature, duration of exposure, and leaf age influenced the susceptibility of plants to powdery mildew in these experiments. The effect of pre-inoculation temperature varied between Cascade and the powdery-mildew-susceptible cultivar 'Symphony' and interacted with leaf age. Powdery mildew also developed differentially on Cascade and Symphony when plants were exposed to supraoptimal temperature directly after inoculation. The number and size of colonies that developed on Cascade was reduced as compared to Symphony at most temperatures, but most notably at temperatures near 26 to $28^{\circ} \mathrm{C}$. Young colonies on Cascade were sensitive to even $12 \mathrm{~h}$ of exposure to 26 to $30^{\circ} \mathrm{C}$, whereas colonies on Symphony became progressively more tolerant to supraoptimal temperature with increasing time from inoculation. These findings indicate that the high temperature threshold in the current hop powdery mildew risk index should be revised downward to estimate disease hazard more accurately in Cascade.

Influence of Meloidogyne arenaria on nicotine accumulation in conventional and low-alkaloid flue-cured tobacco N. ADAMO (1), C. S. Johnson (1), J. D. Eisenback (2), T. D. Reed (1), (1) Virginia Tech, Blackstone, VA, USA; (2) Virginia Tech, Blacksburg, VA, USA

Nicotine, the principal alkaloid in flue-cured tobacco, is synthesized in root tips and translocated to leaves. It has been shown to play an important role in defense against some plant pathogens, and may be involved in resistance to root-knot nematodes (RKN, Meloidogyne spp.). The FDA has proposed rulemaking that would substantially reduce the nicotine content of consumer tobacco products. As such, research efforts have been directed to the development of low-alkaloid tobacco lines. RKN parasitism may inhibit translocation of rootderived plant products to because parasitism includes induction of specialized feeding sites (giant cells) that can significantly diminish vascular function. One 28-day and two 45-day greenhouse pot tests assessed the impact of Meloidogyne arenaria on the accumulation of nicotine in leaves, as well as roots, of conventional and low-alkaloid tobacco lines. Relative resistance to RKN was also evaluated. 
Alkaloid levels varied among conventional and low-alkaloid lines in all trials. Inoculation with M. arenaria increased alkaloid accumulation in roots in the 28 -day trial $(\mathrm{P}=0.030)$ and diminished accumulation in leaves in one 45 -day trial $(\mathrm{P}=0.085)$. Tobacco line and inoculation with $M$. arenaria did not significantly impact other metrics of agronomic performance in consistent manner. in the 28-day trial $(\mathrm{P} \leq 0.001)$. Alkaloid status did not exert a significant effect on RKN parasitism in any of the trials.

Species prevalence and fungicide resistance in Colletotrichum spp. from strawberry fields in the Mid-Atlantic Q. LUO, M. Hu, University of Maryland, College Park, MD, USA

Colletotrichum spp. cause strawberry anthracnose, and resistance to the primary class of fungicides, quinone outside inhibitors (QoIs), has been reported in the southeastern U.S. A total of 200 Colletotrichum isolates were obtained from strawberry plants in Maryland and adjacent states in the mid-Atlantic. Analysis of $g 3 p d h$, tub2, and ITS sequences showed that three Colletotrichum species, $C$. nymphaeae, C. fioriniae, and C. siamense, were responsible for strawberry fruit and crown rots. C. nymphaeae was the most predominant species, representing $90 \%$ of all isolates collected. All Colletotrichum isolates were screened for resistance to QoIs (a.i.: azoxystrobin) in vitro, and all $C$. siamense isolates were additionally detected for resistance to methyl benzimidazole carbamate (MBC) fungicide (a.i.: thiophanate-methyl). Overall frequency of resistance to azoxystrobin and thiophanate-methyl was $41.9 \%$ and $63.6 \%$, respectively. G143A in the cytochrome b gene $(c y t b)$ was found in all $C$. nymphaeae and $C$. siamense isolates with high level of resistance $\left(\mathrm{EC}_{50}>100 \mu \mathrm{g} / \mathrm{ml}\right)$, while $\mathrm{F} 129 \mathrm{~L}$ was found in two of the five $C$. nymphaeae isolates with moderate resistance $\left(\mathrm{EC}_{50} \mathrm{values}\right.$ between 2.6 to $7.8 \mu \mathrm{g} / \mathrm{ml}$ ). All $C$. fioriniae isolates were shown less sensitivity to azoxystrobin (EC50 values between 9.7 to $14.4 \mu \mathrm{g} / \mathrm{ml}$ ), but no mutations in $c y t$ b were detected. Moreover, E198A in $t u b 2$ was linked with $C$. siamense isolates resistant to thiophanate-methyl $\left(\mathrm{EC}_{50}>100 \mu \mathrm{g} / \mathrm{ml}\right)$. These results revealed that fungicide resistance in Colletotrichum spp. is widespread in the mid-Atlantic strawberry fields.

Exploration of the antifungal mechanism and application of antimicrobial peptide LsGRP1 ${ }^{\mathrm{C}}$ on strawberry anthracnose H. C. LU, Y. H. Shih, C. Y. Chen, National Taiwan University, Taipei, TAIWAN

Colletotrichum acutatum infects the fruits, stems, leaves and crown of strawberry (Fragaria $\times$ ananassa Duchesne). Currently, chemical agents are mainly used for prevention and management. Antimicrobial peptides are considered as emerging disease management materials for combating microbial infections. Our laboratory found a gene from Lilium 'Star Gazer' encoding a glycine-rich protein during the researches on the induced systemic resistance, which named LsGRP1 (Lilium 'Star Gazer' glycine-rich protein 1). LsGRP1 C-terminal region $\left(L s G R P 1^{C}\right)$ can effectively inhibit the spore germination of various plant pathogenic fungi and has lethal effect on a variety of bacteria. In this study, chemically synthesized LsGRP $1^{\mathrm{C}}$ was used to perform in vitro antifungal assays on a $C$. acutatum complex isolate SWHL-1 (SWHL-1). LsGRP1 ${ }^{\mathrm{C}}$ of high concentration could inhibit spore germination of SWHL-1 but restricted germ tube elongation at a lower concentration. When chelating agent EDTA was added, LsGRP $1^{\mathrm{C}}$ of low concentration could inhibit spore germination. The mixture of EDTA and LsGRP1 $1^{\mathrm{C}}$, but not EDTA or LsGRP1 $1^{\mathrm{C}}$ singly, caused apoptosis-like programmed cell death in SWHL-1. The experiment on strawberry fruits indicated that LsGRP1 ${ }^{\mathrm{C}}$ with EDTA as adjuvants could significantly reduce the size of symptom area. This study reveals an optimum condition for the application of LsGRP1C to control strawberry anthracnose, facilitating the development of new plant-originated natural compounds good for agricultural production and environmental protection.

Complete genomic analysis of plant pathogenic Pectobacterium species found associated with soft rot disease of kale G. BOLUK, S. Dobhal, A. M. Alvarez, M. Arif, University of Hawaii at Manoa, Honolulu, HI, USA

Bacterial pathogens in the genus Pectobacterium are responsible for soft rot and blackleg of many plants in field crops, vegetables, fruits, and ornamentals worldwide. Recently, we isolated three strains of Pectobacterium sp. (PL64, PL63, and PL48) from kale showing soft rot symptoms. The pathogenicity tests and phylogenetic analyses - using three housekeeping genes $(d n a A$, gyr $B$ and gapA) - confirmed a Pectobacterium sp. as the causal agent of soft rot on kale. For genomic analyses, complete genome of a representative strain PL64 was sequenced through Oxford Nanopore MinION sequencer and de novo assembled using CLC genomics workbench. Interestingly, the PL64 genome showed almost similar homology percentage with two previously described species, $P$. brasiliense and $P$. polaris. The genome homology values of PL64 with strains of $P$. polaris and $P$. brasiliense were 94.20 to $94.97 \%$ and 94.63 to $94.87 \%$, respectively, based on ANI analysis with the MUMmer algorithm, whereas digital DNA-DNA hybridization (diDDH) values of PL64 with strains of $P$. polaris and $P$. brasiliense ranged from 55.9 to $60.2 \%$ and 57.7 to $58.8 \%$, respectively. The extensive analyses of pathogenicity determinants and evolutionary relationships are underway, but based on ANI, phylogenetic data and $d i \mathrm{DDH}$, we anticipate that either this is a new species within the genus Pectobacterium or it is a recombination of $P$. brasiliense and $P$. polaris.

Etiology of emerging leaf spot diseases on industrial hemp (Cannabis sativa) in Florida M. MARIN, N. Y. Wang, J. Coburn, J. Desaeger, N. Peres, University of Florida - Gulf Coast Research and Education Center, Wimauma, FL, USA

Hemp could be a valuable crop for Florida as sources of fiber, building materials, forages, human food products, and medicinal oil products. During a greenhouse (July to September) and a field trial (October to December) in 2019, leaf spots were observed on up to $70 \%$ of plants of different cultivars. Symptoms were classified into two groups. For group 1, the initial symptoms appeared on older leaves that eventually spread throughout the canopy. Infections started as small, individual yellow flecks that later developed into light tan or white spots with a yellow halo and contained dark conidiophores at the center. Group 2 had symptoms starting on either young or old leaves with yellow spots that eventually turned tan to brown with a yellow halo. Severely affected leaves of group 1 usually 
developed chlorosis, leading to premature defoliation, whereas leaves of group 2 remained attached. Isolations were made from diseased tissue for both symptom groups, and isolates recovered were characterized morphologically and molecularly using multilocus sequence analysis. Isolates were identified as Cercospora cf. flagellaris (group 1) and Curvularia pseudobrachyspora (group 2). Pathogenicity tests were conducted in the greenhouse by spraying spore suspensions to run-off, and plants were covered with plastic bags for $72 \mathrm{~h}$. Symptoms of Cercospora and Curvularia infections were observed 3 and 2 weeks after inoculation, respectively. As an emerging crop in Florida, the profitable production of hemp will require more studies on epidemiology and disease management strategies in the future.

Understanding disease development in cultivated American wild rice (Zizania palustris) fields

C. V. CASTELL-MiLleR (1), S. Senait (2), J. Kimball (3), D. A. Samac (4), (1) Plant Pathology Department, University of Minnesota, Saint Paul, MN, USA; (2) University of Minnesota, Saint Paul, MN, USA; (3) Department of Agronomy and Plant Genetics, University of Minnesota, St. Paul, MN, USA; (4) USDA ARS, St Paul, MN, USA

Wild rice is a native, aquatic species cultivated for its nutritious and gourmet grain. Minnesota industry produces over 9 million pounds of finished grain. Fungicides are an essential component of the disease management system, reducing foliar disease severity (DS) and enhancing grain yield. Tracking disease progression (DP) in fungicide-treated and untreated fields in conjunction with associated environmental variables $(\mathrm{EV})$ can provide information for rational fungicide use. DP in the upper wild rice canopy was recorded under two sprays of an azoxystrobin-based fungicide (F) and an untreated-control (NF) in a RCBD with two replicates at Waskish, Aitkin, and Gully, Minnesota. Leaf samples taken four times during the growing season were used to estimate DS, the area under disease progress curve (AUDPC), and AUDP stairs. From the first to the last sampling times DS increased $13.0 \%$ and $5.5 \%$ (Waskish), 17.2\% and 2.4\% (Aitkin), and 22.9\% and 7.7\% (Gully) in NF and F plots, respectively. AUDPC and AUDPS were significantly reduced under F treatments $(\mathrm{P}=0.05)$. AUDPC in F plots was reduced 2.7-fold (Waskish), 3.2-fold (Aitkin), and 2.3-fold (Gully) compared to the NF plots. AUDPS in F plots was similarly reduced 2.5-fold (Waskish), 4.1-fold (Aitkin), and 2.6-fold (Gully). A multivariate linear regression analysis of EV logged when recording DS showed that temperature and relative humidity had significant effects $\left(\mathrm{R}^{2}=45 \%\right.$; $\mathrm{P}=0.01$ ) on DS whereas dew point temperature did not. Understanding DP and its interaction with EV will allow a more efficient timing of fungicide sprays.

Developing a consistent inoculation method for Alternaria leaf spot in sugar beet for the laboratory, greenhouse, or field M. A. JAYAWARDANA (1), T. Goodwill (2), L. E. Hanson (3), (1) Michigan State University, East Lansing, MI, USA; (2) USDAARS-SBRU, East Lansing, MI, USA; (3) USDA ARS, East Lansing, MI, USA

Alternaria leaf spot (ALS), caused by isolates in the Alternaria alternata species complex (AASC) is a foliar disease of sugarbeet. ALS has emerged as an increasing issue in Michigan. Therefore, it is important to develop consistent inoculation methods to study host pathogen interactions. To develop inoculation methods for ALS, a virulent AASC strain was grown on $1 / 2$ strength V8 agar in the dark. A detached leaf assay droplet inoculation was performed in the lab and a spray inoculation was used in the greenhouse and field. A susceptible (C059) and resistant (HM9616) sugarbeet variety at the 8-10 leaf growth stage or older were used. Conidia were suspended in sterile distilled water or a $0.2 \%$ malt extract broth, with sterile water or broth as controls. For detached leaves, inoculated and control leaves were kept in moist chambers for the duration of the experiment. In the greenhouse, plants were kept in misting chambers for three days. Rating was done seven days after inoculation. Effect of wounding was compared to unwounded leaf tissue. In the field, disease rating was initiated three weeks after inoculation and rating was continued biweekly. Lesions developed on all inoculated leaves in the detached leaf assay, with or without wounding. The greenhouse inoculation with malt extract broth were significantly higher than the control, but there was no significant difference in disease between resistant and susceptible plants. Field inoculation has been inconsistent. Testing continues to get differences in disease development between susceptible and resistant varieties.

\section{Virulence phenotypes of soybean cyst nematode populations in North Dakota and reproduction abilities in soybean cultivars and breeding lines \\ I. CHOWDHURY, G. Yan, North Dakota State University, Department of Plant Pathology, Fargo, ND, USA}

Soybean cyst nematode (SCN; Heterodera glycines) continues to be the greatest threat to soybean production in the United States. To assess the prevalence of SCN and characterize their virulence phenotypes, soil samples were collected in 2015, 2016, and 2017 from 410 soybean fields across 19 counties in North Dakota (ND). Thus far, the virulence phenotypes of SCN from 73 infested fields in five counties were successfully characterized using HG type tests conducted in a growth chamber. The new HG types detected in ND were HG types 7, 2.5.7, 5.7, 1.2.5.7, and 2.7, in addition to HG type 0 reported previously. A total of 33 field populations were further confirmed for their HG types suggesting that there are SCN populations in ND that can successfully reproduce on PI 88788, the most widely used source of resistance. The reproduction abilities of two common SCN populations (HG type 2.5.7 and HG type 0) on selected commercial soybean cultivars and breeding lines from ND and Minnesota were also evaluated in this study. Of the 85 soybean cultivars and breeding lines screened against HG type 2.5.7, only seven had female index (FI) values $\leq 30$ compared to susceptible soybean (Barnes). Nevertheless, 24 of the 48 soybean entries screened against HG type 0 had FI $\leq 30$. These results indicate that host resistance is still an effective management strategy for ND growers against the commonly found virulence phenotypes. However, an integrated approach that includes crop rotation, cover crops, and rotation between sources of resistance is recommended for sustainable SCN management.

Genomic analysis of the Eastern Filbert Blight fungus, Anisogramma anomala, provides insight to pathogen biology A. B. COHEN (1), G. Cai (2), D. Price (1), L. He (1), N. Zhang (1), T. J. Molnar (1), B. I. Hillman (1), (1) Rutgers University, New Brunswick, NJ, USA; (2) USDA-ARS / Purdue University, West Lafayette, IN, USA 
Eastern Filbert Blight (EFB) is a devastating disease of European hazelnut (Corylus avellana), limiting commercial production of hazelnut in the United States. The biology of the causal agent of EFB, Anisogramma anomala, has remained poorly understood due to experimental constraints with growing the pathogen in a laboratory setting. Here, we report the sequencing, assembly, annotation, and analysis of the genome of $A$. anomala. The genome was assembled into 112 scaffolds with an $\mathrm{N}_{50}$ of $33.3 \mathrm{Mb}$ and total size of 342.5 $\mathrm{Mb}$. Independent verification of genome size using flow cytometry indicated a genome size of $370 \mathrm{Mbp}$. The overall genome had a GC ratio of $34.46 \%$, which was separated into GC rich coding regions (59\% GC) and GC poor repetitive regions (32\% GC). It encoded 9,179 predicted genes with a large set of 762 secreted proteins, including 165 predicted effector molecules, and 442 carbohydrate-active enzymes. The $A$. anomala genome also hosted a massive transposable element population that accounted for $88 \%$ of the genome. Analysis of the mating type region revealed genetic basis for homothallism as genes for both MAT1-1 and MAT1-2 idiomorphs were present. Orthologous gene comparison with related fungal pathogens including Fusarium, Valsa, Cryphonectria, Magnaporthe, and others revealed 1,253 genes (14\%) unique to $A$. anomala. Functions of unique gene families, as well as those shared amongst canker pathogens were explored further. These analyses provide us with an understanding of $A$. anomala biology and its pathogenesis in EFB.

Tobacco ringspot virus in highbush blueberries (Vaccinium corymbosum) in Washington State A. MITRA (1), S. Jarugula (1), G. Hoheisel (2), R. Naidu (1), (1) Washington State University - Prosser, Prosser, WA, USA; (2) Washington State University Extension, Prosser, WA, USA

Washington State is one of the leading producers of highbush blueberries (Vaccinium corymbosum) in the U.S.A. Between 2015 and 2019, several blueberry plants in two cultivars planted in an organic farm in Eastern Washington showed early-season stunting with almost no leaves and small, poorly-ripened berries. Although symptomatic plants produced a flush of new leaves during mid-season indicating possible recovery, they gradually declined leading to death within 2-3 years after planting. High-throughput sequencing (HTS) of total RNA from symptomatic plants revealed the presence of viral sequences aligning with Tobacco ringspot virus (TRSV, Secoviridae: Nepovirus). The presence of TRSV was further confirmed by ELISA using antibodies to a panel of nepoviruses documented in blueberries and RT-PCR assays using primers specific to a region of TRSV RNA1 and the RNA2-encoded Coat Protein gene. Sequence analysis of amplicons specific to RNA1 and RNA2 showed $89.27 \%$ and $98.1 \%$ nucleotide (nt) identity with corresponding sequences of TRSV isolates available in GenBank. The complete sequence of RNA1 and RNA2 genome segments was determined to be 7,512 nt and 3,925 nt, respectively. In phylogenetic analysis, RNA1 and RNA2 of TRSV from blueberries aligned closely with virus isolates infecting soybean from South Korea. TRSV spread was demonstrated from symptomatic blueberry plants to healthy cucumbers used as transmission bait plants, indicating transmission by nematode vectors present in the soil. To our knowledge, this is the first report of TRSV infecting blueberry in Washington State.

Evaluation of hemp powdery mildew host resistance and host range

A. R. CALA (1), C. T. C. Day (1), G. Giles (2), C. Carlson (3), G. Stack (3), M. Ullrich (4), J. Crawford (5), L. Smart (3), C. D. Smart (1), (1) Plant Pathology and Plant-Microbe Biology Section, Cornell AgriTech, Geneva, NY, USA; (2) Cornell University, Geneva, NY, USA; (3) Horticulture Section, Cornell AgriTech, Geneva, NY, USA; (4) Cornell Cooperative Extension Orange County, Middletown, NY, USA; (5) Plant Breeding and Genetics Section, Cornell University, Ithaca, NY, USA

Golovinomyces spadiceus (causal agent of hemp powdery mildew) has been reported on industrial hemp across North America, but there is little known about this pathogen or effective management strategies. One goal of our work is to determine whether powdery mildew host resistance is present in the available hemp germplasm. During the summer of 2019, 30 high cannabidiol (CBD) cultivars were screened in two field locations for powdery mildew disease severity. We identified a wide range of susceptibility including cultivars that were highly susceptible, and one cultivar that had little to no disease. This cultivar will be used by the breeding program to create a mapping population to identify candidate resistance genes. Additional cultivars are currently being screened for resistance to several isolates of $G$. spadiceus. We are also looking at the host range of G. spadiceus isolates collected from hemp. G. spadiceus has been reported on several other crop species including sunflower, okra, and the ornamental flower Green and Gold. Current studies are aimed at determining the host range of the hemp powdery mildew fungus through mist chamber and field inoculations.

Management performance maps: Identifying system traits for seed degeneration management in vegetatively-propagated crops S. THOMAS-SHARMA (1), R. I. Alcala-Brise (2,3,4), C. Almekinders (5), K. F. Andersen (2,3,4), J. L. Andrade-Piedra (6), A. Bonaventure Omondi (7), C. Buddenhagen (2,3,4,8), M. Carvajal-Yepes (9), R. Choudhury $(2,3,4,10)$, W. Dantes (2,3,4), J. Fayette $(2,3,4)$, J. Fulton (2,3,4), J. F. Hernandez Nopsa (11), J. F. Kreuze (6), P. Kromann (12), J. P. Legg (13), M. McEwan (14), P. S. McManus (15), L. Nkengla (16), K. Ogero (17), O. Ortiz (6), G. Thiele (6), Y. Xing (2,3,4), J. E. Yuen (18), G. A. Forbes (6), K. A. Garrett (2,3,4), (1) Louisiana State University Agricultural Center, Baton Rouge, LA, USA; (2) Emerging Pathogens Institute, University of Florida, Gainesville, FL, USA; (3) Plant Pathology Department, University of Florida, Gainesville, FL, USA; (4) Institute for Sustainable Food Systems, University of Florida, Gainesville, FL, USA; (5) Wageningen University \& Research, NETHERLANDS; (6) CGIAR Research Program on Roots, Tubers and Banana, International Potato Center, Lima, PERU; (7) CGIAR Bioversity International, BURUNDI; (8) AgResearch, Ltd., Ruakura, Hamilton, NEW ZEALAND; (9) International Center for Tropical Agriculture (CIAT), Cali, COLOMBIA; (10) University of Texas Rio Grande Valley, Edinburg, TX, USA; (11) Corporación Colombiana de Investigación Agropecuaria, AGROSAVIA, Mosquera-Bogotá, FL, COLOMBIA; (12) CGIAR Research Program on Roots, Tubers and Banana, International Potato Center, Quito, ECUADOR; (13) International Institute of Tropical Agriculture, Dar es Salaam, TANZANIA; (14) International Potato Center, KENYA; (15) University of Wisconsin-Madison, Madison, WI, USA; (16) International Institue of Tropical Agriculture, CAMEROON; (17) International Potato Center, TANZANIA; (18) Swedish Univ of Agricultural Sciences, Uppsala, SWEDEN 
An important problem for most vegetatively-propagated crops is the ease of transmitting pathogens via seed material. Repeated propagation of diseased plants can lead to significant yield reductions, a problem called 'seed degeneration'. This is a complex agricultural problem and involves interactions across multiple dimensions, spatial resolutions, time-frames, pathogens, and often among many stakeholders. Management of seed degeneration is handled distinctly in different systems, often with varying degrees of success. We compare these disparate systems using an influence diagram framework, to identify common themes that could cross-fertilize research and development efforts. This framework captures the biophysical dimension (environmental and biotic factors) and human dimension (socio-economic, policy and regulatory factors) of seed degeneration, and addresses their influence on two interdependent elements: management efficacy and management adoption. We use this to identify system traits that increase the probability of success of new/improved innovations, and evaluate the geographic probabilities in 'management performance maps' (MPMs). MPMs represent the expected 'performance' of disease management practices, defined as the ability of management practices to reduce the effects of disease, while simultaneously improving profits from production. MPMs can guide decision-making by farmers, extension personnel, actuarial scientists, and policy makers. MPMs have broad applicability across vegetatively-propagated crops as well as for other disease management scenarios.

Visual assessment of bacterial leaf scorch symptoms: Association with Xylella fastidiosa incidence and yield impacts on southern highbush blueberry

D. Di Genova (1), K. Lewis (1), E. Smith (1), P. Severns (2), J. E. OLIVER (3), (1) University of Georgia, Tifton, GA, USA; (2) University of Georgia, Athens, GA, USA; (3) Department of Plant Pathology, University of Georgia, Tifton, GA, USA

Xylella fastidiosa (Xf) is an emerging vector-transmitted bacterial pathogen, capable of infecting many economically important crops across the globe. In recent years, $\mathrm{Xf}$ has been confirmed as the cause of bacterial leaf scorch (BLS), which causes relatively rapid decline and death of susceptible southern highbush (SHB) blueberry cultivars in the southeastern United States. Due to the recent emergence of Xf on blueberry, basic questions on its epidemiology, establishment, and dispersal dynamics in field conditions remain largely unanswered, and currently there are few practical management options for this disease. To limit losses, blueberry growers rely on the identification, removal, and replacement of symptomatic plants. To better understand the impact and spread of BLS, SHB blueberry cultivars 'Rebel' and 'Star' were visually assessed for BLS symptom severity in two plantings in Bacon County, Georgia during fall 2018 and 2019. Leaves collected from these plants were tested for Xf via molecular testing, and yield and fruit quality were assessed in spring 2019 on a subset of plants assessed in 2018. Results of these assessments indicated an increase in observable disease severity over two years, a significant association between visual assessment and Xf incidence, and a measurable impact of BLS on yield. The results of this case study provide valuable preliminary information regarding the usefulness of visual assessment for the identification of Xf infection in blueberry and the implementation of management strategies such as plant removal and replacement.

A metagenomics approach to study pecan bacterial leaf scorch disease associated with Xylella fastidiosa in Georgia J. CHEN (1), J. Huang (2), M. Bao (2), M. W. Hotchkiss (3), C. H. Bock (4), (1) USDA-ARS, Parlier, CA, USA; (2) South China Agricultural University, Guangzhou, CHINA; (3) USDA ARS, Byron, GA, USA; (4) USDA ARS, Southeastern Fruit and Tree Nut Research Laboratory, Byron, GA, USA

Pecan bacterial leaf scorch disease (PBLSD), caused by Xylella fastidiosa (Xf), is endemic to pecan in the southern USA. Research is challenging as $\mathrm{Xf}$ is difficult to culture and pecan is a woody perennial. Total leaf DNA was extracted from 40 suspect PBLSD samples from 3 orchards in Georgia. Xf was detected by SYBR green rt-PCR with 2 primer sets, A: Teme150fc-Teme454rg specific to subsp. fastidiosa, and B: Dixon454fa-Dixon1261rg specific to subsp. multiplex. Only 2 samples (1 site, 1 tree each of cvs. Elliott and Forkert) were confirmed $(\mathrm{Ct}<30)$ as subsp. fastidiosa, and 19 samples ( 2 sites, several trees of cvs. Cape Fear and Candy) as subsp. multiplex. Two samples, $\mathrm{T} 1$ (cv. Elliott; subsp. fastidiosa - $\mathrm{Ct}=25.62$ for set $\mathrm{A}$ and $\mathrm{Ct}=35.18$ for set $\mathrm{B}$ ) and T9 (cv. Cape Fear; subsp. multiplex $\mathrm{Ct}=32.64$ for set $\mathrm{A}$ and $\mathrm{Ct}=21.89$ for set $\mathrm{B}$ ), were selected for high throughput sequencing (Illumina HiSeq 3000 ). Over $300 \mathrm{M}$ short reads (101 bp each; >30 Gbp data) were generated from each sample. A partial genome (10,338 bp) was obtained from T1 referenced to the whole genome sequence (WGS) of strain M23 (NC_010577.1, subsp. fastidiosa). A close to complete genome (2,322,390 bp) was obtained from T9 referenced to the WGS of strain M12 (NC_010513.1, subsp. multiplex). Thus, PCR and genome sequence analyses shows both Xf subsps. fastidiosa and multiplex are associated with PBLSD, but subsp. multiplex is dominant in the studied samples. Metagenomic analyses (using software Kaiju) also detected other bacterial and fungal endophytes in the two samples.

Diseases affecting sunn hemp (Crotolaria junceae) in the Lower Rio Grande Valley of Texas Q. Rai, P. Soti, A. Racelis, R. CHOUDHURY, University of Texas Rio Grande Valley, Edinburg, TX, USA

Sunn hemp (Crotolaria junceae) is an emerging cover crop with many reported benefits such as nitrogen fixation, carbon sequestration, and the suppression of weeds and nematodes. Relatively little is known about the diseases that affect sunn hemp within the lower Rio Grande Valley of Texas, an important agricultural region. The objective of this study were to survey sunn hemp diseases and their fungal associations along with characterizing the role of sunn hemp as a potential 'green bridge' for pathogens that affect agronomically important crops. Field surveys revealed plants with necrotic lesions and premature abscission on lower leaves, leading to poor nodulation in infected plants. Isolates of Fusarium, Bipolaris, and Stemphilium were identified from diseased leaf tissue. Pathogenicity assays are underway to determine if these pathogens are able to infect sunn hemp as well as their ability to infect sorghum, a common agronomic crop. Field surveys also revealed sunn hemp plants with distinct white mycelia on leaves and green stems, consistent with powdery mildew disease. Microscopic examination of conidia identified the pathogen as a Golovinomyces species, and pathogenicity assays confirmed pathogenicity on sunn hemp. These results suggest that sunn hemp may serve as a green bridge capable of hosting and spreading pathogens to agronomically important crops. 
Elucidation of FliA regulation of methyl-accepting chemotaxis protein genes in Dickeya dadantii 3937 A. S. B. NASARUDDIN, A. O. Charkowski, Colorado State University, Fort Collins, CO, USA

Compared to the animal pathogens in the order 'Enterobacteriales', plant pathogenic Pectobacteriaceae have higher numbers of methyl-accepting chemotaxis protein genes $(\mathrm{mcp})$ in their genomes. Approximately 1\% of Pectobacteriaceae genomes encode mcp. Dickeya dadantii 3937 encodes $47 \mathrm{mcp}$ in its genome. The FliA sigma factor is the transcriptional regulator of the flagellar and chemotaxis genes in E. coli. However, the regulatory cascade of $m c p$ in soft rot bacteria is unknown. The purpose of this study is to determine if FliA regulates $m c p$ in $D$. dadantii 3937. The wild type (WT) and fliA mutant strains of $D$. dadantii 3937 were grown to early $\log$ phase under aerobic condition at $30^{\circ} \mathrm{C}$ in $\mathrm{M} 9$ minimal medium supplied with either $0.2 \%$ glucose or $0.2 \%$ glycerol. Total RNA was extracted from the bacterial cultures and RNA-sequencing was carried out using the Illumina NextSeq platform. The differentially expressed genes were identified using DESeq2. As expected, the class III flagellar genes were differentially regulated in the fli $\mathrm{A}$ mutant compared to the WT in both glucose and glycerol, which indicates that FliA regulates the transcription of flagellar genes. Only two $m c p$ were downregulated in the fliA mutant compared to the WT grown in glycerol. Two mcp genes were downregulated in the fliA mutant compared to the WT grown in glucose. However, approximately $50 \% \mathrm{mcp}$ had basal level expression in the fliA mutant. Our results suggest that sigma factors beside FliA regulate the transcription of $m c p$ genes in D. dadantii 3937.

A comparative analysis of ATP-binding cassette (ABC) transporters provides insight into the evolution of pathogenicity in softrot Pectobacteriaceae

Y. ZENG, A. O. Charkowski, Colorado State University, Fort Collins, CO, USA

ATP-binding cassette (ABC) transporters are a major group of cellular translocation machinery in all bacterial species for supporting their growth in various environments. A functional ABC transporter generally has two components: the ATP-binding domains and the transmembrane domains, with an addition of a periplasmic binding protein for importers. Here, we identified orthologs of ATP-binding domains of $\mathrm{ABC}$ transporters in the complete genomes of 71 type strains representing the order of Enterobacterales, using ATP-binding proteins of Pectobacterium atrosepticum SCRI 1043 as reference sequences. The structure of each transporter unit was also examined by comparing the conservation of the operon structure. Through the analysis, we found Pectobacteriaceae pathogens tend to encode over 70 ABC transporters per genome, which is approximately $20 \%$ more than closely related animal pathogens. These highly represented transporters in Pectobacteriaceae are mainly importing sugars, peptides, amino acids, or siderophores, or exporting antimicrobials. In addition, the shared ABC transporter operons are strongly conserved between Pectobacteriaceae and other enterobacteria. However, $\mathrm{ABC}$ transporters in plant pathogens that mediate the transport of amino acids, siderophores, or antimicrobials have more diverse operon structures. These results suggest that acquisition of some types of $\mathrm{ABC}$ transporters is important for evolution of plant pathogenesis.

Genetic screen to identify temperature dependent regulation in cool-virulent strains of the bacterial phytopathogen, Ralstonia solanacearum

J. K. SCHACHTERLE, Q. Huang, Floral and Nursery Plants Research Unit, U. S. National Arboretum, USDA/ARS, Beltsville, MD, USA

Ralstonia solanacearum causes a devastating bacterial wilt with broad host range, including economically important vegetable crops such as potatoes and tomatoes. Although most $R$. solanacearum lineages cause disease in tropical climates, certain strains retain virulence at cool temperatures. These strains are listed as select agent strains of $R$. solanacearum in the United States. Despite several efforts to determine what mechanisms differentiate cool-virulent strains from tropical strains in causing disease at cool temperature, the underlying mechanisms have remained elusive. In order to better understand these mechanisms, we have performed a genetic screen to identify temperature responsive regulatory elements in cool-virulent $R$. solanacearum. We randomly mutagenized the cool-virulent $R$. solanacearum strain UW551 with a transposon carrying a promoter-less bacterial luciferase (luxCDABE) operon as a reporter. By screening these transposon mutants at cool $\left(20^{\circ} \mathrm{C}\right)$ and tropical $\left(28^{\circ} \mathrm{C}\right)$ temperatures, we have identified specific mutants in which expression of the reporter is temperature dependent, indicating that the transposon inserted downstream of a temperature dependent promoter. Further characterization of the transposon insertion sites in these mutants will provide greater understanding of the temperature responsive elements that contribute to cool-virulence in select-agent strains of $R$. solanacearum. This in turn would facilitate accurate definition, detection and control of the select agent strains of $R$. solanacearum to safeguard U. S. Agriculture.

\section{Reclassification of Chromobacterium violaceum ATCC 31532 to Chromobacterium subtsugae ATCC 31532 A. HARRISON, S. Soby, Midwestern University, Glendale, AZ, USA}

Quorum sensing is a bacterial cell density-dependent communication system. In many gram negative bacteria, N-acyl-homoserine lactones (AHLs) serve as auto-inducers that coordinately regulate gene expression within a population. Chromobacterium, a gramnegative betaproteobacterium, utilizes AHLs to promote the expression of the characteristic purple pigment violacein, as well as virulence factors, and many other cellular functions. CV026, a mini-Tn5 mutant of $C$. violaceum ATCC 31532, has been extensively used and widely published as a quorum sensing biosensor for the production of AHLs in many bacterial species. The type isolate of the species, C. violaceum ATCC 12472 produces N-decanoyl-L-homoserine lactone (C10-HSL), and C. violaceum ATCC 31532 produces N-hexanoyl-homoserine lactone (C6-HSL), suggesting that these two organisms differ substantially in their gene regulation systems, and therefore are likely not members of the same species. Subsequent molecular phylogeny based on whole genome sequences of the type strain C. violaceum ATCC 12472 and ATCC 31532 indicate that these two isolates are different species. Additionally, ATCC 31532 is part of the same clade as C. subtsugae, which also produces N-hexanoyl-homoserine lactone (C6-HSL). Based on these data 
and additional phenotypic analyses, ATCC 31532, and therefore the biosensor CV026, reclassification from C. violaceum to $C$. subtsugae is proposed.

Baseline sensitivity of Cercospora sojina, causal agent of soybean frogeye leaf spot, to pydiflumetofen D. NEVES, C. A. Bradley PhD, University of Kentucky, Princeton, KY, USA

Pydiflumetofen is a new fungicide that belongs to the succinate dehydrogenase inhibitor (SDHI) class. This novel fungicide provides broad spectrum action against different phytopahogenic fungi, including Cercospora sojina, causal agent of frogeye leaf spot of soybean. To establish a baseline sensitivity, 88 C. sojina isolates that have never been exposed to SDHI fungicides were tested for their sensitivity to pydiflumetofen. In vitro assays were conducted using different doses of pydiflumetofen $(0,0.001,0.01,0.1$, and $10 \mathrm{ppm})$. The effective concentration of pydiflumetofen in which $C$. sojina isolates were inhibited by 50\% (EC50), mean and median were calculated. The EC50 values ranged from 0.335 to $4.335 \mu \mathrm{g} / \mathrm{ml}$, the mean value was $1.616 \mu \mathrm{g} / \mathrm{ml}$, and median value was $1.309 \mu \mathrm{g} / \mathrm{ml}$. This baseline sensitivity will be used when monitoring the sensitivity of $C$. sojina from soybean fields that have been sprayed with pydiflumetofen to determine if any shifts in sensitivity have occurred.

Understanding tolerance to virus infections and consequences for plant-pollinator interactions C. HINSHAW, M. López-Uribe, C. Rosa, The Pennsylvania State University, University Park, PA, USA

The inability to replicate in resistant hosts may place selective pressures on pathogen populations for increased virulence, and resistance-breaking pathogen strains have been identified in many systems. In contrast to resistance, host tolerance allows for pathogen accumulation with few to no visual symptoms and may therefore provide more durable disease management. Preliminary research has identified vine crops in the genus Cucurbita with differing levels of tolerance to Zucchini yellow mosaic virus (ZYMV). Here I use plant species with different levels of tolerance to evaluate the effects of ZYMV infection on metrics of plant fitness in the field including flower production, pollinator visitation, fruit yield, and seed viability. Results indicated that the most tolerant species maintained high virus titers, yet had mild symptoms and did not significantly differ in any of the measured traits (ANOVA, $P>0.05$ ), however, these plants were more attractive to pollinators compared to uninfected plants (GLMM, $P<0.05$ ). In contrast, less tolerant species had high virus titers and produced significantly fewer flowers, smaller fruits, and were less likely to be visited by pollinators. This study provides evidence of the feasibility of virus tolerance as a management strategy in agriculture by demonstrating that virus tolerance does not impact the retention of fitness traits in infected plants. Additionally, we show that plant pathogens may alter pollinator visitation which can reduce yields in insect-pollinated crops.

Xylella fastidiosa transmission and biofilm formation influenced by fluid dynamics D. WHITE (1), I. Marcus (2), S. Walker (2), M. C. Roper (1), (1) University of California, Riverside, Riverside, CA, USA; (2) Drexel University, PA, USA

Xylella fastidiosa is a global plant pathogenic bacterium vectored between plants by sharpshooter leafhoppers (Hemiptera: Cicadellidae: Cicadellinae) and other insects. The bacteria form biofilms in plant xylem and insect preoral cavities. There are varying hypotheses for mechanisms of $X$. fastidiosa transmission reported in the literature. In this study, transmission via flow driven detachment mechanisms are supported by comparing 3D fluid dynamic simulations with previously reported colonization patterns of insect preoral cavities. The simulations were developed for both grapevine xylem and the blue-green sharpshooter preoral cavity. Results are combined with an estimate for water hammer to infer a cohesive narrative on $X$. fastidiosa acquisition, transmission, and biofilm formation. Based on the narrative, acquisition and transmission are likely to occur in the course of sharpshooter $\mathrm{X}$ wave feeding. This novel mechanistic understanding clarifies reasons for previously reported transmission variables while emphasizing new targets for disease prevention.

Lessons from the wild: A unique chemistry of wild potato that enables resistance to bacterial disease J. R. JOSHI, A. L. Heuberger, A. O. Charkowski, Colorado State University, Fort Collins, CO, USA

Potato is the primary global vegetable crop and potato plant health is largely affected by a bacterial disease known as soft rot. Soft rot bacteria such as Pectobacterium cause damage in the field as well as in storage, causing an estimated annual loss of \$40 million to the United States potato industry, and even more in the global market. Efforts to control the disease with chemicals or agronomic practices have been largely ineffective, however resistance has been identified in wild potato, specifically Solanum chacoense. Here, we elucidated a unique chemistry of Solanum chacoense that enables resistance to soft rot bacteria. S. chacoense chemical extracts inhibited virulence of $P$. brasiliense $\mathrm{Pb} 1692$, essentially by reducing motility and exoenzyme activity, thus preventing them from infecting their host. The chemical extracts were analyzed using liquid chromatography mass spectrometry combined with bioassay-guided fractionations of the chemical extracts. The analysis identified several compounds that can enable defense, specifically phenol/polyphenols, terpenes, and glycoalkaloids. Taken together, these data provide novel information that wild potato has the capacity to interfere with the pathogenic behavior of soft rot bacteria, and that there is a chemical basis for this phenomenon in $S$. chacoense stems and tubers. Future work involves isolating these compounds from $S$. chacoense and identifying genetic regulators of these molecules that can aid breeding for soft rot bacterial resistance in cultivated potato.

Efficacy of fungicide applications to manage anthracnose of spinach in the Texas Winter Garden K. A. COCHRAN (1), M. Phillips (2), L. Stein (1), (1) Texas A\&M University, Uvalde, TX, USA; (2) South Texas Ag Research, TX, USA 
Spinach producers in the Texas Winter Garden are regularly faced with both yield and quality challenges due to foliar leaf spots such as anthracnose caused by Colletotrichum dematium. In the past five production seasons, anthracnose has been of significant concern in the area and caused cumulative losses in the millions of dollars. To date, producers have not been able to achieve satisfactory control in the field with recommended foliar fungicide applications. The objective of this study was to determine the efficacy of repeated application fungicide regimens to manage anthracnose of spinach. In the 2019-2020 winter production season two fungicide field trials were planted and then inoculated with $C$. dematium infested oats after emergence. Plots were sprayed with various fungicide rotation regimens approximately every week including Miravis Prime, Merivon, Luna Tranquility, Inspire Super, Regalia, Oso, and Switch. Plots were rated using a $0-100 \%$ scale for disease symptoms as percent of symptomatic leaves within the plot throughout the season starting $\sim 30$ dpi and again $\sim 60$ dpi. Data were analyzed across replications and separately for each disease rating date. The fungicide regimens that included at least one application of Switch had consistently lower disease plot ratings relative to other spray regimens. While additional work is needed in this area to reveal the best integrated approach for managing this challenging disease, this research will assist producers in making the most prudent and efficacious fungicide applications in the future.

Whole genome sequences of plant-associated strains of known and putative new species of the genus Rathayibacter and Other Microbacteriaceae

S. Tarlachkov (1), I. Starodumova (1), L. Dorofeeva (1), N. Prisyazhnaya (1), S. Leyn (2), J. Zlamal (2), M. Elan (2), A. Osterman (2), S. Nadler (3), S. SUBBOTIN (4), L. Evtushenko (5), (1) Institute of Biochemistry and Physiology of Microorganisms, Pushchino, Russia, RUSSIA; (2) Sanford Burnham Prebys Medical Discovery Institute, La Jolla, CA, USA; (3) University of California, Davis, CA, USA; (4) California Department of Food and Agriculture, Sacramento, CA, USA; (5) Institute of Biochemistry and Physiology of Microorganisms, Pushchino, RUSSIA

Complete and draft genome sequences of 26 bacteria mostly belonging to Rathayibacter (18 strains) and a few other genera of Microbacteriaceae were generated using Oxford Nanopore and Illumina technologies. The genus Rathayibacter currently comprises 8 species with validly published names, including the well-known plant pathogens ( $R$. rathayi, $R$. iranicus, $R$. tritici, and $R$. toxicus) vectored to their host plants by seed gall nematodes of the genus Anguina (Anguinidae). Members of the other valid Rathayibacter species were originally found in various plants, including those with no symptoms of bacterial or nematode infections. The newly isolated strains of this genus originate from Tanacetum vulgare (Asteraceae) infested by foliar nematode Aphelenchoides fragariae (Aphelenchoididae) and from several healthy plants without any signs of diseases. These strains showed 97.5-100\% 16S rRNA gene sequence identities with valid Rathayibacter species. Analyses of MALDI-TOF mass spectra and gyrB gene sequences revealed that most of these strains may belong to new species. The calculated ANI and dDDH values (well below the borderlines for species differentiation) provided robust evidence for the presence of seven new species. The genome sizes and the $\mathrm{G}+\mathrm{C}$ contents of these putative new species range from 3.21 to $4.61 \mathrm{Mb}$ and from 70.7 to $72.7 \%$, respectively. Further phenotypic study and genome-wide analyses will facilitate valid descriptions of the revealed new species and insight into molecular mechanisms involved in interactions between plants, bacteria and nematodes.

Managing oat crown rust and wheat stripe rust with varietal resistance in New York

M. FULCHER (1), D. Benscher (2), M. Sorrells (2), G. C. Bergstrom (2), (1) University of Minnesota, Saint Paul, MN, USA; (2) Cornell University, Ithaca, NY, USA

Oat crown rust (Puccinia coronata f. sp. avenae) and wheat stripe rust (P. striiformis $\mathrm{f}$. sp. tritici) are episodic but often significant diseases in New York. They can be managed by appropriate cultivar selection, though the impact of each disease on yield is unclear. Using multi-year, multi-site crop variety trials, we evaluated commercially available cultivars and university breeding lines for susceptibility to naturally occurring rust populations. Severity ratings were estimated visually as percent flag leaf damage at the early grain filling growth stages (Zadoks GS 83-85). Susceptible through resistant ratings were assigned from maximum observed severity with arbitrary cutoffs and supported by recording disease progress at three trials. Yields were estimated in small plots where ratings were taken, and the relationship of rust severity and yield was determined with a meta-analysis using linear mixed models. Several currently or soon-to-be available varieties were identified as resistant to local rust populations in both systems. Wheat yields were not impacted by rust severity, possibly due to the late timing of infection. Oat yields were highly correlated to crown rust severity with an average loss of $34.56 \mathrm{~kg} \mathrm{ha}^{-1}$ for every one percent increase in rust severity. The gap between New York spring oat yields and the national average ( $360 \mathrm{~kg} \mathrm{ha}^{-1}$ ) during this time could be explained by loss to disease given the mean severity observed on a widely planted cultivar ('Corral'). Replacement of this variety with resistant cultivars could significantly increase crop value.

\section{Saponin-enriched root extracts of Medicago truncatula inhibit Magnaporthe oryzae appressorium formation and mitigate rice blast disease \\ K. HAYDON, K. Korth, University of Arkansas, Fayetteville, AR, USA}

Plant saponins are specialized metabolites that provide defense against insects and pathogens. Medicago truncatula, a relative of alfalfa (Medicago sativa), is a potent saponin producer, especially in the root tissue of Mt accession ESP105. We have previously demonstrated that saponin-enriched root extracts of Mt ESP105 inhibit growth of Magnaporthe oryzae in culture, and reduce rice blast lesion area when applied to foliar tissue of rice (Oryza sativa) cultivar CO39 seedlings prior to inoculation. Here we present further in vitro and in planta results investigating the mode of action of these extracts. Mo spores were incubated overnight in root extract solutions and then observed by microscopy; spore germination and appressorium formation were reduced with increasing extract concentrations, with complete inhibition at $2.5 \mathrm{mg} / \mathrm{ml}$. We tested for induction of systemic resistance in rice by treating 3-leaf stage plants with extracts and inoculating 72 hours later when the untreated $4^{\text {th }}$ leaf had emerged, and comparing lesion area 7 days later on the $4^{\text {th }}$ leaves with a 
positive control. There was no reduction in disease on the $4^{\text {th }}$ leaf of treated plants. However, there was a significant reduction in disease on the $3^{\text {rd }}$ (treated) leaves, indicating that extract efficacy on treated tissue persisted at least 3 days after inoculation. These results suggest saponin-enriched root extracts of $M t$ reduce rice blast disease by directly inhibiting spore infectivity, and do not promote a systemic plant defense response when applied as a foliar treatment.

Laccase 3 activity is regulated by small heat shock protein 24 of the chestnut blight fungus Cryphonectria parasitica J. CHUN, Y. H. Ko, K. K. So, D. H. Kim, Jeonbuk National University, Jeonju, SOUTH KOREA

As an important virulence factor of the chestnut fungus Cryphonectria parasitica, laccase 3 was induced in the presence of tannic acid, a major barrier in the bark of chestnut tree against the pathogen infection. Reduction in laccase 3 activity has been associated with hypovirulence in virus-infected hypovirulent strains of $C$. parasitica. Heat shock protein 24 in C. parasitica contributes to the virulence through regulating the stress response. Interestingly, the accumulation of $\mathrm{CpHsp24}$ transcript was induced in response to tannic acid, but was decreased by the hypovirus infection. These expression patterns were similar to those of lac3. Moreover, lac3 induction in response to tannic acid supplementation depended on the CpHsp24 gene in that the lac 3 induction by the tannic acid was inhibited in the CpHsp24-null mutant. Based on these results, we tested whether $C p H s p 24$ might function as a molecular chaperone for the protein product of the lac3 gene. Functional interaction between two genes was investigated by Saccharomyces cerevisiae heterologous expression system. Heterologous expression revealed that lac 3 activity was significantly higher when the $C p H s p 24$ gene was coexpressed, which suggested the function of the protein product of $\mathrm{CpHsp24}$ gene as a molecular chaperone of LAC3 protein. Pull-down experiment using Escherichia coli-expressed proteins suggested that the protein products of $C p H s p 24$ and lac 3 genes physically interact as well. Our findings suggest that the molecular association of HSP24 with LAC3 may play an important role in the regulation of pathogenesis.

Characterizing differential phenotypes of two Trichoderma harzianum isolates upon interaction with Fusarium virguliforme M. FILGUEIRA PIMENTEL, L. F. Rocha, A. Y. Srour, J. P. Bond, A. M. Fakhoury, Southern Illinois University, Carbondale, IL, USA

Trichoderma species are well-studied biocontrol agents. However, Trichoderma strains may vary significantly in their behavior and efficacy against a specific fungal pathogen due to the intra- and inter-specific variations in Trichoderma. While studying the antagonistic effect of Trichoderma against Fusarium virguliforme, the causal agent of sudden death syndrome of soybean (SDS), we observed that two $T$. harzianum isolates that were effective against $F$. virguliforme exhibited distinct behaviors upon interacting with the pathogen. $T$. harzianum 3G1 quickly overgrew $F$. virguliforme, whereas $T$. harzianum $1 \mathrm{G} 5$ formed an extensive inhibition zone with the pathogen. In the current study, we aimed to resolve mechanisms potentially involved in the interaction between $F$. virguliforme and $T$. harzianum by analyzing gene expression patterns in the BCAs and the pathogen. A dual plate assay using PDA-covered cellophane was carried out, and mycelia were harvested before, during, and after the complete establishment of the inhibition zone or overgrowth. The comparison between the differentially expressed genes during the interaction of both $T$. harzianum isolates with $F$. virguliforme can provide valuable insights into the mechanisms of recognition between these fungi, and how that affects the antagonistic mechanisms governing the interaction. These results provide a deeper understanding of the biology of the Trichoderma-F. virguliforme interaction, which is the basis for the development of applied biocontrol tools to help manage SDS.

New datasheets on plant pathogens of concern published in the CABI Invasive Species Compendium R. W. HAMMOND (1), J. E. Fajardo (2), T. L. Widmer (3), L. McGillivray (4), H. Diaz-Soltero (5), (1) USDA ARS Molecular Plant Pathology Laboratory, Beltsville, MD, USA; (2) USDA Office of Pest Management Policy, Washington, DC, USA; (3) USDA ARS, Beltsville, MD, USA; (4) CABI, Wallingford, UNITED KINGDOM; (5) USDA APHIS, Washington, DC, USA

Invasive plant pathogens represent a threat to agriculture, forestry and the environment worldwide. Accurate information on their biology, distribution range, pathways, hosts, impacts, diagnosis, and management are required to prevent their introduction, eradicate new incursions or mitigate the damage they cause. The Invasive Species Compendium (ISC) is an open access encyclopedic resource published by CABI (www.cabi.org/isc) that brings together a wide range of different types of science-based information to support decision-making in invasive species management worldwide. It comprises over 2300 detailed invasive species datasheets that have been written by experts, edited by CABI, peer reviewed, and enhanced with data from specialist organizations, images, maps, and a bibliographic database of abstracts and full text articles. The Plant Pathogens Subcommittee of the US Federal Interagency Committee on Invasive Terrestrial Animals and Pathogens (ITAP) has identified the worst plant pathogens that are either in the US and have potential for further spread, or represent a new threat if introduced. Of these, 50 requiring new detailed ISC datasheets were commissioned from experts, edited, peer reviewed and published in the ISC. We present an update of the newest datasheets in the Compendium.

\section{Differential roles in natural competence and twitching motility for pil genes in the plant pathogen Xylella fastidiosa} M. V. MERFA, L. De La Fuente, Auburn University, Auburn, AL, USA

Xylella fastidiosa (Xf), a gram-negative bacterium that harms economically important crops worldwide, is naturally competent, allowing it to take up and incorporate exogenous DNA into its genome via homologous recombination. This potentially leads to emergence of novel pathogenic variants, capable of host range expansion. Type IV pili (TFP) play a central role in natural competence and also twitching motility of this bacterium. Since only a few pil genes have been evaluated for their role in Xf, in this study we assessed the functional role of the complete set of 37 pil genes on both of these features through site-directed mutagenesis. Individual pil genes either stimulated or inhibited movement and natural competence of Xf. Higher movement phenotypes usually correlated with 
higher recombination rates and thus natural competence. Interestingly, paralogous pairs of pil genes and genes in the same pathway showed different roles. pilY1-1 and pilY1-2 genes, which encode structural proteins of TFP, showed less movement and higher recombination when pilY1-1 was deleted, while deletion of pilY1-2 increased movement and did not affect natural competence. Likewise, mutations in the two-component regulatory system pilR/pilS showed that deletion of pilR repressed movement and natural competence, while deletion of pilS increased both features. Understanding the mechanisms by which Xf moves within plant hosts, acquires exogenous DNA and evolves, will contribute to the development of new management approaches to control outbreaks and prevent emergence of novel pathogenic strains.

Meta-analysis on the efficacy of the endophytic entomopathogenic fungus, Beauveria bassiana, against plant pathogens S. Yerukala, B. H. OWNLEY, Department of Entomology and Plant Pathology, University of Tennessee, Knoxville, TN, USA

Beauveria bassiana $(\mathrm{Bb})$, an endophytic entomopathogenic fungus, can protect plants against insects, but less is known about its efficacy against plant pathogens. To fill this knowledge gap, we conducted a meta-analysis of 1,287 publications by screening for reports on endophytic $\mathrm{Bb}$ and percent pathogen suppression, as measured by reduced pathogen growth, disease severity, or disease incidence. Factors that influenced plant pathogen suppression by endophytic $\mathrm{Bb}$ were identified. Across all studies, endophytic $\mathrm{Bb}$ suppressed plant pathogens by $31 \%$. Total variation across studies due to heterogeneity was $98.26 \%$. A Bb rate of $1 \times 10^{8}$ spores $/ \mathrm{ml}$ gave $60 \%$ pathogen suppression. Isolates from soil and plants were more effective than those from insects $(67 \%, 63 \%$, and $30 \%$, respectively). Foliar sprays were more effective than other application types. Commercial isolates were more effective than natives, but more studies included native isolates. Among different pathogens, endophytic Bb suppressed viruses (64\%), oomycetes (32\%), fungi $(31 \%)$ and bacteria (15\%). Among fungal and fungal-like pathogens, Bb suppressed ascomycetes (33\%), oomycetes (32\%) and basidiomycetes (22\%). Among plants, Bb suppressed pathogens of squash most (64\%), and was more effective on monocots (42\%) than eudicots (27\%), and annuals (59\%) than perennials (19\%). Bb was more effective in protected culture (greenhouse, $43 \%)$ than field studies $(8.6 \%)$. Our findings provide a quantitative review of the ability of $\mathrm{Bb}$ to suppress plant pathogens and increase our knowledge of its potential as a plant disease management tool.

Fungicide sensitivity of Calonectria spathulatum, causal agent of yerba mate leaf black spot M. Maidana-Ojeda (1), M. Medina (1), D. Haupenthal (1), M. Fernandez-Gamarra (1), A. D. Sanabria (2), G. ENCISO (1), (1) Centro de Desarrollo e Innovación Tecnológica, Hohenau, PARAGUAY; (2) North Carolina State University, Raleigh, NC, USA

Leaf black spot, caused by Calonectria spathulatum, is a fungal disease that causes premature defoliation and significantly reduces the yield of yerba mate (Ilex paraguariensis). Information on the sensitivity of $C$. spathulatum to fungicides has not been determined and will be important for the design of a management program. The objective was to evaluate in vitro sensitivity of $C$. spathulatum to different fungicide concentrations. Experiments were carried out with an isolate of $C$. spathulatum from Itapúa, Paraguay. A completely randomized design was used with a $4 \times 7$ factorial arrangement with four replications. The fungicides carbendazim, tebuconazole, propiconazole, and azoxystrobin + cyproconazole were mixed with potato dextrose agar culture medium at concentrations of $0 ; 0.01$; $0.1 ; 1 ; 5 ; 10$ and $100 \mathrm{ppm}$ of active ingredient. The experimental units were 5 -mm mycelial plugs transferred to the center of 90 -mm Petri dishes with $20 \mathrm{ml}$ of PDA. Mycelial radial growth was evaluated at 3,6 and 9 days after incubation and percentage of growth inhibition (PGI) was calculated. Fungicides significantly $(\mathrm{P}<0.0001)$ reduced the growth of $C$. spathulatum by $100 \%$ with 1 ppm carbendazim, $5 \mathrm{ppm}$ tebuconazole, and $100 \mathrm{ppm}$ propiconazole. However, the fungicide azoxystrobin + cyproconazole at $100 \mathrm{ppm}$ inhibited only $73 \%$. Since the appearance of $C$. spathulatum the use of fungicide was not been studied, these results will serve as baseline data for going forward and developing an application program.

Post-harvest apple rots and implications for cider production

T. W. WANG (1), K. T. Hodge (2), (1) Cornell University, Ithaca, NY, USA; (2) Cornell University - Ithaca, NY, USA

Quality of apple cider relies on healthy apples. One problematic contaminant of apple products is patulin, an FDA-regulated mycotoxin. Patulin is harmful to ingest and is produced by a handful of fungi, including a number of plant pathogens associated with apples and apple products. These patulin producers can grow and reproduce in apple fruits, leading to both spoilage and patulin contamination. Here, we document the varying levels of susceptibility of four cider apple cultivars to Paecilomyces rot, a post-harvest disease of apples caused by Paecilomyces niveus, as well as susceptibility of two cider apple cultivars to three additional apple-associated, patulinproducing fungi (Penicillium carneum, Penicillium expansum, and Penicillium griseofulvum). We also present data on the persistence of each of these fungi through fermentation and discuss implications for the cider industry.

The effects of $\mathrm{pH}$ and temperature on growth of Rhizoctonia solani isolated from symptomatic cotton using in vitro assays A. M. MADDOX (1), R. B. Hutmacher (2), M. Ulloa (3), M. L. Ellis (4), (1) California State University, Fresno, Clovis, CA, USA; (2) University of California, Davis, Shafter Research and Extension Center, Five Points, CA, USA; (3) USDA ARS, Lubbock, TX, USA; (4) California State University, Fresno, Fresno, CA, USA

Rhizoctonia solani is a common soilborne pathogen that causes seedling disease in cotton. Seedling disease caused by $R$. solani in cotton has been found to be most prevalent in sandy acidic soils, and under cool and wet conditions. However, $R$. solani has also been found causing disease in heavier alkaline soils in California. Therefore, this study evaluated the effect of $\mathrm{pH}$ and temperature in an in vitro assay using two $R$. solani isolates from cotton. Each isolate was evaluated on an artificial culture medium at six $\mathrm{pH}$ levels $(4,5,6$, $7,8,9)$ and incubated at four temperatures $\left(15,20,25,30^{\circ} \mathrm{C}\right)$. There were three replicate plates per treatment combination and the experiment was repeated. Data for average colony diameter for each plate was taken at $72 \mathrm{~h}$. There was a significant difference for $\mathrm{pH}$ 
and temperature $(\mathrm{P}<0.0001)$, but not for isolate. The two $R$. solani isolates were found to grow best at a lower $\mathrm{pH}$ of 4 and 5 with colony dimeter averages for $\mathrm{pH}$ at 5.9 and $5.8 \mathrm{~cm}$, respectively. The isolates did not grow at the higher $\mathrm{pH}$ levels of 8 and 9 . For temperature, the most growth occurred at $30^{\circ} \mathrm{C}$ with an average colony diameter of $5.4 \mathrm{~cm}$, while the least amount of growth was observed at $15^{\circ} \mathrm{C}$ with an average colony diameter of $1.5 \mathrm{~cm}$. These results, do not support the observation of disease development in cotton caused by $R$. solani in more alkaline soils, suggesting other factors may be contributing to the success of disease development in these soils. Future greenhouse studies will be used to examine the effect of soil texture and $\mathrm{pH}$ on disease development in cotton.

\section{Biological control potential of Bacillus velezensis with orange peel amendment against Meloidogyne incognita} M. HASSAN (1), M. Boersma (2), B. Lawaju (1), K. S. Lawrence (1), E. J. Sikora (3), M. Liles (4), J. W. Kloepper (1), (1) Entomology and Plant Pathology, Auburn University, Auburn, AL, USA; (2) Mass Spectrometry Center, Auburn University, Auburn, AL, USA; (3) Department of Entomology and Plant Pathology, Auburn University, Auburn, AL, USA; (4) Biological Sciences, Auburn University, Auburn, AL, USA

Bacillus velezensis $(\mathrm{Bv})$ strain AP203 was examined with and without orange peel (OP) amendment for their potential biological control of the root-knot nematode, $M$. incognita on soybean and cotton. LC-MS tests were performed to evaluate the bioactive metabolites produced by strain AP203 grown in media amended with OP. The overall hypothesis was that the model Bv strain, grown in OP amended media, will produce different bioactive metabolites and thereby will inhibit M. incognita in vitro and in planta. An in vitro test included the second-stage juveniles (J2) of M. incognita inoculated with Bv spores plus orange peel suspension (OPS), J2 with OPS, and non-inoculated control. The greenhouse test included seeds planted in soil then inoculated with cell-pellet suspension (CPS), culture broth (CB), and cell-free supernatant (CFS) of strain AP203 grown in OP amended media and a non-inoculated control. In vitro test results showed that spores AP203 with the OPS significantly increased J2 mortality compared to other treatments $(P \leq 0.05)$. The J2 mortality rate significantly increased with Bv strain AP203 plus the OPS (94\%) compared to the same strain without OPS (53\%), the OPS alone (59\%), and the non-inoculated control (7\%). The greenhouse test results showed that there were no significant differences between CPS, CB, or CFS of Bv strain AP203, but CPS, CB, or CFS significantly reduced the M. incognita population compared to the positive control. LC-MS/MS test results suggest that the combination of Bv strain AP203 and OPS produced four bioactive metabolites.

\section{Global cropland connectivity: A risk factor for invasion and saturation by emerging pathogens and pests} Y. Xing (1,2,3,4), J. F. Hernandez Nopsa (2,3,4,5,6), K. F. Andersen (1,2,3), J. L. Andrade-Piedra (7), F. Beed (8), G. Blomme (9), M. Carvajal-Yepes (10), D. L. Coyne (11), W. Cuellar (10), G. A. Forbes (7), J. F. Kreuze (7), J. Kroschel (12), P. L. Kumar (13), J. P. Legg (14), M. Parker (15), E. Schulte-Gelderman (15), K. Sharma (15), K. A. GARRETT (1,2,3,4), (1) Institute for Sustainable Food Systems, University of Florida, Gainesville, FL, USA; (2) Plant Pathology Department, University of Florida, Gainesville, FL, USA; (3) Emerging Pathogens Institute, University of Florida, Gainesville, FL, USA; (4) Department of Plant Pathology, Kansas State University, Manhattan, KS, USA; (5) Corporación Colombiana de Investigación Agropecuaria, AGROSAVIA, Mosquera-Bogotá, FL, COLOMBIA; (6) Corporación Colombiana de Investigación Agropecuaria, AGROSAVIA, Mosquera-Bogota, COLOMBIA; (7) CGIAR Research Program on Roots, Tubers and Banana, International Potato Center, Lima, PERU; (8) Plant Production and Protection Division (FAO), Rome, ITALY; (9) Bioversity International, c/o ILRI, Addis Ababa, ETHIOPIA; (10) International Center for Tropical Agriculture (CIAT), Cali, COLOMBIA; (11) International Institute of Tropical Agriculture (IITA), Nairobi, KENYA; (12) International Potato Center (CIP), Lima, PERU; (13) International Institute of Tropical Agriculture, Ibadan, NIGERIA; (14) International Institute of Tropical Agriculture, Dar es Salaam, TANZANIA; (15) International Potato Center, Nairobi, KENYA

The geographic pattern of cropland is an important risk factor for invasion and saturation by crop-specific pathogens and arthropods. Understanding cropland networks supports smart pest sampling and mitigation strategies. We evaluate global networks of cropland connectivity for key vegetatively-propagated crops (banana and plantain, cassava, potato, sweetpotato, and yam) important for food security in the tropics. For each crop, potential movement between geographic location pairs was evaluated using a gravity model, with associated uncertainty quantification. For example, in addition to locations with high risk due to high cropping density, high risk locations due to their role as bridges for cassava include locations in southcentral Nigeria, central Ghana, and southwestern Brazil. For potato, bridges occur in central and southern Poland and central and northern Ukraine. The highly linked hub and bridge locations in cropland connectivity risk maps are likely priorities for surveillance and management, and for tracing intra-region movement of pathogens and pests. Important locations are identified beyond those locations that simply have high crop density. Cropland connectivity risk maps provide a new risk component for integration with other factors - such as climatic suitability, genetic resistance, and trade routes - to inform Pest Risk Assessment and mitigation strategies for local and regional activities, and for global initiatives such as the Global Surveillance System (GSS).

\section{Development of an isothermal AmplifyRP XRT assay for rapid real-time detection of hop stunt viroid through recombinase polymerase amplification \\ S. ZHANG (1), B. Davenport (2), R. Li (2), J. Matousek (3), D. Groth-Helms (2), K. Schuetz (2), (1) Agdia Inc., Elkhart, IN, USA; (2) Agdia, Inc., Elkhart, IN, USA; (3) Institute of Plant Molecular Biology, České Budějovice, CZECH REPUBLIC}

Hop stunt viroid (HSVd), the type member of the genus Hostuviroid in the family Pospiviroidae, infects a wide range of economically important crops including apricot, cherry, citrus, grapevine, hop, peach and plum. HSVd occurs worldwide and its infection causes diseases such as hop stunt in hops and affects production and quality of host crops. Like any other viroids, HSVd does not code for any proteins and is a naked single-stranded RNA of approximately 297 nucleotides without encapsidation. Thus, there are no serological detection methods available and the few reported nucleic acid-based detection methods, such as reverse transcription-polymerase chain reaction (RT-PCR), require RNA purification and a thermal cycler. In this report, we describe development of a rapid, real-time isothermal AmplifyRP ${ }^{\circledR}$ XRT assay based on reverse transcription-recombinase polymerase amplification (RT-RPA). This AmplifyRP ${ }^{\circledR}$ 
assay utilizes crude plant extracts as template and a constant temperature of $42^{\circ} \mathrm{C}$ for all reactions including recombination, amplification and reverse transcription to occur in a single PCR tube. The assay results are reported in real time within 25 minutes using a portable, battery-operable fluorescence reader AmpliFire ${ }^{\circledR}$. This enables the assay to be deployable both in laboratories and in the field. Furthermore, the assay can specifically detect all three types (citrus-, hop- and plum-) of HSVd and has a detection sensitivity of as low as $10 \mathrm{fg}$ in vitro HSVd transcript. This is the first report of HSVd detection through real-time RT-RPA.

\section{Laboratory examination of Colorado potato cultivar field tolerance to blackleg disease} D. TAYLOR, Y. Zeng, A. O. Charkowski, Colorado State University, Fort Collins, CO, USA

Seed potato certification data collected in Colorado was used to rank potato cultivar tolerance to blackleg. Five cultivars with different tolerance levels (Chipeta $<$ Algeria $<$ Lamoka $<$ Classic Russet $<$ Yukon Gem) to blackleg were tested to determine if we could validate field data with laboratory assays. A strain isolated from Colorado, Pectobacterium atrosepticum CW1-4, and the P. atrosepticum type strain, 33260 , were used to infect plants through vacuum infiltration and stem inoculation. Disease was assessed with time-lapse video and by measuring lesion length and disease incidence. Through tuber vacuum infiltration, we demonstrated that $P$. atrosepticum 33260 and CW1-4 caused soft rot in all cultivars with different levels of tolerance (Lamoka $<$ Classic Russet $<$ Yukon Gem@Alegria $<$ Chipeta). The incidence of blackleg on the stems of the five cultivars also varied after vacuum infiltration (Lamoka $<$ Classic Russet $<$ Alegria $<$ Chipeta $<$ Yukon Gem). All cultivars were susceptible after stem inoculation with either strain and showed varying lesion lengths. Time-lapse video was used to assess Classic Russet and Yukon Gem. CW1-4 resulted in faster occurrence of disease symptoms than 33260 in Classic Russet. Blackleg incidence from either CW1-4 and 33260 in Yukon Gem was insignificant. These results indicate that relative susceptibility of the five cultivars to $P$. atrosepticum is significantly different than data gathered from field observations, suggesting that greenhouse assays do not accurately predict blackleg susceptibility in the field.

Leveraging genome-wide SNPs to understand emergence of boxwood blight disease

N. LEBLANC (1,2,3), M. A. Cubeta (2), J. A. Crouch (1), (1) USDA-ARS, Mycology and Nematology Genetic Diversity and Biology Laboratory, Beltsville, MD, USA; (2) North Carolina State University, Raleigh, NC, USA; (3) Oak Ridge Institute for Science and Education, Oak Ridge, TN, USA

Boxwood blight is an emerging disease of Buxus sp. first described in Europe in the 1990s prior to detection in Asia, New Zealand, and North America. The introduced clonal fungus Calonectria pseudonaviculata $(\mathrm{Cps})$ is the primary causal agent of this disease. Minimal genetic diversity in existing Cps molecular markers (AFLPs, housekeeping genes, and SSRs) limits our ability to identify genetic changes or migration events underlying the global expansion of boxwood blight. The goal of this research was to overcome this limitation through identification and analysis of genome-wide SNPs. Genomic data were generated from $67 \mathrm{Cps}$ isolates using the Illumina MiSeq, then aligned to a reference Cps genome assembly to identify SNPs. Genetic differentiation was evaluated using nonparametric clustering and phylogenetic methods. Models of divergence among genetic lineages were compared using approximate Bayesian computation. After quality filtering, 1607 SNPs were identified across the $54 \mathrm{Mb}$ reference genome. Four genetic lineages were identified, three of which were found on multiple continents. The most probable divergence model showed one of the lineages found in the U.S. was derived from an unknown geographic location, while the other resident lineage likely has a European origin. These results show that multiple clonal lineages have arisen in Cps populations since 1990s, the majority of which have migrated among continents. In addition, evidence of multiple origins of Cps in the U.S. indicate future introductions are likely to occur and should be considered in quarantine efforts.

The transcriptional regulator MucR from 'Candidatus Liberibacter asiaticus' mediates exopolysaccharides synthesis W. WANG (1), N. Wang (2), (1) University of Florida, Lake Alfred, FL, USA; (2) Citrus Research and Education Center, University of Florida, Lake Alfred, FL, USA

Huanglongbing (HLB), caused by Candidatus Liberibacter asiaticus (Las), is the most devastating disease of citrus worldwide. Las is vectored by Asian citrus psyllid Diaphorina citri. Las is a non-culturable bacteria that makes it difficult to understand the pathogenic mechanisms. Las has undergone reductive evolution and its genome size is much smaller than its relatives such as Sinorhizobium. Las has very few regulatory genes probably due to its lifestyle in the nutrient-rich environments of the phloem and in psyllids. Interestingly, Las encodes a transcriptional regulator MucR that has been reported to regulate the expression of transporter genes, control exopolysaccharide synthesis, and be involved in resistance to oxidative, detergent, and saline stresses and cell envelope modifications. We hypothesize that characterization of MucR of Las will help us understand the biology of Las. In this study, we used Sinorhizobium meliloti as a surrogate to investigate the function of MucR. MucR represses the exp genes for EPS II synthesis and activates the exo genes for EPS I synthesis in S. meliloti. In S. meliloti, mutation of mucR resulted in increased production of EPS II and reduced synthesis of EPS I. We will test whether the $m u c R_{\text {Las }}$ complements $m u c R$ mutant of $S$. meliloti. We will further characterize its targets in both $S$. meliloti and Las.

Acibenzolar-S-methyl controls bacterial blight disease of cabbage and Japanese radish by activating stomatal-based defense T. Ishiga (1), N. Sakata (1), T. Ugajin (2), S. Taniguchi (2), T. Hirata (2), Y. ISHIGA (1), (1) University of Tsukuba, Tsukuba, JAPAN; (2) Syngenta Japan, JAPAN

Bacterial blight disease of Brassicaceae caused by Pseudomonas cannabina pv. alisalensis (Pcal) is an economically important disease worldwide. Management of bacterial blight caused by Pcal based on copper fungicides and antibiotics resulted in the occurrence of a Pcal resistant strain, and severe bacterial blight outbreaks have been reported on cabbage in Japan. Therefore, there is an increasing 
demand to develop new bacterial blight management strategies. Plant defense activators not only protect plants from invading pathogens, but also help reduce the amount of copper fungicides and antibiotics. In this work, we focused on the well-known plant defense activator, acibenzolar-S-methyl (ASM), and cruciferous plants including cabbage and Japanese radish to control bacterial blight caused by Pcal. A soil drench with ASM effectively suppressed Pcal lesion formation which was associated with reduced bacterial populations on cabbage leaves. We also demonstrated that ASM controlled bacterial blight disease not only on the treated leaves, but also on the upper and lower leaves of Japanese radish. Interestingly, we found that ASM activated stomatal-based defense against Pcal, resulting in reduced bacterial populations as well as reduced disease symptoms on cabbage and Japanese radish leaves. These results indicate that ASM protects cabbage and Japanese radish from Pcal by activating stomatal-based defense.

\section{Determining the stage of maturation when fruit of Ilex serrata $\times$ I. verticillata become susceptible to colonization by Diaporthe ilicicola \\ I. EMANUEL (1), F. Peduto Hand PhD (2), (1) Ohio State University, Columbus, OH, USA; (2) Department of Plant Pathology, The} Ohio State University, Columbus, OH, USA

Diaporthe ilicicola is a major contributor to deciduous holly fruit rot (Ilex spp.). The fungus infects open flowers mid-Spring but symptoms do not develop until early-Fall. Changes in phenolic compounds during fruit maturation are negatively correlated with disease incidence, suggesting latent infections may result from changes in physiological factors as fruit ripen. This study expanded our understanding of this pathosystem by determining the point in the maturation process disease resistance was lost and fruit became susceptible to colonization by $D$. ilicicola. An in vitro bioassay was conducted with Ilex serrata $\times I$. verticillata cv. Sparkleberry fruit from outdoor potted plants collected at 13 timepoints (June to November 2019) from two locations. At each collection, 20 fruit were selected randomly from each experimental unit, surface sterilized, wounded, and inoculated with a spore suspension of $D$. ilicicola $\left(10^{5}\right.$ spores $/ \mathrm{ml}$ ) or water. Fruit were incubated in moist chambers in a randomized complete block design and monitored for the formation of pycnidia, indicating the pathogen's ability to colonize the tissues. By late-summer, detached fruit lost their resistance while fruit that developed from flowers inoculated at bloom in the outdoor trials, and that were not detached from the plants, remained asymptomatic until early-Fall, suggesting additional factors contribute to delayed symptom development. Future research will investigate metabolites contributing to resistance in immature fruit and putative relationships between environmental factors and symptom development.

Population structure, evolution, and detection of Lychnis mottle virus in peony

C. SHAFFER (1), D. Michener (2), N. B. Vlasava (3), I. E. Tzanetakis (1), (1) University of Arkansas, Fayetteville, AR, USA; (2) University of Michigan, Ann Arbor, MI, USA; (3) Central Botanical Gardens NAS, Minsk, BELARUS

Lychnis mottle virus (LycMoV) has been detected in $\sim 40 \%$ of the more than 300 peonies tested. The population structure of the virus was elucidated after sequencing the RNA 2 of 48 isolates collected across the United States. Phylogenetic analyses revealed a strong correlation between host and virus isolates, with nearly all isolates grouping with their respective host. The virus population is very homogeneous, and proteins are under strong purifying selection. The sequence information generated was used to design detection primers able to detect all peony isolates. Peony is an excellent example of how ornamentals can act as a vehicle of virus dispersal. For this reason, ornamentals must be screened because they may harbor asymptomatic viruses which may cause an epidemic if they jump to a susceptible host.

Crops in rotation with potato that predict the occurrence of mefenoxam-resistant Pythium species I. KUMBHAKAR (1), M. Kleber (2), K. Frost (1,3), (1) Oregon State University, Department of Botany and Plant Pathology, OR, USA; (2) Oregon State University, Department of Crop and Soil Sciences, Corvallis, OR, USA; (3) Oregon State University, Hermiston Agricultural Research and Extension Center, Hermiston, OR, USA

The crop history of a field can significantly influence the population biology of soil-borne pathogens, affecting their abundance, diversity, and development of resistance to fungicides. Mefenoxam is a systemic fungicide commonly used for potato and crops in rotation with potato to prevent diseases caused by Pythium. Resistance to mefenoxam has developed in several oomycete pathogens important to potato and we hypothesized that mefenoxam-resistance relates to a field's cropping history. Soils were collected from 35 georeferenced potato fields in the Pacific Northwest. Estimates of Pythium spp. and mefenoxam-resistant Pythium spp. (CFU/g soil) were obtained by culturing soil. The USDA Cropland Datalayer was used to determine the 10-year cropping rotation and crop frequency for each field. Correlation and regression analyses were used to determine which crop frequencies described the most variation in the proportion of mefenoxam-resistant Pythium spp. in a field. The abundance of mefenoxam-resistant Pythium was correlated with the rate of wheat $(\mathrm{R}=0.35, \mathrm{p}<0.04)$, sweet corn $(\mathrm{R}=0.44, \mathrm{p}<0.01)$, pea $(\mathrm{R}=0.32, \mathrm{p}<0.06)$, and field corn $(\mathrm{R}=-0.41, \mathrm{p}<0.02)$ in the rotation. A 2-variable regression model with sweet corn and pea described approximately $30.1 \%$ of the variation in proportion of mefenoxam-resistant Pythium $(\mathrm{F}=6.894 ; \mathrm{df}=2,32 ; \mathrm{p}=0.003)$ present. Our investigation suggests that crop rotations can be optimized to reduce fungicide resistance development and emphasizes the usefulness of long-term management records for the development of health management strategies.

Defensive response of loblolly pine (Pinus taeda) to fungal symbionts in the Southern Pine Beetle (Dendroctonus frontalis) System

J. G. DE SOTO (1), K. Gandhi (2), K. D. Klepzig (3), C. Villari (2), (1) University of Georgia, GA, USA; (2) D.B. Warnell School of Forestry, University of Georgia, Athens, GA, USA; (3) The Jones Center at Ichauway, Newton, GA, USA 
Loblolly pine (Pinus taeda) is a key species in southeastern pine plantations and accounts for most of the timber harvested in the southern US. Its most destructive pest is southern pine beetle (Dendroctonus frontalis) (SPB), which is associated with two mutualistic fungi, Ceratocystiopsis ranaculosus and Entomocorticium sp. A, serving as nutrient sources for SPB larvae. The beetles also carry a reportedly antagonistic species, Ophiostoma minus, that competes for space with the other fungi and SPB larvae. Direct competition with the two mutualists is likely the main reason for the antagonistic behavior of $O$. minus, but other mechanisms may exist. We hypothesize that $O$. minus, a moderately virulent pathogen, triggers a stronger defense response in the host tree than mutualists. This tree defense response is likely detrimental to mutualists, as they are not pathogens adapted to such induced defenses. Our objectives are to determine: (i) tree defense compounds (i.e. terpenoids and phenolics) induced in mature loblolly pine after inoculation with the three SPB associated fungal species, and (ii) the susceptibility, tolerance, or detoxification ability of fungi each against compounds identified in objective (i). A pine stand was selected at Whitehall Forest (Athens, Georgia), and 50 trees were inoculated. Phloem samples were collected before and after inoculation, lesion length measured, and defense compounds are being analyzed. Results will help elucidate and improve our understanding of the chemical ecology and complex interactions in the SPB system.

The healthy phyllosphere microbiome of greenhouse crops

M. LEGEIN (1), R. Samson (2), S. Lebeer (2), (1) UAntwerpen, Antwerpen, BELGIUM; (2) UAntwerpen, BELGIUM

The phyllosphere, the above-ground surface of plants that are in contact with the atmosphere, is inhabited by a diverse community of bacteria, fungi and other micro-organisms. These micro-organisms interact closely with the plant and can have an effect on plant health and plant growth. Beneficial bacteria can inhibit pathogen growth directly, trough microbe-microbe interactions or indirectly, by triggering the plants defence system. Phyllosphere bacteria can also enhance plant growth through the modulation of plant hormones. This research aims to gain insights into the taxonomic composition of a healthy phyllosphere microbiome and what factors shape these communities. The phyllosphere communities from seven tomato cultivars and three strawberry cultivars were sampled during eight weeks at two research greenhouses. 16s rRNA gene amplicon sequencing was used to determine the bacterial community structure of the phyllosphere and its dynamics over time. The bacterial communities on the leaves were highly variable over time and in space. This is in contrast with the stable greenhouse environment that is highly controlled to minimize biotic and abiotic stresses on the crops. In addition, the diversity of the communities was low. These results suggest that the bacteria on the phyllosphere did not reach a stable community. Interestingly, a high amount of bacteria on the leaves were associated with the insects present in the greenhouse. This indicates that insects play an important role in dispersing bacteria and shaping phyllosphere communities.

\section{The diverse mycoflora present on dried cannabis (Cannabis sativa $\mathrm{L}$.) inflorescences in commercial production} Z. K. PUNJA, Simon Fraser Univ, Burnaby, BC, CANADA

Harvested inflorescences (buds) of cannabis (Cannabis sativa L.) were assessed for fungal presence and diversity in drying rooms of three licensed production facilities in British Columbia during 2017-2019. Sterile cotton Q-tips were gently swabbed over the bud surfaces and streaked onto potato dextrose agar $+140 \mathrm{mg} / \mathrm{L}$ streptomycin sulfate. Petri dishes were incubated at $21-24^{\circ} \mathrm{C}$ for $5-6$ days and developing colonies were enumerated. Representative colonies of each unique morphological type were identified to genus and species by PCR of the ITS1-5.8-ITS2 region of rDNA and sequence analysis. The testing was repeated to provide $>40$ cumulative sampling times over a 2-yr period. Among the 33 different fungal species identified, the most prevalent group was Penicillium (comprising 17 different species), followed by species of Cladosporium, Botrytis, Aspergillus, Fusarium, Talaromyces and Alternaria. All samples had several fungal species present. The swab method provided a qualitative assessment of viable mold contaminants on cannabis buds and reflected the diversity of mycoflora present, many of which are previously unreported. The number and species composition varied at different sampling times and also within different facilities. Fungi on cannabis buds could originate from spores released from diseased or decomposing plant materials, from the growing substrates used in cannabis production, or as air-borne contaminants in the post-harvest trimming and drying rooms. Exposure of dried buds to electrobeam (e-beam) radiation reduced fungal contamination to undetectable levels.

Bacterial biocontrol agents against fungal and oomycete pathogens of rice and other field crops S. A. SHARFADINE (1), L. Delgado (2), S. A. Winters (1), C. M. Rojas (1), A. Rojas (1), (1) University of Arkansas, Fayetteville, AR, USA; (2) Universidad de los Andes, Bogota, COLOMBIA

Rice is the primary staple for more than half of the global population and is the second most important cereal worldwide. In the United States, Arkansas is the major producer of rice and this crop is affected by several fungal and bacterial diseases. The major diseases that affect the rice crop are the sheath blight whose causal agent is Rhizoctonia solani; rice blast by Magnaporthe oryzae; Fusarium graminearum, the causal agent of the fusarium head blight in wheat, but it also infects rice; and the oomycete Pythium sylvaticum, which causes seedling diseases in this cereal. Most management tactics for these diseases rely on the use of chemicals and tolerant cultivars. The use of novel strategies to manage these disease could provide new approaches to minimize the impact on yields of this important crop. Recently, two potential biological control agents (BCA) were identified in Arkansas, Pseudomonas protegens and Burkholderia cenocepacia, showed inhibitory effect against the panicle blight pathogen B. glumae. The aim of this study was to determine the potential biocontrol activity of these BCAs against the prevalent fungal diseases on rice. These BCAs were challenged in dual culture assays against $R$. solani, $F$. graminearum and $P$. sylvaticum on three different media optimizing the biocontrol effect. The results showed an antagonistic activity of $B$. cenocepacia against the panel of fungi evaluated, suggesting that this bacteria could be used as a biocontrol agent. An extra set of seven bacteria are currently being tested for their biocontrol potential. 
Fungal community on lodgepole pine (Pinus contorta) needles changes in the presence of Lophodermella needle cast pathogens in Colorado, USA

J. P. ATA (1), J. Ibarra Caballero (2), K. Burns (3), S. Marchetti (4), J. J. Worrall (4), Z. Abdo (1), J. Stewart (1), (1) Colorado State University, Fort Collins, CO, USA; (2) Colorado State University-Agricultural Biology, Fort Collins, CO, USA; (3) USDA Forest Service, Golden, CO, USA; (4) USDA Forest Service, Gunnison, CO, USA

Plant species host a diverse community of foliar fungal endophytes, whereby many species have unknown ecological functions. With the increasing needle disease incidence worldwide, do foliar endophytes play an important role in plant protection from pathogens? Studying the community structure and diversity of these endophytes amidst various environmental conditions will provide clues for understanding their ecology. The change of fungal endophytic communities of conifer needles after infection by needle pathogens have yet to be characterized. Metagenomics and metatranscriptomics allow characterization of hidden microbial diversity and the identification of potential genes expressed and exchanged between host and microbes. This study aims to understand the interaction of Lophodermella needle cast pathogens, Lophodermella concolor and L. montivaga, with the needle mycobiome on lodgepole pine (Pinus contorta) using next-generation sequencing. We hypothesize that community structure differs significantly between symptomatic and asymptomatic needles where fungal endophytic diversity in symptomatic needles are lower compared to the asymptomatic ones. Further, this also helps elucidate whether or not Lophodermella species are latent pathogens shifting from an endophytic lifestyle. Results of this study provide additional insights on host-pathogen-microbe interactions and may also aid in developing efficient disease management strategies using beneficial microbial communities.

Occurrence and host associations of an Armillaria root disease pathogen, Armillaria gallica, in Central Mexico E. Duarte-Mata (1), R. D. Elías-Román (1), J. W. Hanna (2), M. S. Kim (3), N. B. KLOPFENSTEIN (2), (1) Department of Agronomy, DICIVA, CIS, University of Guanajuato, Irapuato, MEXICO; (2) USDA Forest Service, Rocky Mountain Research Station, Moscow, ID, USA; (3) USDA Forest Service, Pacific Northwest Research Station, Corvallis, OR, USA

During summer of 2019, preliminary root surveys were conducted for Armillaria associated with symptomatic and non-symptomatic Quercus, Pinus, and Malus species in the Central Mexico states of Jalisco, Guanajuato, and Michoacan. Rhizomorph samples were collected from seven trees and established in culture. Based on translation elongation factor 1-alpha sequence comparisons and somatic pairing tests against known tester isolates, all collected isolates were identified as $A$. gallica. In Jalisco, A. gallica was associated with root disease signs/symptoms (e.g., mycelial fans, wilting, chlorosis) on Pinus pseudostrobus and symptomless $P$. devoniana. In Michoacan, A. gallica was associated with root disease-killed Malus domestica, and root diseased P. devoniana and $P$. teocote. In Guanajuato, A. gallica was associated with root disease of Quercus jonesii. Ecological behavior of Armillaria differs both within and among species, and Armillaria root disease is predicted to become more damaging due to climate change and host tree maladaptation. Armillaria roots disease poses a major threat to woody plants in forests, orchards, and urban areas of Central Mexico. For these reasons, it is essential to continue documenting the current distribution and host associations of Armillaria spp., so that appropriate management strategies can be developed and implemented.

Utilizing cover crop residues as carbon sources for anaerobic soil disinfestation in tomato J. GARCIA GONZALEZ (1), J. Pollok (2), M. Reiter (3), S. L. Rideout (2), (1) ESAREC, Virginia Tech, Painter, VA, USA; (2) Virginia Tech - Eastern Shore AREC, Painter, VA, USA; (3) Virginia Polytechnic Institute \& State University, Painter, VA, USA

Anaerobic soil disinfestation (ASD) is an alternative ecological strategy to control soilborne pathogens. Identifying suitable local carbon sources is crucial for ASD to be successful. A field trial was laid out in randomized block design with four replicates in 2019 on sandy loam soils. Cereal rye (Scale cereal L.), rapeseed (Brassica napus L.), and hairy vetch (Vicia villosa Roth) were grown and incorporated into the soil $\left(2.5,1.3\right.$, and $1.2 \mathrm{Mg} \mathrm{ha}^{-1} \mathrm{DW}$, respectively), and compared to pelletized poultry litter $\left(10.6 \mathrm{Mg}\right.$ ha $\left.{ }^{-1} \mathrm{DW}\right)$ and a nonamended soil control (aerobic and anaerobic). Raised-beds were covered with polyethylene mulch and 20-day ASD treatment was applied. Tomato seedlings ('BHN602') were transplanted 9 days post-ASD. Soilborne diseases did not develop during the growing season. There were no significant differences in final weed counts; however, nutsedge counts were significantly lower (72\%) in all ASD treatments compared to aerobic control 13 days post-ASD. Plant vigor was significantly better for pelletized poultry litter (9.3), followed by hairy vetch (7.8). Total marketable yield was significantly greater for the pelletized poultry litter $\left(7.9 \mathrm{Mg}^{-1}\right)$, followed by hairy vetch $\left(4.3 \mathrm{Mg} \mathrm{ha}^{-1}\right)$, while intermediate yield was observed but higher than the aerobic control for ryegrass and rapeseed (2.2 -2.8 $\mathrm{Mg} \mathrm{ha}^{-1}$ ). The ASD effect on tomato crops varied among carbon sources. Poultry litter and legumes exhibited increased plant vigor and marketable yield; which helps to elucidate effective carbon sources for ASD in plasticulture systems in the mid-Atlantic region.

Characterization of antifungal activity of plant endophytic bacterium for control of aflatoxin contamination J. JIA, E. Ford, S. Baird, S. Lu, Mississippi State University, Mississippi State, MS, USA

Aflatoxins are one group of the major mycotoxins and produced by the fungi including Aspergillus flavus, which contaminate agricultural products and thread food safety. Different strategies have been used to prevent aflatoxin contamination in field and storage conditions. Strain A was isolated from charcoal rot associated soybean plants and identified as a member of Burkholderia pyrrocinia using multilocus sequence typing. Strain A shows a significant antimicrobial activity against common soil-borne fungal and bacterial pathogens, including A. flavus. Genomic analysis revealed that the genome of strain A consists of three chromosomes and one plasmid. Mutants MT36 (ORF11::Tn5) and MT37 (ORF17::nptII), which were generated using a random mutation and site-specific approach, respectively, lost its antifungal activity against $A$. flavus as compared with the wild type strain. A 57-kb genetic locus harboring the genes disrupted in two mutants shares $90.77 \%$ identity to the ocf gene cluster of Burkholderia contaminans MS14. The ORF17 was predicted to encode a LuxR regulatory protein, which shares $87.23 \%$ identity to the LuxR family transcriptional regulator AmbR 1 of $B$. 
contaminans MS14. Quantitative PCR and RNASeq analyses demonstrated that transcription of the NRPS/PKS genes of the gene cluster was significantly reduced (5.57-8.95 fold lower) in the mutant MT37 as compared with that of the wild type strain. This work will provide potential for development of bacterial antagonists as biological control agents and agricultural fungicides.

Etiology and phenology of infection by Anthracnose blight disease in California avocado orchards H. F. AVENOT (1), D. Felts (2), J. Lake (3), D. Vega (4), A. Tako (5), T. J. Michailides (6), (1) University of California, Kearney Agricultural Research \& Extension Center, Parlier, CA, USA; (2) UC Davis, Parlier, CA, USA; (3) California State University, Fresno, Fresno, CA, USA; (4) California State University, Pomona, Ventura, CA, USA; (5) Agricultural University of Athens, Greece, GREECE; (6) University of California Davis, Kearney Agricultural Research \& Extension Center, Parlier, CA, USA

Colletotrichum spp., specifically C. gloeosporioides is typically a postharvest pathogen that causes avocado fruit rot. However, recent sampling of avocado orchards and analyses of branch cankered tissues revealed the presence, although at a lesser magnitude, of Colletotrichum spp. in addition to that of Botryosphaeria spp. There is no evidence that Colletotrichum invasion of avocado branches is pathogenic nor there is information on their diversity, geographical distribution, phenology of infection and contributing factors. Thus, their possible contribution and the epidemiology of avocado branch canker need to be investigated. Using DNA sequence data of $\beta$ tubulin 2, ApMAT, and GAPDH genes, several Colletotrichum (from the C. gloeosporioides species complex) were identified to species from symptomatic tissues, with $C$. fructicola being the most common species associated with anthracnose blight. Pathogenicity assays on potted trees (cv. Hass) using a mycelium plug placed in wounded stems of trees showed that two months after inoculations, Colletotrichum species caused lesions on the inoculated branches, with $C$. fioriniae (isolated from pistachio) being highly virulent on avocado stems. Similar inoculations in the field showed that Colletotrichum could colonize the xylem tissues two months after inoculation and throughout the sampling period, regardless of the phenological stage of avocado.

\section{Impact network analysis: A framework for evaluating regional disease management strategies} K. A. GARRETT, Plant Pathology Department, University of Florida, Gainesville, FL, USA

Managing the regional spread of pathogens is key to agricultural productivity and protection of wildlands. The success of regional disease management depends on the decisions of many types of stakeholders who may have different priorities. Impact network analysis (INA) is a framework for evaluating how disease management programs and technologies may need to be modified for regional success. INA evaluates regional scenarios composed of (1) management technologies, (2) socioeconomic networks that influence technology adoption, and (3) biophysical networks of pathogen dispersal. This poster introduces the INA package in the R programming environment, which provides tools for scenario analysis to evaluate the likely outcomes from a range of regional management strategies, for application before, during, and after regional management projects. These strategies may include investment in surveillance, communication and capacity development, subsidies supporting management technology adoption, and improvements to the management technologies to make them more effective. The INA framework is designed to provide input for decision-making by users such as extension agents, policymakers, and development organizations. More generally, INA is designed for a community of practice of researchers and practitioners who address similar problems in regional management of invasive pathogens, pests, and other species or important subpopulations such as antibiotic/pesticide-resistant populations. This poster provides examples of the application of INA and links to other examples and resources.

\section{Nematode resistance in cowpea and its effects on rhizobial symbiosis} G. ORTIZ, S. Neal, F. Khairi, J. Sachs, University of California Riverside, Riverside, CA, USA

Breeding for disease resistance in crops is critical to reduce disease incidence and pathogen populations while enhancing other fundamental traits that increase yield or that improve plant performance. However, there are often costs to resistance associated with these traits, especially when defense mechanisms can affect associations with beneficial partners. Root knot nematodes infect plant hosts by entering roots and using their stylets to create feeding sites, called giant cells, where cells differentiate into tumor-like nutrient sinks used by the pathogen. On this same tissue, legumes evolved a mutualistic interaction with rhizobia, where bacteria instigate the formation of tumor-like structures called nodules wherein they fix atmospheric nitrogen for the plant in exchange for photosynthates. We investigated whether breeding for resistance against root knot nematodes degrades symbiosis between legumes and rhizobia. We tested four near-isogenic cowpea genotypes that differed in their resistance genes against root knot nematodes and inoculated them with two root nodule forming Bradyrhizobium strains that were either efficient or inefficient nitrogen fixers. We measured and compared host performance traits and host investment into symbiosis for the four genotypes. Our data uncovered no evidence of costs to resistance to root-knot nematodes, as there was no performance difference among the plant genotypes tested. These data suggest that cowpeas exhibit separate genetic and physiological pathways to regulate beneficial and harmful associates of roots.

\section{Evolution Xanthomonas euvesicatoria, a major pathogen of pepper}

F. IRUEGAS-BOCARDO, S. Timilsina, G. V. Minsavage Jr., J. B. Jones, E. M. Goss, University of Florida, Gainesville, FL, USA

Bacterial leaf spot (BLS) is one of the most destructive diseases of tomato and pepper in Florida. Xanthomonas euvesicatoria (Xe), one of four taxonomic groups causing the disease, was prevalent in both hosts until the 1980s. By 1982, the pathogen experienced race shifts in pepper. In the $1990 \mathrm{~s}, X e$ was displaced from tomato by $X$. perforans $(X p)$. To better understand the evolutionary and demographic history of $X e$ in Florida, we analyzed whole-genome sequences of 76 strains collected from tomato and pepper in Florida over 53 years, and 33 strains available in public databases that were collected worldwide. We used core genome sequences to reconstruct the consensus phylogeny of $X e$. To better understand $X e$ host-pathogen interactions, we examined effector variation over time. Our 
phylogenetic tree shows multiple clades in Florida, consistent with multiple introductions of strains. We also found some genetic separation of tomato and pepper strains. Selection analysis of Xe effectors, revealed 12 effectors with at least 1 site under positive selection (XopK, XopAA, XopAF, XopAD, XopAE, XopP, XopAP, XopE1, XopF1, XopM, XopAU and AvrBs2), and 5 effectors strongly conserved (XopAX, XopB, XopD, XopE3 and AvrBs1). Our results advance our knowledge of the evolution of Xe on pepper and tomato and point to genes potentially relevant for pathogenicity that can inform breeding and management approaches of BLS pathogens affecting pepper in Florida.

Pythium species associated with crown and root rot on cannabis (Cannabis sativa L., marijuana) plants grown under commercial greenhouse conditions

Z. K. PUNJA, Simon Fraser Univ, Burnaby, BC, CANADA

Cannabis (Cannabis sativa L., marijuana) plants with symptoms of crown rot, root decay, wilting and plant death were sampled during 2018 and 2019 from seven greenhouses. Tissue pieces from 140 diseased plants were surface-sterilized and plated onto potato dextrose agar. Colonies of Pythium species displaying unique morphologies were identified to genus and species by PCR of the ITS1-5.8-ITS2 region of ribosomal DNA followed by sequence analysis. Five Pythium species were recovered (in order of decreasing frequency) $: P$. myriotylum, $P$. dissotocum, $P$. catenulatum, $P$. aphanidermatum and $P$. ultimum. All species were present in licensed production facilities in British Columbia, $P$. dissotocum was found in two facilities in Ontario, and $P$. myriotylum was present in one facility in northern California. Phylogenetic analyses clearly separated the different species and they grouped with isolates from different hosts and geographical regions worldwide. On PDA, growth of $P$. ultimum, $P$. aphanidermatum and $P$. myriotylum was the fastest, $P$. dissotocum was intermediate, and $P$. catenulatum was slow growing. Pathogenicity tests conducted on rooted cuttings in a coco:vermiculite potting medium demonstrated that all species caused root browning and decay and stunting of plants, while $P$. aphanidermatum, P. myriotylum, and P. ultimum were the most aggressive and caused plant death. Wounding of the roots and prolonged periods of saturation enhanced disease development. Pythium catenulatum is reported for the first time to cause root browning and stunting and yellowing of cannabis plants.

Assessing Medicago spp. genetic resources for disease reaction to Phoma medicaginis, causal agent of spring black stem and leaf spot of alfalfa

B. M. IRISH (1), S. Shellenberger (2), E. Cervantes (2), L. D. Porter (3), D. A. Samac (4), (1) USDA ARS Western Regional Plant Introduction Station, Prosser, WA, USA; (2) USDA, WA, USA; (3) USDA ARS, Prosser, WA, USA; (4) USDA ARS, St Paul, MN, USA

Alfalfa (Medicago sativa) is the fourth most widely grown agricultural crop and the most significant forage in the U.S. Alfalfa diseases reduce yield and quality impacting production and producers' economic gains. Spring black stem and leaf spot, caused by Phoma medicaginis, is an important fungal leaf spot pathogen for which good resistance is lacking in commercial cultivars. To identify potential sources of resistance, alfalfa germplasm was screened in greenhouse seedling inoculation protocols. Several isolates were evaluated for pathogenicity and spore concentrations were determined for ideal level of disease pressure. A modified rating scale incorporating additional half values was developed to account for differences observed in disease phenotypes. Following these modifications, a group of 80 standard check alfalfa cultivars and 189 alfalfa-related Medicago species accessions were screened for disease reaction in replicated randomized complete block designs. Preliminary results suggest that an inoculum concentration of 50,000 spores/ml was ideal as at this concentration susceptible 'Lahontan' and moderately resistant 'Ramsey' standard check cultivars performed as expected. 'Travois' and '5411' appeared to be more resistant than 'Ramsey' with several of the related Medicago species showing very little disease. Additional accessions are currently being evaluated for disease reaction and resistant germplasm will be selected for development of advanced populations. The modified protocol, summarized data, and resistant germplasm will become publicly available.

Virulence, aggressiveness, and fungicide sensitivity of Phytophthora spp. associated with root rot of soybeans in Illinois D. CERRITOS-GARCIA, S. Y. Huang, N. M. Kleczewski, S. X. Mideros Mora, University of Illinois, Urbana, IL, USA

Phytophthora root rot is one of the most devastating oomycete diseases of soybean in the Midwest U.S. Phytophthora sojae is the main pathogen associated with this disease, but recent reports indicate that Phytophthora sansomeana could also cause root rot on soybeans. The objectives of this study were to evaluate virulence, aggressiveness and fungicide sensitivity for isolates of these two oomycetes from Illinois. Baiting of soils was used to obtain 24 isolates of $P$. sojae and 7 of $P$. sansomeana from surveys conducted from $2016-$ 2018. Pathogenicity of all isolates was evaluated using the hypocotyl inoculation technique in 13 soybean differentials. Phytophthora spp. aggressiveness was evaluated in layer tests by measuring root dry weight, root length, and root area. Aggressiveness assays were performed in the greenhouse with an average temperature of $24.3^{\circ} \mathrm{C}\left(20.9-29.6^{\circ} \mathrm{C}\right)$ and 16 hours photoperiod. Sixty percent of $P$. sojae isolates were virulent to Rps $1 \mathrm{k}$ and thirty percent were virulent to Rps 1a and Rps $1 \mathrm{c}$. No consistent results were observed for virulence of $P$. sansomeana. P. sojae isolates were more aggressive compared to $P$. sansomeana under the conditions of this study. No insensitive isolates to mefenoxam, metalaxyl, azoxystrobin or ethaboxam were observed based on EC50 values.

In vitro screening of fungicides against Colletotrichum theobromicola, causal agent of boxwood dieback H. KAUR, R. Singh, Louisiana State University AgCenter, Baton Rouge, LA, USA

Boxwoods are one of the most widely used ornamental plants in home and commercial landscape and are infected by several plant diseases. Recently, a new disease called boxwood dieback, caused by Colletotrichum theobromicola, was first identified in Louisiana in 
2015. Since then the disease was reported from several U. S. states. This disease can be confused with others such as Phytophthora root rot. Although current management practices include removing symptomatic plants and disinfesting pruning tools in nurseries, information on fungicides for managing boxwood dieback is lacking. Consequently, fungicides that were ranked good to excellent in effectiveness against Colletotrichum species were tested based on reports from 2018 Guide to Ornamental Diseases. Efficacy tests were conducted against $C$. theobromicola isolate PDC14313 with seven fungicides at 0, 0.1, 1, 5, 10, 25 and 50 ppm of active ingredient and LD 50s were calculated after 7 days. All fungicides applied at $0.1 \mathrm{ppm}$ were not effective. Azoxystrobin + benzovindiflupyr, difenoconazole + pydiflumetofen, fluoxapyroxad + pyraclostrobin and pyraclostrobin + boscalid showed 78\%, 78\%, 56\% and 54\% mycelial growth inhibition at $1 \mathrm{ppm}$, respectively. Thiophanate-methyl + iprodione showed $79 \%$ reduction at 5 ppm, whereas propiconazole + chlorothalonil inhibited mycelial growth by $56 \%$ at $10 \mathrm{ppm}$. Fluopyram + trifloxystrobin was least effective with an inhibition rate of $53 \%$ at $50 \mathrm{ppm}$. Further testing includes screening of C. theobromicola isolates collected from different U. S. states as well as in vivo screening of fungicides.

Investigating the sensitivity of Phytophthora infestans isolates to copper hydroxide P. M. SPYCHALLA, T. Wu, Y. Chen, A. J. Gevens, University of Wisconsin-Madison, Madison, WI, USA

Phytophthora infestans is an oomycetous plant pathogen that causes late blight in potatoes and tomatoes. For certified organic production systems, copper-containing fungicides are considered the most effective in managing disease. The sensitivity of $P$. infestans populations to copper has not been routinely assessed. To better inform management practices, an amended media assay was conducted to characterize the sensitivity of $P$. infestans isolates to copper hydroxide. We evaluated the growth response of 107 isolates collected in Wisconsin from diseased potato and tomato foliage from the 2017-2019 growing seasons on Rye A media amended with varying concentrations ( $300 \mathrm{ppm}, 100 \mathrm{ppm}, 50 \mathrm{ppm}, 10 \mathrm{ppm}$ and $0 \mathrm{ppm}$ ) of copper hydroxide. After a two-week incubation period at $18 \mathrm{C}$ and under ambient lighting, radial mycelial growth and mycelial biomass were measured and compared to the controls. Results indicated that all $P$. infestans isolates were sensitive to copper hydroxide at $300 \mathrm{ppm}$, which is equivalent to the highest label field application rate. Contemporary $P$. infestans isolates from Wisconsin are sensitive to copper hydroxide, making this fungicide a good, continued option for late blight management in tomato and potato crops.

Effects of Tsunami 100 treatment on cucumber green mottle mosaic virus (CGMMV) viability R. PEDROZO, D. A. Mayfield, T. Bruns, G. P. Munkvold, Iowa State University, Ames, IA, USA

CGMMV can severely reduce fruit yield and quality in cucurbits, especially in cucumber, melon, and watermelon crops. Tsunami 100 is used as a seed disinfectant and there are some claims that it can affect seed transmission of viruses. The objective of this study was to assess and describe the impacts of Tsunami 100 treatment on the integrity and infectivity of CGMMV. Purified CGMMV particles were exposed to Tsunami 100 at $1.2 \%$ for 15 minutes. Particles treated with water and hydrochloric acid (5\%) were used as negative and positive controls, respectively. Treated particles were examined for the integrity of virus coat protein using transmission electron microscopy (TEM). Random breakages were observed in some particles treated with Tsunami 100 when compared with the negative control particles, which did not display any visual damage. The coat protein of particles treated with $\mathrm{HCl}(5 \%, 15 \mathrm{~min})$ however was completely denatured. Furthermore, bioassays were used to test the infectivity of treated particles on Cucumis sativus (Cucumber), cv. 'Straight $8^{\prime}$ and tests plants were maintained in growth chamber at $25^{\circ} \mathrm{C}$. The development of typical leaf symptoms and systemic spread of the virus was assessed 25 days post inoculation. Leaf symptoms and systemic spread of the virus were observed in plants inoculated with the water- and Tsunami 100-treated particles only. Tsunami 100 at this concentration and exposure time does not appear to be an effective treatment for reducing CGMMV transmission.

\section{Control of Globodera pallida using Brassica juncea seed meal extract and the trap crop Solanum sisymbriifolium B. BHATTA, L. M. C. Dandurand, M. J. Morra, I. Popova, University of Idaho, Moscow, ID, USA}

Globodera pallida, commonly known as the pale cyst nematode, is a regulated potato pest that potentially causes negative economic impacts. Individually, the trap crop species Solanum sisymbriifolium and biofumigation by Brassica juncea seed meal extract are effective in controlling G. pallida. However, the large amount of $B$. juncea extract needed may prove technically difficult for large-scale field application. Although the trap crop S. sisymbriifolium induces hatch of G. pallida, a small reserve of viable eggs remaining after the trap crop could potentially serve as a nematode reservoir in the subsequent growing season. Greenhouse and field experiments were conducted to evaluate the efficacy of B. juncea seed meal extract alone versus when combined with S. sisymbriifolium to control G. pallida. Six rates $(0,125,250,500,1000$, and $2000 \mathrm{lbs} /$ acre $)$ of $B$. juncea extract in the greenhouse and three rates $(0,1000$, and 2000 $\mathrm{lbs} / \mathrm{acre}$ ) in the field were tested. Fewer encysted eggs were found after treatment with S. sisymbriffolium alone than when in combination with biofumigation implying that the nematodes hatched in the presence of the trap crop, but that the biofumigant inhibited hatch of the nematode at all rates. Hatch and reproduction of the nematode were further reduced when $B$. juncea extract applied at a rate of $125 \mathrm{lbs} /$ acre was combined with $S$. sisymbriifolium. Results demonstrate that lower doses of $B$. juncea seed meal extract in combination with $S$. sisymbriifolium reduce G. pallida populations more effectively than the individual treatments.

ToxA presence and haplotype diversity in the Kansas Bipolaris sorokiniana population

D. MANGEL (1), M. Bruce (1), E. D. De Wolf (1), R. L. Bowden (2), J. Rupp (1), (1) Kansas State University, Manhattan, KS, USA; (2) USDA ARS, Manhattan, KS, USA

Kansas wheat fields are challenged with multiple pathogens under field conditions. The wheat leaf spot complex is an active pathogen group that is quickly evolving. The most virulent pathogen in the complex is Pyrenophora tritici-repentis, the causal agent of tan spot. 
There is strong evidence that $P$. tritici-repentis emerged as an economically important wheat pathogen around 1940 upon receiving the host selective toxin gene ToxA through horizontal transmission. There is also evidence that the same toxin responsible for the emergence of the $P$. tritici-repentis as an important pathogen has recently been transferred to the pathogen Bipolaris sorokiniana. Our research objective was to characterize the leaf spot complex, determine if $B$. sorokiniana present in Kansas wheat production contained ToxA, and if its ToxA haplotype matched those previously reported. A statewide survey of leaf spot pathogens in the Kansas wheat production region has yielded leaf spot isolates from 55 counties. Isolates were characterized using PCR to amplify and sequence the ToxA coding region. Amplicons were compared to known ToxA haplotypes. We have identified ToxA containing B. sorokiniana in the Kansas leaf spot population. Yield loss to the leaf spot complex can be severe. With the addition of ToxA, B. sorokiniana could become an economically significant pathogen in wheat production.

New techniques exploring relationships between pathogen pressure and disease progression in strawberry M. MATSON (1), M. Hang (2), A. M. Pastrana Leon (3), U. Crouch (1), P. Goldman (1), P. Henry (4), F. Melton (2), F. N. Martin (1), (1) USDA ARS, Salinas, CA, USA; (2) California State University-Monterey Bay/NASA ARC-SGE, Seaside, CA, USA; (3) University of California, Davis, Davis, CA, USA; (4) United States Department of Agriculture, Salinas, CA, USA

Resurgences of soilborne diseases in strawberry production due to the phaseout of methyl bromide has necessitated a better understanding of how different levels of pathogen presence contribute to subsequent disease. We modeled the distribution of Verticillium dahliae microsclerotia (MS) prior to fumigation in a 10 acre field in Watsonville CA. 96 sample points were arranged in a grid, and samples were collected via pooling five individual subsamples originating from the center and edges of a $5 \mathrm{ft}$ radius circle. Following fumigation, plant health was monitored by remote sensing drone flights and there was an end-of-season soil recollection of 36 soil samples to reassess pathogen presence and distribution. We identified a modest correlation between $\mathrm{MS} / \mathrm{g}$ values and disease progression in prefumigation soil samples, but a poor correlation between end-of-season values and disease progression, likely due to low inoculum levels. A similar experiment in fields showing disease due to Fusarium oxysporum f.sp. fragariae utilized both direct plating colony counts and a new method for extracting DNA from large volume soil samples for the purpose of qPCR based prediction of CFU/g. These end-of-season soil samples showed a better correlation between measured CFU/g levels and remote sensing NDVI values than from $V$. dahliae. These results validate that pre-fumigation of modelling of pathogen distribution can predict subsequent disease severity, but that some pathogens can persist following fumigation to levels below meaningful detection thresholds while still contributing to disease.

\section{Multitrophic interactions of chromosomally labelled Pectobacterium and Dickeya species with their host and analysis of pathogenicity determinants \\ S. DOBHAL, G. Boluk, D. Arizala, A. M. Alvarez, M. Arif, University of Hawaii at Manoa, Honolulu, HI, USA}

Soft rot pathogenic bacteria, Dickeya and Pectobacterium, are associated with the economically important blackleg disease. It is common to find more than one pectolytic bacterial species on the same potato plant showing symptoms of blackleg. In this study, we aimed to determine the multitrophic molecular interactions between Pectobacterium carotovorum (Pc) and Dickeya dianthicola (Dd), and their host when isolated from the same infected plant. Chromosomally integrated mutants of Pc and Dd with mScarlet-I and sGFP2 were generated using both $\operatorname{Tn} 5 / \operatorname{Tn} 7$. The chromosomal integration was validated using multiplex PCR and TaqMan qPCR, demonstrating 100\% integration and maintenance into the genome. Transmission electron microscopy showed no differences in the cell morphology of the parental and labelled bacteria. Furthermore, mutants generated during the study did not interfere bacterial fitness. Observations using Leica FCA fluorescent stereomicroscope revealed that the Pc strain was very aggressive when potato was infected alone or in combination with both Pc and Dd. Pc showed no difference in infectivity capability in the presence or absence of Dd. The genome of Pc was sequenced using Oxford Nanopore MinION sequencer, de novo assembled using CLC genomics workbench and evaluated for pathogenicity determinants. Among the six type secretion systems reported in Pectobacterium, only T4SS was absent; Pc also harbored CRISPR-Cas $1 \mathrm{E}$ and $1 \mathrm{~F}$. This study has potential implications for understanding the disease development and bacterial behavior in the course of co-infection.

Iron-limitation induced tomato root exudation and interactions with the bacterial wilt pathogen Ralstonia solanacearum V. RAI (1), A. Loucanides (2), A. Schopps (2), O. Baars (2), (1) North Carolina State University, Raleigh, NC, USA; (2) North Carolina State University, Raleigh, NC, USA

Iron is an essential but poorly bioavailable micronutrient due to its low mobility in soil. Plants and microbes can secrete small molecule iron chelators and reductants that mobilize ferric iron by chelation or reduction. Recent studies suggest metabolic overlaps between abiotic and biotic interactions around iron in the rhizosphere and these overlaps are are emerging as critical controls on plant resistance. In this study, we investigate iron-limitation induced exudation and effects on pathogen resistance or susceptibility with tomato and the bacterial wilt pathogen Ralstonia solanacearum. Untargeted metabolomics of iron-limitation induced root exudates from different tomato varieties (Solanum lycopersicum 'Heinz 1706', genetically sequenced / 'Hawaii line 7996', high bacterial wilt resistance / wild type tomato S. pimpinellifolium) revealed strong secretion of several phenylpropanoid pathway metabolites under iron limitation. Our ongoing studies suggest that iron limitation and iron-limitation induced exudates lead to increased resistance of tomato against infection by $R$. solanacearum. The outcomes of our study provide first links between iron-availability as an abiotic control on root exudation and pathogen resistance with tomato as an important model crop.

Influence of liquid seed-applied fungicide on potato crop emergence and health

S. Hansen, S. A. Jordan, J. Hammel, S. Meyers, A. J. GEVENS, University of Wisconsin-Madison, Madison, WI, USA 
Freshly cut seed potato pieces are susceptible to infection by Fusarium spp. (causing dry rot) and Pectobacterium carotovorum (causing soft rot). Liquid seed-applied fungicides, like fludioxonil, have been developed to replace the standard treatment of mancozeb dust. Liquid seed treatments can result in delayed emergence or reduced stand of potato. The purpose of this study was to optimize the use of liquid seed treatments to manage seed decay without delaying emergence or reducing stand. A 2019 field trial was conducted with Russet Burbank (RB) and Dark Red Norland (DRN) potato cultivars. The trial examined the influence of suberization, treatment with liquid fungicide (fludioxonil), and treatment with a drying agent (fir bark). When planting without a suberization period, treatment with fludioxonil resulted in delayed emergence, reduced stand, and decreased yield for RB and DRN. There was a significant reduction in yield for DRN, and numerical reduction in yield for RB. Additionally, for RB, reduced stand resulted in a significant increase in culls. Our results indicate that when using a liquid seed treatment, a suberization period pre-plant and/or application of a drying agent is advisable to maintain stand and yield.

Genome sequencing, host range and Real-time RT-PCR detection of Tomato brown rugose fruit virus B. CHANDA (1), Y. Rosado-Rivera (2), S. Nunziata (2), M. Galvez (3), A. Gilliard (4), K. S. Ling (4), (1) USDA ARS, Charleston, SC, USA; (2) USDA-APHIS-PPQ-S\&T, Beltsville, MD, USA; (3) Department of Entomology and Plant Pathology, NC State University, Raleigh, NC, USA; (4) USDA-ARS, Charleston, SC, USA

Tomato brown rugose fruit virus (ToBRFV) has caused serious disease outbreaks in greenhouse tomatoes from many countries in Asia, Europe and North America ever since its first identification in 2014 in the Middle East. With its seed-borne nature, easy mechanical transmission and resistance breaking to the popular $T m 2^{2}$ gene, ToBRFV could potentially cause serious economic losses to tomato industries worldwide if not managed timely. In the U.S., the first ToBRFV outbreak on tomato was identified in 2018 in a greenhouse facility in California. In the current study, using high throughput sequencing, a complete genome sequence of the US isolate of ToBRFV was obtained. The high nucleotide sequence identity $(99 \%)$ to other known ToBRFV isolates around the world suggests a high possibility in sharing the same or similar origin. In a comparative evaluation of potential host ranges among tomato-infecting tobamoviruses (ToMV, ToMMV and ToBRFV), despite of many similarities, some unique host species were also identified for each respective virus. Many plant species belonging to four families (Amaranthaceae, Apocynaceae, Asteraceae and Solanaceae) were hosts, while plants of three other families (Brassicaceae, Cucurbitaceae and Verbenaceae) were non hosts for ToBRFV. Due to cross serological reactivity among tomato-infecting tobamoviruses, the current serological tests (ELISA, immunostrips) are good for general diagnosis of a possible tobamovirus infection. A species-specific real-time RT-PCR was developed for ToBRFV which was shown to be useful for plant health and seed health assays.

Application of high-throughput sequencing for detection of viruses causing disease of previously unknown etiology on Iris sp. in Serbia

A. Vučurović (1,2), D. Kutnjak (2), N. Mehle (2), Z. Kogej (2), I. Stanković (1), B. Krstic (1), M. RAVNIKAR (2,3), (1) University of Belgrade -Faculty of Agriculture, Belgrade, REPUBLIC OF SERBIA; (2) National Institute of Biology, Ljubljana, SLOVENIA; (3) University of Nova Gorica, Nova Gorica, SLOVENIA

Cultivation of ornamentals in Serbia has been increasing constantly over the last decade. Serbia's trade value of ornamentals market exceeds 8 million EUR. Several viruses are known to infect irises, some of which cause significant damage. During 2013 at Krnjača locality (City of Belgrade District, Serbia) iris plants exhibiting severe stunting and leaf mosaic, accompanied by delay or total absence of flowering. Disease incidence was estimated at more than $80 \%$ with market losses up to $95 \%$. Collected samples were tested for the presence of tospoviruses using ELISA and RT-PCR methods but none of these viruses were detected. Three selected samples were pooled and subjected to high throughput sequencing of small RNAs. Samples were sent for sequencing of small RNAs on Illumina HiSeq platform. Data analysis was performed using CLC Workbench software (Qiagen) using automated analysis workflow developed by National Institute of Biology, Slovenia. Using this approach, the presence of two viruses belonging to genus Potyvirus was detected, ornithogalum mosaic virus, for the first time in Serbia, and iris mild mosaic virus. Results were confirmed with conventional RT-PCR revealing mixed infections in all three samples. This study confirmed that sequencing of sRNAs using HTS platform could be a very powerful tool for detection of plant viruses in ornamentals. HTS based methods can be used as a "generic" tool for simultaneous detection of plant viruses in ornamentals.

The effect of tractor speed and spray application volume on severity of scab at different heights in tall pecan trees C. H. Bock (1), M. W. HOTCHKISS (2), (1) USDA ARS, Southeastern Fruit and Tree Nut Research Laboratory, Byron, GA, USA; (2) USDA ARS, Byron, GA, USA

Scab (caused by Venturia effusa) is the most yield-limiting disease of pecan in the southeastern USA. On susceptible cultivars, the disease is managed using fungicides, but spray coverage is an issue in tall trees. We used an air-blast sprayer to compare scab severity on fruit at $5-15 \mathrm{~m}$ height in trees receiving the same dose of fungicide at 468,935 and $1871 \mathrm{~L} / \mathrm{ha}$ at 2.4 and $3.2 \mathrm{kph}$. An air-blast sprayer was used for the applications, which included a typical series of active ingredients (a.i.). Nozzles were selected to provide similar proportions of spray to the upper and lower canopy. The treatments (or subsets thereof) were repeated 2015-17 on cv Schley, and in 2017 and 2019 on cv Desirable. All treatments reduced scab compared to the control. But overall there was little difference among the treatments on severity of scab on mature fruit. Fungicide applied in $468 \mathrm{~L} / \mathrm{ha}$ at 2.4 or $3.2 \mathrm{kph}$ was as effective at reducing disease in most seasons as were the higher volumes (sometimes more so). There were height effects related to volume: higher volumes generally had slightly better control higher in the canopy. The scab epidemic severity appeared to affect control efficacy. These preliminary observations indicate no single volume or speed was consistently superior; this suggests in most seasons' low volumes 
(high concentration of a.i.) may be similarly efficacious as high volumes (low concentration of a.i.) for controlling scab in tall pecan trees.

Evaluation of the uptake efficacy of streptomycin by citrus leaves after foliar spray

D. LEE (1), J. Li (2), N. Wang (2), (1) University of Florida, Lake Alfred, FL, USA; (2) Citrus Research and Education Center, University of Florida, Lake Alfred, FL, USA

Citrus huanglongbing (HLB) or citrus greening is one of the most devastating diseases worldwide. Candidatus Liberibacter asiaticus (CLas) is the causal agent of HLB in the USA. Two antibiotics oxytetracycline and streptomycin (STM) are allowed as a bactericide ingredient to control HLB via foliar spray application in Florida. However, bactericides are not easily absorbed by citrus leaves because of the cuticular wax layer. In this study, we investigated the uptake of STM by citrus leaves and its effect on suppression of CLas. STM was applied via foliar spray. Several different leaf washing methods were tested including shaking and ultrasonication to remove STM on the leaf surface. STM residues in leaves were detectable from both old and young leaves at 30 days after application, and the residue level was higher in young than old leaves. However, all treatments did not suppress CLas population effectively. The minimum inhibitory concentrations (MICs) of STM were evaluated against three bacterial species closely related to CLas to estimate a minimum concentration necessary for the suppression of $C$ Las. These results demonstrate that a foliar spray was able to deliver STM inside citrus leaves, but the concentration maybe did not reach a MIC to suppress CLas population by a one-time application.

\section{Chimeric infectious clones between Basal-BR and World-B isolates of turnip mosaic virus (TuMV) from Korea identify determinants of host specificity}

Z. X. SONG (1), W. X. Hu (2), E. Y. Seo (2), J. Kim (2), J. Hammond (3), H. S. Lim (2), (1) Chungnam National University, Daejeon, SOUTH KOREA; (2) Applied Biology, Chungnam National University, Daejeon, SOUTH KOREA; (3) USDA ARS Floral and Nursery Plant Research Laboratory, Beltsville, MD, USA

Turnip mosaic virus (TuMV), a member of the genus Potyvirus, mainly infects crops of plant species in the family Brassicaceae worldwide. In recent years, infectious clones of multiple isolates have been generated, which has enhanced our understanding of the interaction between this virus and host plants. Infectious clones of isolates KRS and KBC, collected from radish (Raphanus sativus) and Chinese cabbage (B. rapa ssp. pekinensis) respectively, were constructed and introduced into Nicotiana benthamiana. Their infectivity to radish and Chinese cabbage were assessed via rub inoculation with sap prepared from the corresponding infected $N$. benthamiana. The results indicate that KRS and KBC have different phenotypes in these two plants; KRS induced symptoms in radish but not in Chinese cabbage, whereas KBC infected Chinese cabbage but failed to infect radish. Phylogenetic analysis shows KRS and KBC are grouped into different clades, belonging to Basal-BR and World-B, respectively. Pairwise analysis indicates that they share $87.11 \%$ genomic nucleotide and $94.41 \%$ polyprotein amino acid identity. To identify the genetic determinant that plays a critical role on overcoming resistance in radish or Chinese cabbage, a series of chimeric clones were constructed by exchange of fragments including the $\mathrm{P} 3$ region that is known to be a symptom and movement determinant in radish, and the CI region responsible for breaking resistance conferred by Rntl-l in Chinese cabbage and by TuRBO3 in B. napus. Infectivity of the chimeras was determined by sap inoculation to radish and Chinese cabbage.

Advances in biological control of Xylaria sp., the causal agent of taproot decline of soybean A. BADIAL (1), N. S. Nejat (2), M. Tomaso-Peterson (2), S. Popescu (2), (1) Mississippi State University, Starkville, MS, USA; (2) Mississippi State University, Mississippi State, MS, USA

Taproot decline (TRD) is an emerging disease of soybean caused by Xylaria sp. This fungus infects the emerging soybean hypocotyl and taproot as it develops. Mummified soybean seedlings may be found in the field, but typical symptoms include foliar chlorosis followed by necrosis in the late vegetative to early reproductive stages. TRD is intractable to current management practices. Biocontrol strategies for fungal diseases are emerging as alternative control methodologies, however, insufficient knowledge exists on soybean root microbial communities and their inherent potential to suppress TRD. Our long-term goals are to discover and deploy management techniques against soybean TRD. We are taking a two-pronged approach: (1) High-throughput characterization of the rhizobiome and endophytes associated with TRD-symptomatic and asymptomatic soybean roots, and (2) Targeted characterization of microbial strains with potential Xylaria-biocontrol activity. Thus far, in a comparative study, we defined the taxonomic units present in root microbiome of healthy, low, and heavily-symptomatic (necrotic) soybean roots from plants located $3.0 \mathrm{~m}$ apart, from a north-central MS farm, and quantified the impact of Xylaria sp. on the composition of soybean microbiome communities. A screen of a library of previouslyisolated endophytes identified several bacterial strains with inhibitory activity on Xylaria sp. growth. Our study highlights the potential of a transdisciplinary data-driven approach to developing sustainable microbiome-derived biologicals to control intractable fungal diseases.

Genome-wide analysis of genetic diversity of Rhizoctonia solani AG-11 isolates from rice in the southern United States S. P. GAIRE (1), X. G. Zhou (1), Y. K. Jo (2), S. Wang (3), (1) Texas A\&M AgriLife Research Center, Beaumont, TX, USA; (2) Texas A\&M University, College Station, TX, USA; (3) Genomics and Bioinformatics Service, Texas A\&M AgriLife Research, College station, TX, USA

Rhizoctonia solani AG-11 is one of the most important fungal pathogens causing seedling disease in rice in the southern U.S. No information is available on the genetic diversity of populations of this pathogen in the rice-growing region. The objectives of this study were to examine the population diversity of $R$. solani AG-11 and determine if there were genotypic clusters associated with 
geographical origins. We sequenced the whole genomes of 78 isolates collected from rice fields in Arkansas, Louisiana, Mississippi, Missouri, and Texas via Illumina sequencing platform. De novo sequence assembly was conducted using the DNA sequence reads of two isolates from Arkansas, and subsequently all other isolates were aligned using this newly assembled genome as a reference sequence. High genetic variations were found in the populations of $R$. solani AG-11, with a total of $605.4 \mathrm{~K}$ single nucleotide polymorphisms (SNPs) identified. A phylogenetic tree was constructed based on the genome wide SNPs using maximum-likelihood algorithm. Surprisingly, clades and subclades were defined nearly by geographical origins of the isolates. The Arkansas and Mississippi isolates were more diverse than the other isolates. These results demonstrate high levels of genetic diversity among the populations of $R$. solani AG-11 in rice in the southern U.S.

\section{A Colletotrichum spp. population from the Mid-Atlantic apple growing region with reduced sensitivity to 10 of 16 fungicide active ingredients}

P. L. MARTIN (1), K. Pierce (2), C. Thomas (3), T. Krawczyk (4), K. A. Peter (5), (1) Penn State University Fruit Research and Extension Center, Biglerville, PA, USA; (2) North Carolina State University, NC, USA; (3) Temple University, PA, USA; (4) Penn State Fruit Research and Extension Center, Biglerville, PA, USA; (5) Pennsylvania State University, Fruit Research and Extension Center, Biglerville, PA, USA

Apple bitter rot is a major problem in the Mid-Atlantic region, and fungicide resistance was suspected as a factor for its increased incidence. A 2018 sampling of $>500$ bitter rot infected apples from 36 orchards in PA, VA, MD, DE, and OH resulted in 8 Colletotrichum spp., with C. fioriniae (66\%) of the acutatum complex and C. chrysophilum (22\%), C. siamense (4.5\%), and C. sp. nov. (3.4\%) of the gloeosporioides complex being the most prevalent. Representative isolates were used to obtain EC $\mathrm{E}_{50}$ values via mycelial growth, and resulting discriminatory doses were used to screen 255 isolates. Resistance was detected in C. siamense to FRAC groups 1 (13/22 had the E198A $\beta$-tubulin mutation) and 11 (2/22 had the G143A cytochrome-b mutation), but these were not widespread. However, across species and orchards, $C$. spp. isolates had relatively poor sensitivity to many active ingredients. E.g. fluxapyroxad, fluopyram, inpyrfluxam, pydiflumetofen, boscalid, and isofetamid (FRAC 7), pyrimethanil and cyprodinil (FRAC 9), and trifloxystrobin and kresoxim-methyl (FRAC 11) showed less growth inhibition in-vitro than benzovindiflupyr and penthiopyrad (FRAC 7), pyraclostrobin (FRAC 11), fludioxonil (FRAC 12), and fluazinam (FRAC 29). Sensitivity to thiophanate methyl (FRAC 1) varied by spp. complex. Results from this investigation show the Colletotrichum spp. diversity from apple in the Mid-Atlantic region, their fungicide resistance profiles, and emphasizes that selection of active ingredient, especially within a FRAC group, is important in choosing a fungicide for optimal bitter rot control.

Dead or alive: Plant pathogens influence the production of lipopeptides by Bacillus subtilis strain PTB185 L. Cossus (1), F. Roux-Dalvai (2), I. Kelly (2), T. T. A. Nguyen (3), H. Antoun (3), A. Droit (4), R. J. TWEDDELL (5), (1) Département de médecine moléculaire, Université Laval, Québec, QC, CANADA; (2) Proteomics platform, CHU de Québec Université Laval Research Center, Québec, CANADA; (3) Département des sols et de génie agroalimentaire, Université Laval, Québec, CANADA; (4) Département de médecine moléculaire, Proteomics platform, Université Laval, Québec, CANADA; (5) Département de phytologie, Université Laval, Québec, QC, CANADA

Lipopeptides are closely linked to the biocontrol activity of Bacillus spp. The objective of the study was to investigate the effect of different plant pathogens on the production of lipopeptides by Bacillus subtilis strain PTB185. The bacterium was co-cultured for 5-7 days on agar with either Botrytis cinerea, Mucor sp., Pythium ultimum, Rhizoctonia solani or Sclerotinia sclerotiorum. Lipopeptides (surfactins, iturins, and fengycins) produced by $B$. subtilis were then extracted from agar and quantified using MALDI-TOF mass spectrometry. Bacillus subtilis was also cultivated in liquid media supplemented with plant pathogen mycelium $\left(1 \mathrm{~g} \mathrm{~L}^{-1}\right)$ of each tested pathogen (B. cinerea, Mucor sp., P. ultimum, R. solani, S. sclerotiorum). After 3 days of growth, lipopeptides were quantified in the filtrate cultures. The results obtained show that lipopeptides production by $B$. subtilis was influenced by the pathogens in dual culture (agar) and by the presence of autoclaved mycelium of the pathogens (liquid medium). Significantly higher quantities of iturins and fengycins were produced in dual culture with Mucor sp., P. ultimum, and $R$. solani. Fengycins and iturins were produced in significantly higher amounts in presence of autoclaved mycelium of $B$. cinerea, Mucor sp., and $R$. solani. In addition, incorporation of autoclaved pathogen mycelium in the liquid medium was shown to influence $B$. subtilis biocontrol activity. This study provides new insights into the production of $B$. subtilis for biotechnological applications.

In vitro sensitivity of Sclerotinia sclerotiorum isolates from the mid-Atlantic region of the United States to six fungicides registered on lima bean

H. DEMISSIE (1), K. L. Everts (2), (1) University of Maryland, College Park, MD, USA; (2) University of Maryland, College Park, Salisbury, MD, USA

Management of white mold on lima bean in the mid-Atlantic region relies on fungicides; however, no studies have examined the regional population of Sclerotinia sclerotiorum for fungicide sensitivity. In-vitro bioassays were conducted in 2017 and 2019 to determine the sensitivity of $40 \mathrm{~S}$. sclerotiorum isolates to boscalid (FRAC code 7), fludioxonil (12), cyprodinil (9), thiophanate-methyl (1), prothioconazole (3), and fluazinam (29). Fungicides were diluted in dimethyl sulfoxide and amended to potato dextrose agar at two concentrations. Three, 5-mm diameter plugs of S. sclerotiorum were transferred to fungicide-amended and non-amended plates. Diameter of the mycelial growth after three days, compared to the non-amended plates, was used to calculate percent reduction in mycelial growth (PRMG). Isolates varied in PRMG to all fungicides, and PRMG differed at the two concentrations, except for cyprodinil and fludioxonil. There were significant interactions between isolate and concentration except for boscalid and thiophanatemethyl. Correlations identified associations between fungicide sensitivities. Isolate sensitivity to fluazinam was positively correlated to sensitivity to prothioconazole $(\mathrm{r}=0.35695 ; P<0.0001)$ and thiophanate methyl $(\mathrm{r}=0.46247 ; P=<0.0001)$, but negatively correlated to 
boscalid sensitivity $(\mathrm{r}=-0.20119 ; P=0.0136)$. Sensitivity to fludioxonil was positively correlated to sensitivity to boscalid $(\mathrm{r}=$ $0.19309 ; P=0.0179)$ and thiophanate methyl $(0.28760 ; P=0.0004)$. Correlation among fungicide sensitivities may impact disease management and should be further examined.

Development of multiplex conventional and real-time RT-PCR assays to detect Tomato black ring virus J. ZHOU (1), G. Wei (2), V. A. Mavrodieva (3), M. K. Nakhla (4), (1) USDA, Beltsville, MD, USA; (2) USDA-APHIS-PPQ-S\&TCPHST, Beltsville, MD, USA; (3) USDA-APHIS-PPQ S\&T CPHST, Beltsville, MD, USA; (4) USDA-APHIS-PPQ-S\&T, Beltsville Laboratory, Beltsville, MD, USA

Tomato black ring virus (TBRV) is a nepovirus widely distributed in Europe and has expanded its territory to more continents in recent years. TBRV infects a wide range of economically important crops including Solanaceous, Fragaria, Prunus and vegetable species. The virus can be transmitted through seeds and by nematodes Longidorus elongatus and Longidorus attenuates that facilitate the shortand long-distance dispersal of the virus. Given these epidemiological characteristics and the economic importance, availability of molecular tools for detection of TBRV is vital in the event of an incursion. For this purpose, conventional and real-time RT-PCR assays were developed in the PPQ S\&T Beltsville laboratory to detect currently known TBRV isolates including newly emerged ones, such as TBRV-Pi, -P1 and -MJ isolates from Poland. The two assays were optimized as multiplex assays by the addition of a plant internal control. Validation analysis using nine (9) TBRV isolates collected from four (4) countries revealed both assays were sensitive and repeatable. Specificity of the assays were tested against nine (9) common tomato viruses and nepoviruses, and selectivity of the assays were assessed in six (6) different plant species. Results showed that both assays were specific to TBRV and capable of detecting TBRV in the tested host species.

Transcriptional response of sweet sorghum lines varying in resistance to stalk rot pathogens M. KHASIN (1), P. O’Neill (2), D. L. Funnell-Harris (2), S. Sattler (2), (1) USDA-ARS, LINCOLN, NE, USA; (2) USDA-ARS, Lincoln, NE, USA

Sweet sorghum is grown as source sugars for ethanol and syrup production. Stalk rots caused by Fusarium thapsinum (Fusarium stalk rot) and Macrophomina phaseolina (charcoal rot) can significantly impact yields and quality of sorghum. This study compared lesion lengths and transcriptomes of moderately susceptible (Colman) and moderately resistant (M81-E) lines following inoculation with each pathogen. M81-E developed significantly shorter lesions than Colman in response to M. phaseolina, but not to F. thapsinum. Network and differential expression analysis of the transcriptomes of Colman and M81E in response to each fungus and to a broth control revealed that genes encoding phenylpropanoid and flavonoid biosynthetic and modification enzymes were a core part of the early wounding response. These phenylpropanoid biosynthetic genes were differentially coexpressed with SbMyb76, a transcription factor predicted to target them, in Colman with all inoculations and in M81E only with M. phaseolina inoculation. Thus, SbMyb76 is a candidate transcription factor controlling defense lignin biosynthesis. Coexpression analysis also highlighted the role of auxin, jasmonic acid, abscisic acid, and cytokinin signal transduction and protein turnover in the early response and RNA processing in the late response. Several pathogenesis-related (PR)-like proteins were more highly expressed in M81E than in Colman in the broth controls, suggesting that components of the uninoculated wound response in M81E may provide a protective effect, particularly in response to $M$. phaseolina.

Pathogenicity and aggressiveness of Pestalotiopsis-like species isolates on strawberry J. BAGGIO, M. Marin, N. Peres, University of Florida - Gulf Coast Research and Education Center, Wimauma, FL, USA

Pestalotiopsis-like species are common plant pathogens but are also frequently isolated as endophytes in a variety of plants worldwide. In strawberry, it has been considered a secondary pathogen, known to cause fruit, root and crown rots. Recently, severe and unprecedented outbreaks have been reported in Florida, where root, crown, petiole, fruit and leaf symptoms were observed, and yield was severely affected. Strawberry plants of 'Florida127' were inoculated with spore suspensions of Pestalotiopsis-like isolates originating from alternative hosts (Limonium sp., Quercus sp., Smilax sp., Kalmia sp., Rhododendron sp., Rubus sp., and Punica granatum) and three strawberry isolates collected prior and after the recent outbreaks. Also, six Florida cultivars were screened using a mixture of strawberry isolates from the outbreaks. Foliar and fruit disease incidences were assessed throughout the season. Only plants inoculated with an isolate originating from Rhododendron sp., sampled around a strawberry nursery field associated with the outbreak, showed incidence levels similar to those caused by strawberry isolates from recent outbreaks. Incidence on plants inoculated with past strawberry isolates and isolates from all other hosts were low and did not differ from the non-inoculated control. All cultivars were susceptible to the pathogen, but 'Florida Beauty' and 'Florida Brilliance' were significantly more affected. The disease is difficult to control and an integrated disease management approach involving strawberry nursery and production fields needs to be developed.

Endobacteria of Fusarium fujikuroi W343 affects host virulence, macroconidia and fumonisin production, and expression of fumonisin biosynthetic genes

K. OBASA (1), A. O. Adesemoye (2), R. Obasa (3), D. Moraga-Amador (3), H. Shinogle (4), K. Kelley (3), S. Alvarez (5), (1) Texas A\&M University, Amarillo, TX, USA; (2) Terramera Inc., Vancouver, CANADA; (3) University of Florida, FL, USA; (4) University of Kansas, KS, USA; (5) University of Nebraska, NE, USA

Until recently, Fusarium fujikuroi was widely described as a producer of little to none of the B-series fumonisin toxins despite having a complement of fumonisin biosynthetic genes similar to those in $F$. verticillioides and $F$. proliferatum. While high and low fumonisinproducing $F$. fujikuroi strains have been shown to induce typical disease symptoms, the relationship between fumonisin production and 
virulence in F. fujikuroi has not been fully established. This study investigated the association of two strains of bacteria of the genus Enterobacter with a F. fujikuroi strain, W343, isolated from wheat in Nebraska. Results of confocal, scanning, and transmission electron microscopy analysis demonstrated the endohyphal (intracellular) localization of the bacteria within F. fujikuroi W343. Investigations of the association of the bacteria with the fungus also showed significantly higher $(\alpha=0.05)$ virulence, fumonisin and macroconidia production in the bacteria-associated fungus compared with the bacteria-free fungal strain. RNA-seq transcript data showed that fumonisin production correlated with higher expressions of fumonisin biosynthetic genes fum 1, fum8, fum 15, and fum 21 under fumonisin-inducing and non-inducing conditions. These findings suggest the symbiotic association has implications for the fungus' phenotypes. Exploiting the ability of endohyphal bacteria to influence important fungal phenotypes could lead to the development of alternative management approaches for diseases and fumonisin-related issues by virulent and mycotoxigenic strains of Fusarium in field crops.

Evaluation of factors that affect fungicide coverage of wheat heads and their impact on control of Fusarium head blight N. WHITE (1), T. Stombaugh (1), C. A. Bradley PhD (2), (1) University of Kentucky, Lexington, KY, USA; (2) University of Kentucky, Princeton, KY, USA

Fusarium head blight (FHB), caused by Fusarium graminearum, reduces wheat yields and grain quality. In addition, infected grain may become contaminated by the mycotoxin, deoxynivalenol (DON). Fungicides are commonly used to manage FHB and DON contamination in wheat, but good coverage of the wheat head is needed to achieve the best efficacy. A field research trial was conducted in 2017, 2018, and 2019 in Princeton, KY to evaluate the effect of application ground speed, nozzle type and nozzle orientation on fungicide coverage of wheat heads and control of FHB and DON. Fungicide coverage was determined by analyzing water-sensitive cards that were constructed to simulate wheat heads and placed in wheat plots. Results indicated that both coverage and fungicide efficacy were impacted by nozzle type, and that DON contamination could be reduced by approximately $70 \%$ with an appropriate fungicide application compared to a non-treated control.

\section{Influence of cultural practices and soybean maturity group on soil and root microbial community composition I: The fungal perspective \\ A. Y. BANDARA, D. K. Weerasooriya, R. Trexler, T. Bell, P. Esker, The Pennsylvania State University, University Park, PA, USA}

Cultural practices are key tactics in integrated pest management. They can possibly alter soil and root microbial composition, which can have direct impacts on soil/plant health. At V1 (one trifoliate) soybean growth stage, bulk soil (BS), rhizosphere soil (RS), and roots (R) were sampled from 14 farms in Pennsylvania with histories of different cultural practices. DNA was extracted from samples, PCR amplified targeting ITS region (ITS1F/58A2R primers), and sequenced. Samples classified based on cultural practices and total MiSeq read counts were subjected to factor analysis of mixed data (FAMD). Cover crops and tillage contributed the most to variation among BS samples. Variation among RS and R samples were primarily explained by crop rotation and soybean maturity group. For BS, cover crops decreased the relative abundance (RA) of Fusarium, Verticillium, and Mortierella. Tillage reduced the RA of Monographella, Corynespora, and Preussia while increasing Verticillium. Corn-soybean-corn (CSC) rotation increased RA of Corynespora, Fusarium, and Monographella in RS samples compared to non-CSC rotations. CSC increased the RA of Fusarium, Monographella, and Mortierella while decreasing the Corynespora in R samples. RS from maturity group two (MG2) soybean contained greater \% of Fusarium, Mortierella, and Preussia compared to late-three (MGL3). R from MG2 contained lower \% of Corynespora, Monographella, Mortierella, and greater \% of Fusarium compared to MGL3. Our findings showed how cultural practices can be used to manipulate soil and root fungal communities of soybean.

USDA Cochran Fellowship Program: Food safety in Pakistan through biological control of aflatoxins H. L. Mehl (1,2), J. OPOKU (2), A. Ortega-Beltran (3), K. A. Callicott (1), C. G. Aguilar (2), N. Kaur (2), M. P. Braverman (4), D. Hamilton (5), (1) USDA Agricultural Research Service, Tucson, AZ, USA; (2) Virginia Tech Tidewater AREC, Suffolk, VA, USA; (3) International Institute of Tropical Agriculture, Ibadan, NIGERIA; (4) Interregional Research Project Number 4(IR-4), Rutgers University, Princeton, NJ, USA; (5) USDA Foreign Agricultural Service, Washington, DC, USA

Crop aflatoxin contamination is a worldwide problem impacting food and feed safety as well as international trade. The USDA Foreign Agricultural Service is making investments to develop aflatoxin biocontrol technologies for use in Pakistan, but there is lack of local expertise in regulatory and research aspects of aflatoxin mitigation using atoxigenic isolates of Aspergillus flavus as biocontrol agents. Through a Cochran Fellowship capacity building program hosted by Virginia Tech, a team of scientists from Pakistan received training on Aspergillus section Flavi identification and atoxigenic strain characterization, evaluation of aflatoxin biocontrol efficacy, development and registration of aflatoxin biocontrol products, and implementation of an aflatoxin mitigation program through grower education and adoption of biocontrol technologies. A diverse group of scientists with expertise in aflatoxin biocontrol and biopesticide registration from Virginia Tech, the IR-4 Project, USDA Agricultural Research Service, and International Institute of Tropical Agriculture led the program which combined lectures, field demonstrations, and hands-on laboratory training. The program concluded with a trip to Washington, DC to meet with representatives of government agencies involved in regulatory aspects of food safety and biopesticide registration. Following the Cochran Fellowship program, the Aflatoxin Control Laboratory at Crop Diseases Research Institute was established in Islamabad, and progress has been made towards registration of an aflatoxin biocontrol product for use in Pakistan.

Fungicide sensitivity in strawberry powdery mildew caused by Podosphaera aphanis in California

M. PALMER (1), G. J. Holmes (2), (1) California Polytechnic State University, San Luis Obispo, CA, USA; (2) Strawberry Center, California Polytechnic State University, San Luis Obispo, CA, USA 
Reduced fungicide sensitivity in strawberry powdery mildew (SPM) is a suspected problem in California. SPM is one of the most common diseases in strawberry production that is controlled with foliar fungicide applications. This study characterizes the sensitivity of 7 Podosphaera aphanis isolates to the most common fungicides used against SPM in California. Isolates were collected from Santa Maria, Oxnard, and Turlock, California. Clean, unfurled strawberry leaves (cv. Monterey) were treated with one of 6 fungicides using the minimum labeled rate and inoculated with conidia of $P$. aphanis. Inoculated leaves were incubated at $20^{\circ} \mathrm{C}$ under $16 / 8$ hours of day/night lighting and assessed for disease incidence (\%) and severity (\%) at 14 days. The 6 treatments and the average resulting disease incidence for the 7 isolates are: cyflufenamid (11\%), myclobutanil (41\%), penthiopyrad (35\%), quinoxyfen (28\%), trifloxystrobin $(17 \%)$, trifloxystrobin + fluopyram $(2 \%)$. The average disease incidence for the trifloxystrobin treatment was raised significantly by a single isolate considered to be resistant to the product (disease incidence $=89 \%$ ). An isolate from an organic production system was sensitive to all fungicides. This documents that $P$. aphanis in California can develop partial to complete resistance to most of the fungicides used for its control. Characterization of additional isolates is ongoing.

Use of Alliums as trap crops to reduce white rot inoculum in infested field soil K. Hua, J. K. S. DUNG PHD, Oregon State University, Madras, OR, USA

White rot, caused by Sclerotium cepivorum, is a destructive disease of Alliums that can render infested fields unsuitable for production. The fungus persists in soil as sclerotia that germinate in response to Allium root exudates. This study investigated whether earlyterminated Allium trap crops can reduce white rot sclerotia populations in soil. Seeds of white-, red-, or sweet onion $(A$. cepa), or bunching onion (A. fistulosum), were sown in pots filled with white rot-infested soil. Fallow soil served as a control. Seedlings were manually terminated at 3-, 7- and 11 weeks post-emergence (wpe) and disease incidence, soil sclerotia populations, and sclerotia viability were assessed. The remaining soil, together with plant materials, was kept for another 4 weeks and sclerotia populations and viability were re-evaluated. Disease symptoms developed on $8 \%$ and $45 \%$ of seedlings at 7 wpe and at 11 wpe, respectively. All Alliums significantly $(P \leq 0.046)$ reduced soil sclerotia populations by up to $55 \%$ at 3 wpe and $59 \%$ at 7 wpe. However, the number of sclerotia in soil cultivated with bunching and white onion for 11 weeks increased and was greater than the control. Analyses of soil samples collected 4 weeks after crop termination showed that sclerotia populations remained unchanged when seedlings were terminated at 3 wpe, but sclerotia counts significantly increased in soil cultivated with bunching, white and sweet onions after seedling termination at 11 wpe. This study demonstrates the potential for early termination of onion trap crops to help reduce white rot inoculum in soil.

\section{Spatiotemporal patterns of Impatiens necrotic spot virus (INSV) and its thrips vector in lettuce agroecosystems in the Salinas Valley of California \\ D. K. HASEGAWA (1), L. Hladky (1), A. Del Pozo (2), (1) USDA-ARS, Crop Improvement and Protection Research Unit, Salinas, CA, USA; (2) University of California Cooperative Extension, Monterey County, Salinas, CA, USA}

Since first reported in 2008, research and monitoring of the tospovirus, impatiens necrotic spot virus (INSV) and its primary vector, the western flower thrips, Frankliniella occidentalis, has been limited in the Salinas Valley of California, despite increasing reports of INSV outbreaks in commercial lettuce production. Only limited strategies exist for thrips management in lettuce and there are no direct methods for managing INSV under commercial conditions. Furthermore, the large host range of plant species that can support thrips populations and INSV creates a need to identify epidemiological factors that impact lettuce production in the Salinas Valley. Here, we sought to: 1) Understand the timing of INSV infection and thrips infestation in commercial lettuce fields, 2) Identify host plants that serve as significant reservoirs for INSV, and 3) Monitor thrips populations throughout the Salinas Valley. Together, the data demonstrate a need to extend management strategies beyond lettuce fields, and to consider factors that are dependent on the location and timing of lettuce plantings. The outcomes of these studies provide knowledge that will improve thrips and INSV management in commercial lettuce production in coastal lettuce production regions of California.

\section{The plant-soil-microbe interactions mediate variation in dollar spot severity in turfgrass}

M. Y. CHOU (1), P. L. Koch (2), (1) University of Wisconsin, Madison, Madison, WI, USA; (2) University of Wisconsin, Madison, WI, USA

Dollar spot (Clarireedia jacksonii) in turfgrass often occurs in a highly variable manner, even on a local scale where many of the environmental confounding factors are uniform. This study aims to investigate how plant-associated bacterial communities and soil chemical properties contribute to the observed disease variability. Eighteen equally healthy-looking turfgrass samples were taken in the fall of 2019. Endophytic, rhizosphere, and bulk soil subsamples were taken from within each sample prior to inoculation with $C$. jacksonii and incubation in a growth chamber. Bacterial communities were profiled from each segment using an Illumina MiSeq platform targeting the 16s rRNA gene v4 region, and bulk soil chemical properties of each sample was also analyzed. Disease severity following inoculation was used to categorize the 18 samples into high, medium, and low severity. The initial rhizosphere bacterial communities prior to $C$. jacksonii inoculation from the high disease turf differed significantly from that of low disease turf during the peak disease development. In addition, bulk soil chemical property did not correlate with the bulk soil bacterial community but significantly correlated with the rhizosphere bacterial community, which suggested that plant and soil interactions co-shaped the rhizosphere bacterial community. Further research in this area may provide important clues as to why plant disease development can be so scattered over a small scale, which may lead to new and innovative disease control strategies. 
Tomato biotic stress induced root exudation and effects on bacterial wilt resistance

A. LOUCANIDES (1), V. Rai (2), O. Baars (3), (1) North Carolina State University, Raleigh, NC, USA; (2) North Carolina State

University, Raleigh, NC, USA; (3) North Carolina State University, Raleigh, NC, USA

The similarities between induced systemic resistance (ISR) and iron limitation in plants has been a growing area of study with Arabidopsis. Preliminary research in our lab on tomato showed that iron limitation lead to a defense priming phenotype when tomato plants were challenged with the pathogen Ralstonia solanacearum. We had further observed iron-limitation induced root-secretion of phenylpropanoid metabolites with antimicrobial effects. In this study, we investigated tomato ISR and biotic stress responses focusing on effects on the environment through root exudation. Tomatoes grown in hydroponics were either left untreated or given rhizosphere or foliar applications of ISR elicitors (Lifegard ${ }^{\mathrm{TM}}$ ). Secreted root exudates were collected and analyzed via liquid chromatography tandem mass spectrometry. Subsequently, we analyzed root exudates secreted in response to $R$. solanacearum infection without and with prior elicitation of ISR. We are currently analyzing the chemical identity and bioactivity of these biotic stress induced tomato root exudates to better understand potential antimicrobial activity and overlaps between ISR and iron-limitation induced exudation. The outcomes provide insight into the biochemical role of root exudation during biotic stress of tomato and overlaps between ISR and iron-limitation.

Early detection of maize dwarf mosaic using near-infrared spectroscopy

A. O. CONRAD (1), M. Jones (2), K. Willie (2), D. Massawe (3), L. R. Stewart (2), P. Bonello (1), (1) The Ohio State University, Department of Plant Pathology, Columbus, OH, USA; (2) USDA-ARS Corn, Soybean and Wheat Quality Research Unit, Wooster, OH, USA; (3) The Ohio State University, Department of Plant Pathology, Wooster, OH, Wooster, OH, USA

The need for ever more sensitive, rapid, effective and inexpensive diagnostic tools is ever present in plant pathology. Near-infrared spectroscopy (NIRS) is a method for rapidly measuring the chemical composition of plant tissues that could fit such needs. When combined with predictive modeling, NIRS can be a powerful tool for identifying diseased but presymptomatic plants. We evaluated whether NIRS, combined with machine learning, can be used to identify presymptomatic corn plants infected with maize dwarf mosaic virus (MDMV). Maize dwarf mosaic disease caused by MDMV or related potyviruses occurs across the United States and globally, and in synergistic coinfections contributes to maize lethal necrosis, a devastating emerging disease. We artificially inoculated corn plants in the field with MDMV, and collected NIR spectra from foliage prior to development of disease symptoms. Then, we used support vector machine to develop a model for classifying plants as either inoculated with MDMV, mock-inoculated, or non-inoculated. Based on field experiments, we were able to correctly classify $96.3 \%(\mathrm{~N}=80)$ of all plants in the testing set at three days post-inoculation using a region of the NIR spectrum from $1898-2551 \mathrm{~nm}$, with $100.0 \%(\mathrm{~N}=28)$ of inoculated plants in the testing set correctly classified. This indicates that it is possible to identify presymptomatic MDMV-infected corn plants in the field using NIRS and support vector machine. Additional work is needed to refine and validate existing models, and to test this approach in settings of natural disease development.

Genetic diversity of Wheat streak mosaic virus in the wheat growing regions of Western Canada H. S. BENNYPAUL (1), I. Abdullahi (2), R. Aboukhaddour (3), M. W. Harding (4), (1) Canadian Food Inspection Agency, North Saanich, BC, CANADA; (2) Canadian Food Inspection Agency, North Saanich, CANADA; (3) AAFC, Lethbridge, AB, CANADA; (4) Alberta Agriculture and Forestry, Crop Diversification Center South, Brooks, AB, CANADA

Wheat streak mosaic virus (WSMV) causes a potentially devastating disease of wheat in western Canada. Understanding the genetic diversity of WSMV is critical for developing management options. WSMV isolates have been divided into four clades based on nucleotide sequence of the coat protein (CP) gene. Clade A contains isolates from Mexico, clade B from Europe and Russia, clade C from Iran, and clade D from the U.S., Turkey, Australia, and Argentina. We explored the genetic diversity of WSMV isolates collected from the wheat growing regions of western Canada, primarily southern Alberta, during 2016 and 2017. Phylogenetic analysis of 16 isolates and the isolates available in GenBank was performed using the 1,043-bp sequence corresponding to nucleotide positions 8,192 to 9,234 of the Sidney 81 strain coat protein gene using neighbour-joining analysis. Half of the isolates clustered in clade B while the other half in clade D. Of the eight isolates in cluster D, five showed evidence of recombination. More polymorphic sites, parsimony informative sites, and increased diversity were observed in clade D isolates than clade B, suggesting more recent establishment of the virus in the latter. The observed genetic diversity could pose challenges to breeding durable resistance in wheat in case there is a differential response of WSMV resistance genes to isolates from different clades. Tested CP-based assay was unreliable in detecting all the isolates. Sequence information generated is this study will be useful in developing sensitive and reliable diagnostic assays.

Fungal endophyte Acremonium alternatum helps to mitigate biotic and abiotic stress in plants S. AUER, TU Dresden, Dresden, GERMANY

The use of biocontrol agents and beneficial microbes in integrated pest management helps to secure crop yields. Reports from the last decades indicate that the endophytic fungus Acremonium alternatum can control various pathogens (mildew, tar spot) but the mechanism behind this was not clear. Our goals were to identify molecular markers behind the biocontrol action of A. alternatum against pathogens and assess general plant performance with this endophyte under stress conditions. For the root pathogen Plasmodiophora brassicae (clubroot) we found that $A$. alternatum reduced clubroot symptoms by $30 \%$. The endophyte established systemic acquired resistance in clubroot-infected roots, delayed clubroot development, enhanced reproductive success and survival of clubroot-inoculated plants. For the foliar pathogen Botrytis cinerea we found that $A$. alternatum reduced lesion size by $50 \%$ and $70 \%$ when applied on leaves directly or indirectly through soil. The fungus also increased salt stress tolerance in plants grown in axenic culture. We started to look at performance of specific crops (oilseed rape, tomato, wheat, corn) for their response to A. alternatum under stress conditions. For the near future we look for solutions to (1) further elucidate which molecular markers are crucial for the 
biocontrol effect of the fungus against pathogens using RNAseq and - omics techniques and (2) acquire funding and cooperation partners to carry out field-trial like experiments with natural soils to confirm our findings from greenhouse trials.

National survey of grower knowledge and perceptions of Erysiphe necator fungicide resistance in US vineyards C. OLIVER (1), M. M. Moyer (1), W. Mahaffee (2), M. Cooper (3), M. L. Lewis Ivey (4), T. D. Miles (5), P. M. Brannen (6), (1) Washington State University, Prosser, WA, USA; (2) USDA ARS, Corvallis, OR, USA; (3) Univ of California, Napa, CA, USA; (4) Department of Plant Pathology, The Ohio State University, Wooster, OH, USA; (5) Michigan State University, East Lansing, MI, USA; (6) University of Georgia, Athens, GA, USA

Grape powdery mildew (Erysiphe necator) fungicide resistance has become a persistent concern in the US. The Fungicide Resistance Assessment, Mitigation and Extension Network for Wine, Table and Raisin Grapes (FRAME network), was created to detect, predict, and mitigate the rise of fungicide resistance through research and extension. In 2019, a national survey of grape growers was conducted by FRAME network to identify foci for fungicide resistance extension programming. The survey grouped responses from 20 states by production region and assessed US grape grower fungicide resistance knowledge and perceptions. Of the thirteen knowledge-specific questions, grower responses to five were significantly influenced by production region. A perception shift was observed regarding resistance concerns within a personal vineyard compared to within the US. Nationally, 145 of 252 responses graded concerns of national fungicide resistance a four, or serious problem. However, within a personal vineyard, the national response shifted to a minor to moderate problem with 70 and 75 respondents providing a grading of two or a three, respectively. Additionally, a regional effect $(P=$ 0.02 ) was observed with north- and southeastern US growers expressing more concern compared to Midwest and southwestern. These responses provide insight into the effect of production region on growers' baseline knowledge and perception of fungicide resistance. This information will allow the FRAME network and other extension personnel to regionally tailor programming to address fungicide resistance knowledge gaps.

Progress in Metabarcoding High-Throughput Sequencing (HTS) long-amplicon reads for identification of Phytophthora species in environmental DNA samples

Z. G. ABAD (1), S. Srivastava (1,2), L. M. Knight (1,2), K. A. Zeller (1), V. A. Mavrodieva (1), M. K. Nakhla (3), (1) USDA-APHISPPQ S\&T Beltsville Laboratory, Beltsville, MD, USA; (2) Department of Entomology and Plant Pathology, NC State University, Raleigh, NC, USA; (3) USDA-APHIS-PPQ-S\&T, Beltsville Laboratory, Beltsville, MD, USA

The genus Phytophthora includes many species of regulatory concern. Systems for identification of species in DNA from environmental samples (e-DNA) are important to be-examined with traditional sequencing tools and state-of-the-art nova technologies. We have examined a rapid system for identification of Phytophthora species from e-DNA samples that exploits some of the unique properties of the MinION Nanopore long-read HTS technology. In this work, we evaluated six sets of modified PCR primers that we generated based on the ribosomal operon $(12 \mathrm{~Kb})$ of Phytophthora spp. and tested using MinION Flow cells. Although, we did achieve amplification for DNA fragments of up to $6 \mathrm{~Kb}$, we found that our most consistent results were with fragments of up to $2000 \mathrm{bp}$. We then selected primers for ITS-rDNA ( $\sim 800-850 \mathrm{bp})$ for more extensive testing. For DNA fragments of this size we could achieve a minimum detection level is of $50 \mathrm{fg} / \mu \mathrm{l}$ of target in genomic DNA. We extended this testing to include amplified DNA of 20 Phytophthora species from cultures and mock environmental samples of both individual species, and from mixed samples that included DNA of $P$. ramorum in combination with two or three other Phytophthora species. When we compared our results to a robust local reference database of the ITS-rDNA sequence data including data from 139 Ex-types and 22 well-authenticated Phytophthora species we were able to unambiguously identify the species in these mock samples. We have been extending this line of research by testing e-DNAs from field samples provided to us by national collaborators.

Does deficit irrigation influence vine decline diseases of processing tomato in California? J. BEAULIEU, I. Herrera, N. Tautges, K. Scow, C. L. Swett, University of California, Davis, CA, USA

California produces $90 \%$ of the nation's processing tomatoes, with over $30 \%$ of production in water-scarce regions. In water-scarce counties, growers commonly irrigate to $100 \%$ of crop evapotranspiration (ET) until fruit set, then reduce irrigation to $80-60 \%$ ET; growers refer to this as deficit irrigation (DI). Recent studies indicate that DI may affect impacts of soil borne vine decline diseases in processing tomato production. This study aimed to monitor soil borne disease development under two irrigation treatments: $100 \%$ ET (well-watered) and 60\% ET (DI). Because compost amendments are postulated to improve plant water relations, we examined irrigation treatments under two soil management regimes: synthetic fertilizer (180lb N/acre) and composted poultry manure ( 2 tons/acre). Compared to well-watered plots, plant decline and foot rot incidence were 53\% and $8 \%$ greater, respectively, in tomatoes grown under DI with synthetic fertilizer. With the compost amendment, foot rot incidence was increased by $25 \%$ and $32 \%$ under well-watered and DI, respectively. Stomatal conductance was 30\% lower under DI, indicating plant stress. Two pathogens were recovered from plants with vine decline: Fusarium falciforme and putative $F$. oxysporum $\mathrm{f}$. sp. radicis lycopersici. These studies suggest that DI may pose a risk for Fusarium diseases, and that this risk is enhanced by use of compost. In order to develop disease and water co-management recommendations for growers, further studies will examine the additive effects of compost and DI over two years, with specific attention to $F$. falciforme.

Development of a quadruplex TaqMan real-time RT-qPCR assay for simultaneous detection of seed-borne Tobamoviruses infecting cucurbits

C. PADMANABHAN (1), T. Pitman (2), T. Tian (3), B. W. Falk (2), V. A. Mavrodieva (4), M. K. Nakhla (5), A. Roy (6), (1) North Carolina State University, Beltsville, MD, USA; (2) University of California, Davis, CA, USA; (3) California Dept of Food \& Agric, 
Out of six known cucurbit infecting tobamoviruses (CITV), Cucumber green mottle mosaic virus (CGMMV), Cucumber mottle virus $(\mathrm{CMoV})$ and Watermelon green mottle mosaic virus (WGMMV) are considered as most probable seed transmitted viruses. All three viruses produce leaf crinkle, mottle, mosaic, necrotic and stunted growth symptoms in plants grown in the field and greenhouses. Phylogenetic analysis of complete genome sequences of CITV displayed two distinct sub-clades in the phylogenetic tree; one for potential seed-transmitted and another for non-seed-transmitted viruses. Both CGMMV and WGMMV are transmitted through seed, while transmissibility of CMoV through seed is not yet confirmed. CGMMV is one of the most important seed borne viral pathogens worldwide, whereas CMoV and WGMMV are emerging viruses. The main objective of this study was to prevent the dispersal of CITV by developing a reliable, sensitive and specific diagnostic assay. Here, we report the development of a simplex and quadruplex one step real-time RT-qPCR assay for the detection of seed transmitted viruses using CITV isolates from UC Davis, CA and synthetic DNA of $\mathrm{CMoV}$. The reference gene Nad5 was selected as a host internal control. Primers and TaqMan probes generic to WGMMV and CMoV and specific to CGMMV and WGMMV were designed. Both simplex and multiplex RT-qPCR assays successfully detected all three CITV. Specificity of these assays were evaluated using CITV and other tobamoviruses. Newly developed real-time RT-qPCR assays will be useful tools for routine diagnosis in surveys and quarantine programs.

Detection of Spongospora subterranea in commercial peat-based potting mix and potting mix sanitation A. C. FULLADOLSA, Y. Zeng, A. O. Charkowski, Colorado State University, Fort Collins, CO, USA

The potato pathogen Spongospora subterranea is a broad-host, soil-borne pathogen that can also be found in peat-based potting mix (PPM), a medium for growing plants in most commercial and research greenhouse operations. S. subterranea posed a problem for seed potato production in recent years and may impact other greenhouse crops. S. subterranea detection in PPM samples is done with a quantitative real-time PCR (qPCR) assay, but results can be inconclusive due to low levels of the pathogen in PPM, the small subsample used in testing, and difficulty in homogenizing samples. Our first goal was to develop a reliable assay to detect S. subterranea in PPM. We performed qPCR assays on 6-10 subsamples of each of six PPM samples and a negative control (pathogen-free PPM). Subsamples showed high variation (e.g. 0-78 sporosori/g of PPM), making it difficult to determine pathogen presence in the sample. We also performed tomato bioassays consisting of two tomato plants grown in six pots with each PPM samples. The bioassay was more effective than qPCR, confirming $S$. subterranea presence in 4/6 samples. Our second goal was to evaluate two methods of PPM sanitation. We found that baking PPM at $105^{\circ} \mathrm{C}$ for 2,5 , or 8 days was not effective in reducing S. subterranea inoculum, but tripleautoclaving resulted in low to no detectable levels of sporosori. Although it is effective, autoclaving to sanitize PPM may not be practical at a commercial scale. Thus, reliable $S$. subterranea detection using qPCR in combination with a tomato bioassay remains critical for clean seed and plant production.

Evaluation of small molecules for management of bacterial spot of tomato and metabolomic insights into their mechanisms M. JIBRIN (1), Q. Liu (1), J. B. Jones (2), T. Garret (2), S. Zhang (1), (1) University of Florida, Homestead, FL, USA; (2) University of Florida, Gainesville, FL, USA

Small molecules are organic compounds of low molecular weight, often less than 900 Daltons, capable of permeating intracellular components where they interact and interfere with biological processes. They can function directly as a drug or serve as 'leads' in the discovery of new chemicals. Small molecules were evaluated for their effects on bacterial spot of tomato, an important disease of tomato in Florida and worldwide. These compounds included carvacrol, eugenol, and three cinnamic acids (i.e., transcinnamic acid, caffeic acid, and fumaric acid). The compounds were evaluated at various concentrations, alone and in combination with surfactants, for their potential to manage bacterial spot in tomato. Eugenol at $0.2 \%(\mathrm{v} / \mathrm{v})$, carvacrol at $0.75 \mathrm{mM}$, and cinnamic acids at minimum inhibitory concentrations significantly $(\mathrm{P}<0.05)$ reduced bacterial spot severity compared to the untreated control in the greenhouse. Preliminary results from field evaluations confirmed the greenhouse results. Metabolomic studies of $X$. perforans strains treated with carvacrol and eugenol were conducted using high resolution mass spectrometer coupled with positive and negative phase liquid chromatography. Preliminary results from metabolomics analyses revealed differential changes in metabolites at 1 and $6 \mathrm{~h}$ after treatment compared to the untreated control, providing insights into metabolomic changes that occurred in nontreated $X$. perforans bacterial cells by these small molecule compounds. Results from this study are important in advancing management of bacterial spot in tomato.

\section{Development and application of multiplex-PCR to specifically detect Xanthomonas translucens pvs. undulosa and translucens in infected field samples \\ W. Shoaf (1), N. MEZACHE (1), E. K. Luna (2), J. M. Lang (2), S. Carpenter (3), K. E. Ledman (4), R. Curland (4), J. E. Leach (2), A. J. Bogdanove (3), R. Dill-Macky (4), J. A. Pierzynski (5), J. M. Jacobs (1,6), V. Roman-Reyna (1,6), (1) Department of Plant Pathology, The Ohio State University, Columbus, OH, USA; (2) Colorado State University, Fort Collins, CO, USA; (3) Cornell University, Ithaca, NY, USA; (4) Department of Plant Pathology, University of Minnesota, Saint Paul, MN, USA; (5) The Ohio State University, Reynoldsburg, OH, USA; (6) Infectious Diseases Institute, The Ohio State University, Columbus, OH, USA}

Bacterial streak and black chaff, caused by Xanthomonas translucens pathovars, are major diseases affecting small grains. X. translucens pv. undulosa (Xtu) and X. translucens pv. translucens (Xtt) are seedborne pathogens that cause similar leaf necrosis symptoms on barley. Xtt is a more diverse group representing three distinct clades (A, B, C), while Xtu strains form one genomic group. Outbreaks of Xtu and Xtt have been a concern for wheat and barley growers in the Northern Great Plains and Upper Midwest, however, there are limited diagnostic tools for pathovar differentiation. We developed primers based on whole-genome differences for two 
multiplex-PCR reactions: one to distinguish between pathovars and another to differentiate Xtt subgroups. We validated the primers across more than twenty $X$. translucens isolates representing genetic diversity and global distribution. For bacteria in culture, instead of colony PCR, we implemented a twenty-minute genomic extraction from single colony that could be used for PCR and for nextgeneration sequencing. We examined the primers efficacy using seeds and leaves from infected barley plants. For infected leaf tissue PCR, we used water from leaf suspensions to ensure simplicity, avoiding both genomic extraction and bacterial culture. From US field samples, we identified Xtt subgroups A and B. To our knowledge, these are the first primers to distinguish Xtu and Xtt pathovars and Xtt subgroups. These molecular tools will support disease management strategies enabling detection and pathovar incidence analysis of X. translucens.

\section{Designing primers to improve detection of Pectobacterium species} M. REYNOSO, A. C. Fulladolsa, A. O. Charkowski, Colorado State University, Fort Collins, CO, USA

Pectobacterium sp. cause blackleg of potato and soft rot on a broad range of hosts. Several species and subspecies of the plant pathogenic genus Pectobacterium have recently been discovered, reclassified, renamed, or elevated to species. Currently available primers do not distinguish among species. For example, primers routinely used to detect $P$. carotovorum also amplify $P$. parmentieri DNA. Moreover, there are no PCR-based diagnostic methods for several newly described Pectobacterium species. The aim of this study is to improve and develop conventional PCR detection methods for several species of Pectobacterium by designing specific primers that differentiate the species. The Uniqprimer pipeline was used to align complete genomes, isolate distinct sequences in target strains, and design species-specific primers for $P$. parmentieri, $P$. brasiliense, $P$. odoriferum and $P$. carotovorum detection. We will present the results of primer set evaluation, parameter optimization for the PCR assays and their respective specificity and sensitivity of detection. These assays will improve diagnostics and epidemiological studies for this genus.

\section{Urgent problems call for collaboration: Growers, industry, and academia working together on citrus greening} M. IOTT, Bayer Crop Science, West Sacramento, CA, USA

Citrus greening, or Huanglongbing (HLB), is one of the most devastating diseases to hit the citrus growing community. It has cost the industry billions of dollars in production and jobs. There is currently no control of the disease, and research for finding solutions is fractured between universities, private companies, and grower consortiums which leads to lack of effective advances towards a solution. An integrated approach in methodology and collaboration is needed to find innovative solutions for management. In 2017, the Citrus Research Development Foundation (CRDF) partnered with Bayer to design an integrated platform to screen for potential candidates to help manage HLB in the field. This partnership consisted of a dual technical approach: targeting the pathogenic bacteria with an antibacterial solution using a beneficial microbe and an induced plant host defense solution using a synthetic chemical. To set this approach up for success, Bayer established collaborations with the University of California - Davis, Southern Gardens Citrus, Texas A\&M University, and University of Florida to evaluate advanced leads selected from high-throughput in vitro assays and in planta test systems. Currently, pilot field studies are being conducted to determine the best field methodology needed to determine effectiveness of the platform candidates. Using the strengths that each institution brings to the table, this collaborative partnership has fostered the ongoing development of a screening pipeline in a short amount of time that aims to address an urgent threat to the industry.

Competition between two criniviruses for transmission by the whitefly, Bemisia tabaci, and subsequent infection of melon plants S. MONDAL, L. Hladky, W. M. Wintermantel, USDA-ARS, Salinas, CA, USA

Two criniviruses, cucurbit yellow stunting disorder virus (CYSDV) and cucurbit chlorotic yellows virus (CCYV) (Crinivirus, Closteroviridae), are now both established in the low desert melon (Cucumis melo) production region of Yuma, AZ and Imperial Valley, CA. A 2019 field survey identified frequent co-infections in melon, but seasonal differences in prevalence among locations. Experiments were conducted to determine the influence of inoculation timing on whitefly transmission from single and co-infections in melon. Whiteflies (B. tabaci MEAM1) were given a 48-h acquisition access period on melon leaves infected with CYSDV, CCYV, and CYSDV+CCYV, then were transferred in clip cages to 2-4 leaf stage melon plants. Forty viruliferous whiteflies from each virus source were provided a 48-h inoculation access period simultaneously on the same leaf using separate clip cages or separately 7 days apart on neighboring leaves. Multiplex RT-PCR demonstrated that when melon plants were simultaneously inoculated with each virus, CCYV was predominantly transmitted. When CYSDV was inoculated prior to CCYV, CYSDV was transmitted either alone or with CCYV. In contrast, only CCYV was transmitted when it was inoculated prior to CYSDV. This transmission bias may result from preferential virus accumulation in melon following inoculation or from competition for binding within the whitefly. Ongoing studies are comparing differential accumulation of each virus throughout vines in co-infected plants and preferential virus binding in whiteflies.

Photolyase plays a key role in UV-mediated management of powdery mildew R. PATHAK (1), Å. Ergon (1), A. Stensvand (1,2), H. R. Gislerød (1), K. A. Solhaug (3), L. E. Cadle-Davidson (4), A. Suthaparan (1), (1) Faculty of Biosciences, Norwegian University of Life Sciences, Ås, NORWAY; (2) Norwegian Institute of Bioeconomy Research, Ås, NORWAY; (3) Norwegian University of Life Sciences, Ås, NORWAY; (4) USDA Grape Genetics Research Unit, Geneva, NY, USA

Powdery mildews are caused by obligate biotrophic fungi and can cause severe yield losses in a range of crops. Fungicides are the primary tool to control the disease, but ultraviolet (UV) and light-mediated disease management could be an environmentally friendly alternative. Previous studies showed that short wavelength UV $(<290 \mathrm{~nm})$ was effective against powdery mildews, but the effect depended on the UV dose and the subsequent light environment. UV application was effective if applied alone, but with an increased 
efficacy in combination with red light. However, it was ineffective if applied together with UVA/blue light. This indicated a possible role of photolyase-mediated repair of DNA lesions (photoreactivation) in determining the treatment efficacy. Whole genome and transcriptome sequencing of Pseudoidium neolycopersici, the cause of tomato powdery mildew, revealed the presence of three cryptochrome photolyase family (CPF) like genes. CPF protein cofactors harvest energy from UVA/blue light for electron transfermediated repair of UV-induced lesions. Complementation assays with $E$. coli ( $p h r$ mutant) carrying these genes confirmed that one of these three genes plays a key role in photoreactivation activity. Furthermore, this candidate photolyase expressed broad action spectra $(365-454 \mathrm{~nm})$ with high action efficiency within the UVA range. The finding of photoreactivation in $P$. neolycopersici provides insights for planning an effective and accurate irradiation-based disease management strategy.

Incidence and abundance of Helminthosporium solani, causal agent of potato silver scurf, in Wisconsin potato production J. SPYCHALLA, S. Macchiavelli-Girón, Y. Chen, S. A. Jordan, A. J. Gevens, University of Wisconsin-Madison, Madison, WI, USA

Silver scurf is a potato blemish disease caused by the fungal pathogen Helminthosporium solani. This disease affects periderm quality, negatively impacting potato marketability and storability in Wisconsin and other potato-producing regions. Improved understanding of the pathogen's biology will help prescription of optimum timing for treatments or cultural practices to further reduce inoculum and disease. We investigated the presence and quantity of $H$. solani through the time course of the potato production season by surveying soil and plant tissues for the pathogen at key growth stages. We subjected tuber and soil samples collected from 6 fungicide regimes, with 4 replicates, from the field at four different times during the growing season in 2017 and 2018 to DNA extraction and quantitative PCR. Results from 2017 were analyzed using a repeated measures ANOVA. There were no significant differences in $H$. solani DNA quantity among the 6 different fungicide treatments or application timings $(p=0.8941)$. However, there were differences detected between sampling times $(\mathrm{p}=0.0610)$. Pathogen DNA was detected at planting (in the seed tubers) and at harvest. In 2018, pathogen DNA was not detected until the last time point (at harvest), so these data were analyzed using a one-way ANOVA. There was no significant fungicide treatment effect on $H$. solani quantity $(\mathrm{p}=0.08467)$. Results indicated that pathogen quantity was not differentially affected by fungicide treatments and that the pathogen in our system was predominantly seedborne and only infected tubers later in the season.

An intact cuticle regulates apoplastic transport of salicylic acid and systemic acquired resistance H. LIU (1), G. H. Lim (2), K. Yu (2), R. Liu (2), M. B. Shine (2), A. Kachroo PhD (2), P. Kachroo (2), (1) University of Kentucky, Lexington, KY, USA; (2) University of Kentucky, Lexington, KY, USA

The plant cuticle is a hydrophobic layer that covers the aerial surface of plants and forms the first line of contact with the environment. The mature cuticle is composed of cutin and cuticular wax. The cuticular wax is a complex mixture of very-long-chain fatty acid derivatives formed upon elongation of fatty acids (FA), which are biosynthesized in the plastids. The C16 and C18 FAs are also required for biosynthesis of cutin, a polymer of $\mathrm{C} 16$ and $\mathrm{C} 18$ diacids and w- and mid-chain hydroxy FAs. Due to the importance of C16 and C18 FA levels in both wax and cutin biosynthesis, reductions in the flux of these FA s impairs cuticle development. Earlier we showed that an intact cuticle is important for the broad-spectrum defense signaling mechanism, systemic acquired resistance (SAR). SAR is a form of systemic immunity that protects distal uninfected parts of the plant against secondary infections. A number of chemical SAR inducers have been identified including salicylic acid (SA), which accumulates in both inoculated and uninoculated leaves and is essential for SAR. It is generally assumed that despite the fact that SA biosynthesis in the distal tissue is absolutely essential for SAR, SA is not a translocated mobile signal during SAR. We show that transport of SA from local to distal tissues is indeed essential for SAR and this transport is governed by cuticle facilitated water potential in the infected tissue. We also show that intracellular partitioning of SA plays an important role in SAR.

Improving fungicide efficacy for management of Cercospora leaf spot (Cercospora beticola Sacc.) in sugarbeet (Beta vulgaris L.) K. THORNTON (1), A. W. Schaafsma (2), J. Deveau (3), C. Trueman (2), (1) University of Guelph, Ingersoll, ON, CANADA; (2) University of Guelph, Ridgetown, ON, CANADA; (3) Ontario Ministry of Agriculture, Food, and Rural Affairs, Simcoe, CANADA

Cercospora leaf spot (CLS; Cercospora beticola Sacc.) has a devastating economic impact on Ontario sugarbeet (Beta vulgaris L.) production due to reduced yield and sugar quality. Mancozeb is a contact fungicide that relies on good canopy coverage in order to be effective. InterLock, a vegetable oil-based spray adjuvant (VOA), is designed to improve spray deposition by optimizing spray droplet size. In a previous study, greater application carrier volumes reduced disease severity when disease intensity was high. The potential of VOA and higher carrier volumes to improve efficacy of mancozeb for CLS management was evaluated in two field trials in Ontario, Canada. Trials were arranged in a randomized complete block with four replications. Applications of water, mancozeb, VOA, and mancozeb in combination with VOA using carrier volumes of 115, 235, 350, and $470 \mathrm{~L} \mathrm{ha}^{-1}$ were made on a 14-day interval. Adding VOA to mancozeb did not reduce disease severity or improve sugar recovery or quality more than applications of mancozeb alone. Carrier volume did not have an effect on disease severity or sugar quality, however recoverable white sucrose acre ${ }^{-1}$ was greater with $350 \mathrm{~L} \mathrm{ha}^{-1}$ compared to $115 \mathrm{~L} \mathrm{ha}^{-1}$. No treatment increased sugarbeet yield compared to the water control. Identifying practices that improve fungicide efficacy will provide growers with the information needed to develop strategic disease management programs. A continuation of this research is planned for 2020 to further evaluate effects of VOA and carrier volume on CLS.

\section{Management thresholds based on weather data provide cost-effective control of citrus and blueberry diseases caused by} Colletotrichum spp.

A. B. GAMA (1), L. Cordova (2), N. Peres (3), M. M. Dewdney (1), (1) University of Florida, Lake Alfred, FL, USA; (2) Corteva Agriscience, Indianapolis, IN, USA; (3) University of Florida - Gulf Coast Research and Education Center, Wimauma, FL, USA 
The disease triangle is a pivotal concept studied by plant pathologists. A disease can only occur when the pathogen is virulent, the host is susceptible, and the environmental conditions are favorable. Most disease management practices focus on the pathogen and the host. The environment is seldom the core of management programs. Florida growers control postbloom fruit drop (PFD) of citrus and anthracnose fruit rot (AFR) of blueberry based on a fungicide application calendar, regardless of environmental conditions. PFD and AFR are mostly caused by Colletotrichum acutatum and C. gloeosporioides species complexes in Florida, respectively. Our hypothesis was that weather data and epidemiological models developed for Colletotrichum spp. could aid growers decide when disease control was required. We used threshold values of the models and site-specific weather data to time fungicide applications and compared their efficacy to calendar-based strategies. Five and nine field experiments were conducted in different citrus and blueberry groves in Florida, respectively. The efficacy of the threshold treatments was consistently equal to or better than that of the calendar-based strategy. Treatment effect was not significant on citrus $(P=0.053)$ and blueberry $(P=0.64)$ yield or PFD $(P=0.236)$ and AFR $(P=0.79)$ incidence in our trials. The number of applications following the thresholds was reduced up to $100 \%$ for PFD and $43 \%$ for AFR. In conclusion, the often-forgotten environmental vertex of the disease triangle can be the core of PFD and AFR management programs in Florida.

\title{
Bacterial stem blight of alfalfa: An emerging disease in the United States caused by Pseudomonas syringae pv. syringae and Pseudomonas viridiflava
}

S. LIPPS (1), D. A. Samac (2), (1) University of Minnesota, Saint Paul, MN, USA; (2) USDA ARS, St Paul, MN, USA

In the Intermountain West, alfalfa producers have observed significant damage from frosts in both spring and fall, setting back growth and reducing forage yields. Associated with this damage is bacterial stem blight (BSB), a disease caused by the ice nucleation-active bacterium Pseudomonas syringae pv. syringae (phylogroup $2 \mathrm{~b}$ of the $P$. syringae complex). The bacterium promotes frost formation, penetrates stems at the injury sites, and decays leaves and stems. Recently, Pseudomonas viridiflava (Pv) (phylogroup 7a), was also identified as a causal agent BSB. Although $P v$ has low ice nucleation activity, it produces high levels of pectolytic enzymes, which likely contribute to the disease. Here, the genetic diversity of a population of Pss isolates from several locations across the United States was analyzed via multi-locus sequence analysis using the housekeeping genes gltA, gap $A$, $r p o D$, and gyr $B$. Limited $P v$ sequence data is shown here as it is still in progress. Isolate virulence data from both species was also collected. The Pss pathogen population is widespread on alfalfa in the U.S. and genetically similar, suggesting that there has not yet been enough selection to induce major changes. Although the population is largely homogenous, there is still a fair amount of diversity in the population given by SNPs and the grouping of isolates to several reference strains within phylogroup 2 . This could suggest that the pathogen was not recently introduced. Future work will address diversity of the associated $P v$ species, and its involvement in causing BSB symptoms in alfalfa.

\author{
Brown root rot and crown rot of cannabis (Cannabis sativa L., marijuana) plants caused by Fusarium (Cylindrocarpon) \\ lichenicola \\ Z. K. PUNJA, Simon Fraser Univ, Burnaby, BC, CANADA
}

Young flowering plants of cannabis (Cannabis sativa L., marijuana) with symptoms of yellowing, root browning and crown rot were sampled during 2018-2019. From tissue isolations, slow-growing colonies with gray-white aerial mycelium and a distinct chestnutbrown colony pigmentation were obtained. The colonies produced cylindrical 1-3 septate conidia without a distinctive foot cell and chlamydospores were formed in culture. Microconidia were absent. Phylogenetic analysis of elongation factor (EF -1) and ITS1-5.8SITS2 sequences of three isolates placed them in the Fusarium (Cylindrocarpon) lichenicola subclade of the Fusarium solani species complex (FSSC). Pathogenicity tests using a mycelial and spore suspension on cannabis cuttings and rooted plants produced symptoms of yellowing and wilting after 3 weeks. The origin of $F$. lichenicola was the cocofibre (coconut coir) growing substrate commonly used in cannabis cultivation, as serial dilutions showed inoculum of $F$. lichenicola was present in cocofibre samples. Previous reports of $F$. lichenicola (Cylindrocarpon lichenicola) are from tropical climates, where the fungus is associated with dermal and occular infections of human tissues, with one report of it causing a fruit rot on pomelo. Members of the FSSC cause opportunistic infection of human tissues as well as diseases of plants worldwide, and many are placed in the genus Neocosmaspora. The recovery of Fusarium (Neocosmaspora) lichenicola from cannabis plants represents the first occurrence worldwide and it is considered to be a weak introduced pathogen on cannabis plants.

High resolution DNA melting analysis for detection of potato mop-top virus and its fungal vector, Spongospora subterranean, in soil and plant samples

X. NIE (1), M. Singh PhD (2), D. Chen (3), C. Gilchrist (4), Y. Soqrat (5), M. Shukla (5), A. Creelman (5), J. Lavoie (6), V. S. Bisht (7), (1) Agriculture and Agri-Food Canada, Fredericton, NB, CANADA; (2) Agricultural Certification Services, Fredericton, NB, CANADA; (3) AAFC-Fredericton Research and Development Centre, Fredericton, NB, CANADA; (4) Fredericton Research and Development Centre, CANADA; (5) Fredericton Research and Development Centre, Fredericton, CANADA; (6) New Brunswick Department of Agriculture, Aquaculture and Fisheries, Wicklow, CANADA; (7) Manitoba Agric Foods \& Rural Initatives, Carman, MB, CANADA

A high resolution DNA melting (HRM) analysis was developed for the detection of potato mop-top virus (PMTV) and its fungal vector, Spongospora subterranea f.sp. subterranea (Sss). To achieve this, we first developed a tobacco-based PMTV baiting system to assess the infestation of soil with PMTV. We then evaluated various nucleic acid extraction procedures and identified the most reliable and suitable protocol for PCR-based detection of Sss and PMTV. New primer pairs specific to PMTV RNA 1, 2 and 3 were designed in order to obtain amplicons that can be efficiently amplified by real-time PCR and differentiated by HRM. Indeed, under real-time PCR, successful amplifications of the target amplicons were achieved. The amplicons exhibited different melting profiles, and thus can be 
differentiated readily. All three duplex PCR combinations (i.e., Sss + PMTV RNA 1, Sss + PMTV RNA 2 and Sss + RNA 3 ) led to successful detection of respective PMTV RNA species and Sss in the samples. The assay was then applied to soil samples collected from three fields; two were known to be infested with PMTV and one was believed to be PMTV-free. Indeed, HRM assay demonstrated that most samples from the PMTV-infested fields were positive for both PMTV and Sss and the samples from the PMTV-free field were negative for the organisms. These results were further validated by the tobacco-based baiting assay. When the HRM assay was applied to 250 field potatoes collected from PMTV-infested areas, 8 tested PMTV-positive, which is consistent with the ELISA and the conventional RT-PCR assays.

Expression profiling of lettuce and tomato root response genes after crab shell chitin treatment S. L. KANDEL, A. Anchieta, B. Mou, S. J. Klosterman, USDA-ARS, Salinas, CA, USA

Chitin and other components released from fungal cell walls are well known elicitors of plant defense responses. Exogenous applications of chitin induce expression of defense-related genes, stimulate the biosynthesis of antioxidant compounds, regulate signaling pathways that transduce signals for resistance, and can increase resilience to plant stress. But the full scope of plant gene expression affected in chitin-specific responses, particularly in root tissues, has not been established. In this study, we applied RNA sequencing for evaluation of the expression profile of chitin-responsive genes in stem lettuce line PI 251246 and tomato cultivar 'Bonny Best' at $0 \mathrm{hr}$ and at 24 hours post chitin application. RNA-Seq analysis revealed that 147 and 158 genes were significantly differentially expressed in lettuce and tomato, respectively. The highly up-regulated genes in lettuce included those genes encoding endoglucanase 1like protein, putative lipid transfer protein, bifunctional riboflavin biosynthesis protein, and an auxin-responsive protein. In tomato roots, significantly up-regulated genes included those encoding a serine/threonine-protein phosphatase, plant cysteine oxidase, serine/threonine-protein kinase, a LRR receptor-like serine/threonine-protein kinase, and a probable glutathione S-transferase. This study provides information on innate responses of lettuce and tomato to chitin, and thus represents a valuable resource to those investigating the molecular basis of chitin-mediated responses in plants and candidate genes associated with defense.

A novel boron-containing compound, BAG20, to control soilborne plant pathogenic nematodes and fungi C. HAHNE (1), W. Cheng (2), M. Mackowiak (2), C. McGregor (2), M. Samuels (2), L. Steere (2), Y. Zhang (2), (1) Boragen, Durham, NC, USA; (2) Boragen, Research Triangle Park, NC, USA

Boron chemistry has been shown to possess unique fungicidal properties in both human health and agriculture. Boragen utilizes boroncontaining compounds to find solutions to pressing issues in these areas. A novel dual-action fungicide/nematicide, BAG20, has demonstrated the ability to effectively control a broad spectrum of agronomically relevant soilborne plant pathogens. BAG20 has exhibited both in vitro and in vivo efficacy against the two most important nematode crop pests in North America - Meloidogyne incognita (Southern root-knot nematode) and Heterodera glycines (Soybean cyst nematode). BAG20 has also demonstrated fungicidal activity against common soilborne pathogens Rhizoctonia solani and Pythium ultimum, outperforming established nematicide/fungicides - fluopyram and tioxazafen - in agar-based assays. Greenhouse trials have shown that an in-furrow, at planting application of BAG20 significantly reduces nematode reproduction in maize (85\% inhibition of RKN eggs/gram of root compared to control) and squash (98\%). This treatment also has resulted in increased biomass in cotton (79\% increase in root + shoot dry weight compared to control) and soybean (331\%) inoculated with RKN and SCN, respectively. In regard to product safety, BAG20 has shown low cytotoxicity $(\mathrm{AC} 50>200 \mathrm{uM})$ and no genotoxicity in screening level studies. Overall, BAG20 is a promising new dual-action fungicide/nematicide that offers root system protection in soilborne disease management.

Calcium sprays and dips as a management strategy for Botrytis blight in cut roses M. MUNOZ, J. Faust, G. Schnabel, Clemson University, Clemson, SC, USA

Botrytis blight caused by Botrytis cinerea is a devastating disease of cut rose flowers worldwide. Fungicide applications are used as the main disease management strategy. Preharvest fungicide applications and postharvest fungicide dips and showers are common strategies to avoid disease development. $B$. cinerea is known to develop resistance to fungicides making alternative strategies attractive. The

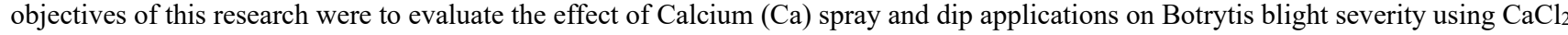
as the source of $\mathrm{Ca}$; and to evaluate changes in $\mathrm{Ca}$ concentration in rose tissue. Ca sprays were applied weekly for 24 weeks to 'Orange Crush' flowers in two commercial greenhouses in Colombia at rates of 0, 500, 1000 and $1500 \mathrm{mg} / \mathrm{L}$. Roses from each treatment were harvested and shipped to Clemson University for analysis. Additionally, dips of commercial flower bouquets of 'Freedom' and 'Orange Crush' with 0, 1000 and $2000 \mathrm{mg} / \mathrm{L} \mathrm{Ca}$ were investigated. Upon arrival, roses of each treatment were placed at 100\% RH and Botrytis blight severity was evaluated. Tissue analysis using Inductively Coupled Plasma Spectroscopy and Energy Dispersive X-ray Spectroscopy techniques was performed to assess Ca concentration in the petals. Calcium sprays and dips decreased disease severity; however, the dips were more effective. Ca dips but not sprays increased Ca content in petals. Our results suggest that postharvest Ca dips are more effective compared to sprays, likely due to larger amount of solution reaching flower tissue resulting in an increased Ca content.

\section{Distribution and population structure of Rhizoctonia zeae in the North Central United States}

N. GAMBHIR (1), S. Kodati (2), A. O. Adesemoye (3), O. O. Ajayi (4), K. Bissonnette (5), C. A. Bradley PhD (6), M. Chilvers (7), A. M. Fakhoury (8), T. A. Jackson-Ziems (1), L. F. S. Leandro (9), C. R. Little (10), D. K. Malvick (11), F. M. Mathew (12), B. D. Nelson Jr. (13), G. Sassenrath (14), D. L. Smith (15), D. E. P. Telenko (16), K. A. Wise (6), S. E. Everhart (1), (1) University of Nebraska, Lincoln, NE, USA; (2) University of Nebraska Lincoln, North Platte, NE, USA; (3) Terramera Inc, Vancouver, BC, CANADA; (4) Monsanto Company, Chesterfield, MO, USA; (5) University of Missouri, Columbia, MO, USA; (6) University of Kentucky, Princeton, 
KY, USA; (7) Michigan State University, East Lansing, MI, USA; (8) Southern Illinois University, Carbondale, IL, USA; (9) Iowa State University, Ames, IA, USA; (10) Dept. of Plant Pathology, Kansas State University, Manhattan, KS, USA; (11) University of Minnesota, Department of Plant Pathology, St. Paul, MN, USA; (12) South Dakota State University, Brookings, SD, USA; (13) Plant Pathology NDSU, Fargo, ND, USA; (14) Kansas State University, Parsons, KS, USA; (15) University of Wisconsin-Madison, Madison, WI, USA; (16) Department of Botany and Plant Pathology, Purdue University, West Lafayette, IN, USA

Corn (Zea mays) and soybean (Glycine max) are the major commodities in the North Central United States. Rhizoctonia zeae was recently identified as the dominant Rhizoctonia species in Nebraska and was shown to have pathogenicity to corn and soybean. Knowledge of the pathogen population structure is relevant for designing effective disease management strategies. Plant and soil samples were collected in 2015-2017 and 2019 from 118 corn and soybean fields in 12 states (IA, IL, IN, KS, KY, MI, MN, MO, ND, $\mathrm{NE}, \mathrm{SD}$, and WI). A total of 347 Rhizoctonia isolates were obtained from plant and soil samples, of which $65.4 \%$ were $R$. zeae. Five $R$. zeae isolates were analyzed by whole genome sequencing and 1,594 candidate microsatellite loci were identified. Forty-three microsatellite loci that showed in silico polymorphisms were used to design primers, which were subsequently evaluated on $13 R$. zeae isolates. Preliminary results showed seven primers produced consistent amplification and polymorphisms, with an average of 5.86 alleles, medium-high gene diversity $\left(H_{\exp }=0.72\right)$, and medium-high evenness $(E .5=0.78)$. These markers will be used to further characterize the population structure of $R$. zeae, which will allow inference of the evolutionary potential, mating system, and dispersal mechanism in populations of this species. This information will help to devise better control strategies for $R$. zeae in the North Central United States.

\section{Evaluation of resistance of USDA spinach germplasm to Stemphylium vesicarium} B. LIU, G. Bhattarai, A. Shi, J. Correll, C. Feng, University of Arkansas, Fayetteville, AR, USA

Leaf spot diseases of spinach, caused by Stemphylium vesicarium, can reduce yield and quality, and are a major constraint in spinach production in the U.S. Screening spinach germplasm to identify resistant material will be a critical component for disease management. A total of 148 USDA spinach lines and 169 commercial cultivars, including a subset of contemporary cultivars, were screened for disease severity by inoculating 34 to 39-day-old spinach plants with an isolate $S$. versicarium (Sb-1-St001) at a spore concentration of 1 $\times 10^{6}$. Non-inoculated controls (Viroflay) were sprayed with distilled water and subjected to the same conditions. After inoculation, plants were incubated in a dew chamber for $48 \mathrm{~h}$ at 20 to $22^{\circ} \mathrm{C}$. Plants were then transferred to the greenhouse $\left(20\right.$ to $\left.30^{\circ} \mathrm{C}\right)$ and disease severity was rated on scale of 0 to 4.0 from $7-16$ days after inoculation. The disease severity (DS) scale used was $0=$ no symptoms on leaves; $1=1-25 \%$ leaf area infected; $2=26-50 \%$ leaf area infected; $3=51-75 \%$ leaf area infected; $4=76-100 \%$ leaf area infected. Of the lines and cultivars tested, 35\% were considered highly susceptible (DS $=4.0), 35 \%$ were susceptible $(\mathrm{DS}=3.0-4.0), 17 \%$ were moderately resistant ( $\mathrm{DS}=2.0-3.0)$, and $14 \%$ were highly resistant $(\mathrm{DS}=1.0-2.0)$. Phenotypic evaluation of the USDA germplasm identified a number of lines that could be used to accelerate disease resistance breeding to Stemphylium leaf spot.

Dampening of wound-induced jasmonate burst in Arabidopsis grown at eCO $\mathrm{C}_{2}$; does nitrogen fertilizer make a difference? J. Martinez Henao (1), E. Demers (1), K. Grosser (2), A. Schedl (2), N. van Dam (2), J. BEDE (3), (1) McGill University, CANADA; (2) German Centre for Integrative Biodiversity Research (iDiv), GERMANY; (3) McGill University, Ste-Anne-de-Bellevue, QC, CANADA

Leaf damage by chewing insects or necrotrophic pathogens results in an increase in jasmonate (JA) phytohormones that leads to induced defense responses; however, the wound-induced JA burst is dampened in Arabidopsis thaliana plants grown at elevated $\mathrm{CO}_{2}$ $\left(\mathrm{eCO}_{2}\right)$, particularly in well nitrate fertilized plants. This may, in part, explain why caterpillar and beetle herbivores are often more destructive at $\mathrm{eCO}_{2}$. We hypothesize that the reduction in photorespiration in $\mathrm{C} 3$ plants grown at $\mathrm{eCO}_{2}$ changes the cellular distribution of reductant resulting in increased competition for reductant between pathways, such as the Calvin-Benson-Bassham cycle, nitrate assimilation and the Halliwell-Asada cycle, which results in a shift in cellular oxidative balance and, thus, plant defense responses. Nitrate assimilation requires more reductant than ammonium. Therefore, the effect of these two fertilizers on wound-induced responses were compared in plants grown at ambient or $\mathrm{eCO}_{2}$. As expected, foliar levels of pyridine nucleotides and redox pools indicate a slightly more oxidative cellular environment in nitrate-fertilized plants. Compared to plants grown at ambient conditions, the woundinduced, fertilizer-rate associated increase in jasmonyl-L-isoleucine was muted in plants grown at eCO $\mathrm{C}_{2}$; however, a fertilizer-dependent difference in JA levels was not observed. JAs are key regulators of developmental and defensive processes. Understanding how phytohormones are impacted by nitrogen fertilization and $\mathrm{CO}_{2}$ is critical for crop management practices under future environmental conditions.

Prevalence and characterization of Wheat streak mosaic virus in the Kansas winter wheat N. B. RANABHAT (1), M. Bruce (1), J. Fellers (2), J. Rupp (1), (1) Kansas State University, Manhattan, KS, USA; (2) USDA-ARS, Manhattan, KS, USA

Wheat streak mosaic virus (WSMV), one of the most common wheat viruses, causes significant yield loss in wheat production. The key management strategy against WSMV is the use of genetic resistance, which is limited. Frequent identification and characterization of field isolates of WSMV are essential to determine the population structure of the virus in the field and develop a relevant collection of isolates to test the reaction of wheat breeding lines. A statewide survey was conducted to collect the WSMV isolates in the Kansas winter wheat fields. In 2019, a total of 101 symptomatic wheat leaves were collected from 60 different counties of Kansas. EnzymeLinked Immunosorbent Assay (ELISA) results showed that there was a high prevalence of WSMV incidence. Out of 101 samples, 72 samples (71.3\%) were positive for WSMV, representing 42 counties. Field surveys will continue in 2020. Nanopore sequencing 
technology will be used for whole genome sequencing of the positive WSMV samples for characterization. The knowledge of unique WSMV isolates present in the Kansas wheat fields will enable plant breeders to use relevant isolates in their future breeding program, while providing a more complete picture of WSMV prevalence and population structure in Kansas.

\section{Draft whole-genome sequence of the sugarcane rust fungal pathogen Puccinia kuehnii}

D. E. CARDENAS (1), J. Cheema (2), D. Oppelaar (3), M. Hincapie (4), S. Sood (5), J. C. Huguet-Tapia (1), D. G. O. Saunders (6), J. C. Comstock (7), L. M. Cano (8), P. C. Rott PhD (4,9), (1) University of Florida, Gainesville, FL, USA; (2) John Innes Centre, Norwich Research Park, Norwich, UK., Norwich, UNITED KINGDOM; (3) University of Florida, Fort Pierce, FL, USA; (4) University of Florida, Belle Glade, FL, USA; (5) Sugarcane Field Station, USDA-ARS, Belle Glade, FL, USA; (6) John Innes Centre, Norwich, UNITED KINGDOM; (7) USDA-ARS (Retired), Pensacola, FL, USA; (8) University of Florida, UF/IFAS, IRREC, Plant Pathology, Fort Pierce, FL, USA; (9) CIRAD-Département BIOS UMR BGPI, Montpellier, FRANCE

Puccinia kuehnii, the causal agent of orange rust, is a fungal pathogen that has conduced to significant losses of the sugarcane crop in Florida since 2007. During plant infection, rust fungi deliver effector proteins into host tissues using a specialized feeding structure called a haustorium to manipulate plant functions and promote parasitic growth. Identification of these effectors is, therefore, an essential step to understand host-pathogen interactions and to develop new control methods. Genomic DNA was extracted from spores of isolates P. kuehnii 1040 and P. kuehnii 2143 that were collected from sugarcane cultivars CL 85-1040 and CP 89-2143, respectively. Short read Illumina and long read SMRT-Pacbio sequencing was used to generate hybrid genome assemblies of the two orange rust isolates. The P. kuehnii $1040 \mathrm{draft}$ genome has a size of $73.9 \mathrm{Mb}$ with 5,648 scaffolds and a N50 value of $93 \mathrm{~Kb}$. The draft genome of P. kuehnii 2143 has a size of $113 \mathrm{Mb}$ with 8,502 scaffolds and a N50 value of $22 \mathrm{~Kb}$. BUSCO analyses of coding spaces revealed that genome completeness reached $97.1 \%$ for isolate 1040 and $90.3 \%$ for isolate 2143 . Downstream gene annotations will be performed using the 24,945 and 17,143 predicted gene calls for 1040 and 2143, respectively. These two rust genome resources are useful databases for the sugarcane community and for downstream genome analysis including secretome and disease-related effector annotations.

\section{Temporal dynamics of tar spot of corn in the U.S. and Honduras}

C. C. GONGORA-CANUL (1), C. E. Puerto Hernandez (2), D. E. P. Telenko (1), N. M. Kleczewski (3), L. Henriquez-Dole (4), M. C. Avellaneda Barbosa (5), T. J. Ross (6), J. Valle-Torres (7), C. D. Cruz (6), (1) Department of Botany and Plant Pathology, Purdue University, West Lafayette, IN, USA; (2) Zamorano Escuela Agricola Panamericana Biblioteca, San Antonio de Oriente, Francisco Moraz, HONDURAS; (3) University of Illinois, Urbana, IL, USA; (4) Escuela Agrícola Zamorano, HONDURAS; (5) Louisiana State University, Baton Rouge, LA, USA; (6) Purdue University, West Lafayette, IN, USA; (7) Purdue University, West Lafayette, IN, USA

Tar spot, caused by Phyllachora maydis, is a major disease of corn native to countries in Latin America and the Caribbean. The disease emerged in the U.S. in 2015, and severe outbreaks in 2018 and 2019 caused an economic impact throughout the Midwest.

Epidemiological studies on tar spot are scarce; here, we describe and compare the temporal dynamics of tar spot epidemics in various locations within the U.S. and Honduras. In 2019, four experiments were conducted, with three field sites in the U.S. (two in Indiana, $\mathrm{n}=516, \mathrm{n}=576$, and one in Illinois, $\mathrm{n}=360)$, and one in Honduras $(\mathrm{n}=432)$. Plants were visually rated over time for tar spot severity at the lower, middle, and upper canopy. Gompertz (G) and logistic (L) models fit disease progress curves (DPC's) data better than exponential (E) and monomolecular (M) in the U.S. ( ${ }_{G} R^{2}=0.93, p=0.005 ; \mathrm{L}^{2}=0.92, p=0.005 ; \mathrm{E}^{2}=0.90, \mathrm{p}=0.005$; $\mathrm{M}^{2}=0.77$, $\mathrm{p}=0.02$ ) and Honduras (G $\mathrm{R}^{2}=0.79, \mathrm{p}=0.04 ; \mathrm{L} \mathrm{R}^{2}=0.78, \mathrm{p}=0.04 ; \mathrm{E} \mathrm{R}^{2}=0.74, \mathrm{p}=0.05 ; \mathrm{m}^{2}=0.79, \mathrm{p}=\mathrm{ns}$ ). Moreover, the Gompertz model fit well the data collected from the lower, middle and upper canopy in the U.S. $\left(R^{2}=0.91, p=0.01 ; R^{2}=0.94, p=0.006 ; R^{2}=0.93, p=0.001\right)$ and Honduras $\left(R^{2}=0.70\right.$, $\mathrm{p}=0.04 ; \mathrm{R}^{2}=0.81, \mathrm{p}=\mathrm{ns} ; \mathrm{R}^{2}=0.87, \mathrm{p}=0.03$ ). Middle and upper canopy DPC's data resulted in better model description than the lower canopy. Overall, the rate of tar spot change over time was similar in the U.S. $\left(\mathrm{r}_{\mathrm{G}^{*}}=0.051\right.$ day $\left.^{-1}\right)$ and Honduras $\left(\mathrm{r}_{\mathrm{G}^{*}}=0.050\right.$ day $\left.{ }^{-1}\right)$. Results are foundational to developing tar spot epidemic prediction and surveillance models.

\section{Using the Oxford Nanopore MinION'M sequencer to detect the boxwood blight pathogen, Calonectria pseudonaviculata (Cps), in infected plant tissue}

S. YANG (1), M. Aguilera (1,2), M. A. Hansen (1), E. A. Bush (1), S. Li (1), B. A. Vinatzer (1), (1) School of Plant and Environmental Sciences, Virginia Tech, Blacksburg, VA, USA; (2) Genetics, Bioinformatics, and Computational Biology program, Virginia Tech, Blacksburg, VA, USA

Boxwood is one of the most popular ornamentals in the U.S. Boxwood blight, caused by Calonectria pseudonaviculata (Cps), is a devastating fungal disease of great concern to the horticulture industry. The best way to protect boxwood from boxwood blight is to prevent $C p s$ dissemination through infected plants. However, infected boxwood is initially asymptomatic, and coinfection with other fungi complicates diagnosis. The Oxford Nanopore MinIONTM is a portable sequencing platform, which can be used for fast pathogen detection using DNA directly extracted from plant tissue. Since rapid detection is critical for effective disease management, the MinION sequencer could be advantageous compared to other diagnostic methods that are more time-consuming or less reliable. In this study, several DNA extraction methods from boxwood blight-infected boxwood were compared followed by sequencing with the MinION. DNA was extracted either from homogenized boxwood or from filtrate recovered from sonicated boxwood. After sequencing, data were analyzed using a customized bioinformatics pipeline. While DNA sequences of $C p s$ were identified in all samples regardless of the extraction method, the number of $C p s$ sequences varied. Overall, homogenization resulted in more $C p s$ sequences than sonication. Since our goal is to detect $C p s$ in asymptomatic plants, next the detection limit of sequencing with the MinION will be determined using the most efficient DNA extraction method. Our work provides a promising new approach to detect $C p s$ and could be used for rapid detection of other fungal pathogens. 
Developing DNA-based tests to breed fire blight resistant pears

J. D. ZURN, M. Driskill, N. Bassil, USDA-ARS National Clonal Germplasm Repository, Corvallis, OR, USA

Fire blight, caused by Erwinia amylovora, is one of the most devastating diseases of pears (Pyrus spp.) and is a persistent problem in major production regions in the U.S. The most commonly grown scions and rootstocks are susceptible to the disease and new resistant cultivars are needed. Very little work has been done to identify fire blight resistance quantitative trait loci (QTLs) in pear. QTLs have been consistently detected in a similar region on chromosome 2 in the resistant cultivars Moonglow, Potomac, Old Home, and the selection NJA2R59T69. Recent advancements in pear genomics have shown that approximately 400 genes are present in this region. In order to characterize the QTL region and develop tools for DNA-informed breeding, genes were sequenced in resistant and susceptible progeny of Potomac, Old Home, and NJA2R59T69 and the parental varieties. Average coverage of the genes in this region was approximately 50X per sample and 14,020 polymorphisms were identified. The resistance in 'Moonglow', 'Potomac', and 'Old Home' was found to have 13 shared polymorphisms in the receptor-like kinase gene pycom02g05250. This is in agreement with previous hypotheses that the same gene mediates resistance to fire blight in these cultivars. Interestingly, these polymorphisms were not identified in resistant progeny derived from NJA2R59T69 supporting the hypothesis that a different gene is responsible for resistance. Further work is needed to develop diagnostic markers, validate these markers and pycom02g05250, and identify the gene responsible for NJA2R59T69 mediated resistance.

\section{Comparative genomic analysis of the lettuce bacterial leaf spot pathogen}

E. R. ROSENTHAL (1), A. Sebastian (1), N. Potnis (2), I. Albert (1), C. Bull (1), (1) Pennsylvania State University, University Park, PA, USA; (2) Department of Entomology and Plant Pathology Auburn University, Auburn, AL, USA

Xanthomonas campestris pv. vitians (Xcv), the causal agent of lettuce bacterial leaf spot, was first identified in the US in 1918 but reemerged as a significant threat in the 1990s. Prior research demonstrated that the bacteria are potentially seedborne, cause race-specific responses upon inoculation in lettuce cultivars, and are poorly controlled using chemical treatment. This study sought to predict the molecular basis of bacterial race-specificity using whole genome sequences. 19 isolates of $X c v$ and 8 related type and pathotype strains were sequenced via Illumina MiSeq, generating $250 \mathrm{nt}$ paired-end reads at $185 \mathrm{Mb}$ yield per sample. For these $5 \mathrm{Mb}$ genomes, this is $37 x$ coverage. Raw reads were trimmed to remove poor quality data, trimmed reads were assembled de novo, and assembled genomes were annotated. Trimmed reads were used to construct a maximum likelihood phylogeny that mostly corroborates the multi-locus sequence analysis of the $X c v$ strains and related strains. Genome size, number of scaffolds, coverage, and $\mathrm{N}_{50}$ for each assembly are reported. Predicted features, including plasmids and secretion systems, are also reported. From 6,400 genes annotated in the $X c v$ pangenome, over 100 predicted race-specific genes were identified. Further genome mining predicted unique effector repertoires for each of the three $\mathrm{Xcv}$ races. Future studies will demonstrate the role of these predicted genes in race-specificity through empirical testing and use genome comparisons to make a race-specific detection method for $X c v$ for disease monitoring in fields and potential seed detection.

Interactions between Aspergillus flavus and stored-grain insects in conventional and transgenic maize J. A. L. Mandap (1), R. Hellmich (2), G. P. MUNKVOLD (3), (1) University of the Philippines, Los Baños, IA, PHILIPPINES; (2) USDA ARS, Ames, IA, USA; (3) Iowa State University, Ames, IA, USA

Uncontrolled grain temperature and moisture during storage can lead to insect and mold infestations that result in mycotoxin contamination. Stored-grain insects cause damage that predisposes grain to fungal colonization and mycotoxin contamination. Transgenic insect resistance in maize has been shown to indirectly reduce mycotoxin contamination in the field, but this has not been demonstrated in storage. In this study, interactions between Aspergillus flavus and Indianmeal moth (Plodia interpunctella) or maize weevil (Sitophilus zeamais) were assessed in grain from $B t$ and non-Bt maize hybrids under storage conditions that are common in tropical and sub-tropical areas $\left(32^{\circ} \mathrm{C}\right.$ and $\left.80-85 \% \mathrm{RH}\right)$. Indianmeal moth larvae or maize weevil adults were infested into grain in separate jars with and without $A$. flavus. After 28 days, no insects survived in the transgenic hybrids with lepidopteran and coleopteran resistance, respectively. A. flavus increased mortality and reduced survivorship and growth of both insects. Hence, grain damage and weight loss were higher in the uninoculated grain because of greater insect feeding than in the inoculated grain. A. flavus-insect interactions were influenced by the presence of $B t$ proteins in the maize grain. Insect infestations increased levels of aflatoxin contamination in the non- $B t$ hybrid inoculated with $10^{6}$ spores $/ \mathrm{ml}$, but did not affect aflatoxin levels in $B t$ hybrids. These results indicate that transgenes that target field pests also provide protection against storage insects and can reduce the risk of aflatoxin development in stored grain.

Quick field-adapted detection of Stagonosporopsis spp., the causal agent of gummy stem blight in watermelon J. A. REYES (1), C. Villari (2), M. T. Brewer (3), B. Dutta (4), C. McGregor (3), (1) University if Georgia, Athens, GA, USA; (2) D.B. Warnell School of Forestry, University of Georgia, Athens, GA, USA; (3) University of Georgia, Athens, GA, USA; (4) University of Georgia, Tifton, GA, USA

Gummy stem blight (GSB), caused by three Stagonosporopsis species is a major fungal disease of watermelon that leads to severe yield losses. Commercial cultivars with high levels of genetic resistance to GSB are not currently available and thus, its management relies on cultural practices and costly fungicide applications. Efficient methods for pathogen detection have become a necessity to aid management decisions in an attempt to reduce the frequency and costs associated with such fungicide applications. In this study, a DNA-based quick pathogen diagnostic system for Stagonosporopsis was developed and tested using naturally and artificially infected watermelon plants. The system consisted of a simple pathogen sampling device, quick DNA extraction protocols and Stagonosporopsis- 
specific loop-mediated isothermal amplification (LAMP) assays. DNA was extracted from leaves using cellulose dipsticks and from spore traps using a chelating resin protocol. Pathogen DNA could be detected from low, mild, and severely infected samples as well as in air samples collected with the spore trap system. The pathogen sampling device, DNA extraction methods and LAMP assays can be adapted for field deployment and show potential for point-of-care diagnosis. This would allow growers and county agents to make informed decisions in an efficient and expedited way based on the actual presence or absence of the pathogen.

\section{Effect of Solanaceous glycoalkaloids on hatch and pre-parasitic juvenile motility of potato cyst nematode Globodera pallida S. SIVASANKARA PILLAI, L. M. C. Dandurand, University of Idaho, Moscow, ID, USA}

Glycoalkaloids are biologically active plant secondary metabolites within the Solanaceae family which function as natural defense substances against pathogens and insects. These metabolites have the potential to act as effective natural pesticides because they have many cellular targets that cover a diverse array of metabolic pathways, macromolecules and organelles. Four pure steroidal glycoalkaloids (SGA) solamargine, solasodine, solamarine and solasonine were tested for their nematicidal activity on $G$. pallida. In vitro studies showed that the SGAs in the presence of a potato root diffusate, inhibited hatch by 87, 64, 62 and 53 percent respectively. Solamargine and solasodine were further evaluated for their effect on G. pallida juvenile (J2) motility. After a 24 hour exposure to the SGAs, PCN motility was reduced by 26 and 33 percent by solamargine (100 and $200 \mu \mathrm{g} / \mathrm{ml}$ concentrations), while solasodine reduced motility by 20 and 25 percent. When exposed for 7 days, both SGAs reduced J 2 motility by 99 percent compared to a 3.5 percent decline in motility in the control. Efforts are underway to test the toxicity of these compounds to reproduction of PCN in the presence of a susceptible potato plant.

\section{Dynamic changes in plum pox virus population structure during leaf and bud development}

Y. TAMUKOnG (1), T. Collum (2,3), A. L. Stone (3), D. Sherman (3), M. Kappagantu (4), E. E. Rogers (3), C. D. Dardick (5), J. N. Culver (4,6), (1) University of Maryland College Park, College Park, MD, USA; (2) USDA ARS FDWSRU, Ft. Detrick, MD, USA; (3) USDA, Agricultural Research Service, Foreign Disease-Weed Science Research Unit, Frederick, MD, USA; (4) Institute of Bioscience and Biotechnology Research, University of Maryland, College Park, MD, USA; (5) USDA, Agricultural Research Service, Appalachian Fruit Research Station, Kearneysville, WV, USA; (6) Department of Plant Science and Landscape Architecture, University of Maryland, College Park, MD, USA

Known sequences of plum pox virus (PPV), a potyvirus and the most devastating threat to stone fruit worldwide, may be grouped into 10 different strains. Each strain is able to generate and maintain new variants with different pathologies, especially over multiple growing seasons in perennial hosts. To investigate PPV variant dynamics, we analyzed NGS data generated from ribosome-associated (translatome) RNA, which are likely being translated and thus actively contributing to the infection process. RNA was isolated from infected plum leaves 2, 4, 6 and 12 weeks post leaf emergence during two separate growth periods (GP). Bud samples were collected pre- and post-simulated dormancy. PPV translation levels were consistent with expected viral infection levels. Within GPs, PPV variants occur at proportionately higher levels in newly developing plum tissue with low viral titers while more mature tissues with high infection levels have a lower fraction of variants. Analysis of individual variants revealed distinct groups as some variants were maintained at either high or low levels in all samples while others showed dynamic changes. Potential tissue-specific variants in the phloem or whole leaf translatomes were also identified. Our results suggest that PPV variant population structure expands during seasonal bud break and shrinks upon rapid viral replication; also, variants may display high, low, or fluctuating levels during leaf development.

Trichoderma isolates collected from arid region of Nepal help alleviate drought stress in susceptible tomato genotypes M. S. Benitez Ponce (1), J. Scheerens (2), R. RAWAL (3), (1) The Ohio State University, Wooster, OH, USA; (2) Department of Department of Plant Pathology, Ohio State University, Wooster, OH, USA; (3) Department of Horticulture and Crop Science, Ohio State University, Wooster, OH, USA

Drought stress (DS) is the most destructive abiotic stress responsible for limiting global food security. The use of symbiotic fungi of the genus Trichoderma has been established as a tool for inducing stress tolerance. However, the beneficial effects of Trichoderma on DS are poorly understood and may be isolate-specific. The overall goal of this research is to evaluate the ability of Trichoderma isolates, primarily native to different regions in Nepal, to improve growth in tomato genotypes exposed to DS. We hypothesized that the isolates collected from the dryer regions will have greater impact on DS response. Out of 41 Nepalese Trichoderma isolates studied, 3 isolates (T. viride-T3, T. asperellum-T16, and T33) were selected based on their capacity to tolerate osmotic stress and to stimulate shoot biomass in tomato (Roma) under DS in greenhouse. Further, the response of these 3 selected isolates were tested on tomato genotypes with differing tolerance to drought $\{$ Jaune Flamme (JF), Roma, Punta Banda (PB)\}. The result showed that the drought susceptible genotype JF inoculated with isolate T-33 recorded 50\% more shoot biomass while drought tolerant PB had only $12 \%$ increase compared to non-inoculated control under DS. In addition, isolate-T33, collected from the arid region of Nepal, increases root biomass and chlorophyll content in plants and changes the root architecture (higher lateral root density) of seedlings denoting a potential adaptation strategy. However, the complex mechanisms involved in DS amelioration under this microbial symbiosis need to be better understood.

\section{Identification of the roselle wilt disease and development of biocontrol strategies} Y. H. YU, National Taiwan University, Taipei, TAIWAN

The roselle field disease survey conducted recently by Taitung District Agricultural Research and Extension Station in Taiwan reported that roselle wilt disease widely occurred, and the disease incidence of several fields was approximately $90 \%$. The stems of the wilted 
roselle were browned and slightly constricted, and white aerial hyphae were observed. The rot pith was found in the vertically dissected stem base and typical macroconidia and microconidia of Fusarium species were observed under microscope, indicating that the roselle wilt disease might be caused by Fusarium species. However, there are no scientific reports documenting this disease in Taiwan. In this study, we collected diseased samples from six roselle plantations in Taitung county. 63 strains were isolated, 40\% are Fusarium solani, $21 \%$ are Phytophthora nicotianae, $9 \%$ are $F$. equiseti, $6 \%$ are $F$. oxysporum, $24 \%$ are others. Koch's postulate was used to test $F$. solani strain $\mathrm{K} 2$ and $P$. nicotianae strain CWR-42. We found that $F$. solani $\mathrm{K} 2$ can cause wilting and rot pith of the plants, while CWR-42 result in leaf yellowing and root rot. We confirmed that $F$. solani $\mathrm{K} 2$ can cause the roselle wilt symptoms similar to those observed in the roselle fields, and $P$. nicotianae may only play minor role in developing the disease. It is the first time to demonstrate that $F$. solani can cause roselle wilt in Taiwan. Meanwhile, we found that Bacillus amyloliquefaciences Ba01 and Streptomyces sp. KHY11 can inhibit the growth of $F$. solani $\mathrm{K} 2$, serving as potential biocontrol strategies against roselle wilt disease.

\section{Development of strawberry gray mold management tool for California}

S. S. HEWAVITHARANA (1), J. Smith (2), S. M. Mansouripour (3), G. J. Holmes (4), (1) California Polytechnic University San Luis Obispo, San Luis Obispo, CA, USA; (2) California Polytechnic State University, CA, USA; (3) Strawberry Center, California Polytechnic State University, San Luis Obispo, CA, USA; (4) California Polytechnic State University, San Luis Obispo, CA, USA

Gray mold of strawberry caused by Botrytis cinerea is an important pre- and post-harvest fruit disease in California that has the potential to cause significant losses. It can manifest in the field, storage, and transit to distant markets. In conventional production, it is primarily managed by fungicides. However, due to high genetic variability, abundant inoculum, and multiple cycles, the fungus can readily become resistant to many fungicides. The long production season and the need for repeated fungicide applications in California increases selection pressure. The objectives of this project were to better understand the interaction of cultural practices, time of the year, weather conditions, and timing of fungicide applications, that contributes to disease development. Marketable fruit were obtained from 6 grower fields of cvs. Monterey and Portola from July to November 2019. The fruit clamshells were transported mimicking cold chain and incubated at $1{ }^{\circ} \mathrm{C}$ for 4 days and $16^{\circ} \mathrm{C}$ for 3 days and assessed for Botrytis incidence at both timings. Fungicide application records were obtained. Until rainy weather occurred in October, there was no observed Botrytis infection after incubation for 4 days at $1{ }^{\circ} \mathrm{C}$ and $1.3 \%$ was the highest incidence observed. Botrytis incidence at $16^{\circ} \mathrm{C}$ after rainy weather period ranged from $0.2 \%$ to $39 \%$ in four farms. Fungicide application timings were analyzed against gray mold incidence. In certain farms, based on the non-conducive weather parameters, the number of fungicide applications could have been reduced without affecting Botrytis management.

\section{A sweet tale of sugar-binding proteins: The contribution of lectins to virulence in Ralstonia solanacearum} M. CARTER, J. Prom, C. Allen, University of Wisconsin-Madison, Madison, WI, USA

Lectins are adhesive proteins that bind carbohydrates. In some pathogenic bacteria, carbohydrate-lectin interactions are critical for virulence functions, including adhesion to host surfaces and biofilm formation. The genome of the bacterial wilt pathogen Ralstonia solanacearum $(R s)$ encodes three lectins that are among the most highly expressed genes in planta: LecM, LecF, and LecX. LecM has affinities for mannose and fructose, LecF has affinities for fucose and galactose, and LecX has affinities for xylose, mannose and fucose. While some features of these proteins have been characterized, the function of $R s$ lectins in disease is understudied. We previously showed that LecM is required for plant colonization, symptom development and survival in potato tubers. Since polysaccharides are components of both plant and bacterial cell surfaces, we hypothesize that LecF also facilitates host colonization and disease development by mediating adhesion to the host and/or cohesion to other $R s$ cells. A $\Delta l e c F$ mutant formed wild-type levels of biofilm an in vitro biofilm assay, but this function must also be tested in planta. LecF was not required for internal colonization of tomato roots, but it was required for normal colonization of tomato stems. This tissue specificity may reflect an affinity for specific xylem wall components. Thus like LecM, LecF contributes to virulence. Future studies will elucidate how LecF contributes to Rs attachment behaviors in planta and overall disease progress.

\section{A naturally occurring genetic variant of grapevine virus $L$ from Texas Blanc du Bois grapevines}

O. J. ALABI (1), S. A. McBride (2), D. N. Appel (3), M. Al Rwahnih PhD (4), (1) Texas A\&M University, Weslaco, TX, USA; (2) Texas A\&M AgriLife Extension Service, College Station, TX, USA; (3) Texas A\&M University, College Station, TX, USA; (4) University of California-Davis/FPS, Davis, CA, USA

The virome of a 32-yr. old Blanc du Bois (Vitis spp.: 'Florida D 6-148' × 'Cardinal') vineyard in Texas was explored during 2018 season. Fifty-one samples were collected and equimolar amounts of total RNA from these samples were pooled for highthroughput sequencing on the Illumina NextSeq 500 platform. Analyses of the obtained $\sim 23.5$ million raw reads (76 nucleotides [nt] each) revealed several virus and viroid specific contigs, five (206 to 4,405 nt) of which shared significant (73-83\%) nt identity to corresponding sequences of several isolates of grapevine virus L $(\mathrm{GVL})$, a recently characterized Vitivirus species. The complete $(+)$ ssRNA genome of GVL was amplified from two samples as two pieces of overlapping DNA fragments, and the 5' and 3' UTRs were verified by RACE assays. The fragments were cloned into pJET1.2 vector and Sanger sequenced using vector and virus-specific primers. The derived 7,594 nt (excluding the polyA tail) complete genome of GVL from Blanc du Bois (GVL-TX-WAT20 and GVL-TX-WAT39) encoded five open reading frames (ORFs) that are homologous and phylogenetically related to ORFs of grapevine-infecting vitiviruses. The replication-associated protein $(73 \%$ nt \& $81-82 \%$ amino acids [aa]) and coat protein $(83 \%$ nt $\& 89-93 \%$ aa) cistrons of both isolates were most identical and phylogenetically related to those of isolates GVL-KA (MH643739), GVL-RS (MH248020), GVL-VL (MH681991) and GVL-SB (MH686191), indicating that both represent a naturally occurring genetic variant of the virus. This is the first report of GVL in Texas. 
Coffee Leaf Rust (Hemileia vastatrix Berk. \& Br.) control with Cyproconazole + Trifloxystrobin fungicide in Coffea arabica L. cv. Caturra in Colombia

C. A. Rivillas, J. Cardona, C. A. Zuluaga, C. A. ANGEL, CENICAFE, FNC Colombia, Manizales, COLOMBIA

Coffee Leaf Rust (CLR) is the most important coffee disease worldwide, causing severe defoliation, yield and quality losses up to $50 \%$ in susceptible varieties. Colombia still has $17 \%$ out 854,000 ha in susceptible varieties requiring chemical control. This experiment evaluated Cyproconazole $160 \mathrm{~g} / \mathrm{L}+$ Trifloxystrobin $375 \mathrm{~g} / \mathrm{L}$ (Sphere ${ }^{\circledR} \mathrm{Max}$, BAYER) fungicide to control CLR on cv. Caturra plants in an open sun exposure field, 7,142 plants/ha, 1,350 m.a.s.1., at Cenicafé's La Catalina Station (Pereira, Colombia), from 2016 to 2019. Fungicide was sprayed at 0.4 and $0.45 \mathrm{~L} /$ ha with two criteria: fixed calendars (FC) based on historical regional flowering pattern, and the adjustment to the field main flowering date applying 60 and 120 days after it, and compared to a reference commercial fungicide and to a negative check without applications. After three seasons, there were statistical differences in CLR incidence, severity and defoliation in favor of fungicide treatments. Maximum CLR monthly incidence was 31\% (2017), 49\% (2018) and 41\% (2019) in nonapplied plants versus $9 \%, 36 \%$ and $16 \%$ for Sphere ${ }^{\circledR}$ Max treatments. There were not consistent differences between the two doses, the two spraying criteria, and plant's growth variables. There were yield statistical differences in 2018 , completing a cumulative $21 \%$ losses between negative check and the best treatment $(0.4 \mathrm{~L} / \mathrm{ha}, \mathrm{FC})$. No negative effects on bean's physical and beverage quality, and no fungicide traces were detected when applied 15 and 30 days before harvesting. To conclude, Sphere ${ }^{\mathbb{R}}$ Max is an alternative to control CLR in Colombia.

The influence of infection timing and fungicide mode of action on suppression of downy mildew on hop B. CLAASSEN (1), D. H. Gent (2), (1) Oregon State University, Corvallis, OR, USA; (2) US Department of Agriculture, Agricultural Research Service, Corvallis, OR, USA

Downy mildew, caused by Pseudoperonospora humuli, is a limiting factor for hop production in wet, humid production regions. The pathogen overwinters in buds and crowns of hop plants as systemic mycelia within the plant. Disruption of pathogen overwintering could improve management of the disease, however the impact of infection timing on pathogen survival and disease development in the next year is not well understood. To describe the overwintering process, batches of hop plants were inoculated monthly from June to October and the following year emerging shoots were rated for downy mildew. Perennation occurred at some level regardless of when plants were infected, although delaying infection till after September significantly reduced disease levels in following year in two of three years of the experiment. In parallel, studies were conducted to quantify disease suppression when using different fungicide modes of action and timing of applications in spring. Fungicide mode of action and timing interacted to influence disease levels. In general, using more efficacious fungicides in the first two sprays of the season versus later in spring improved disease control. Together, these studies indicate that delaying disease onset in the previous season and controlling disease at the earliest stages of the epidemic in spring are important in overall management programs for downy mildew.

First report of leaf spot caused by Corynespora citricola on citrus seedlings in nurseries in Florida S. GEBBEN (1), O. Batuman (2), (1) University of Florida, IMMOKALEE, FL, USA; (2) University of Florida, Southwest Florida Research and Education Center, Immokalee, FL, USA

Citrus seedlings of Swingle citrumelo and X-639 rootstocks with lesions that were circular to irregular and reddish-brown with a yellow halo were brought to the University of Florida-IFAS, Southwest Florida Research and Education Center located in Immokalee, FL. Isolates from excised leaf lesions were identified molecularly using the internal transcribed spacer (ITS), beta-tubulin, elongation factor, and actin genes. Based on sequences of the ITS gene, the isolates were $100 \%$ identical to Corynespora citricola. However, due to the absence of $C$. citricola sequences in GenBank, other gene sequences analyzed showed $\sim 85-93 \%$ homology to $C$. smithii. In order to confirm pathogenicity, healthy citrus seedlings of both five-month-old Swingle and two-month-old rough lemon rootstocks were inoculated in the greenhouse. Within seven days, symptoms like those described above were observed. C. citricola was readily isolated from lesions, fulfilling Koch's postulates, and were confirmed morphologically and by sequencing. To the best of our knowledge, this is the first report of $C$. citricola infecting citrus seedlings in nurseries in Florida. The presence of this disease in citrus groves in Florida is yet to be determined. The establishment of any new disease, such as $C$. citricola, could have a significant impact on the citrus industry, which is already suffering with Huanglongbing (HLB) and citrus black spot (Phyllosticta citricarpa) epidemics. This work will assist future diagnosis of the disease and reduce its spread in Florida and to other citrus producing regions in the United States.

Spore trapping of trunk canker pathogens in California almond orchards

I. JIMENEZ LUNA (1), P. E. Rolshausen (2), (1) University of California, Riverside, CA, USA; (2) University of California, Riverside, Riverside, CA, USA

Fungal canker diseases are major limiting factors to woody perennial crop productivity and longevity. The causal agents are airborne and invade the plant through wounds. In orchards within Merced County, California, severe symptoms of gumming and wood cankers located on trunks and primary limbs were observed. These cankers often led to limb loss or tree death. The goal of the study was to assess the seasonal dynamics of spore release and identify the main pathogens present in commercial orchards. These affected almond orchards with different locations and management practices were selected and the experiment was conducted from 2012-2014. Microscope glass slides coated with petroleum jelly were used to trap airborne spores. Spore-traps were placed at the periphery and center of each orchard and collected weekly during the winter season, from November to March. Slides were brought back to the laboratory to be washed and resulting slur was cultured on Potato Dextrose Agar medium with antibiotics. Pure fungal cultures were identified to species by sequencing three DNA loci. Our results showed that fungi in the Diaporthales and Botryosphaeriales were the most common pathogens spreading in orchards, with over 10 species identified. Results also showed that spores of fungal pathogens 
were trapped after rainy events mostly at the periphery of the orchards, and that the pathogen profile was orchard specific. This information is useful for developing guidelines to manage trunk canker diseases of almond in California.

Sensitivity of Botrytis cinerea and Calonectria pseudonaviculata to different disinfectants

R. Bika (1), W. E. Copes (2), F. BAYSAL-GUREL (3), (1) Tennessee State University, Mc Minnville, TN, USA; (2) USDA ARS, Poplarville, MS, USA; (3) Tennessee State University, McMinnville, TN, USA

There are several potential critical control points in nursery crop production systems where there is high risk of introduction and spread of plant pathogens. Specifically, pruning, collecting cuttings and harvesting could be major routes of introduction for mechanically transmissible pathogens like Botrytis cinerea $(B c)$ and Calonectria pseudonaviculata $(C p s)$. The objective of this study was to examine sensitivity of $B c$ and $C p s$ to disinfectants. Fungal spore suspensions were prepared by flooding 10 to 14 day- and 3 to 4 week-old cultures of $B c$ and $C p s$, respectively, and adjusted to $10^{5}$ conidia/ml. Disinfectants were proportionally mixed with the respective fungal spore suspension to achieve the proper test concentration; $100 \mu \mathrm{L}$ aliquots were withdrawn at 1 (immediately), 30 and 60 sec and plated on PDA. Cultures were incubated at room temperature and pathogen sensitivity was determined by the absence or reduction of fungal colonies. The effectiveness of prepared disinfectant solutions was evaluated for one-month period. Lysol (undiluted), Clorox (10\%), Alcohol (70\%), Vinegar (100\%), Simple Green d Pro 5 (7.8 ml/L), Simple Green d Pro 3 plus (15.6 ml/L), KleenGrow (7.8 ml/L and $15.6 \mathrm{ml} / \mathrm{L})$, and Green-Shield $(15.6 \mathrm{ml} / \mathrm{L})$ substantially inhibited germination of $B c$ and $C p s$ spores at all three contact times. Virkon $\mathrm{S}$ $(2 \%)$ and Vinegar (50\%) were also very effective at inhibiting conidia germination of Cps. Additional trials are underway to determine effectiveness of these disinfectants in preventing plant-to-plant spread of both pathogens via pruning tools.

\section{Aecial host specificities of two forms of Puccinia coronata}

N. GREATENS (1), P. D. Olivera Firpo (2), Y. Jin (3), (1) Department of Plant Pathology, University of Minnesota, St. Paul, MN, USA; (2) University of Minnesota, St. Paul, MN, USA; (3) USDA-ARS Cereal Disease Laboratory, Saint Paul, MN, USA

Most assessments of crown rust (Puccinia coronata) host ranges have focused on the telial hosts in Poaceae, including cereal crops, forages, and turfgrasses. Two plants of European origin, common buckthorn (Rhamnus cathartica) and glossy buckthorn (Frangula alnus) are often cited as the alternate (aecial) hosts of crown rust, but many species within Rhamnaceae and Eleagnaceae are also known to be hosts. Since the last published tests of crown rusts' aecial host ranges were conducted, the taxonomy has changed significantly, and multiple new species have been described. P. coronata is today considered a species complex that includes various formae speciales with different, sometimes poorly defined host specificities. We conducted experiments to inoculate five species of buckthorns, native and introduced, with two forms of $P$. coronata: crown rust of oats (P.c. var. avenae f.sp. avenae) and of reed canarygrass (P.c. var. coronata), the latter likely a recent introduction to North America that cycles between telial host Phalaris arundinacea and aecial host $F$. alnus. Oat crown rust produced infection on $R$. cathartica, and on two Frangula spp. native to North America: $F$. rubra and $F$. carolinana, but not on $F$. alnus or $F$. purshiana, another native. The crown rust of reed canarygrass produced infection on $F$. alnus and $F$. carolinana, but not on $R$. cathartica, F. rubra, or F. purshiana. Ongoing investigations of aecial host specificities will include additional potential hosts and more forms of $P$. coronata.

\section{Understanding the role of bacteriocins in mediating competition among Pseudomonas spp. that cause bacterial blotch of white} button mushroom

R. HERSCHLAG (1), S. Martins (2), L. Ramos-Sepulveda (3), C. Bull (4), K. Hockett (5), (1) Department of Plant Pathology and Environmental Microbiology Penn State, University Park, PA, USA; (2) The Pennsylvania State University, University Park, PA, USA; (3) Millersville University, Millersville, PA, USA; (4) Pennsylvania State University, University Park, PA, USA; (5) The Pennsylvania State University, University Park, PA, USA

Bacteriocins are narrow spectrum proteinaceous toxins that are produced by bacteria and kill closely related species. Although bacteriocins may provide a competitive advantage, killing activity can come at a high cost, often requiring lysis of the producer cell. Determining which bacteria species kill which other closely related species can be crucial for disease complexes, such as bacterial blotch of white button mushroom, Agaricus bisporus, caused by at least eleven Pseudomonas species. Previous surveys have shown that multiple pathogenic Pseudomonas species may be found on individual mushroom cap surfaces, but more intensive sampling is needed to understand which species commonly co-exist, in order to determine if killing activity via bacteriocins may play a role. An in vitro approach was used to investigate the killing competition via bacteriocin production among twenty-nine blotch inciting Pseudomonas strains collected from four organic mushroom farms in Pennsylvania. We also conducted a more in-depth sampling of at least ten mushroom caps, collecting multiple isolates in three blotch lesions per cap. Overall, we observed multiple members of a single clade often killing the same pathogenic Pseudomonas strain, and demonstrated that there is a greater diversity of Pseudomonas on a single cap than previously understood. Our goal is to use observed killing activity via bacteriocins, along with our sampling data to better understand the blotch disease complex and inform future biocontrol strategies.

\section{Meta-analysis on usage of quaternary ammonium compounds to inactivate plant pathogens W. E. COPES, USDA ARS, Poplarville, MS, USA}

Quaternary ammonium compounds (QACs) have been used as disinfestants in plant production systems since the mid-twentieth century. A systematic review was conducted to evaluate the overall efficacy of three generations of QAC chemistry, which included products with different mixtures of active ingredients. Meta-analysis was performed using Hedges' g, which is a standardized mean difference between QAC-treated and untreated substrates corrected for small sample size bias. Studies included a diverse collection of 
38 plant pathogens, including 21,16,86, and 6 cases with bacteria, oomycetes, fungi and viruses, respectively, for a total of 129 cases from agricultural and horticultural crop production systems. Effect sizes represented a heterogeneous population based on a significant Q-value and width of $95 \%$ prediction intervals. A funnel plot showed a Gaussian distribution for most cases, but the largest effect sizes deviated outside the funnel plot and had higher standard errors. A meta-regression procedure was used to evaluate two continuous moderator variables, dose and contact time; two categorical moderator variables, treatment target and type of organism; and their interactions. Between-study variance was partially explained by contact time, the dose and contact time interaction and the target being treated. Strengths and weaknesses of inactivation of plant pathogens using QACs will be discussed as well as research prospects.

\section{Pectinolytic activity and soft rot suppression of bacteria isolated from a greenhouse hydroponic potato minituber production} system

J. Knoke (1), J. Rach (1), B. Babler (2), R. RIOUX (1), (1) University of Wisconsin-Madison, Madison, WI, USA; (2) Wisconsin Seed Potato Certification Laboratory, Madison, WI, USA

In some locations, production of minitubers for seed potato propagation has recently shifted from artificial peat-based media to hydroponic systems. The potential for introduction of water-borne pathogens, including soft rot causing bacteria in the genera Dickeya and Pectobacterium, is not well understood nor is it known how bacteria introduced through the nutrient solutions used in hydroponic production interact with soft rot pathogens. In this study, water samples were collected from a hydroponic system for minituber production at weekly intervals throughout the summer of 2019. While some bacteria from these samples caused pitting on crystal violet pectate (CVP) medium, molecular methods determined that D. dianthicola and Pectobacterium were not present. Tuber slice inoculation assays indicated that four of twenty (20\%) bacterial isolates obtained from plating on CVP exhibited weak pectinolytic activity but none were as aggressive as $D$. dianthicola. A separate 25 isolates obtained from plating on nutrient agar were tested for their ability to antagonize $D$. dianthicola in tuber slice inoculation assays. One isolate reduced $D$. dianthicola lesion size by more than $57.9 \%$ $(P<0.05)$. Overall, these data provide new insights on bacteria present in hydroponic system nutrient solution and their potential interactions with soft rot pathogens. Future research will focus on identifying isolates of interest and further exploring their interactions with potatoes and soft rot pathogens under more agronomically relevant conditions.

Development and validation of a V6 race-specific differentiation assay for Podosphaera macularis in the Pacific Northwest M. H. BLOCK (1), B. J. Knaus (1), M. S. Wiseman (1), N. J. Grunwald (2), D. H. Gent (3), (1) Oregon State University, Corvallis, OR, USA; (2) USDA ARS, Corvallis, OR, USA; (3) US Department of Agriculture, Agricultural Research Service, Corvallis, OR, USA

Hop powdery mildew (Podosphaera macularis) was confirmed in the Pacific Northwest in 1996. Before 2012, the most common race of $P$. macularis was able to infect plants that possessed powdery mildew resistance based on the R-genes Rb, R3, and R5. Post 2012, two additional races of $P$. macularis were discovered that can overcome the resistance gene R6 and the partial resistance found in the cultivar Cascade. Current methods for race determination for $P$. macularis are slow, costly, and labor intensive. We sought to develop a molecular assay to differentiate race V6 isolates of $P$. macularis from other races. The transcriptomes of 46 isolates of $P$. macularis were sequenced to identify loci and variants unique to V6-isolates. Fourteen primer pairs were designed for ten candidate loci that contained single nucleotide polymorphisms (SNP) and indel variants. Two differentially labeled locked nucleic acid probes were designed for a contig that contained a conserved SNP. The multiplexed real time PCR assay was tested against 49 V6 and 51 non-V6 P. macularis isolates collected from the United States and Europe. The limit for detection of PNW derived V6-isolates was 10 picograms of DNA. The specificity of the assay was tested with other powdery mildew species and pathogens found on hop. Weak non-specific amplification occurred with powdery mildew collected from grape, strawberry and zinnia; however, this is not a concern with the practical application of this assay. This assay has practical applications in hop breeding, epidemiological studies, and as a research tool.

\section{Novel gemycircularvirus rescued and causes hypovirulence in Sclerotiniaceae fungi} C. FENG, S. Y. L. Marzano, South Dakota State University, Brookings, SD, USA

Gemycircularviruses are being quickly discovered by metagenomics approaches from samples associated with various environments, animals, plants, and fungi, but information related to these viral exact host range and biocontrol potential are not available. In this study, a reverse genetic system of Soybean leaf-associated gemycircularvirus 1 (SlaGemV-1) was established and the virus was successfully rescued by transfecting a plant pathogenic fungus, Sclerotinia sclerotiorum. Then the infectivity of the viral particles on other members of Sclerotiniaceae, including Botrytis cinerea, and Monilinia fruticola was determined positive. SlaGemV-1 infection significantly reduced the virulence of these Sclerotiniaceae fungi. SlaGemV-1 genome codes for two proteins, capsid protein (CP) or replicase (Rep). To determine the protein causes hypovirulence, CP and Rep were expressed separately in B. cinerea (BCP-SL and BRep-SL) via a sitespecific integration method. On potato dextrose agar, BRep-SL grew much slower than BCP-SL $\left(\mathrm{P}<10^{-4}\right)$, whereas BCP-SL had no difference in growth from the wild type. Consistently, the plant pathogenicity assays showed BRep-SL had reduced virulence compared to BCP-SL and the wild type $(\mathrm{P}<0.01)$. The expression of the single Rep protein confers anti-fungal activity equivalent to the viral infection. The rescue of SlaGemV-1 virus, the determination of its host range in Sclerotiniaceae fungi and the identification the Rep protein as the sole contributor to hypovirulence pinpoint multiple utilities of gemycircularvirus in fungal pathogen control.

Population diversity and dynamics of stripe rust pathogen Puccinia striiformis on wheat and barley in the United States from 2010 to 2017

Q. BAI (1), A. Wan (1), M. Wang PhD (1), D. See (1,2), X. Chen (1,2), (1) Washington State University, Pullman, WA, USA; (2)

USDA-ARS, Pullman, WA, USA 
Puccinia striiformis (Ps) causes stripe rust on cereal crops and related wild grasses, and the formae speciales $P$. striiformis f. sp. tritici $(P s t)$ and $P$. striiformis f. sp. hordei $(P s h)$ are especially destructive to wheat and barley crops, respectively. To study the differentiation, diversity, and dynamics of the Ps populations in the United States, 2,247 (Pst) and 167 (Psh) isolates collected in 2010 to 2017 were genotyped into 1,445 and 154 multi-locus genotypes (MLGs), respectively using 14 simple sequence repeat markers. The Pst and Psh MLGs were separated into two clusters, with only 7 MLGs shared by the two forms. The Pst population was clustered into two genetic lineages. Isolates from the western U.S. were relatively evenly in both lineages with 700 isolates (42\%) in lineage 1 and 967 isolates $(58 \%)$ in lineage 2, whereas most of the isolates (542 isolates, 93\%) from the eastern U.S. were in linage 2 and only 38 isolates (7\%) in linage 1. The western U.S. subpopulation was more diverse than the eastern U.S. subpopulation. During the eight years, the national Pst population had the highest diversities in 2010 and 2011, but became less diverse afterwards, suggesting that the Pst population was under host and possible environmental selections. The study provides the information on distributions and dynamics of various genetic groups of the stripe rust pathogen in various epidemiological regions in the U.S., which is useful to develop strategies for managing stripe rust.

\section{Evolutionary genomics reveals recombination events involved in speciation, host specificity and pathogenicity in the genus Clavibacter

\author{
D. ARIZALA, S. Dobhal, S. Paudel, H. Seo, A. M. Alvarez, M. Arif, University of Hawaii at Manoa, Honolulu, HI, USA
}

Clavibacter is comprised of six species and three subspecies. In this study, we aimed to unveil the role of evolutionary events in diversification, speciation, host range and pathogenicity in the genus Clavibacter. In this study 69 genomes were retrieved from the NCBI GenBank database and other representative genomes were sequenced using PacBio. Clavibacter phylogeny was reconstructed and recombination regions were identified using ClonalFrameML. The fastGear algorithm was used to investigate recent and ancestral recombination events. Our results revealed widespread recombination sites and distinct degrees of homoplasy, suggesting high recombination rates. Three lineages were detected; greatest recombined fragment lengths were predicted in tomato endophyte strains. These recombinant strains could be the result of genome plasticity due to multiple introduction events. Various recombination hotspots were observed upon divergence of the closely related species $C$. californiensis and $C$. michiganensis, suggesting that non-pathogenic $C$. californiensis might have acquired the pathogenicity island $\operatorname{chp} /$ tom $A$ and speciated in C. michiganensis, the causal agent of canker and wilt on tomato. Moreover, we found relevant molecular events of gene flow and genetic exchange, probably linked with the lifestyle and specific niche adaptations in Clavibacter. The current research revealed the evolutionary dynamics within the genus Clavibacter, and provides novel insights into homologous recombination events which has fostered genome structure, host adaptability, and pathogenicity development.

Examination of SDHI fungicide resistance mechanism in phytopathogenic fungi through a heterologous expression system in Sclerotinia sclerotiorum

J. PENG (1), H. Sang (1), T. J. Proffer (2), J. C. Gleason (1), C. A. Outwater (1), G. W. Sundin (1), (1) Michigan State University, East Lansing, MI, USA; (2) Kent State Univ, Salem, OH, USA

The succinate dehydrogenase inhibitor (SDHI) is a class of broad-spectrum fungicides for management of many diseases caused by phytopathogenic fungi, including Botrytis cinerea, Clarireedia homoeocarpa, Sclerotinia sclerotiorum, and Monilinia fructicola, and Blumeriella jaapii. In many cases, reduced sensitivity to SDHI fungicides has been shown to be correlated with point mutations in the $S d h$ target genes; however, the genetic basis of SDHI fungicide resistance mechanisms has been characterized in very few fungi. $S$. sclerotiorum is a fast-growing and SDHI-sensitive phytopathogenic fungi and can be genetically modified. Given the high sequence similarity and potentially overlapping SDHI target sites between S. sclerotiorum and other common phytopathogenic Ascomycetes fungi, we developed a heterologous expression system in $S$. sclerotiorum to examine the function of mutations in $s d h B$ or $s d h C$ from several Ascomycetes fungi in conferring resistance to boscalid, fluopyram or fluxapyroxad in vitro. Using this system in $S$. sclerotiorum, we successfully validated the function of different mutations in the $s d h$ genes to SDHI fungicide resistance that have been previously identified in field isolates of $B$. cinerea, B. jaapii and C. homoeocarpa and that yet not known to exist in $M$. fructicola population. The approach developed in this study can be widely applied to investigate SDHI fungicide resistance mechanisms in other phytopathogenic Ascomycetes fungi.

Assessment of American sycamore resistance to canker pathogens C. FARINAS (1), D. Sydnor (1), A. Wooten (1), P. Bonello (2), F. Peduto Hand PhD (3), (1) The Ohio State University, Columbus, OH, USA; (2) The Ohio State University, Department of Plant Pathology, Columbus, OH, USA; (3) Department of Plant Pathology, The Ohio State University, Columbus, OH, USA

American sycamore (Platanus occidentalis), a tree species native to Eastern North America, is a valuable timber resource. The main problem associated with the cultivation of American sycamore is its susceptibility to anthracnose, leaf spot, and canker pathogens. Over the years, a sycamore tree growing on the campus of The Ohio State University (OSU; Columbus, OH) was observed to have superior anthracnose resistance compared to neighboring trees. The tree was clonally propagated by a commercial nursery and clones were denoted 'Davis'. In April 2018, we established two trials by planting six four-year-old 'Davis' ramets and six four-year-old wildtype genets, under each of two mature American sycamores that had displayed clear anthracnose and canker symptoms for several years. Incidence and severity of leaf necrosis was assessed every two weeks from May through September 2018 and 2019. At the end of the experiment, we also performed isolations from all symptomatic tissues and characterized the fungal isolates through sequencing of four loci (APN2, ITS, EF1- $\alpha$, TUB). Several canker pathogens were recovered, such as Apiognomonia veneta, the pathogen causing sycamore anthracnose, and Diaporthe eres, the pathogen causing cankers and dieback on many woody hosts. While the significance of incidence and severity of leaf necrosis varied between years, significantly lower dieback and tree mortality were observed on 'Davis' 
sycamores at the end of the study. Considering these results, the American sycamore 'Davis' appears to be a better option for urban landscapes than the wildtype.

Are avocados toast? Managing the threat of laurel wilt disease at a regional level R. CHOUDHURY (1,2,3,4), Y. Xing (3,5,6), J. H. Crane (7), D. Carrillo (7), E. Evans (7), J. Wasielewski (8), L. L. Stelinski (9), R. C. Ploetz (7), K. A. Garrett (2,4,10), (1) University of Texas Rio Grande Valley, Edinburg, TX, USA; (2) Emerging Pathogens Institute, University of Florida, Gainesville, FL, USA; (3) University of Florida, Institute for Sustainable Food Systems, Gainesville, FL, USA; (4) Plant Pathology Department, University of Florida, Gainesville, FL, USA; (5) University of Florida, Gainesville, FL, USA; (6) University of Florida, Emerging Pathogens Institute, Gainesville, FL, USA; (7) University of Florida - Tropical Research and Education Center, Homestead, FL, USA; (8) University of Florida, Homestead, FL, USA; (9) University of Florida, Lake Alfred, FL, USA; (10) Institute for Sustainable Food Systems, University of Florida, Gainesville, FL, USA

Managing diseases at regional scales is critical for diseases that spread rapidly, have multiple modes of spread, and can persist in the environment. Avocado production in Florida is threatened by laurel wilt disease, which is caused by the ascomycete fungus Raffaelea lauricola and is vectored by several ambrosia beetles. Avocado trees are killed shortly after infection, where the fungus spreads rapidly throughout the vascular system of the tree and moves between neighboring trees through rootgrafts. We used publicly available satellite images that date back to prior to the outbreak in Florida to estimate the rate of focus expansion within avocado groves and use these parameters to model the effects of different management strategies on within- and between-grove spread. Preliminary analyses indicate that foci expand at a rate of approximately one tree per week on average. Management strategies that rapidly reduce inoculum and limit spread both within and between groves can help to reduce the overall epidemic. Grower decision-making determines the potential for successful regional management, and we compare scenarios for economic and social influences on decision-making, with implications for constructing effective regional policies. Managing avocado laurel wilt at a regional scale will require rapid action and coordination among many stakeholders.

\author{
Comparative and functional genomics uncovers evolutionary dynamics and pathogenesis mechanisms of citrus huanglongbing- \\ associated bacteria \\ D. ZHANG, Saint Louis University, Saint Louis, MO, USA
}

Huanglongbing (HLB) is a worldwide citrus disease caused by several Candidatus Liberibacter species. In this study, we utilize the available genome information of HLB-associated pathogens and their relatives to systematically identify the unique genomic features and changes which accompany the evolution of these species in terms of their transition from free-living to intracellular habitats and their transition from being non-pathogenic to pathogenic. By operonic analysis, we discovered and annotated all potential prophage regions, and classified them into four types. We showed that each prophage type originated from an independent gene transfer during evolution and that they are among the most fast-evolving genes on the genome. By ortholog analysis, we identified two major gene groups which were either lost or gained during evolution. We analyzed and predicted the function of many of these genes. The genes lost in the decedents provide a molecular foundation of the intracellular habitats, whereas the genes gained during evolution point to mechanisms of pathogenicity. Several potential virulence factors were predicted, and their toxicities were verified using In vitro experiments. The genomic features and changes that we identified through comparative genomics provide functional insight into the biology and evolution of HLB-associated bacteria. The newly identified virulence factors will help the current understanding of the pathogenesis mechanisms of the HLB disease.

Pythium spp. as causal agents of root and crown rot of winter wheat in North Carolina

E. REEVES (1), J. P. Kerns (1), C. Cowger (1,2), B. B. Shew (1), (1) Department of Entomology and Plant Pathology, NC State University, Raleigh, NC, USA; (2) USDA-ARS Plant Science Unit, Raleigh, NC, USA

Soft red winter wheat was valued at over $\$ 66$ million in North Carolina in 2019. Mild to severe stunting and root and crown rot are frequent problems during wet years, especially in eastern NC. In 2016, these symptoms were associated with infection by several Pythium spp. and Koch's postulates were completed for P. irregulare (Pi), P. vanterpoolii (Pv), and P. spinosum (Ps) (Lookabaugh et al., 2017). To characterize the prevalence of Pythium root rot in wheat, 100 isolates of Pythium were collected from symptomatic plants in 8 counties and the same 3 species predominated. Symptoms ranged from slight to severe root necrosis in seedling pathogenicity assays. Experiments investigated the influence of environment ( $3 \mathrm{~N}$ levels x 2 temps) and pathogen species $(P i, P s$, or $P i+P s)$ on disease severity, plant height, root length, and biomass (fresh wt). $P i, P s$, or $P i+P s$ caused severe root rot under all conditions tested. Mean disease severity was greater at $12 / 14^{\circ} \mathrm{C}$ than at $18 / 20^{\circ} \mathrm{C}$, but this difference was not significant $(\mathrm{P}>0.05)$. Temperature interacted with pathogen species for biomass, with the lowest biomass recorded for plants inoculated with $P i$ at $12 / 14^{\circ} \mathrm{C}$. Five isolates of each of the 3 species were grown on $\mathrm{V} 8$ media at $14^{\circ} \mathrm{C}$ and $20^{\circ} \mathrm{C}$. All isolates of $P s$ grew faster than the other species at both temperatures. The aggressiveness of these isolates on a single cultivar of winter wheat was compared at $14^{\circ} \mathrm{C}$. The fastest growing and most aggressive isolates belonged to $P i$ and $P s$, but intraspecific variation was observed and highly aggressive isolates were found among all 3 species.

Effects of potato age-related resistance to Potato virus $Y$ on virus systemic movement, yield, quality, and virus translocation into progeny tubers

M. CHIKH ALI, L. Tran, A. V. Karasev, University of Idaho, Moscow, ID, USA

The recombinant strain of Potato virus $Y$, PVY ${ }^{\mathrm{NTN}}$, is the main cause of the potato tuber ringspot disease (PTNRD) in susceptible potato cultivars which reduces the quality of potato tubers, in addition to the yield loss. Here, the effects of the age-related resistance (ARR) 
were investigated in transplants of a potato cultivar Yukon Gold, to the infection with PVYNTN strain, in greenhouse experiments. Within the first three weeks after transplanting into soil (W1-3), Yukon Gold plants developed ARR that impaired the systemic movement of PVY ${ }^{\mathrm{NTN}}$ into upper, non-inoculated leaves and concomitant translocation into progeny tubers starting from week four (W4) after transplanting. The yield and quality of tubers from PVY-infected plants with the established ARR (W5-W8) were comparable to the healthy controls, suggesting late PVY infection would not significantly affect commercial potato production. Potato plants inoculated early (W1-W2), prior to the establishment of the ARR, exhibited a $100 \%$ primary systemic infection with PVYNTN and produced fewer tubers of smaller sizes, exhibiting PTNRD, which resulted in up to $70 \%$ yield reduction compared to plants inoculated later in the season, W5-W8. This ARR greatly restricted the systemic movement of PVY in potato foliage and limited virus translocation into progeny tubers at 7.8\% and 4.1\% in 2017 and 2018, respectively, of all plants inoculated later in the season, W5-W8. This study suggests that PVY management programs should focus more on the early stages of the potato development, prior to the onset of the ARR to PVY.

\section{A Medicago truncatula cytochrome 450 gene is involved in penetration resistance against Asian soybean rust} U. GILL (1), J. Nakashima (2), B. Chandrashekhar (2), S. Mittal (2), Y. Ishiga (3), S. R. Uppalapati (4), K. S. Mysore (2), (1) North Dakota State University, Fargo, ND, INDIA; (2) Noble Research Institute, Ardmore, OK, USA; (3) University of Tsukuba, Tsukuba, JAPAN; (4) FMC, Newark, DE, USA

Asian soybean rust (ASR), caused by Phakopsora pachyrhizi, is a significant disease of soybean in the world. A model legume species, Medicago truncatula, which is a nonhost to P. pachyrhizi, shows incompatible interactions with this fungus. Using forward genetics screening, we identified a M. truncatula mutant, eprl (Enhanced Penetration of Rust 1), with altered response to ASR preinfection structure formation. The eprl mutant showed small germ-tube formation from urediniospores upon germination and enhanced penetration of $P$. pachyrhizi on the leaf surface. The mutant plants also exhibited stunting and water stress under the greenhouse conditions. Further investigations with scanning electron microscopy revealed leaf surface changes on both abaxial and adaxial leaf surfaces of eprl mutant, hence indicating a role of leaf surface topology in altering rust pre-infection structure formation. The genetic co-segregation analysis revealed a mutation in the cytochrome 450 gene, which is responsible for the phenotype. Transcript analysis of the cytochrome $p 450$ gene detected a negligible transcript level in leaves but significant transcript abundance in leaf epidermis, indicating its role in leaf surface structure formation. A detailed characterization of the cytochrome p450 gene will be presented.

\section{Citrus proteins mediate the transmission of Citrus tristeza virus by aphid}

T. SHILTS (1), C. A. El-Mohtar (2), W. O. Dawson (1), N. Killiny (3), (1) University of Florida, Lake Alfred, FL, USA; (2) University of Florida, CREC, Lake Alfred, FL, USA; (3) Department of Plant Pathology, Citrus research and education center, IFAS, UF, Lake Alfred, FL, USA

Citrus tristeza virus (CTV) is transmitted by several aphid species in a semipersistent manner. While aphid transmission has been well documented, the molecular mechanisms implicated in aphid transmission remain undetermined. Multiple factors affect the efficiency of aphid transmission. In this study, we explored the role of citrus proteins in aphid transmission of CTV. Far-western assays enabled us to identify four plant proteins (1) a miraculin-like protein-1, (2) an iron superoxide dismutase, (3) a ubiquitin-conjugating enzyme variant, and (4) a cyclophilin protein, that interact with CTV virions. We used CTV-T36 infectious clone-based RNAi vectors to knockdown each of the identified genes individually and assess the aphid transmissibility from those plants. It is important to mention the CTV-T36 vector is poorly transmitted by aphids with less than $1 \%$ transmission rate. The brown citrus aphid (Aphis citricidus), the most efficient vector of CTV, was used for transmission. Interestingly, knocking down these four genes did not affect CTV replication or its movement within citrus hosts. On the other hand, the RNAi of the iron superoxide gene significantly increased aphid transmission of CTV to approximately 25\%. RNAi of Miraculin-like protein-1 and cyclophilin resulted in increased aphid transmission rates to $6 \%$ and $8 \%$ respectively. Ubiquitin-conjugating enzyme did not affect aphid transmission. Understanding the molecular mechanisms of CTV transmission by aphids will open new avenues to control Citrus tristeza disease.

\section{Potentiation of plant-derived anti-quorum sensing compounds}

I. YEDIDIA (1), Z. Kerem (2), M. Pun (3), (1) Agriculture Research Organization, Rishon Lezion, ISRAEL; (2) Hebrew University of Jerusalem, Rehovot, ISRAEL; (3) Hebrew University of Jerusalem, Rehovot, ISRAEL

For a decade, bacterial quorum sensing (QS) systems gained recognition as new targets for antimicrobial compounds. Plant derived molecules including several phenolics were shown to inhibit QS and virulence traits regulated by it. The genus Pectobacterium is a major cause of soft rot and blackleg on potato and ornamentals worldwide, which uses QS to synchronize virulence. We hypothesized that the anti QS action of some phenolics can be intensified by blocking other cellular targets such as efflux pumps. Membrane transporters may be involved in export of QS signaling molecules as well as diverse antimicrobial compounds, including phenolics. By inhibiting efflux, the cellular concentrations of some antivirulence compounds are expected to rise. We aim to identify compounds that when combined will either completely block virulence, or in-planta bacterial survival. To test this, phloretin, a less studied QS inhibitor from apple leaves, was tested and shown to be an efficient inhibitor of pectobacteria virulence. We further tested inhibitors of AcrB component of the efflux transporter AcrAB-TolC, and present their antimicrobial/ anti-virulence activities. Finally, we tested various combinations of these compounds, to reveal their potential to act synergistic. The mechanisms underlying the interaction between these virulence systems, are now further studied by transcriptomic analysis, mutagenesis and in planta expression. The results will provide better insight into mechanisms by which plant molecules interfere with bacterial virulence. 
Transposon and deletion mutagenesis of Pseudomonas cannabina pv. alisalensis identifies genetic forces driving pathogenicity N. SAKATA, T. Ishiga, Y. Ishiga, University of Tsukuba, Tsukuba, JAPAN

Pseudomonas cannabina pv. alisalensis (Pcal), which causes bacterial blight of Brassicaceae, is an economically important pathogen worldwide. To identify Pcal genes involved in pathogenesis, we conducted a screen of $P$ cal KB2 $11 \mathrm{Tn} 5$ mutants with reduced virulence on cabbage plants using a dip-inoculation method. We identified several potential virulence factors including the type III secretion system, membrane transporters, transcriptional factors, and amino acid metabolism. Importantly, Pcal can infect not only a wide range of Brassicaceae but also green manure crops such as oat ${ }^{[2]}$. Therefore, we also carried out the inoculation assay on oat plants. As a result, we successfully identified the core virulence factors involved in pathogenicity on both plants, cabbage and oat. Furthermore, we also focused on function of coronatine (COR). Firstly, we generated a COR-defective mutant $(\triangle c m a A)$ which was then dip-inoculated onto cabbage and oat plants with wild-type Pcal KB211. $\triangle \mathrm{cmaA}$ showed reduced disease symptoms and bacterial populations on both plants. Furthermore, we also performed syringe-infiltration with wild-type Pcal KB211 and $\triangle c m a A$ into both plants, and $\triangle c m a A$ showed reduced virulence on both plants. Taken together, we demonstrated that COR is an important virulence factor in overcoming both stomatal and apoplastic defenses. We believe our continuous study will elucidate the pathogenicity of Pcal.

\section{Characterization of cell wall proteins in chickpea that inhibit fungal hydrolytic enzymes} V. ELLUR (1), W. Wei (2), G. J. Vandemark (3), W. Chen (3), (1) Washington State University, Pullman, WA, USA; (2) Washingtron State University, Pullman, WA, USA; (3) USDA ARS, Pullman, WA, USA

The plant cell wall is the first barrier against pathogenic fungi; in order to breach it, fungal pathogens secrete several cell wall-degrading enzymes. Polygalacturonases (PGs) are one of them and the most critical virulence enzymes that hydrolyze polygalacturonic acid in pectin, which is the main component of a plant cell wall. To counteract these PGs, plants produce polygalacturonase-inhibiting proteins (PGIPs). They interact with fungal PGs to inhibit PG activity. The objective of this study is to test the significance of the PGIP gene from chickpea, Capgip 1, in resistance against pathogenic fungi. Capgip1 contains domains of ten imperfect tandemly arranged leucinerich repeats (LRRs), which are characteristic of immune receptors. Real-time PCR analysis showed that Capgip1 is upregulated after infection by Fusarium oxysporum f.sp. ciceris, Ascochyta rabiei, and treatment with defense hormones such as salicylic acid, methyl jasmonate, abscisic acid, and wounding, indicating its potential role during infection. Also, fluorescent microscopy in Nicotiana benthamiana plant expressing Capgip1-GFP indicated that CaPGIP1 protein localizes on the cell wall. Moreover, CaPGIP1 protein is heterologously expressed in methylotrophic yeast Pichia pastoris for studying its inhibitory functions against polygalacturonase of chickpea pathogenic fungi. Results of this study indicate CaPGIP1 is a cell wall-bound protein that is induced during the fungal infection and has an inhibitory effect against pathogen PGs, suggesting its role in defense of chickpea against fungal invasion.

Invasive alien forest pathogens and pests devastate ecosystems and economies - Time to fix a flawed response framework P. BONELlO (1), F. T. Campbell PhD (2), D. Cipollini (3), A. O. Conrad (4), C. Farinas (1), K. Gandhi (5), F. Hain (6), D. Parry (7), D. Showalter (8), C. Villari (5), K. Wallin (9), (1) The Ohio State University, Columbus, OH, USA; (2) Center for Invasive Species Prevention, Bethesda, MD, USA; (3) Wright State University, Dayton, OH, USA; (4) The Ohio State University, Department of Plant Pathology, Columbus, OH, USA; (5) D.B. Warnell School of Forestry, University of Georgia, Athens, GA, USA; (6) North Carolina State University (emeritus) and Forest Restoration Alliance, Raleigh, NC, USA; (7) SUNY ESF, Syracuse, NY, USA; (8) University of Minnesota, St. Paul, MN, USA; (9) North Dakota State University, Fargo, ND, USA

One outcome of global connectivity is the widespread mortality of forest trees due to invasive, alien pathogens and pests, with significant ecosystem and economic disruption. While important ongoing initiatives aimed at solid wood packing material and other prominent sources of invasion may reduce the incursion of new alien species, current policy and management strategies directed at established invasive pathogens and pests have a theme in common: in the long run, they almost invariably fail. This is due to the reactive, short-term, poorly coordinated and underfunded nature of our responses. This outcome is also due to intrinsic properties of the invaded ecosystems, specifically due to the lack of a co-evolutionary history, naïve host trees provide a largely defense-free space where alien pathogens and pests can prosper. Thus, the breeding and deployment of resistant tree genotypes is a priority as we devise ways to respond and eventually restore ecosystems. Here we propose that this insidious problem would be best managed with a command-and-control structure that could deal with these issues end-to-end, from prevention to restoration: the federal Centers for Forest Pest Control and Prevention.

\section{Root rot mitigation in pulse crops C. MURPHY, M. E. Burrows, Montana State University, Bozeman, MT, USA}

Aphanomyces root rot on pulse crops, caused by Aphanomyces euteiches, was first discovered in Montana in 2016, during statewide field pea disease surveys. This oomycete pathogen has now presented in pea and lentil fields in six Montana counties, in a pulse root rot species complex, causing crop failure in a few cases. A. euteiches is troublesome to manage as there are no effective fungicides for its control on the market, it can boom in seasons when moisture is high, and the resting oospore can live in the soil for ten years. The first objective of this research was to assess different crop species and weeds for their ability to host Montana isolates of $A$. euteiches. The second objective was to observe the plant species impact on the residual soil inoculum potential of $A$. euteiches. Plant species grown in inoculated soil differed in hosting ability, and results were consistent with those reported in the literature. Peas, lentils, and vetch were good hosts for $A$. euteiches, while cereal crops and chickpeas were non-hosts, and the cultivar of faba bean that was tested was resistant to $A$. euteiches. Plant species varied in residual A. euteiches soil inoculum potential $(\mathrm{p}<0.001)$, measured using pea baiting techniques. Peas and lentils increased soil inoculum potential compared to controls, while non-host species either reduced the inoculum potential of 
the pathogen in the soil or had minimal impact on pathogen levels. Research is ongoing, to determine appropriate crop rotation strategies in Montana to combat the disease.

\author{
Elucidating the role of DIAGEOTROPICA in salicylic acid-auxin crosstalk during responses to Ralstonia solanacearum in \\ tomato \\ K. RIVERA-ZULUAGA, P. Choudhary, D. Caldwell, M. Ghaste, J. Widhalm, A. Iyer-Pascuzzi, Purdue University, West Lafayette, IN, \\ USA
}

Bacterial Wilt (BW) is among the most devastating plant diseases in the world. This disease is caused by the soilborne plant pathogen Ralstonia solanacearum $(R s)$ and affects more than 200 species of plants. In tomato, resistance to $R s$ is quantitative and the result of many genes, however, the molecular basis of resistance is still unclear. No resistance genes to the US Rs strain K60 have been identified. Transcriptomic analysis of resistant tomato roots showed that at 48 hours post inoculation with $R s$ K60, genes involved in auxin transport and signaling pathways are downregulated. Furthermore, a tomato mutant defective in auxin transport and signaling, known as diageotropica (dgt 1-1) has enhanced resistance to RsK60. RsK60 root vasculature colonization is delayed in dgt 1-1 compared to wild type. Treating wild type susceptible tomato plants with the auxin transport inhibitor naphthylphthalamic acid (NPA) enhances resistance to RsK60 in a dose-dependent manner. Auxin acts antagonistically with the plant hormone salicylic acid (SA), and we found that $d g t$ 1-1 has higher levels of SA and increased expression of SA-response genes. A strain of Rs that can degrade SA is partially virulent on $d g t$ 1-1. Our research suggests that the $D G T$ gene is required for susceptibility to RsK60, and proper auxin transport and signaling is important for susceptibility to RsK60 in tomato plants. This may be in part due to interactions with the SA pathway. Understanding the role of DGT, auxin, and SA in defense responses to $R s$ in tomato is important for Solanaceae crop improvement.

A novel role of a fungal alcohol oxidase $(A O X)$ in plant colonization N. M. WESTRICK (1), M. Roth (2), D. Shao (3), D. L. Smith (3), M. Kabbage (3), (1) University of Wisconsin - Madison, Madison, WI, USA; (2) University of Wisconsin - Madison, Madison, WI, USA; (3) University of Wisconsin-Madison, Madison, WI, USA

Sclerotinia sclerotiorum is a broad-host range necrotrophic pathogen capable of causing devastating disease in a range of economically important crops. Through a time-course transcriptome analysis of S. sclerotiorum infection in soybean, an alcohol oxidase (SsAOX) was identified as one of the most highly expressed genes during infection and was up-regulated $\sim 1,200$-fold during late-stage colonization. A knockout mutant was generated $(\triangle S S A O X)$ which showed significantly reduced virulence in detached leaf assays of several plant hosts when compared to the wild-type 1980 strain (WT). Whole soybean plants were also inoculated through cut petioles. Similarly, $\triangle S s A O X$ infected plants demonstrated a significantly reduced mortality rate $(\sim 10 \%)$ when compared to the WT strain ( $90 \%)$ at 9 days post inoculation, seemingly due to its inability to properly colonize soybean stem tissue. Chemical genomics suggest that SsAOX may function as an aromatic alcohol oxidase, and phylogenic analysis demonstrates a high level of conservation of putative AOX orthologues throughout the phylum Ascomycota. Interestingly, SsAOX homologs are largely associated with the genomes of plant associated fungi, and a review of existing transcriptome datasets highlights its utilization by a broad range of fungal pathogens during pathogenesis. From this data, we hypothesize that $A O x$ is a key component in the detoxification of antifungal aromatic compounds released from plant cell walls during infection, an important process that appears to be conserved across broad evolutionary distances of plant associated fungi.

Understanding white mold in Pennsylvania soybeans: Spatial distribution of Sclerotinia sclerotiorum sclerotia at the field scale T. MCFEATERS (1), K. Luong (2), A. A. Collins (3), A. Murillo-Williams (4), P. Esker (1), (1) The Pennsylvania State University, University Park, PA, USA; (2) The Pennsylvania State University, State College, PA, USA; (3) Southeast Agricultural Research \& Extension Center, Manheim, PA, USA; (4) Penn State Extension-Centre County, Bellefonte, PA, USA

White mold (WM) is an economically important disease that negatively impacts Pennsylvania (PA) soybean production. Sclerotinia sclerotiorum $(S s)$, the soil-borne pathogen that causes WM, thrives in cool, wet weather. Therefore, the unique and variable microclimates throughout PA drive sporadic epidemics. The goal of this project is to increase knowledge about the field scale spatial distribution of $S s$ in different regions of PA in order to improve field trial design and provide better management recommendations for growers. Fields in a corn-soybean rotation were selected from the northwest, west, central, east, and southeast regions of PA. Individual fields were gridded, and a stratified random sampling design was used to sample soil. Approximately 35 soil subsamples were taken from every field, with GPS coordinates recorded for each subsample plot. Samples were sieved to capture and harvest $S s$ sclerotia. Sclerotia were weighed and germinated on PDA plates to test viability of the Ss isolates. Preliminary results show individual plots within the same field ranged from approximately 0 to 14 viable sclerotia/kg of soil sampled. Mapped results suggest an aggregated distribution is present at the field level. These preliminary results are important to work with growers to consider field variation of $S s$ when developing management strategies. Future work will focus on the genetic analysis of the $S s$ isolates to look at within field and regional genotypic diversity and genetic differentiation.

Mapping of a broad-spectrum rust resistance locus in Andean common bean PI 260418

G. VALENTINI (1), L. Xavier (1,2), M. A. Pastor-Corrales (1), (1) ARS-USDA, Beltsville, MD, USA; (2) Universidade Estadual de Maringa, Beltsville, MD, USA

Rust, caused by Uromyces appendiculatus, is a major disease of common bean (Phaseolus vulgaris) in the world. PI 260418 is an Andean common bean landrace from Bolivia with resistance to all but one of the 90 races of $U$. appendiculatus maintained at the USDA-ARS Bean Project, Beltsville, MD. The objective of this study was to map the resistance locus in PI 260418 using phenotypic, 
genomic, and high-throughput genotyping technologies. An F2 population from the Pinto $114 \times$ PI 260418 cross was inoculated with races 31-1 and 31-22 of $U$. appendiculatus. This resulted in 211 resistant and 62 susceptible plants, suggesting the presence of a single and dominant resistance locus $(P$ value $=0.38)$ in PI 260418. A bulk segregant analysis (BSA) performed with the BARCBean12K SNP Chip was used to determine the genomic region containing the resistance locus in PI 260418. We then used KASP markers specifically designed to target the genomic region. A total of 25 polymorphic SNPs from the BSA positioned the resistance locus on a genomic region from 275,483 to 1'401,732 bp on the upper arm of chromosome Pv04 (genome v.2.1). Further genotyping positioned the locus in a 1'019,458 bp genomic region between KASP markers SS130 (302,160 bp) and SS61 (1'321,618 bp), a genomic region characterized by low recombination. Additional efforts focus on fine mapping the region containing the rust resistance locus to develop markers that would enable the introgression of the PI 260418 resistance locus into dry and snap bean cultivars.

\title{
Whole genome sequences of 281 Xanthomonas perforans strains reveal structure of population in relation to tomato production chain variables
}

J. KLEIN (1), S. Timilsina (1), P. Abrahamian (2), Y. Xing (3,4,5), K. A. Garrett (3,4,5), G. V. Minsavage Jr. (1), M. L. Paret (6), J. B. Jones (1), E. M. Goss (1), G. E. Vallad (7), (1) University of Florida, Gainesville, FL, USA; (2) USDA ARS Molecular Plant Pathology Laboratory, Beltsville, MD, USA; (3) Plant Pathology Department, University of Florida, Gainesville, FL, USA; (4) Institute for Sustainable Food Systems, University of Florida, Gainesville, FL, USA; (5) Emerging Pathogens Institute, University of Florida, Gainesville, FL, USA; (6) North Florida Research \& Education Center, University of Florida, Quincy, FL, USA; (7) Gulf Coast Research and Education Center, University of Florida, Wimauma, FL, USA

Bacterial spot of tomato (BST), one of the most economically important diseases of tomato, is caused by four Xanthomonas species. In Florida, $X$. perforans $(X p)$ is the primary cause of BST. We characterized $585 X p$ isolates from symptomatic tissue in 70 commercial tomato fields throughout Florida in fall 2017 to determine the population composition. To identify possible points of $X p$ spread, we then sequenced the genomes of 281 representative strains using the Illumina NextSeq 550 platform. Whole-genome single nucleotide polymorphisms (SNPs), core genomes, and type III secretion system effector profiles were tested for associations with production chain variable metadata, inlcuding 'seed producer', 'cultivar', 'transplant facility', 'county', 'operation', and 'farm'. Dramatic genetic differences among phylogenetic groups were observed, similar to previous studies from our research group, requiring association testing be performed separately for each phylogenetic group. Using SNP data, AMOVA tests revealed that the variables 'transplant facility', 'county', and 'farm' explained significant proportions of the genetic variation within phylogenetic groups ( $p$-values ranged from 0.001 to 0.015 ). While the composition of the $X p$ population in Florida is complex, with the majority of fields containing strains from more than one phylogenetic group, our results demonstrate there is some structure across production chain variables. Understanding these associations will lead to improved management strategies for Xanthomonas in the production system.

\section{Development of a Venturia effusa pangenome to aid in identification of cultivar specificity genes}

J. SHILLER (1), C. H. Bock (2), N. D. Charlton (3), N. Krom (3), Y. Alarcon (3), M. J. Monteros (3), C. Graham (1), C. A. Young (3), (1) Noble Research Institute, Ardmore, OK, USA; (2) USDA ARS, Southeastern Fruit and Tree Nut Research Laboratory, Byron, GA, USA; (3) Noble Research Institute, LLC, Ardmore, OK, USA

\begin{abstract}
The most destructive disease of pecan (Carya illinoinensis) in the southeastern U.S. is scab, caused by Venturia effusa. Symptoms include lesions on nuts, leaves and shoots that reduce overall yield. Growers rely heavily on fungicide use to control the disease, applying ten or more sprays per season. Pecan cultivars with resistance to scab isolates exist but are not widely grown. However, the mechanisms and potential durability of scab resistance are unknown. Multiple races of $V$. effusa with different cultivar specificities have been identified and these interactions are likely to be governed by gene-for-gene interactions similar to those observed in other Venturia species (e.g., $V$. inaequalis, the apple scab pathogen). We used comparative genomics among multiple scab isolates to identify genes that are potentially involved in cultivar specificity. The genomes of 11 isolates of $V$. effus $a$ were sequenced and assembled, including one full-length chromosome level assembly. The isolates were collected from five pecan cultivars from five growing regions, and two isolates from native Carya spp. A $V$. effusa pangenome was generated to find conserved and unique genes. Genes commonly involved in plant-host interactions were identified, including small secreted proteins, carbohydrate-active enzymes and gene clusters involved in secondary metabolism. Meta-transcriptome data from naturally infected pecan in the field was also incorporated into the analysis. The analysis provides a basis for broader exploration at the population level to identify key genes that determine host specificity.
\end{abstract}

Structural and functional characterization of an atypical rice blast resistance gene $\boldsymbol{P t r}$ Y. JIA (1), H. Zhao (2), X. Wang (3), M. Jia (4), Y. Liu (5), H. Box (4), (1) USDA ARS DBNRRC, Stuttgart, AR, USA; (2) USDA ARS, 130, AR, USA; (3) UA/USDA, Stuttgart, AR, USA; (4) USDA ARS Dale Bumpers National Rice Research Center, Stuttgart, AR, USA; (5) University of Arkansas Rice Research and Extension Center, Stuttgart, AR, USA

Rice blast disease caused by the fungus, Magnaporthe oryzae, is one of the most damaging rice diseases worldwide. Effective resistance $(R)$ genes and fungicides have been used to manage blast with limited success due to frequent genetic changes of the fungus. In the present study, we characterized a new type of disease resistance genes, Ptr, in rice. The Ptr gene, from a blast resistant tropical japonica rice variety Katy, was map-based cloned with the help of fast neutron mutagenesis and CRISPR-CAS9. The rice variety with Ptr is resistant to all known blast races in the US except for the races, IE1k and IB33. The Ptr gene has 4 exons and 3 introns encoding two predicted protein isoforms with 864 and 905 amino acids each carrying 4 armadillo repeats, but lacking a U box implying that the Ptr protein is an atypical E3 ligase. The absence of polyubiquitination signals at the Ptr protein in vitro suggests that Ptr does not have E3 ligase activity. Black hull weedy red rice, RR20, is resistant to IB33 and the $R$ gene was mapped at the Ptr locus. There were two amino acid alterations of the Ptr protein in RR20 when compared with that of the susceptible mapping parent, Dee-Gee-Woo-Gen, and one of the two is also different when compared with that of Katy. These findings suggest that a single amino acid change of the Ptr protein in 
RR20 may determine pathogen signal recognition specificity. Ptr in rice varieties was found to be induced by blast pathogen and abiotic stresses including chilling, heat, drought and salt. The role of Ptr in stress tolerance will be presented.

\title{
Potential host range and effects of taproot decline on rotation cover crops
}

J. Moscoso (1), S. A. Winters (2), A. ROJAS (2), (1) Entomology and Plant Pathology, University of Arkansas - Division of Agriculture, Fayetteville, AR, USA; (2) University of Arkansas, Fayetteville, AR, USA

Taproot decline, caused by Xylaria spp. is an emergent disease of soybean in the southern United States. The pathogen colonizes the root system causing necrotic symptoms on the taproot and continuous infection causes yellowing of the foliage that turns into necrotic lesions followed by foliage desiccation. Xylaria is a well-known genus of saprophytic fungi, but often members of this genus are also associated with the functional guild of endophytes. Taproot decline has recently gained attention and cultivar screening and seed treatments have been assessed as strategies to manage this disease. However, other cultural practices such as crop rotation and enhancement of soil health by using cover crops could have the potential on changing the environment where this disease occurs. The hypothesis is that cover crops could serve as potential hosts for this pathogen or the increase of organic matter will favor the prevalence of Xylaria. In order to test this hypothesis we adapted inoculation methods to study the potential of Xylaria to survive and colonize different cover crops used in Arkansas. The cover crops studied includes winter pea, radish, wheat, mustard and cereal rye. Two isolates were tested on seed plate assays and seedling assays. The responses varied among the cover crops, either reducing, not affecting or increasing biomass. The potential survival of Xylaria spp. on cover crops will be useful in considering rotations for management of taproot decline.

\author{
The use of nanotechnology in spray-induced gene silencing (SIGS) provides a steady RNAi effect against Botrytis cinerea \\ infection on plant material \\ J. NIÑO SANCHEZ (1), R. Hamby (2), L. Quian (2), H. Jin (2), (1) University of California, Riverside, Riverside, CA, USA; (2) \\ University of California, Riverside, CA, USA
}

Fungal pathogens are responsible for many plant diseases and cause severe crop losses worldwide, threatening global food security. $B$. cinerea, the causal agent of grey mold disease on over 1000 plant species, can deliver small RNAs (sRNAs) to its host during the infection process to silence host defense genes, and the host plant is able to send sRNAs to the fungal pathogen to inhibit virulence genes. This mechanism was described by our group and called "cross-kingdom RNA interference". The subsequent discovery that $B$. cinerea can efficiently take up environmental sRNAs that silence fungal genes with complementary sequences led to the development of an innovative and eco-friendly fungal disease management strategy: spray-induced gene silencing (SIGS). Although this spray application of RNAs is effective in controlling several fungal pathogens on pre- and post-harvest plant material, the durability of crop protection is not clear, because SIGS is limited by the stability of double-stranded RNA (dsRNA) and its uptake efficiency by the fungus. Here, we made use of nanotechnology to develop coated-dsRNA sprays against key virulence-related (Dicer-like, DCL1 and $D C L 2)$ and essential fungal growth genes (VPS51, SAC1, DCTN1) on various plant materials, including leaves, flowers and fruits. The use of nanotechnology provides dsRNA protection, as well as higher silencing efficacy than naked dsRNA application over time, therefore offering more potential and broader applications to control fungal plant diseases through sequence-specific SIGS technology.

The newly emerging powdery mildew management challenge of sweet cherry orchards: Fungicide resistance P. S. SWAMY, G. G. Grove, Washington State University, Prosser, WA, USA

The Podosphaera cerasi, incitant of the sweet cherry powdery mildew remains one of the continuing threats to sustainable high-quality cherry production. The situation is exacerbated due to the high selection pressure exerted by various synthetic fungicides that results in fungicide resistance. Notably, Group 3 (DMI) and Group 11 (strobilurins) fungicides are applied in the commercial orchards and are among fungicides categories with medium-high to high risk of developing resistance. To determine the presence and quantify the extent of fungicide resistance, several P. cerasi isolates were collected from commercial orchards in the Pacific Northwest (PNW). The protocols were developed to identify the mutant allele (G461S) of the CYP51/ ERG11 gene that corresponds to fungicide resistance by using hydrolysis probe-based qPCR techniques. Due to a large sequence variability in the CytB gene among various $P$. cerasi isolates, the mutation (G143A) that correlates with the Group 11 resistance was detected by PCR amplification followed by DNA sequencing. In an initial survey, about $35 \%$ of the $P$. cerasi isolates exhibited G461S mutation while more than $40 \%$ isolates contained G143A mutation. Representative isolates were subjected to leaf-disc bioassays per the field dosage of azoxystrobin and myclobutanil. The molecular data confirmed that most of the fungicide-insensitive colonies contained respective mutations hence deemed fungicide resistant isolates. The data suggested a need for revised fungicide spray programs to exclude Group 3 or 11 fungicides in affected cherry growing orchards.

\section{Suppression of Aspergillus flavus infection through host-induced gene silencing reduced aflatoxin contamination in transgenic maize lines \\ O. O. OMOLEHIN (1), Y. Raruang (1), Q. Wei (2), K. Rajasekaran (3), Z. Y. Chen (1), (1) Department of Plant Pathology and Crop Physiology, Louisiana State Univ AgCenter, Baton Rouge, LA, USA; (2) USDA-ARS, SRRC, New Orleans, LA, USA; (3) U.S. Department of Agriculture-Southern Regional Research Center, New Orleans, LA, USA}

Aflatoxin is a carcinogenic secondary metabolite produced by the fungus Aspergillus flavus. Aflatoxin contamination in maize poses a health threat to 4.5 billion people, mainly in developing nations, whose staple food is maize. Therefore, enhancing maize resistance to A. flavus infection or aflatoxin contamination has been the focus of many researches. In the present study, the $A$. flavus alkaline protease 
gene $(A l k)$, involved in the infection of maize kernels, was selected as a possible target for suppression through host-induced gene silencing (HIGS) to mitigate aflatoxin accumulation in maize. An RNAi vector containing a portion of the Alk gene was introduced into immature B104 maize zygotic embryos. Homozygous transgenic maize plants developed from four events carrying the Alk genesilencing construct reduced up to $80 \%$ of aflatoxin production in repeated laboratory and field studies when compared to those without the $A l k$ gene (P-values between 0.04 and 0.003 ). Histological study using a GFP-transformed $A$. flavus demonstrated reduced fungal infection in the transgenic maize kernels compared to the non-transgenic control. In addition, significantly higher levels of $A l k$-specific small RNAs were detected in transgenic maize kernels, confirming the role of Alk-specific small RNAs in enhancing aflatoxin resistance. Future studies will focus on transferring this trait into elite inbred lines for developing commercial aflatoxin resistant maize lines.

Transcriptional expression pattern in effector genes of Phytophthora betacei C. BUITRAGO, S. Restrepo, A. J. Bernal, Universidad de Los Andes, Bogotá, COLOMBIA

Effectors are proteins produced by plant pathogens in order to manipulate their hosts and successfully infect them. Studying these genes is critical for basic understanding plant-pathogen interactions and finding possible targets to control infection. Phytophthora genus includes important plant pathogens such as P. infestans, $P$. capisci, $P$. sojae among others. Phytophthora betacei is a recently described species, whose preferred host is Solanum betaceum (tree tomato). In previous studies, we observed multiple gene copies of effector genes in strains of $P$. betacei. In order to understand more about these genes, we characterized the transcription expression pattern for Avr3a, Sne1, Npp1 and Nlp1 effector genes at different time points during infection to S. betaceum. We infected two varieties of tree tomato (Común and Rojo) with two strains of $P$. betacei that vary in aggressiveness. We observed different expression patterns for each gene, each strain and each host variety. Avr3a and Sne1 effectors are highly expressed during the biotrophic phase and Npp1 and Nlp1 effectors are highly but not uniquely expressed in the necrotrophic phase. These results indicated a highly regulated system that depends on multiple factors that must be taking into account in order to study the effect of these genes on disease development.

\section{Characterizing heterogeneity and defining optimum sample sizes for estimation of wheat Fusarium head blight index in research plots \\ W. BUCKER MORAES, L. V. Madden, P. A. Paul, Department of Plant Pathology, The Ohio State University, Wooster, OH, USA}

Since Fusarium head blight (FHB) intensity is usually highly variable within a plot, the number of spikes rated for FHB index (IND) quantification must be considered when designing experiments. In addition, quantification of sources of IND heterogeneity is crucial for defining sampling protocols. Two field experiments were conducted to quantify the variability of IND at different spatial scales and to investigate the effects of sample size on estimated plot-level mean IND. A total of 2167 -row $\times 6$-m-long plots of a moderately resistant and a susceptible cultivar were spray inoculated with different $F$. graminearum spore concentrations at anthesis to generate a range of IND levels. A two-stage cluster sampling approach was used to estimate IND, with an average of 32 spikes rated at each of 10 equallyspaced points per plot. Plot-level mean IND ranged from $0.9 \%$ to $37.9 \%$. Heterogeneity of IND, quantified by fitting unconditional hierarchical linear models, was greater among spikes within clusters then among clusters within plots or among plots. Predicted coefficient of variation (CV) of mean IND increased as mean IND decreased, and as sample size decreased below 100 spikes per plot. Simple random samples were drawn with replacement 50,000 times from the original dataset for each plot $(\mathrm{n}=320)$ and used to estimate the effects of sample sizes on mean IND. Samples of 100 or more spikes resulted in more precise estimates of mean IND than smaller samples. Poor sampling may result in inaccurate estimates of IND, and this could affect interpretation of results in FHB experiments.

Optimizing soil microbiomes and physiochemical characteristics to enhance potato health, productivity, and quality through crop management

M. BOLTON (1), J. Crants (2), C. Rosen (2), L. L. Kinkel (1), (1) University of Minnesota, MN, USA; (2) University of Minnesota, Saint Paul, MN, USA

Soil microbes are essential for building and maintaining soil structure, nutrient cycling, plant growth promotion and pathogen suppression. However, our understanding of how to manage microbial communities for agronomic benefits remains limited. In this study, we utilize a national platform of research plots across eight different states to identify reliable potato soil health indicators and determine effective methods for managing soil microbiomes and physicochemical properties to enhance potato health and productivity. Data from 2019 provide insights into the impacts of potato cultivar, soil fumigants, and crop rotation on soil microbiomes and soil physicochemical characteristics. Soil samples were collected at pre-planting and 60 days post-planting. Soil physicochemical characteristics and bacterial and fungal community composition and diversity were determined for every sample. In addition, potato crop yields and disease intensities were evaluated for all treatments. In Minnesota, we did not observe significant variation in potato yields among treatments, however certain treatments did differ in percent common scab pitting. The microbiome characteristics most strongly correlated with healthy, high-yielding potatoes, including microbial community composition and diversity, will be determined for individual states and across the national platform. We will specifically highlight Minnesota's results. Relationships between crop management practices, microbiomes and soil physicochemical characteristics will provide insights into optimizing soils to enhance potato production.

Utilization of agro-industrial waste tobacco dust for sustainable agricultural intensification and improvement of soil biological health

S. BANU, A. Saha, M. Alam, K. Alam, Bangladesh Agricultural Research Institute, Gazipur, BANGLADESH 
Tobacco dust, an agro-industrial waste, is traditionally used in soil by farmers of northern Bangladesh for potato cultivation. The dry dust can act as repellent against soil-borne pests and diseases, especially nematodes, and can reduce turnaround time between crops. As a plant product, tobacco dust is readily degradable in soil, including elimination of nicotine by soil micro-organisms. This study was carried out to identify the practical effects of tobacco dust soil treatment for potato production during 2017-18 and 2018-19 cropping seasons in both research and farmer's field of northern Bangladesh. Tobacco dust from the local market was compared at three different doses with cow dung for the cultivation of potato variety Cardinal. Significantly higher tuber yield in both seasons were obtained with tobacco dust at a dose of $850 \mathrm{~kg} \mathrm{ha}^{-1}$. The lowest numbers of fungal and bacterial colonies and pathogenic nematodes were found at this dose. The incidence and severity of common scab was also significantly lower. A similar effect was also observed for black scurf disease. In addition, plant senescence was delayed by 21-23 days which may have contributed to higher tuber yields. The percentage of organic matter in soil was higher in all three doses of tobacco dust 55 days after planting. Tobacco dust could be a good alternative to cow dung, the traditional source of organic matter. Thus, the use of tobacco dust in soil at $850 \mathrm{~kg} \mathrm{ha}^{-1}$ is a sustainable and eco-friendly solution for potato cultivation, improvement of soil biological health and conversion of waste into wealth.

Insect-bacteria-plant interactions: Microbiomes in honeydew of Russian wheat aphids increase aphid virulence to wheat E. K. LUNA (1), J. Hardin (1), D. Caldwell (2), A. Iyer-Pascuzzi (2), J. E. Leach (1), (1) Colorado State University, Fort Collins, CO, USA; (2) Purdue University, West Lafayette, IN, USA

Phenotypic responses of plants to biotic stresses are often studied as interactions between two species; however, in the phytobiome, these responses frequently result from complex interactions involving several organisms. We previously demonstrated that variation in chlorosis on wheat caused by feeding of Russian wheat aphid (Diuraphis noxia) is determined, in part, by aphid-associated bacteria. Although saliva from aphids contains culturable bacteria, using scanning electron microscopy (SEM), bacteria were not detected in high numbers in the salivary glands or foregut of the aphids. Culturing studies show that bacteria in the same genera of those identified in aphid saliva are also present in aphid honeydew, including bacteria in the families Enterobacteriaceae and Erwiniaceae. Honeydew collected from aphid colonies that had been reared from nymphs isolated at birth contained lower titers of bacteria than honeydew collected from non-isolated aphid populations reared with adults. The microbiome of whole aphids as well as aphid honeydew are dominated by bacteria from the families Enterobacteriaceae and Erwiniaceae, after the removal of sequences from the aphid's main symbiont, Buchnera aphidicola. Currently, studies are underway to determine the role of honeydew in initiation of plant defenses. We hypothesize that these honeydew-associated bacteria contribute to aphid virulence by suppressing the plants' insect defense mechanisms, allowing the aphid to feed freely.

\section{Biological strategy for Rhizoctonia solani mediated damping-off in sugar beet using Penicillium pinophilum}

M. E. HAQUE (1), D. K. Lakshman (2), A. Qi (3), M. Z. R. Bhuiyan (4), Y. Liu (4), P. Hakk (5), M. F. R. Khan (6), (1) North Dakota State University, Dept of Plant Pathology, Fargo, ND, USA; (2) USDA ARS, Beltsville, MD, USA; (3) University of Hertfordshire, Hatfield, Hertfordshire, UNITED KINGDOM; (4) NDSU, Fargo, ND, USA; (5) North Dakota State University, Fargo, ND, USA; (6) North Dakota State Univ \& Univ of MN, Fargo, ND, USA

Rhizoctonia solani is a major pathogen of sugar beet (Beta vulgaris L.) which causes seedling damping-off, and root and crown rot of older plants. Growers use cultural practices, partially resistant cultivars, and fungicides to manage $R$. solani. There has not been much success in using biological control for this pathogen in sugar beet. Our objective was to investigate the bio-control potential of $P$. pinophilum on $R$. solani AG2-2. In vitro co-culture of both fungi showed that $R$. solani growth was inhibited by $P$. pinophilum. A greenhouse study was done using sclerotia of $R$. solani and conidia suspension of $P$. pinophilum. Treatments included one sclerotium $/ \mathrm{seed}$, conidia suspension $\left(1 \times 10^{6} \mathrm{conidia} / \mathrm{ml}\right) / \mathrm{seed}$, sclerotium $/ \mathrm{seed}+$ conidia suspension $\left(1 \times 10^{6} \mathrm{conidia} / \mathrm{ml}\right)$, and mockinoculation/seed. One 2-cm deep furrow was made in the middle of peat filled trays into which 10 seeds were planted. Independent treatments were placed adjacent to each seed and covered with peat. There were four replicates per treatment arranged in a completely randomized design. The sclerotia mediated damping-off were observed by $75 \%$, while the combined treatment of sclerotia and conidia reduced damping-off by $75 \%$. No damping-off were observed in conidia suspension and mock inoculated check. This study suggests that $P$. pinophilum has the potential to reduce damping-off caused by $R$. solani and further studies are needed to determine the mechanism of suppression.

\section{Understanding the genotypic diversity of Sclerotinia sclerotiorum in Pennsylvania and New York}

K. LUONG (1), T. McFeaters (2), S. J. Pethybridge (3), P. Esker (4), (1) The Pennsylvania State University, State College, PA, USA; (2) Penn State Department of Plant Pathology, Fredericksburg, PA, USA; (3) Cornell University, Geneva, NY, USA; (4) The Pennsylvania State University, University Park, PA, USA

White mold, caused by Sclerotinia sclerotiorum, is an important disease in the Northeast USA. However, there is limited knowledge of the genotypic diversity and genetic differentiation of $S$. sclerotiorum populations in this region. The objective of this study is to determine the extent of white mold disease incidence across Pennsylvania (PA), analyze the genotypic diversity of these populations, and compare with those from New York (NY). During the summer of 2019, field samples were collected from seven counties in various production regions in PA. In the laboratory, sclerotia were harvested from diseased plants and soil, surface sterilized with $4 \%$ sodium hypochlorite and $80 \%$ ethanol, bisected, and placed cut-side-down onto potato dextrose agar. Single isolates were obtained via hyphal tipping. A total of 185 putative isolates were obtained, 103 from PA and 82 from NY on soybean and snap bean, respectively. Multilocus genotypes (MLGs) will be determined using nine microsatellite markers and genetic diversity analyses will be performed using R statistical software. These findings will give insight on the composition of MLGs contributing to the population in PA and 
movement of MLGs between PA and NY. With this knowledge, dominant MLGs can be identified and mapped out, and the durability of fungicides as management tools can be evaluated.

Population structure and genetic diversity of Phytophthora capsici in Tennessee T. SIEGENTHALER, K. H. Lamour, Z. R. Hansen, University of Tennessee, Knoxville, TN, USA

Phytophthora capsici is the causal agent of Phytophthora blight of cucurbits and peppers. Migration of $P$. capsici is limited mainly to surface water, soil transmission, and movement of infected plant material. Following introduction to a field, management of $P$. capsici is difficult due to the production of hardy sexually produced oospores, although asexually produced sporangia and zoospores are responsible for rapid epidemic progress. Together, these are the main drivers of $P$. capsici population structure, an important idea that shows how the genetics differ within this species in a region. To explore population structure in Tennessee (TN), $298 P$. capsici isolates were collected from fields in five counties across Middle and East TN in 2004, 2007, 2018 and 2019. Not all locations were sampled every year, but some were sampled multiple years. DNA was extracted from cultures or directly from infected plant tissue and used in a multiplexed PCR reaction containing primers targeting 39 previously identified single nucleotide polymorphisms (SNPs). The products from these reactions were sequenced on an Illumina platform and were used to identify multilocus genotypes for each isolate. Using the R package Poppr, summary statistics were generated, and structure analyses were conducted. These indicate that populations in TN were structured by geographic region and did not shift over time, showing that fields contain isolated populations and that selection pressures applied by growers likely drive genetic changes in TN . capsici populations, rather than outside introductions.

Large-scale transposon mutagenesis of Pseudomonas syringae pv. actinidiae using a kiwifruits seedling assay T. ISHIGA (1), N. Sakata (2), Y. Ishiga (1), (1) University of Tsukuba, Tsukuba, JAPAN; (2) University of Tsukuba, Tsukuba, Japan, JAPAN

Bacterial canker of kiwifruit caused by Pseudomonas syringae pv. actinidiae (Psa) is a serious threat to kiwifruit production worldwide. Enhanced virulence strains of Psa biovar 3 (Psa3) were reported and spread rapidly to kiwifruit production areas worldwide. Therefore, there is an urgent need to develop critical management strategies for bacterial canker based on dissecting the dynamic interactions between Psa and kiwifruit. However, there are only a few studies which screened for Psa3 virulence genes. To elucidate the molecular mechanism of Psa3 infection, we developed a rapid and reliable flood-inoculation method using kiwifruit seedlings grown on Murashige and Skoog medium and a high-throughput inoculation method using kiwifruit seedlings. Using this inoculation method, we screened 3000 Psa3 transposon insertion mutants and identified virulence related genes. We isolated 105 reduced virulence mutants and identified several potential virulence factors including the type III secretion system (T3SS), membrane transporters, transcriptional factors, motility, and amino acid metabolism. These virulence factors include not only the T3SS and type III secretion effectors, but also many unknown factors. These results suggest that this inoculation method is effective for analyzing the interaction between kiwifruit and Psa3, as well as for elucidating Psa3 infection mechanisms.

Profiling N-acyl-Homoserine Lactones produced by various Chromobacterium spp. L. WANG, A. Harrison, C. Vetlri, S. Soby, Midwestern University, Glendale, AZ, USA

Quorum sensing (QS) is used by bacteria to synchronize group behavior, including the regulation of virulence genes, by responding to the production of small molecules known collectively as autoinducers (AIs). In Gram negative bacteria N-acyl-homoserine lactones (AHLs) serve as AIs. We identified the AHLs produced by Chromobacterium spp. by liquid chromatography-mass spectrometry (LC/MS) of supernatant extracts. N-Decanoyl-homoserine lactone (C10-HSL) is produced by $C$. vaccinii and the proposed new species C. altocapite; N-Octanoyl-homoserine lactone (C8-HSL) is produced by C. aquaticum; and N-Hexanoyl-homoserine lactone (C6-HSL) is produced by proposed new species $C$. alloccove. In contrast to what has previously been published, there was no evidence of $\mathrm{N}$ Dodecanoyl-homoserine lactone (C12-HSL) or N-Octanoyl-homoserine lactone (C8-HSL) in C. haemolyticum extracts, or N-Octanoylhomoerine lactone(C8-HSL) or N-Butyryl-homoserine lactone (C4-HSL) in C. piscinae extracts. QS is a global gene regulator that regulates cell behavior, which may help plants by reducing pathogen, nematode, and insect populations.

Tobacco mosaic virus mediated suppression of age related resistance

M. KAPPAGANTU (1), T. Collum (1), J. N. Culver (1,2), (1) Institute of Bioscience and Biotechnology Research, University of Maryland, College Park, MD, USA; (2) Department of Plant Science and Landscape Architecture, University of Maryland, College Park, MD, USA

TMV is a major virus pathogen on several horticulturally important crops worldwide. Our previous studies have shown that in Arabidopsis, the helicase domain of TMV interacts with an Aux/IAA protein, IAA26, a transcriptional regulatory factor involved in auxin mediated response. IAA26 is expressed in the phloem companion cells of older leaves and is functionally disrupted by its interaction with TMV. This interaction is essential for the phloem loading of the TMV in an age dependent manner, suggesting association with age related resistance (ARR). To understand the potential role of IAA26 in the ARR, we used IAA26 overaccumulating and IAA26 knockout lines in a comparative analysis with wild type Arabidopsis. Our results show that mutation in IAA26 leads to alteration in the timing for the onset of flowering, a generally used physiological marker for ARR. TMV infection of mutant Arabidopsis plants before and after flowering showed significantly higher levels of TMV in IAA26 knockout compared to overaccumulating line, but only after flowering. To understand if stabilization of IAA26 primes the plants with defense responses towards viral infections, transcriptome analysis of healthy IAA26 over-accumulating and knockout lines were compared with wild type Arabidopsis. Results showed that IAA26 acts as a trigger for several defense pathways even in the absence of the pathogen. Combined 
these findings link IAA26 to the induction of ARR and suggest that TMV's directed disruption of this factor is involved in suppressing this resistance phenomenon.

\author{
Oomycete mitochondrial genomics - development of a resource for phylogenetics and marker development \\ F. N. MARTIN, USDA ARS, Salinas, CA, USA
}

Mitochondrial genomes for a wide range of oomycetes were assembled to develop a database useful for identifying loci for phylogenetic studies, rapid identification of taxa, systematic development of diagnostic capabilities and cytoplasmic markers for population studies. Genomes representing over 500 isolates of 160 taxa have been completed. Analyses of the 35 genes and two ribosomal RNA sequences have been useful for conducting oomycete phylogenetic studies. Comparative analyses of gene order identified loci that are useful for development of highly specific genus and species-specific diagnostic markers for Phytophthora and Pythium, with preliminary data identifying targets for Phytopythium and Aphanomyces detection. Multiplexed assays that are both genus and species specific have been developed for some genera. Gene order differences have been useful for downy mildew diagnostics but targeting unique putative open reading frames has been successful as well. Analyses of gene order in oomycetes, plants and Eumycotan fungi identified a highly conserved unique gene order of tRNAs flanking the rps 10 gene in Oomycetes. Conserved primers with minimal background amplification were developed; sequence analysis of the amplicon confirmed utility of the locus as a barcode for species identification. Comparative mitochondrial genomics has also been useful for designing highly conserved primers for amplification of specific regions of the genome exhibiting intraspecific polymorphisms, thereby facilitating development of mitochondrial haplotype markers for population studies.

Tattle-TALEs: Tools for testing type three targeting

S. P. COHEN (1), J. Butchacas (1), V. Roman-Reyna (1,2), J. Long (1), C. Pesce (3,4,5), T. Vancheva (3,5), F. Bini (6), I. Otto (6), B. Szurek (3), J. E. Leach (7), J. Kumlehn (6), C. G. Bragard (5), R. Koebnik (3), J. M. Jacobs (1,2), (1) Department of Plant Pathology, The Ohio State University, Columbus, OH, USA; (2) Infectious Diseases Institute, The Ohio State University, Columbus, OH, USA; (3) IRD, CIRAD, Université Montpellier, IPME, 34000 Montpellier, FRANCE; (4) HM.CLAUSE (Limagrain group), Davis, CA, USA; (5) Earth \& Life Institute, UCLouvain, Louvain-la-Neuve, BELGIUM; (6) Leibniz Institute of Plant Genetics \& Crop Plant Research (IPK), Gatersleben, GERMANY; (7) Colorado State University, Fort Collins, CO, USA

During infection, bacterial plant pathogens secrete effector proteins to promote virulence and overcome host immunity. Some effectors disrupt plant immunity upon direct injection into host cells through a Type III secretion system. The spatiotemporal dynamics of Type III effector activity during early infection are not well characterized. Here, we developed a method of visualizing in situ Type IIIeffector activity of Xanthomonas translucens during infection of barley. Transgenic barley plants were developed with an inducible gene encoding green fluorescent protein (GFP). The plant promoter is activated by $X$. translucens injecting an artificial transcriptional activator-like effector, designated as dTALE-GFP. $X$. translucens (dTALE-GFP) also expresses a gene encoding red fluorescent protein that can be simultaneously detected and differentiated from the plant GFP with confocal microscopy. We detected TALE-induced GFP expression in plant cells as early as six hours post-inoculation. With this system, we demonstrate that stomatal guard cells and mesophyll cells are targeted by Type III effectors as disease progresses, and that epidermal cells are likely not targeted by bacteria from the leaf surface. This system provides a valuable tool for research into the dynamics of plant-microbe interactions during infection.

Analyzing the efficacy of QM practices on phytosanitary risk reduction along the seed production pathway D. POSNY (1), W. Luo (1), J. Laborde (1), S. Ippolito (1), A. B. Kriss (2), R. L. Dunkle (3), G. P. Munkvold (4), T. R. Gottwald (1), (1) USDA ARS, Ft Pierce, FL, USA; (2) Syngenta, Greensboro, NC, USA; (3) American Seed Trade Association, Alexandria, VA, USA; (4) Iowa State University, Ames, IA, USA

Seed companies routinely establish their own quality management $(\mathrm{QM})$ programs to monitor seed production and reduce pathogen contamination risks. However, different practices may be adopted individually or as a comprehensive QM system, without quantification of the supposed benefits. This inherent variability across the industry, coupled with the rapid growth of international seed trade, provides numerous pathways for widespread pathogen and pest introductions and spread via transported and traded seed. This can lead to an unpredictable trade environment if phytosanitary issues are not quickly identified, as consumers of seed products expect healthy, disease-free seeds for plant propagation. A 2013 outbreak of Cgmmv in CA, initiated by contaminated imported seed, highlights the substantial vulnerability of the industry to high risk introductions via seed. Therefore, we developed a probabilistic riskbased model to quantitatively assess the effects of management practices on phytosanitary risk for bacterial, viral and fungal pathosystems (Case studies: Cmm of tomato, Cgmmv of cucurbits, Black leg of Brassica, Gummy stem blight of cucurbits). The Bayesian modeling approach, formulated and calibrated using expert opinion and experiences, provides methods to conduct pathway analyses and a framework on which to develop/justify international phytosanitary standards. Decision makers can use the interactive, front-end interface to investigate how critical control points and practices affect risk, through 'what-if' scenarios (e.g. contingency planning) and QM comparative analyses.

Transcriptomic analysis of transgenic Arabidopsis expressing the rolB-genes from Agrobacterium rhizogenes C. CHAGAS DE FREITAS, C. G. Taylor, The Ohio State University, Wooster, OH, USA

Agrobacterium rhizogenes is the causative agent of crazy-root (hairy-root) disease in a wide variety of plants. The disease manifests as the over-proliferation of roots at the expense of shoot and fruit production. The root-inducing plasmid of $A$. rhizogenes harbors a region that is transferred from the bacteria to the plant called the transfer DNA (T-DNA). Among the genes encoded on the T-DNA, rol (root 
oncogenic loci) genes have been shown to be critical for the formation of hairy roots. Little is known about the mechanism(s) of hairy root formation. In this study, we have created over-expression constructs for rolA, rolB and rolC from A. rhizogenes strain K599 and generated transgenic Arabidopsis lines for each individual rol gene. We selected three expressing homozygous Arabidopsis lines for each rol gene and conducted transcriptomic analysis of isolated root tissue. RNAseq was conducted on extracted RNA samples and the subsequent sequence was processed and bioinformatically analyzed to identify genes that were differentially expressed. Arabidopsis lines expressing rolB exhibited significant changes in gene expression. Of the 609 genes differentially expressed in roots of rolB plants, 251 were up-regulated and 358 were down-regulated. Up-regulated genes were found to be involved with primary metabolism, root system development and auxin transport while down-regulated genes were associated with defense responses and the abscisic acid pathway. Further experiments examining the role these genes have in hairy root function will be presented.

Lipopolysaccharide primes Vitis vinifera for enhanced defense against Xylella fastidiosa and suppresses Pierce's disease C. A. CASTRO (1), M. Massonnet (2), D. Cantu (2), C. Roper (1), (1) University of California, Riverside, Riverside, CA, USA; (2) University of California, Davis, Davis, CA, USA

Plants have developed complex mechanisms to defend themselves from constant biotic and abiotic challenges presented by the environment. One of these mechanisms, called priming, is a strategy that exploits plant 'memory' to efficiently counteract pathogens and abiotic stress. Microorganisms have signature molecules called microbe-associated molecular patterns (MAMPs) that can act as stimuli to induce the primed state. This 'memory' allows plants to quickly recognize pathogens and activate robust immune responses that result in disease resistance or tolerance. Xylella fastidiosa $(X f)$ is a Gram-negative, xylem-limited bacterium that causes Pierce's disease of grapevine. Lipopolysaccharide (LPS) covers most of the cell surface in Gram-negative bacteria and is a well-described MAMP that elicits host defense responses. The goal of this project was to characterize $X f$ LPS treatment before $X f$ inoculation in vines and to explore early host transcriptional responses underlying the LPS-mediated priming phenomenon against $X f$. LPS significantly reduces symptom development and bacterial titer in vines. Analysis of enriched functional categories indicates that the functional categories "MAPK cascade," "WRKY family transcription factor," "R proteins from plant-pathogen interaction," "Callose biosynthesis," and other categories related to secondary metabolism in local and systemic tissue are significantly up-regulated postpathogen challenge in plants that received LPS. These results indicate that LPS reprograms the vine's transcriptome and primes the vine for enhanced defense against $X f$.

Integrating cover crops with anaerobic soil disinfestation to manage Fusarium oxysporum in strawberry production U. SHRESTHA (1), B. H. Ownley (2), D. Butler (1), (1) University of Tennessee, Knoxville, TN, USA; (2) Department of Entomology and Plant Pathology, University of Tennessee, Knoxville, TN, USA

Field trials of crop rotation integrated with anaerobic soil disinfestation (ASD) for strawberry production were conducted to assess survival of Fusarium oxysporum (Fo) inoculum, anaerobic conditions, and volatile fatty acid (VFA) concentration in treated soils. Twoyear crop rotations were a) pumpkin/winter wheat cover crop (PWC)-summer fallow/strawberry, b) sorghum sudangrass cover crop (SSC)/strawberry-SSC/strawberry, and c) continuous strawberry (Cs, summer fallow/strawberry) applied as whole plot treatments. Soil treatments using ASD (dry molasses mixed with wheat bran as carbon-sources) or non-ASD (control) were applied as a split-plot in each rotation prior to strawberry planting. There was no interaction between rotation and ASD on $F o$, but ASD reduced survival of $F o$ by $2 \log _{10}(\mathrm{CFU}+1)$ regardless of rotation. Iron paint removal from IRIS tubes indicated more reduced (i.e., anaerobic) conditions in ASD in the rotation with SSC (42\%) compared to other ASD (16\%) and non-ASD $(<4 \%)$ treatments in all rotations. A significant interaction between rotation and ASD was observed for soil VFA concentration. The ASD effect on total VFA production increased with the SSC (11 and $20 \mathrm{mmol} / \mathrm{kg}$ soil) and PWC (4 and $14 \mathrm{mmol} / \mathrm{kg}$ soil) compared to ASD with Cs (10 and $2 \mathrm{mmol} / \mathrm{kg}$ soil) at 3 and 7 days respectively. SSC in strawberry crop rotations integrated with ASD is a promising tactic to suppress $F o$ as evident from higher anaerobic conditions and production of fungitoxic VFAs during the incubation period, although more work is needed to evaluate $F O$ disease incidence in these systems.

\section{Exploring suppression of SCN (Heterodera glycines) populations by wheat in double-cropping soybeans: A metagenomics approach \\ L. F. ROCHA, M. Filgueira Pimentel, A. Y. Srour, J. P. Bond, A. M. Fakhoury, Southern Illinois University, Carbondale, IL, USA}

Double cropping (DC) is defined as producing more than one crop on the same parcel of land in a single growing season. DC is reported to have many benefits when incorporated in cropping systems, including improving soil health. In some DC systems, soybeans are planted following winter wheat. The soybean cyst nematode (SCN) (Heterodera glycines) is a major soybean pathogen, and several reports indicate suppressive effects of wheat on SCN populations. A field trial was conducted in 2017 to assess the effect of wheat on SCN populations in DC soybeans. Nine fields with 3 levels of initial SCN populations (low, moderate, and high) were selected in Illinois. In each location, wheat (WT) was planted in strips alternating with strips maintained in fallow (FL) over winter. Soybeans followed all strips after wheat harvest. SCN counts were assessed at four timepoints: pre-wheat planting, post-wheat/pre-soybeans, midsoybeans (R1) and post-soybeans. Wheat strips had reduced SCN counts compared to fallow strips at R1 and after soybean harvest. Field locations with noted SCN suppression were selected for a metagenomics study. Locations selected were fields 3 (low), 6 (high) and 8 (high), all located in Washington Co., Illinois. A total of 10 subplots were selected (5 WT and 5 FL) in each field location. A total of 90 soil samples were selected: 3 fields $\times 2$ treatments $\times 3$ timepoints $\times 5$ replications. DNA was extracted from samples using a commercial kit and sequencing was performed using an Illumina MiSeq platform. Bacterial and fungal communities were explored using a Mothur pipeline. 
The regulation of sclerotium initiation and virulence in Sclerotinia sclerotiorum by cyclic AMP-protein kinase A signaling pathway

P. L. YU, J. A. Rollins, University of Florida, Gainesville, FL, USA

Fungi have developed diverse mechanisms to facilitate dispersal and survival. Sclerotinia sclerotiorum forms sclerotium, a mass of compacted hyphae, which serves as a primary source of inoculum and long-term survival structure. Previous research has demonstrated that sclerotia production is negatively regulated by the cyclic AMP (cAMP) in S. sclerotiorum. To demonstrate the role of the cAMPdependent pathway in the regulation of fungal development and virulence, two genes, encoding a catalytic subunit ( $p k a 2)$ and a regulatory subunit ( $p k a R$ ) of protein kinase A (PKA), were disrupted using CRISPR-Cas9 and functionally characterized. Vegetative growth and virulence were greatly attenuated in $\Delta p k a 2$ and $\Delta p k a R$ mutants. The $\Delta p k a 2$ mutants formed compact colonies and produced sclerotia in concentric rings similar to adenylate cyclase-coding gene-deletion mutants, which produce little cAMP. Carpogenic germination of the $\Delta p k a 2$ mutant was delayed compared with WT. Unlike $\Delta p k a 2$ mutants, the ability to form sclerotia was completely abolished in $\triangle p k a R$ mutants. In addition, the $\Delta p k a R$ mutants produced appressoria without melanization. Both $\Delta p k a 2$ and $\Delta p k a R$ mutants developed symptoms much slower than WT on multiple hosts. The results reveal that the cAMP-PKA signaling pathway regulates the sclerotial development and virulence in S. sclerotiorum. This study provides new targets for disease management based on a reduction in primary inoculum of this sclerotium-producing fungus.

Rapid detection of QoI fungicide resistance in Cercospora sojina and characterization of populations in Nebraska A. MANE (1), T. A. Jackson-Ziems (2), C. A. Bradley PhD (3), S. E. Everhart (2), (1) University of Nebraska-Lincoln, Lincoln, NE, USA; (2) University of Nebraska, Lincoln, NE, USA; (3) University of Kentucky, Princeton, KY, USA

In 2019, QoI fungicide resistance was detected in 10 Cercospora sojina isolates from 10 counties in Nebraska. This fungus causes frogeye leaf spot, a foliar disease of soybean, and up to 35 percent yield loss. Continuous planting, no-till, and use of single-mode of action fungicides contribute to disease severity and development of fungicide resistance. Presently, we do not have an in-field, rapid detection method for QoI resistant C. sojina. It is also unknown whether resistance has emerged due to fungicide over-use or seedborne transmission. In the present study, we are developing a highly sensitive isothermal DNA amplification method based on ligation-rolling circle amplification. This method will allow rapid detection from leaf samples in 2-3 hours, without culturing. A single reaction will be able to detect three possible mutations (G143A, F129L, and G137R) in the cytochrome b gene that confer QoI resistance. This assay will be used for rapid detection of resistance and timely recommendations to growers. Complementary knowledge of the pathogen population structure will be used to determine if fungicide resistance in the pathogen originates from contaminated seed or if in-field applications and survival of the pathogen in residue. A survey of the pathogen from Nebraska will be conducted and genotyping will be used to characterize the pathogen population. Multiple leaf samples will be collected from selected fields known or suspected to have QoI resistance and the pathogen will be characterized to establish relationships within and between fields in Nebraska.

\section{Benefit of biological products in integrated resistance management programs alongside 'at risk' conventional fungicides to control Botrytis cinerea \\ S. Hovinga (1), J. DERPMANN (2), (1) Bayer Crop Science, West Sacramento, CA, USA; (2) Bayer AG - Crop Science Division, Monheim, GERMANY}

Biological products have multiple modes of action that are different to conventional chemistries, and thus have the potential to control pathogenic strains carrying different mutations leading to reduced sensitivity or resistance against such chemistries. New research sought to better define how biological products in integrated programs can lower frequencies of resistant strains in populations and therefore increases the understanding of effective product use. Both Bacillus amyloliquefaciens strain QST 713 (synonym Bacillus subtilis strain QST 713) and its SERENADE ${ }^{\circledR}$ commercial product, were investigated for their effects on $s d h$ - or erg 27 mutant strains of Botrytis cinerea, which are selected by fluopyram or fenhexamid, respectively. In vitro and in planta experiments compared the effect of conventional and biological products on the evolution of mixed populations and differences were seen in the selection for mutants and the stabilization of wild-type isolates, respectively. Additionally, fitness penalties caused by pleotropic effects associated with mutations were observed, which gives insight into hypotheses for effective application timings. Lab, greenhouse, and field trial data will be discussed in the context of real-world use and when managing resistance in Botrytis. These results suggest that using microbials, such as the SERENADE product, in an integrated program for B. cinerea control has the potential to increase the lifespan of 'at risk' fungicides.

Cell biology of Candidatus Liberibacter asiaticus in its plant and insect-vector hosts D. Achor (1), M. Ghanim (2), S. Welker (3), S. K. Ben-Mahmoud (4), A. LEVY (5), (1) University of Florida, Lake Alfred, FL, USA; (2) Volcani Center, Bet Dagan, ISRAEL; (3) University of Florida Plant Pathology Department, Auburndale, FL, USA; (4) University of California, Davis, Davis, CA, USA; (5) University of Florida, Citrus Reseach and Education Center, Lake Alfred, FL, USA

Citrus greening is caused by the phloem-restricted bacterium Candidatus Liberibacter asiaticus (CLas) and is transmitted by the Asian citrus psyllid (Diaphorina citri). CLas is an intracellular bacterium, but very little is known about its interactions with the plant host and insect vector at the cellular level. We used transmission electron and confocal microscopy to study and compare the accumulation and movement of CLas inside its hosts. We demonstrated that CLas enters the D. citri gut cells by endocytosis and that it forms a large replicative vacuole inside the cells. The formation of these vacuoles is accompanied by dramatic re-organization of the psyllid endoplasmic reticulum (ER) that associates with these intracellular bodies. In citrus, CLas propagates in the cytoplasm of the phloem sieve elements and moves through the phloem pores. In the flush, pores were plugged by callose that reduced their open space. In the roots, pores were surrounded by dark material, with little accumulation of callose. The expression of CALLOSE SYNTHASE7 and 
PHLOEM PROTEIN2 genes was upregulated in the shoots, but downregulated in roots. In seed coats, no phloem occlusion was observed, and CLas accumulated to high levels. CLas movement seems to depend on the bacterium's ability to change its form and to bind to the host cell membrane. Our results show that important communication is taking place between CLas and its vector and plant hosts at the cellular level, which effects both the bacteria and their hosts.

Understanding the impact of Gibberella ear rot and stalk rot on deoxynivalenol accumulation in silage corn H. REED, J. Goeser, D. L. Smith, University of Wisconsin-Madison, Madison, WI, USA

Corn silage, made from Zea mays, is a high energy feed that provides much of the caloric and nutritional demands of dairy cows. Gibberella zeae (anamorph Fusarium graminearum) is an ascomycete fungus that causes Gibberella ear and stalk rot. G. zeae produces deoxynivalenol (DON), a secondary metabolite toxic to humans and animals. An understanding of the distribution of DON and $G$. zeae throughout the corn plant is important for determining the prevalence of the toxin in silage. A partitioned sample experiment was conducted in research plots located in Arlington, WI in 2018 and 2019 that included two silage hybrids, P0956AMX ${ }^{\circledR}$ and F2F627 ${ }^{\circledR}$, and three fungicide treatments, Headline AMP ${ }^{\circledR}$, Proline ${ }^{\circledR}$, and a non-treated control. At harvest, stalk and ear parts were physically separated and dried for analysis. DON concentration (ppm) was determined using ELISA test kits and G. zeae DNA (pg/ng) within plant material was determined using qPCR. Both DON and fungal DNA were detected in all samples, showing their ability to accumulate throughout the plant. In 2018, DON contamination was high, up to $30 \mathrm{ppm}$, but varied by plant part and hybrid. In 2019 , DON was much lower $(<5 \mathrm{ppm})$, but consistently higher in stalk samples than ear samples. Across both years we found that DON and fungal accumulation were highly correlated within the separated stalk $\left(\mathrm{R}^{2}=0.91\right)$ and ear portions $\left(\mathrm{R}^{2}=0.89\right)$. Since $G$. zeae accumulates throughout the plant, disease management strategies should focus on fungicide applications and selecting stalk rot-resistant hybrids to keep whole plant DON levels low.

Genomic acquisitions in emerging populations of Xanthomonas

A. L. PEREZ-QuinTERO (1), R. Steele (1), M. C. Ortiz-Castro (1), J. M. Lang (1), J. M. Jacobs (2), K. Broders (3), J. E. Leach (1), (1) Colorado State University, Fort Collins, CO, USA; (2) Department of Plant Pathology, The Ohio State University, Columbus, OH, USA; (3) Smithsonian Tropical Research Institute, PANAMA

New plant pathogen epidemics can occur due to sudden changes in environmental conditions or to exposures to new susceptible hosts. However, these spreads are often also associated to genetic changes in the pathogen: mutation or acquisition of genetic features that confer a fitness advantage. To have a streamlined way to identify these features, we created a pipeline to perform comparative genomics to identify genetic factors associated to epidemics. We applied this pipeline to understand genetic mechanisms behind the recent fast spread of Xanthomonas vasicola pv. vasculorum $(X v v)$, causing bacterial leaf streak on corn, in South America and in the U.S. We sequenced 41 genomes of Xvv and the related sorghum-infecting $X$. vasicola pv. holcicola $(X v h)$, and performed comparative analyses against all available $X$. vasicola genomes. Time-measured phylogenetic analyses showed that $X v v$ strains from the U.S. and Argentina are closely related and arose from two introductions to the Americas. Gene content comparisons identified clusters of genes enriched in corn $X v v$ that showed evidence of horizontal transfer including one cluster corresponding to a prophage found in all $X v v$ strains from the Americas, possibly transferred from $X v h$. The acquisitions identified may contain virulence determinants associated with the spread of $X v v$ in the Americas which will be the subject of future work. We are using the pipeline to identify candidate virulence factors associated to other emergence events, including populations of $X$. oryzae pv. oryzae shown to breakdown resistance in rice.

New insights into the wheat seed microbiome: An in silico approach

L. E. BECKER, M. A. Cubeta, North Carolina State University, Raleigh, NC, USA

Wheat (Triticum aestivum L.) is grown worldwide and accounts for $19 \%$ of total available calories globally. During wheat seed development, physiological changes are likely to shape seed microbial communities, but little is known about the microbiome of developing wheat seeds. The recent release of the wheat genome allows for comprehensive examination of seed microbial communities and facilitates targeted isolation of seed associated bacteria and fungi. In this study, an in silico approach was used to examine microbial community diversity of developing wheat seeds. Our dataset included publicly available RNAseq reads from internal wheat seed compartments sampled at 10, 20, and 30 days post anthesis. Quality filtered reads were mapped to the reference wheat genome to remove plant host reads and microbial reads were assembled in metaSPAdes. Kaiju webserver taxonomically classified reads into 69, 126, and 81 bacterial species and 99, 114, and 104 fungal species associated with the wheat seed microbiome for 10, 20, and 30 days post anthesis, respectively. Kaiju identified 26 and 37 bacterial and fungal species across all three seed developmental stages sampled. The use of in silico analysis for targeted identification and isolation of bacteria and fungi across wheat seed generations will be presented.

The dilemma of coordination for huanglongbing management in California S. GARCIA FIGUERA (1), N. McRoberts (2), (1) University of California, Davis, Davis, CA, USA; (2) University of California, Davis, CA, USA

Plant disease management often requires the coordination of control measures across a region because properties that don't participate can be sources of inoculum for the rest. As individuals cannot be excluded from the benefits of the control efforts of others, they might be tempted to free-ride on others' efforts while avoiding the costs of control. This dilemma is an obstacle for huanglongbing (HLB) management in California. HLB is an invasive disease of citrus associated with the bacterium "Candidatus Liberibacter asiaticus", which is transmitted by the Asian Citrus Psyllid (ACP), Diaphorina citri. It was first detected in 2012 and to date more than 1,800 
HLB-positive trees have been removed from residences in Southern California. To suppress ACP populations and limit HLB spread, citrus growers are being asked to coordinate insecticide treatments, but participation in this area-wide management (AWM) program has been uneven. We studied individual factors influencing decisions to participate, institutional arrangements to promote coordination and the benefits of coordination. Surveys of citrus growers showed that while they believe in the efficacy of AWM, trust that their neighbors will participate is highly variable. Areas with more mandatory institutional arrangements are achieving higher coordination, but this depends on the size of the group, and simulations with an agent-based model have shown that very high participation levels might be required to effectively prevent HLB spread, highlighting the relevance of addressing this dilemma for HLB and other plant diseases.

Promoters: Key elements for broad-spectrum disease resistance in rice

A. M. BOSSA-CASTRO (1,2), B. W. Tonnessen (2,3), A. L. Perez-Quintero (2), V. M. Verdier (4), J. E. Leach (2), (1) Universidad de los Andes, Bogota, COLOMBIA; (2) Colorado State University, Fort Collins, CO, USA; (3) New Mexico State University, Las Cruces, NM, USA; (4) IRD, Cirad, Univ Montpellier, IPME, Montpellier, FRANCE

Xanthomonas oryzae pv. oryzicola (Xoc) and Xanthomonas oryzae pv. oryzae (Xoo) are responsible for major losses in rice production in Asia and Africa. Controlling these pathogens is particularly important in Sub-Saharan Africa, where no sources of broad-spectrum resistance (BSR) are available in currently deployed varieties. We hypothesize that potential convergence points of PAMP-triggered immunity (PTI) and effector-triggered immunity (ETI) can be found in promoters of genes within resistance quantitative trait loci (QTL). On one hand, promoters contain groups of cis-acting elements that determine gene transcription, such as cis-regulatory modules (CRMs). CRMs are structural organizations of motifs involved in disease resistance, which can be used to predict BSR in rice. On the other hand, rice promoters can be hijacked by specialized bacterial effectors, transcription activator-like (TAL) effectors, to favor pathogen fitness. Both Xoc and Xoo are equipped with these type of bacterial proteins. Previously, we identified 14 disease resistance QTL effective against multiple $X$. oryzae in an indica rice MAGIC population. In this study, loci within those QTL were evaluated for promoters with multiple CRMs, promoters with putative targets for TAL effectors from multiple $X$. oryzae strains, and SNP markers associated with resistance to $X$. oryzae strains. The identification of genes implicated in both PTI and ETI responses is a powerful resource to understand plant immunity and to aid the development of improved elite varieties with durable resistance to $X$. oryzae.

Wheat genotypes recruit suppressive rhizosphere microbiomes against Rhizoctonia solani AG8

C. J. D. ERMITA (1), R. W. Lewis (2), T. S. Sullivan (2), T. C. Paulitz (3), S. H. Hulbert (1), (1) Department of Plant Pathology, Washington State University, Pullman, WA, USA; (2) Department of Crop and Soil Sciences, Washington State University, Pullman, WA, USA; (3) USDA ARS, Wheat Health, Genetics and Quality Research Unit, Pullman, WA, USA

The root rot disease caused by Rhizoctonia solani AG8 in wheat reduces yield up to $15 \%$ in the dryland areas of the Pacific Northwest, where no-till cropping or direct-seeding is practiced to reduce soil erosion and operating costs. Despite efforts to identify resistance to this disease, host resistance is rare in wheat germplasm. Thus, discovering a disease suppressive microbiome in wheat is desirable, as disease suppressive soils have been documented after 8-10 years of wheat monoculture. Studies have demonstrated that winter wheat genotypes recruit specific Pseudomonas populations exhibiting varying levels of suppression against $R$. solani AG8. To explore more diverse genotypes that recruit suppressive microbiomes, we screened seven winter and 31 spring wheat genotypes cultivated at Lind, WA for two-years in the field. Loosely-bound soils previously cultivated with different wheat genotypes were inoculated with $R$. solani AG8 to identify wheat genotypes recruiting suppressive microbiomes. Rhizosphere soil DNA was then sequenced (16S V4 rDNA) to identify bacterial taxa associated with disease suppression. Among the 38 wheat genotypes, three spring wheat genotypes recruited microbiomes exhibiting disease suppression against $R$. solani AG8, and were further validated in growth chamber experiments. Culturebased characterization of the suppressive microbiome is still underway. Identification of wheat genotypes differentially recruiting suppressive microbiomes holds promise in manipulating rhizosphere microbial communities to suppress $R$. solani AG8 in dryland cropping systems.

Tad pili in 'Candidatus Liberibacter' spp. are retractile and play a dynamic role in bacterial motility, survival and colonization L. CAI (1), M. Jain (1), A. Munoz Bodnar (1), M. Sena Velez (2), K. M. M. Jones (2), L. A. Fleites (3), M. Heck (4), D. W. Gabriel (1), (1) University of Florida, Gainesville, FL, USA; (2) Florida State University, Tallahassee, FL, USA; (3) USDA-ARS, Emerging Pests and Pathogens Research Unit, Ithaca, NY, USA; (4) USDA-ARS, Emerging Pest and Pathogen Research Unit, Ithaca, NY, USA

'Ca. Liberibacter' sp. are associated with four serious psyllid-transmitted plant diseases such as citrus greening and potato zebra chip. Nonpathogenic L. crescens (Lcr) is the only cultured member of Liberibacter genus and a useful surrogate for functional genomic studies of the uncultured ' $\mathrm{Ca}$. Liberibacter' sp. All Liberibacters have the complete repertoire of genes annotated for encoding flagella and Tight Adherence (Tad) pili. Presence of both surface appendages in Lcr was confirmed by ultrastructural evidence. Marker interruption mutagenesis of flgK (B488_RS04530) and flgF (B488_RS00930), encoding flagellar hook-associated protein and basalbody rod protein, respectively, abolished swimming behavior. Interruption of cpaF (B488_RS06240), encoding the Tad pilus ATPase motor, reduced twitching motility and severely compromised bacterial growth. Interruption of comEC (B488_RS05330), encoding the inner membrane channel protein for the import of intact extracellular DNA, had no effect on motility or Lcr growth but completely abolished natural transformation competence of Lcr. Since Liberibacter genomes lack any other Type IV pili, these data indicate that Lcr Tad pili are atypically dynamic and retractable. Pilin depolymerization facilitated by the bidirectional CpaF ATPase motor activity is a prerequisite for both twitching and natural competence. Tad pili-mediated uptake of extracellular DNA is demonstrated here to be critical for Lcr survival and by extension, host colonization by ' $\mathrm{Ca}$. Liberibacter' sp., that are deficient in nucleotide biosynthetic pathways. 
Characterizing the xylem environment during Ralstonia Solanacearum infection of resistant hosts C. HAMILTON, C. Allen, University of Wisconsin-Madison, Madison, WI, USA

The soilborne pathogen Ralstonia solanacearum (Rs) causes bacterial wilt disease (BWD) by colonizing plant xylem, triggering wilt and death. Rs reduces crop yields at every level of production from small- to large-scale farming operations, affecting over 200 plant species, including tomato. The best source of resistance in tomato is breeding line Hawaii7996(H7996), but we don't understand the mechanism(s) of this key QTL-mediated trait. Moreover, some $R s$ strains unlike RsGMI1000 already break H7996 resistance including select agent, $R s \mathrm{UW} 551$ which increases our need to understand this valuable form of resistance. Previous reciprocal grafting experiments with roots and scions from susceptible and resistant plants reasserted that BWD-resistant cultivar H7996 limits Rs growth not only in the roots but in the stem too. We found that ex vivo xylem sap from RsGMI1000 infected H7996 plants inhibited rather than supported bacterial growth like sap from susceptible cultivar Bonny Best. We hypothesized that xylem sap from BWD-resistant cultivars restricts $R s$ virulence by chemical inhibition, not carbon starvation. We isolated subsets of compounds found in H7996 xylem sap that contribute to BWD-resistance. To sharpen the focus, we also use $R s$ mutagenesis to remove several $R s$ strains ability to fully efflux common toxins. These findings further our understanding how this devastating plant pathogen succeeds or fails in the harsh resistant host xylem environment.

Genome scale metabolic models of Phytophthora infestans and Phytophthora betacei: A key tool to understand phytopathogens metabolic capabilities

M. J. RODRIGUEZ-CUBILlOS (1), S. Restrepo (2), A. Gonzalez-Barrios (2), D. Botero (2), (1) Universidad de Los Andes, BOGOTA DISTRITO CAPITAL, CO, COLOMBIA; (2) Universidad de Los Andes, Bogotá, COLOMBIA

The genus Phytophthora includes species of pathogens with the ability to infect a wide range of hosts. This is the case of Phytophthora infestans causing late blight in plants of the Solanaceae family, specifically Solanum lycopersicum and Solamun tuberosum. The new disease outbreaks found in Solanum betaceum warned researchers about a new species able of infecting solanaceous crops. Molecular studies and several infection tests allowed Phytophthora betacei to be identified as a new species. Its description generated questions about the differences between $P$. infestans and $P$. betacei, because of the latter specific host preference. One possible explanation for these differences might reside on the metabolisms of both organisms. Metabolic dissimilarities could be linked to metabolic pathways that favor the production of pathogenicity and/or virulence factors in a particular host. In order to explore the complete metabolism of $P$. infestans and $P$. betacei we performed genome scale metabolic reconstructions. We identified the unique and shared metabolism between the two organisms using these reconstructions. By generating metabolic models for each one, we found 1.858 shared reactions and a set of 85 unique reactions for $P$. infestans and 68 unique reactions for $P$. betacei. Finally, we identified the purine and pyrimidine pathways as a possible way to explain the metabolic differences between them and we evaluated the models in order to predict other metabolic pathways related to the phytopathogenic response of each organism.

Excessive callose formation and presence of Candidatus Liberibacter asiaticus linked to phloem transport dysfunction in citrus S. WELKER (1), M. Dutt (2), A. Levy (3), C. Vincent (4), (1) University of Florida Plant Pathology Department, Auburndale, FL, USA; (2) University of Florida, Lake Alfred, FL, USA; (3) University of Florida, Citrus Reseach and Education Center, Lake Alfred, FL, USA; (4) University of Florida, FL, USA

Huanglongbing (HLB) disease devastates citrus production throughout the world. In infected plants, sugars are stored in source leaves as starch granules, while the fruit and roots become deficient in sugar. HLB leads to a massive yield loss due to fruit drop, smaller fruit size and fruit bitterness. These symptoms suggest yield reduction may result from a disruption of the normal sugar transport. To test whether sugar transport is inhibited in HLB-positive plants, we explored a novel use of a ${ }^{14} \mathrm{CO}_{2}$ tracer to measure live phloem translocation in HLB-positive and negative trees. Our results indicated that translocation speeds were slower in infected trees. We hypothesized that this reduction is associated either with the presence of the HLB-causing bacterium (Candidatus Liberibacter asiaticus; Las) or with callose plugging of the phloem pores. To test this, we developed a staining method to quantify the quantity and density of phloem callose. We also estimated the Las population with qPCR. We found that callose quantity and density increased with increasing amounts of Las genetic material, and that rootstock type influences the amount of callose formed. A significant negative correlation was identified between the quantity of callose and both Las genetic material and phloem translocation speed. These findings support the hypothesis that local presence of Las leads to callose deposition in the phloem which disrupts carbon transport. Genetic modification or rootstock selection to mitigate callose plugging in the phloem may improve yield of citrus groves in the presence of HLB.

\section{Identification of host specificity genes in Setosphaeria turcica}

P. SINGH, S. Y. Huang, T. Jamann, S. X. Mideros Mora, University of Illinois, Urbana, IL, USA

The hemibiotrophic pathogen Setosphaeria turcica causes sorghum leaf blight (SLB) and northern corn leaf blight (NCLB). On sorghum, the disease may result in over 50\% grain yield loss, while NCLB is recognized as one of the most important foliar diseases of maize globally. Setosphaeria turcica strains vary in their host specificity, which has been reported due to a single locus for sorghum and a second distinct locus for maize. This facilitates host jumps between maize and sorghum and may render sorghum susceptible to novel strains adapted to maize. Our research seeks to identify $S$. turcica genes responsible for host specificity. A fungal bi-parental mapping population was created by crossing maize and sorghum specific strains. A total of 190 ascospores were isolated from 35 ascocarps. MAT locus typing of 22 randomly selected progeny showed 1:1 segregation among the two mating types, MAT1-1 and MAT1-2 ( $p=$ $0.198)$. Phenotyping of the progeny $(\mathrm{n}=72)$ on maize and sorghum in the greenhouse showed independent inheritance of virulence, as indicated by a 1:1:1:1 segregation for virulence to maize, sorghum, both maize and sorghum, and avirulence to both crops $(p=0.53)$. 
Preliminary results indicate that pathogenicity on sorghum may be attributed to a single locus. An additional 72 progeny will be phenotyped, all progeny will be genotyped, and linkage mapping will be conducted to identify sorghum and maize specificity genes in S. turcica. Results have implications in improving resistance to NCLB and SLB, and for the study of host specificity in the Dothideomycetes.

Virulence of Blumeria graminis f.sp. tritici in South Africa, Turkey, Russia, and Brazil T. KLOPPE (1), C. Cowger (2,3), R. Whetten (3), (1) North Carolina State Univ, Raleigh, NC, USA; (2) Department of Entomology and Plant Pathology, NC State University, Raleigh, NC, USA; (3) USDA-ARS Plant Science Unit, Raleigh, NC, USA

Blumeria graminis f.sp. tritici (BGT), cause of powdery mildew of wheat (Triticum aestivum), is managed with fungicides or host genetic resistance. Due to frequent sexual reproduction, transposable element proliferation and large population sizes, BGT can readily adapt and overcome both methods of control. Many of the powdery mildew resistance (Pm) genes in wheat have been defeated relatively quickly by BGT populations. Effective resistance breeding involves incorporating into quantitatively resistant backgrounds Pm genes that are still effective in the regions for which specific cultivars are bred. BGT populations in South Africa, Turkey, Russia and Brazil were investigated for virulence to Pm genes to help elucidate how virulence is distributed across the globe. A total of 369 isolates were tested against a differential set of 19 wheat lines, each carrying a single Pm gene. Among the Pm genes in commercial use a relatively long time, generally high frequencies of virulence to $P m 6, P m 8$, and $P m 17$ confirmed a widespread defeat of those genes, while $P m 1 b$ remained effective. By contrast, most sub-populations had low virulence frequencies to Pm genes more recently introgressed from wild relatives. Isolates from South Africa had significantly lower virulence complexity (VC, percentage of the 19 Pm genes defeated) at $16 \%$ than isolates from Turkey (45\%), Russia (44\%), and Brazil $(57 \%)(P<0.0001)$. Virulence to Pm1a, Pm2 and $P m 3 a$ varied widely, ranging from low (1-10\%) in South Africa to fixed in Brazil, illustrating the large global differences in efficacy of these older genes.

\section{Cyclopiazonic acid production by S-morphology Aspergillus section Flavi fungi isolated from maize and groundnut in Northern Mozambique}

L. R. L. ARONE (1), J. Augusto (2), K. A. Callicott (3), R. Bandyopadhyay (4), M. Orbach (1), P. J. Cotty (5), (1) University of Arizona, Tucson, AZ, USA; (2) Intl Inst of Tropical Agriculture (IITA), Nampula, MOZAMBIQUE; (3) USDA Agricultural Research Service, Tucson, AZ, USA; (4) IITA, NIGERIA; (5) USDA ARS, Tucson, AZ, USA

Fungal species within Aspergillus section Flavi have the potential to produce several mycotoxins, including aflatoxins which are regulated in food and feed. Another non-regulated mycotoxin produced by some members of section Flavi is cyclopiazonic acid (CPA). CPA acts as a neurotoxin, and co-occurrence of aflatoxins and CPA may have additive negative health effects. Mycotoxin-producing potential varies among lineages within this group of fungi, so the goal of this project was to determine CPA-producing abilities of highly aflatoxigenic S-morphology Aspergillus isolated from maize and groundnut samples collected in Northern Mozambique. Isolates resolved into four distinct phylogenetic lineages based on bayesian analysis of $4.3 \mathrm{~kb}$ of three partial gene sequences (niaD, BenA, $\mathrm{CaM}$ ). Isolates from each lineage collected across several agro-ecologies were examined for ability to produce CPA. Fungi were grown on autoclaved maize ( 7 days, $31 \mathrm{C}$ ), and CPA was extracted with chloroform and quantified directly on TLC with a scanning densitometer. Three of the S-morphology lineages produced CPA, with the most frequently isolated lineage (49\%) producing up to 9 ppm. A lineage comprising $8 \%$ of isolates did not produce detectable levels of CPA. Variation in CPA production among lineages of S morphology Aspergillus in Mozambique has important implications for understanding etiology of symptoms associated with aflatoxicosis outbreaks where fungi with ability to produce both toxins occur.

Silencing suppression of a polerovirus P0 protein associated to Maize lethal necrosis N. M. HOLSTE, H. Garcia-Ruiz, University of Nebraska-Lincoln, Lincoln, NE, USA

Maize lethal necrosis is an intense viral disease spreading across sub-Saharan Africa. Maize is the staple crop grown in sub-Saharan Africa, but most crops infected with maize lethal necrosis will not survive to harvest. This causes immense economic hardship and starvation within the population. Maize lethal necrosis consists of a combination of two viruses, Maize chlorotic mottle virus (MCMV) and a virus from the genus potyvirus. In a recent study, a Maize yellow dwarf virus-RMV (MYDV-RMV)-like polerovirus, was repeatedly detected in plants with maize lethal necrosis. Poleroviruses have a silencing suppressor, P0 protein, and the mechanism of suppression is poorly understood. In order to understand the mechanisms of silencing suppression, P0 was cloned and tagged. Transient analysis showed it is a strong suppressor of transgene silencing. P0 also restored pathogenicity in trans-complementation assays with two suppressor-deficient viruses, Turnip mosaic virus (TuMV), a potyvirus, and Turnip crinkle virus (TCV), a carmovirus, elucidating that $\mathrm{P} 0$ is a suppressor of antiviral silencing. $\mathrm{P} 0$ lead to the depletion of secondary small-interfering RNAs (siRNAs) possibly due to the degradation of Argonautes (AGO). P0 was co-expressed with tagged AGO 1, 2, 4, 5, 7, and 10 and caused a drop in all AGOs except AGO 4. Dual suppressor assays show that P0 affects the biogenesis of secondary virus-derived siRNAs. Our results provide novel insights on the mechanism of siRNA silencing suppression by polerovirus P0.

Comparative population structure and chemotype diversity of Fusarium graminearum in wheat fields in Canadian prairies A. Oghenekaro (1), S. Kannangara (1), X. Wang (2), A. Oviedo-Ludena (3), H. R. Kutcher (4), D. G. FERNANDO (5), (1) University of Manitoba, Winnipeg, CANADA; (2) AAFC, Morden, CANADA; (3) University of Saskatchewan, Saskatoon, CANADA; (4) Univ of Saskatchewan, Saskatoon, SK, CANADA; (5) University of Manitoba, Winnipeg, MB, CANADA 
Fusarium head blight (FHB) in small grain cereals is a serious problem in Canada. The disease is caused by a plethora of Fusarium species of which Fusarium graminearum sensu stricto is the major species causing FHB in Canada. The fungus reduces yield and contaminates grains with several trichothecene mycotoxins with significant economic losses to the Canadian agriculture sector. In this study, 508 F. graminearum isolates were collected from wheat fields in 2018 and 2019 from Alberta, Manitoba and Saskatchewan. The isolates were investigated for population structure and trichothecene chemotype diversity using 10 variable number tandem repeat (VNTR) markers. The population genetic analysis of 251 F. graminearum isolates collected in 2018 in Manitoba and Saskatchewan showed low genetic differentiation between the provinces $\left(F_{S T}>0.0208\right.$ : $\left.\mathrm{P}<0.001\right)$ as there was significant gene flow between the populations. The genetic structure of the isolates grouped according to chemotypes (3ADON and 15ADON) showed a significant level of genetic differentiation $\left(F_{S T}>0.1073 ; \mathrm{P}<0.001\right)$ suggesting distinct chemotype populations. Of the 251 isolates, $87 \%$ had the $3 \mathrm{ADON}$ trichothecene chemotype and $13 \%$ were $15 \mathrm{ADON}$. There was significant gene flow among populations from the crop districts within each province. Knowledge of the genetic structure of the pathogen will assist in the development of marker-assisted selection of resistant wheat cultivars. The Alberta isolates are analyzed at the moment and will be presented along with Manitoba, Saskatchewan with a compare and contrast.

Quantifying stability of maize hybrid reaction to Gibberella ear rot and deoxynivalenol accumulation in grain F. DALla LANA (1), R. Minyo (2), P. Thomison (3), L. V. Madden (4), P. A. Paul (4), (1) The Ohio State University, Wooster, OH, USA; (2) OARDC/OSU, Wooster, OH, USA; (3) Department of Horticulture and Crop Science, Ohio State University, Columbus, OH, USA; (4) Department of Plant Pathology, The Ohio State University, Wooster, OH, USA

Multilocation trials were conducted to quantify the stability (lack of G x E interaction) of 15 maize hybrids to Gibberella ear rot (GER; Fusarium graminearum) and deoxynivalenol contamination of grain (DON) across 30 Ohio environments (3 years $\times 10$ locations). In each environment, one plot of each hybrid was planted and 10 ears per plot were inoculated via the silk channel with a spore suspension of F. graminearum. GER severity (mean proportion of ear area diseased) and DON contamination of grain (ppm) were quantified. Several statistical approaches were used to quantify hybrid stability, including nonparametric methods, a clustered residual-based method for additivity (Malik's test), and linear mixed models (LMMs) with different variance-covariance structures. Although all nonparametric tests detected variations among within-environment rankings, no evidence of instability was detected, with Piepho's $U$ statistics of 13.2 and 16.3 for GER and DON, respectively $(P>0.2)$. Malik's test also indicated no evidence of G x E interaction (or non-additivity) for either variable $(P>0.4)$. Based on information criteria and likelihood ratio tests, LMMs with more complex variance-covariance structures fitted better than the model with a simpler variance-covariance structure, indicating that individual hybrids were not stable across environments. Overall, hybrids were stable based on nonparametric and residual-clustering methods, but the LMMs identified a few hybrids that were sensitive to environment.

\author{
Three-year soybean-wheat-corn rotation benefits on soybean production, soil health and soil bacterial community are site and \\ year dependent \\ D. Huo (1), L. Lindsey (2), M. S. BENITEZ PONCE (1), (1) The Ohio State University, Wooster, OH, USA; (2) The Ohio State \\ University, Columbus, OH, USA
}

Greater crop rotational diversity can improve crop productivity, soil health and soil microbial diversity. This research hypothesized that 3-year (3Y) rotation of soybean-wheat-corn would have higher soybean productivity, better soil health, and more diverse soil bacterial communities compared to 2-year (2Y) soybean-corn rotation. A rotation experiment was established in 2013 at two research sites in Ohio. Soybean seedling establishment and biomass, crop yield, and soil were collected in 2018 and 2019. Higher seedling stand, seedling biomass, and soybean yield were observed in the $3 \mathrm{Y}$ rotation, but results were not consistent across all sites and years. Soil organic matter was higher in the $3 \mathrm{Y}$ rotation at three site-years. Similarly, higher soil carbon, nitrogen and active carbon was detected in the $3 \mathrm{Y}$ rotation at one site-year. The diversity of soil bacteria, sampled at soybean seedling stage, did not differ between the $2 \mathrm{Y}$ and $3 \mathrm{Y}$ rotation. Six bacterial sequence variants were common in all treatments. However, compared to the $2 \mathrm{Y}$ rotation, the $3 \mathrm{Y}$ rotation had $22 \%$ and $14 \%$ higher relative abundance of the genus Pseudomonas and $89.97 \%$ and $93.75 \%$ lower relative abundance of the genus Ralstonia at each site, respectively. Most rotational benefits were detected in site-year combinations where environment and weather conditions were unfavorable to soybean growth, including poorly drained soil, high precipitation, and fewer growing degree days. Hence, under unfavorable environments, 3 Y rotation of soybean-corn-wheat is recommended for improved soybean production and soil health.

\title{
Leveraging population genetics to develop disease control practices: A study in crop-specific management of cucurbit downy mildew
}

K. D’ARCANGELO (1), A. Rahman (2), T. D. Miles (3), L. M. Quesada-Ocampo (4), (1) North Carolina State University, Raleigh, NC, USA; (2) Corteva Agrisciences, Raleigh, NC, USA; (3) Michigan State University, East Lansing, MI, USA; (4) Department of Entomology and Plant Pathology, North Carolina State University, Raleigh, NC, USA

A recent population shift in Pseudoperonospora cubensis resulted in the loss effective cucumber host resistance and efficacy of many commercial fungicide products, leading to devastating yield losses. Population studies determined that two host-adapted clades occur in $P$. cubensis (Clade 1 and 2) and a TaqMan clade-specific assay was developed to differentiate isolates. A follow-up study determined that single nucleotide polymorphisms known to confer resistance to FRAC 40 fungicides were more prevalent in Clade 2 isolates, with specific mutations occurring preferentially based on clade. To further study this phenomenon while controlling for genetic differentiation by clade, field trials including 18 fungicide treatments and four non-treated varieties were conducted in 2016 and 2017 on cucumber (Clade 2 isolates) with the objective of determining the genetic structure of $P$. cubensis populations immediately following single-product fungicide applications. Isolates were genotyped using the clade-specific assay, FRAC 11 and FRAC 40 TaqMan qPCR 
assays and microsatellites. Genetic structure analyses revealed distinct population shifts following fungicide applications as well as similar genetic clusters present in non-treated varieties with the same source of host resistance. Additionally, it was observed that different FRAC groups were stratifying the population similarly. These results provide valuable insight into how fungicides and host tolerance affect $P$. cubensis populations and highlight the necessity for crop-specific management strategies to preserve fungicide efficacy.

Organic management of ascochyta blight caused by Didymella rabiei on Cicer arietinum using five essential oils L. PARIKH (1), S. Moparthi (1), B. Agindotan (2), M. E. Burrows (1), (1) Montana State University, Bozeman, MT, USA; (2) CPHST Beltsville Laboratory, USDA APHIS PPQ, Beltsville, MD, USA

There is interest in developing non-synthetic fungicide management options for Ascochyta blight of Cicer arietinum caused by Didymella rabiei. In a test of 38 essential oils (EOs), those from palmarosa, oregano, clove, cinnamon, and thyme were the most effective at inhibiting growth of D. rabiei in-vitro. Efficacy was evaluated on naturally infected seeds. Seeds were treated with different dilutions of EOs and plated on potato dextrose agar. Four replications with 10 seeds each $(n=40 /$ treatment $)$ were used in randomized complete block design (RCBD). Percent of seed infected with D. rabiei was scored after 7 days. A linear decrease was observed in percent infection with an increase in EO concentration. EO efficacy against foliar ascochyta blight was tested in the greenhouse at 1:250 (5.6 L EO/A), 1:500 (2.8 L EO/A), and 1:1000 (1.4 L EO/A) dilutions. Controls included treatment with water and Headline SC (0.38 $\mathrm{kg}$ ai/A). Treatments were sprayed on 8 seedlings each $(\mathrm{n}=8 /$ treatment $)$ of $C$. arietinum cultivars Sierra, Orion and Leader followed by D. rabiei spore inoculation $\left(10^{5}-10^{7}\right)$ after $48 \mathrm{~h}$ and arranged in a RCBD. Disease severity was scored as the percent leaf area affected 14 days post inoculation. Headline SC control, and highest concentration of oregano and thyme oils reduced D. rabiei infection in Sierra significantly $(P<0.05)$ to $33.8 \% \pm 2.0,35 \% \pm 2.3$ and $25 \% \pm 2.1$ as compared to water control at $75 \% \pm 3.5$. The results from the study suggests use of thyme and oregano oils for the management of ascochyta blight may be a viable alternative to fungicide use.

Managing apple scab through the integration of biological controls with SDHIs to decrease use of broad-spectrum fungicides K. AYER, M. W. Choi, K. D. Cox, Cornell University, Geneva, NY, USA

Apple scab requires considerable fungicide input to achieve adequate control. Broad-spectrum fungicides such as mancozeb and captan are integral to chemical management programs. In efforts towards sustainability, regulatory restrictions have been placed on these materials in some countries. To curtail use of broad-spectrum fungicides, we tested the potential of using of biological control with the succinate dehydrogenase inhibitor (SDHI), benzovindiflupyr. Programs included; 1. unmanaged control, 2. protectants alternated with SDHIs on a calendar schedule, 3. Bacillus subtilis alternated with SDHIs on a calendar schedule, 4. protectants alternated with SDHIs applied with disease forecasting, and 5. B. subtilis alternated with SDHIs applied with forecasting. Programs were conducted on 'Gala' apples trained to both a vertical axis and super spindle. Disease incidence was rated biweekly and relative area under the disease progress curve was analyzed. No significant differences were observed between management programs, however there were trends towards an increase in efficacy using forecasting or using mancozeb/captan. Isolates of the pathogen Venturia inaequalis were collected from treatment programs and assessed for SDHI sensitivity after the final fungicide application. No differences in sensitivity were observed in isolates collected from plots treated with protectants and biological control when on the same application schedule. Hence, sustainable disease and resistance management may be achieved without protectants. Continued evaluation is needed to optimize programs.

A decision support model for landscape-level management of cassava mosaic disease in Southeast Asia K. F. ANDERSEN (1), E. Delaquis (2), J. Newby (2), C. T. L. Thuy (3), S. de Haan (2), J. P. Legg (4), W. Cuellar (5), R. I. AlcalaBrise (1), K. A. Garrett (6), N. Minato (7), (1) University of Florida, Gainesville, FL, USA; (2) International Center for Tropical Agriculture (CIAT), Vientiane, LAOS; (3) International Center for Tropical Agriculture (CIAT), Hanoi, Vietnam, Hanoi, VIETNAM; (4) International Institute of Tropical Agriculture, Dar es Salaam, TANZANIA; (5) International Center for Tropical Agriculture (CIAT), Cali, COLOMBIA; (6) Plant Pathology Department, University of Florida, Gainesville, FL, USA; (7) Niigata University, Niigata, JAPAN

Sri Lankan cassava mosaic virus (SLCMV), a causal agent of cassava mosaic disease (CMD), was first reported in Cambodia in 2015 and more recently in Vietnam, China, and Thailand. Rapid spread of CMD through this region has resulted in large production losses and has negatively impacted the cassava industry. SLCMV has two modes of dispersal in the landscape: it is vectored short distances by whiteflies and also spread through the movement of infected planting material. We use a network meta-population model to simulate the spread of this epidemic in the landscape and to assess the likely influence of management strategies on spread. We estimated environmental suitability for CMD establishment using machine learning methods. We conducted simulation analyses to ask the follow questions: 1) how does complete trade restriction limit the spread of CMD; 2) how does adding clean seed into the system each year impact disease, as a function of volume, and; 3 ) how should locations for clean seed interventions be selected? We compare static and adaptive management strategies. Results suggest that a reduction in both prevalence and distribution can be achieved through trade restrictions. Interestingly, when we compared distributing a large amount of seed ( 80 ha) to each of a restricted number of districts (20), this was less successful at reducing incidence than the opposite strategy ( 20 ha of clean seed to each of 80 districts). Findings will inform planning for SLCMV interventions in Southeast Asia. This framework can be used in a range of pathosystems to combat invading pathogens.

Gene expression of Geosmithia morbida and Juglans nigra during pathogen infection under restricted and well-watered regimes R. Sitz (1), J. IBARRA CABALLERO (2), J. Stewart (1), (1) Colorado State University, Fort Collins, CO, USA; (2) Colorado State University-Agricultural Biology, Fort Collins, CO, USA 
Geosmithia morbida is the fungal causal agent of thousand cankers disease of walnut trees, a disease especially threatening to black walnut (Juglans nigra). The disease results from the activity of both the fungus and its vector, the walnut twig beetle (Pityophthorus juglandis). In this study, we artificially inoculated black walnut saplings with mycelium of G. morbida to determine what gene transcripts are expressed by the host and pathogen during infection and whether their response differs when exposed to well-watered and drought stressed conditions. Saplings were maintained in a greenhouse and either well-watered (maintained at $100 \%$ pot capacity) or drought conditions (maintain at $60 \%$ pot capacity). Samples were taken from the infection zone at different times points, from $12 \mathrm{~h}$ to up to 16 days post-inoculation. We profiled the gene expression from the fungus and trees using TagSeq of RNA extracted from the obtained samples. For the control group, we included samples obtained from un-inoculated saplings (well-watered and drought stressed) and G. morbida mycelium grown in broth. Numerous differentially expressed transcripts were found when comparing the different treatments. Among them, several genes coding for glycosyl hydrolases, peptidases, transcription factors, and other proteins that could be involved in the pathogenicity of the fungus and as a response mechanism in the tree host.

\section{Characterization of Fusarium species causing mycotoxin contamination of Michigan wheat and corn in order to inform disease management strategies}

M. R. BREUNIG (1), A. Byrne (2), T. J. Ward (3), M. Chilvers (2), (1) Michigan State Univ, East Lansing, MI, USA; (2) Michigan State University, East Lansing, MI, USA; (3) USDA/ARS/NCAUR, Peoria, IL, USA

Fusarium species are major pathogens of concern. Besides yield losses, they damage wheat and corn grain quality with mycotoxin contamination. A wide diversity of Fusarium species and toxins are reported worldwide. Therefore, we sought to characterize the populations in Michigan in order to inform management strategies. Over 500 isolates were collected and species composition, chemotype (3-ADON, 15-ADON, NIV) and fungicide sensitivity determined. F. graminearum 15-ADON dominated the wheat samples, and a greater diversity of species were found in corn. In vitro sensitivity to demethylase inhibitor (DMI) fungicides (metconazole, tebuconazole, and prothioconazole) were assessed with mycelial growth assays for all isolates. Interestingly, differences in sensitivity between Fusarium species were revealed, with F. tricinctum less sensitive to DMIs, while species such as $F$. subglutinans were more sensitive in vitro. A larger variation of sensitivity was found in F. graminearum, so a field trial was conducted in 2019 and 2020 to investigate if practical DMI resistance exists in vivo. Plots were inoculated with different spore suspensions of individual isolates representing those with greatest and least sensitivity in vitro. In 2019, no differences in the relative fungicide efficacy were found, signaling no practical DMI resistance currently exists in F. graminearum, despite differences in vitro and widespread use in Michigan wheat for the last 10 years. For a subset of $F$. graminearum isolates, baseline in vitro sensitivity was also determined for the SDHI chemistry, pydiflumetofen.

\section{Fungicide resistance in Podosphaera xanthii and impact on managing cucurbit powdery mildew} M. T. MCGRATH, Cornell University, Riverhead, NY, USA

Effectively managing powdery mildew (PM) in cucurbit crops necessitates applying fungicides with risk for resistance development (due to their single-site mode of action) because their mobility enables movement to lower leaf surfaces where Podosphaera xanthii develops best. In 2018 and 2019 fungicide efficacy was assessed on naturally-infected field-grown plants and pathogen sensitivity to fungicides was determined using an in-field seedling bioassay and a laboratory leaf disk bioassay. Resistance to MBC (FRAC Code 1) and QoI (11) fungicides and the SDHI fungicide boscalid (7) were common. Resistance to cyflufenamid (U6) and quinoxyfen (13) were more commonly found near the end of the growing season; thus these fungicides are an option early in PM development. Cross resistance is partial with DMI (3) fungicides and pathogen sensitivity to DMIs has not changed for several years; thus newer DMI fungicides remain important for managing PM. In contrast, while partial cross resistance also is observed for some SDHI fungicides, notably fluopyram, it has exhibited decreased efficacy. There has been no indication of resistance developing to metrafenone (50) thus it, along with recently registered flutianil (U13) and a DMI fungicide, are recommended as the main fungicides to manage PM. Isolates with resistance to multiple fungicides in different chemical groups were detected on research plants treated with a recommended fungicide program, only metrafenone, or biopesticides, and on plants not treated with fungicides. Multi-fungicide resistance challenges PM management.

\section{Combined effects of inundative biocontrol and anaerobic soil disinfestation (ASD) using non-host cover crops as carbon sources} for clubroot management

R. B. KHADKA (1), S. A. Miller (2), (1) The Ohio State University, Wooster, OH, USA; (2) Department of Plant Pathology, The Ohio State University, Wooster, OH, USA

Combinations of commercially available biocontrol agents and anaerobic soil disinfestation (ASD) with non-host cover crops as carbon sources were evaluated for clubroot disease management utilizing a factorial randomized complete block design with three replications. The first factor was ASD amendment (winter rye, ryegrass, sudangrass or leek cover crop, wheat bran (standard)), and covered (anaerobic) and uncovered (aerobic) non-amended controls. The second factor was biocontrol treatment (Prestop (Gliocladium catenulatum); Actinovate (Streptomyces lydicus), Serenade (Bacillus subtilis) or no product). Cover crops were grown in 350-ml pots containing muck soil inoculated with the clubroot pathogen (Plasmodiophora brassicae) for 45 days in growth chambers. Then cover crops were uprooted, chopped into small pieces, and mixed with soil in the same pot. Each pot was saturated with tap water before inoculation of biocontrol product and then enclosed in two Ziploc ${ }^{\mathbb{B}}$ bags. After three weeks bags were removed, soil aired for one week, and mustard greens 'Green Wave' seeded. Prestop and Serenade consistently reduced the clubroot index alone and in combination with wheat bran- and sudangrass-amended ASD treatment. Furthermore, synergy in reducing clubroot indices was observed with the combination of ASD-winter rye with Serenade or Actinovate, and ASD-sudangrass with Actinovate, Prestop or Serenade. Combining 
ASD amended with selected cover crops with Serenade, Actinovate or Prestop may enhance ASD efficacy for clubroot management in organic and conventional farming systems.

Population genetic structure of Puccinia striiformis f. sp. tritici in southwestern and northwestern China B. JIANG, X. Lv, W. Gong, C. Wang, Z. Ma, China Agricultural University, Beijing, CHINA

Wheat stripe rust, caused by Puccinia striiformis f. sp. tritici (Pst), is a destructive disease worldwide, particularly in southwestern and northwestern China. In order to understand the relationships among the regional subpopulations in northwestern and southwestern China, we collected 2702 wheat stripe rust samples from ten provinces in these regions and tested the isolates using 12 simple sequence repeat markers. Of the 791 multi-locus genotypes (MLGs), 252 MLGs were shared by the subpopulations in the ten provinces. The Qinghai subpopulation had the highest diversity, followed by the Ningxia and Tibet subpopulations, while the Xinjiang subpopulation had the lowest diversity. Analysis of molecular variance showed that $72 \%$ variation was from within subpopulations. The Pst isolates were clustered into three linages, Qinghai, Ningxia, Gansu, and Shaanxi in linage 1; Sichuan, Chongqing, Yunnan, Xizang, and Guizhou in linage 2; and Xinjiang in linage 3, indicating that the Pst subpopulations were related to geographic regions. The study provides the information about the epidemiology of stripe rust, which is useful to manage the disease in China.

\section{Population biology of Melampsora americana and eQTL mapping for rust resistance in Salix purpurea shrub willow} C. R. CROWELL (1), D. Wilkerson (2), P. McMullen (3), A. R. Cala (4), L. Smart (5), C. D. Smart (4), (1) Plant Pathology and PlantMicrobe Biology Section, Cornell Agrtech, Geneva, NY, USA; (2) Horticulture Section, Cornell Agritech, Geneva, NY, USA; (3) Lebanon Valley College, Tamaqua, PA, USA; (4) Plant Pathology and Plant-Microbe Biology Section, Cornell AgriTech, Geneva, NY, USA; (5) Horticulture Section, Cornell AgriTech, Geneva, NY, USA

Salix spp. shrub willow, a bioenergy feedstock, shows promise in the northeast US due to its robust woody growth on marginal lands. However Melampsora willow rust, caused by M. americana, can directly account for up to 50\% yield loss in susceptible Salix cultivars. This has spurred investigation into both genotypic diversity of Melampsora spp. in the northeast, as well as the identification of resistance within Salix spp. breeding populations. We collected and genotyped, using genotyping-by-sequencing (GBS), 255 isolates from the northeast US. Multi-year sampling revealed that the rust population is highly diverse over large geographic space, however clonal spread of few genotypes dominates infection within individual plantations. Additionally, we discovered evidence of large geographic spread of clonal lineages, as well as evidence of unexpected asexual overwintering. We explored disease resistance by performing a greenhouse expression QTL mapping project on a single breeding family to discover transcription factors associated with rust resistance. Pools of resistant and susceptible progeny (30 each) were inoculated with a single rust isolate and leaf tissue was collected at 42 and 66 hpi for RNA extraction. In total, we discovered 40,000 cis and 87,000 trans eQTLs. A subset of these correlate with genomic regions of previously identified QTLs for rust resistance and others serve as novel candidates for resistance. This work has led to further understanding of the population biology of Melampsora willow rust, while providing crucial information for future willow breeders.

\section{Copper tolerance and oxytetracycline resistance in Xanthomonas arboricola pv. pruni} B. COX (1), G. Schnabel (1), H. Wang (2), (1) Clemson University, Clemson, SC, USA; (2) Clemson University, Blackville, SC, USA

Bacterial spot caused by Xanthomonas arboricola pv. pruni (Xap) is a major disease of peach. Management is largely based on preventative sprays of copper and to a lesser degree oxytetracycline, but little is known about potential selection for tolerance or resistance to these products. A total of 298 Xap strains were collected from cankers, leaves, and fruit of four commercial O'Henry orchards in South Carolina throughout the growing season in 2018 and 2019, respectively. Xap strains were considered tolerant to copper when they grew on media amended with copper sulfate pentahydrate at $150 \mathrm{mg} / \mathrm{L}$ or higher, and resistant to oxytetracycline when they could grow on media amended with oxytetracycline or higher. Cankers were collected at the phenological stage bud break and fruit and leaf samples were collected at pit hardening and final swell. The bacterial strains collected over the two-year span were mostly sensitive to copper and oxytetracycline, but strains (58 total) with tolerance to copper were identified in each orchard. More strains with copper tolerance were found in 2019 compared to the previous year. Two were resistant to oxytetracycline and tolerant to copper. The study illustrates the existence of Xap with tolerance to copper and resistance to oxytetracycline in commercial orchards. Such isolates could play a role in the inability to effectively control the disease in South Carolina peach orchards. 